ADVERTIMENT. La consulta d'aquesta tesi queda condicionada a l'acceptació de les següents condicions d'ús: La difusió d'aquesta tesi per mitjà del servei TDX (www.tesisenxarxa.net) ha estat autoritzada pels titulars dels drets de propietat intel-lectual únicament per a usos privats emmarcats en activitats d'investigació i docència. No s'autoritza la seva reproducció amb finalitats de lucre ni la seva difusió i posada a disposició des d'un lloc aliè al servei TDX. No s'autoritza la presentació del seu contingut en una finestra o marc aliè a TDX (framing). Aquesta reserva de drets afecta tant al resum de presentació de la tesi com als seus continguts. En la utilització o cita de parts de la tesi és obligat indicar el nom de la persona autora.

ADVERTENCIA. La consulta de esta tesis queda condicionada a la aceptación de las siguientes condiciones de uso: La difusión de esta tesis por medio del servicio TDR (www.tesisenred.net) ha sido autorizada por los titulares de los derechos de propiedad intelectual únicamente para usos privados enmarcados en actividades de investigación y docencia. No se autoriza su reproducción con finalidades de lucro ni su difusión y puesta a disposición desde un sitio ajeno al servicio TDR. No se autoriza la presentación de su contenido en una ventana o marco ajeno a TDR (framing). Esta reserva de derechos afecta tanto al resumen de presentación de la tesis como a sus contenidos. En la utilización o cita de partes de la tesis es obligado indicar el nombre de la persona autora.

WARNING. On having consulted this thesis you're accepting the following use conditions: Spreading this thesis by the TDX (www.tesisenxarxa.net) service has been authorized by the titular of the intellectual property rights only for private uses placed in investigation and teaching activities. Reproduction with lucrative aims is not authorized neither its spreading and availability from a site foreign to the TDX service. Introducing its content in a window or frame foreign to the TDX service is not authorized (framing). This rights affect to the presentation summary of the thesis as well as to its contents. In the using or citation of parts of the thesis it's obliged to indicate the name of the author 


\title{
Spectrum USAge Models for the Analysis, Design and Simulation of Cognitive RAdio Networks
}

By

Miguel López Benítez

\author{
A Thesis Submitted to the \\ Department of Signal Theory and Communications \\ of the Universitat Politècnica de Catalunya \\ in Partial Fulfillment of the Requirements for the Degree of \\ Doctor OF PHILOSOPHY
}

Barcelona, May 2011

Thesis Advisor: Prof. Fernando Casadevall

Mobile Communication Research Group (GRCM) Department of Signal Theory and Communications (TSC)

Universitat Politècnica de Catalunya (UPC) 

Essentially, all models are wrong, but some are useful.

George E. P. Box 

To my beloved family for their unconditional love and support.

A mi querida familia por su cariño y apoyo incondicionales. 



\section{ABSTRACT}

The owned spectrum allocation policy, in use since the early days of modern radio communications, has been proven to effectively control interference among radio communication systems and simplify the design of hardware for use at a known and fixed range of frequencies. However, the overwhelming proliferation of new operators, innovative services and wireless technologies during the last years has resulted, under this static regulatory regime, in the depletion of all spectrum bands with commercially attractive radio propagation characteristics. The vast majority of spectrum regarded as usable has already been allocated, thus hindering the commercial rollout of new emerging services. An important number of spectrum measurement campaigns have demonstrated, however, that spectrum is mostly underutilized, thus indicating that the virtual spectrum scarcity problem actually results from static and inflexible spectrum management policies rather than the physical scarcity of usable radio frequencies. The owned spectrum allocation policy was once appropriate, but nowadays it has become obsolete and new spectrum management paradigms are therefore required in order to efficiently exploit the precious radio resources.

This situation has motivated the emergence of more flexible spectrum access policies. In this context, the Dynamic Spectrum Access (DSA) concept, based on the Cognitive Radio (CR) paradigm, has gained popularity as a promising solution to conciliate the existing conflicts between the ever-increasing spectrum demand growth and the currently inefficient spectrum utilization. The basic underlying idea of DSA/CR is to allow unlicensed (secondary) users to access in an opportunistic and non-interfering manner some licensed bands temporarily unoccupied by the licensed (primary) users. Unlicensed secondary terminals monitor the spectrum in order to identify time gaps left unused by primary users, perform transmissions and vacate the channel as soon as primary users return. Secondary unlicensed transmissions are allowed following this operating principle as long as they do not result in harmful interference to primary radios. 
Due to the opportunistic nature of the DSA/CR principle, the behavior and performance of a secondary network depends on the spectrum occupancy patterns of the primary system. A realistic and accurate modeling of such patterns becomes therefore essential and extremely useful in the domain of DSA/CR research. The potential applicability of spectrum usage models ranges from analytical studies to the design and dimensioning of secondary networks as well as the development of innovative simulation tools and more efficient DSA/CR techniques. Nevertheless, the utility of such models depends on their realism and accuracy. Unfortunately, the models for spectrum use commonly used to date in DSA/CR research are limited in scope and based on oversimplifications or assumptions that have not been validated with empirical measurement data. Spectrum occupancy modeling in the context of DSA/CR constitutes a rather unexplored research area that still requires more effort.

This dissertation addresses the problem of modeling spectrum usage in the context of DSA/CR networks by contributing a comprehensive and holistic set of realistic models capable to accurately capture and reproduce the relevant statistical properties of spectrum usage in real radio communication systems, in the time, frequency and space dimensions, for its application in the development and improvement of the future DSA/CR technology. A distinguishing feature of the models developed in the context of this dissertation is the demonstrated ability to accurately capture and reproduce the statistical properties of spectrum usage observed in real systems of various radio technologies. The development and validation of such models rely on spectrum occupancy data that have been obtained from extensive spectrum measurement campaigns performed with state-of-the-art equipment.

The first part of this dissertation addresses the development of a unified methodological framework for spectrum measurements in the context of DSA/CR and presents the results of an extensive spectrum measurement campaign performed over a wide variety of locations and scenarios in the metropolitan area of Barcelona, Spain, in order to identify potential bands of interest for the future deployment of the DSA/CR technology. To the best of the author's knowledge, this is the first study of these characteristics performed under the scope of the Spanish spectrum regulation and one of the earliest studies in Europe. The second part deals with various specific aspects related to the processing of the measurements to extract the spectrum occupancy patterns, which is largely similar to the problem of spectrum sensing in DSA/CR systems. The performance of energy detection, the most widely employed spectrum sensing technique in DSA/CR, is first assessed empirically. The outcome of this study motivates the development of a more accurate theoretical-empirical model for the performance of energy detection as well as an improved energy detection scheme capable to outperform the conventional method while preserving a similar level of complexity, computational cost and field of application. The findings of these studies are finally applied in the third part of the dissertation to the development of innovative spectrum usage models for the time domain (in its discrete- and continuous-time versions), the frequency domain and the space domain. The proposed models can been combined and integrated into a unified modeling approach where the time, frequency and space dimensions of spectrum usage can simultaneously be taken into account and accurately reproduced, thus providing a complete and holistic characterization of spectrum usage in real systems for the analysis, design and simulation of the future DSA/CR networks. 


\section{ACKNOWLEDGMENTS}

An important stage in my life comes to an end and when I look back I realize how many people I have met along the way and how much I have been helped, in one way or another, to get here. I would like to express my gratitude to all those who were with me and gave me their support over these nearly five years.

First of all, I want to thank my advisor Professor Fernando Casadevall for guiding me along this journey in an exemplary manner. He has been really encouraging allowing me freedom in exploring new ideas while giving me directions, suggestions and support whenever needed. He was always able to make time for me whenever I needed, for reading, commenting and discussing my work, even in difficult times, on weekends, and the days before the deadline for many conferences... His valuable and insightful comments have indubitably contributed to improve the quality of this dissertation. The freedom I have had to submit the work to journals and conferences without restrictions, and the required financial support I received, is something for which I also am very grateful. I feel deeply indebted for everything. It has been a great pleasure to make this long journey under his guidance.

I would also like to express my gratitude to the rest of members of the Mobile Communication Research Group (GRCM) of the Universitat Politècnica de Catalunya (UPC), who somehow have also contributed to forge what is in the reader's hands. The results of many fruitful collaborations in the context of various research projects have been embodied in this thesis as well. Over these years I have had the opportunity to teach university courses, thus discovering an exciting world hitherto unknown to me. Although most of my predoctoral research period was supported by the Spanish Ministry of Science and Innovation (MICINN) under FPU grant AP2006-848, and for a brief initial period by the Catalan Regional Government under FI grant 2007FI-00524, I have also had during the last months the financial support of the GRCM to finish my dissertation, which of course I sincerely appreciate. I am very glad and very happy to have had the opportunity to work with them. 
I could not forget to mention here Dr. Javier Gozálvez, Universidad Miguel Hernández. He introduced me to the fascinating world of research during my undergraduate studies. During those years, I learned from his motivation and enthusiasm for research, without which I would not probably have been able to get here. Somehow, I feel indebted to him as well.

Certainly, some of the results presented in this dissertation would not exist without the support and dedication of several M.Sc. students. Concretely, I want to thank Susana Molina Corbacho (UPC), Ramsès García Calafí (UPC), Jesús Manuel Pérez Sotelo (UPC), María Huidobro Manilla (UPC), Luis Daniel López Aguado (UPC), Emanuele Tassinello (University of Padova), and Corrado Martella (University of Bologna) for their helpful support.

All this time has become a better experience thanks to the company of my colleagues at the GRCM. We worked together to turn ourselves into "ingenieros de tenazas" but we also laughed together, comparing the different ways in which dogs bark, cats meow and cows moo in Spanish, Catalan, Serbian, Romanian, Italian, Portuguese, Polish, Arabic, Urdu and some other languages. Thank you for making lunch breaks, dinners and many other events a delightful time for freakstorming. You have been excellent travel companions and I wish you all the best for the rest of the trip.

Last but not least, I am deeply thankful to my family for being there for me, supporting me despite the distance that separated us. Their unconditional love and support have been a constant source of strength and motivation to go always on. I am specially grateful to my parents, for the education they have given to me. They taught me a thousand lessons that cannot be found in books. They have been and will always be the inspiration of my life. 


\section{CONTENTS}

List of Tables

xviii

List of Figures $\quad$ xxiii

List of Abbreviations $\quad$ xxv

1 Introduction $\quad 1$

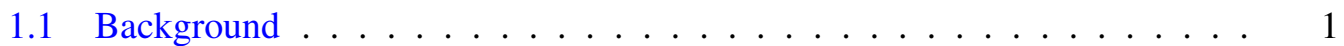

1.1.1 Radio communications and spectrum regulation . . . . . . . . . 1

1.1.2 Dynamic spectrum access ............... 3

1.1.3 Software defined radio and cognitive radio . . . . . . . . . . . . 6

1.1.4 Network architecture and functions . . . . . . . . . . . . . . 7

1.1.5 Standardization activities . . . . . . . . . . . . . 11

1.2 Motivation and scope . . . . . . . . . . . . . . . . . . 14

1.3 Thesis contributions . . . . . . . . . . . . . . . . . . . . . . . . . . . . .

1.4 Thesis outline . . . . . . . . . . . . . . . . . . . . . . . . . . . . . . . . . . 17

1.5 List of publications . . . . . . . . . . . . . . . . . . . . . . . . . . . . . . . . . . .

1.6 Project involvement . . . . . . . . . . . . . . . 23

I Low Time Resolution Measurements 27

2 Methodological aspects of spectrum occupancy evaluation 29

2.1 Introduction . . . . . . . . . . . . . . . . . . . . . . . . . . . . . . . 29

2.2 Measurement setup . . . . . . . . . . . . . . . . . . . . 34

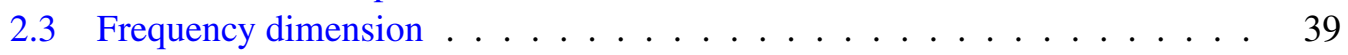


2.4 Time dimension . . . . . . . . . . . . . . . . . . . . . . . . . . 44

2.5 Data post-processing . . . . . . . . . . . . . . . . . 42

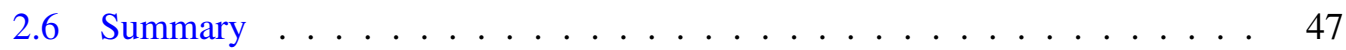

3 Evaluation of spectrum occupancy 49

3.1 Introduction . . . . . . . . . . . . . . . . . . . . . . . . 49

3.2 Previous spectrum measurement campaigns . . . . . . . . . . . . 50

3.3 Measurement configuration . . . . . . . . . . . . . . . . . 52

3.4 Measurement scenarios . . . . . . . . . . . . . . . . . . . . . 53

3.5 Measurement results and analysis . . . . . . . . . . . . . . . 54

3.5 .1 Occupancy metrics . . . . . . . . . . . . . . . 54

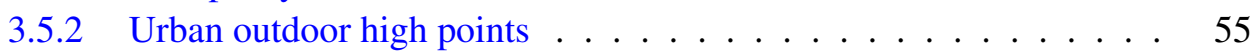

3.5 .3 Urban indoor locations . . . . . . . . . . . . . . . . 60

3.5.4 Urban narrow streets and open areas . . . . . . . . . . . . . . 64

3.5.5 Sub-urban environments . . . . . . . . . . . . . . 66

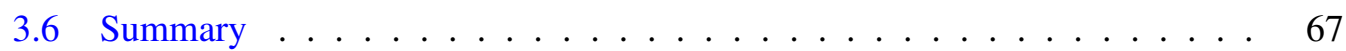

II High Time Resolution Measurements $\quad 69$

4 Experimental evaluation of energy detection performance 71

4.1 Introduction . . . . . . . . . . . . . . . . . . . 71

4.2 Novelties of this study . . . . . . . . . . . . . . . . . . . 72

4.3 System model, problem formulation and evaluation metrics . . . . . . . . 73

4.4 Energy detection . . . . . . . . . . . . . . . . . . . . 75

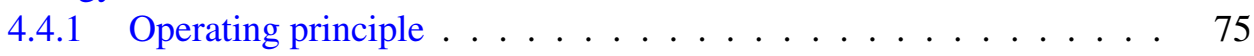

4.4.2 Theoretical performance . . . . . . . . . . . . . 75

4.4 .3 Threshold setting . . . . . . . . . . . . . . 76

4.4 .4 Noise uncertainty . . . . . . . . . . . . . . . . . . . 77

4.5 Measurement platform . . . . . . . . . . . . . . . . . . 78

4.6 Evaluation methodology . . . . . . . . . . . . . . . . . . . . 79

4.7 Experimental performance results $\ldots \ldots \ldots \ldots$

4.7 .1 Validation . . . . . . . . . . . . . . . . . . . . 84

4.7 .2 Analysis . . . . . . . . . . . . . . . . . 84

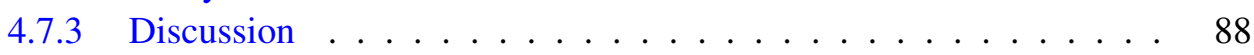

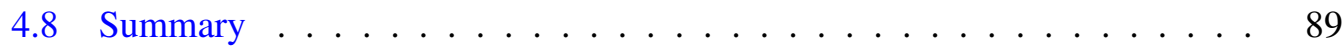

5 Empirical model for energy detection performance $\quad 91$

5.1 Introduction . . . . . . . . . . . . . . . . . . . . 91

5.2 Signal uncertainty model . . . . . . . . . . . . . . . . . . . . . 92

5.2 .1 Problem formulation . . . . . . . . . . . . . . . . . 92

5.2.2 Approximation for the SNR statistics _. . . . . . . . . . . . 93

5.2.3 Approximation for the probability of detection . . . . . . . 97

5.2.4 Closed-form expressions . . . . . . . . . . . . . . . . . . 98 
5.2.5 Impact of the sensing period . . . . . . . . . . . . . 101

5.2.6 Model validation . . . . . . . . . . . . . . 103

5.3 Signal and noise uncertainties . . . . . . . . . . . . . . . . . . . . . . . . . . . . . 103

5.3.1 Performance analysis without noise uncertainty . . . . . . . . 105

5.3.2 Performance analysis with noise uncertainty . . . . . . . . . 105

5.3.3 Design and configuration of energy detection . . . . . . . . . . 107

5.4 Summary . . . . . . . . . . . . . . . . . 108

6 Improved Energy Detection 111

6.1 Introduction . . . . . . . . . . . . . . . . 111

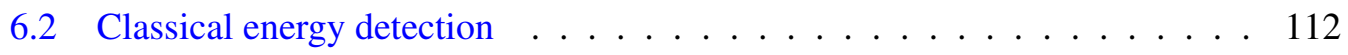

6.3 Modified energy detection . . . . . . . . . . . . . . . . . . . 114

6.3.1 MED operating principle . . . . . . . . . . . . . . . 114

6.3.2 MED theoretical performance . . . . . . . . . . . . 116

6.4 Improved energy detection . . . . . . . . . . . . . . . . 118

6.4.1 IED operating principle . . . . . . . . . . . . . . 118

6.4.2 IED theoretical performance . . . . . . . . . . . . 120

6.4 .3 IED threshold setting . . . . . . . . . . . . . . . . . . 122

6.4.4 IED experimental performance . . . . . . . . . . . . 123

6.5 Complexity analysis . . . . . . . . . . . . . . . . 126

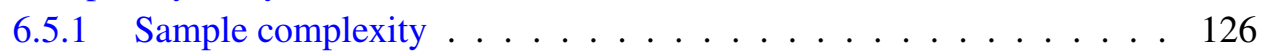

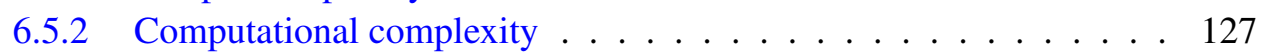

6.6 Discussion . . . . . . . . . . . . . . . . . 127

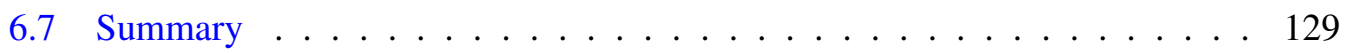

III Spectrum Usage Models $\quad 131$

7 Discrete Time Models 133

7.1 Introduction . . . . . . . . . . . . . . . . 133

7.2 Related work and motivation . . . . . . . . . . . . . . . . 134

7.2.1 Previous work based on continuous-time Markov chains . . . . . . 134

7.2.2 Previous work based on discrete-time Markov chains . . . . . . . . 134

7.3 Measurement setup and methodology . . . . . . . . . . . . . . 135

7.4 Discrete-time Markov chain . . . . . . . . . . . . . . . . . 137

7.5 Deterministic duty cycle models . . . . . . . . . . . . . . . . . . 142

7.5.1 Deterministic duty cycle model for low/medium loads . . . . . . . 143

7.5.2 Deterministic duty cycle model for medium/high loads . . . . . . . 144

7.5.3 Deterministic duty cycle models validation and applicability . . . . 145

7.6 Stochastic duty cycle models . . . . . . . . . . . . . . . . . . 147

7.7 Model validation . . . . . . . . . . . . . . . . . . . . . . . . . . . . . . . . . . . . . . . . .

7.8 Case study . . . . . . . . . . . . . . . . . . . . 152

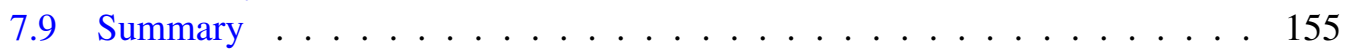


8 Continuous Time Models 157

8.1 Introduction . . . . . . . . . . . . . . . . . . . . 157

8.2 Previous work . . . . . . . . . . . . . . . . . . . . . . . . . . . . . . . . . . . . . .

8.3 Novelties of this study . . . . . . . . . . . . . . . . . . . . . . . . . . . . . . . . . . . . . . .

8.4 Measurement setup and methodology . . . . . . . . . . . . . . . . 160

8.5 Considered probability distributions . . . . . . . . . . . . . . . . 161

8.6 Goodness-of-fit metrics . . . . . . . . . . . . . . . . 165

8.7 Low time resolution models . . . . . . . . . . . . . . . 166

8.8 High time resolution models . . . . . . . . . . . . . . . . 170

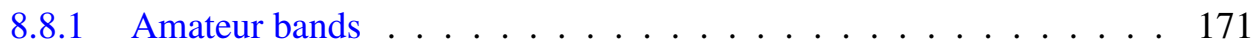

8.8 Paging bands .................... 171

8.8.3 Private/public access mobile radio bands . . . . . . . . . . 171

8.8.4 Cellular mobile communication bands . . . . . . . . . . . . . . 174

8.8.5 Cordless telephone bands . . . . . . . . . . . . . . . 179

8.9 Time correlation models . . . . . . . . . . . . . . . . . . . 181

8.9 .1 Correlation metrics . . . . . . . . . . . . . . 181

8.9.2 Correlation properties of spectrum usage . . . . . . . . . . 182

8.9.2.1 Correlation between busy and idle periods . . . . . . . . 182

8.9.2.2 Autocorrelation of busy and idle periods . . . . . . . . 183

8.9.3 Random variate generation principles . . . . . . . . . . . 185

8.9.3.1 The inversion method . . . . . . . . . . . 185

8.9.3.2 Generation of correlated random variates . . . . . . . . 186

8.9.3.3 Relation among correlation metrics . . . . . . . . . . 186

8.9.4 Simulation of correlation properties . . . . . . . . . . . . . 187

8.9.4.1 Simulation method . . . . . . . . . . . . . 187

8.9 .4 .2 Validation ................. 188

8.10 Proposed modeling approaches . . . . . . . . . . . . . . . . . . . . . . . . . . . . . . . . . . . . . . .

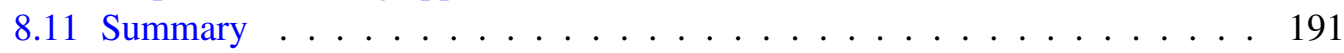

9 Time-Frequency Models $\quad 195$

9.1 Introduction . . . . . . . . . . . . . . . . . . . 195

9.2 Previous work . . . . . . . . . . . . . . . . 196

9.3 Measurement setup and methodology . . . . . . . . . . . . . . . . 197

9.4 System model . . . . . . . . . . . . . . . . . . . . . . . . 198

9.5 Statistical time-frequency characteristics of spectrum usage . . . . . . . . 198

9.5.1 Correlation metrics . . . . . . . . . . . . . . . . . . . . 198

9.5 .2 Correlation results . . . . . . . . . . . . . . . 199

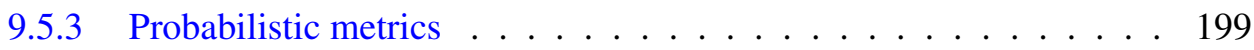

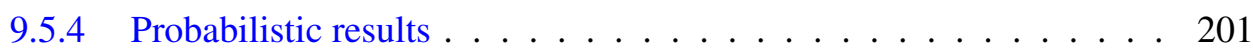

9.6 Statistical characteristics of spectrum usage over frequency . . . . . . . . . 202

9.6.1 Duty cycle distribution . . . . . . . . . . . . . . . . . 202

9.6.2 Duty cycle clustering ...................... 204

9.7 Applicability of the models and simulation method . . . . . . . . . 207

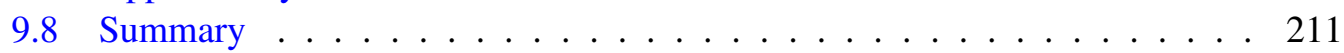


10 Spatial Models $\quad 213$

10.1 Introduction . . . . . . . . . . . . . . . . . . 213

10.2 Previous work . . . . . . . . . . . . . . . . 214

10.3 Measurement setup and methodology . . . . . . . . . . . . . . 215

10.4 Models for average spectrum occupancy perception . . . . . . . . . . . 215

10.4.1 Received average power distribution . . . . . . . . . . . . 216

10.4.2 Spatial duty cycle models . . . . . . . . . . . . . . 218

10.4.2.1 Constant-power continuous transmitters . . . . . . . 220

10.4.2.2 Constant-power discontinuous transmitters . . . . . . . 223

10.4.2.3 Variable-power discontinuous transmitters . . . . . . . 224

10.5 Models for concurrent snapshots observations . . . . . . . . . . . . 230

10.6 Applicability of the models . . . . . . . . . . . . . . . 235

10.6.1 Statistical prediction of spectrum occupancy perception . . . . . . . 235

10.6.1.1 Statistical prediction approach . . . . . . . . . . 236

10.6.1.2 Considered scenario and propagation models . . . . . . . 238

10.6.1.3 Numerical results . . . . . . . . . . . . . 239

10.6.2 Snapshot-based simulation of spatial spectrum usage . . . . . . . 245

10.7 Summary . . . . . . . . . . . . . . . . . . 248

11 Unified Modeling Approach

11.1 Introduction . . . . . . . . . . . . . . . . 251

11.2 Integration of the proposed models in analytical studies . . . . . . . . . 251

11.3 Integration of the proposed models in simulation tools . . . . . . . . . . . . 252

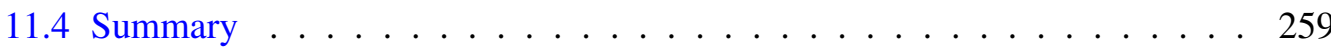

IV Conclusions and Future Work 261

12 Conclusions and Future Work 263

12.1 Conclusions . . . . . . . . . . . . . . . . . . . . 264

12.2 Future work . . . . . . . . . . . . . . . 266

V Appendices $\quad 271$

$\begin{array}{ll}\text { A Spectrum Measurement Platform } & 273\end{array}$

A.1 Introduction . . . . . . . . . . . . . . . . 273

A.2 Measurement platform overview . . . . . . . . . . . . . . 273

A.3 Antenna subsystem . . . . . . . . . . . . . . . . . 276

A.4 Radio frequency subsystem . . . . . . . . . . . . . . . . . 276

A.5 Capturing subsystem . . . . . . . . . . . . . . . . 277

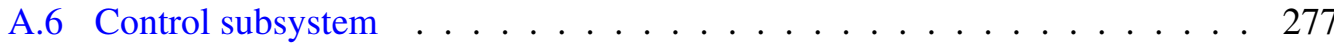

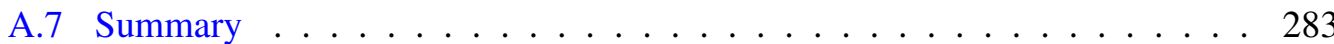

B Duty Cycle Computation $\quad 285$ 
C Approximation to the Gaussian Q-function 289

C.1 Introduction . . . . . . . . . . . . . . . . . . . . . . . . . . . . . . . 289

C.2 Previous work . . . . . . . . . . . . . . . . . . . . . . . . . . . 290

C.3 Proposed approximation . . . . . . . . . . . . . . . . . . . . . 291

C.4 Accuracy analysis and comparison . . . . . . . . . . . . . . . . 291

C.5 Applicability example . . . . . . . . . . . . . . . . . . . . . . . . . 296

C.6 Summary . . . . . . . . . . . . . . . . . . . . . . . . . . . . . . 299

$\begin{array}{ll}\text { References } & 326\end{array}$

$\begin{array}{lr}\text { Short biographical sketch } & 329\end{array}$ 


\section{LIST OF TABLES}

2.1 Previous spectrum measurement campaigns (Part I) . . . . . . . . . . . 31

2.2 Previous spectrum measurement campaigns (Part II). . . . . . . . . . 32

2.3 Previous spectrum measurement campaigns (Part III) . . . . . . . . . . 33

2.4 Impact of the amplification configuration. . . . . . . . . . . 38

2.5 Impact of the resolution bandwidth on the activity detected between 146 and $235 \mathrm{MHz} . \ldots \ldots \ldots \ldots \ldots$. . . . . . . . . . . . . 41

3.1 Spectrum analyzer configuration. . . . . . . . . . . . . . 53

3.2 Average duty cycle statistics in location $1 \ldots \ldots \ldots \ldots \ldots$

3.3 Cases considered in Figure $3.7 \ldots \ldots$. . . . . . . . . . . . . 61

4.1 Channels measured in the study of Chapter $4 \ldots \ldots$. . . . . . . 81

7.1 Spectrum bands considered in this study. . . . . . . . . . . . 136

7.2 Fitted values of the deterministic DC models parameters. . . . . . . . . . 146

7.3 Channel access statistics for DECT channel 9 (stochastic DC model and $K=5$ ) 154

7.4 Channel access statistics for TETRA DL channel 340 (stochastic DC model and $K=5) \quad \ldots \ldots \ldots \ldots \ldots \ldots$

8.1 Distributions considered in this study and estimation of their parameters based on the method of moments. . . . . . . . . . . . . . . 164

8.2 KS metric of idle periods for various distributions and bands based on the MLE method. . . . . . . . . . . . . . . . . . . . . . . . 167

8.3 GOF metrics for low time resolution measurements. . . . . . . . . . . . 167

8.4 Parameters of the generalized Pareto distribution for busy and idle periods extracted from empirical measurement results by means of MLE. . . . . . . 170 
8.5 GOF metrics for amateur bands. . . . . . . . . . . . . . . . . 172

8.6 Parameters of busy and idle period distributions for amateur bands. . . . . . 172

8.7 GOF metrics for paging bands. . . . . . . . . . . . . . . . . 173

8.8 Parameters of busy and idle period distributions for paging bands. . . . . . 173

8.9 GOF metrics for PMR/PAMR bands. . . . . . . . . . . . . . . . . 175

8.10 Parameters of busy and idle period distributions for PMR/PAMR bands. . . 175

8.11 GOF metrics for cellular mobile communication bands. . . . . . . . . . . 176

8.12 Parameters of busy and idle period distributions for cellular mobile communication bands. . . . . . . . . . . . . . . . 176

8.13 Discrete distributions considered in this study and estimation of their parameters based on the method of moments. . . . . . . . . . . . . . . 177

8.14 GOF metrics for cellular mobile communication bands (discrete-time models). 178

8.15 Busy-idle correlation coefficients for the measured bands. . . . . . . . . . . 184

9.1 Fitted values for the Beta and Kumaraswamy DC distributions. . . . . . . . 203

9.2 Fitted values for the geometric distribution of number of channels per cluster. 206

10.1 Joint and conditional probabilities of simultaneous observations. . . . . . . 232

10.2 Experimental values of $\sigma_{S}$ and $\sigma_{N} \ldots \ldots \ldots \ldots \ldots \ldots \ldots$

C.1 Optimum values of fitting parameters $\left(a^{*}, b^{*}, c^{*}\right)$ for different fitting criteria and optimized argument ranges $\hat{x}$. . . . . . . . . . . . . . 292

C.2 Comparison of the exact and approximated probability of detection of an energy detector under Rayleigh fading $\left(P_{f a}=0.01\right.$ and $\left.N=1000\right) \ldots \ldots 298$ 


\section{LIST OF FIGURES}

1.1 Classification of dynamic spectrum access schemes. . . . . . . . . . . 4

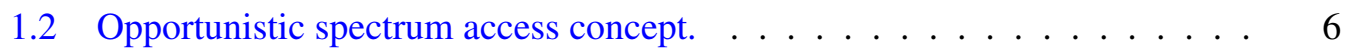

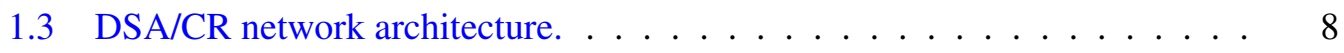

1.4 Organization of this Ph.D. dissertation. . . . . . . . . . . . 18

2.1 Measurement setup employed in this study. . . . . . . . . . . . 35

2.2 Impact of amplification on the activity detected for GSM. . . . . . . . . 37

2.3 Impact of amplification on the activity detected for BWA. . . . . . . . . 37

2.4 Impact of the frequency bin on the detected occupancy. . . . . . . . . . . 40

2.5 Average duty cycle per hour for DCS downlink. . . . . . . . . . . . . 43

2.6 Average duty cycle as a function of the decision threshold for different systems. 45

2.7 Various criteria to determine the decision threshold. . . . . . . . . . . 45

2.8 Impact of different decision threshold selection criteria on the detected activity for various licensed frequency bands. . . . . . . . . . . . 47

3.1 Measurement locations in urban environment. . . . . . . . . . . . . . . 54

3.2 Spectrum occupancy in location $1(75-960 \mathrm{MHz}) \ldots \ldots \ldots \ldots \ldots$

3.3 Spectrum occupancy in location $1(960-3100 \mathrm{MHz}) . \ldots \ldots \ldots$

3.4 Spectrum occupancy in location $1(3100-7075 \mathrm{MHz}) \ldots \ldots \ldots \ldots$

3.5 Spectrum occupancy in location 1 for specific bands. . . . . . . . . . . 58

3.6 Band-by-band average duty cycle statistics in location $1 . \ldots . . . . .660$

3.7 Average duty cycle statistics in locations 1 and 2 for specific bands. . . . . 62

3.8 Spectrum occupancy in location $2(75-235 \mathrm{MHz}) \ldots \ldots \ldots$. . . . . . . 63

3.9 Normalized average duty cycle statistics in locations 3 to 12 for specific bands 65

3.10 Spectrum occupancy in urban and sub-urban environments. . . . . . . . . 67 
4.1 Measurement platform (scheme) employed in the study of Chapter 4. . . . . 79

4.2 Measurement platform (picture) employed in the study of Chapter 4. . . . . 80

4.3 Average power spectrum for some of the captured signals. . . . . . . . . . 82

4.4 Experimental $P_{d}(\gamma)$ for $10 \log _{10} \alpha=0 \mathrm{~dB}$. . . . . . . . . . . . 83

4.5 Experimental $P_{d}(\gamma)$ for $10 \log _{10} \alpha=1 \mathrm{~dB} \ldots \ldots \ldots$

4.6 Experimental $P_{d}(\gamma)$ for $10 \log _{10} \alpha=2 \mathrm{~dB}$. . . . . . . . . . 83

4.7 Moving-averaged normalized power received for some captured signals. . . 86

4.8 Impact of the signal power variability on the ED performance. . . . . . . . 87

4.9 Probability of detection as a function of the sensing period for various radio technologies. ....................... 88

5.1 Empirical and approximated PDF for the received SNR. . . . . . . . . . . 95

5.2 Dependence between the mean and the variance of the received SNR. . . . 96

5.3 Probability of detection for constant- and variable-power transmitters when the SNR is modeled as modified Gaussian $(\beta \in[0.01,0.27])$, Rayleigh and gamma distributions. . . . . . . . . . . . . . . . 100

5.4 Empirical and approximated results for $\rho$ and $\tau(N) \ldots \ldots$. . . . . . . . . 102

5.5 Probability of detection as a function of the SNR $\left(P_{f a}=0.01,10 \log _{10} \alpha=0\right.$ $\mathrm{dB}, N=100)$. . . . . . . . . . . . . . . . . .

5.6 Probability of detection as a function of the SNR $\left(P_{f a}=0.01,10 \log _{10} \alpha=1\right.$ $\mathrm{dB}, N=100) . \ldots \ldots \ldots \ldots \ldots$

5.7 Probability of detection as a function of the signal uncertainty for various convergence rates and sensing periods $\left(\bar{P}_{d}^{\text {min }}=0.9, P_{f a}=0.01,10 \log _{10} \alpha=\right.$ $0.0 \mathrm{~dB})$.

5.8 Probability of detection as a function of the signal uncertainty for various convergence rates and sensing periods $\left(\bar{P}_{d}^{\min }=0.9, P_{f a}=0.01,10 \log _{10} \alpha=\right.$ $0.1 \mathrm{~dB})$.

5.9 Required $N^{\text {min }}$ as a function of the experienced SNR without $\left(10 \log _{10} \alpha=\right.$ $0.0 \mathrm{~dB})$ and with $\left(10 \log _{10} \alpha=0.1 \mathrm{~dB}\right)$ noise uncertainty $\left(\bar{P}_{d}^{\min }=0.9, P_{f a}=\right.$ $0.01, \beta=1.00) \ldots \ldots \ldots \ldots \ldots$

6.1 ROC curve for the CED and MED algorithms $(L=3, M \in[0, L], N=1000$,

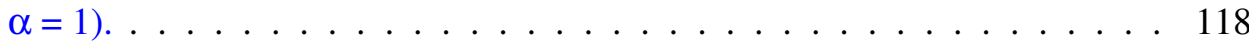

6.2 Rationale for the IED proposal. . . . . . . . . . . . . . . . . . 119

6.3 ROC curve for the CED and IED algorithms $(L=3, M \in[0, L], N=1000, \alpha$

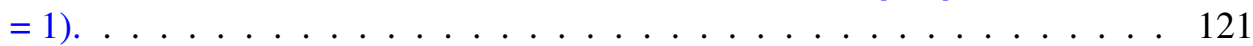

6.4 Theoretical performance for the CED, MED and IED algorithms $(\alpha=1) . \quad$. 124

6.5 Experimental detection and false alarm performance for the IED algorithm. 125

6.6 Experimental ROC for the CED (solid line) and IED (dashed line) algorithms. 126

6.7 Complexities of the CED and IED algorithms (upper graph: sample complexity; lower graph: computational complexity). . . . . . . . . . . . . 128

7.1 Discrete-Time Markov Chain (DTMC) model. . . . . . . . . . . . . . . . . 138 
7.2 Empirical and DTMC-simulated distributions of busy and idle periods along with DC time evolution for DCS 1800 DL channel 70.

7.3 Empirical and DTMC-simulated distributions of busy and idle periods along with DC time evolution for E-GSM 900 DL channel 23. . . . . . . . . . . .

7.4 Empirical and DTMC-simulated distributions of busy and idle periods along with DC time evolution for TETRA DL channel 340.

7.5 Empirical and DTMC-simulated distributions of busy and idle periods along with DC time evolution for TETRA UL channel 375. . . . . . . . . . . . . 141

7.6 Parameters of the deterministic DC model. . . . . . . . . . . . . . . . . . . 144

7.7 Validation of the deterministic DC model. . . . . . . . . . . . . . . . 145

7.8 Stochastic DC models: case L.I. . . . . . . . . . . . . . . . . . . . . . . 149

7.9 Stochastic DC models: case L.II. . . . . . . . . . . . . . . . . . . . . . 149

7.10 Stochastic DC models: case M.I. . . . . . . . . . . . . . . . . . . . . . . 149

7.11 Stochastic DC models: case M.II. . . . . . . . . . . . . . . . . . . . . . . 149

7.12 Stochastic DC models: case H.I. . . . . . . . . . . . . . . . . . . . . . . . 149

7.13 Stochastic DC models: case H.II. . . . . . . . . . . . . . . . . . . . . . . . 149

7.14 Empirical and DTMC-simulated distributions of busy and idle periods along with DC time evolution for DCS 1800 DL channel 70.

7.15 Empirical and DTMC-simulated distributions of busy and idle periods along with DC time evolution for E-GSM 900 DL channel 23. . . . . . . . . . .

7.16 Empirical and DTMC-simulated distributions of busy and idle periods along with DC time evolution for TETRA DL channel 340 . . . . . . . . . . . . . 152

7.17 Case study description. . . . . . . . . . . . . . . . . . . . . . . . . . . . . . . . . . . . . .

7.18 Interference results for DECT channel 9. . . . . . . . . . . . . . 155

8.1 Comparison of channel occupancy patterns extracted by the CED and IED algorithms. . . . . . . . . . . . . . . . . 162

8.2 Low versus high time resolution measurements. . . . . . . . . . . . . . 162

8.3 Empirical CDF and fitted distributions based on the MLE approach. . . . . 169

8.4 Empirical CDF and fitted distributions based on the MOM approach. . . . . 169

8.5 Empirical CDF and fitted distributions for amateur bands. . . . . . . . . . . 172

8.6 Empirical CDF and fitted distributions for paging bands. . . . . . . . . . . 173

8.7 Empirical CDF and fitted distributions for PMR/PAMR bands. . . . . . . . 175

8.8 Empirical CDF and fitted distributions for cellular mobile communication bands. . . . . . . . . . . . . . . . . . 176

8.9 Empirical CDF and fitted distributions for cellular mobile communication bands (discrete-time models). . . . . . . . . . . . . . . . . . . . 178

8.10 Empirical CDF and fitted distributions for cordless telephone bands (discrete-time models). . . . . . . . . . . . . . . . . . . . . . . 180

8.11 Empirical PDF of the correlation coefficients for DCS 1800 DL. . . . . . . 183

8.12 Spearman's autocorrelation functions of idle periods. . . . . . . . . . . 185

8.13 Validation of the proposed simulation method. . . . . . . . . . . . . . . 189

8.14 Proposed modeling approaches for spectrum occupancy patterns in the time domain. 
9.1 Elements of matrix $\mathbf{R}$ for TETRA DL based on Pearson's correlation coefficient. . . . . . . . . . . . . . . . . . 200

9.2 Elements of matrix abs $(\mathbf{P}-\mathbf{Q})$ for TETRA DL. . . . . . . . . . . . 201

9.3 Empirical DC distributions and their corresponding beta and Kumaraswamy fits.

9.4 Empirical spectrum data for the TETRA UL band. . . . . . . . . . . . . . . 205

9.5 Empirical spectrum data for the TETRA DL band. . . . . . . . . . . . . . 205

9.6 Empirical distributions of the number of channels per cluster and their corresponding geometric fits. . . . . . . . . . . . . . . . 206

9.7 Artificially generated spectrum data for the TETRA UL band. . . . . . . . 210

9.8 Artificially generated spectrum data for the TETRA DL band. . . . . . . . 210

10.1 Validation of the Gaussian approximation for the PDF of the received average power. . . . . . . . . . . . . . . . 218

10.2 Model considered to compute the DC (shaded area). . . . . . . . . . . 219

10.3 Validation of DC model for constant-power continuous transmitters (analogical TV). . . . . . . . . . . . . . . . . . . . . 221

10.4 Validation of DC model for constant-power continuous transmitters (digital TV).

10.5 Validation of DC model for constant-power continuous transmitters (E-GSM 900 broadcast channels).

10.6 Validation of DC model for constant-power continuous transmitters (DCS 1800 broadcast channels).

10.7 Validation of the approach employed to estimate the signal parameter's for constant-power discontinuous transmitters (TETRA channels).

10.8 Validation of the DC model for constant-power discontinuous transmitters (TETRA channels).

10.9 Validation of the approach employed to estimate the signal parameter's for variable-power discontinuous transmitters (TETRA channels).

10.10Validation of the DC model for variable-power discontinuous transmitters (TETRA channels).

10.11Empirical and fitted PDF of the received power for a time-shared TETRA channel.

10.12 Validation of the DC model for variable-power discontinuous transmitters.

10.13 Validation of the joint probabilities.

10.14 Validation of the conditional probabilities. . . . . . . . . . . . 233

10.15Empirical validation of the joint probabilities. . . . . . . . . . . . . . . . 234

10.16Empirical validation of the conditional probabilities. . . . . . . . . . 234

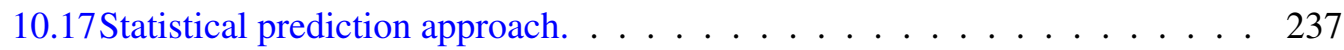

10.18Considered scenario. . . . . . . . . . . . . . . . . . . . 239

10.19Primary signal power $P_{S}(\mathrm{dBm})$ received at various locations in the area un-

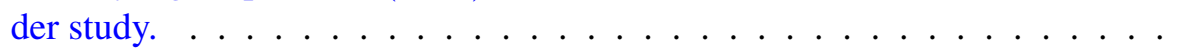

10.20Binary spectrum occupancy pattern perceived at various locations in the area under study. 
10.21 Probabilistic spectrum occupancy pattern perceived at various locations in the area under study . . . . . . . . . . . . . . . . . . . . . . . . 242

10.22Probabilistic spectrum occupancy pattern perceived at various locations in the area under study. . . . . . . . . . . . . . . . . . . . . . 242

10.23Perceived spectrum occupancy at the ground level. . . . . . . . . . . . . 244

10.24Perceived spectrum occupancy at the ground level. . . . . . . . . . . . . . 244

10.25Perceived spectrum occupancy at indoor locations. . . . . . . . . . . . 245

10.26Random simulation snapshot when the channel is observed as idle at the reference location. . . . . . . . . . . . . . . . . . . 247

10.27Random simulation snapshot when the channel is observed as busy at the reference location. . . . . . . . . . . . . . . . . . 247

11.1 Generic simulation scenario. . . . . . . . . . . . . . . . 253

11.2 Unified simulation approach. . . . . . . . . . . . . . . . . . . . . . . . . . . . . . . . . . . . .

11.3 Time-frequency map of spectrum occupancy. . . . . . . . . . . . . . . 257

11.4 Time-frequency map of spectrum occupancy as perceived at 10-dB SNR. . 257

11.5 Time-frequency map of spectrum occupancy as perceived at 3-dB SNR. . . 258

11.6 Time-frequency map of spectrum occupancy as perceived at 0-dB SNR. . . 258

A.1 Detailed scheme of the measurement platform. . . . . . . . . . . . . . . . 274

A.2 Modules of the measurement platform. . . . . . . . . . . . . . . 275

A.3 Control subsystem. . . . . . . . . . . . . . . . . . . . . . . . . . . . . . . . . . . . . . . . . .

A.4 Data formats. . . . . . . . . . . . . . . . . 283

C.1 Absolute relative error of the proposed exponential approximations for various optimized argument ranges $\hat{x}$. . . . . . . . . . . . . . . . . 292

C.2 Absolute relative error of the proposed exponential, Börjesson et al., and Chen et al. approximations $(\hat{x} \in[0,6]) \ldots \ldots$. . . . . . . . . . . . . 294

C.3 Absolute relative error of the proposed exponential, Karagiannidis et al., and

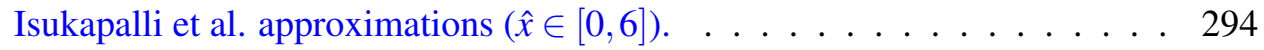

C.4 Absolute relative error of the proposed exponential, Chiani et al., and Loskot et al. approximations $(\hat{x} \in[0,6])$. 



\section{LIST OF ABBREVIATIONS}

$2 \mathrm{G}$

$3 \mathrm{G}$

$4 \mathrm{G}$

AC

ADC

$\mathrm{AF}$

ARIMA

AWGN

BB

BS

BW

BWA

CCDF

$\mathrm{CDF}$

CDMA

CED

CORVUS

CPC

CPE

CR

CTMC

CTSMC

DAB

DAC $2^{\text {nd }}$ Generation

$3^{\text {rd }}$ Generation

$4^{\text {th }}$ Generation

Alternating Current

Analogical to Digital Conversion

Activity Factor

Auto-Regressive Integrated Moving Average

Additive White Gaussian Noise

Base Band

Base Station

BandWidth

Broadband Wireless Access

Complementary Cumulative Distribution Function

Cumulative Distribution Function

Code Division Multiple Access

Classical Energy Detection

COgnitive Radio approach for usage of Virtual Unlicensed Spectrum

Cognitive Pilot Channel

Consumer Premise Equipment

Cognitive Radio

Continuous-Time Markov Chain

Continuous-Time Semi-Markov Chain

Digital Audio Broadcasting

Digital to Analogical Conversion 


$\begin{array}{ll}\text { DARPA } & \text { Defense Advanced Research Projects Agency } \\ \text { DC } & \text { Duty Cycle } \\ \text { DCS } & \text { Digital Cellular System } \\ \text { DC-T } & \text { Downlink-Continuous Transmission } \\ \text { DECT } & \text { Digital Enhanced Cordless Telecommunications } \\ \text { DFS } & \text { Dynamic Frequency Selection } \\ \text { DL } & \text { DownLink } \\ \text { DIMSUMNet } & \text { Dynamic Intelligent Management of Spectrum for Ubiquitous Mobile } \\ & \text { NETworks } \\ \text { DRiVE } & \text { Dynamic Radio for IP-Services in Vehicular Environments } \\ \text { DS } & \text { DownStream } \\ \text { DSA } & \text { Dynamic Spectrum Access } \\ \text { DSP } & \text { Digital Signal Processor } \\ \text { DTMC } & \text { Discrete-Time Markov Chain } \\ \text { ED } & \text { Energy Detection } \\ \text { E-GSM } & \text { Extended Global System for Mobile communications } \\ \text { ETSI } & \text { European Telecommunications Standards Institute } \\ \text { FCC } & \text { Federal Communications Commission } \\ \text { FDD } & \text { Frequency Division Duplex } \\ \text { FFT } & \text { Fast Fourier Transform } \\ \text { FIR } & \text { Finite Impulse Response } \\ \text { FM } & \text { Frequency Modulation } \\ \text { FPGA } & \text { Field-Programmable Gate Array } \\ \text { FTP } & \text { File Transfer Protocol } \\ \text { GMDSS } & \text { Global Maritime Distress and Safety System } \\ \text { GMSK } & \text { Gaussian Minimum Shift Keying } \\ \text { GOF } & \text { Goodness-Of-Fit } \\ \text { GPS } & \text { Global Positioning System } \\ \text { GSM } & \text { Global System for Mobile communications } \\ \text { HF } & \text { High Frequency } \\ \text { HTTP } & \text { HyperText Transfer Protocol } \\ \text { IED } & \text { Improved Energy Detection } \\ \text { IEEE } & \text { Institute of Electrical and Electronics Engineers } \\ \text { IETF } & \text { Internet Engineering Task Force } \\ \text { IF } & \text { Intermediate Frequency } \\ \text { IMT } & \text { International Mobile Telecommunications } \\ \text { ISM } & \text { Industrial, Scientific and Medical } \\ \text { ITU } & \text { International Telecommunication Union } \\ \text { KL } & \text { Kullback-Leibler } \\ \text { KS } & \text { Kolmogorov-Smirnov } \\ \text { LC } & \text { Large City } \\ \text { MAC } & \text { Medium Access Control } \\ \text { MARE } & \end{array}$




\begin{tabular}{|c|c|}
\hline MC & Multi Carrier \\
\hline MED & Modified Energy Detection \\
\hline MLE & Maximum Likelihood Estimation \\
\hline MOM & Method Of Moments \\
\hline MSC & Medium-Small City \\
\hline NSF & National Science Foundation \\
\hline NTIA & National Telecommunications and Information Administration \\
\hline OSA & Opportunistic Spectrum Access \\
\hline OT & Opportunity Time \\
\hline OverDRiVE & $\begin{array}{l}\text { Spectrum Efficient Uni- and Multi-cast Services over Dynamic } \\
\text { Multi-Radio Networks in Vehicular Environments }\end{array}$ \\
\hline PAMR & Public Access Mobile Radio \\
\hline PAWS & Protocol to Access White Space \\
\hline PC & Personal Computer \\
\hline PDF & Probability Density Function \\
\hline PFA & Probability of False Alarm \\
\hline PHY & Physical layer \\
\hline PMR & Private/Professional Mobile Radio \\
\hline PSD & Power Spectral Density \\
\hline PTM & Point-To-Multipoint \\
\hline QoS & Quality of Service \\
\hline RAN & Radio Access Network \\
\hline RBW & Resolution BandWidth \\
\hline REM & Radio Environment Map \\
\hline RF & Radio Frequency \\
\hline RFID & Radio Frequency IDentification \\
\hline R-GSM & Railway Global System for Mobile communications \\
\hline RMSE & Root Mean Square Error \\
\hline ROC & Receiver Operating Characteristic \\
\hline ROSHT & Recursive One-Sided Hypothesis Testing \\
\hline RRS & Reconfigurable Radio Systems \\
\hline SCC & Standards Coordinating Committee \\
\hline SCPI & Standard Commands for Programmable Instruments \\
\hline SDR & Software Defined Radio \\
\hline SFDR & Spurious-Free Dynamic Range \\
\hline SNR & Signal-to-Noise Ratio \\
\hline S-PCS & Satellite Personal Communication Systems \\
\hline SPDT & Single-Pole Double-Throw \\
\hline SSE & Sum of Square Errors \\
\hline TDD & Time Division Duplex \\
\hline TDMA & Time Division Multiple Access \\
\hline TETRA & TErrestrial Trunked RAdio \\
\hline TPC & Transmit Power Control \\
\hline
\end{tabular}




$\begin{array}{ll}\text { TV } & \text { TeleVision } \\ \text { UDP } & \text { User Datagram Protocol } \\ \text { UL } & \text { UpLink } \\ \text { UMTS } & \text { Universal Mobile Telecommunications System } \\ \text { US } & \text { UpStream } \\ \text { USB } & \text { Universal Serial Bus } \\ \text { USRP } & \text { Universal Software Radio Peripheral } \\ \text { UWB } & \text { Ultra Wide Band } \\ \text { VBW } & \text { Video BandWidth } \\ \text { VHF } & \text { Very High Frequency } \\ \text { VISA } & \text { Virtual Instrument Standard Architecture } \\ \text { WCDMA } & \text { Wideband Code Division Multiple Access } \\ \text { WiFi } & \text { Wireless Fidelity } \\ \text { WiMAX } & \text { Worldwide Interoperability for Microwave Access } \\ \text { WLAN } & \text { Wireless Local Area Network } \\ \text { WRAN } & \text { Wireless Regional Area Network } \\ \text { WRC } & \text { World Radio Conference } \\ \text { xG } & \text { Next Generation }\end{array}$






\section{Chapter}

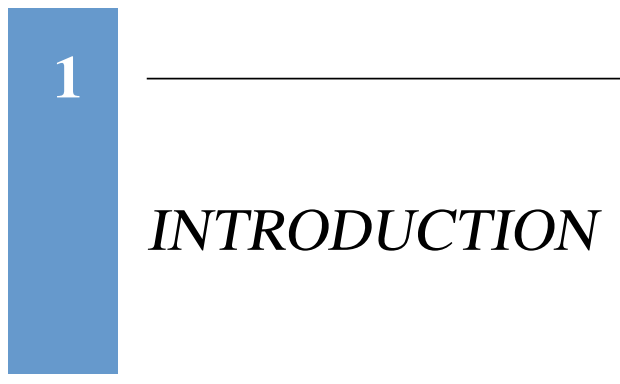

\subsection{Background}

\subsubsection{Radio communications and spectrum regulation}

In 1865 a Scottish physicist, James Clerk Maxwell, unified all previously unrelated observations, experiments and equations of electricity, magnetism and even optics into a consistent mathematical theory of electromagnetic energy [1]. Maxwell's work predicted that electricity, magnetism and even light are all manifestations of the same phenomenon, namely the electromagnetic field, which travels at the constant speed of light through space in the form of waves. This was confirmed by a set of experiments performed between 1886 and 1888 [2] by a German physicist, Heinrich Rudolf Hertz, who caused an electrical discharge between two small spheres (used as poles of a spark gap) and projected the charge from the space between the spheres across a short distance to a detection device consisting on a copper wire loop with a similar gap. Hertz had demonstrated the possibility to convert electricity into another form of energy that could be conducted over distance without wires.

Motivated by Hertz's experimental findings, an Italian inventor, Guglielmo Marconi, created a practical system for wireless telegraphy (i.e., the transmission of telegraph messages without wires) capable to use electromagnetic waves to send information. After numerous public demonstrations, Marconi accomplished the first long range radio transmissions via electromagnetic waves: in 1899 over the English Channel and in 1901 spanning the Atlantic ocean. Although he was implicated in a series of disputes with other contemporaneous experimenters and inventors (Sir Oliver Joseph Lodge, Nikola Tesla, Alexandr Stepánovich Popov and Édouard Branly) who claimed that Marconi had employed many of their patentprotected inventions, and Tesla was legally credited for the invention of radio, Marconi developed and exploited commercial radio worldwide. Radio communications had been born. 
Radio communications gained popularity as a result of Marconi's pioneering demonstrations and their role in the rescue of hundreds of lives during the tragic sinking of the Titanic ocean liner in 1912. However, the spark-gap transmission technology employed in the first designs was inefficient and could only be used for radiotelegraph applications. With the advent of the vacuum tube and the more efficient continuous-wave transmission technology, radio communications experienced a major boost. Vacuum tubes were critical to the development of electronic technology and the resulting expansion and commercialization of new radio communication applications such as entertainment audio and television broadcasting, large telephone networks, and radar systems.

With the growth and deployment of radio communications, the first interference problems arose. Interference issues were already noticed with the first radio designs. The frequency of early radio transmitters and receivers was not able to be controlled to any significant degree, which greatly restricted the number of simultaneous spark-gap transmitters within a geographical area without causing mutually disruptive interference. With the advent of tunable continuous-wave transmitters, the concept of frequency management emerged as a means to enable simultaneous radio communications and unleash the full potential of the radio technology. Radio spectrum gradually emerged as a public and economic resource, which highlighted the importance of controlling interference among transmitters and hence the necessity of governmental agencies in charge of its management [3].

Nowadays, use of the electromagnetic spectrum is regulated by governments in most countries in a spectrum management process known as frequency allocation or spectrum allocation. Under the coordination of the International Telecommunication Union (ITU), the representatives of the national regulatory agencies meet at the World Radio Conference (WRC) on a regular basis to develop the international fundamentals for national frequency use, coordinating the global use of the spectrum and establishing worldwide standards.

Since the first spark-gap transmitters, the radio communications technology has been growing at an impressive pace, subject to a ceaseless process of transformation and evolution in line with the most recent discoveries such as the invention of the transistor, the development of the digital signal processing theory and technology, and the subsequent emergence of the digital communications paradigm. Nevertheless, the rules currently applied by regulatory agencies are not so different from those that began to be applied in the early twentieth century. Since the early days of radio communications, wireless communication systems have been exploited under a fixed and strict spectrum management policy where portions of spectrum, separated by guard bands, have been allocated to particular radio standards or services over large geographical regions (e.g., whole countries), on a long term basis (e.g., several years), and under exclusive exploitation licenses.

Static spectrum allocation policies commonly applied by regulatory agencies have been proven to effectively control interference among radio communication systems and simplify the design of hardware for use at a known and fixed range of radio frequencies. However, the overwhelming proliferation of new operators, innovative services and wireless technologies during the last years has resulted, under this static regulatory regime, in the exhaustion of spectrum bands with commercially attractive radio propagation characteristics. The vast majority of spectrum regarded as usable has already been allocated, thus hindering the 
commercial rollout of new emerging services. This situation gave rise to a common belief about depletion of usable radio frequencies, which was certainly strengthened by the overly crowded frequency allocation charts of many countries and the multi-millionaire prize of a $20 \mathrm{MHz}$ frequency band in the European spectrum auction for third-generation cellular mobile communication systems. Nevertheless, some preliminary field measurements of spectrum usage indicated that most of the allocated spectrum was vastly underutilized [4], with temporal and geographical variations in the utilization of the assigned spectrum ranging from $15 \%$ to $85 \%$ [5]. More recent spectrum measurement campaigns performed all over the world have corroborated the underutilization of spectrum, thus indicating that the virtual spectrum scarcity problem actually results from static and inflexible spectrum management policies rather than the physical depletion of usable radio frequencies. The owned spectrum allocation policy was once appropriate. Nowadays, this outdated scheme has become obsolete. New spectrum management paradigms are therefore required for a more efficient exploitation of the precious radio resources. This situation has motivated the emergence of flexible spectrum access policies aimed at overcoming the drawbacks and shortcomings of the currently inefficient static allocation schemes.

\subsubsection{Dynamic spectrum access}

The current spectrum underutilization has motivated an important number of activities and initiatives in the regulatory [6-12], economic [13-17] and research communities in searching for better spectrum management policies. In this context, the term Dynamic Spectrum Access (DSA) has been coined to refer to innovative solutions proposing a procedure or scheme to share spectrum among wireless operators, technologies and services in order to increase the overall spectrum utilization. Although spectrum sharing is not a new idea [18], it has gained popularity in the last years. The diversity of envisioned spectrum reform ideas and spectrum access models can be broadly categorized under three different models [19, 20] as shown in Figure 1.1 (a more detailed classification is provided in [21, 22]):

- Dynamic exclusive use model. This model maintains the basic structure of the static spectrum allocation policy. Spectrum bands are licensed to operators, technologies and/or services for exclusive use, but some flexibility is introduced in order to improve spectrum efficiency. Two different approaches have been proposed under this model:

- Spectrum property rights [23]. This approach allows licensees to sell and lease some portions of its licensed spectrum and to freely choose the technology to be employed as well as the service to be provided in that band. Economy and market would thus play a more important role in driving the most profitable use of spectrum under this scheme. Note that even though licensees have the right to sell or lease the spectrum for economic profit, such spectrum sharing is not mandated by the regulatory organism.

- Dynamic spectrum allocation [24, 25]. This approach was brought forth by the European DRiVE project (Dynamic Radio for IP-Services in Vehicular Environments) and its successor the OverDRiVE project (Spectrum Efficient Uni- and 


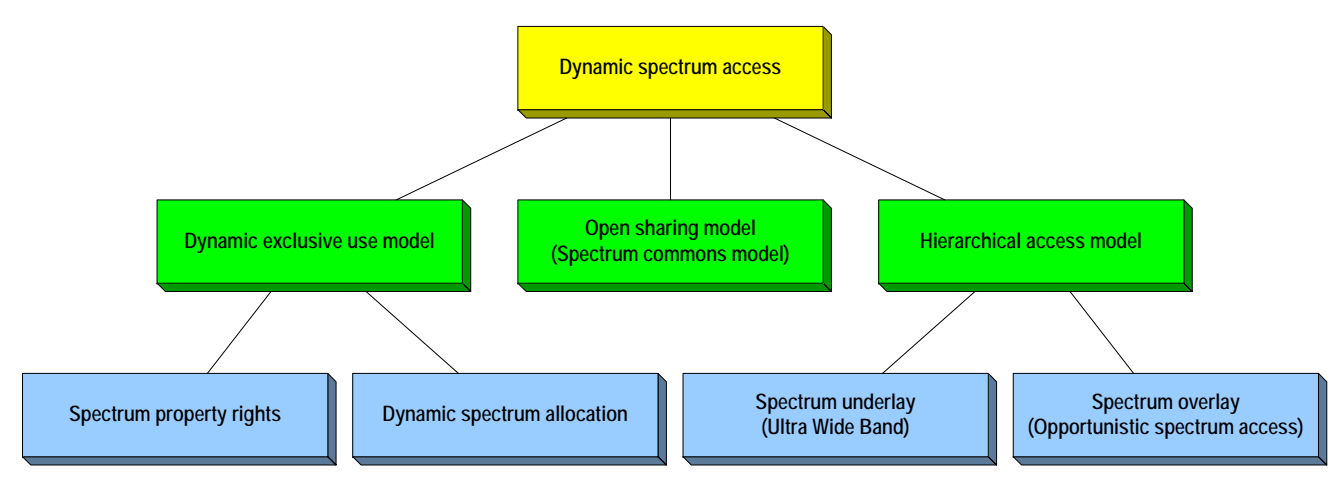

Figure 1.1: Classification of dynamic spectrum access schemes [20].

Multi-cast Services over Dynamic Multi-Radio Networks in Vehicular Environments) in the Fifth Framework Programme of the European Community. It is aimed at managing the spectrum utilized by a converged radio system and share it between participating Radio Access Networks (RANs) over space and time to increase overall spectrum efficiency. Most radio communication systems are subject to space- and time-dependent load characteristics that lead to variations in the degree to which spectrum is utilized. A bandwidth reservation as close to the expected maximum load as possible will result in unused spectrum for long periods of time and along large geographical areas. Therefore, spectrum efficiency can be improved by exploiting the spatial and temporal traffic variations of different services. The underlying idea of dynamic spectrum allocation methods is to allow two or more networks of a converged radio system to share an overall block of spectrum in such a way that spectrum allocations can adapt to either temporal or spatial variations in demand on the networks. Similar to the current static spectrum allocation policy, such strategies allocate, at a given time and region, a portion of the spectrum to a RAN for its exclusive use. This allocation, however, varies at a much faster scale than the current policy (e.g., traffic demands usually exhibit a periodic daily pattern). This approach received great attention within the research community [26-32].

- Open sharing model [33]. Also referred to as spectrum commons, this model employs open sharing among peer users as the basis for managing a spectral region in a similar way to that of wireless services operating in the unlicensed Industrial, Scientific and Medical (ISM) band. Advocates of this approach argue that the success of WiFistyle devices is proof that unlicensed devices can coexist in a largely unregulated way. Spectrum commons proponents assert that wireless transmissions can be regulated by baseline rules that allow users to coordinate their use, to avoid interference-producing collisions, and to prevent congestion. On the other hand, opponents of this proposal argue that it ultimately would result in a depletion of the shared limited resource in a 
scenario where too many nodes over-exploit the common good and degrade its quality, which is known as the tragedy of commons [34, 35]. However, there has been limited research into whether highly heterogeneous wireless services can coexist using selfinterested coordination. Centralized [36-38] and distributed [39-41] spectrum sharing strategies have been investigated to address technological challenges under this model.

- Hierarchical access model. This model can be regarded as a hybrid of the above two. It is built upon a hierarchical access structure that distinguishes between primary or licensed users, and secondary or license-exempt users. The basic idea is to open licensed spectrum to secondary users limiting the interference perceived by primary users. Two approaches to spectrum sharing between primary and secondary users have been considered:

- Spectrum underlay. This approach allows overlapping transmissions from secondary users but imposes severe constraints on their transmission power so that they operate below the noise floor of primary users. To meet such requirements, secondary transmissions must spread over a wide frequency band, which can be achieved by means of technologies such as Code Division Multiple Access (CDMA) or Ultra Wide Band (UWB). This approach allows secondary users to potentially achieve short-range high data-rates with extremely low transmission power. Another advantage is that the activity of primary users does not need to be tracked by secondary users; overlapping transmissions are allowed provided that power constraints are met. Despite the aforementioned benefits, this approach suffers from some practical drawbacks that mitigate its importance [19], the most important one being that the low transmission power still limits the applicability of spectrum underlays to short-range applications.

- Spectrum overlay. Differing from spectrum underlay, this approach is not necessarily characterized by severe restrictions on the transmission power of secondary users, but rather on when and where they may transmit. The basic idea of this approach is to identify spatial and temporal spectrum gaps not occupied by primary users, referred to as spectrum holes or white spaces, and place secondary transmissions within such spaces. The aim of this scheme is therefore to identify and exploit local and instantaneous spectrum availability in a nonintrusive and opportunistic manner as illustrated in Figure 1.2. This approach is also referred to as Opportunistic Spectrum Access (OSA) [42].

Although the dynamic exclusive use model is able to improve the spectrum efficiency offered by legacy management policies, this approach cannot eliminate white spaces in spectrum resulting from the bursty nature of wireless traffic. The interest of the open sharing model has recently been decreasing due to the interference among multiple heterogeneous wireless technologies on this band (e.g., wireless local and personal area networks, cordless telephones, and so on). On the other hand, the hierarchical access model is perhaps the most promising alternative and the most compatible with the current spectrum management policies and legacy wireless systems. In particular, the spectrum overlay/OSA approach is one 


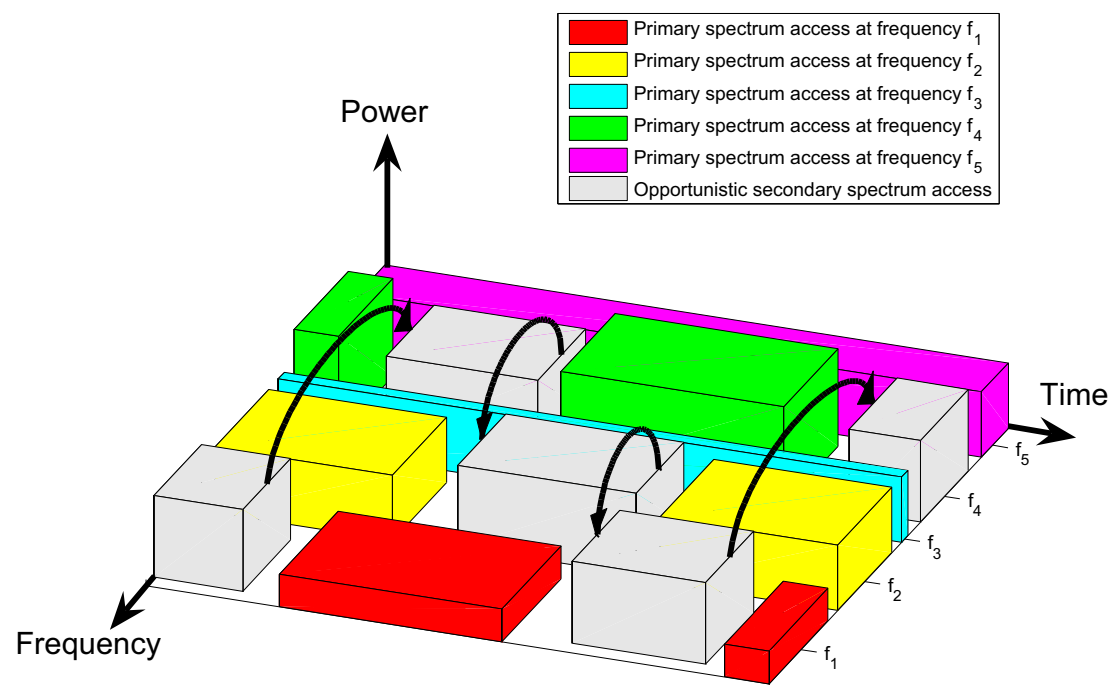

Figure 1.2: Opportunistic spectrum access concept.

of the most popular frameworks nowadays. The spectrum overlay/OSA scheme has received large attention during the last years and also constitutes the DSA model considered in this dissertation. Although spectrum overlay/OSA is a particular model for DSA, it is frequently referred to as DSA. This has been a common convention in existing literature and it also is adopted in this dissertation. Therefore, the term DSA will be used in the remainder of this dissertation to refer to the spectrum overlay/OSA model.

\subsubsection{Software defined radio and cognitive radio}

DSA has been paid much attention in regulatory, industrial and research communities not only for its promising capability to solve the existing conflicts between spectrum demand growth and spectrum underutilization but also for its potential to unleash new products and applications in leased, ad hoc, mesh, emergency and military networks [43-46]. The key enabler of the DSA concept is the Cognitive Radio (CR) paradigm, which usually, but not necessarily, is supported by the Software Defined Radio (SDR) technology.

The terms SDR and CR were introduced by J. Mitola in 1992 [47] and 1999 [48], respectively. SDR, sometimes shortened to software radio, is generally a multi-band radio that supports multiple air interfaces and protocols, and is reconfigurable through software run on a Digital Signal Processor (DSP), Field-Programmable Gate Array (FPGA), or generalpurpose microprocessor [49]. CR, usually built upon an SDR platform, is a context-aware intelligent radio capable of autonomous reconfiguration by learning from and adapting to the surrounding communication environment [50]. CRs are capable of sensing their Radio Frequency (RF) environment, learning about their radio resources, user and application 
requirements, and adapting their behavior accordingly. Other definitions of the CR concept:

- J. Mitola and G. Q. Maguire [48]: "Radio etiquette is the set of RF bands, air interfaces, protocols, and spatial and temporal patterns that moderate the use of radio spectrum. Cognitive radio extends the software radio with radio-domain model-based reasoning about such etiquettes."

- S. Haykin [51]: "Cognitive radio is an intelligent wireless communication system that is aware of its surrounding environment (i.e. its outside world), and uses the methodology of understanding-by-building to learn from the environment and adapt its internal states to statistical variations in the incoming RF stimuli by making corresponding changes in certain operating parameters (e.g. transmit power, carrier-frequency and modulation strategy) in real-time, with two primary objectives in mind: highly reliable communications whenever and wherever needed and efficient utilization of the radio spectrum."

- F. K. Jondral [52]: "A CR is an SDR that additionally senses its environment, tracks changes, and reacts upon its findings. A CR is an autonomous unit in a communications environment that frequently exchanges information with the networks it is able to access as well as with other CRs."

From these definitions, two main characteristics of CR can be identified: cognitive capability, i.e. the ability to capture information from the radio environment, and reconfigurability, which enables the transmitter parameters to be dynamically programmed and modified according to the radio environment.

CRs are therefore a powerful tool for solving the spectrum usage problem in wireless systems [53]. Such radios are capable of sensing spectrum occupancy and opportunistically adapting transmission parameters to utilize empty frequency bands without causing harmful interference to primary networks. A CR is able to reconfigure several parameters such as the operating frequency (to take profit of spectrum holes detected on different frequency bands), modulation and/or channel coding (to adapt to the application requirements and the instantaneous channel quality conditions), transmission power (to control interference), and communication technology (to adapt to specific communication needs). Based on the characteristics of the detected spectrum holes, these parameters can be reconfigured so that the $\mathrm{CR}$ is switched to a different spectrum band, transmitter and receiver parameters are reconfigured and the appropriate communication protocol parameters and modulation schemes are used. While DSA is certainly an important application of CR, the latter represents a much broader communications paradigm where many aspects of wireless communication systems can be improved via cognition and reconfiguration.

\subsubsection{Network architecture and functions}

The generic architecture of a DSA/CR network is shown in Figure 1.3, where different possible scenarios are considered. The components of such network architecture can be classified in two groups as primary network and secondary network [54, 55]: 


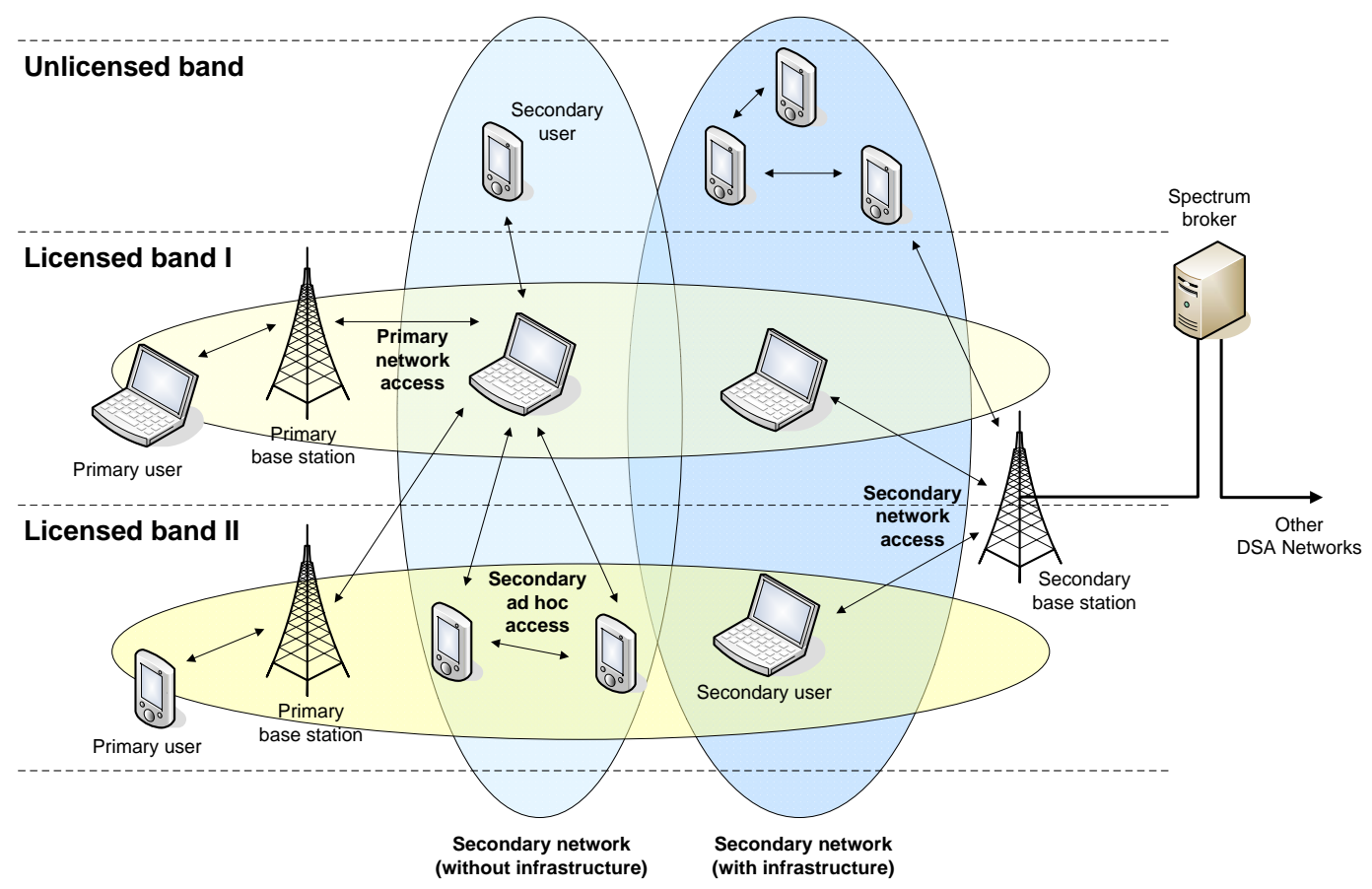

Figure 1.3: DSA/CR network architecture $[54,55]$.

- Primary network. A primary network is defined as an existing network infrastructure that has a license for exclusively accessing a certain spectrum band. Examples include the common cellular and TV broadcast networks. A primary network if composed of:

- Primary users. Primary or licensed users have a license to operate in a certain spectrum band. This access can only be controlled by the primary base station and should not be affected by the operation of any other unlicensed user. Primary users do not need any modification or additional functions to coexist with a secondary network.

- Primary base stations. Primary or licensed base stations are part of the fixed infrastructure of the primary network and thus they have a spectrum license. In principle, primary base stations are not designed to support any capability for sharing spectrum with a secondary network. However, primary base stations may support both legacy and new protocols for the primary network access of secondary users.

- Secondary network. A secondary or unlicensed network is defined as a network, with fixed infrastructure or based on ad hoc communication principles, without license to operate in a desired band. Hence, spectrum access is allowed only in an opportunistic 
manner. To this end, some special functions are required, which will be discussed later on. A secondary network is composed of:

- Secondary users. Secondary or unlicensed users have no spectrum license and make use of the detected spectrum holes in an opportunistic manner.

- Secondary base stations. A secondary or unlicensed base station is a fixed infrastructure component providing DSA capabilities. Secondary base stations provide single-hop connections to secondary users without spectrum license.

- Spectrum broker. A spectrum broker is a central network entity that controls the spectrum sharing among different secondary networks. A spectrum broker can be connected to each network and can serve as a spectrum information manager to enable coexistence of multiple secondary networks.

In this reference network architecture, three different access types are considered: primary network access (primary users access primary base stations through licensed bands), secondary network access (secondary users access secondary base stations in both licensed and unlicensed bands), and secondary ad hoc access (secondary users communicate among them in both licensed and unlicensed bands without the support of any base station). When operating in licensed bands, the interference avoidance with primary users is the most important issue for the secondary network. In this case the spectrum available to secondary users will depend on the traffic pattern of the primary users and the interference at the nearby primary users. On the other hand, when operating on unlicensed bands, all network entities have the same right to access the spectrum bands. In this case, a spectrum broker would play an important role in fairly sharing the available spectrum among secondary entities.

The DSA/CR concept, though simple in theory, poses exceptional challenges in practice. To guarantee interference-free and efficient spectrum access, a set of specific functions are required, which can broadly be summarized as follows [54, 55]:

- Spectrum sensing. This function is in charge of determining which portions of the spectrum are available to secondary users for opportunistic use (spectrum holes or white spaces), and detecting the presence of licensed users if they appear while a secondary user operates in a licensed band.

Some authors [56] have discussed the possibility of network-aided approaches based on an active agreement between the secondary network and the original spectrum licensee whereby the primary network shares real-time information with the secondary network regarding spectrum utilization. This information would be broadcast to secondary users by means of appropriate signaling channels such as the proposed Cognitive Pilot Channel (CPC) [57-59]. This approach would allow secondary systems to conceptually have perfect knowledge of current spectrum usage as well as possibly knowledge about traffic trends and future frequency usage. However, if the primary system is based on a legacy radio technology, enabling such interaction would require additional modifications to the already existing primary network, which might not be possible or economically feasible. In such a case, it is the secondary network's responsibility to track the primary network activity, performing its own observations 
of spectrum utilization and vacating the channel whenever the primary network resumes its activity. Under such circumstances, spectrum sensing becomes a function of paramount importance to enable coexistence.

Spectrum sensing may be performed in cooperative and non-cooperative fashions. In non-cooperative schemes, secondary users detect the primary transmitter signal independently based on local observations of spectrum usage. On the other hand, cooperative solutions rely on the exchange of sensing information from multiple secondary users. Cooperation among secondary users is theoretically more accurate and provides several advantages such as relaxation of the detection performance requirements for individual secondary terminals and mitigation of the degrading effects of multi-path fading and shadowing $[60,61]$, which theoretically enables practical sensitivity levels close to the nominal path loss with a relatively small number of cooperating users [60]. The work reported in $[62,63]$ demonstrates that cooperative sensing can also be used to reduce the detection time of weak primary signals, thereby increasing the frequency agility of the overall network. However, cooperation also has some disadvantages. First, it requires a reliable control channel to exchange sensing information. Second, it introduces additional signaling overhead, which might be significant depending on the amount of cooperating nodes and the type of information exchanged (e.g., binary busy/idle decisions or more advanced signal statistics). The required signaling data rates might be infeasible in primary networks with fast varying spectrum usage. Third, cooperation implies additional energy consumption, which is an important aspect in battery-powered terminals.

- Spectrum decision. This function analyzes the characteristics of the detected spectrum holes and decides the most suitable spectrum band to meet the communication requirements of the secondary user. The spectrum decision function is divided into two steps. First, the characteristics of the spectrum holes detected in different spectrum bands are analyzed in terms of interference level, path loss, channel error rate, link layer delay, expectable vacancy duration, and other appropriate parameters. After properly characterizing the detected spectrum holes, the operating band is then selected based on the user Quality of Service (QoS) requirements (e.g., data rate, error rate, delay, etc.) and the spectrum characteristics.

- Spectrum sharing. This function is aimed at providing a fair spectrum access to the coexisting secondary users and/or networks by coordinating the access to the available spectrum holes. To some extent, spectrum sharing shows some analogies with the Medium Access Control (MAC) problem in traditional communication systems. However, substantially different challenges exist for spectrum sharing in DSA/CR networks, which claims for specific DSA/CR MAC protocols [64].

- Spectrum mobility. To avoid harmful interference to licensed users, secondary users must vacate a channel when a licensed primary user is detected and move to an alternative spectrum hole. The transfer of the secondary communication to a different available channel and/or spectrum band may also be triggered by other reasons (e.g., 
preservation or improvement of the QoS). This event results in a transition from one spectrum band to another, which is referred to as spectrum handover. The objective of the spectrum mobility function is to ensure a seamless communication during transitions between different spectrum bands while avoiding harmful interference.

Other schemes have been proposed to classify the functions required by a DSA/CR network. For instance, according to [20], the main functions are categorized into spectrum opportunity identification, spectrum opportunity exploitation, and regulatory policy. These functions can be related to the previous classification as follows.

The opportunity identification function defined in [20] is responsible for accurately identifying and intelligently tracking idle frequency bands that are dynamic in both time and space. Thus, this function is equivalent to the spectrum sensing function described above. The opportunity exploitation function takes input from the opportunity identification function and decides whether and how a transmission should take place. This function comprises the aforementioned spectrum decision and sharing functions. The spectrum mobility function is not explicitly considered in the classification criterion proposed in [20], but it could be included within the opportunity exploitation function.

The regulatory policy function considered in [20] defines the basic etiquette for secondary users, dictated by a regulatory body, to ensure compatibility with legacy systems. An early example of such DSA policy is Dynamic Frequency Selection (DFS) [65]. DFS allows unlicensed 802.11 communications devices in the $5 \mathrm{GHz}$ band to coexist with legacy radar systems. The policy specifies the sensor detection threshold as well as the timeline for radar sensing, usage, abandoning the channel, and a non-occupancy time after detection. This policy allows limited but minimal harm to legacy radar systems by accounting for the specific form of sensor for detection and prescribing the timeline for channel use and departure. Another example is the listen-before-talk strategy developed in the Next Generation $(\mathrm{xG})$ program of the Defense Advanced Research Projects Agency (DARPA). Further DSA/CR systems have been envisaged, such as e.g. spectrum pooling [66-72], CORVUS (COgnitive Radio approach for usage of Virtual Unlicensed Spectrum) [73-75], and DIMSUMNet (Dynamic Intelligent Management of Spectrum for Ubiquitous Mobile NETworks) [76]. Some other DSA/CR systems have been developed and specified in the framework of standardization activities, which are reviewed in the next section.

\subsubsection{Standardization activities}

An important and essential aspect in the commercial feasibility and deployment of the DSA/CR concept is its standardization. The most important standardization initiatives to date have been brought forward by the Institute of Electrical and Electronics Engineers (IEEE). Nevertheless, other international standardization organizations or industry associations such as the ITU, the Wireless Innovation Forum (formerly known as SDR Forum), the European Telecommunications Standards Institute (ETSI), and the European association for standardizing information and communication systems (ECMA international) have been or are working in the development of DSA/CR standards as well [77]. 
The most popular standards on DSA/CR are the IEEE 802.22 standard for Wireless Regional Area Network (WRAN) using white spaces in the TV frequency spectrum and the IEEE P1900 series of standards in the area of dynamic spectrum management [78] developed by the IEEE Standards Coordinating Committee (SCC) 41, formerly known as IEEE P1900 Standards Committee. However, there are several other, lesser known, related activities within IEEE as well. Many other completed IEEE 802 standards already include DSA/CRlike capabilities or related building blocks that evolved from coexistence activities [79].

IEEE 802.22 is the first worldwide standard based on the DSA/CR technology [8082]. The most prominent target application of IEEE 802.22 is wireless broadband access in rural and remote areas, with performance comparable to those of existing fixed broadband access technologies serving urban and suburban areas. The standard defines the physical (PHY) and MAC layers of a DSA/CR-based air interface [83, 84] for use by license-exempt devices on a non-interfering basis in spectrum allocated to the TV broadcast service. The standard considers the utilization of TV bands for two main reasons, namely the favorable propagation characteristics of the lower frequency bands allocated to the TV service and the consequent larger coverage areas, and the considerable amount of available TV white space [85-87]. The 802.22 project initially identified the North American frequency range of operation between 54 and $862 \mathrm{MHz}$, which was extended between 47 and $910 \mathrm{MHz}$ in order to meet additional international regulatory requirements. Since there is no worldwide uniformity in channelization for TV services, the standard also accommodates the various international TV channel bandwidths of 6,7, and $8 \mathrm{MHz}$. The 802.22 system defines a fixed Point-To-Multipoint (PTM) architecture where each Base Station (BS) communicates with up to 255 associated stations, referred to as Consumer Premise Equipment (CPEs), by means of time division multiplexing in the DownStream (DS) direction (BS to CPE) and a demand assigned Time Division Multiple Access (TDMA) scheme in the UpStream (US) direction (CPE to BS). The specified BS coverage range is $30 \mathrm{~km}$ (with some additions to support 100 $\mathrm{km}$ under favorable radio propagation conditions) and targets peak throughput rates at the edge of the coverage area of $1.5 \mathrm{Mbit} / \mathrm{s}$ per user in DS and $384 \mathrm{kbit} / \mathrm{s}$ in US.

While IEEE 802.22 focuses on the development of specific mechanisms for the PHY and MAC layers, the IEEE P1900 series concentrates on the development of architectural concepts and specifications for policy-based network management with dynamic spectrum access in a heterogeneous wireless access network composed of incompatible wireless technologies such as $3^{\text {rd }} / 4^{\text {th }}$ Generation $(3 \mathrm{G} / 4 \mathrm{G})$, WiFi, and Worldwide Interoperability for Microwave Access (WiMAX). The series is composed of six standards defining terminology and concepts (IEEE P1900.1), recommended practice for interference and coexistence analysis (IEEE P1900.2), dependability and evaluation of regulatory compliance (IEEE P1900.3), architectural building blocks (IEEE P1900.4 [88]), policy language and policy architectures (IEEE P1900.5), as well as spectrum sensing aspects (P1900.6).

Other IEEE standardization activities have been initiated to address coexistence issues among various systems or to make amendments to existing standards with the aim of supporting coexistence with license-exempt devices. For instance, several amendments have been made to the PHY and MAC layers of the IEEE 802.11 standard to support channel access and coexistence in TV white spaces. Some examples include the introduction of new 
functionalities such as sensing of other transmitters (IEEE 802.11af, also known as Wi-Fi 2.0, White-Fi or "Wi-Fi on steroids" [89, 90]), Transmit Power Control (TPC) and DFS (IEEE 802.11h), and some extensions thereof (IEEE 802.11y). Similarly, the IEEE 802.16h amendment develops improved mechanisms to enable coexistence among license-exempt systems based on the IEEE 802.16 standard, and to facilitate the coexistence of such systems with primary users [91]. IEEE 802.19 deals with coexistence issues between unlicensed wireless networks, such as IEEE 802.11/15/16/22. The focus of the IEEE 802.19 standard is on the development of technology-independent methods for supporting coexistence among potentially dissimilar networks that will operate in a common TV white space channel.

In addition to the work performed by the IEEE, some other organizations are working on the definition of standards for DSA/CR systems. ITU-R published two technical reports $[92,93]$ on the application of SDR to the International Mobile Telecommunications (IMT) 2000 global standard for 3G mobile communications and other land mobile systems. In September 2009 the Wireless Innovation Forum (formerly known as SDR Forum) initiated a test and measurement project in order to develop a set of use cases, test requirements, guidelines and methodologies required for secondary opportunistic access to TV white space. The project addresses critical functions such as spectrum sensing, interference avoidance, database performance and policy conformance. In October 2009 the ETSI Technical Committee for Reconfigurable Radio Systems (RRS) published a series of technical reports [94] summarizing various feasibility studies and examining standardization needs and opportunities. In December 2009 ECMA released the first DSA/CR standard for personal/portable devices operating in TV white space $[95,96]$. The standard specifies the PHY and MAC layers of a DSA/CR system with flexible network formation, mechanisms for protection of primary users, adaptation to different regulatory requirements, and support for real-time multimedia traffic. The ECMA-392 standard is expected to enable new applications such as in-home high-definition video streaming and interactive TV broadcasting services. More recently in February 2011, and after the decision of the Federal Communications Commission (FCC) to remove spectrum sensing requirements and use TV white space data bases to identify unused TV channels, the Internet Engineering Task Force (IETF) has joined the standardization efforts by releasing a draft on a Protocol to Access White Space (PAWS) databases [97, 98]. The protocol will explore the various aspects of a messaging interface between white space devices and (potentially multiple) white space databases.

Despite the number of initiatives and activities carried out so far, standardization of DSA/CR systems constitutes an exciting challenge still requiring much more effort. Different countries may have different spectrum regulations. While this appears to be reasonable as a result of different social and economic environments, this situation complicates the standardization of DSA/CR systems and the development of worldwide standards. Moreover, there exists a variety of organizations working independently on different standards. Furthermore, the evolving trend towards converged heterogeneous wireless access networks poses unique challenges. Although some consolidation is required in this area, the fact is that regulation, standardization and evolution towards heterogeneity is ongoing and the final impact remains unknown. How these aspects can be harmonized constitutes a big question yet to be answered in the foreseeable future. 


\subsection{Motivation and scope}

During the last years, the DSA/CR concept has gained popularity as a promising solution to conciliate the existing conflicts between the ever-increasing spectrum demand growth and the demonstrated spectrum underutilization. As explained in Section 1.1.2, the basic underlying principle of DSA/CR is to permit unlicensed (secondary) users to access in an opportunistic and non-interfering manner some licensed bands temporarily unoccupied by licensed (primary) users. Unlicensed secondary terminals sense the spectrum to detect spectrum gaps/holes in the activity patterns of primary users, opportunistically transmit on them and vacate the channel as soon as a primary user reappears. Secondary unlicensed transmissions are allowed following this operating principle provided that no harmful interference levels are experienced by the primary network.

While conceptually simple, the realization of the DSA/CR concept poses great difficulties in practice. To date, various testbeds [99-102], prototypes [103-109] and experiments [110-112] have already demonstrated that the DSA/CR vision is not a mere theoretical concept, but a real promising solution. However, many critical aspects still remain open. How to sense and identify spectrum opportunities, coordinate opportunistic spectrum access among a number of nodes, and guarantee interference-free coexistence with heterogeneous primary systems, are simple questions still requiring definitively satisfactory solutions. More efforts must therefore be devoted to the development of new schemes enabling an optimum opportunistic usage of the available spectrum without causing harmful interference to the licensees. There is still a long way to go before the DSA/CR vision becomes a reality.

The development of the DSA/CR technology has successfully been relying on a combination of analytical models, computer simulations and network prototypes. The DSA/CR solutions proposed to date have commonly been envisaged, designed, developed and evaluated by means of analytical studies and simulations, which unavoidably rely on assumptions and models required to describe certain aspects of the system and scenario under study. The purpose of such models is to simplify some parts of the real environment in order to provide a tractable, yet realistic representation thereof, that can adequately be employed in analytical studies or implemented in simulation tools for the performance evaluation of DSA/CR techniques. Due to the opportunistic nature of the DSA/CR principle, the behavior and performance of a secondary network depends on the spectrum occupancy patterns of the primary users. A realistic and accurate modeling of such patterns becomes therefore essential and extremely useful in the domain of DSA/CR research. The potential applicability of spectrum usage models ranges from analytical studies to the design and dimensioning of DSA/CR networks, as well as the development of innovative simulation tools and more efficient DSA/CR techniques.

Modeling of spectrum usage for the study of radio communication systems has been an important concern for several decades. Early models on spectrum usage date from the late 1970s, when various authors analyzed and characterized spectrum usage in the High Frequency (HF) bands [113-121]. Although such works provide a valuable reference in the history of spectrum usage modeling, their applicability in the framework of the recent DSA/CR concept is very limited. The unique features of DSA/CR systems, bands of operation and modern primary radio communication systems, result in a particularly complex scenario that 
requires tailored modeling approaches. Recently, some simple spectrum usage models appear to have been widely accepted by the DSA/CR research community. Unfortunately, the models of spectrum usage commonly used to date in DSA/CR research are limited in scope and based on oversimplifications or assumptions that have not been validated with empirical measurement data. Spectrum occupancy modeling in the context of DSA/CR constitutes a rather unexplored research area that still requires much more effort.

In this context, this Ph.D. dissertation addresses the problem of realistically and accurately modeling spectrum usage in the context of DSA/CR networks. The ultimate objective of this Ph.D. dissertation is to contribute a holistic set of realistic models capable to accurately capture and reproduce relevant statistical properties of spectrum usage in real radio communication systems, in the time, frequency and space dimensions, for its application in the development and improvement of the future DSA/CR technology.

\subsection{Thesis contributions}

The main contribution of this dissertation is the development of realistic and accurate spectrum usage models for its application in the design and evaluation of DSA/CR systems. A distinguishing feature of the models developed in the context of this dissertation is the demonstrated ability to accurately capture and reproduce the statistical properties of spectrum usage observed in real channels of various radio technologies. The development and validation of such models rely on spectrum data that have been obtained from extensive spectrum measurement campaigns performed with state-of-the-art equipment.

The unique characteristics of the ambitious problem under consideration, and the inherent complexity of studies involving theoretical concepts and empirical results, highlighted the necessity of various preliminary studies for an adequate collection and processing of the spectrum data on which the developed models rely. These preliminary studies ended up making use of a multidisciplinary body of knowledge embracing the fields of RF engineering, digital signal processing, optimization methods, curve-fitting procedures, probability theory and statistical analysis. As a result of such preliminary studies, which finally constitute two of the three parts of this dissertation, several contributions have been performed in other fields in addition to the field of spectrum usage modeling.

The main contributions of this dissertation follow:

[TC.1] Design and implementation of an advanced radio spectrum measurement platform. The developed platform has been specifically envisaged and developed for the explicit purpose of spectrum occupancy evaluation in the context of DSA/CR and incorporates several advanced and powerful features.

[TC.2] Development of a unified methodological framework for spectrum occupancy evaluation in the context of DSA/CR. The developed framework unifies the methodologies of previous spectrum studies and embraces aspects related to the design of the measurement equipment, its configuration, and spectrum data processing procedures, with explicit characterization of the impact of individual aspects on the resulting spectrum occupancy statistics along with several useful guidelines. 
[TC.3] Evaluation of spectrum occupancy and identification of potential spectrum bands for the future deployment of the DSA/CR technology. The development of worldwide standards and rollout of new products on a large scale based on the DSA/CR technology requires a realistic understanding of spectrum usage under various spectrum regulations. To the best of the author's knowledge, this is the first study of these characteristics performed under the scope of the Spanish spectrum regulation and one of the earliest studies in Europe.

[TC.4] Assessment of the performance of energy detection-based spectrum sensing with field measurements of various radio technologies. This study contributes a more realistic and accurate knowledge of the real performance of energy detection, the most widely employed spectrum sensing technique in DSA/CR research, when applied to a wide variety of relevant radio technologies.

[TC.5] Development of an empirical model for energy detection performance. An accurate model for energy detection performance, based on theoretical reasoning complemented with empirical approximations, is developed. The model is able to reflect the impact of particular radio technology features on the experimental performance of energy detection in the real world, an aspect not captured by the classical theoretical results. Moreover, this study introduces the novel concept of signal uncertainty and its impact on the detection performance of energy detection.

[TC.6] Design and evaluation of an improved energy detection algorithm. The proposed method outperforms the conventional energy detection algorithm while retaining a similar level of complexity and range of applicability.

[TC.7] Development of a versatile, accurate and analytically tractable approximation to the Gaussian $Q$-function. While numerous approximations to the Gaussian $Q$ function exist in the literature, the proposed solution provides a more adequate balance between applicability, accuracy and analytical tractability, which is particularly useful in the context of some DSA/CR studies.

[TC.8] Development of a realistic and accurate discrete time model of spectrum usage with deterministic and stochastic duty cycle models. The conventional discrete time model widely employed in the literature is extended with appropriate deterministic and stochastic duty cycle models in order to capture and reproduce not only the mean channel occupancy level but also more advanced features such as the statistical distributions of busy and idle periods.

[TC.9] Development of realistic and accurate continuous time models, at both short and long time scales, along with a combined modeling approach. Accurate models for reproducing the statistical distributions of busy and idle periods in real channels, at various time scales, are developed and combined into a unified modeling approach.

[TC.10] Development of realistic and accurate time correlation models. The developed models capture and reproduce not only the mean channel occupancy and statistical distributions of busy and idle periods but also the correlation level between busy and idle periods along with the autocorrelation properties of busy or idle periods. 
[TC.11] Development of a realistic and accurate time-frequency model of spectrum usage. The model is able to capture and reproduce the joint time-frequency properties and statistics of spectrum usage in real channels of various radio technologies.

[TC.12] Development of realistic and accurate spatial models of spectrum usage. The developed modeling approach is able to capture and reproduce the spectrum occupancy perception of DSA/CR terminals as a function of the geographical location and some basic primary signal parameters, not only terms of average occupancy levels but also in terms of concurrent snapshot observations.

\subsection{Thesis outline}

The core of this Ph.D. dissertation is structured in three parts along with the corresponding conclusions in Part IV, which are complemented with three appendices in Part V. Parts I and II deal with specific methodological aspects of the two spectrum measurement platforms employed in the context of this dissertation, while Part III presents the developed spectrum usage models. The organization of this dissertation is illustrated in Figure 1.4.

Part I treats various aspects related to the first employed measurement platform, which relies on a spectrum analyzer setup (an in-depth description of this platform is provided in Appendix A [TC.1]). Spectrum analyzers have the advantage of enabling high dynamic ranges, high sensitivities and wideband measurements. However, their swept nature results in poor time resolutions. The particular features of these measurement devices and their impact in the context of DSA/CR are discussed in this part. First, Chapter 2 analyzes the impact of individual aspects of the measurement equipment, its configuration, and spectrum data processing procedures on the obtained occupancy statistics, thus developing a methodological framework for spectrum measurements in the context of DSA/CR [TC.2]. Chapter 3 then presents the results of an extensive spectrum measurement campaign that was carried out, taking into account the findings of Chapter 2, from 2008 to 2009 over a wide variety of locations and scenarios in the metropolitan area of Barcelona, Spain. Measurement results are exhaustively analyzed in order to identify potential bands of interest for the future deployment of the DSA/CR technology [TC.3]. Spectrum occupancy in Chapters 2 and 3 is quantified in terms of the duty cycle, which is computed as detailed in Appendix B.

Part II deals with various specific aspects related to the second measurement platform, which is based on the Universal Software Radio Peripheral (USRP) and GNU Radio architecture. The processing of the signals captured with this high time resolution platform is largely similar to the problem of spectrum sensing in DSA/CR systems. As a result, various spectrum sensing studies are carried out in this part. Concretely, Chapter 4 assesses the performance of energy detection-based spectrum sensing with field measurements of various radio technologies [TC.4]. The outcome of this study will be highly useful in the processing of the field measurements for various bands. Motivated by the findings of this study, Chapter 5 develops an empirical model for energy detection performance based on theoretical reasoning complemented with empirical approximations [TC.5], which makes use of the novel approximation to the Gaussian $Q$-function presented in Appendix C [TC.7]. Moreover, an improved energy detection scheme is presented and evaluated in Chapter 6 [TC.6]. 


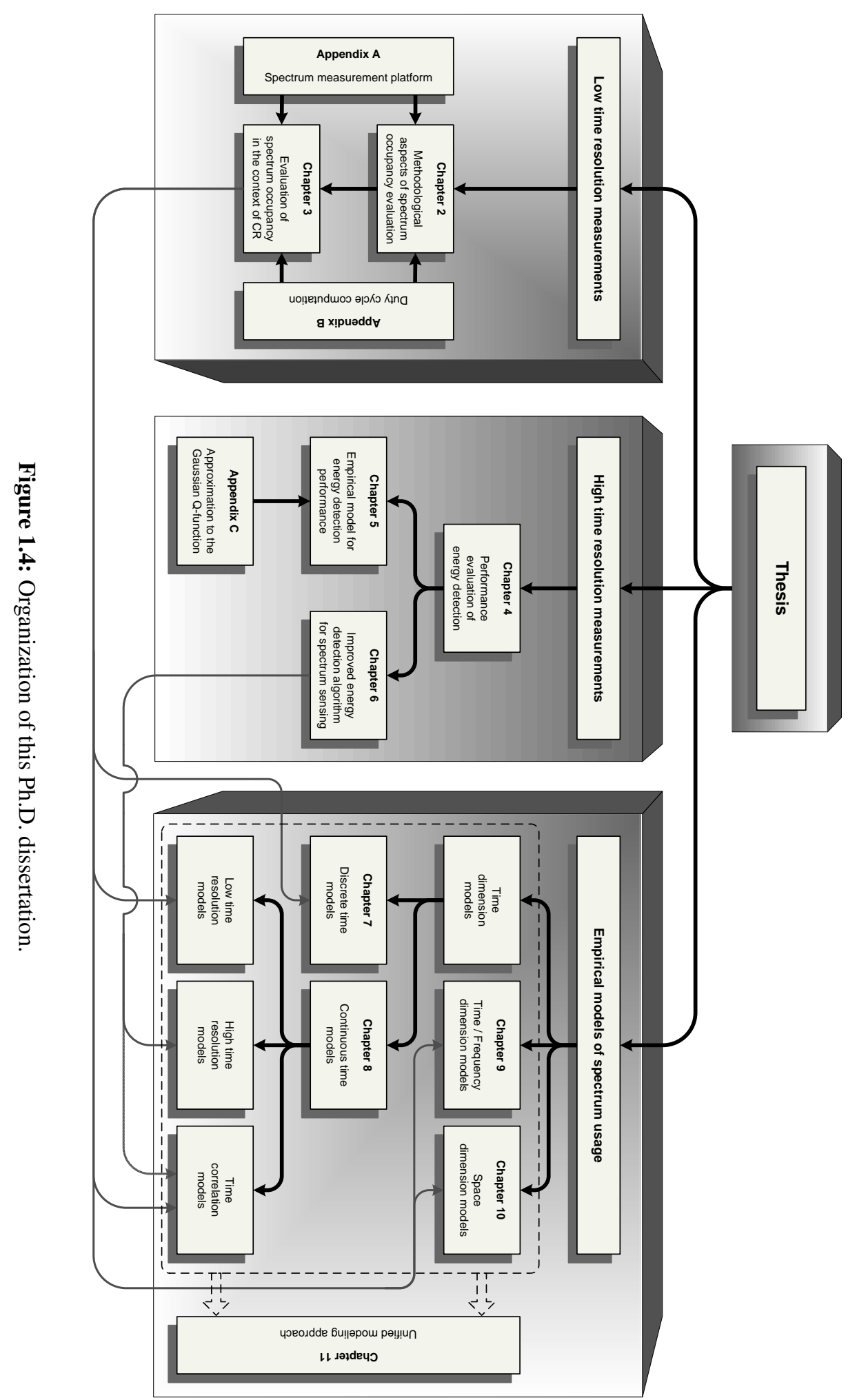


Part III presents a holistic set of spectrum usage models, which have been developed based on the spectrum data captured with the measurement platforms considered in Parts I and II. The contributed models have been envisaged to describe the statistical properties of spectrum usage observed in various domains, namely the time dimension in its discrete (Chapter 7 [TC.8]) and continuous (Chapter 8 [TC.9]) versions along with time correlation properties (Chapter 8 [TC.10]), the time and frequency dimensions from a joint perspective (Chapter 9 [TC.11]) and the spatial dimension (Chapter 10 [TC.12]). The integration of the proposed models into a unified modeling approach is discussed in Chapter 11.

Finally, Chapter 12 summarizes the main conclusions derived from the investigation carried out in this dissertation and suggests possible directions for future work.

\subsection{List of publications}

The contributions of this thesis have been published in several journals, international conferences and Spanish national conferences. The active participation in various research projects (see Section 1.6) offered the author the opportunity to present the work to project partners, from both industry and academia, and develop fruitful collaborations in the framework of such projects. The outcome of this project involvement is reflected not only in the corresponding contributions towards project deliverables (see Section 1.6) but also in a number of joint publications with project partners. Additionally, a technical report has been made publicly available and various exhibitions and demonstrations have been performed in conferences and other scientific events as well. A complete list of publications and dissemination activities along with the related contributions is listed below.

\section{Journals (published/in press)}

[J.1] M. López-Benítez, F. Casadevall, "Methodological aspects of spectrum occupancy evaluation in the context of cognitive radio," EUROPEAN TRANSACTIONS on Telecommunications, Special Issue on Selected Papers from the European Wireless 2009 Conference, vol. 21, no. 8, pp. 680-693, December 2010 [TC.1][TC.2].

[J.2] M. López-Benítez, F. Casadevall, "Spectrum occupancy in realistic scenarios and duty cycle model for cognitive radio," AdVANCES IN ELECTRONICS AND TELECOMmunications, Special Issue on Radio Communication Series: Recent Advances and Future Trends in Wireless Communication, vol. 1, no. 1, pp. 26-34, April 2010 [TC.3][TC.12].

[J.3] J. Pérez-Romero, D. Noguet, M. López-Benítez, F. Casadevall, "Towards a more efficient spectrum usage: Spectrum sensing and cognitive radio techniques," URSI RAdio SCiEnCE Bulletin, no. 336, pp. 59-74, March 2011 [TC.3].

[J.4] M. López-Benítez, F. Casadevall, "Improved energy detection spectrum sensing for cognitive radio," To appear in IET CommuniCATIONS, Special Issue on Cognitive Communications (in press) [TC.6]. 
[J.5] M. López-Benítez, F. Casadevall, "Versatile, accurate and analytically tractable approximation for the Gaussian $Q$-function," IEEE TRANSACTIONS ON COMMUNICATIONS, vol. 59, no. 4, pp. 917-922, April 2011 [TC.7].

[J.6] M. López-Benítez, F. Casadevall, "Empirical time-dimension model of spectrum use based on discrete-time Markov chain with deterministic and stochastic duty cycle models," To appear in IEEE TRANSACTIONS ON VEHICULAR TECHNOLOGY (in press) [TC.8].

\section{Journals (submitted)}

[J.7] M. López-Benítez, F. Casadevall, "Signal uncertainty in spectrum sensing for cognitive radio," submitted for consideration of publication, October 2010 (undergoing second round of review) [TC.5].

\section{Journals (in preparation)}

[J.8] M. López-Benítez, F. Casadevall, "Time-dimension models of spectrum usage for the analysis, design and simulation of cognitive radio networks" [TC.9][TC.10].

[J.9] M. López-Benítez, F. Casadevall, "Space-dimension models of spectrum usage for the analysis, design and simulation of cognitive radio networks" [TC.12].

[J.10] M. López-Benítez, F. Casadevall, "Spectrum usage in cognitive radio networks: From field measurements to empirical models" [TC.1]-[TC.12].

\section{International conferences}

[IC.1] M. López-Benítez, F. Casadevall, "A radio spectrum measurement platform for spectrum surveying in cognitive radio," in Proceedings of the 7th International ICST Conference on Testbeds and Research Infrastructures for the Development of Networks and Communities (TridentCom 2011), Shanghai, China, 17-19 April 2011, pp. 1-16 [TC.1].

[IC.2] M. López-Benítez, F. Casadevall, "Methodological aspects of spectrum occupancy evaluation in the context of cognitive radio," in Proceedings of the 15th European Wireless Conference (EW 2009), Aalborg, Denmark, 17-20 May 2009, pp. 199-204 [TC.1][TC.2].

[IC.3] M. López-Benítez, A. Umbert, F. Casadevall, "Evaluation of spectrum occupancy in Spain for cognitive radio applications," in Proceedings of the IEEE 69th Vehicular Technology Conference (VTC 2009 Spring), Barcelona, Spain, 26-29 April 2009, pp. 1-5 [TC.3]. 
[IC.4] M. López-Benítez, F. Casadevall, A. Umbert, J. Pérez-Romero, R. Hachemani, J. Palicot, C. Moy, "Spectral occupation measurements and blind standard recognition sensor for cognitive radio networks," in Proceedings of the 4th International Conference on Cognitive Radio Oriented Wireless Networks and Communications (CrownCom 2009), Special session on Flexible and opportunistic wireless access, Hannover, Germany, 22-24 June 2009, pp. 1-9 [TC.3].

[IC.5] M. López-Benítez, F. Casadevall, A. Umbert, R. Hachemani, J. Palicot, C. Moy, "Spectral occupancy measurements in urban areas and their applicability to the blind standard recognition sensor concept," in Proceedings of the NEWCOM++/ACoRN Joint Workshop (NAW 2009), Barcelona, Spain, 30 March-1 April 2009, pp. 1-5 [TC.3].

[IC.6] M. López-Benítez, F. Casadevall, “On the spectrum occupancy perception of cognitive radio terminals in realistic scenarios," in Proceedings of the 2nd IAPR International Workshop on Cognitive Information Processing (CIP 2010), Special session on NEWCOM++, Elba Island, Italy, 14-16 June 2010, pp. 99-104 [TC.3].

[IC.7] M. López-Benítez, F. Casadevall, C. Martella, "Performance of spectrum sensing for cognitive radio based on field measurements of various radio technologies," in Proceedings of the 16th European Wireless Conference (EW 2010), Special session on Cognitive Radio, Lucca, Italy, 12-15 April 2010, pp. 969-977 [TC.4].

[IC.8] W. Jouini, A. Nafkha, M. López-Benítez, J. Pérez-Romero, "Joint learning-detection framework: an empirical analysis," in Proceedings of the Joint COST2100 \& IC0902 Workshop on Cognitive Radio and Networking, Bologna, Italy, 23 November 2010, pp. 1-6 [TC.4].

[IC.9] M. López-Benítez, F. Casadevall, "Discrete-time spectrum occupancy model based on Markov chain and duty cycle models," in Proceedings of the IEEE 5th International Symposium on Dynamic Spectrum Access Networks (DySPAN 2011), Aachen, Germany, 3-6 May 2011, pp. 1-10 [TC.8].

[IC.10] M. López-Benítez, F. Casadevall, "Modeling and simulation of time-correlation properties of spectrum use in cognitive radio," in Proceedings of the 6th International ICST Conference on Cognitive Radio Oriented Wireless Networks (CrownCom 2011), Osaka, Japan, 1-3 June 2011, pp. 1-5 [TC.10].

[IC.11] M. López-Benítez, F. Casadevall, "Spatial duty cycle model for cognitive radio," in Proceedings of the 21st Annual IEEE International Symposium on Personal, Indoor and Mobile Radio Communications (PIMRC 2010), Istanbul, Turkey, 2629 September 2010, pp. 1631-1636 [TC.12].

[IC.12] M. López-Benítez, F. Casadevall, "Statistical prediction of spectrum occupancy perception in dynamic spectrum access networks," in Proceedings of the IEEE International Conference on Communications (ICC 2011), Kyoto, Japan, 5-9 June 2011, pp. 1-6 [TC.12]. 
[IC.13] M. López-Benítez, F. Casadevall, "An overview of spectrum occupancy models for cognitive radio networks," in Proceedings of the International Workshop on Performance Evaluation of Cognitive Radio Networks (PE-CRN 2011), Valencia, Spain, 13 May 2011, pp. 1-10 (Invited Paper) [TC.8]-[TC.12].

\section{National conferences}

[NC.1] M. López-Benítez, F. Casadevall, A. Umbert, "Evaluación del nivel de utilización del espectro: Posibilidades de la tecnología cognitive radio en España," en Actas del XXIV Simposium Nacional de la Unión Científica Internacional de Radio (URSI 2009), Santander, 16-18 septiembre 2009, pp. 154-155 [TC.3].

[NC.2] M. López-Benítez, F. Casadevall, "Oportunidades de despliegue para redes radio cognitivas de acceso dinámico al espectro en diversos escenarios realistas," en Actas de las XIX Jornadas Telecom I+D (Telecom I+D 2009), Madrid, 24-26 noviembre 2009, pp. 1-7 [TC.3].

\section{Technical reports}

[TR.1] M. López-Benítez, F. Casadevall, "Spectrum Survey in Urban Environment: UPC Campus Nord, Barcelona, Spain," Technical Report, Mobile Communication Research Group (GRCM), Department of Signal Theory and Communications (TSC), Universitat Politècnica de Catalunya (UPC), December 2010 [TC.3].

\section{Exhibitions and demonstrations}

[ED.1] M. López-Benítez, F. Casadevall, A. Umbert, R. Hachemani, C. Moy, J. Palicot, "Spectral occupancy measurements in urban areas," NEWCOM++/ACoRN Joint Workshop (NAW 2009), Dissemination Day and Workshop Poster Session, Barcelona, Spain, 31 March 2009 [TC.3].

[ED.2] M. López-Benítez, F. Casadevall, A. Umbert, R. Hachemani, C. Moy, J. Palicot, "Spectral occupancy measurements in urban areas," 18th ICT Mobile and Wireless Communications Summit (ICT-MobileSummit 2009), Exhibition, Santander, Spain, 10-12 June 2009 [TC.3].

[ED.3] M. López-Benítez, F. Casadevall, A. Umbert, R. Hachemani, C. Moy, J. Palicot, "Blind standard recognition sensor validation with data from measurement campaign," 18th ICT Mobile and Wireless Communications Summit (ICT-MobileSummit 2009), pre-conference workshop on Dynamic Spectrum Management in Cognitive Radio Networks, Santander, Spain, 9 June 2009 [TC.3].

[ED.4] M. López-Benítez, F. Casadevall, C. Martella, "Empirical study of energy detection-based spectrum sensing for different radio technologies," Future Network \& Mobile Summit 2010 (FuNeMS 2010), Poster, Florence, Italy, 18 June 2010 [TC.4]. 


\subsection{Project involvement}

The work carried out during the doctoral research period has been inevitably linked to various research projects and initiatives being funded by both Spanish and European Union entities, which are detailed below:

- "Flexible and spectrum-Aware Radio Access through Measurements and modelling In cognitive Radio systems" (FARAMIR), funded by the European Union in the Seventh Framework Programme (FP7), Information and Communication Technologies (ICT), Small or Medium Scale Focused Research Project (STREP), Ref. ICT-248351.

- "Network of Excellence in Wireless COMmunications++" (NEWCOM++), funded by the European Union in the Seventh Framework Programme (FP7), Information and Communication Technologies (ICT), Network of Excellence (NoE), Ref. INFSOICT-216715.

- "Advanced Resource Management Solutions for Future All IP Heterogeneous Mobile Radio Environments" (AROMA), funded by the European Union in the Sixth Framework Programme (FP6), Information Society Technologies (IST), Specific Targeted Research Projects (STREP), Ref. IST-4-027567.

- "Opportunistic and Cognitive Radio Access," original title in Spanish: "Acceso Radio Cognitivo y Oportunista" (ARCO), funded by the Spanish Research Council (MICINN) in the framework of Plan Nacional de Investigación Científica, Desarrollo e Innovación Tecnológica 2008-2011, Ref. TEC2010-15198.

- "Cognitive Spectrum and Radio Resource Management in Heterogeneous Mobile Networks with End-to-End Quality of Service Provisioning," original title in Spanish: “Gestión Cognitiva de Recursos Radio y Espectro Radioeléctrico en Redes Móviles Heterogéneas con Provisión de Calidad de Servicio Extremo a Extremo" (COGNOS), funded by the Spanish Research Council (MICINN) in the framework of Plan Nacional de Investigación Científica, Desarrollo e Innovación Tecnológica 2004-2007, Ref. TEC2007-60985.

The outcome of this project involvement can be measured not only in a number of joint publications with project partners (see Section 1.5) but also in several contributions towards the following project deliverables:

\section{Project deliverables}

[PD.1] J. Riihijärvi, R. Agustí (Editors), "State of the art review," FARAMIR Project, Deliverable D2.1, 30 April 2010.

[PD.2] L. Gavrilovska, V. Atanasovski (Editors), "Prototype requirement analysis," FARAMIR Project, Deliverable D6.1, 28 February 2011. 
[PD.3] A. Serrador, L. M. Correia (Editors), "Identification of relevant scenarios, use cases and initial studies on JRRM and ASM strategies," NEWCOM++ Network of Excellence, Deliverable DR9.1, 6 January 2009.

[PD.4] P. Sroka (Editor), "Definition and evaluation of JRRM and ASM algorithms," NEWCOM++ Network of Excellence, Deliverable DR9.2, 12 January 2010.

[PD.5] J. Pérez-Romero (Editor), "Final report of the JRRM and ASM activities," NEWCOM++ Network of Excellence, Deliverable DR9.3, 1 February 2011. 




\section{Part I}

\section{Low Time Resolution Measurements}

We don't see things as they are, we see them as we are.

Anais Nin 



\section{Chapter}

2

\section{METHODOLOGICAL ASPECTS OF SPECTRUM OCCUPANCY EVALUATION IN THE CONTEXT OF COGNITIVE RADIO}

\subsection{Introduction}

The DSA/CR paradigm is expected to dramatically increase the efficiency of spectrum use in radio communication systems. However, before this paradigm can become reality, a full understanding of the dynamic use of spectrum in real deployments is firstly required. To this end, spectrum measurements become an essential and unavoidable step.

By means of field measurements of the radio environment, regulatory agencies have been gathering frequency usage data for spectrum management purposes for several decades [122]. The measurement of real network activities constitutes an important step towards a realistic understanding of dynamic spectrum usage and hence towards the practical deployment of the future DSA/CR technology. One of the most important uses of spectrum data is not only to convince regulatory bodies and policy makers on the necessity of new spectrum access policies but also to provide a quantitative support to enhance the use of the currently underutilized spectral resources. The investments required to develop the DSA/CR technology may be misapplied if they do not address the current and future spectrum usage situation. Understanding the current use trends in the spectrum is required for the knowledgeable furtherance of these efforts. Spectrum measurements are therefore critical to ensure that research investments target the evolving real-world technical issues.

From a research perspective, spectrum measurements are useful in detecting what bands are subject to low utilization levels, thus assessing and characterizing the availability of underutilized spectral resources in terms of time, frequency and space. This information can be very helpful to the research community in order to identify the most suitable and interesting bands for the future deployment of the DSA/CR technology. Besides this, the empirical data captured in spectrum measurements can find many other interesting practical applications. One example is the evaluation and validation of existing and novel DSA/CR techniques with real-world data. While a large amount of theoretical analyses and simulations on the per- 
formance of DSA/CR techniques has been performed, little is known on the applicability of the existing schemes in reality. Empirical measurements can be employed to evaluate and validate the proposed solutions. Another application is the development of realistic spectrum usage models. A significant body of DSA/CR research is based on excessively simple channel models. Empirical data can be exploited in the development of more sophisticated and realistic models of spectrum usage.

From the previous discussion it is clear that the practical development of the DSA/CR technology can significantly benefit from empirical measurements of the spectrum occupancy. The success of such enterprise, however, depends on the availability of reliable and accurate spectrum utilization statistics. Several spectrum measurement campaigns covering wide frequency ranges [123-141] as well as some specific licensed bands [142-146] have already been performed in diverse locations and scenarios in order to determine the degree to which allocated spectrum bands are used in real wireless communication systems. Tables 2.1-2.3 summarize the main technical aspects of various broadband spectrum measurement campaigns ${ }^{1}$ [123-141] (the meaning of some parameters will be clarified later on). Although previous spectrum measurement campaigns followed similar approaches, a detailed analysis of Tables 2.1-2.3 highlights the lack of a common and appropriate evaluation methodology. As pointed out in [147], different measurement strategies can result in widely divergent answers. Therefore, the availability of a common and reliable evaluation methodology would be desirable not only to prevent inaccurate results but also to enable the direct comparison of results from different sources.

In this context, this chapter presents a comprehensive and in-depth discussion of several important methodological aspects that need to be carefully taken into account when evaluating spectrum occupancy. Certain issues discussed in this chapter are rather intuitive but they have never been assessed in a formal, rigorous and quantitative manner in the context of DSA/CR. This chapter presents various useful results that quantify the impact of different individual factors on the obtained occupancy statistics. The evaluated factors can be grouped into aspects related to the design of the measurement setup (Section 2.2), the frequency (Section 2.3) and time (Section 2.4) dimensions ${ }^{2}$ as well as the employed data post-processing procedures (Section 2.5). Based on the obtained results, various useful and practical guidelines for future spectrum measurement campaigns are also provided. The main objective of this chapter is to cope with the major drawback of previous spectrum occupancy studies (i.e., the lack of a rigorous evaluation methodology) by providing a unifying methodological framework for future spectrum measurement campaigns. The results presented in this chapter highlight the importance of carefully following such an appropriate methodology when evaluating spectrum occupancy in the context of DSA/CR.

\footnotetext{
${ }^{1}$ The information displayed is for existing measurement campaigns at the time of this study was conducted. Several additional works were published later on, which are described in Chapter 3.

${ }^{2}$ In the context of spectrum occupancy evaluation for DSA/CR, the spatial domain may refer to the impact of the selected measurement location on the occupancy statistics. The choice of the measurement location, however, can be regarded as an aspect more related to the particular evaluated scenario rather than the evaluation methodology itself. Therefore, the spatial domain is not treated as a methodological aspect in this chapter. A detailed analysis and discussion of this aspect is carried out in Chapter 3.
} 


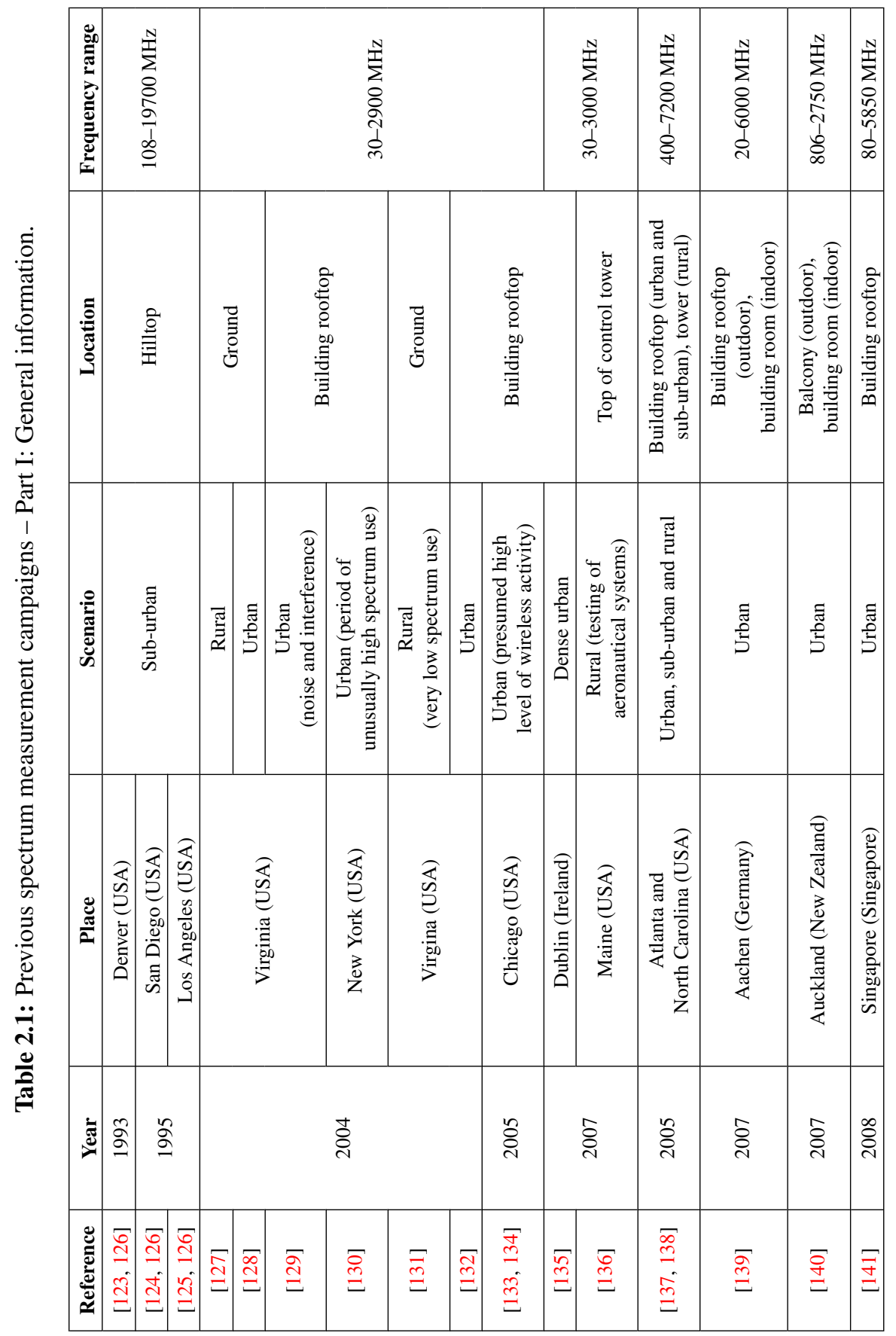




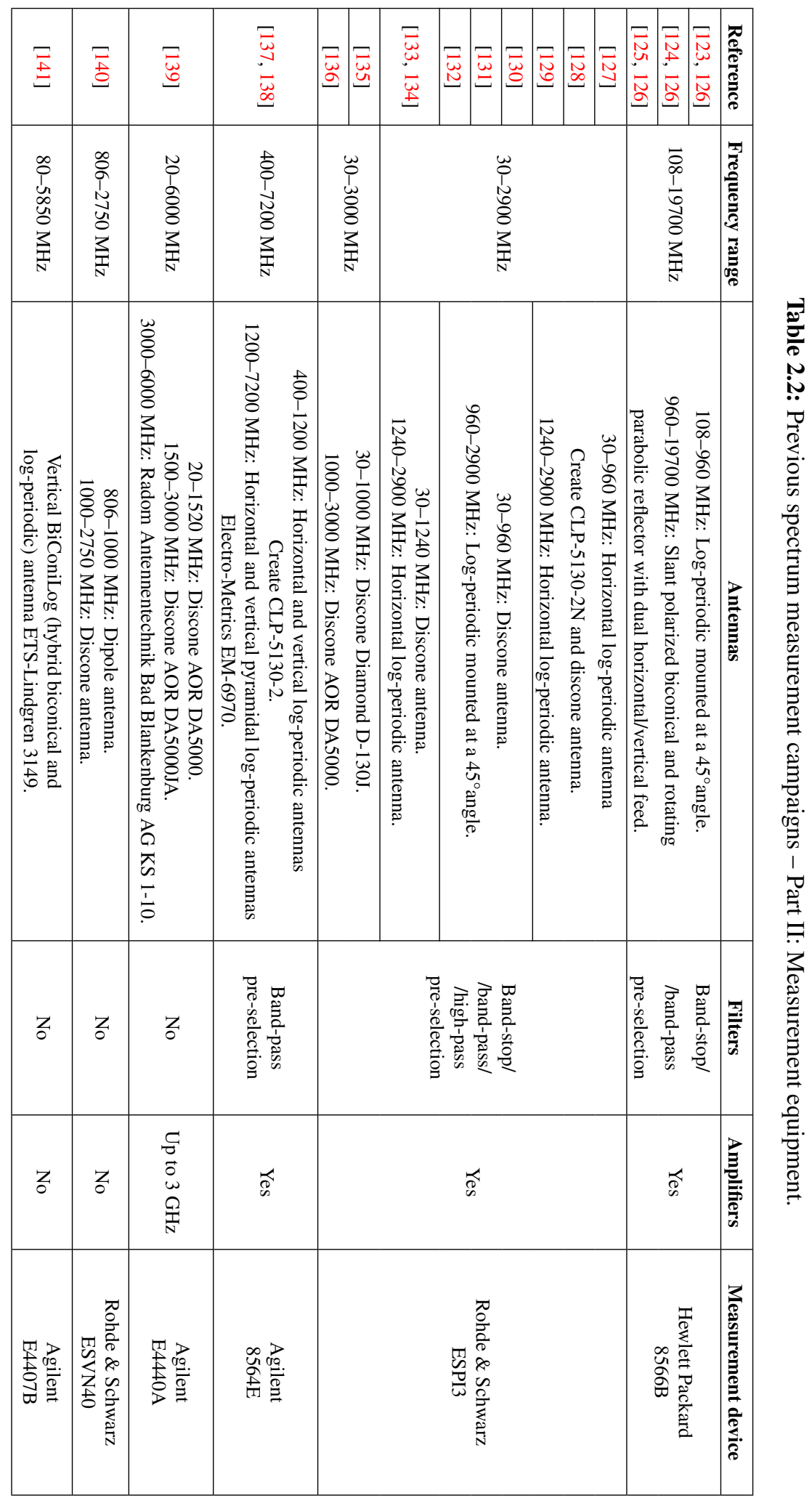




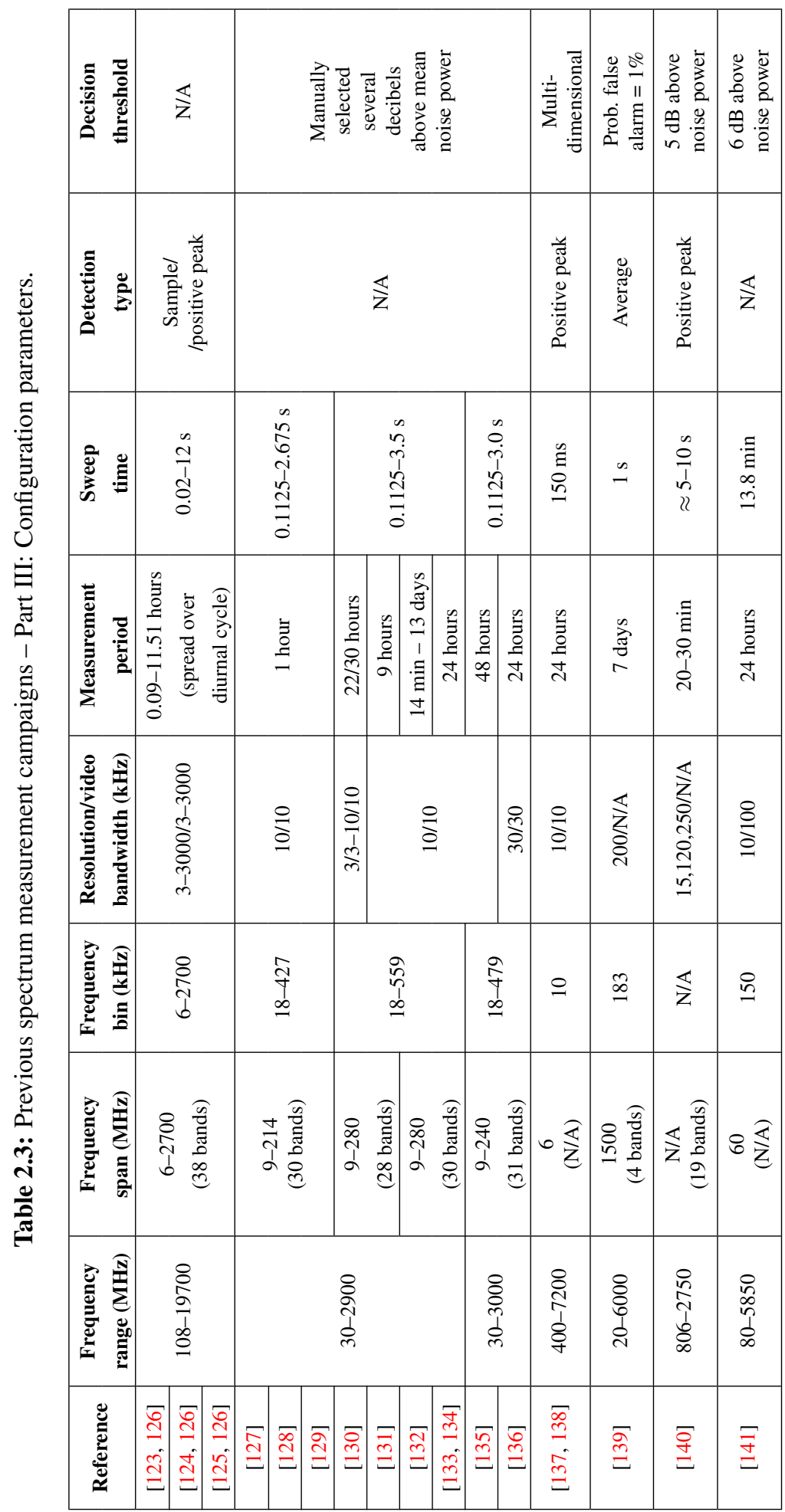




\subsection{Measurement setup}

Many factors need to be considered when defining a strategy to meet a particular spectrum measurement need. As detailed in [147], there are some basic dimensions that every spectrum measurement strategy should clearly specify: frequency (frequency span and frequency points to be measured), location (measurement site selection), direction (antenna pointing angle), polarization (receiving antenna polarization) and time (sampling rate and measurement period). The measurement setup employed in the evaluation of spectrum occupancy should be designed taking into account the previous factors since they play a key role in the accuracy of the obtained results. The measurement setup should be able to detect, over a wide range of frequencies, a large number of transmitters of the most diverse nature, from narrow- to wide-band systems and from weak signals received near the noise floor to strong signals that may overload the receiving system.

Depending on the purposes of the study, different configurations have been used in previous spectrum measurements ranging from simple setups with a single antenna directly connected to a spectrum analyzer [145] to more sophisticated and complex designs [126, 137]. Different configurations between both extreme points may determine various trade-offs between complexity and measurement capabilities. The present study is based on a spectrum analyzer setup where different external devices have been added in order to improve the detection capabilities of the system and hence obtain more accurate and reliable results. Figure 2.1 shows a simplified scheme. A complete scheme along with an exhaustive description is provided in Appendix A. The design is composed of two broadband discone-type antennas that cover the frequency range from 75 to $7075 \mathrm{MHz}$, a Single-Pole Double-Throw (SPDT) switch to select the desired antenna, several filters to remove undesired signals, a low-noise pre-amplifier to enhance the overall sensitivity and thus the ability to detect weak signals, and a high-performance spectrum analyzer to record the spectral activity.

When covering small frequency ranges or specific licensed bands a single antenna may suffice. However, in broadband spectrum measurements from a few megahertz up to several gigahertz, two or more broadband antennas are required in order to cover the whole frequency range. Most of spectrum measurement campaigns have been based on omnidirectional measurements in order to detect licensed signals coming from any directions. To this end, omni-directional vertically polarized antennas have been the most common choice. Our antenna system comprises two broadband discone-type antennas, which are wideband antennas with vertical polarization and omni-directional receiving pattern in the horizontal plane. Even though some transmitters are horizontally polarized, they usually are highpower stations, such as e.g. TV stations, that can be detected even with vertically polarized antennas. The exceptionally wideband coverage (allowing a reduced number of antennas in broadband spectrum studies) and the omni-directional feature (allowing the detection of licensed signals coming from any directions) make discone antennas an attractive alternative in radio scanning and monitoring applications, and have been a preferred option for many past spectrum occupancy measurement studies.

In studies where the direction of the incoming signal needs to be resolved, it is possible to use multiple antenna arrays along with beam forming techniques in order to selectively receive or suppress (filter) signals in the angular domain. Directive antennas (e.g., log-periodic 


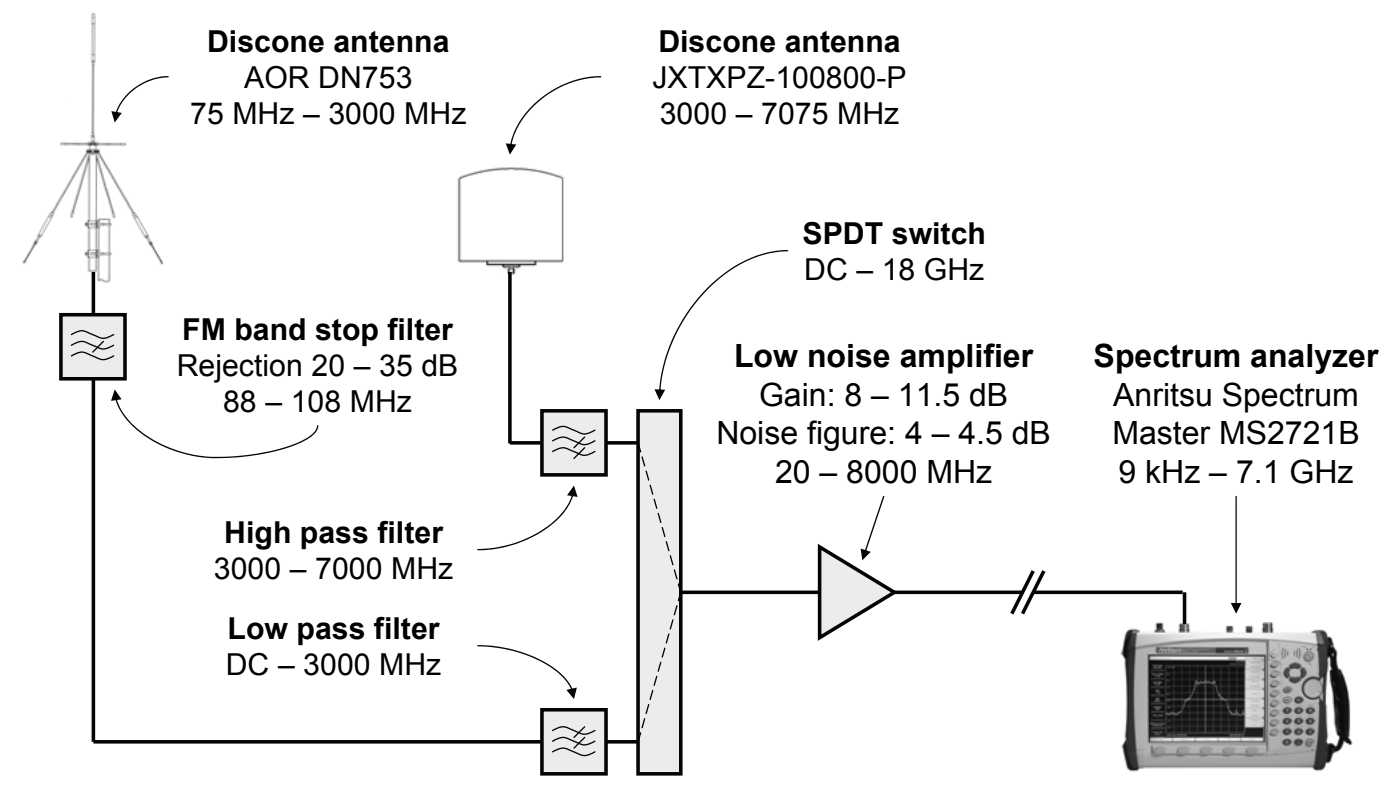

Figure 2.1: Measurement setup employed in this study.

antennas) may also be used to this end as well as in order to improve the system's sensitivity at the cost of an increased complexity in the measurement procedures. For example, if a directive antenna with $\alpha$-degree beamwidth is used in order to provide an additional $G$-dB gain with respect to an omni-directional antenna, it would be necessary to repeat the measurements $N=\lceil 360 / \alpha\rceil$ times in order to cover the entire 360-degree range of azimuths.

An alternative option to obtain additional gain is the use of amplification. Most spectrum analyzers include built-in high-gain pre-amplifiers. Nevertheless, in some measurement conditions there may be high losses between the antenna port and the spectrum analyzer. In this case a better option to improve the system's noise figure is to place a low-noise pre-amplifier right after the antenna system, as show in Figure 2.1. This amplifier compensates for device and cable losses and increases the system's sensitivity. It is worth noting that choosing an amplifier with the highest possible gain not always is the best option in broadband spectrum surveys, where very different signal levels may be present. The existing trade-off between sensitivity and dynamic range must be taken into account. Thus, the correct pre-amplifier has to be chosen based on the specific measurement needs. If the best possible sensitivity is absolutely wished and there are no concerns about measurement range, a high-gain, lownoise pre-amplifier should be chosen. However, if an improved sensitivity is desired but the resulting measurement range is relevant, a lower-gain pre-amplifier would be a more adequate choice. A reasonable design criterion when selecting the pre-amplifier is to guarantee that the different received signal strengths lie within the overall system's Spurious-Free Dynamic Range (SFDR), which is defined as the difference between a threshold or lower limit at which signals can be detected without excessive interference by noise (constrained by the system's noise floor) and the input signal level that produces spurs at levels equal to the 
noise power [148]. If the maximum input level is exceeded, some spurs might arise above the system's noise floor and be detected as signals in truly unoccupied bands, thus resulting in inaccurate results and erroneous conclusions on the spectral occupancy. As shown in Figure 2.1, the use of band-stop filters to remove undesired overloading signals, such as those coming from FM audio broadcast stations, as well as low/high-pass filters to remove out-of-band components, which might create harmonics or intermodulation products, can be very helpful in satisfying the SFDR criterion without loss in sensitivity at other frequencies.

Figures 2.2 and 2.3 quantitatively exemplify the impact of the overall system's sensitivity on the detected spectral activity. In each subfigure, the upper graph shows the Power Spectral Density (PSD) in average value (thick line) as well as minimum and maximum values. When considered together, average, minimum and maximum PSD provide a simple characterization of the temporal behavior of a channel. For example, if the results are quite similar, it suggests a single transmitter that is always on, experiences a low level of fading and is probably not moving. At the other extreme, a large difference among average, minimum and maximum PSD suggests a more intermittent use of the spectrum [145]. To more precisely quantify the detected spectral activity, the lower graph of each subfigure shows the Duty Cycle (DC). For each measured frequency point, the DC is computed as the fraction of PSD samples, out of all the captured PSD samples, indicating the presence of a licensed signal (a more formal definition is provided in Appendix B). This metric represents the fraction of time a given frequency is observed as busy and, for the purposes of this chapter, it also serves as a metric to quantify the ability to detect the presence of a licensed signal within a certain frequency range ${ }^{3}$.

Figure 2.2 shows the results obtained for the Global System for Mobile communications (GSM). As depicted in Figure 2.2(a), when the uplink direction is measured without any amplification (external pre-amplifier of Figure 2.1 or spectrum analyzer's built-in amplifier), some signals are detected (see PSD) resulting in an overall DC of $1.07 \%$ for the entire band. When only the external amplifier is connected, a higher number of licensed signals are detected and the resulting average DC is $7.03 \%$ in this case, as shown in Figure 2.2(b). These results indicate that, when measuring the GSM uplink spectral activity in the considered experiment, an absolute estimation error of nearly $6 \%$ is observed due to insufficient amplification. In the case of GSM downlink, poor sensitivity levels result in severe underestimation of spectral activity. While the results obtained without amplification in Figure 2.2(c) conclude that the GSM downlink band is subject to moderate/high usage levels $(67.95 \%)$, the results obtained with amplification in Figure 2.2(d) reveal that such band is actually overcrowded with an average DC of $96.51 \%$, thus resulting in an absolute estimation error of $96.51 \%-67.95 \%=28.56 \%$. These results highlight the importance of the sensitivity: if the measurement setup is not sensitive enough, the obtained occupancy statistics may be subject to high estimation errors, thus leading to wrong conclusions.

Figure 2.3 shows the results obtained for Broadband Wireless Access (BWA) systems operating in the 3.4-3.6 GHz band. Without amplification, Figure 2.3(a) shows that the band

\footnotetext{
${ }^{3}$ Under appropriate evaluation conditions, higher DCs can be associated to a better capability to detect the presence of a licensed signal. Based on this principle, the signal detection capabilities of different measurement setups, configurations, methods or algorithms can be evaluated and compared in terms of the DC, inferring better signal detection capabilities for those approaches providing higher DC values.
} 


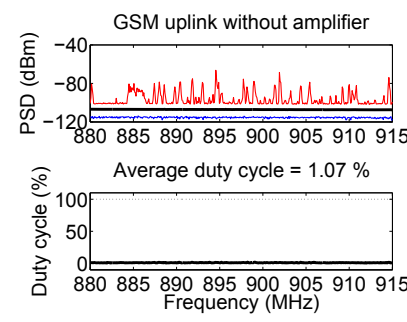

(a)

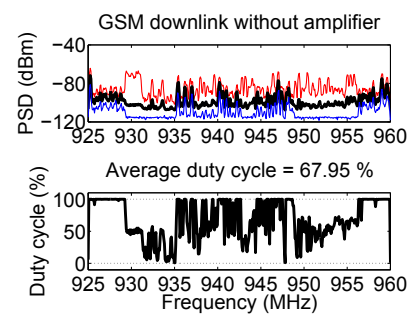

(c)

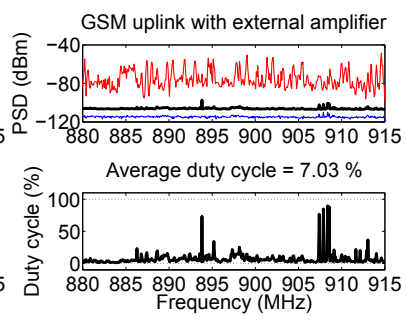

(b)

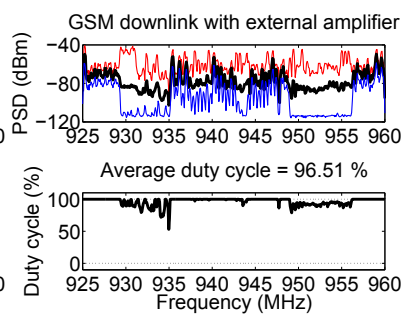

(d)

Figure 2.2: Impact of amplification on the activity detected for GSM: (a) uplink without amplifier, (b) uplink with external amplifier, (c) downlink without amplifier, and (d) downlink with external amplifier.

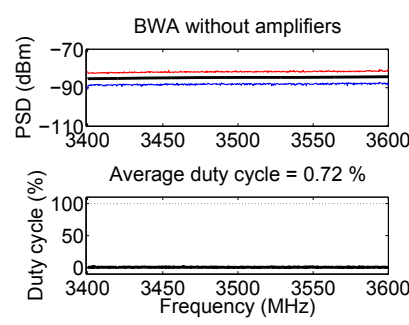

(a)

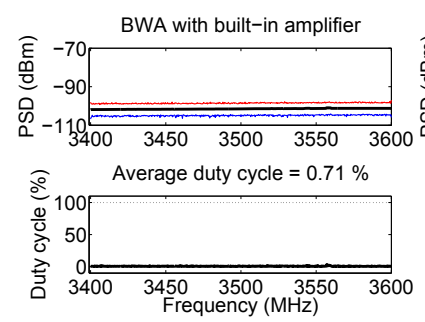

(c)

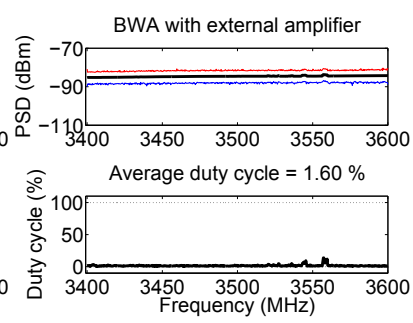

(b)

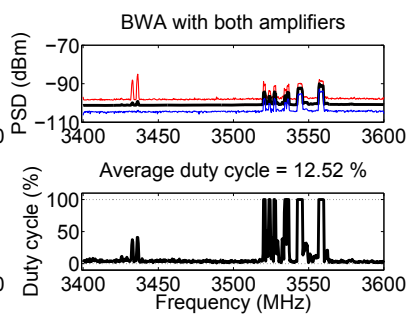

(d)

Figure 2.3: Impact of amplification on the activity detected for BWA: (a) without amplifiers, (b) with external amplifier, (c) with built-in amplifier, and (d) with both amplifiers. 
Table 2.4: Impact of the amplification configuration.

\begin{tabular}{|l|r|r|r|r|}
\hline Amplifiers & None & Internal & External & Both \\
\hline Noise figure (dB) & 24.0 & 13.2 & 10.2 & 5.0 \\
\hline Sensitivity (dBm/10 kHz) & -110.0 & -120.8 & -123.8 & -129.0 \\
\hline Dynamic range (dB) & 80.0 & 79.2 & 79.2 & 74.7 \\
\hline Maximum input (dBm/10 kHz) & -30.0 & -41.6 & -44.6 & -54.4 \\
\hline
\end{tabular}

is detected as idle (the average DC of $0.72 \%$ is due to the criterion employed to select the decision threshold, which is explained in Section 2.5). By comparing the average DC of Figures 2.3(b) and 2.3(c) it can be confirmed that the use of pre-amplifiers near the antenna system provides better sensitivity improvements than the use of the spectrum analyzer's built-in amplifier. Although the external pre-amplifier's gain is only 8-11.5 dB, it enables the detection of some signals that are not detected with the spectrum analyzer's 25 -dB gain built-in amplifier. However, Figure 2.3(d) demonstrates that both amplifiers are required in order to properly detect the presence of licensed systems. These results indicate that amplification by itself is not enough: an appropriate amplification configuration is required in order to accurately estimate spectrum usage.

Table 2.4 illustrates the detection capabilities of the developed spectrum measurement platform under different amplification configurations. In particular, the table shows the overall noise figure of the measurement configuration, the minimum detectable power in a bandwidth of $10 \mathrm{kHz}$ (sensitivity), the SFDR (dynamic range) and the maximum input power in a bandwidth of $10 \mathrm{kHz}$ that can be tolerated without undesired spurs above the noise floor. As it can be appreciated, spectrum analyzers are in general characterized by high noise figures, which limits the minimum signal power that can be detected. By using amplifiers with lower noise figures it is possible to reduce the overall noise figure and hence improve the overall system's sensitivity. As previously mentioned, the use of pre-amplifiers close to the antenna system can provide better sensitivity improvements, which is clearly appreciated by comparing the sensitivities obtained with separate internal and external amplifiers. However, the best sensitivity is attained when both amplifiers are simultaneously employed. It is worth noting that the use of amplifiers not only can reduce the overall noise figure but also the system's dynamic range. As it can be appreciated in Table 2.4, the use of either the internal or external amplifier does not reduce the effective SFDR significantly. However, when both amplifiers are simultaneously activated, the resulting overall gain causes an appreciable reduction of the dynamic range, thus imposing a trade-off between the sensitivity and the dynamic range (and consequently the maximum input power). Based on Table 2.4, an adequate measurement strategy is to employ both amplifiers whenever possible (this provides the best possible sensitivity) and deactivate the internal or external amplifiers (preferably in this order) in the presence of overloading signals received above the maximum tolerable input power (this can avoid the appearance of spurs while still improving sensitivity). In the eventual case that some signals were still received at excessive powers it might be possible to deactivate both amplifiers but it would be more convenient to consider the use of band-stop filters to remove undesired signals and avoid having to further sacrifice sensitivity. 
Since the different operating modes of spectrum analyzers can significantly alter the results of a measurement, proper parameter selection is crucial to produce valid and meaningful results. Although the basic principles of spectral analysis [149] have to be taken into account, some particular aspects specific to DSA/CR need to be considered as well. Sections 2.3 and 2.4 discuss on the proper selection of configuration parameters related to the frequency and time domains, respectively.

\subsection{Frequency dimension}

When the measurement equipment is designed, the next required step is to decide the frequency blocks (frequency spans) to be measured. This task basically consists in dividing the entire frequency range under study into smaller frequency blocks/spans over which measurements are performed individually. This is necessary, especially in broadband measurement campaigns, because measuring the whole frequency range under study as a single measurement block would result in an extremely poor frequency resolution and hence in a very coarse spectrum occupancy estimation. In previous spectrum measurement campaigns, the division into frequency blocks/spans has been performed according to arbitrary criteria and following a single-stage measurement procedure. However, when little is known about the spectrum bands to be measured and their spectral activity, a more reasonable approach is to follow a two-stage measurement procedure. In the first stage, the whole frequency range is divided into relatively large frequency blocks/spans to produce a first picture of spectrum occupancy. This information may be useful to determine which spectrum bands are worthy of a more detailed study. Based on this first impression and following the local spectrum allocations, the entire frequency range can then be divided into smaller blocks/spans in such a way that higher frequency resolutions are obtained in those bands where some spectral activity is detected and/or the transmitted signals' bandwidth is narrower.

The relation between the transmitted signal's bandwidth and the frequency resolution is an important aspect to be accounted for that, unfortunately, has received little attention in previous spectrum measurement campaigns. For a given number of measured frequency points per block/span, the frequency bin size (i.e., the separation between two consecutively measured frequencies) increases with the frequency span. In general, higher frequency bins tend to result in higher spectrum occupancy rates, as it is shown in Figure 2.4 for the Digital Cellular System (DCS) and the Universal Mobile Telecommunications System (UMTS) downlink bands. However, the exact behavior in both cases is different. In the case of DCS 1800 , for frequency bins lower than the bandwidth of the transmitted DCS signal (200 kHz), the average DCs (45.16\% and 58.91\%) indicate that the band is subject to moderate usage levels. For a frequency bin of $1 \mathrm{MHz}$, which is quite greater than the signal bandwidth, the obtained DC of $84.68 \%$ incorrectly concludes that the same band experiences a high level of utilization. This phenomenon can be explained as follows. As it can be observed in Figure 2.4(a) for DCS 1800, some regions of the band are busy during the entire measurement period. As a result, the three frequency bin values agree and provide similar DCs (nearly $100 \%$ ) in such portions of the band. In other regions where the activity is lower, different frequency bin values provide very different results. Concretely, large frequency bin values tend 


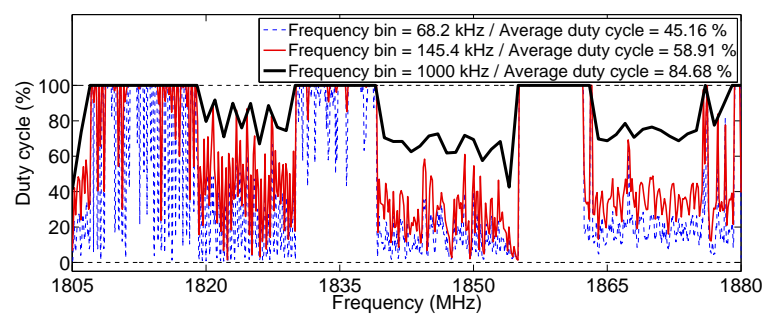

(a)

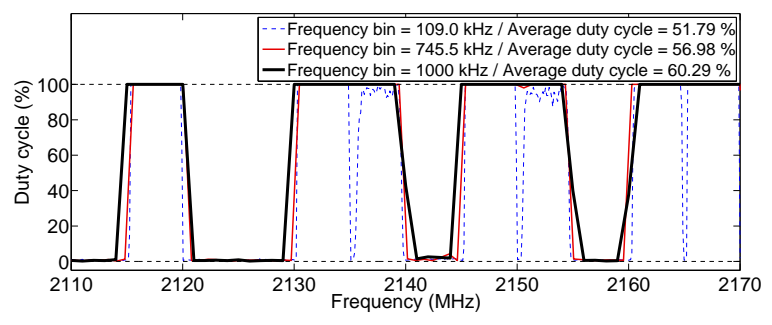

(b)

Figure 2.4: Impact of the frequency bin on the activity detected for: (a) DCS 1800 downlink, and (b) UMTS downlink.

to overestimate spectrum occupancy. For example, if a frequency bin of $1000 \mathrm{kHz}$ is used, a single high-power $200 \mathrm{kHz}$ active channel within the bin may result in the entire $1000 \mathrm{kHz}$ bin being declared as busy. As a result, frequency bin values larger than the signal bandwidth lead to important overestimations of spectrum occupancy in regions with moderate activity levels, which in turn results in greater average DCs for the entire band. In the case of UMTS the studied frequency bins are always lower than the signal bandwidth $(5 \mathrm{MHz})$. Although the average DC increases with the frequency bin, the difference is less significant (only $8.5 \%$ between 109 and $1000 \mathrm{kHz}$ ). This difference can indeed be ascribed to the fact that for the lower frequency bins some frequency points lie within the UMTS channels' guard bands (as shown in Figure 2.4(b)), where the DC is zero. It can therefore be concluded that if the frequency bin is larger than the bandwidth of the signal being measured, spectrum occupancy is notably overestimated. On the other hand, occupancy estimation is reasonably accurate as long as the frequency bin size remains acceptably narrower than the signal bandwidth.

Another aspect related to the frequency dimension is the employed Resolution BandWidth (RBW). Narrowing the RBW increases the system's ability to resolve signals in frequency and decreases the noise floor, which in turn improves the ability to detect weak signals, at the cost of increased measurement times [149]. This trend is illustrated in Table 2.5. As it can be appreciated, decreasing the RBW results in higher average DCs for the same band (enhanced capability to detect the presence of licensed signals within the measured frequency range) as well as higher average sweep times (i.e., longer inter-sample intervals and therefore longer required measurement periods to obtain the same number of samples). The band considered in this example (146-235 MHz) comprises transmissions from radio technologies with various signal bandwidths, including Private Mobile Radio (PMR) networks 
Table 2.5: Impact of the resolution bandwidth on the activity detected between 146 and $235 \mathrm{MHz}$.

\begin{tabular}{|c|c|c|}
\hline RBW & Average duty cycle & Average sweep time \\
\hline $3 \mathrm{kHz}$ & $58.04 \%$ & $7.49 \mathrm{~s}$ \\
\hline $10 \mathrm{kHz}$ & $56.08 \%$ & $2.81 \mathrm{~s}$ \\
\hline $30 \mathrm{kHz}$ & $50.84 \%$ & $1.85 \mathrm{~s}$ \\
\hline $100 \mathrm{kHz}$ & $43.30 \%$ & $0.92 \mathrm{~s}$ \\
\hline $300 \mathrm{kHz}$ & $40.36 \%$ & $0.79 \mathrm{~s}$ \\
\hline
\end{tabular}

$(12.5 / 25 \mathrm{kHz})$, wireless microphones $(200 \mathrm{kHz})$ and Digital Audio Broadcasting (DAB) systems $(1.54 \mathrm{MHz})$. As a result, the occupancy statistics shown in Table 2.5 implicitly include the effects of several RBWs on different signal bandwidths. In the performed experiment, the $10-\mathrm{kHz}$ RBW can be considered as an adequate trade-off between detection capability (represented by the average DC) and measurement time (represented by the average sweep time). For the results shown in Table 2.5, the $10-\mathrm{kHz}$ RBW configuration only misses the detection of $58.04 \%-56.08 \% \approx 2 \%$ of licensed signals with respect to the $3-\mathrm{kHz} \mathrm{RBW}$ configuration while it is able to capture $7.49 \mathrm{~s} / 2.81 \mathrm{~s}=2.67$ times more PSD samples within the same measurement period. Wider RBWs result in shorter average sweep times but higher estimation errors, up to $17.68 \%$ for the $300-\mathrm{kHz}$ RBW configuration. Moreover, since signal bandwidths of less than $10 \mathrm{kHz}$ are unusual, a 10-kHz RBW is sufficient to resolve signals in frequency for most of the existing radio technologies. Based on these observations, a $10-\mathrm{kHz} \mathrm{RBW}$ can be considered as an appropriate choice for broadband spectrum surveys, offering an adequate trade-off between detection capability and required measurement time.

\subsection{Time dimension}

The time dimension of spectrum measurements is mainly defined by two parameters, namely the sampling rate, i.e. the rate at which PSD samples are captured, and the measurement period. While the former is constrained (and in some cases automatically adjusted) by the measurement device, the latter can be easily controlled.

Very different measurement periods have been considered in previous spectrum measurement campaigns, as it can be appreciated in the summary shown in Table 2.2. The selected measurement period depends on the trade-off between the overall time required to complete the measurement campaign and the particular objectives of the measurement study. Some previous studies have been aimed at identifying spectrum usage patterns over long periods and understanding any potential seasonality in the visible spectrum usage. For such kind of studies, long-term measurement campaigns with measurement periods of several years have been suggested [133, 150]. However, from the standpoint of resource utilization, a short-term evaluation and characterization of spectrum usage is frequently more interesting since in practice it has an important impact on the behavior and performance of a DSA/CR network. In such a case, long-term measurements are not necessary. Although the employed measurement periods can be drastically shortened, a minimum number of PSD samples is required to correctly characterize the spectral activity of the measured bands. In this con- 
text, and from a statistical viewpoint, the question is how long should spectrum bands be measured in order to obtain a representative estimate of the actual spectrum usage. This section tries to answer this question by showing the effects of the measurement period on the obtained results in a quantitative manner.

A portion of the DCS downlink band (1862.5-1875.5 MHz) was selected and measured during 24 hours. The average DC for each measured frequency point was computed over 1-hour periods, thus obtaining the time evolution of the DC for different frequencies over one day. The obtained results are shown in Figure 2.5. As it can be appreciated in Figure 2.5 (a), the activity in the measured band is produced by at least two base stations, which can be inferred from the two broadcast channels that can clearly be identified at $1863.2 \mathrm{MHz}$ and 1867.4 MHz for their constant DCs (the latter is lower than $100 \%$ because of the distance from the base station and the experienced propagation loss). Traffic channels are also distinguishable in Figure 2.5(a) for their temporal variation. In the particular case of broadcast channels, the spectral activity is constant and hence the instantaneous DC matches the average value at every time instant. As a result, a 1-hour measurement period would report an acceptable estimate of the actual occupancy rate regardless of the start time. This conclusion is valid not only for broadcast channels of cellular mobile communication systems but in general for transmitters with a constant temporal activity pattern such as TV and FM broadcast stations, among many other types of wireless systems. The rest of the band in Figure 2.5(a) exhibits an oscillating behavior over time. When the entire band is considered, the instantaneous DC then notably differs from the average value. For example, Figure 2.5(b) indicates that a 1-hour measurement period started at 9:32 would report an occupancy rate of $41.58 \%$ while the same time span started at $12: 32$ and 15:32 would report average DCs of $68.37 \%$ and $33.75 \%$, respectively. None of these values is representative of the actual average usage of the band since the true mean over the 24-hour period is $35.60 \%$. Based on this discussion, a reasonable option to obtain representative results without any a priori information of the band to study is to consider periods of at least 24 hours in order to account for potential daily temporal patterns. Longer measurement periods would certainly enable a more reliable estimation of the spectral activity in allocated bands. For example, 48-hour periods would provide more realistic estimates, while 7-day periods would also show the potentially different usage patterns of some spectrum bands in weekdays and weekends. However, a 24-hour period can be considered as a reasonable trade-off between reliability of the obtained results and overall time required to complete the measurement campaign.

\subsection{Data post-processing}

While the previous sections dealt with aspects to be considered before the fieldwork, this section discusses different methods for post-processing the empirical data captured during the measurement stage and the impact of such methods on the obtained spectrum usage statistics. Regardless of the final objective of the measurement campaign (e.g., definition of more efficient DSA/CR policies, identification of sparsely used frequency bands or development of spectrum usage models), one of the very first steps of data post-processing is to determine which captured PSD samples correspond to busy and idle channels. 


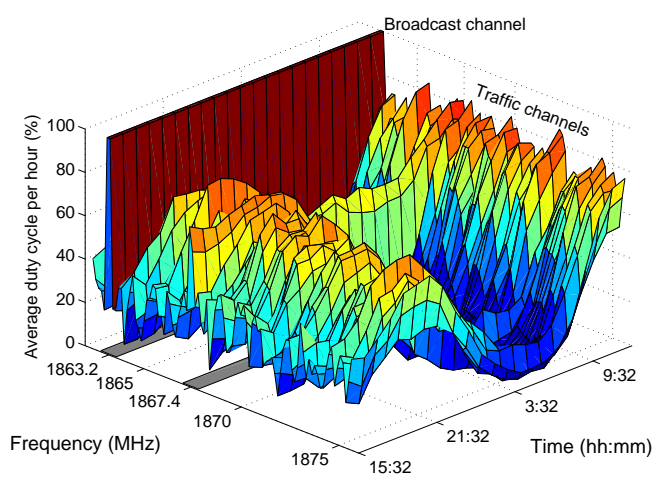

(a)

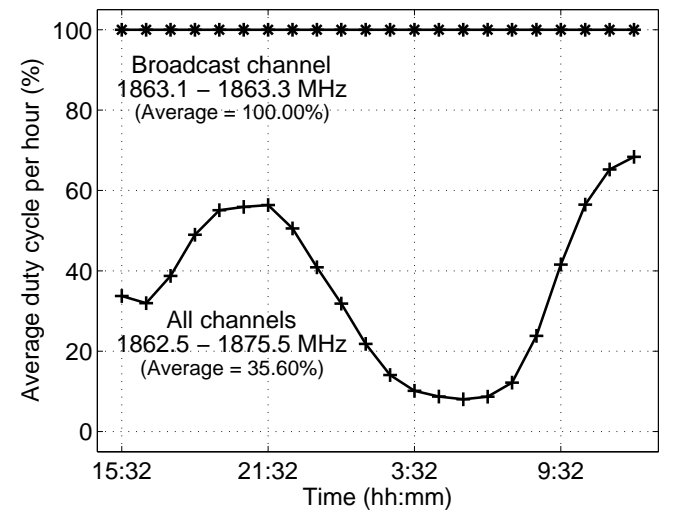

(b)

Figure 2.5: Average duty cycle per hour for DCS downlink.

To detect whether a channel is used by a licensed user, a number of different signal detection methods, referred to as spectrum sensing algorithms in the context of DSA/CR, have been proposed in the literature [151-155]. The existing solutions provide different trade-offs among required sensing time, complexity and detection capabilities. Their practical applicability, however, depends on how much information is available about the primary user signal. In the most generic case no prior information is available. If only low time-resolution power measurements of the spectrum utilization are available, the Energy Detection (ED) method is the only possibility left [156], which is able to work irrespective of the signal to be detected. Due to its simplicity and relevance to the processing of power measurements, ED has been a preferred approach for many past spectrum studies and also constitutes the spectrum sensing method considered in this work. ED compares the received signal energy in a certain channel to a properly set decision threshold. If the signal lies above the threshold the channel is declared to be busy (i.e., occupied by the licensed system). Otherwise the channel is supposed to be idle (i.e., available for secondary usage). Therefore, the measured PSD samples need to be compared to a threshold in order to determine whether they correspond to busy or idle channels.

The decision threshold is a critical parameter in data post-processing since its value severely impacts the obtained occupancy statistics. High decision thresholds may result in underestimation of the actual spectrum occupancy due to the misdetection of faded signals. On the other hand, excessively low decision thresholds may result in overestimation caused by noise samples above the threshold. As shown in Figure 2.6, different systems may exhibit different sensitivities to the variation of the decision threshold. In general, the DC for high-powered transmitters such as TV stations and cellular communication base stations (downlink direction) shows a lower decreasing rate as the decision threshold increases. On the other hand, for bands where the received signal levels are lower the DC is more sensitive to the decision threshold, with changes from $100 \%$ to $0 \%$ in $5 \mathrm{~dB}$ or less. This observation highlights the importance of using an adequate criterion to select the decision threshold. 
Several methods to determine the decision threshold have been employed in previous studies. Most of them are based on a priori knowledge of noise properties. The simplest way to determine the decision threshold based on the noise knowledge is via empirical analysis, where the collected measurements are visually inspected and the threshold is arbitrarily placed somewhere in the middle between the noise and signal curves. For example, the threshold in [127-132] is decided based on visual inspection of PSD curves. An alternative approach is adopted in [146], where the spectral occupancy (average DC) is computed as a function of the decision threshold as in Figure 2.6, and the decision threshold is manually placed between the curves corresponding to noise and busy channels. A similar scheme is employed in [157]. The main shortcomings of these approaches are their arguably lack of rigor, subjectivity and difficulty to be implemented in an automated fashion.

Other more rigorous methods to determine the decision threshold are shown in Figure 2.7. These methods assume a perfect knowledge of the noise properties, at least of the mean $X_{\text {mean }}(f)$, minimum $X_{\min }(f)$ and maximum $X_{\max }(f)$ values at different frequencies $f$, which can easily be measured by simply replacing the antenna with a matched load. A simple possibility to determine the decision threshold would be to select the maximum noise level $X_{\max }(f)$ recorded at each measured frequency point $f$ as the decision threshold $\lambda(f)$ :

$$
\lambda(f)=X_{\max }(f)
$$

This method will be referred to as MaxNoise criterion. This option guarantees that no noise samples lie above the threshold and therefore that spectrum occupancy is never overestimated. However, occupancy may be underestimated due to weak signal samples lying below the maximum noise level. To solve this problem, an alternative option is to fix the decision threshold $m$ decibels above the average noise level:

$$
\lambda(f)=X_{\text {mean }}(f)+m
$$

where $m$ represents a constant quantity added to the mean noise level (e.g., $m=6 \mathrm{~dB}$ as in [141] or $m=10 \mathrm{~dB}$ as suggested in [158]). This method will be referred to as $m-d B$ criterion. The main drawback of this method is that the noise variance and also the maximum noise level $X_{\max }(f)$ may vary band-by-band depending on several configuration parameters such as the frequency span, which makes difficult to precisely control the relation between the noise level and the decision threshold. As illustrated in Figure 2.7, a fixed decision threshold may be appropriate for certain measurement conditions but may not be adequate if the measurement configuration changes from one band to another. Therefore, a constant $\mathrm{m}$ $\mathrm{dB}$ threshold over the entire measurement range may not be appropriate. A different solution conciliating the previous methods is the Probability of False Alarm (PFA) criterion. In the context of DSA/CR, the PFA is defined as the probability that the DSA/CR network declares a channel to be occupied by a licensed signal when it is actually idle. This event may be caused by strong noise samples along with low decision thresholds. Based on a target PFA for a DSA/CR network equal to $P_{f a}$, the decision threshold $\lambda(f)$ at each measured frequency point $f$ is fixed such that only a fraction $P_{f a}$ of the noise samples $X(f)$, measured by replacing the antenna with a matched load, lie above the threshold:

$$
\lambda(f)=F_{X(f)}^{-1}\left(1-P_{f a}\right)
$$




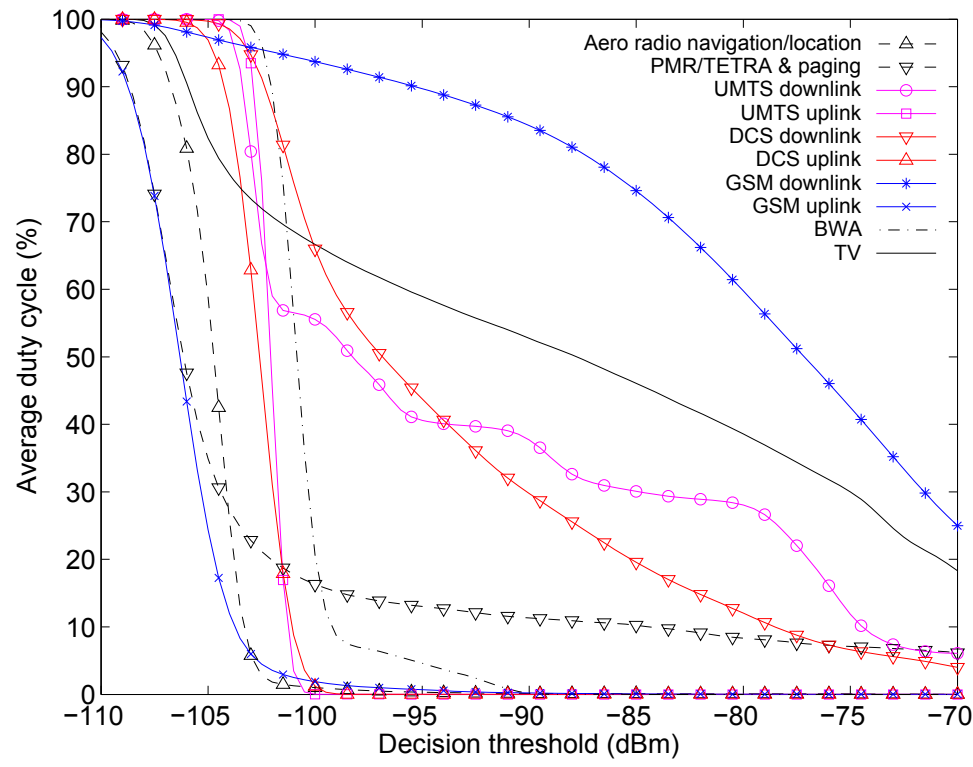

Figure 2.6: Average duty cycle as a function of the decision threshold for different systems: TV (470-862 MHz), BWA (3400-3600 MHz), GSM uplink (880-915 MHz) and downlink (925-960 MHz), DCS 1800 uplink (1710-1785 MHz) and downlink (1805-1880 MHz), UMTS uplink (1920-1980 MHz) and downlink (2110-2170 MHz), PMR/TETRA and paging (406.1-470 MHz), and aeronautical radio navigation/location (960-1350 MHz).

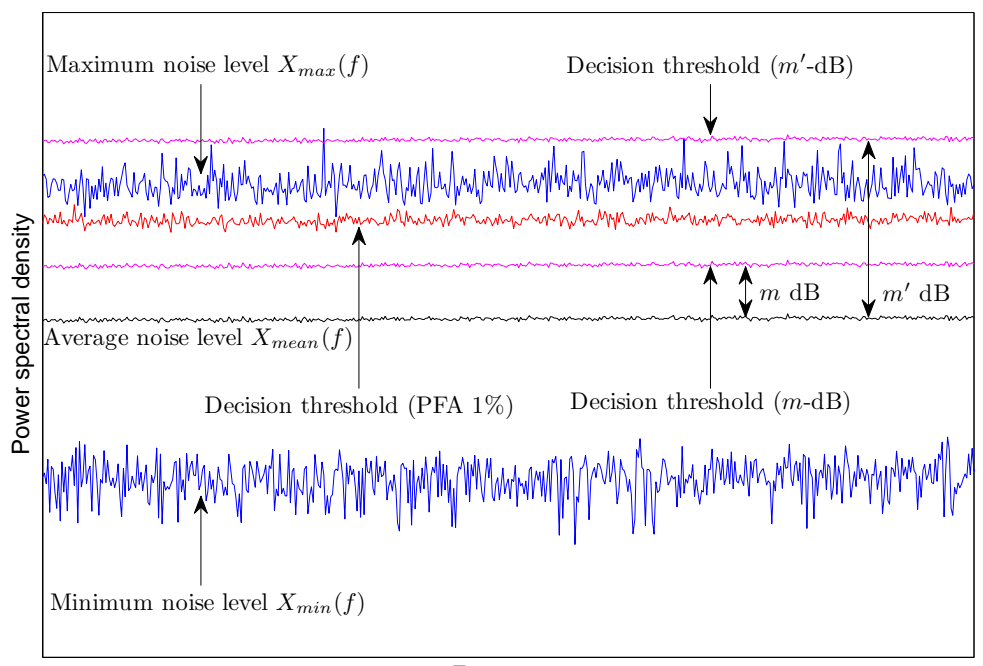

Frequency

Figure 2.7: Various criteria to determine the decision threshold. 
where $F_{X(f)}^{-1}(\cdot)$ is the inverse of $F_{X(f)}(\cdot)$, the Cumulative Distribution Function (CDF) of the noise values $X(f)$. It is worth noting that this alternative can be considered as an intermediate approach between the MaxNoise criterion, where spectrum occupancy is never overestimated, and the $m$ - $\mathrm{dB}$ criterion, where spectrum occupancy may be significantly over/underestimated. The maximum overestimation error in which the PFA criterion may incur is given by the value of $P_{f a}$. In other words, if the maximum fraction of noise samples allowed to lie above the decision threshold $\lambda(f)$ is equal to $P_{f a}$, spectrum occupancy will never be overestimated by an amount greater than $P_{f a}$.

There exists a second category of algorithms to determine the decision threshold without a priori knowledge of the noise properties. Some examples are the Otsu's algorithm [159] and the Recursive One-Sided Hypothesis Testing (ROSHT) algorithm proposed in [160]. The main drawback of these algorithms is that they are more complex and based on some assumptions that may not hold. Moreover, such assumptions are not necessary when noise properties can be known as it is the case. These methods are not considered in this study.

To quantitatively assess the impact of the decision threshold on the obtained occupancy statistics, the same set of empirical data was post-processed based on the ED principle but using the MaxNoise, $m-\mathrm{dB}$ and PFA criteria. The results obtained for various frequency bands are shown in Figure 2.8. Regarding the $m$ - $\mathrm{dB}$ criterion, the performed experiments demonstrate, as previously mentioned, that a constant value of $m$ fails to provide consistent results over the entire measurement range: while for some bands the obtained results are similar to those obtained with MaxNoise and PFA, for other bands the results are completely divergent. This indicates that the suitability of this criterion is highly questionable.

The rest of this section focuses on the comparison of MaxNoise and PFA. The comparison is performed based on the occupancy statistics obtained for four types of bands, namely bands allocated to a wide diversity of licensed systems (146-235 MHz and 235-317 MHz), high-power transmitters (470-870 MHz and 915-960 MHz), low-power transmitters (870$915 \mathrm{MHz}$ ), and sparsely used (2700-3000 MHz) or completely idle (5470-5875 MHz).

When the decision threshold is lowered from the MaxNoise criterion to the PFA $1 \%$ criterion, a maximum amount of $1 \%$ noise samples are allowed to lie above the decision threshold, which may be detected as signal samples. A maximum increase of $1 \%$ in the Dc due to noise samples is therefore expected in this case. However, Figure 2.8 indicates that the average DC for the 146-235 MHz and 235-317 MHz bands, which are allocated to a wide variety of licensed systems, increases $12.76 \%$ (from $43.32 \%$ to $56.08 \%$ ) and $12.55 \%$ (from $28.90 \%$ to $41.45 \%$ ), respectively, when moving from MaxNoise to PFA $1 \%$. Since these increments are higher than $1 \%$ this clearly means that PFA is able to detect some additional weak signals around the noise level in these bands. If the decision threshold is further lowered with the PFA 2\%, 5\% and 10\% criteria, more weak signals are detected and the resulting average DCs increase (and so does the maximum overestimation error). A similar trend is observed for weak GSM uplink signals (870-915 MHz), although in this case the PFA improvement is less significant.

In the 2700-3000 MHz band (where a few military radars are observed) and the 5470$5875 \mathrm{MHz}$ band (completely idle), the PFA criterion increases the average DC by the same amount as the $P_{f a}$ parameter of the algorithm. For example, when moving from PFA 2\% 

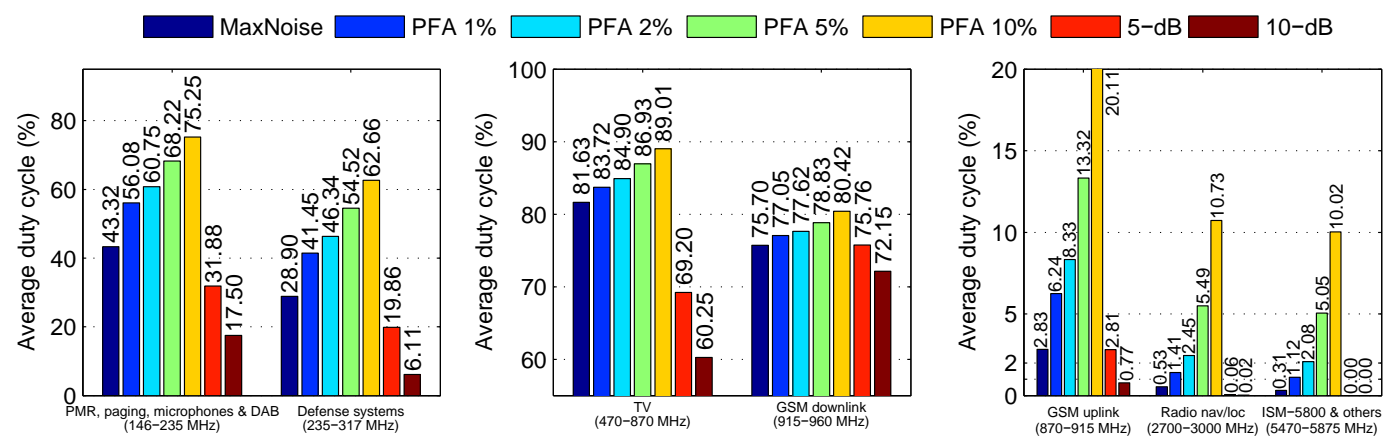

Figure 2.8: Impact of different decision threshold selection criteria on the detected activity for various licensed frequency bands.

to PFA 5\% the DC increases about 3\%. The absence of licensed signals in these bands indicates that such increase is not caused by the detection of true weak signals but noise samples above the threshold. As shown in Figure 2.8, a similar behavior is also observed for bands with high-power transmitters such as TV and GSM downlink. In this case, this means that in such bands lowering the decision threshold below the maximum noise level with the PFA criterion does not result in the detection of some additional weak signals, which indeed do not exist in these bands, but the misinterpretation of some noise samples as signals. In such a case PFA results in an overestimation error equal to $P_{f a}$ with respect to MaxNoise.

It can be concluded that PFA, in general, improves the detection performance of MaxNoise at the cost of a risk of incurring in overestimation errors in bands allocated to highpower transmitters. Increasing the target $P_{f a}$ of the PFA method improves the detection performance but results in an increased maximum overestimation error. Based on the obtained results, the PFA $1 \%$ criterion can be considered as a reasonable trade-off between the improvement in the ability to detect weak signals and the overestimation error in bands allocated to high-power transmitters.

\subsection{Summary}

Although several spectrum measurement campaigns have been performed in the context of DSA/CR, there is a lack of common and appropriate evaluation methodology, which would be desirable not only to prevent inaccurate results but also to enable the direct comparison of results from different sources. This chapter has presented a comprehensive and in-depth discussion of several important methodological aspects that need to be carefully taken into account when evaluating spectrum occupancy. A quantitative evaluation of the impact of different individual factors on the obtained results along with various useful guidelines have been provided as well. The results presented in this chapter highlight the importance of carefully designing an appropriate methodology when evaluating spectrum occupancy in the context of DSA/CR. This chapter has contributed a unifying methodological framework for future spectrum measurement campaigns. The findings of this chapter will be of great utility in the study reported in Chapter 3. 



\section{Chapter}

3

\section{EVALUATION OF SPECTRUM OCCUPANCY IN THE CONTEXT OF COGNITIVE RADIO}

\subsection{Introduction}

The DSA/CR paradigm is expected to improve spectrum usage efficiency in radio communication systems. Nevertheless, before investigating the technical issues of DSA/CR systems, and in order to address the real situation of spectrum usage, a clear picture of how different spectrum bands are used in specific environments is necessary. An appropriate study of spectrum usage enables the characterization of underutilized spectral resources in terms of time, frequency and space as well as the identification of the most suitable and interesting bands for opportunistic access. The measurement of real network activities constitutes a valuable tool towards a realistic understanding of dynamic spectrum usage.

Spectrum utilization has already been evaluated in various measurement campaigns all over the world. However, when the study reported in this chapter was initiated (around the middle of 2008), most of them had been carried out in the United States. Very few studies had taken place in other locations. Some examples are the measurements performed in New Zealand in 2007, Singapore in 2008 and some European cities such as Dublin, Ireland in 2007 and Aachen, Germany in 2007 (see Table 2.1). Thenceforth, the number of reported spectrum occupancy studies in the context of DSA/CR has grown to include a greater number of locations all over the world such as Doha, Qatar [161], Bucharest, Romania [162], Amsterdam, Netherlands [163], Brno, Czech Republic [164], Paris, France [164] and some additional cities in the United States [165].

All these spectrum measurements provide a valuable source of information for a realistic understanding of real spectrum usage. However, to enable a large scale deployment, the DSA/CR technology cannot be developed based on the conclusions derived from the studies conducted in a few geographical areas or under specific spectrum regulations. In contrast, the development of the DSA/CR technology should take into account the possibility to operate under many different spectrum regulations and a wide variety of scenarios. Therefore, 
spectrum occupancy information needs to be consistently collected over a wider range of locations in order to assess the variations of spectrum usage in environments with various spectrum regulations, user profiles and habits, geographical characteristics and population densities. In this context, this chapter presents an extensive and rigorous broadband spectrum measurement campaign performed in the metropolitan area of Barcelona, Spain. The main objective of this study is to evaluate and analyze the availability of free spectral resources in terms of time, frequency and space over a wide range of practical scenarios, thus identifying frequency bands suitable for DSA/CR deployments. A second objective is to capture spectrum data to support the development of spectrum usage models in Part III. The employed measurement campaign relies on a carefully designed measurement equipment and methodology (see Appendix A and Chapter 2) and covers a wide range of frequencies (75-7075 MHz), scenarios (in indoor and outdoor environments) and locations (in urban and sub-urban areas). To the best of the author's knowledge, this is the first study of these characteristics performed under the scope of the Spanish spectrum regulation in the context of DSA/CR, and one of the earliest studies in Europe. The measurements have been analyzed and compared to the official spectrum allocations [166], demonstrating the existence of a significant amount of spectrum available for the deployment of future DSA/CR networks.

The rest of this chapter is organized as follows. First, Section 3.2 reviews some previous spectrum measurement campaigns. The employed measurement configuration, which relies on the findings of the study performed in Chapter 2, is described in Section 3.3. Section 3.4 presents the scenarios considered in this study, while Section 3.5 analyzes the obtained results. Finally, Section 3.6 summarizes and concludes the chapter.

\subsection{Previous spectrum measurement campaigns}

The first larger spectrum occupancy measurement campaign was performed between 1993 and 1995 by the National Telecommunications and Information Administration (NTIA) [123-126], responsible for managing the Federal Government's use of the radio spectrum in the United States. The study measured the spectrum usage in several metropolitan areas across the frequency range from $108 \mathrm{MHz}$ to $19.7 \mathrm{GHz}$. These survey reports show the maximum, minimum and average measured power levels of received signals in different bands. Although this information can be used to qualitatively assess the relative density of channel occupancy on a band-by-band basis, the temporal information of spectrum usage required to infer the statistical occupancy rates is not provided. The main conclusions of the study are the higher spectrum occupancy activities in coastal areas due to the presence of naval radars and the extensive occupancy of ISM bands.

Another large scale spectrum occupancy measurement campaign funded and ordered by the National Science Foundation (NSF) is reported in [127-136]. The spectrum utilization in several American cities and a European city (Dublin, Ireland) was evaluated in outdoor environments in a band-by-band basis between $30 \mathrm{MHz}$ and $3 \mathrm{GHz}$. Average occupancy rates between $1 \%$ and $18 \%$ were observed for the considered locations. This study can be considered as the first large measurement campaign performed in the context of DSA/CR and it certainly is a rigorous and methodical spectrum study covering a wide range of locations 
and environments. However, the different equipments and procedures employed in this study prevent from a fair comparison among various spectrum occupancy results. Moreover, the study covers the frequency range up to $3 \mathrm{GHz}$. Additional bands above $3 \mathrm{GHz}$ may exhibit interesting opportunities for DSA/CR and should therefore be analyzed as well.

An extensive outdoor spectrum survey in urban (Atlanta, USA), sub-urban (Atlanta, USA) and rural (North Carolina, USA) environments between $400 \mathrm{MHz}$ and $7.2 \mathrm{GHz}$ is presented in $[137,138]$. Low occupancy levels were also observed in this survey, namely $6.5 \%$, $5.3 \%$ and $0.8 \%$ for urban, sub-urban and rural scenarios, respectively. Several novelties with respect to previous measurement campaigns were introduced in this study. First, spectrum occupancy was evaluated not only in frequency and time but also for different polarizations (horizontal and vertical) and azimuths (6 directions). Moreover, in addition to threshold decision a more sophisticated decision algorithm was employed to identify busy bands. This decision algorithm was claimed to be able to detect weak signals marginally above the noise floor, exhibiting a much lower false alarm rate than the threshold-based method while retaining a similar probability of detection. Nevertheless, the unusual decision method considered leads to particular occupancy statistics that, although presumably more accurate, may not be comparable with the results of other measurement campaigns.

Some broadband measurement campaigns have also been carried out in Europe. In addition to the study performed in Dublin, Ireland documented in [135], the work reported in [139] describes a study conducted between $20 \mathrm{MHz}$ and $6 \mathrm{GHz}$ in both outdoor and indoor environments in Aachen, Germany. In the outdoor campaign, an unexpected occupancy of nearly $100 \%$ was obtained between $20 \mathrm{MHz}$ and $3 \mathrm{GHz}$. This surprising result was attributed to a high level of out-of-band transmissions, ambient noise and man-made noise that were captured by the highly sensitive measurement system employed and interpreted as licensed user signal because the considered ED scheme is not able to differentiate between signals and ambient noise without further information. In the indoor case several spectrum bands between 1 and $3 \mathrm{GHz}$ appeared to be idle for longer time periods, thus showing some possibilities for opportunistic spectrum access. The observed occupancy in the band from 3 to $6 \mathrm{GHz}$ was very low in both indoor and outdoor environments but the sensitivity of the measurement system in this case was presumably lower since pre-amplification was used up to $3 \mathrm{GHz}$. The study presented in [139] concludes that the main opportunities in outdoor environments reside in the frequency bands above $3 \mathrm{GHz}$. In the case of low-power indoor systems, some lower frequency bands may be usable for secondary access as well.

Broadband measurement campaigns have also been reported in [140] (with occupancy rates of $6.21 \%$ and $5.72 \%$ for outdoor and indoor environments, respectively, between 806 MHz and $2750 \mathrm{MHz}$ in urban Auckland, New Zealand) and [141] (with an overall occupancy level of $4.54 \%$ between 80 and $5850 \mathrm{MHz}$ in an outdoor urban environment in Singapore). Other spectrum studies reported in the literature have exclusively focused on specific aspects of the services operating in narrower spectrum bands [142-146, 167, 168]. Despite the measurements campaigns performed prior to the study presented in this chapter [123-146, $167,168]$, and the number of similar works published subsequently [161-165, 169, 170], the successfull deployment of the DSA/CR technology claims for a larger number of measured locations and spectrum allocations, thus motivating the work described in this chapter. 


\subsection{Measurement configuration}

The measurement equipment employed in this study relies on the same spectrum analyzer setup used in Chapter 2 (see Appendix A). Table 3.1 shows the main configuration parameters, which are based on the results obtained in Chapter 2.

The measured frequency range $(75-7075 \mathrm{MHz})$ was divided into 25 blocks of variable size (from 45 to $600 \mathrm{MHz}$ ) based on the Spanish frequency allocation chart [166]. These frequency blocks were defined to ensure that no spectrum band in [166] is split off and the resulting frequency bins, in accordance with the conclusions of Chapter 2, remain acceptably narrower than the RF bandwidth of the measured signals (the employed spectrum analyzer provides a fixed non-configurable number of 551 frequency points per span, hence the frequency span determines the resulting frequency bin). For example, GSM/DCS bands were measured with frequency spans of $45 \mathrm{MHz}$, which results in a frequency bin of $45 \mathrm{MHz}$ / $(551-1)=81.8 \mathrm{kHz}$ (i.e., narrower than the $200 \mathrm{kHz}$ RF bandwidth of the GSM/DCS signal). Similarly, $727.3 \mathrm{kHz}$ and $745.5 \mathrm{kHz}$ bins were employed to measure TV bands ( $8 \mathrm{MHz} \mathrm{RF}$ bandwidth) and UMTS bands (5 MHz RF bandwidth), respectively.

Based on the results obtained in Chapter 2, a 10-kHz RBW was selected as an adequate trade-off between detection capabilities and required measurement periods. The Video BandWidth (VBW) is a function that dates to analog spectrum analyzers, but is now nearly obsolete. It can be used to reduce the effect of the noise on the displayed signal amplitude. When the VBW is narrower than the RBW, this filtering has the effect of reducing the peakto-peak variations of the displayed signal, thus averaging noise without affecting any part of the trace that is already smooth. With modern digital spectrum analyzers this smoothing effect can be achieved by means of trace averaging. To eliminate this analog form of averaging, the VBW was set equal to the RBW.

Each of the 25 analyzed bands was measured either 1 or 24 hours, depending on the measurement location (see Section 3.4). When the circumstances allowed unattended operation, a 24-hour measurement period was selected as suggested in Chapter 2. In public places, where the presence of an operator was required, measurement periods of 24 hours were infeasible and were therefore shortened to 1 hour. The number of recorded traces/sweeps is a function of the sampling rate (i.e., the sweep time), which is automatically adjusted by the employed spectrum analyzer to the minimum value that ensures reliable results. The resulting range of average sweep times is also shown in Table 3.1.

As mentioned in Appendix A, the spectrum analyzer's built-in pre-amplifier is used only above $3 \mathrm{GHz}$, while the external pre-amplifier is always used. To simplify the presentation of the results obtained in low- and high-frequency bands, the noise floor values in the 75$3000 \mathrm{MHz}$ and $3000-7075 \mathrm{MHz}$ bands were artificially equalized by adding a $20 \mathrm{~dB}$ offset to the reference level in the $3000-7075 \mathrm{MHz}$ band (reference level offset). The reference level was then adjusted in accordance with the maximum power observed in each frequency range, while the amplitude scale was adjusted based on the noise floor. The spectral activity in the analyzed bands was monitored with no attenuation and making use of an average type detector, which reports the average power measured at each frequency bin. The best sensitivity of the measurement equipment is around $-129 \mathrm{dBm} / 10 \mathrm{kHz}$ (i.e., $5 \mathrm{~dB}$ above the absolute lower bound imposed by the thermal noise) as shown in Table 2.4. 
Table 3.1: Spectrum analyzer configuration.

\begin{tabular}{|c|c|c|c|}
\hline & Parameter & & lue \\
\hline \multirow{5}{*}{ 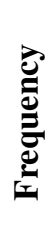 } & Frequency range & $75-3000 \mathrm{MHz}$ & $3000-7075 \mathrm{MHz}$ \\
\hline & Frequency span & \multicolumn{2}{|c|}{$45-600 \mathrm{MHz}$} \\
\hline & Frequency bin & \multicolumn{2}{|c|}{$81.8-1090.9 \mathrm{kHz}$} \\
\hline & Resolution BandWidth (RBW) & \multicolumn{2}{|c|}{$10 \mathrm{kHz}$} \\
\hline & Video BandWidth (VBW) & \multicolumn{2}{|c|}{$10 \mathrm{kHz}$} \\
\hline \multirow{2}{*}{$\stackrel{\mathscr{g}}{\mathrm{g}}$} & Measurement period & \multicolumn{2}{|c|}{1 or 24 hours } \\
\hline & Sweep time & \multicolumn{2}{|c|}{ Auto $(1.55-17.19 \mathrm{~s})$} \\
\hline \multirow{6}{*}{ 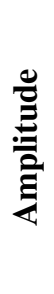 } & Built-in pre-amplifier & Deactivated & Activated \\
\hline & Reference level & $-20 \mathrm{dBm}$ & $-50 \mathrm{dBm}$ \\
\hline & Reference level offset & $0 \mathrm{~dB}$ & $-20 \mathrm{~dB}$ \\
\hline & Scale & \multicolumn{2}{|c|}{$10 \mathrm{~dB} /$ division } \\
\hline & Input attenuation & \multicolumn{2}{|c|}{$0 \mathrm{~dB}$} \\
\hline & Detection type & \multicolumn{2}{|c|}{ Average (RMS) detector } \\
\hline
\end{tabular}

\subsection{Measurement scenarios}

Most of previous spectrum occupancy studies are based on measurements performed in outdoor environments and more particularly in outdoor high points such as building rooftops, balconies and towers (see Table 2.1). The main advantage of high points is that they provide direct line-of-sight to various transmitters and therefore enable a more accurate measurement of the spectral activity. Nevertheless, this scenario may not be representative of the spectrum occupancy perceived by a secondary network in other interesting practical situations where the secondary antenna is not placed in a static high point (e.g., a mobile secondary user communicating inside a building or while walking in the street between buildings). The measurement of real network activities in additional scenarios of practical significance is therefore required for an adequate and full understanding of the dynamic use of spectrum.

The measurement locations considered in this study embrace a rich diversity of practical scenarios in urban and sub-urban areas. The scenarios analyzed in urban areas are illustrated in Figure 3.1 and include various locations within the Campus Nord of Universitat Politècnica de Catalunya (UPC) in urban Barcelona, not only outdoor high points (location 1), but also indoor environments (location 2) as well as outdoor locations at the ground level in narrow streets (locations 3-7), between buildings (locations 8-10) and in open areas (locations 11-12). For outdoor high point measurements (location 1), the equipment was placed in the rooftop of the three-floor building belonging to the Department of Signal Theory and Communications of UPC (latitude: $41^{\circ} 23$ ' 20" North; longitude: $2^{\circ}$ 6' 43" East; altitude: 175 meters). The selected place is a strategic location with direct line-of-sight to several transmitting stations located a few tens or hundreds of meters away from the antenna and without buildings blocking the radio propagation. This strategic location allowed to accurately measure the spectral activity of various transmitters such as TV broadcast stations, several nearby base stations for cellular mobile communications and a military head quar- 


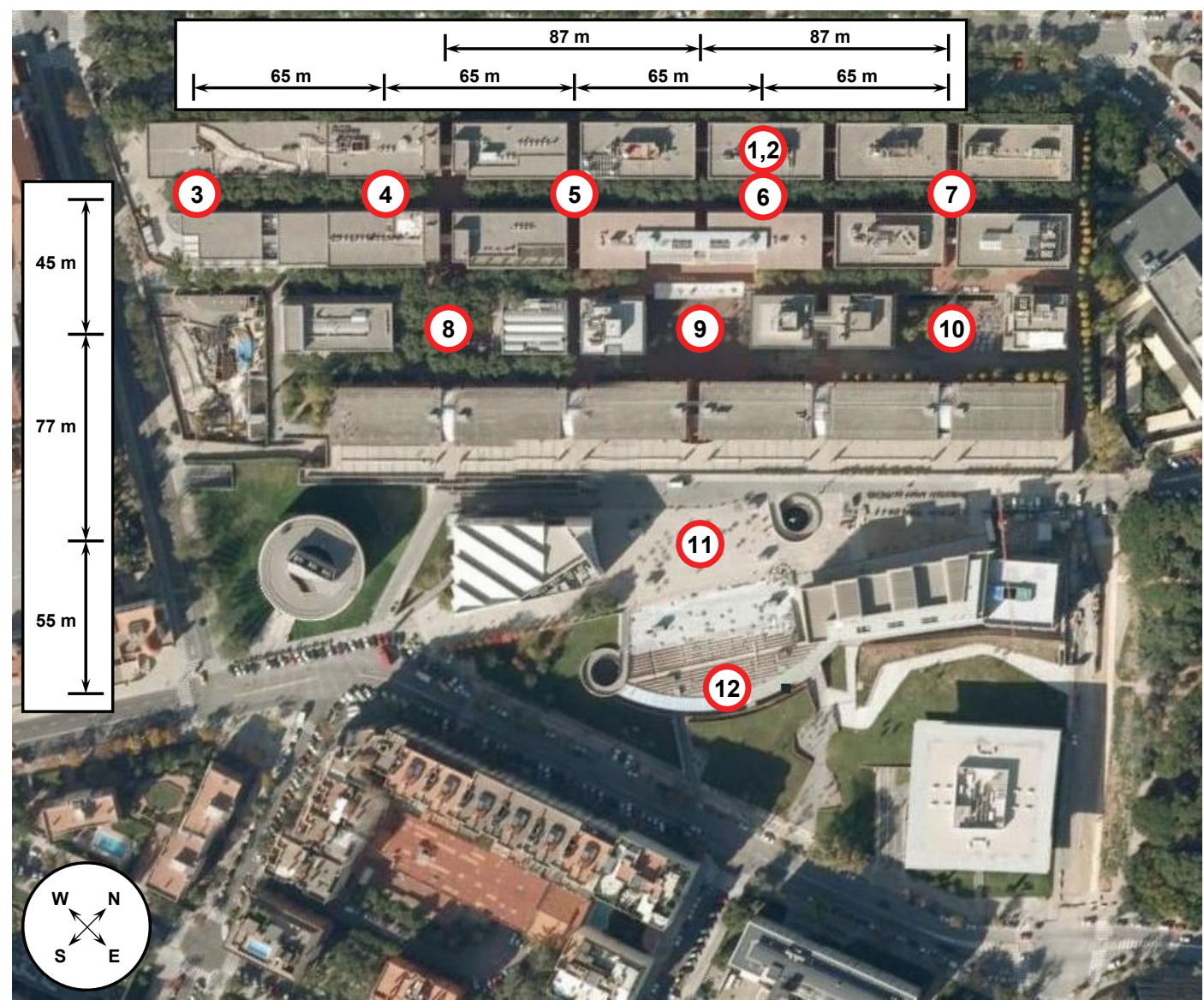

Figure 3.1: Measurement locations in urban environment.

ter as well as some maritime and aeronautical transmitters due to the relative proximity to the harbor and the airport. For indoor experiments, the measurement equipment was placed inside the same building (location 2) in the middle floor. For the rest of measurements at the ground level (locations 3-12) the equipment was moved along the campus as shown in Figure 3.1. This set of urban scenarios is complemented with an additional outdoor high point in UPC Campus Castelldefels (latitude: $41^{\circ} 16^{\prime} 31^{\prime \prime}$ North; longitude: $1^{\circ} 59^{\prime} 13$ " East; altitude: 175 meters), which constitutes a representative example of sub-urban environment. The variety of considered measurement locations provides a broad view and understanding of dynamic spectrum usage under various scenarios of practical interest.

\subsection{Measurement results and analysis}

\subsubsection{Occupancy metrics}

Spectrum utilization is characterized and quantified in this section by means of three occupancy metrics, which are shown in the figures presented in the following sections. The first 
occupancy metric is the Power Spectral Density (PSD), which is shown in the upper graph of each figure in minimum, maximum and average values for each measured frequency. When considered together, minimum, maximum and average PSD provide a simple characterization of the temporal behavior of a channel. For example, if the three PSD values are quite similar, it suggests a single transmitter that is always on, experiences a low level of fading and is probably not moving. At the other extreme, a large difference among minimum, maximum and average suggests a more intermittent use of the spectrum [145].

The second occupancy metric, shown in the middle graph of each figure, represents the instantaneous evolution of the temporal spectrum occupancy over the whole measurement period. Black dots represent samples of busy channels, while the white color indicates the absence of licensed signals. The busy/idle state of each measured frequency is determined following an ED principle. The ED decision threshold has been set for a PFA of 1\%, which provides a reasonable trade-off between detection capability and maximum overestimation error as discussed in Chapter 2.

The third considered occupancy metric is the DC, which is shown in the lower graph of each figure. This metric, computed as described in Appendix B, represents the fraction of time that a certain channel or frequency range is observed as busy over a specified period of time, thus providing a more precise quantification of spectrum occupancy.

\subsubsection{Urban outdoor high points}

The obtained measurement results are shown in Figures 3.2, 3.3 and 3.4. As it can be appreciated, spectrum experiences a relatively moderate use below $1 \mathrm{GHz}$ and a low usage between 1 and $2 \mathrm{GHz}$, while remains mostly underutilized between 2 and $7 \mathrm{GHz}$ (with some clear exceptions that will be discussed later on). In fact, while the average DC between 75 and $2000 \mathrm{MHz}$ is $31.02 \%$, the value of this parameter between 2000 and $7075 \mathrm{MHz}$ is only $2.75 \%$, as shown in Table 3.2. The overall average DC over the considered frequency range is $17.78 \%$, which reveals the existence of significant amounts of unused spectrum that could potentially be exploited by future DSA/CR networks. Although these results clearly indicate low spectrum utilization levels, they do not provide a clear picture of how spectrum is used in frequency bands allocated to different specific services. Therefore, a more detailed discussion on the spectrum usage in some allocated bands of interest is provided next.

Despite the highest spectral activity is observed below $1 \mathrm{GHz}$, some opportunities for DSA/CR networks can still be found in this frequency range, even in those bands with the highest observed average DCs. For example, the frequency band 470-862 MHz (see Figure $3.5(\mathrm{a})$ ), which is allocated to analogical and digital terrestrial TV, shows an average DC of $82.08 \%$, one the highest values observed in this study. Although the sub-band $830-862 \mathrm{MHz}$ (exclusively reserved for digital TV systems) exhibits an intensive usage of nearly $100 \%$ that precludes any DSA/CR applications, the rest of the band between 470 and $830 \mathrm{MHz}$ (allocated to both analogical and digital TV systems) shows some spectrum white spaces ${ }^{1}$. Note that busy TV channels show DCs of about 100\% (i.e. continuous broadcasting), which

\footnotetext{
${ }^{1}$ This frequency organization corresponds to the situation at the moment the measurement campaign was performed. Later on, by the end of 2010, a spectrum refarming process affecting TV bands was initiated.
} 

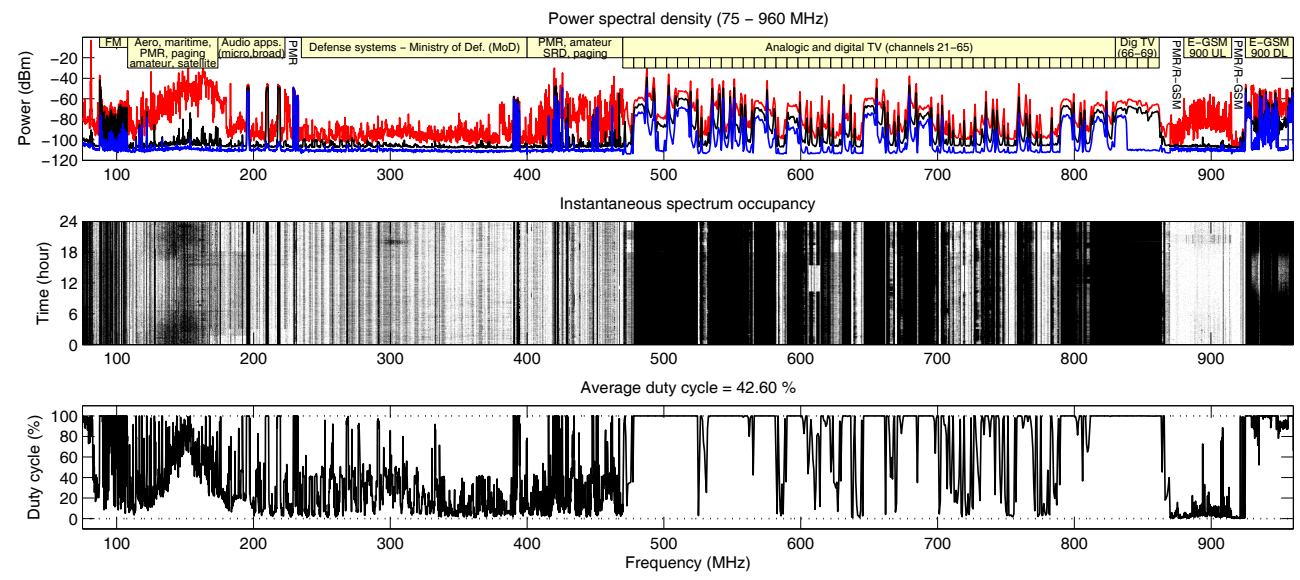

Figure 3.2: Spectrum occupancy in location 1 (75-960 MHz).
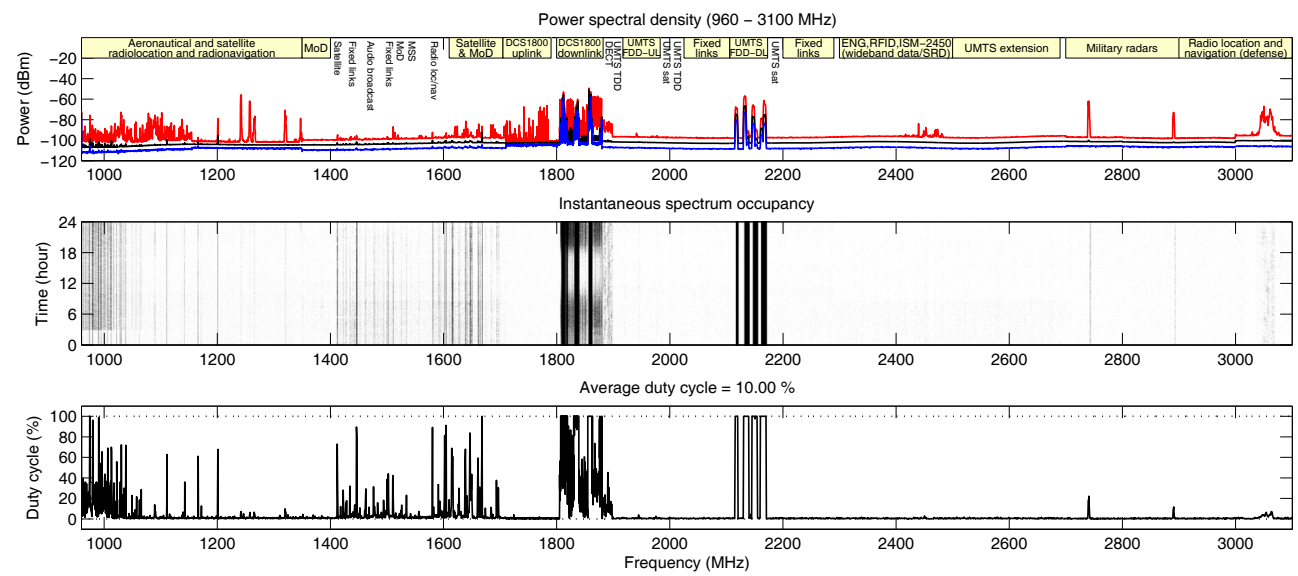

Figure 3.3: Spectrum occupancy in location 1 (960-3100 MHz).
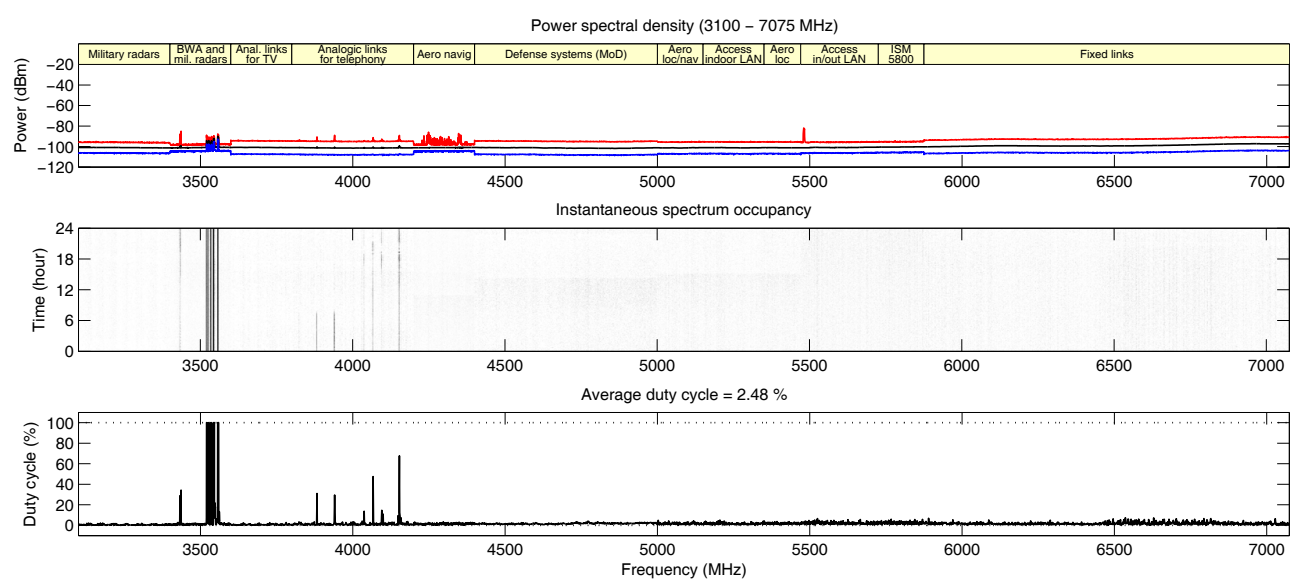

Figure 3.4: Spectrum occupancy in location 1 (3100-7075 MHz). 
Table 3.2: Average duty cycle statistics in location 1.

\begin{tabular}{|c|c|c|c|}
\hline Frequency (MHz) & \multicolumn{3}{|c|}{ Average duty cycle } \\
\hline $75-1000$ & $42.00 \%$ & \multirow{2}{*}{$31.02 \%$} & \multirow{7}{*}{$17.78 \%$} \\
\hline $1000-2000$ & $13.30 \%$ & & \\
\hline $2000-3000$ & $3.73 \%$ & \multirow{5}{*}{$2.75 \%$} & \\
\hline $3000-4000$ & $4.01 \%$ & & \\
\hline $4000-5000$ & $1.63 \%$ & & \\
\hline $5000-6000$ & $1.98 \%$ & & \\
\hline $6000-7075$ & $1.78 \%$ & & \\
\hline
\end{tabular}

impedes temporary opportunistic usage of such channels. Only one TV channel (channel $38,606-614 \mathrm{MHz}$ ) was disconnected during a short period in the night, which might be due to maintenance operations since this behavior was not observed for the same channel in previous experiments. Spectrum opportunities in this band come from TV channels that are received at low power levels. The obtained average DC between 470 and $830 \mathrm{MHz}$ is $80.49 \%$, meaning that one fifth of the TV band (approximately $80 \mathrm{MHz}$ ) can be considered to be idle due to the weak reception of the signals broadcasted from distant TV stations. Therefore, although the TV band appears as considerably populated in this study, it provides some interesting opportunities for secondary usage ${ }^{2}$.

Another interesting case below $1 \mathrm{GHz}$ is observed in the frequency bands allocated to GSM. The Extended GSM (E-GSM) 900 system operates in the 880-915 MHz (uplink) and 925-960 MHz (downlink) bands as shown in Figure 3.5(b). The uplink band appears as a potential candidate for secondary deployments with an average DC equal to $6.26 \%$. However, in this case it is important to highlight that the low activity recorded in this band does not necessarily imply that it could be used by DSA/CR networks. As a matter of fact, the maximum PSD observed in Figure 3.5(b) reaches significant values, revealing the presence of primary signals in uplink. The considerably higher activity in downlink (96.33\%) and the fact that GSM is based on Frequency-Division Duplex (FDD) suggest that the actual usage of the uplink band might be higher than the activity level recorded by the measurement system at the considered location. The unbalanced occupancy patterns observed between uplink and downlink in Figure 3.5(b) can be explained by the considerably higher transmission powers of GSM base stations and the considered measurement location with direct-line-ofsight to several nearby base stations, which flavor the detection of GSM downlink signals. On other hand, the lower transmission powers of GSM cellular phones along with the nonline-of-sight-conditions for terminals at the ground level hinder the detection of GSM uplink signals. As matter of fact, the maximum PSD observed for uplink in Figure 3.5(b) may be due to calls originated from nearby locations (e.g., the upper floors of the building). Therefore, from the obtained results it cannot be concluded that the E-GSM 900 uplink is subject to low activity levels. Similar situations have been observed in previous work (e.g., [141]).

\footnotetext{
${ }^{2}$ After the spectrum refarming process initiated in 2010 the amount of TV white space has decreased. However, at the moment of writing this document about $60 \mathrm{MHz}$ of the band is observed as idle at the same location, which can still be considered as a significant amount of TV white space for DSA/CR systems.
} 


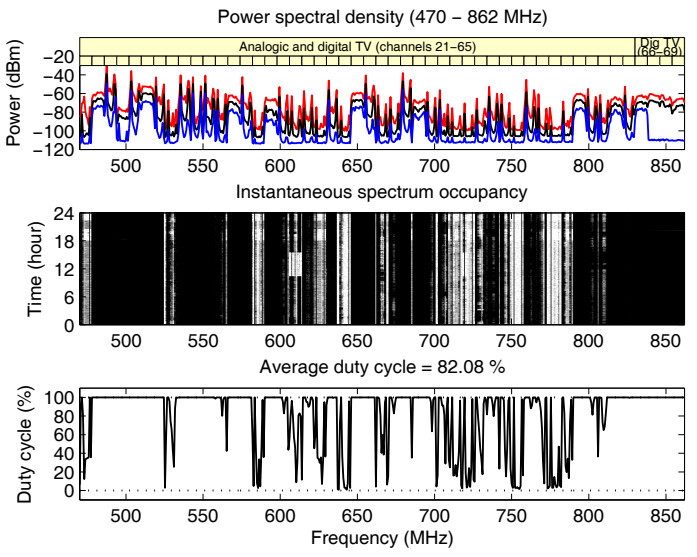

(a)
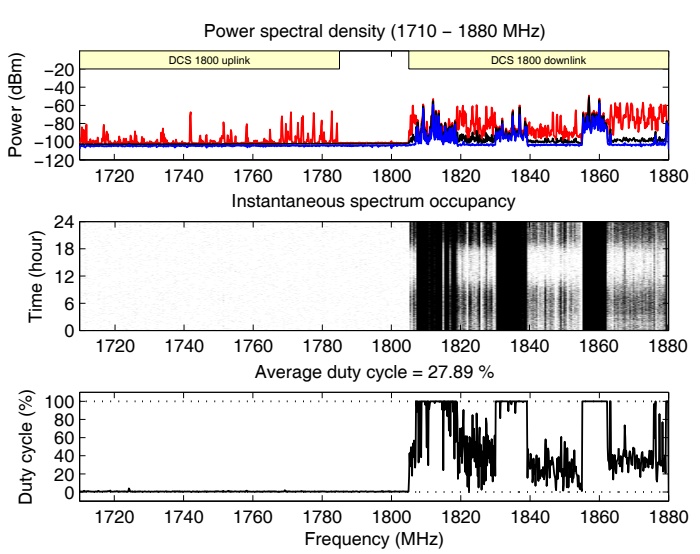

(c)
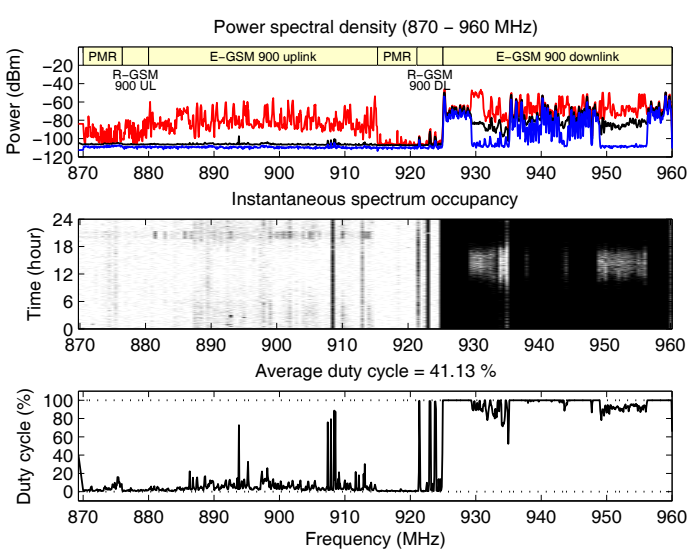

(b)
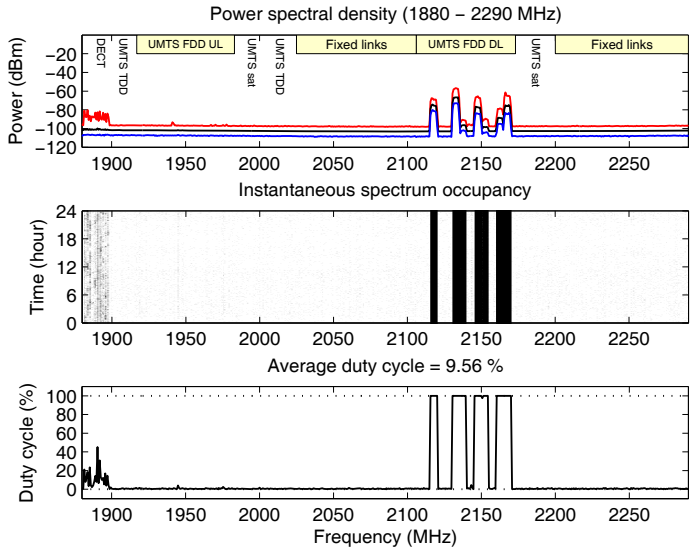

(d)

Figure 3.5: Spectrum occupancy in location 1 for: (a) TV (470-862 MHz), (b) E-GSM 900 (870$960 \mathrm{MHz}$ ), (c) DCS 1800 (1710-1880 MHz), and (d) UMTS (1880-2290 MHz).

In the lower spectrum bands $75-235 \mathrm{MHz}, 235-400 \mathrm{MHz}$ and 400-470 MHz, low to moderate average DCs of $48.59 \%, 24.88 \%$ and $29.85 \%$, respectively, are observed. These bands are populated by a wide variety of narrowband systems, including Private/Professional and Public Access Mobile Radio (PMR/PAMR) systems (75.2-87.5 MHz, 223-235 MHz, 406-430 MHz and 440-470 MHz), FM audio broadcasting (87.5-108 MHz), aeronautical radionavigation and communication systems, maritime systems as the Global Maritime Distress and Safety System (GMDSS), paging systems and fixed links (108-174 MHz), audio applications such as wireless microphones (174-195 MHz) and Digital Audio Broadcasting (DAB) systems (195-223 MHz), satellite systems (137-138 MHz and 400-406 MHz) and amateur systems (144-146 MHz and 430-440 MHz). Although these bands exhibit low to moderate average DCs, there is no significant amount of white space due to the narrowband nature of the systems operating on them. Moreover, the whole band from 235 to $400 \mathrm{MHz}$ 
is exclusively reserved for security services and defense systems of the Spanish Ministry of Defense, which in principle precludes the use of such spectrum bands for DSA/CR applications. Other bands below $1 \mathrm{GHz}$ with low or moderate levels of activity but narrower available free bandwidths are those allocated to wireless microphones and Radio Frequency IDentification (RFID) devices (862-870 MHz), CT1 cordless phones (870-871 and 915-916 $\mathrm{MHz}$ ), cellular access rural telephony (874-876 and 919-921 MHz) and the Railway GSM (R-GSM) 900 system (876-880 and 921-925 MHz).

Between 1 and $2 \mathrm{GHz}$, spectrum is subject to a low level of utilization, while remains mostly underutilized between 2 and $7 \mathrm{GHz}$. Above $1 \mathrm{GHz}$ the highest spectrum usage is observed for the bands allocated to the DCS 1800 system operating on 1710-1785 MHz and 1805-1880 MHz (Figure 3.5(c)), the UMTS system on 1920-1980 MHz and 2110-2170 $\mathrm{MHz}$ (Figure 3.5(d)), and BWA systems operating between 3520 and $3560 \mathrm{MHz}$.

Note that the differences between uplink and downlink usage patterns that have been appreciated for E-GSM 900 are also observed for DCS 1800 and UMTS. In the case of DCS 1800 the differences are more accentuated due to the fact that mobile stations in DCS 1800 have lower transmission powers than in GSM 900, which results in a reduced occupancy for the uplink. In the case of UMTS the difference is higher due to the spread spectrum nature of the Wideband Code Division Multiple Access (WCDMA) radio technology employed by UMTS, which leads to modulated signals that are difficult to detect with spectrum analyzers. As a result, very low activity is observed in UMTS uplink. Although DCS 1800 and UMTS show higher occupancy levels in downlink (58.82\% and 56.93\% respectively), these bands also offer some opportunities for secondary access. In the case of DCS 1800 some portions of the downlink band show a well defined periodic pattern (see Figure 2.5(a)) that could be exploited by some secondary DSA/CR applications by accessing spectrum during lowoccupancy periods. In the case of UMTS, spectrum opportunities can be found in several $5-\mathrm{MHz}$ channels observed as idle. Moreover, the UMTS bands reserved for the Time Division Duplex (TDD) component (1900-1920 and 2010-2025 MHz), the satellite component (1980-2010 MHz and 2170-2200 MHz) and extension (2500-2690 MHz) are not used.

Although the highest activity above $1 \mathrm{GHz}$ is observed for DCS 1800, UMTS and BWA systems, some other bands are also clearly busy but at lower occupancy rates, which in principle offer additional opportunities for DSA/CR applications. Some examples are the 960-1350 MHz band allocated to aeronautical and satellite radio location and navigation systems, the 1400-1710 MHz band allocated to a variety of wireless systems such as Satellite Personal Communication Systems (S-PCS) as well as aeronautical radio navigation, audio broadcasting and defense systems, the $1880-1900 \mathrm{MHz}$ band allocated to Digital Enhanced Cordless Telecommunications (DECT) phones, the 2700-2900 MHz band allocated to military radars, and the $3800-4200 \mathrm{MHz}$ band used by analogical links for telephony.

Finally, it is worth noting as well that some spectrum bands appear as idle when judged by their average DCs. Nevertheless, the maximum PSD reveals that some primary users, although difficult to detect, are present in such bands. Some examples are the uplink bands for mobile communications, the $2400-2500 \mathrm{MHz}$ band (ISM-2450), the 2900-3100 MHz band (radio navigation and location defense systems) and the $4200-4400 \mathrm{MHz}$ band (allocated to aeronautical radio navigation). 


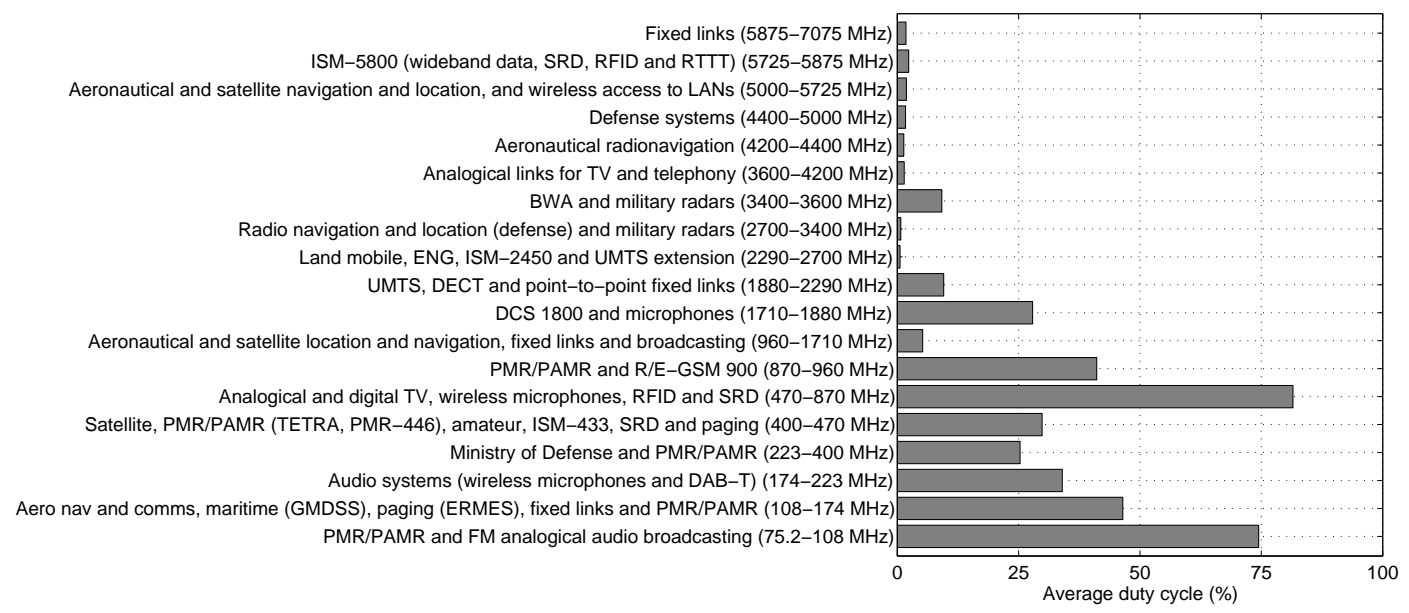

Figure 3.6: Band-by-band average duty cycle statistics in location 1.

To conclude this section, Figure 3.6 summarizes the band-by-band average spectrum occupancy statistics. The obtained results indicate that the actual spectral utilization is not uniform. Some spectrum bands are subject to intensive usage while some others show moderate utilization levels, are sparsely used and, in some cases, are not used at all. The highest occupancy rates are observed for bands allocated to broadcast services (TV as well as analogical and digital audio), followed by digital cellular services such as PMR/PAMR, paging, and cellular mobile communications (E-GSM 900, DCS 1800 and UMTS). Other services and applications, e.g. aeronautical radio navigation and location or defense systems, show different occupancy rates depending on the particular spectrum band. In summary, most of spectrum offers possibilities for secondary usage, even those bands with the highest observed activity levels in terms of average DC. Nevertheless, it is worth emphasizing that the average DC by itself is not a sufficient statistic to reliably declare a spectrum band as truly idle. As a matter of fact, the maximum PSD of some portions of the spectrum have revealed that some allocated frequency bands with approximately null average DCs are actually being used by licensed systems. These aspects should carefully be taken into account when selecting frequency bands for real DSA/CR deployments.

\subsubsection{Urban indoor locations}

This section presents and analyzes the results obtained in a urban indoor environment (location 2) taking as a reference the results obtained in an outdoor high point (location 1). The aim of this section is to determine the impact of an indoor environment on the spectral activity perceived by a secondary user with respect to that observed in an outdoor high point. The qualitative analysis performed in this section will be complemented with a more quantitative treatment of the problem in Chapter 10.

In this study, the measurement configurations employed in both locations were identical (see Section 3.3) but the time instants were different (i.e., locations 1 and 2 were not measured simultaneously). This circumstance introduces some random component in the ob- 
Table 3.3: Cases considered in Figure 3.7.

\begin{tabular}{|c|c|c|}
\hline Case & Transmitter location & Receiver location \\
\hline I & Outdoor & Outdoor \\
\hline II & Outdoor & Indoor \\
\hline III & Indoor & Outdoor \\
\hline IV & Indoor & Indoor \\
\hline
\end{tabular}

tained results since different transmissions were present in each case. However, it is worth noting that the aim of this section is not to characterize the instantaneous spectrum occupancy in the time domain but the average occupancy rate from a statistical point of view. For sufficiently long measurement periods as those considered in this case $(24$ hours for both locations), the impact of different instantaneous transmissions is averaged and the resulting DC constitutes a representative indication of the spectral activity in the analyzed bands.

From a qualitative point of view, the results obtained in location 2 follow the same trend as in location 1. More concretely, the average spectrum occupancy is moderate below $1 \mathrm{GHz}$ $(33.70 \%)$ and very low above $1 \mathrm{GHz}(1.39 \%)$, with an overall occupancy rate of $12.10 \%$. The lower occupancy rates observed for the indoor location can be explained by the fact that most wireless transmitters are located outdoor and the propagation loss due to the outdoor-indoor signal penetration leads to lower signal levels in the indoor scenario, which in turn results in lower occupancy rates. In principle, the lower average DCs obtained indoor suggest the existence of a higher amount of spectrum opportunities. However, this result should be interpreted carefully, taking into account the specific circumstances of particular bands.

To analyze the impact of the indoor location on the occupancy rate for various specific bands, a distinction among four different possible cases is made according to the location of transmitters and receivers as shown in Table 3.3. Based on this classification, the results for various bands of interest are shown in Figure 3.7. Note that for certain bands the classification is not straightforward. For example, in the downlink direction of cellular mobile communication systems the receivers are mobile users that may be located indoor and outdoor simultaneously. In practice, the location of every transmitter and receiver operating on a certain band cannot be reliably determined, which results in some uncertainty. In spite of that, some general trends can be inferred from the results shown in Figure 3.7.

For bands allocated to systems where the transmitters are outdoors (cases I/II), the indoor DCs are in general notably lower, as expected, due to the outdoor-indoor signal penetration loss. In case I (systems with outdoor receivers) the lower indoor occupancy rates indicate the availability of more free spectrum, since an indoor secondary user transmitting in channels sensed as idle would not cause harmful interference to primary outdoor receivers. However, in case II (systems with indoor receivers) the lower indoor DCs do not necessarily imply the existence of more white spaces, since in this case transmitting in a channel sensed as idle could potentially result in interference to primary indoor receivers.

For bands allocated to systems with indoor transmitters (cases III/IV), in general the average DCs tend to be higher in the indoor location (with some exceptions as the E-GSM 900 uplink band, which might be due to the presence of outdoor transmitters in such band). 


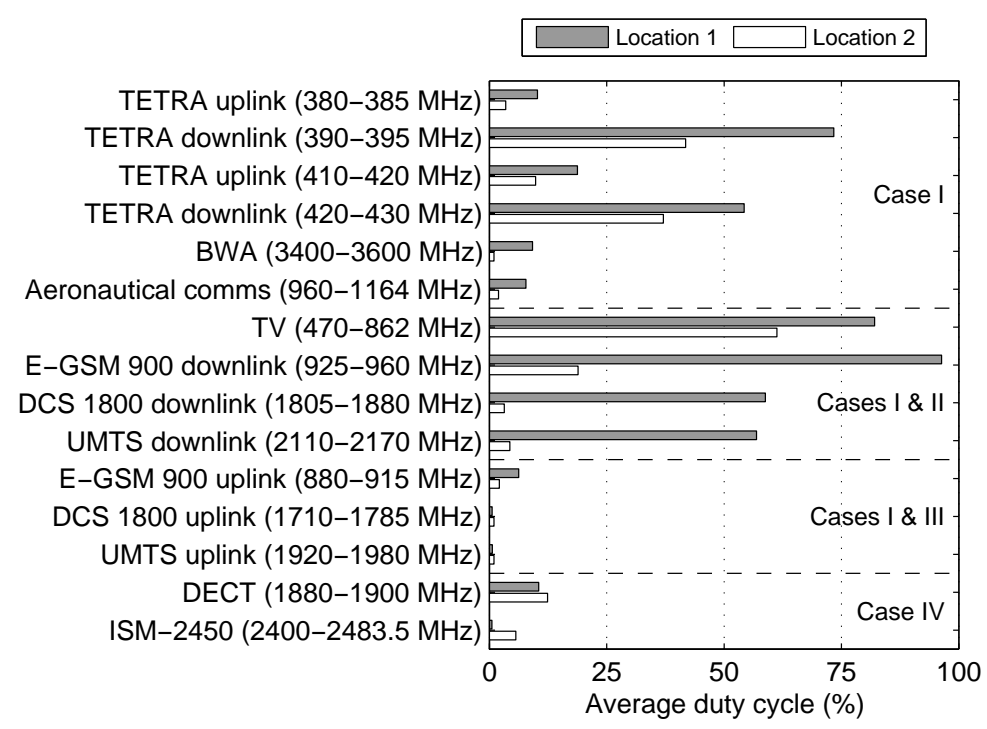

Figure 3.7: Average duty cycle statistics in locations 1 and 2 for specific bands.

Following a similar argument, in case IV (systems with indoor receivers) this indicates the availability of a lower amount of free spectrum, while it could not be necessarily the situation in case III (systems with outdoor receivers). However, the differences between outdoor and indoor locations in these cases are not as significant as in cases I/II.

In summary, although average DCs tend to be lower in indoor locations (with some unimportant particular exceptions), this does not necessarily indicate the existence of more free spectrum. The particular circumstances of the specific bands being sensed and the characteristics of the systems operating on them need to be carefully considered before declaring a band as truly available for potential secondary usage; otherwise, harmful interference could be caused to the primary licensed system. Based on these results, from a practical point of view it can be stated that the output of spectrum sensing procedures may not be enough to declare a band as truly available for secondary access. Some additional techniques may be required such as, for example, sensing both the uplink and downlink directions of FDDbased systems in order to guarantee that the channel can be accessed opportunistically, or employing signal processing techniques as the one described in [171] in order to determine the radio technology present in a certain band before deciding whether a band may be accessed without inducing harmful interference.

Another aspect of practical importance is stressed by the results obtained in the 75-235 $\mathrm{MHz}$ band, where the average DC was significantly lower in the outdoor location (48.43\%) than in the indoor location $(66.56 \%)$, despite that most systems operating in these bands are located outdoor. As observed in Figure 3.8(a), this difference can be ascribed to a higher noise floor above the energy decision threshold in the indoor experiment (high noise levels may be interpreted as primary signals when ED is employed since it is not able to differentiate between intended primary transmissions and external noise sources). Notice that in the FM band (88-108 MHz), where a FM band stop filter is employed, the noise floor is lower 


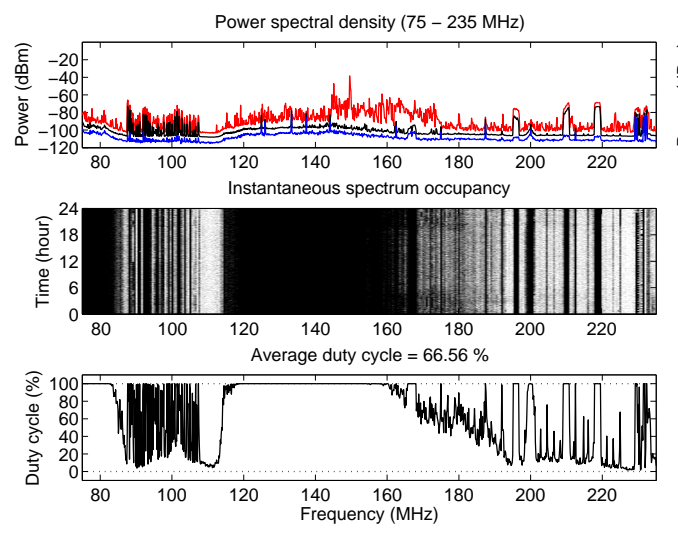

(a)

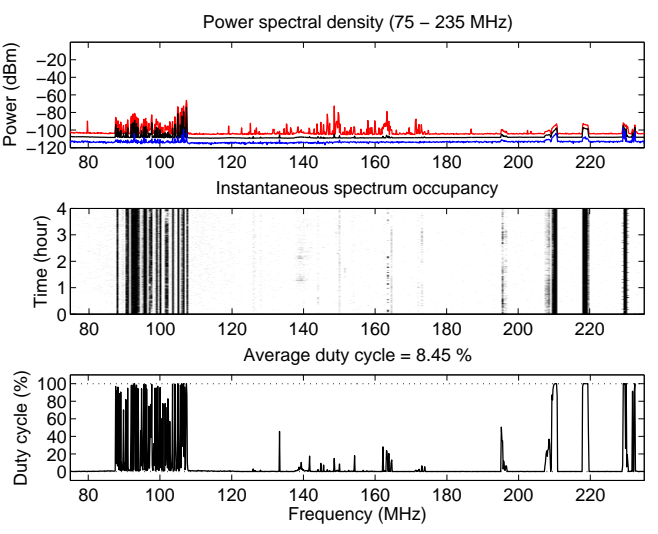

(b)

Figure 3.8: Spectrum occupancy in location 2 (75-235 MHz): (a) In the presence of man-made noise, (b) Without man-made noise.

than in the rest of bands, which indicates that the higher noise floor may be due to external noise sources (e.g., out-of-band transmissions, ambient noise or man-made noise). Manmade noise sources include emissions from electric motors (industrial equipments, trains, buses, etc.), Alternating Current (AC) power systems (consumer products, lighting systems, etc.), microwave ovens and spark plugs of automotive ignition systems, among many others $[172,173]$. Several measurements of the environmental noise floor have shown that the most significant man-made noise component is observed at frequencies below $200 \mathrm{MHz}$ $[174,175]$. At higher frequencies (above $1 \mathrm{GHz}$ ) studies of environmental noise have shown very low levels of noise except for emissions from microwave ovens centered at $2450 \mathrm{MHz}$ [176, 177]. Since man-made noise presents important variations with the environment [174], this could explain the differences observed between locations 1 and 2 in the $75-235 \mathrm{MHz}$ band. To verify this point, the indoor experiment was repeated after deactivating most of the identified potential man-made noise sources in the vicinity of the measurement equipment (computers, air conditioning systems and two racks of computing servers). In this case a maximum measurement period of 4 hours was possible before noise sources became active again. The obtained results are shown in Figure 3.8(b). As it can be appreciated, the noise floor in this case is virtually flat, indicating that man-made noise sources have been removed. Moreover, the obtained average DC is equal to $8.45 \%$, showing that the actual occupancy rate of this band is actually lower in indoor environments, as expected. From a practical point of view, these results show how external noise sources can alter the spectrum occupancy perception of DSA/CR terminals. Under such circumstance, a DSA/CR terminal would incorrectly infer a higher level of spectral activity and thus a lower amount of available spectrum opportunities. Again, this indicates the need to employ more sophisticated spectrum sensing algorithms than the ED scheme considered in this study (similar appreciations were pointed out in [178]) as well as some additional techniques as in [171] in order to minimize the waste of spectrum opportunities. 


\subsubsection{Urban narrow streets and open areas}

This section presents and analyzes the results obtained in urban narrow streets and open areas (locations 3-12) taking as a reference the results obtained in an outdoor high point (location 1). The aim of this section is to determine the impact of considering different outdoor locations at the ground level on the spectral activity perceived by a secondary user with respect to that observed in an outdoor high point. The locations under study in this section can be considered as a representative scenario for secondary mobile users communicating while walking on the street in a urban environment. The qualitative analysis of this section will be complemented with a more quantitative and comprehensive treatment in Chapter 10 .

As in Section 3.5.3, each location was measured at a different time instant. As it has been mentioned, the random component introduced by the presence of different transmissions at different time instants could be averaged by considering a sufficiently long measurement period. However, since the presence of an operator was required in these measurements, periods of 24 hours as in location 1 were infeasible and were therefore shortened to 1 hour in locations 3-12. To reduce the impact of random components and make the results of locations 1 and 3-12 comparable, the average DC, $\Psi_{k}$, obtained in locations $k=3,4, \ldots, 12$ (based on the samples captured during a 1-hour interval), were normalized by the average DC, $\Psi_{1}$, obtained for location 1 when considering the samples corresponding to the same 1-hour time intervals. The normalized average DC for location $k$ is thus computed as:

$$
\bar{\Psi}_{k}=\frac{\Psi_{k}}{\Psi_{1}}
$$

This procedure reduces the randomness of the obtained results and enables a fairer comparison among the outdoor high point and the rest of outdoor locations.

For most of the bands and locations measured in this experiment the obtained normalized average DC is lower than one, meaning that the average DC observed at the ground level is in general lower than at high points. This is a consequence of the radio propagation blocking caused by buildings and other obstacles: under non-line-of-sight conditions, the direct ray (i.e., the strongest signal component) is lost; only multi path propagation components attenuated by reflection, refraction and diffraction are received, thus resulting in lower received signal levels and therefore in lower average DCs. From a practical point of view, this indicates that a secondary user at the ground level would perceive a higher amount of spectrum opportunities. Nevertheless, it is worth highlighting that this observation should be interpreted carefully, taking into account the specific circumstances of each band. In the following, some particular bands of interest are discussed.

Figure 3.9 shows the spatial distribution of the normalized average DC for the TV, UMTS downlink, E-GSM 900 downlink and DCS 1800 downlink bands. The common feature of these bands is that the licensed transmitters are located outside the region under study. In the TV band, it can be clearly appreciated that the normalized average DC is lower in closed regions: in locations 4 and 6 , where radio propagation blocking caused by buildings is more intense, its value is lower than in other less closed regions such as locations 3,5 and 7 . A similar trend is observed for UMTS, E-GSM 900 and DCS 1800 downlink bands. In the two last cases, however, location 5 constitutes an exception, which could be explained by the 


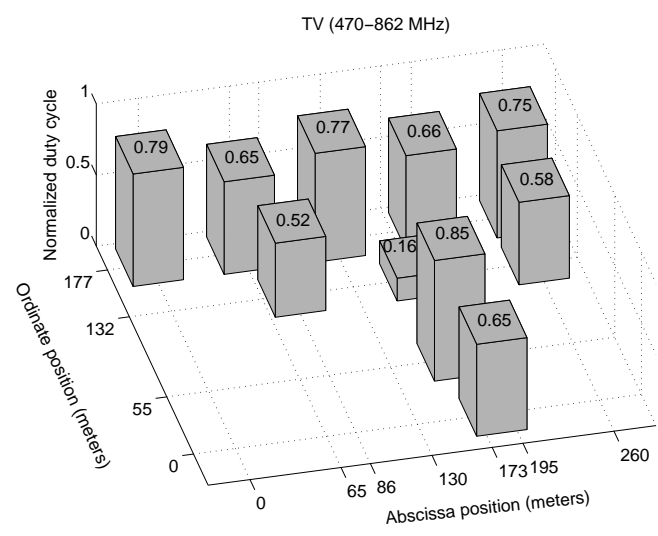

(a)

E-GSM 900 downlink (925-960 MHz)

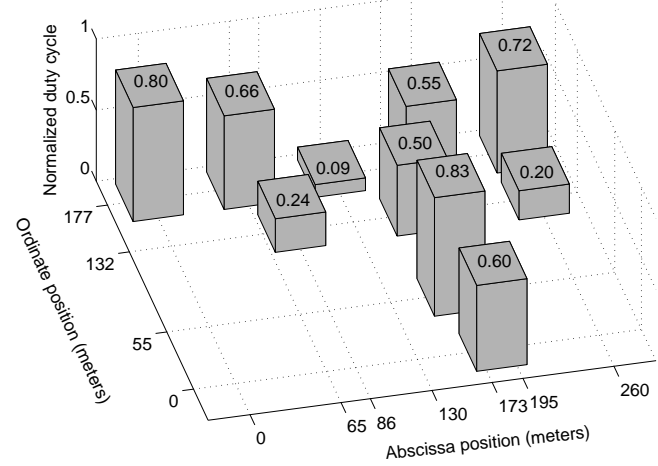

(c)

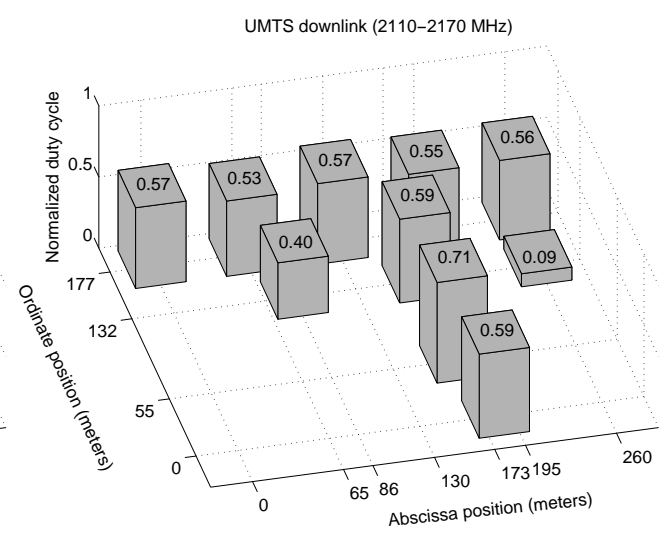

(b)

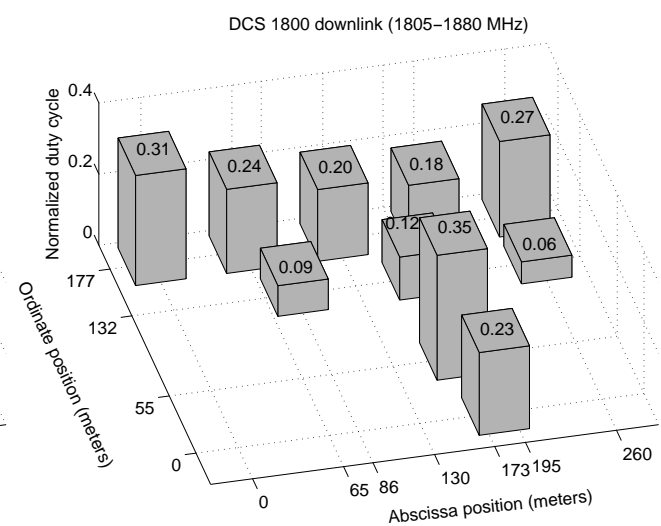

(d)

Figure 3.9: Normalized average duty cycle statistics in locations 3 to 12 for specific bands: (a) TV (470-862 MHz), (b) UMTS downlink (2110-2170 MHz), (c) E-GSM 900 downlink (925-960 MHz), and (d) DCS 1800 downlink (1805-1880 MHz). From left to right and top to bottom, the positions of the bars in each graph correspond to the physical locations of points $3-12$ in Figure 3.1.

different relative position of transmitters and by the fact that the same physical scenario may result in dissimilar propagation scenarios at different frequencies. Comparing locations 8, 9 and 10, the deepest region (location 9) exhibits the lowest normalized average DC in the case of TV, as expected, but the highest value in the case of the cellular mobile communication bands, which could be explained by the deployment of pico-cells and repeaters in shadowed regions as location 9. Regarding locations 11 and 12, it is interesting to note that a higher spectral activity level was recorded in location 11 despite the presence of some surrounding buildings not present around location 12. The detection by the measurement equipment of some additional signal components reflected in such buildings could explain the recording of higher activity levels in a less open region. Although lower DCs are observed at the ground level in the TV, UMTS, E-GSM and DCS 1800 downlink bands, it is worth noting that this does not necessarily imply the existence of more opportunities for secondary access. In 
fact, some faded signals might be undetected at the ground level due to blocking buildings and other obstacles, thus highlighting the importance of detection sensitivity in secondary networks and the need of some additional techniques as mentioned in Section 3.5.3.

In the previous bands, where the transmitters were outside the region under study, the results have shown some spatial patterns. For other bands with transmitters operating inside the region under study (e.g., the uplink direction of cellular communication systems), no particular occupancy patterns were observed, which suggests that in this case the observed spectral activity might mostly depend on the random mobility patterns of the users (transmitters) within the considered area.

In summary, the results presented in this section have demonstrated that the spectral activity perceived by a secondary DSA/CR user for a certain band in realistic urban scenarios strongly depends on the user location, with significant variations even in areas as reduced as the one considered in this study $(\approx 180 \mathrm{~m} \times 260 \mathrm{~m})$. This indicates that the conclusions derived from measurement studies performed in high points may not be well suited to other realistic outdoor scenarios with users at the ground level. Moreover, a qualitative relation has been identified between the level of radio propagation blocking and the level of spectrum occupancy perceived by the DSA/CR terminal. This observation will motivate a more detailed quantitative analysis of the spatial spectrum occupancy perception of secondary users as well as the development of appropriate spatial models of spectrum usage in Chapter 10.

\subsubsection{Sub-urban environments}

This section presents and compares the results obtained in urban and sub-urban environments based on measurements performed in outdoor high points. In this study, two identical measurement suites were deployed in urban (UPC Campus Nord, Barcelona) and sub-urban (UPC Campus Castelldefels) locations, about $23 \mathrm{~km}$ apart. As opposed to Sections 3.5.3 and 3.5.4 where the spectrum occupancy in various locations was evaluated at different time instants, the measurements reported in this section were performed concurrently ${ }^{3}$. Moreover, spectrum occupancy in this section is examined up to $3 \mathrm{GHz}$, since the results shown in Section 3.5.2 indicate that spectrum above $3 \mathrm{GHz}$ remains mostly unused.

The obtained results are shown in Figure 3.10. As it can be appreciated, the overall spectrum occupancy is appreciably higher in the urban environment of Barcelona (average DC of 21.21\%) than in the sub-urban environment of Castelldefels (average DC of 15.15\%). In particular, it is interesting to note that the bands allocated to cellular mobile communication systems such as DCS $1800(1805-1880 \mathrm{MHz})$ and UMTS (2110-2170 MHz) have more channels in use (and subject to a more intensive usage) in the urban environment. A similar trend is observed in other bands at lower frequencies (below $1 \mathrm{GHz}$ ). In some particular bands such as those allocated to broadcast services, e.g. TV (470-862 MHz) and DAB-T (195-223), where spectrum usage does not depend on the number of users of the service, the results are more similar in both environments. It is worth noting that most of the TV channels were observed in the same state (busy or idle) in both cities, which can be explained by the fact that the distance between them $(\approx 23 \mathrm{~km})$ is shorter than the coverage

\footnotetext{
${ }^{3}$ When the studies of Sections 3.5.3 and 3.5.4 were performed, only one measurement suite was available.
} 


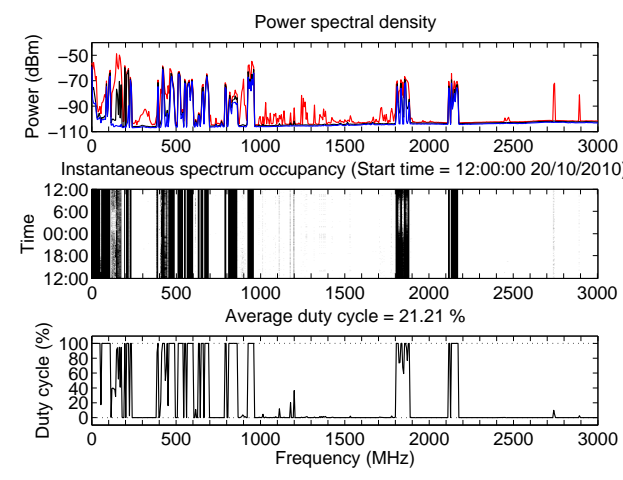

(a)

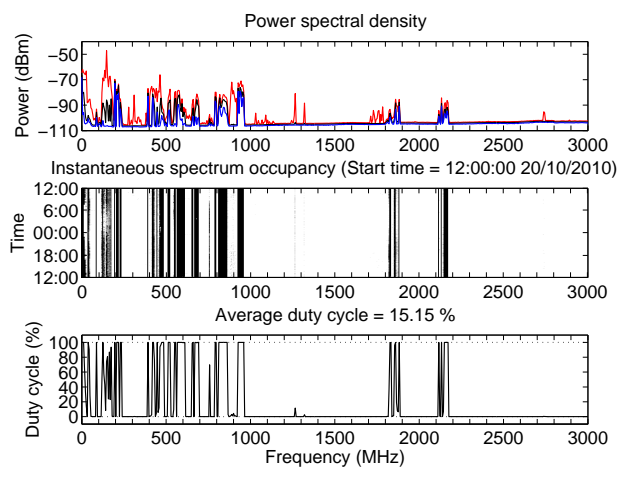

(b)

Figure 3.10: Spectrum occupancy: (a) Urban environment (Barcelona) and (b) Sub-urban environment (Castelldefels).

areas usually intended for TV transmitters and, as a result, both locations observe the same transmitters. An exception was noticed in a few channels, which may be due to local shortrange TV stations. In summary, the results obtained in this study indicate that spectrum usage is generally lower in sparsely populated areas. This indicates the existence of more spectrum opportunities for DSA/CR systems in these environments, which is in accordance with the conclusions of previous spectrum measurement campaigns (see Section 3.2).

\subsection{Summary}

This chapter has presented the results of a broadband spectrum measurement campaign conducted in the frequency range $75-7075 \mathrm{MHz}$ in the metropolitan area of Barcelona, Spain. The study embraces a wide variety of scenarios in indoor and outdoor environments as well as in urban and sub-urban areas. The obtained results have indicated that the actual utilization of spectrum is not uniform: some spectrum bands are subject to intensive usage while some others show moderate utilization levels, are sparsely used and, in some cases, are not used at all. However, the overall level of utilization is significantly low and most of spectrum offers interesting deployment possibilities for DSA/CR systems (even those bands with the highest observed activity levels), specially in sparsely populated areas.

The study reported in this chapter has also indicated that the spectrum occupancy level perceived by a DSA/CR terminal is tightly related to the surrounding radio propagation environment and, as such, depends on the secondary user location. The dependence of the perceived spectral activity with the user location along with the presence of external noise sources (e.g., man-made noise sources) that may alter the observed spectrum occupancy suggest the need for sophisticated signal detection procedures. The use of advanced spectrum sensing methods along with some complementary techniques would ensure a reliable estimation of spectrum occupancy, which would avoid harmful interference levels to licensed systems while maximizing the exploitation of spectrum opportunities. 



\section{Part II}

\section{High Time Resolution Measurements}

Opportunities? They are all around us...

There is power lying latent everywhere

waiting for the observant eye to discover it.

Orison Swett Marden

Learn to listen.

Opportunity could be knocking at your door very softly.

Frank Tyger 



\section{Chapter}

4

\section{EXPERIMENTAL EVALUATION OF ENERGY DETECTION PERFORMANCE}

\subsection{Introduction}

One of the most important challenges for a DSA/CR network is not to cause harmful interference to primary users. To guarantee interference-free spectrum access, secondary users must reliably identify the presence of primary users, which basically implies being able to determine whether a primary signal is present within a certain frequency range [179]. A number of different signal detection methods, referred to as spectrum sensing algorithms in the context of DSA/CR, have been proposed in the literature to identify the presence of primary signal transmissions [151-155]. Some examples of the existing proposals include Energy Detection (ED) [156], matched filter detection [180, 181], feature detection techniques based on cyclostationarity [182, 183], autocorrelation properties [184], statistical covariances [185], and other signal properties [186-189], multi-taper spectrum estimation [190] and filter bank spectrum estimation [191]. The existing solutions provide different trade-offs between required sensing time, complexity and detection capabilities. Their practical applicability, however, depends on how much information is available about the primary user signal. In the most generic case, a DSA/CR user is not expected to be provided with any prior information about the primary signals that may be present within a certain frequency band. When the secondary receiver cannot gather sufficient information, the ED principle [156] can be used due to its ability to work irrespective of the signal to be detected. Despite its practical performance limitations, ED has gained popularity as a spectrum sensing technique for DSA/CR due to its general applicability and simplicity as well as its low computational and implementation costs. ED has been a preferred approach for many past spectrum sensing studies and also constitutes the spectrum sensing method considered in this dissertation.

ED compares the signal energy received in a certain frequency band to a properly set decision threshold. If the signal energy lies above the threshold, the band is declared to be 
busy. Otherwise the band is supposed to be idle and could be accessed by DSA/CR users. Due to the simplicity and generality of its operating principle, the performance of ED is not expected to depend on the type of primary signal to detect. In this context, the aim of this chapter is to evaluate the performance of ED-based spectrum sensing for DSA/CR based on field measurements of various real-world licensed signals. This study is motivated by the need to extract the busy/idle states of the measured channels in order to develop appropriate spectrum usage models. Since such information is extracted by means of the ED principle, and this principle is applied to a wide variety of licensed radio technologies, a realistic and accurate understanding of the real performance of ED when applied to real-world signals is firstly required, which motivates the study performed in this chapter. The results obtained in this study indicate that the detection performance of ED may actually vary with the primary radio technology being considered, but converges under certain conditions. The causes and consequences of the different detection performances for various radio technologies are identified, analyzed and discussed.

The rest of this chapter is organized as follows. First, Section 4.2 summarizes the main novelties of this study, highlighting the differences with respect to other previous works where the performance of spectrum sensing has been studied. Section 4.3 then presents the considered system model, formulates the spectrum sensing problem in rigorous manner and the describes the metrics commonly employed to evaluate and quantify the performance of spectrum sensing algorithms. Afterwards, Section 4.4 describes in detail the operating principle and theoretical results related to ED-based spectrum sensing. The measurement platform and evaluation methodology employed in this study are described in Sections 4.5 and 4.6, respectively. Section 4.7 presents, analyzes and discusses the obtained experimental results. Finally, Section 4.8 summarizes the main conclusions derived from the study presented in this chapter.

\subsection{Novelties of this study}

Previous works have dealt with the study of spectrum sensing in the context of DSA/CR networks. However, there are a number of aspects that have not been covered satisfactorily in the performance evaluation of spectrum sensing. The following points identify some of such deficiencies and explain how this study fills the existing gaps:

- Spectrum sensing has usually been evaluated by means of theoretical studies or simulations considering simple primary user signals such as sine wave carriers or synthetic $M$-PSK/ $M$-QAM modulated signals [192], which lacks of realism in some practical scenarios. By contrast, this study is performed with real-world signals captured by means of a radio measurement platform.

- In theoretical and simulation studies, primary signals have usually been assumed to be only affected by Additive White Gaussian Noise (AWGN), although in practice there exist other noise sources as well as various propagation phenomena impairing the received signal and therefore impacting on the spectrum sensing performance. The performance evaluation of spectrum sensing with real signals, as performed in 
this study, implicitly takes into account all the aspects of the real world that can affect the performance of spectrum sensing algorithms in practice. The need for empirical experiments is stressed by the inability to realistically model all the potential sources of degradation encountered in real receivers and propagation environments.

- Some previous studies have already considered real signals following an approach similar to this study. However, in such cases only TV signals have been considered, and wireless microphone signals in some other cases [193]. Although the first DSA/CR deployments are expected in the TV bands following the IEEE 802.22 standard (see Section 1.1.5), in the future one could expect the deployment of the DSA/CR technology in many other interesting bands once the TV bands become crowded. However, very little is known about the performance of spectrum sensing with other primary signals, which might significantly differ from the performance obtained in the case of TV signals. This study evaluates and analyzes the performance of spectrum sensing when considering not only TV signals but also signals from other radio technologies, thus providing a broader view on the performance of spectrum sensing.

In this context, this study experimentally evaluates and comparatively analyzes the performance of spectrum sensing considering real-world primary signals of different radio technologies under a common evaluation framework. This study will allow not only to corroborate theoretical findings with practical results but also to determine the impact of considering various primary signal technologies on the detection performance of spectrum sensing.

\subsection{System model, problem formulation and evaluation metrics}

The spectrum sensing problem can be formulated as the problem of determining if a primary transmission is present in the sensed band. The primary continuous-time RF signal received at the secondary terminal, $y(t)$, can be expressed as:

$$
y(t)=x(t)+w(t)
$$

where $x(t)$ is the transmitted RF signal to be detected (after passing through the wireless channel, including path loss, fading and multipath effects), $w(t)$ is AWGN noise (some other noise sources could be considered) and $t$ is the temporal variable $\left(t \in \mathbb{R}^{+}\right)$. Note that $x(t)=0$ when there is no primary transmission. The received signal $y(t)$ is then shifted to a lower Intermediate Frequency (IF) at which it is sampled at a rate $f_{s}$. Assuming complex bandpass sampling and RF/IF signal bandwidth $W$, the sampling rate should satisfy $f_{s} \geq W$ if the sensing algorithm needs all the spectral information to be preserved [194-196]. After sampling, the received discrete signal is:

$$
y[m]=x[m]+w[m]
$$

where $y[m], x[m]$ and $w[m]$ represent, respectively, the discrete time versions of $y(t), x(t)$ and $w(t)$ sampled at time instants $t=m T_{s}$, with $m$ being a positive integer $(m \in \mathbb{N}$ ) representing the sample index, and $T_{s}=1 / f_{s}$ the sampling period. 
The received signal is generally passed through a digital filter. Let $f[k], k=0,1, \ldots, K$, be the coefficients of a Finite Impulse Response (FIR) filter, where $K$ is the order of the filter. After filtering, the received signal becomes:

$$
\tilde{y}[m]=\sum_{k=0}^{K} f[k] \cdot y[m-k]
$$

which can be expressed as:

$$
\tilde{y}[m]=\tilde{x}[m]+\tilde{w}[m]
$$

where

$$
\begin{aligned}
\tilde{x}[m] & =\sum_{k=0}^{K} f[k] \cdot x[m-k] \\
\tilde{w}[m] & =\sum_{k=0}^{K} f[k] \cdot w[m-k]
\end{aligned}
$$

If the sampling rate $f_{s}$ is sufficiently larger than the signal bandwidth, it is possible (and convenient in order to reduce the processing load) to down-sample the received signal. Assuming a down-sampling or decimation factor $M \geq 1$, the received signal is:

$$
\tilde{y}[n]=\tilde{x}[n]+\tilde{w}[n]
$$

where $n=M m$.

After properly sampling, filtering and decimating the received signal, the spectrum sensing algorithm has to decide, based on the signal samples $\tilde{y}[n]$, if a primary signal $\tilde{x}[n]$ is present in the received signal or it is only composed of noise samples $\tilde{w}[n]$. Under this approach, the spectrum sensing problem can be formulated as a binary hypothesis testing problem with the following two hypotheses:

$$
\begin{array}{ll}
\mathcal{H}_{0}: \tilde{y}[n]=\tilde{w}[n] & n=1, \ldots, N \\
\mathcal{H}_{1}: \tilde{y}[n]=\tilde{x}[n]+\tilde{w}[n] & n=1, \ldots, N
\end{array}
$$

where $\mathcal{H}_{0}$ is a null hypothesis stating that the received signal samples $\tilde{y}[n]$ correspond to noise samples $\tilde{w}[n]$ and therefore there is no primary signal in the sensed spectrum band, and hypothesis $\mathcal{H}_{1}$ indicates that some licensed user signal $\tilde{x}[n]$ is present. $N$ denotes the number of samples collected during the signal observation interval (i.e., the sensing period), emphasizing that the decision is made based on a limited number of signal samples. According to this formulation, the spectrum sensing function may be regarded as a black box that accepts a sequence of digital signal samples $\tilde{y}[n]$ as input and provides a binary output indicating whether a primary signal is present in the input sequence $\left(\mathcal{H}_{1}\right)$ or not $\left(\mathcal{H}_{0}\right)$.

The ideal spectrum sensor would select hypothesis $\mathcal{H}_{1}$ whenever a primary signal is present and hypothesis $\mathcal{H}_{0}$ otherwise. Unfortunately, spectrum sensing algorithms may fall into mistakes in practice, which can be classified into missed detections and false alarms. A missed detection occurs when a primary signal is present in the sensed band and the spectrum sensing algorithm selects hypothesis $\mathcal{H}_{0}$, which may result in harmful interference to 
primary users. On the other hand, a false alarm occurs when the sensed spectrum band is idle and the spectrum sensing algorithm selects hypothesis $\mathcal{H}_{1}$, which results in missed transmission opportunities and therefore in a lower spectrum utilization. Based on these definitions, the performance of any spectrum sensing algorithm can be summarized by means of two probabilities: the probability of missed detection $P_{m d}=P\left(\mathcal{H}_{0} \mid \mathcal{H}_{1}\right)$, or its complementary probability of detection $P_{d}=P\left(\mathcal{H}_{1} \mid \mathcal{H}_{1}\right)=1-P_{m d}$, and the probability of false alarm $P_{f a}=P\left(\mathcal{H}_{1} \mid \mathcal{H}_{0}\right)$. Large $P_{d}$ and low $P_{f a}$ values would be desirable. Nevertheless, there exists a trade-off between $P_{d}$ and $P_{f a}$, meaning that improving one of these performance metrics in general implies degrading the other one. In this context, Receiver Operating Characteristic (ROC) curves (obtained by plotting $P_{d}$ versus $P_{f a}$ ) are very useful since they allow to explore the relationship between the sensitivity $\left(P_{d}\right)$ and specificity $\left(P_{f a}\right)$ of a spectrum sensing method for a variety of different algorithm parameters and other affecting factors.

\subsection{Energy detection}

\subsubsection{Operating principle}

The ED principle, also referred to as radiometric detection, measures the energy received in a primary band during an observation interval and declares the band as busy (hypothesis $\mathcal{H}_{1}$ ) if the measured energy is greater than a properly set predefined threshold, or idle (hypothesis $\left.\mathcal{H}_{0}\right)$ otherwise [156]. This can be formally stated as:

$$
\mathbb{T}(\tilde{\mathbf{y}})=\sum_{n=1}^{N}|\tilde{y}[n]|^{2} \underset{\mathcal{H}_{0}}{\stackrel{\mathcal{H}_{1}}{\gtrless}} \lambda
$$

where $\mathbb{T}(\tilde{\mathbf{y}})$ is the test statistic computed over the signal vector $\tilde{\mathbf{y}}=(\tilde{y}[1], \tilde{y}[2], \ldots, \tilde{y}[N])$ and $\lambda$ is a fixed decision threshold to distinguish between the two hypotheses in Equation 4.8.

\subsubsection{Theoretical performance}

Closed-form expressions for the detection $\left(P_{d}\right)$ and false alarm $\left(P_{f a}\right)$ probabilities can be obtained based on the statistics of $\mathbb{T}(\tilde{\mathbf{y}})$, which can be derived as follows.

AWGN can be modeled as a zero-mean Gaussian random variable. Assuming that the primary signal varies slowly with respect to the noise, the primary signal can be considered to be quasi-deterministic, although unknown in detail. The assumption of a quasi-deterministic signal means that the input with a primary signal present is Gaussian but not zero-mean [156]. Thus, the test statistic $\mathbb{T}(\tilde{\mathbf{y}})$ follows central (under hypothesis $\mathcal{H}_{0}$ ) and non-central (under hypothesis $\mathcal{H}_{1}$ ) chi-square distributions with $2 N$ degrees of freedom [156]:

$$
\mathbb{T}(\tilde{\mathbf{y}}) \sim \begin{cases}\chi_{2 N}^{2}, & \mathcal{H}_{0} \\ \chi_{2 N}^{2}(2 \gamma), & \mathcal{H}_{1}\end{cases}
$$

where $\gamma=\sigma_{\tilde{x}}^{2} / \sigma_{\tilde{w}}^{2}$ is the Signal-to-Noise Ratio (SNR), and $\sigma_{\tilde{x}}^{2}, \sigma_{\tilde{w}}^{2}$ represent the signal and noise variances, respectively. If only AWGN noise is considered, $P_{d}$ and $P_{f a}$ can be evaluated 
based on the statistics of $\mathbb{T}(\tilde{\mathbf{y}})$ as follows [197]:

$$
\begin{gathered}
P_{d}=P\left(\mathbb{T}(\tilde{\mathbf{y}})>\lambda \mid \mathcal{H}_{1}\right)=Q_{N}(\sqrt{2 \gamma}, \sqrt{\lambda}) \\
P_{f a}=P\left(\mathbb{T}(\tilde{\mathbf{y}})>\lambda \mid \mathcal{H}_{0}\right)=\frac{\Gamma(N, \lambda / 2)}{\Gamma(N)}
\end{gathered}
$$

where $\Gamma(\cdot)$ and $\Gamma(\cdot, \cdot)$ are, respectively, the complete and incomplete gamma functions [198], and $Q_{V}(\cdot, \cdot)$ is the generalized Marcum $Q$-function [199].

Simpler expressions for $P_{d}$ and $P_{f a}$ can be obtained by assuming sufficiently large sensing periods. The non-interference constraint for DSA/CR terminals imposes strict detection performance requirements that must be met even in the worst possible operating case, i.e. low SNR conditions ${ }^{1}$. In low SNR regimes, the number of signal samples required to achieve a certain performance is usually large $(N \gg 1)$. Based on this observation, the central limit theorem can therefore be employed to approximate the test statistic as Gaussian:

$$
\mathbb{T}(\tilde{\mathbf{y}}) \sim \begin{cases}\mathcal{N}\left(N \sigma_{\tilde{w}}^{2}, 2 N \sigma_{\tilde{w}}^{4}\right), & \mathcal{H}_{0} \\ \mathcal{N}\left(N\left(\sigma_{\tilde{x}}^{2}+\sigma_{\tilde{w}}^{2}\right), 2 N\left(\sigma_{\tilde{x}}^{2}+\sigma_{\tilde{w}}^{2}\right)^{2}\right), & \mathcal{H}_{1}\end{cases}
$$

If only AWGN is considered, $P_{d}$ and $P_{f a}$ can then be obtained based on $\mathbb{T}(\tilde{\mathbf{y}})$ as:

$$
\begin{aligned}
P_{d} & =P\left(\mathbb{T}(\tilde{\mathbf{y}})>\lambda \mid \mathcal{H}_{1}\right)=Q\left(\frac{\lambda-N\left(\sigma_{\tilde{x}}^{2}+\sigma_{\tilde{w}}^{2}\right)}{\sqrt{2 N\left(\sigma_{\tilde{x}}^{2}+\sigma_{\tilde{w}}^{2}\right)^{2}}}\right) \\
P_{f a} & =P\left(\mathbb{T}(\tilde{\mathbf{y}})>\lambda \mid \mathcal{H}_{0}\right)=Q\left(\frac{\lambda-N \sigma_{\tilde{w}}^{2}}{\sqrt{2 N \sigma_{\tilde{w}}^{4}}}\right)
\end{aligned}
$$

where $Q(\cdot)$ is the Gaussian tail probability $Q$-function [201, (26.2.3)].

\subsubsection{Threshold setting}

The procedure employed to select the decision threshold is an important aspect since it has a direct impact on the spectrum sensing performance. The decision threshold could be chosen for an optimum trade-off between $P_{d}$ and $P_{f a}$. However, this would require knowledge of noise and detected signal powers. While the noise power can be estimated, the signal power is difficult to estimate since it depends on many varying factors such as transmission and propagation characteristics. In practice, the threshold is normally chosen to satisfy a certain $P_{f a}$ [202], which only requires the noise power to be known. Solving Equation 4.15 for $\lambda$ yields the decision threshold required for a specified target probability of false alarm:

$$
\lambda=\left(Q^{-1}\left(P_{f a}\right) \sqrt{2 N}+N\right) \sigma_{\tilde{w}}^{2}
$$

\footnotetext{
${ }^{1}$ For example, the IEEE 802.22 spectrum sensing requirements specify that a DSA/CR terminal must be able to detect a 6-MHz digital TV signal at a power level of $-116 \mathrm{dBm}$ [200], which corresponds to a SNR of $-21 \mathrm{~dB}$ for a typical TV receiver with a noise figure of $11 \mathrm{~dB}$ [200].
} 
Substituting Equation 4.16 into Equation 4.14 yields:

$$
\begin{aligned}
P_{d}(\gamma) & =Q\left(\frac{Q^{-1}\left(P_{f a}\right) \sqrt{2 N}-N \gamma}{\sqrt{2 N}(1+\gamma)}\right) \\
& \approx Q\left(Q^{-1}\left(P_{f a}\right)-\sqrt{\frac{N}{2}} \gamma\right)
\end{aligned}
$$

which represents the detection probability of the ED algorithm as a function of the SNR. The approximation of Equation 4.18 assumes the case of low SNR regime $(\gamma \ll 1)$.

\subsubsection{Noise uncertainty}

Classical detection theory states that degradation in the detection performance due to reduced SNR can be countered by increasing the sensing time [203, 204]. The minimum number of samples required for a given target performance in terms of $P_{d}$ and $P_{f a}$ can be obtained by solving Equation 4.17 for $N$, which yields:

$$
N=2\left[\frac{Q^{-1}\left(P_{f a}\right)-Q^{-1}\left(P_{d}\right)(1+\gamma)}{\gamma}\right]^{2}
$$

If the number of samples $N$ is not limited, the theoretical result of Equation 4.19 implies that any desired target performance can be met regardless of the experienced SNR. Nevertheless, in practice there exists a limit, referred to as $S N R$ wall, below which a primary signal cannot be reliably detected, irrespective of how large the sensing period is [205, 206]. Such fundamental limitation on the detection performance is imposed by the inability to accurately estimate the noise power as a result of calibration errors as well as changes in the thermal noise caused by temperature variations. These phenomena limit the accuracy with which the noise power can be estimated in practice. The noise power is therefore uncertain within some interval, which is referred to as noise uncertainty. Such uncertainty can be modeled as $\hat{\sigma}_{\tilde{w}}^{2} \in\left[\sigma_{\tilde{w}}^{2}, \alpha \sigma_{\tilde{w}}^{2}\right]$ [207], where $\hat{\sigma}_{\tilde{w}}^{2}$ represents the estimated noise power, $\sigma_{\tilde{w}}^{2}$ is the nominal noise power and $\alpha>1$ is the noise uncertainty. Based on the estimated noise power $\hat{\sigma}_{\tilde{w}}^{2}$, the resulting decision threshold in the worst possible case, i.e. $\hat{\sigma}_{\tilde{w}}^{2}=\alpha \sigma_{\tilde{w}}^{2}$, becomes:

$$
\lambda=\left(Q^{-1}\left(P_{f a}\right) \sqrt{2 N}+N\right) \alpha \sigma_{\tilde{w}}^{2}
$$

Substituting Equation 4.20 into Equation 4.14 yields:

$$
\begin{aligned}
P_{d}(\gamma) & =Q\left(\frac{\alpha Q^{-1}\left(P_{f a}\right) \sqrt{2 N}-N(\gamma+1-\alpha)}{\sqrt{2 N}(1+\gamma)}\right) \\
& \approx Q\left(\alpha Q^{-1}\left(P_{f a}\right)-\sqrt{\frac{N}{2}}(\gamma+1-\alpha)\right)
\end{aligned}
$$


which reduce to Equations 4.17 and 4.18 for the non-uncertainty case $(\alpha=1)$. The counterpart of Equation 4.19 under noise uncertainty can then be derived from Equation 4.21:

$$
N=2\left[\frac{\alpha Q^{-1}\left(P_{f a}\right)-Q^{-1}\left(P_{d}\right)(1+\gamma)}{\gamma+1-\alpha}\right]^{2}
$$

Equation 4.23 indicates that the number of samples required to attain a certain performance tends to infinity as the term $\gamma+1-\alpha$ in the denominator tends towards zero (i.e, $\gamma \rightarrow \alpha-1$ ), which implies that the SNR wall for the ED method is given by [207]:

$$
\gamma_{\text {wall }}=\alpha-1
$$

The existence of SNR walls has been verified experimentally [192, 208], demonstrating that a small noise power estimation error may cause significant performance loss.

\subsection{Measurement platform}

The measurement platform employed in the study of this chapter is based on the Universal Software Radio Peripheral (USRP) hardware (http://www.ettus.com) and the GNU Radio software (http://gnuradio.org). USRP is an openly designed inexpensive SDR hardware platform that provides radio front-end functionalities, Analogical to Digital and Digital to Analogical Conversion (ADC/DAC), decimation and interpolation with filtering as well as a USB interface to connect to an off-the-shelf Personal Computer (PC). The PC runs the GNU Radio software, a free and open source toolkit that provides a library of signal processing blocks for building SDRs. In addition, GNU Radio also provides blocks for communicating with the USRP. The general scheme of this measurement platform is illustrated in Figure 4.1, where the notation introduced in Section 4.3 is included. Figure 4.2 shows a picture.

The licensed signal of interest is captured with an omni-directional discone-type antenna AOR DN753 that covers the frequency range 75-3000 MHz. The USRP RF front-ends are provided in form of daughter boards that can be plugged to the USRP main board. Two receiver-only daughter boards are employed in this study: TVRX (50-860 MHz, $8 \mathrm{~dB}$ typical noise figure) and DBSRX (800-2400 MHz, 3-5 dB typical noise figure). The daughter boards are employed to tune to the carrier frequency of the desired licensed signal and perform down-conversion to the IF at which the USRP main board operates. The USRP main board includes 12-bit ADCs working at $64 \cdot 10^{6}$ samples per second to digitize the received signal and a FPGA to perform filtering and digital down-conversion (decimation) from the IF band to the Base Band (BB). Decimation is required in order to adapt the incoming data rate to the USB and PC computing capabilities. A USB controller sends the digital signal samples to the PC in 16-bit I and 16-bit Q complex format (4 bytes per complex sample), resulting in a maximum rate of $8 \cdot 10^{6}$ complex samples per second and per daughter board. The maximum RF bandwidth that can be handled is therefore $8 \mathrm{MHz}$ (narrower bandwidths can be selected by adjusting the decimation rate). The host PC runs the GNU Radio's usrp_rx_cfile.py script, which collects the digital signal samples sent by the USRP board through the USB interface and saves the received BB digital signal sequence 


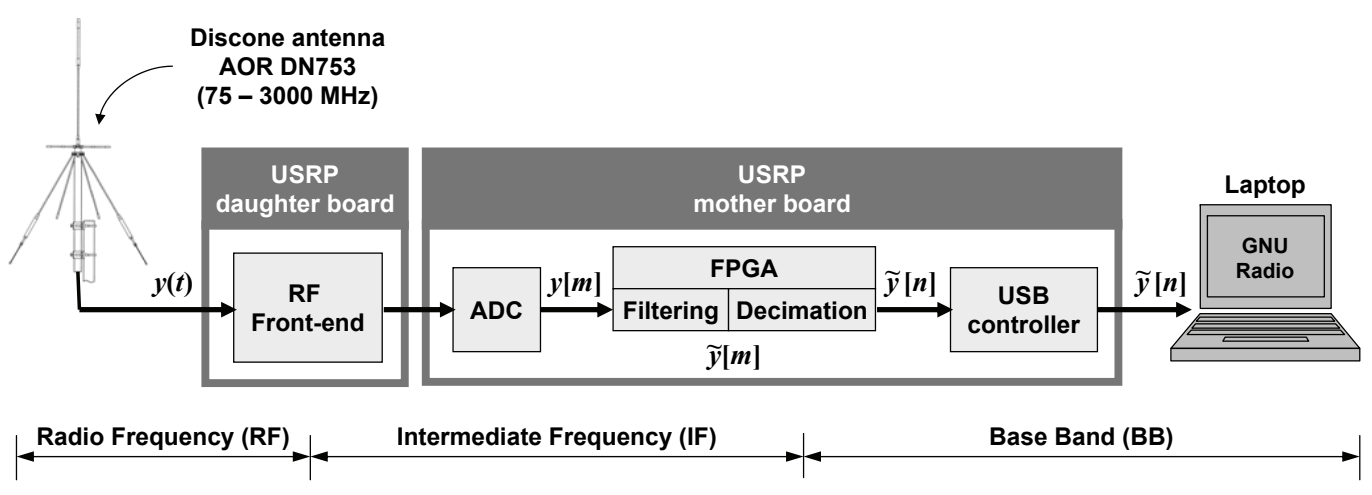

Figure 4.1: Measurement platform employed in this study (scheme).

to a file in the host PC. After the on-line data capturing process, the set of files created by the script are processed off-line. To this end, the ED sensing scheme was implemented in Matlab according to the operating principles and theoretical results described in Section 4.4.

\subsection{Evaluation methodology}

The measurement platform described in Section 4.5 was used to collect digital signal samples of real signals. To this end, the whole measurement platform was placed on a building rooftop in urban Barcelona (see location 1 in Figure 3.1) with direct line-of-sight to several transmitters located a few tens or hundreds of meters away from the antenna and without buildings blocking the radio propagation. This strategically selected measurement scenario enabled to reliably capture the desired signals under high SNR conditions.

With the aid of a spectrum analyzer, various channels were selected for different radio technologies (see Table 4.1). The channels shown in Table 4.1 were selected because of their high power levels (in order to maximize the receiving SNR) and constant activity patterns (i.e., the channels were always busy, which simplifies some computations in the off-line data post-processing phase). For broadcast services (analogical TV, digital TV and DAB-T), the selection was straightforward since these transmitters are always active an transmit at high power levels. For UMTS, the activity factor was not an issue (UMTS base stations transmit broadcast information continuously), but the received signal level was carefully observed, selecting channels from the closest base stations (only a few tens of meters apart). On the other hand, for TETRA, GSM and DCS, the most problematic aspect was the activity factor. In the case of TETRA, transmissions from base stations in Downlink-Continuous Transmission (D-CT) mode [209] were identified. In the case of GSM and DCS, various broadcast channels were selected, which could readily be identified by their high powers.

After identifying the channels to be measured, the required measurements were performed tuning the measurement platform to the center frequency of each channel $\left(f_{\text {center }}\right.$ in Table 4.1) and employing the indicated decimation rates and RF gain factors. Decimation rates were selected so that the effectively sampled signal BandWidth (BW) was equal to or greater than the actual signal BW. Notice that the RF BW $f_{\text {stop }}-f_{\text {start }}$ measured for each 


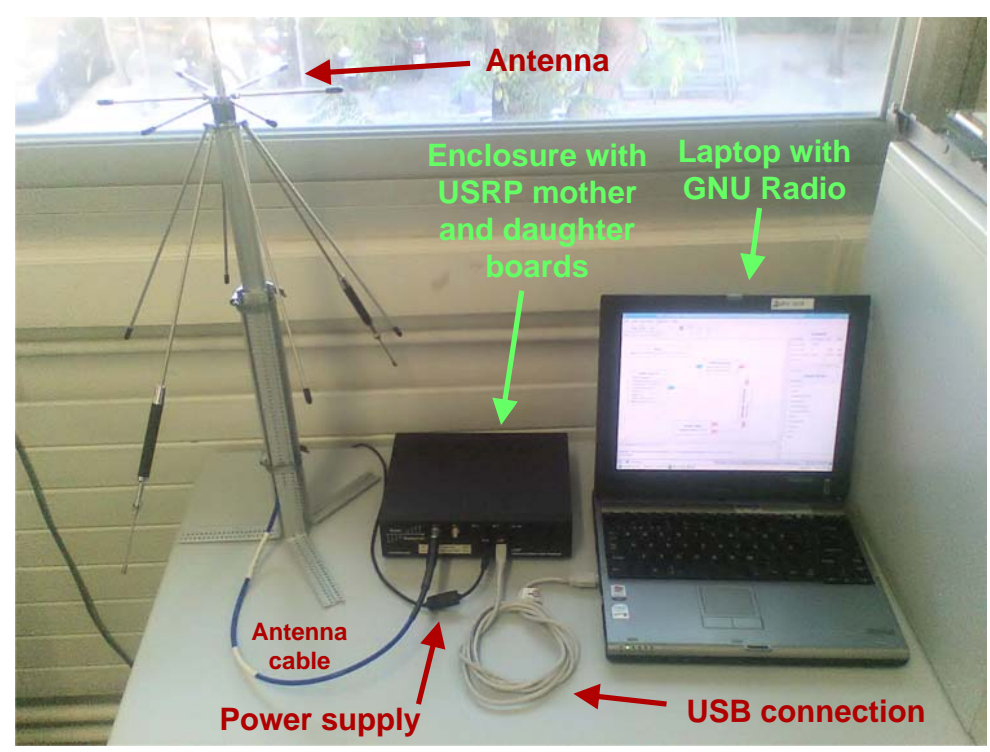

Figure 4.2: Measurement platform employed in this study (picture).

radio technology equals the signal BW except for E-GSM 900 and DCS 1800, where the carrier spacing (signal BW) is $200 \mathrm{kHz}$ but the employed Gaussian Minimum Shift Keying (GMSK) modulation may have power components within a total RF bandwidth of $400 \mathrm{kHz}$. The gain factor was chosen to improve the received signal level without incurring in saturation. In most cases the optimum gain value was $70 \mathrm{~dB}$ but in the particular case of TV the gain was drastically reduced due to the proximity of the TV station $(\approx 3 \mathrm{~km})$.

For each channel, a sequence of $12 \cdot 10^{6}$ signal samples was captured and the first $2 \cdot 10^{6}$ samples were discarded in order to remove any potential transient peaks that may appear during the first capturing instants of the USRP board. The resulting sequence was filtered in software with Matlab using a high-order digital Butterworth filter with no more than $0.1 \mathrm{~dB}$ of losses in the passband and at least $50 \mathrm{~dB}$ of attenuation in the stopband. The normalized cut-off frequencies for each channel are shown in Table 4.1, resulting in passbands equal to or greater than the signal $\mathrm{BW}$, except for $\mathrm{TV}$ channels where some $\mathrm{BW}$ is required to accommodate the filter's transient band (for DAB-T the RF bandwidth is $1.712 \mathrm{MHz}$ but the signal information is confined within a BW of $1.54 \mathrm{MHz}$ ).

Before exploiting the captured signals, the compliance with the two established requirements was verified, namely high signal levels without saturation and activity factors of $100 \%$. The fulfillment of the first requisite can be verified from Figure 4.3, where the average power spectrum of some captured signals is shown as an example. As it can be appreciated, the nearly perfect spectral shapes indicate a high SNR level without distortion by saturation. To verify the accomplishment of the second requirement, the antenna shown in Figure 4.1 was replaced with a matched load and the measurements were repeated in order to measure the receiver's noise. By comparing the instantaneous energy levels of both the signal and noise sequences, the energy levels present in the signal sequences were observed to be significantly higher than those measured for the noise sequences over the whole measurement period, thus indicating that licensed signals were present at all times. 


\begin{tabular}{|c|c|c|c|c|c|c|c|}
\hline 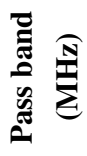 & ñ? & ñ? & $\stackrel{3}{0}$ & $\stackrel{0}{-}$ & $\stackrel{n}{0}$ & $\stackrel{n}{0}$ & $n$ \\
\hline Uે & ڤ్ & ڤ̊. & $\overrightarrow{0}$ & $\stackrel{\infty}{0}$ & $\stackrel{m}{0}$ & $\stackrel{?}{0}$ & $\stackrel{n}{a}$ \\
\hline 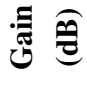 & 으 & 은 & $尺$ & 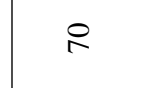 & 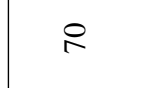 & 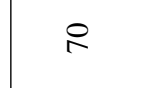 & 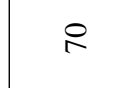 \\
\hline 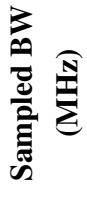 & $\infty$ & $\infty$ & $\stackrel{n}{2}$ & $N$ & 一 & - & $\infty$ \\
\hline 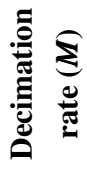 & $\infty$ & $\infty$ & ๗ั & mे & ț & t & $\infty$ \\
\hline 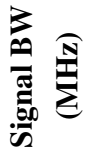 & $\infty$ & $\infty$ & $\stackrel{2}{0}$ & $\stackrel{2}{i}$ & $\stackrel{1}{0}$ & กֶ. & $n$ \\
\hline i & 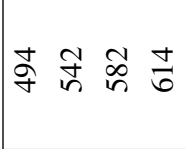 & 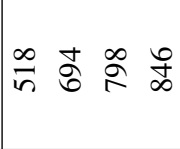 & 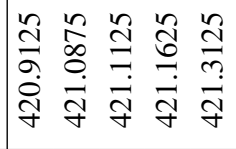 & 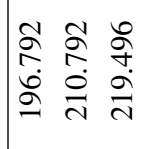 & 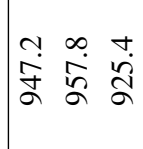 & 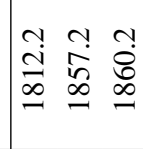 & 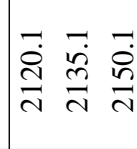 \\
\hline 㺼 & 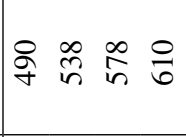 & I & 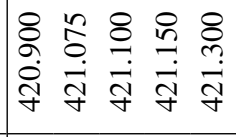 & 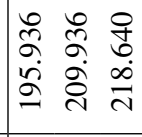 & 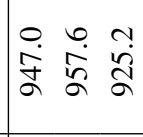 & 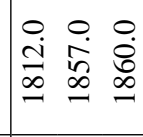 & 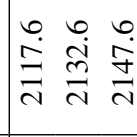 \\
\hline 方 & 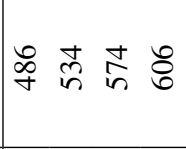 & 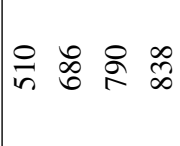 & 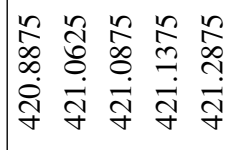 & 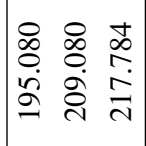 & 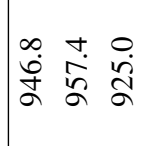 & $\begin{array}{lll}\infty & \infty & \infty \\
\end{array}$ & $\dot{0}$ & 0 \\
$\infty$ & $\infty$ & 0 \\
- & $\infty$ & $\infty$
\end{tabular}$} &{\text { 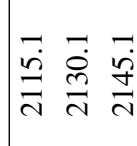 }} \\
{\hline \text { 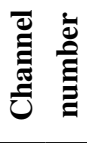 }} &{\text { 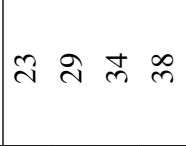 }} &{\stackrel{\sim}{i} \stackrel{\infty}{+} \overline{6}} &{\text { 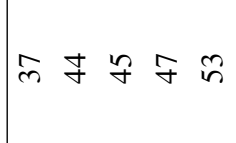 }} &{\text { 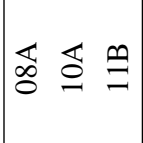 }} &{B \cong \frac{n}{=}} &{\text { 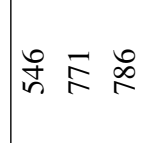 }} &{\begin{array}{lll}\infty & \ddots & \infty \\
\infty & 0 & 0 \\
0 & 0 & 0 \\
0 & 0 & 0\end{array}} \\
{\hline \text { 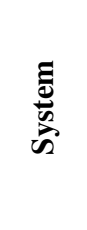 }} &{\text { 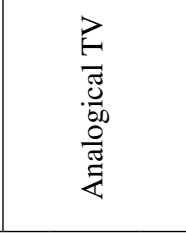 }} &{\text { 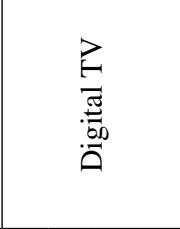 }} &{\underset{\underline{N}}{\mathbb{E}}} &{\text { 客 }} &{\text { 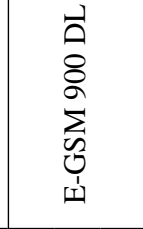 }} &{\begin{array}{l}\vec{D} \\
8 \\
8 \\
\infty \\
\bar{D} \\
0\end{array}} &{\text { 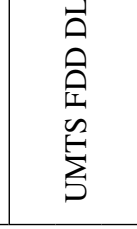 }} \\
$\hline$\end{array}$



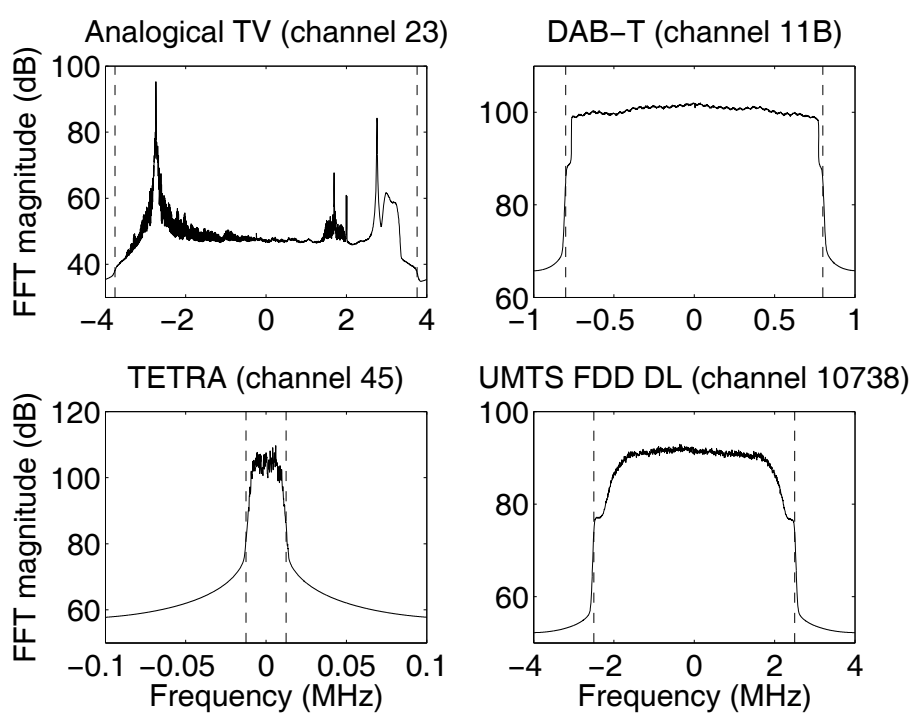

Figure 4.3: Average power spectrum for some of the captured signals. The results shown have been obtained by averaging more than 4800 2048-point Fast Fourier Transforms (FFTs). Dashed lines represent the filter's cut-off frequencies.

To assess the experimental performance of ED, the input sequences were divided into blocks of $N$ samples and a sufficiently high number of such blocks was processed. For low values of $N$, a higher number of blocks was processed in order to keep the statistical accuracy of the obtained results at an acceptable level. Assuming that the signal sequences are noise-free (which is a reasonable approximation for high SNR levels), the power of the captured sequences was considered as the actual signal power $\sigma_{\tilde{x}}^{2}$. AWGN sequences with power levels $\sigma_{\tilde{w}}^{2}=\sigma_{\tilde{x}}^{2} / \gamma$ were then generated and added to the captured sequences in order to simulate various SNRs. The experimental $P_{d}$ was then computed as the fraction of blocks with a detection result $\mathcal{H}_{1}$ when the decision threshold is set for a specified target $P_{f a}$ according to Equation 4.16 (note that a signal was present in all the processed blocks).

\subsection{Experimental performance results}

Based on the measurement platform presented in Section 4.5 and following the methodology described in Section 4.6, the experimental performance of ED was evaluated for the 25 channels shown in Table 4.1. The results obtained for channels belonging to the same radio technology were averaged, since a high similarity was observed among them in most cases. This simplifies the subsequent analysis since the obtained results can be analyzed for each radio technology instead of for each individual channel. The obtained results are shown in Figures 4.4, 4.5 and 4.6 in terms of the experimental detection probability as a function of the experienced SNR. Figure 4.4 shows the results for the case of perfectly calibrated noise, i.e. no noise uncertainty $\left(10 \log _{10} \alpha=0 \mathrm{~dB}\right)$, while Figures 4.5 and 4.6 correspond to the case where noise uncertainty is present $\left(10 \log _{10} \alpha=1 \mathrm{~dB}\right.$ and $\left.10 \log _{10} \alpha=2 \mathrm{~dB}\right)$. 


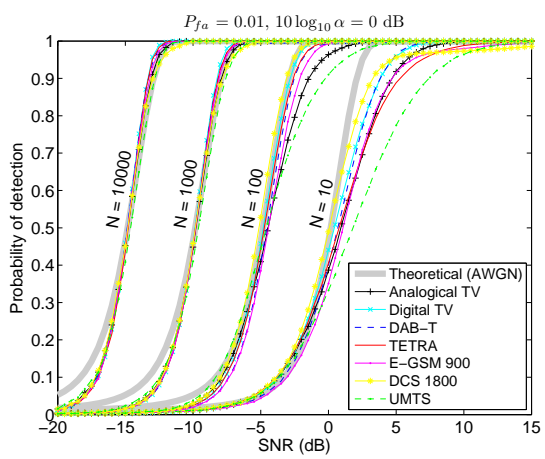

(a)

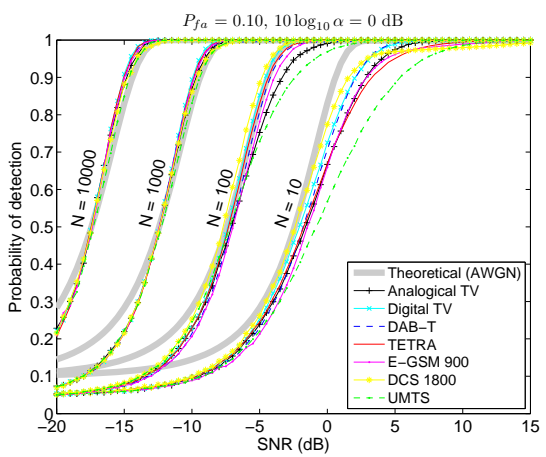

(b)

Figure 4.4: Experimental $P_{d}(\gamma)$ for $10 \log _{10} \alpha=0 \mathrm{~dB}$ : (a) target $P_{f a}=0.01$, (b) target $P_{f a}=0.10$.

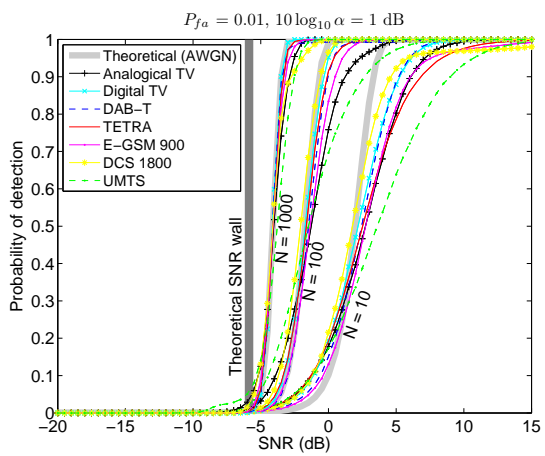

(a)

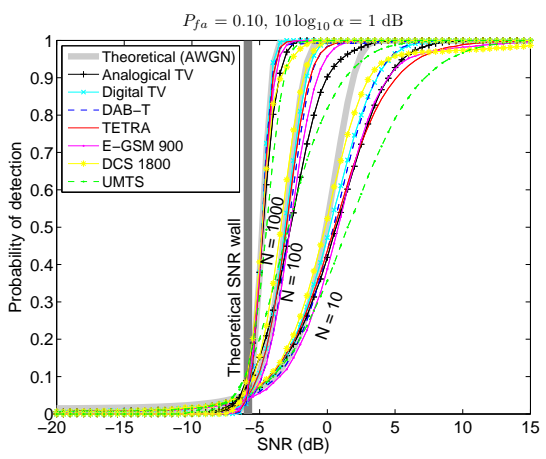

(b)

Figure 4.5: Experimental $P_{d}(\gamma)$ for $10 \log _{10} \alpha=1 \mathrm{~dB}$ : (a) target $P_{f a}=0.01$, (b) target $P_{f a}=0.10$.

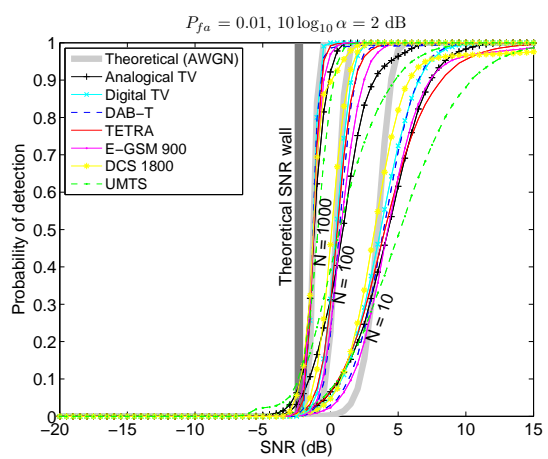

(a)

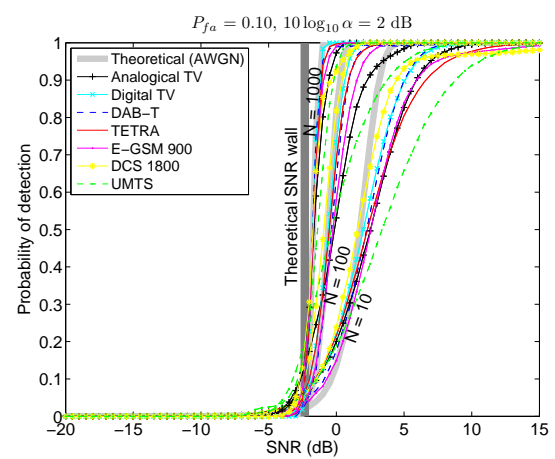

(b)

Figure 4.6: Experimental $P_{d}(\gamma)$ for $10 \log _{10} \alpha=2 \mathrm{~dB}$ : (a) target $P_{f a}=0.01$, (b) target $P_{f a}=0.10$. 


\subsubsection{Validation}

As it can be appreciated in Figures 4.4, 4.5 and 4.6, different performance curves are obtained for different radio technologies although the same ED method is employed in all cases. In principle, this phenomenon might be due to the randomness of the experiments, which are based on measurements performed on an number of arbitrary channels over a short measurement period (from 1.25 to 40 seconds depending on the sampling rate, see Table 4.1). Although any empirical study suffers from some unavoidable random component, it is worth noting that several experiments were performed with a number of iterations lower than that employed to obtain the results shown in this section. To determine the randomness and validate the obtained results the number of iterations was doubled and quite similar results were obtained. Doubling the number of iterations resulted in slightly smoother curves, but it did not change the positions of the curves within the graphs, which indicates the statistical reliability of the results. Moreover, as mentioned above, the performance curves obtained for different channels of the same radio technology did not manifest significant differences. Therefore, the differences observed among different licensed systems have to be ascribed to the particular features of each radio technology.

Figures 4.4, 4.5 and 4.6 also show that the experimental performance of ED is in some cases appreciably lower than the theoretical performance expected from Equations 4.17 and 4.21, specially for short sensing periods (i.e., low values of $N$ ). Although this is an important aspect, it will be treated in more detail in Chapter 5. This chapter focuses on the comparative analysis of the ED performance for various radio technologies and discusses its causes and consequences. In other respects, the obtained results agree with the theoretical predictions. For example, $P_{d}$ decreases with the SNR as expected from Equations 4.17 and 4.21. On the other hand, increasing the target $P_{f a}$ implies a reduction of the energy decision threshold (see Equations 4.16 and 4.20), which in turn results in an improved detection of weak signals and hence an enhanced $P_{d}$. The existing trade-off between $P_{d}$ and $P_{f a}$ can be clearly appreciated by comparing the results obtained for $P_{f a}=0.01$ and $P_{f a}=0.10$. Moreover, Figure 4.4 indicates that for perfectly calibrated noise ED is a robust detection method, i.e. any arbitrary pair $\left(P_{d}, P_{f a}\right)$ can be met by simply increasing the sensing period as indicated in Equation 4.19. In the presence of noise uncertainty, however, there exists a SNR threshold (the SNR wall) below which ED becomes non-robust, meaning that $P_{m d}=1-P_{d}$ and $P_{f a}$ cannot simultaneously be made to go to zero, irrespective of the observation interval. This trend is clearly appreciated in Figures 4.5 and 4.6, where increasing $N$ improves $P_{d}$ only for SNR values above the SNR wall. Below the threshold imposed by the SNR wall, $P_{d}$ approaches zero regardless of the value of $N$, making signal detection impossible. As observed in Figures 4.5 and 4.6, the existence of the SNR wall is verified experimentally with a reasonably accurate match with the theoretical prediction of Equation 4.24.

\subsubsection{Analysis}

The results shown in Figures 4.4, 4.5 and 4.6 indicate that the performance of ED may strongly depend on the considered radio technology. This behavior is not predicted by the classical theoretical results associated to the ED method, which were presented in Section 
4.4. In fact, for a given set of target $P_{f a}$, sensing period $N$, SNR $\gamma$, and noise uncertainty $\alpha$, Equations 4.17 and 4.21 suggest that the resulting performance in terms of $P_{d}$ is unique. However, Figures 4.4, 4.5 and 4.6 clearly demonstrate that for constant $P_{f a}, N, \gamma$ and $\alpha$, the resulting $P_{d}$ may strongly depend on the radio technology being detected. Moreover, it is interesting to note that the observed performance differences are not constant, but increase as the sensing period $N$ decreases. As mentioned in Section 4.7.1, these differences have to be ascribed to the particular features of each radio technology. The objective of this section is to identify and analyze the causes that originate the observed differences.

The performance of any spectrum sensing algorithm depends on the decisions made by such algorithm, which in turn depend on the employed test statistic. Since the ED test statistic is related to the received signal energy (see Equation 4.9), the aforementioned appreciations should be explainable in terms of the characteristics of the received signal energy, or indistinctly the received signal power. The signal power $\mathcal{P}$ is related to the signal energy $\mathcal{E}$ by a scaling factor $N$ :

$$
\mathcal{P}(\tilde{\mathbf{y}})=\frac{1}{N} \mathcal{E}(\tilde{\mathbf{y}})=\frac{1}{N} \mathbb{T}(\tilde{\mathbf{y}})=\frac{1}{N} \sum_{n=1}^{N}|\tilde{y}[n]|^{2}
$$

Therefore, the analysis of the time evolution of the received power indicates how the ED test statistic varies over time.

To evaluate the time evolution of the received signal power, and in order to enable a fair comparison among various signals under the same conditions, the captured sequences $\tilde{y}[n]$ were normalized to obtain zero-mean, unit-variance (unit-power) sequences $\breve{y}[n]$ as follows:

$$
\check{y}[n]=\frac{\tilde{y}[n]-\mu_{\tilde{y}}}{\sqrt{\sigma_{\tilde{y}}^{2}}}
$$

where $\mu_{\tilde{y}}$ and $\sigma_{\tilde{y}}^{2}$ represent the sample mean and sample variance of sequence $\tilde{y}[n]$, respectively. This operation converts the captured sequences $\tilde{y}[n]$ with different absolute signal powers into normalized sequences $\breve{y}[n]$ with the same normalized signal power, which in the presence of the same noise power is equivalent to compare them under the same average SNR. The obtained sequences $\breve{y}[n]$ were divided into blocks of $N$ samples and the average power of each block was computed. The sequence of received power levels was smoothed by means of a moving average. The resulting time evolution of the normalized received power is shown in Figure 4.7 for various channels when $N=100,200,500$ and 1000. Since such signals were captured under perfect line-of-sight and high SNR conditions, it is reasonable to assume that fading effects are negligible and the observed power variations are therefore mainly due to varying transmission power patterns (the measurement conditions were carefully selected to minimize the impairing effects of the channel). The observed patterns can indeed be related to distinctive properties of the received signals that would not have been preserved under severe fading. For example, the highest peaks observed for analogical TV in Figure 4.7(a) occur every 1600 blocks $\times 100$ samples/block $=1.6 \cdot 10^{5}$ samples (the same number of samples can be inferred from Figures 4.7(b)-4.7(d)), which for the employed sampling rate corresponds to the $50-\mathrm{Hz}$ vertical frequency of the PAL system. The UMTS 

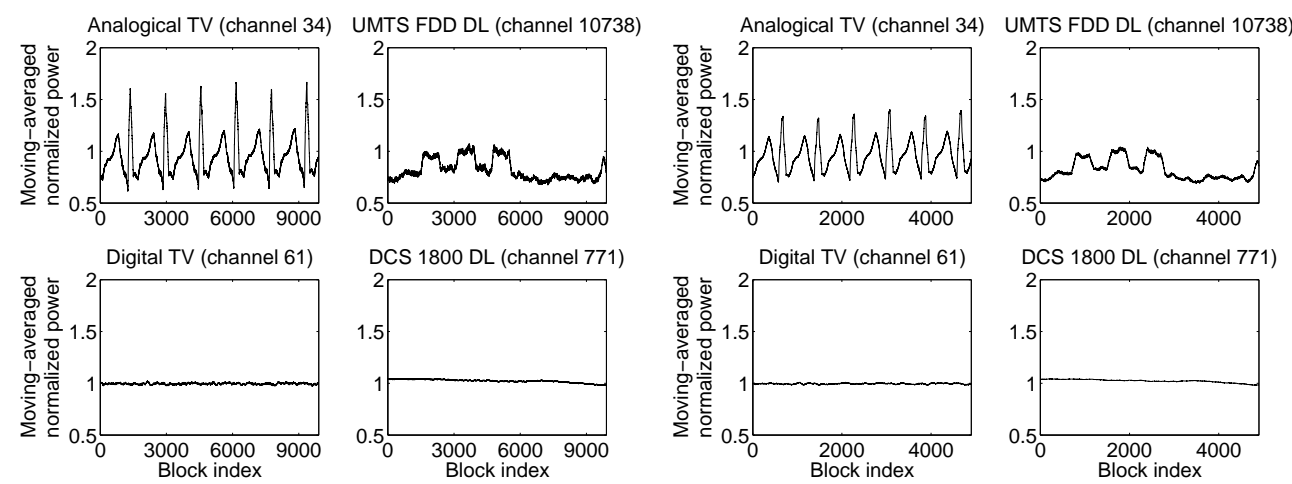

(a)

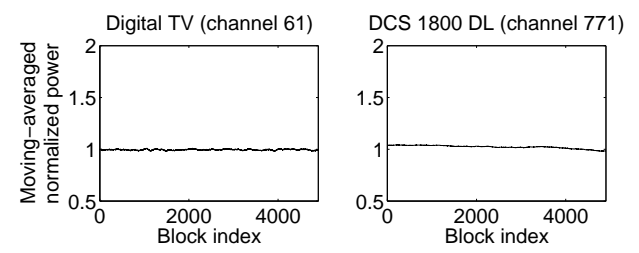

(b)
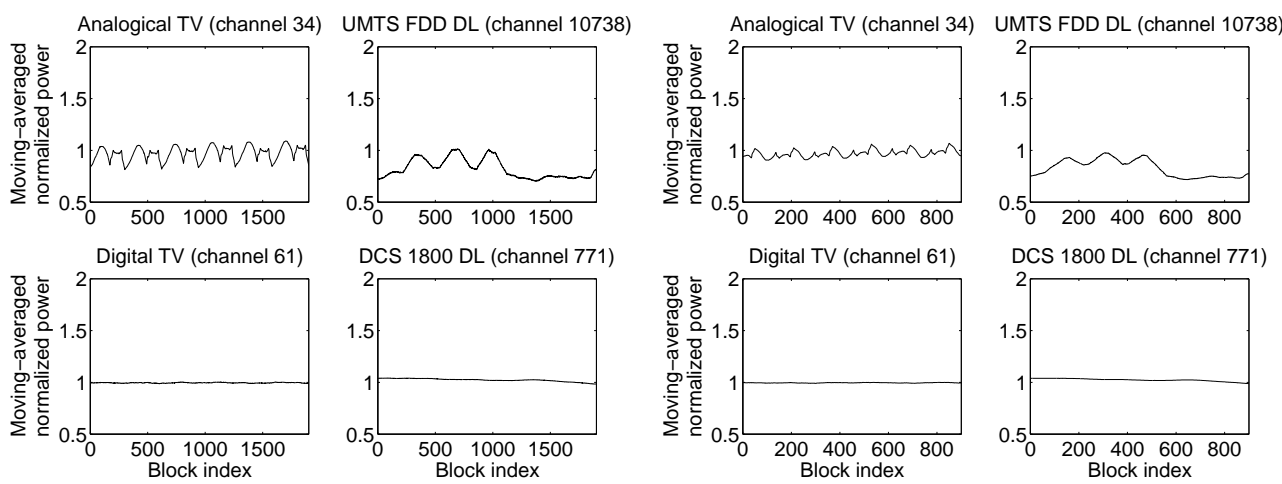

(c)
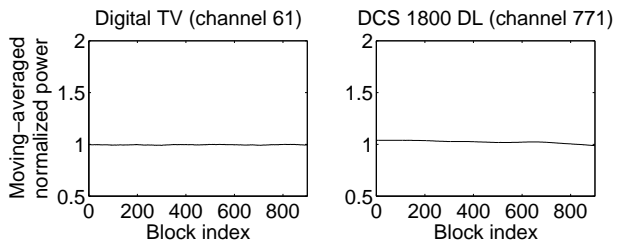

(d)

Figure 4.7: Moving-averaged normalized power received for some captured signals: (a) $N=100$, (b) $N=200$, (c) $N=500$, and (d) $N=1000$.

signal variations can be explained by the use of power control techniques that modify the transmitted power according to the network's load factor. On the other hand, the signals received in digital TV and DCS 1800 (broadcast) channels show a uniform power pattern, as expected. As a matter of fact, for signals with perfectly constant transmission power patterns (e.g., digital TV) the performance observed in Figures 4.4, 4.5 and 4.6 agrees with the theoretical predictions of Equations 4.17 and 4.21 under AWGN, meaning that the variation of the received power levels due to the propagation channel is not significant (except for very short sensing periods, e.g. $N=10$ ). Therefore, the power variations observed in Figure 4.7 can be ascribed to the transmission power patterns of the measured transmitters.

The performance results observed in Figures 4.4, 4.5 and 4.6 can be explained based on Figure 4.7 as follows. For short observation intervals (e.g., $N=100)$ the test statistic $\mathbb{T}(\tilde{\mathbf{y}})$ follows the instantaneous variations of the received signal power. In such a case, if the instantaneous signal power falls below the energy decision threshold, the detection result will be $\mathcal{H}_{0}$, even if it should be $\mathcal{H}_{1}$ due to an average power actually greater than the decision 


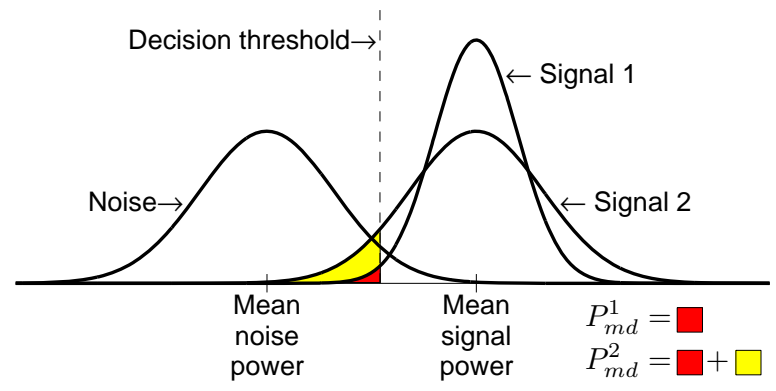

Figure 4.8: Impact of the signal power variability on the ED performance.

threshold. Therefore, under the same average SNR conditions (i.e., signals with the same average power), this means that a higher power variability (variance) implies a higher probability that the instantaneous power level (and therefore the ED test statistic) falls below the decision threshold, which results in a lower detection probability. This idea is illustrated in Figure 4.8. The comparison of the results shown in Figures 4.4, 4.5 and 4.6 for $N=100$ to the power patterns shown in Figure 4.7 for the same value of $N$ indicates that the best detection performance is obtained for DCS 1800, followed by digital TV (the signals with the most uniform power patterns), while the worst detection performance corresponds to UMTS FDD, followed by analogical TV (the signals with the most varying power patterns). Since various radio technologies and transmitters may exhibit different power variation patterns and variances, this explains the different detection performances observed in Figures 4.4, 4.5 and 4.6 for various radio technologies under the same average SNR conditions. As the number of samples $N$ increases, the test statistic $\mathbb{T}(\tilde{\mathbf{y}})$ is computed over larger observation intervals, thus averaging the peculiarities of any instantaneous power variation pattern and reducing its variance as observed in Figure 4.7. In such a case, although the variability of the received power remains the same, the variability of the test statistic $\mathbb{T}(\tilde{\mathbf{y}})$ decreases an so does the probability of missed detection. For sufficiently long observation intervals, the test statistic ceases to follow the instantaneous signal power variations and its value closely resembles the true mean power. When this occurs for all the considered signals, the obtained performance curves converge (i.e., the ED performance does not depend on the considered radio technology). This explains the convergent trend observed in Figures 4.4, 4.5 and 4.6 as the value of $N$ increases.

In summary, the previous analysis indicates that the ED test statistic may follow the instantaneous variations of the received signal power if short sensing periods are selected. When this occurs, the ED performance strongly depends on the radio technology being sensed. In particular, more variable transmission power patterns lead to a lower probability of detection, while the detection performance is affected to a lesser extent when sensing more uniform power patterns. On the other hand, as the sensing period increases with respect to the dynamics of the primary signal, the peculiarities of each transmission power pattern are averaged and the ED performance becomes more independent of the signal to be detected. 


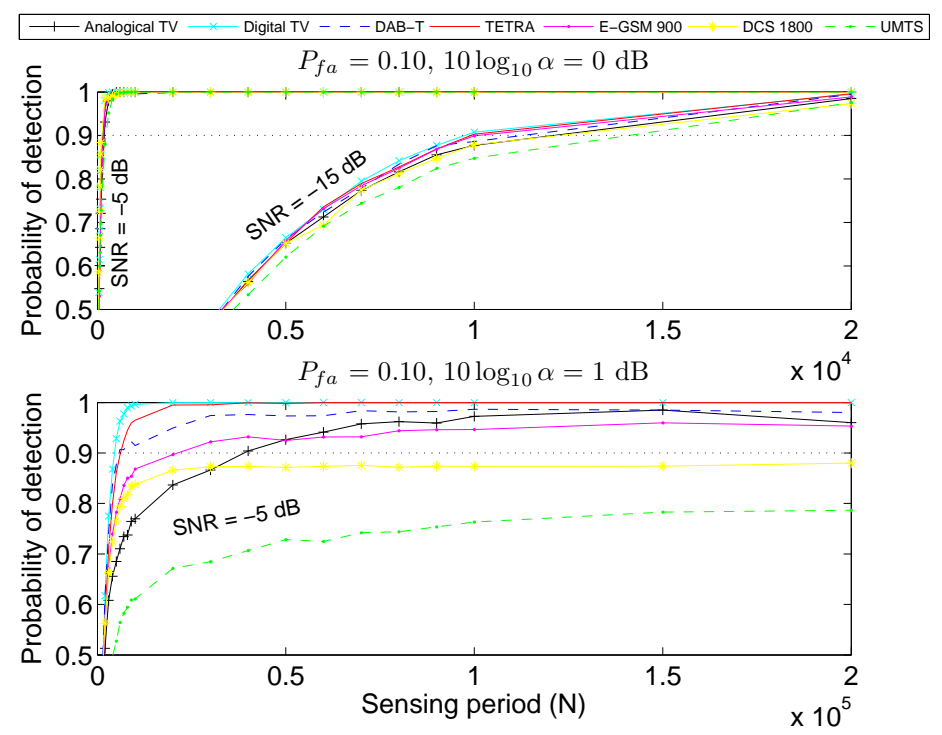

Figure 4.9: Probability of detection as a function of the sensing period for various radio technologies.

\subsubsection{Discussion}

While Section 4.7.2 has analyzed the causes of the varying detection performance of ED depending on the considered radio technology, this section illustrates its consequences in practice. To quantitatively illustrate the impact of this phenomenon, Figure 4.9 shows the detection probability as a function of the sensing period for various licensed signals. As it can be appreciated, under the ideal case of no noise uncertainty (upper graph), the performance is similar for different signals. For example, assuming an average SNR of $-15 \mathrm{~dB}$, a target $P_{d}=0.9$ would require 9745 samples $(\approx 1.22 \mathrm{~ms})$ in the best case (digital TV) and 14099 samples $(\approx 1.76 \mathrm{~ms})$ in the worst case (UMTS). This difference is not very significant, but relies on the assumption that the noise power is perfectly known, which is never true in reality. In the more realistic case of noise uncertainty (lower graph) and assuming an average SNR above the SNR wall of $-5 \mathrm{~dB}$, a target $P_{d}=0.9$ would require 4532 samples $(\approx 0.57 \mathrm{~ms})$ in the best case (digital TV), 38937 samples $(\approx 4.87 \mathrm{~ms})$ in an average case (analogical TV), whereas in the worst case (UMTS) the desired $P_{d}$ would never be reached due to the presence of the SNR wall ( $P_{d}$ for UMTS does not increase beyond 0.8 regardless of the considered sensing interval). These results indicate that different radio technologies may require different sensing periods to attain the same detection performance. Therefore, the sensing period predicted by Equations 4.19 and 4.23 for a given target performance $\left(P_{d}\right.$, $P_{f a}$ ) may be enough to detect the presence of primary signals of certain radio technologies but may actually result insufficient to reliably detect the presence of others. If the maximum sensing period is constrained (by physical layer features, higher layer protocols or other circumstances), this means that some radio technologies would be more susceptible to interference under the same operating conditions. 


\subsection{Summary}

Despite its practical performance limitations, ED has gained popularity during the last years as a spectrum sensing technique for DSA/CR networks. The main advantages of ED are its simplicity, low computational and implementation costs as well as its ability to work irrespective of the signal to be detected. Since no prior knowledge is required, ED can be employed when the secondary receiver cannot gather sufficient information about the primary user signal. However, the detection performance of ED depends on the primary signal being detected in spite of the generality of its operating principle. The results presented in this chapter have indicated that certain inherent technology-dependent properties may result in different detection performances for various radio technologies. The detection performance differences are more noticeable for short sensing periods, where it has been observed that transmission power patterns with higher (lower) levels of variability result in a lower (higher) detection performance. As the sensing interval increases, the ED performance becomes more similar for different radio technologies and thus more independent of the signal to be detected. Although increasing the sensing period improves the probability of detection, different radio technologies may require different sensing periods to attain the same detection performance in practice. For a fixed sensing period, some radio technologies would therefore be more susceptible to interference under the same operating conditions.

Based on the results obtained in this study, there are two important practical aspects to be highlighted. Firstly, the classical theoretical results associated to ED are not able to capture and predict the dependence of the ED performance on the variability of the primary transmission power pattern, which limits the applicability of such theoretical results in the design of real DSA/CR systems. Secondly, the ED performance differs to a greater extent as the sensing period decreases. If a minimum detection performance needs to be provided, the sensing period cannot be shortened below a certain limit, which imposes a fundamental trade-off between detection performance and spectral agility. While the former is treated in Chapter 5 by developing an empirical model for the detection performance of ED, the latter will be addressed in Chapter 6 by proposing an enhanced ED scheme capable to cope with the degraded performance of the classical ED scheme for short sensing periods while providing the same advantages and benefits. 



\section{Chapter}

5

\section{SIGNAL UNCERTAINTY IN SPECTRUM SENSING: EMPIRICAL MODEL FOR ENERGY DETECTION PERFORMANCE}

\subsection{Introduction}

The study conducted in Chapter 4 has demonstrated that the detection performance of ED may strongly vary with the primary radio technology being detected. Certain inherent technology-dependent properties, more concretely the variability (variance) of the primary transmission power pattern, may result in different detection performances for various primary signals under the same operating conditions. In other words, the probability of detection experienced for a fixed set of operating parameters may suffice to reliably detect some primary signals but not some others, thus making certain radio technologies more susceptible to interferences under the same operating conditions. The inability of a DSA/CR terminal to perfectly know beforehand the primary signals that may be present in the sensed band and the properties thereof, henceforth referred to as signal uncertainty, results in a detection performance degradation since the non-interference requirement for the secondary network implies a worst-case design where DSA/CR terminals must guarantee a minimum detection performance regardless of the primary signal being detected.

While the consequences of noise uncertainty (i.e., the inability to perfectly calibrate the noise power) have widely been studied, verified experimentally and distilled into tractable mathematical models [205, 206], the impact of signal uncertainty on the detection performance of ED, which can have important practical consequences as experimentally demonstrated in Chapter 4, has not been studied and analyzed yet. The existing classical theoretical results for ED describe the resulting probability of detection when the received primary signal is impaired by AWGN or wireless propagation environments such as Nakagami or Rayleigh fading channels [197]. Nevertheless, the impact of particular signal properties has not been taken into account in the analysis and performance evaluation of ED in DSA/CR networks. In this context, the study reported in this chapter covers such deficiencies and fills the existing gaps by developing a model for signal uncertainty and analyzing its im- 
pact on the resulting detection performance of ED. Starting with the classical theoretical results associated to ED, a model for signal uncertainty is developed in Section 5.2 based on theoretical reasonings complemented with empirical approximations. The model is capable to capture and reproduce the impact of the variability of the primary transmission power pattern on the experimental performance of ED as observed in Chapter 4. Based on such model, Section 5.3 discusses and exemplifies with numerical results the impact of signal uncertainty on the detection performance of ED, and compares the degrading effect of signal uncertainty with that of noise uncertainty. Finally, Section 5.4 summarizes the main findings of the investigation carried out in this chapter.

\subsection{Signal uncertainty model}

The study carried out in Chapter 4 has highlighted the existence of two important aspects that can neither be predicted nor reproduced with the classical theoretical results associated to ED. First, the experimental results indicate that the ED performance may notably vary with the radio technology being detected. Second, the performance differences among various radio technologies are not constant but depend on the considered sensing period. Both phenomena can be related to the variability (variance) of the primary transmission power pattern as discussed in Chapter 4. However, the potential effect of this particular signal property on the resulting ED performance has not been studied and cannot be predicted with the existing analytical results. The main objective of this chapter is to develop a novel model for the detection performance of ED that takes into account the variability of the primary transmission power pattern and is capable to reproduce the two aspects mentioned above.

\subsubsection{Problem formulation}

In practice, the SNR is not constant at the secondary receiver since it is the result of the combined effects of the primary transmission power pattern and the propagation environment. As a result, the probability of detection varies with the instantaneous SNR ${ }^{1}$. In such a case, Equation 4.21-4.22 gives the probability of detection $P_{d}$ conditioned on the instantaneous value of $\gamma$. Under varying SNR, a more useful performance parameter is the average probability of detection $\bar{P}_{d}$ experienced for an average SNR $\gamma_{0}$, which can be derived by averaging Equation 4.21-4.22 over the SNR statistics:

$$
\bar{P}_{d}\left(\gamma_{0}\right)=\mathbb{E}\left[P_{d}(\gamma)\right]=\int_{\gamma} P_{d}(\gamma) f_{\gamma}(\gamma) d \gamma
$$

where $P_{d}(\gamma)$ is given by Equation 4.21-4.22 and $f_{\gamma}(\gamma)$ is the Probability Density Function (PDF) of the received SNR. Closed-form expressions for the integral in Equation 5.1 under Nakagami and Rayleigh fading environments have been derived in the literature [197] in order to reflect the impact of radio propagation channels on the resulting detection performance of ED. In contrast, the focus of this study is on the impact of the primary transmission power

\footnotetext{
${ }^{1}$ The probability of false alarm remains unchanged since under hypothesis $\mathcal{H}_{0}$ there is no primary signal present and $P_{f a}$ is therefore independent of $\gamma$ as indicated by Equations 4.12 and 4.15.
} 
pattern. Therefore, it will be assumed that $f_{\gamma}(\gamma)$ is mostly the result of the primary transmission power pattern, which was shown in Chapter 4 to strongly affect the ED performance. Under such circumstance, the particular shape of $f_{\gamma}(\gamma)$, and hence the detection performance $\bar{P}_{d}\left(\gamma_{0}\right)$, depend on the primary transmission power pattern and its variability (variance). The main objective of this section is to solve Equation 5.1 in order to establish a direct relation between the primary power variability and the resulting detection performance of ED. To this end, a model for $f_{\gamma}(\gamma)$ is required, which is developed in Section 5.2.2.

\subsubsection{Approximation for the SNR statistics}

From a realistic point of view, it is reasonable to assume that $f_{\gamma}(\gamma)$ cannot be perfectly known in practice. Determining an exact expression for the distribution of received SNR values, $f_{\gamma}(\gamma)$, would require a perfect knowledge of the transmission power statistics, which are in general unknown and cannot easily be determined in reality since they may depend on many factors such as the particular radio technology being detected and its signaling format as well as the instantaneous conditions of the primary network (e.g., a cellular mobile communication system with load-based power control). Even if the transmission power statistics of any primary radio technology could be assumed to be known, the obtained expression for $f_{\gamma}(\gamma)$ would be valid for a single radio technology, thus requiring a separate analysis for every possible primary radio technology to be detected. Such analysis would result impractical.

As an alternative, and given that deriving an individual analytical expression of $f_{\gamma}(\gamma)$ for every possible case is infeasible, this section is aimed at developing a general, technologyindependent approximated expression that can be determined without a perfect knowledge of the primary transmission power statistics and that can be used to describe the distribution of the received SNR values over a wide range of cases with a reasonable level of accuracy. This approach would be valid as long as the resulting expression in Equation 5.1 is able to describe, with a sufficient level of accuracy, the experimental probability of detection and the performance differences observed in Figures 4.4, 4.5 and 4.6 for real-world signals of different radio technologies.

In order to find a realistic model for $f_{\gamma}(\gamma)$, the empirical SNR PDFs of various real-world signals were computed based on the measurements performed in Chapter 4 for different radio technologies (analogical/digital TV, DAB-T, TETRA, E-GSM 900, DCS 1800 and UMTS). In particular, the empirical PDF of the receiving SNR was computed for each individual channel of the analyzed radio technologies and the obtained PDFs were then extensively compared to a wide range of theoretical PDF models. The obtained results indicated that there is no univocal relation between the radio technology to which a channel belongs and the PDF model that best fits the empirical PDF of the SNR values received in that channel. As a matter of fact, channels belonging the same radio technology were best fitted by some theoretical PDF models in some cases but better fitting results were obtained with other PDF models in some other cases. On the other hand, it is also worth noting that the same theoretical PDF models were observed to provide accurate fits for the empirical SNR PDFs of channels belonging to different radio technologies. More concretely, it was found that most of the obtained empirical PDFs, irrespective of their corresponding radio technology, 
can acceptably be approximated by either Rayleigh distributions:

$$
f_{\gamma}^{R}(\gamma)=\frac{\gamma}{s^{2}} \exp \left(-\frac{\gamma^{2}}{2 s^{2}}\right), \quad \gamma \geq 0
$$

as in the example shown in Figure 5.1(a), or gamma distributions:

$$
f_{\gamma}^{G}(\gamma)=\frac{\gamma^{k-1}}{\theta^{k} \Gamma(k)} \exp \left(-\frac{\gamma}{\theta}\right), \quad \gamma \geq 0
$$

with $k=2$, where $\Gamma(\cdot)$ is the gamma function [198], as in the example shown in Figure 5.1(b). For these distributions, the parameters $s$ and $\theta$ can be computed as [210]:

$$
\begin{aligned}
& s=\sqrt{\frac{2}{\pi}} \gamma_{0}=\sqrt{\frac{2 \sigma_{\gamma}^{2}}{4-\pi}} \\
& \theta=\frac{1}{2} \gamma_{0}=\sqrt{\frac{\sigma_{\gamma}^{2}}{2}}
\end{aligned}
$$

where $\gamma_{0}$ and $\sigma_{\gamma}^{2}$ represent the average SNR and the SNR variance, respectively. The values of $\gamma_{0}$ and $\sigma_{\gamma}^{2}$ for the captured signals were respectively estimated as the sample mean and sample variance of the instantaneously received SNR values after normalizing the signal sequences as indicated in Equation 4.26. Based on such estimations, the corresponding PDF models were derived based on either $\gamma_{0}$ (denoted as mean-based) or $\sigma_{\gamma}^{2}$ (denoted as variance-based). As it can be observed in Figures 5.1(a) and 5.1(b), the Rayleigh and gamma distributions provide reasonably good fits for empirical SNR PDFs observed in reality and could therefore be considered as models for the SNR distribution $f_{\gamma}(\gamma)$.

For the Rayleigh and gamma distributions, the SNR mean and variance are related as $\sigma_{\gamma}^{2}=(4 / \pi-1) \gamma_{0}^{2} \approx 0.27 \gamma_{0}^{2}$ for the former and $\sigma_{\gamma}^{2}=0.5 \gamma_{0}^{2}$ for the latter [210]. This indicates the existence of a relation of the form $\sigma_{\gamma}^{2}=\beta \gamma_{0}^{2}$ in practice, where $\beta$ can be regarded as a normalized variance $\left(\beta=\sigma_{\gamma}^{2} / \gamma_{0}^{2}\right)$ and thus as a metric representing the variability of the primary transmission power pattern. As mentioned before, the aim is to establish a direct relation between the primary power variability, which can be quantified by means of $\beta$, and the resulting detection performance $\bar{P}_{d}\left(\gamma_{0}\right)$. In the case of the Rayleigh and gamma distributions the value of $\beta$ is fixed ( $\beta \approx 0.27$ for the former, $\beta=0.5$ for the latter). This means that the resulting expression for $\bar{P}_{d}\left(\gamma_{0}\right)$ in such cases would not allow to analyze the ED performance for any arbitrary primary power variability $\beta$, but only for those cases where the received SNR can adequately be modeled with a Rayleigh or gamma distribution. This observation motivates the search of an alternative model for $f_{\gamma}(\gamma)$ where $\beta$ can be configured in order to analyze the impact of arbitrary primary power variabilities (variances) on the detection performance $\bar{P}_{d}\left(\gamma_{0}\right)$. A simple and analytically tractable PDF model verifying this property is the Gaussian distribution, where the variance is independent of the mean. It is important to note, however, that the Gaussian PDF is defined for any real value while $\gamma \geq 0$. This issue can be resolved by truncating the Gaussian PDF to positive values and introducing 


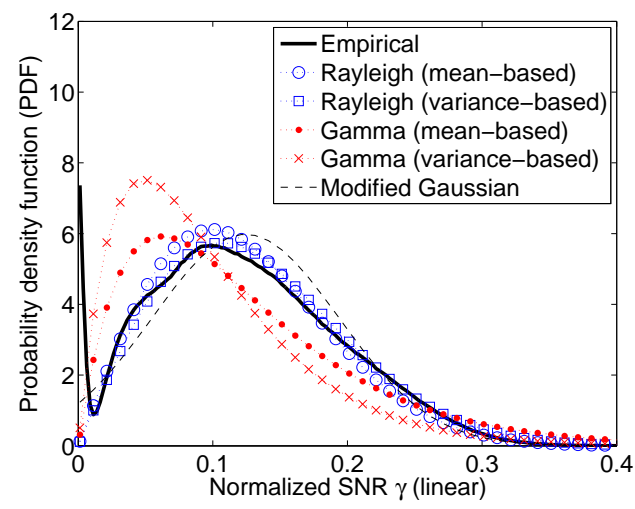

(a)

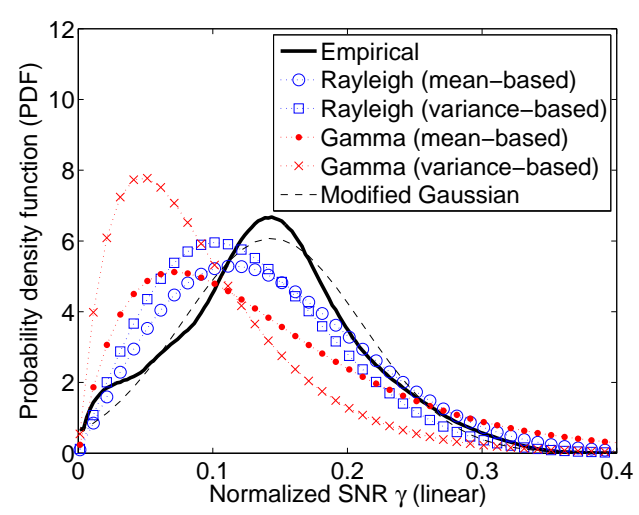

(c)

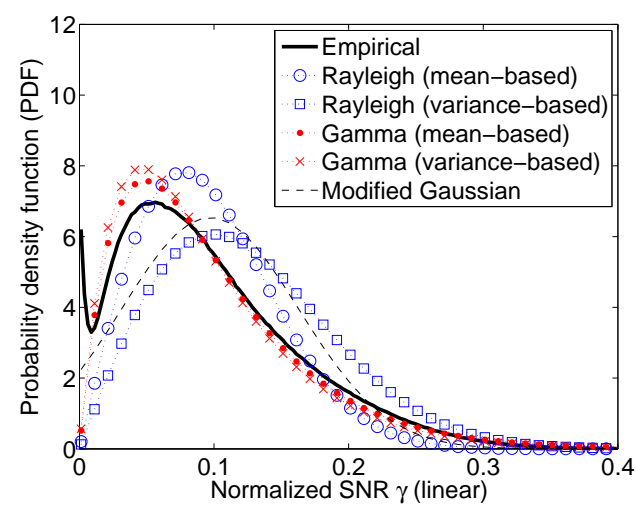

(b)

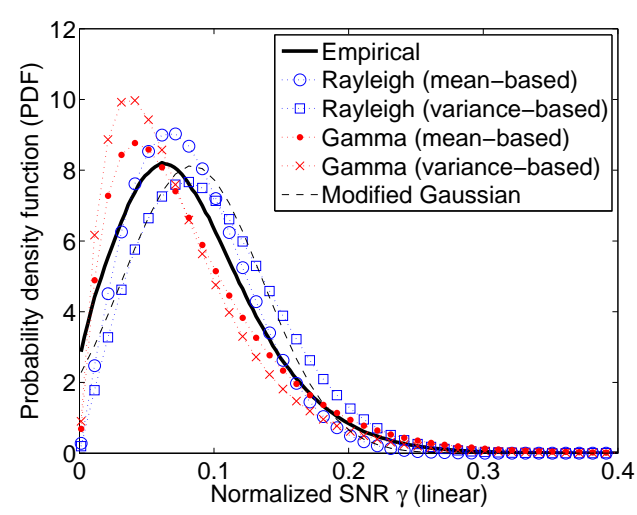

(d)

Figure 5.1: Empirical and approximated PDF for the received SNR.

a normalization factor $\kappa$ such that $\int_{0}^{\infty} f_{\gamma}(\gamma) d \gamma=1$, which results in the following modified Gaussian PDF model:

$$
f_{\gamma}^{M G}(\gamma) \approx \frac{\kappa}{\sqrt{2 \pi} \sigma_{\gamma}} e^{-\frac{1}{2}\left(\frac{\gamma-\gamma_{0}}{\sigma_{\gamma}}\right)^{2}}, \quad \gamma \geq 0
$$

where the normalization factor is given by:

$$
\kappa=\frac{2}{1+\operatorname{erf}\left(\frac{\gamma_{0}}{\sqrt{2} \sigma_{\gamma}}\right)}
$$

with $\operatorname{erf}(\cdot)$ being the error function [201, (7.1.1)]. As a result of the constraint $\gamma \geq 0, \sigma_{\gamma}^{2}$ and $\gamma_{0}$ are not completely independent in practice as illustrated in Figure 5.2. When the received SNR spreads out over a wide range of values (i.e., the received SNR is characterized by a high variance $\sigma_{\gamma}^{2}$ ), the average SNR $\gamma_{0}$ is larger than in the case where the variance of the received SNR is lower. Thus, a low mean value $\gamma_{0}$ cannot be associated with an arbitrarily 

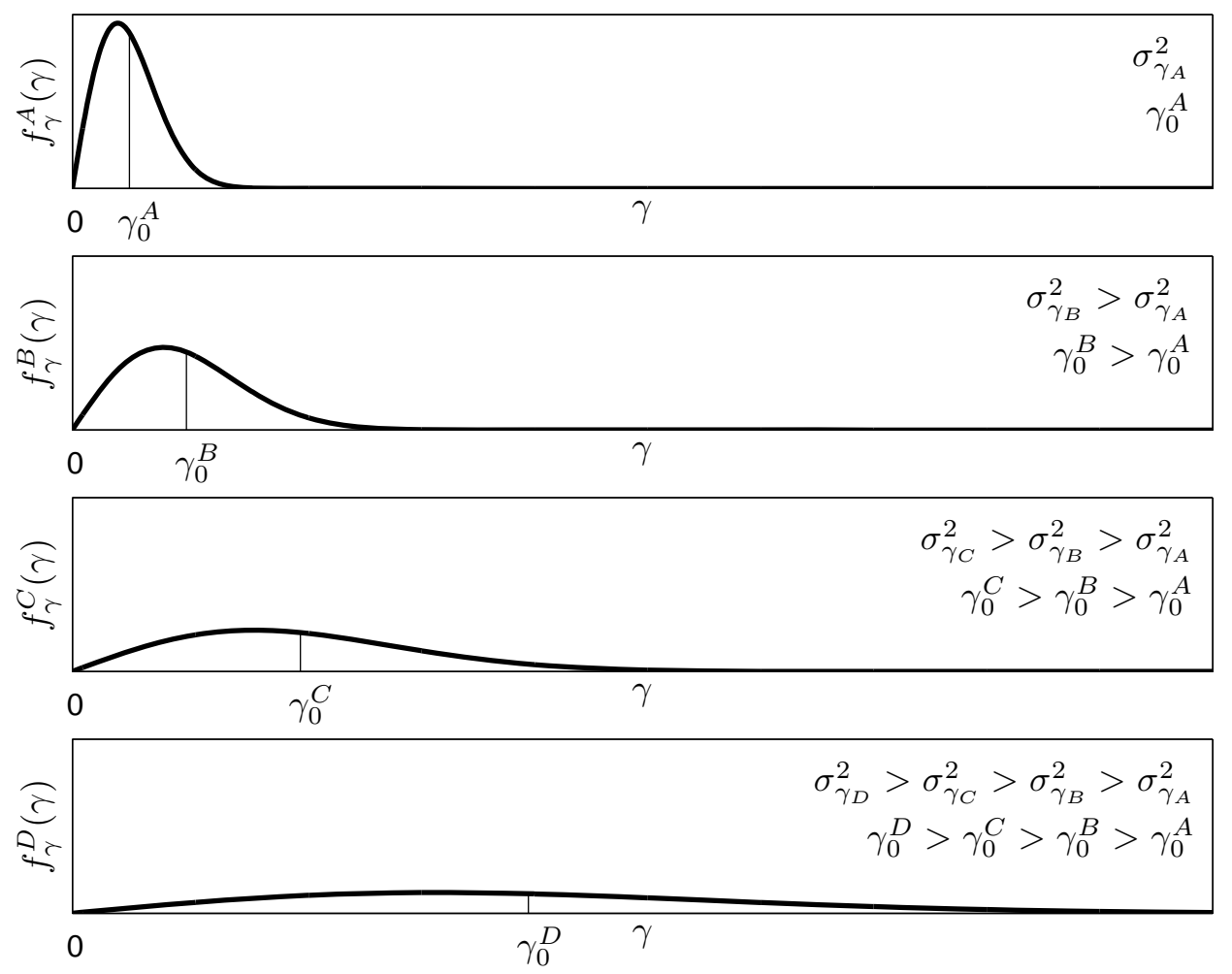

Figure 5.2: Dependence between the mean and the variance of the received SNR.

large variance $\sigma_{\gamma}^{2}$ and vice versa. The dependence between $\sigma_{\gamma}^{2}$ and $\gamma_{0}$ suggests that the relation $\sigma_{\gamma}^{2}=\beta \gamma_{0}^{2}$ also applies in Equations 5.6 and 5.7. As it will be demonstrated later on, the model developed under this consideration provides accurate results. The main advantage of the proposed model with respect to the Rayleigh and gamma models is that the resulting expression for $\bar{P}_{d}\left(\gamma_{0}\right)$ will be obtained as a function of $\beta$, thus enabling the performance analysis of ED for any arbitrary primary power variability. The exact value of $\beta$ can be assumed to be unknown to the secondary receiver due to the inability of a secondary user to perfectly know beforehand the primary signals that might be present in the sensed band and their properties. This parameter will henceforth be referred to as signal uncertainty. The validity of this model is corroborated in Figure 5.1. For the cases where the received SNR can be approximated by Rayleigh or gamma distributions as in Figures 5.1(a) and 5.1(b) respectively, the model of Equations 5.6 and 5.7 provides a reasonable fit. Moreover, it is interesting to mention that, for some cases where the empirical value of $\beta$ is neither 0.27 (Rayleigh case) nor 0.5 (gamma case), the proposed model is able to provide more accurate fits, as illustrated in the example of Figure 5.1(c). In a few particular cases none of the considered approximations is perfect although all of them are able to qualitatively describe the empirical SNR distribution as shown in Figure 5.1(d). In conclusion, the model of Equations 5.6 and 5.7 can be employed to mathematically describe, in an approximated but acceptable manner, the empirical distribution of received SNR values. 


\subsubsection{Approximation for the probability of detection}

In order to facilitate the analytical resolution of Equation 5.1 with the proposed SNR PDF models, the term $P_{d}(\gamma)$ is approximated as follows. According to Equation 4.21-4.22, the probability of detection can be expressed as:

$$
P_{d}(\gamma)=Q(\zeta(\gamma))
$$

where $\zeta(\gamma)$ is given by:

$$
\zeta(\gamma)=\frac{\alpha Q^{-1}\left(P_{f a}\right) \sqrt{2 N}-N(\gamma+1-\alpha)}{\sqrt{2 N}(1+\gamma)} \approx \alpha Q^{-1}\left(P_{f a}\right)-\sqrt{\frac{N}{2}}(\gamma+1-\alpha)
$$

The Gaussian $Q$-function can be approximated by a second-order exponential function:

$$
Q(x) \approx e^{-\left(a x^{2}+b x+c\right)}, \quad x \geq 0
$$

with fitting coefficients $a=0.3845, b=0.7635$ and $c=0.6966$ (see Appendix C). Notice that $\zeta(\gamma)$, the argument of the $Q$-function in Equation 5.8, may take both positive and negative values even though $\gamma \geq 0$ (see Equation 5.9). Since the approximation in Equation 5.10 is valid for positive arguments only, the property $Q(x)=1-Q(-x)$ must therefore be used for negative values of $\zeta(\gamma)$. Applying this approximation to Equation 5.8 it finally yields:

$$
P_{d}(\gamma)=Q(\zeta(\gamma)) \approx \begin{cases}e^{-\left(a[\zeta(\gamma)]^{2}+b \zeta(\gamma)+c\right)}=e^{-\left(\Omega \gamma^{2}+\Psi \gamma+\Phi\right)}, & \zeta(\gamma) \geq 0 \Rightarrow \gamma \leq \xi \\ 1-e^{-\left(a[\zeta(\gamma)]^{2}-b \zeta(\gamma)+c\right)}=1-e^{-\left(\Omega \gamma^{2}+\Xi \gamma+\Theta\right)}, & \zeta(\gamma) \leq 0 \Rightarrow \gamma \geq \xi\end{cases}
$$

where

$$
\begin{aligned}
& \Omega=\frac{a N}{2} \\
& \Psi=-a \alpha Q^{-1}\left(P_{f a}\right) \sqrt{2 N}-a N(\alpha-1)-b \sqrt{\frac{N}{2}} \\
& \left.\Xi=-a \alpha Q^{-1}\left(P_{f a}\right) \sqrt{2 N}-a N(\alpha-1)+b \sqrt{\frac{N}{2}}\right]^{2}+b\left[\alpha Q^{-1}\left(P_{f a}\right)+\sqrt{\frac{N}{2}}(\alpha-1)\right]+c \\
& \left.\Phi=a\left[\alpha Q^{-1}\left(P_{f a}\right)+\sqrt{\frac{N}{2}}(\alpha-1)\right]+\sqrt{\frac{N}{2}}(\alpha-1)\right]+c \\
& \xi=a\left[\alpha Q^{-1}\left(P_{f a}\right)+\sqrt{\frac{N}{2}}(\alpha-1)\right]^{2}-b\left[\alpha Q^{-1}\left(P_{f a}\right)+\sqrt{\frac{2}{N}} Q^{-1}\left(P_{f a}\right)+\alpha-1\right.
\end{aligned}
$$




\subsubsection{Closed-form expressions}

Substituting the SNR PDF model of Equations 5.6-5.7 and the approximation of Equations 5.11-5.17 into Equation 5.1 yields:

$$
\begin{aligned}
& \bar{P}_{d}^{M G}\left(\gamma_{0}\right)=\int_{\gamma} P_{d}(\gamma) f_{\gamma}^{M G}(\gamma) d \gamma \\
& \approx \frac{\kappa}{\sqrt{2 \pi} \sigma_{\gamma}}\left[\int_{0}^{\xi} e^{-\left(\Omega \gamma^{2}+\Psi \gamma+\Phi\right)} e^{-\frac{1}{2}\left(\frac{\gamma-\gamma_{0}}{\sigma \gamma}\right)^{2}} d \gamma+\int_{\xi}^{\infty}\left[1-e^{-\left(\Omega \gamma^{2}+\Xi \gamma+\Theta\right)}\right] e^{-\frac{1}{2}\left(\frac{\gamma-\gamma_{0}}{\sigma \gamma}\right)^{2}} d \gamma\right] \\
& \approx \frac{\kappa}{2} \operatorname{erfc}\left(\frac{\xi-\gamma_{0}}{\sqrt{2} \sigma_{\gamma}}\right) \\
& +\frac{\kappa}{2} \sqrt{\frac{1}{2 \sigma_{\gamma}^{2} \widetilde{\Omega}}} \exp \left(\frac{\widetilde{\Psi}^{2}}{4 \widetilde{\Omega}}-\widetilde{\Phi}\right)\left[\operatorname{erf}\left(\frac{2 \widetilde{\Omega} \xi+\widetilde{\Psi}}{2 \sqrt{\widetilde{\Omega}}}\right)-\operatorname{erf}\left(\frac{\widetilde{\Psi}}{2 \sqrt{\widetilde{\Omega}}}\right)\right] \\
& -\frac{\kappa}{2} \sqrt{\frac{1}{2 \sigma_{\gamma}^{2} \widetilde{\Omega}}} \exp \left(\frac{\widetilde{\Xi}^{2}}{4 \widetilde{\Omega}}-\widetilde{\Theta}\right) \operatorname{erfc}\left(\frac{2 \widetilde{\Omega} \xi+\widetilde{\Xi}}{2 \sqrt{\widetilde{\Omega}}}\right)
\end{aligned}
$$

where

$$
\begin{aligned}
& \widetilde{\Omega}=\Omega+\frac{1}{2 \sigma_{\gamma}^{2}} \\
& \widetilde{\Psi}=\Psi-\frac{\gamma_{0}}{\sigma_{\gamma}^{2}} \\
& \widetilde{\Xi}=\Xi-\frac{\gamma_{0}}{\sigma_{\gamma}^{2}} \\
& \widetilde{\Phi}=\Phi+\frac{\gamma_{0}^{2}}{2 \sigma_{\gamma}^{2}} \\
& \widetilde{\Theta}=\Theta+\frac{\gamma_{0}^{2}}{2 \sigma_{\gamma}^{2}}
\end{aligned}
$$

and $\operatorname{erfc}(\cdot)$ is the complementary error function [201, (7.1.2)]. It is worth noting that the two last terms of Equation 5.18 lead to similar numerical values, specially for high values of SNR, so that they approximately cancel out each other. The result of Equation 5.18 can therefore be simplified to:

$$
\bar{P}_{d}^{M G}\left(\gamma_{0}\right) \approx \frac{\kappa}{2} \operatorname{erfc}\left(\frac{\xi-\gamma_{0}}{\sqrt{2} \sigma_{\gamma}}\right)=\frac{\operatorname{erfc}\left(\frac{\xi-\gamma_{0}}{\sqrt{2} \sigma_{\gamma}}\right)}{1+\operatorname{erf}\left(\frac{\gamma_{0}}{\sqrt{2} \sigma_{\gamma}}\right)}
$$

Equation 5.24 provides an approximated, simpler expression for the ED performance under variable transmission power patterns where the variability of the transmitted power is characterized by means of the signal uncertainty parameter $\beta$, which expresses the SNR variance $\sigma_{\gamma}^{2}$ as a function of the average SNR $\gamma_{0}$. The approximation of Equation 5.24 is valid over a wide range of SNR values and becomes tighter for higher $N$ and lower $P_{f a}$ values. 
For comparison purposes, Equation 5.1 was also solved when the SNR follows Rayleigh (Equation 5.2) and gamma (Equation 5.3) distributions. In such cases, the resulting $\bar{P}_{d}\left(\gamma_{0}\right)$ can be expressed as:

$$
\begin{aligned}
\bar{P}_{d}^{R, G}\left(\gamma_{0}\right) \approx & \Upsilon\left(\gamma_{0}\right) \\
& +\frac{1}{v^{2}} \frac{\bar{\Psi}}{4} \sqrt{\frac{\pi}{\bar{\Omega}^{3}}} \exp \left(\frac{\bar{\Psi}^{2}}{4 \bar{\Omega}}-\Phi\right)\left[\operatorname{erf}\left(\frac{\bar{\Psi}}{2 \sqrt{\bar{\Omega}}}\right)-\operatorname{erf}\left(\frac{2 \bar{\Omega} \xi+\bar{\Psi}}{2 \sqrt{\bar{\Omega}}}\right)\right] \\
& +\frac{1}{v^{2}} \frac{\bar{\Xi}}{4} \sqrt{\frac{\pi}{\bar{\Omega}^{3}}} \exp \left(\frac{\bar{\Xi}^{2}}{4 \bar{\Omega}}-\Theta\right) \operatorname{erfc}\left(\frac{2 \bar{\Omega} \xi+\bar{\Xi}}{2 \sqrt{\bar{\Omega}}}\right) \\
& +\frac{1}{v^{2}} \frac{e^{-\Phi}-e^{-\left(\bar{\Omega} \xi^{2}+\bar{\Psi} \xi+\Phi\right)}-e^{-\left(\bar{\Omega} \xi^{2}+\bar{\Xi} \xi+\Theta\right)}}{2 \bar{\Omega}}
\end{aligned}
$$

where for the Rayleigh distribution:

$$
\begin{aligned}
\Upsilon\left(\gamma_{0}\right) & =\exp \left(-\frac{\pi}{4}\left(\frac{\xi}{\gamma_{0}}\right)^{2}\right) \\
v & =\sqrt{\frac{2}{\pi}} \gamma_{0} \\
\bar{\Omega} & =\Omega+\frac{\pi}{4 \gamma_{0}^{2}} \\
\bar{\Psi} & =\Psi \\
\bar{\Xi} & =\Xi
\end{aligned}
$$

while for the gamma distribution:

$$
\begin{aligned}
\Upsilon\left(\gamma_{0}\right) & =\left(1+\frac{2 \xi}{\gamma_{0}}\right) \exp \left(-\frac{2 \xi}{\gamma_{0}}\right) \\
\nu & =\frac{1}{2} \gamma_{0} \\
\bar{\Omega} & =\Omega \\
\bar{\Psi} & =\Psi+\frac{2}{\gamma_{0}} \\
\bar{\Xi} & =\Xi+\frac{2}{\gamma_{0}}
\end{aligned}
$$

As in Equation 5.18, the last terms of Equation 5.25 approximately cancel out each other, leading to the approximations:

$$
\begin{aligned}
& \bar{P}_{d}^{R}\left(\gamma_{0}\right) \approx \exp \left(-\frac{\pi}{4}\left(\frac{\xi}{\gamma_{0}}\right)^{2}\right) \\
& \bar{P}_{d}^{G}\left(\gamma_{0}\right) \approx\left(1+\frac{2 \xi}{\gamma_{0}}\right) \exp \left(-\frac{2 \xi}{\gamma_{0}}\right)
\end{aligned}
$$




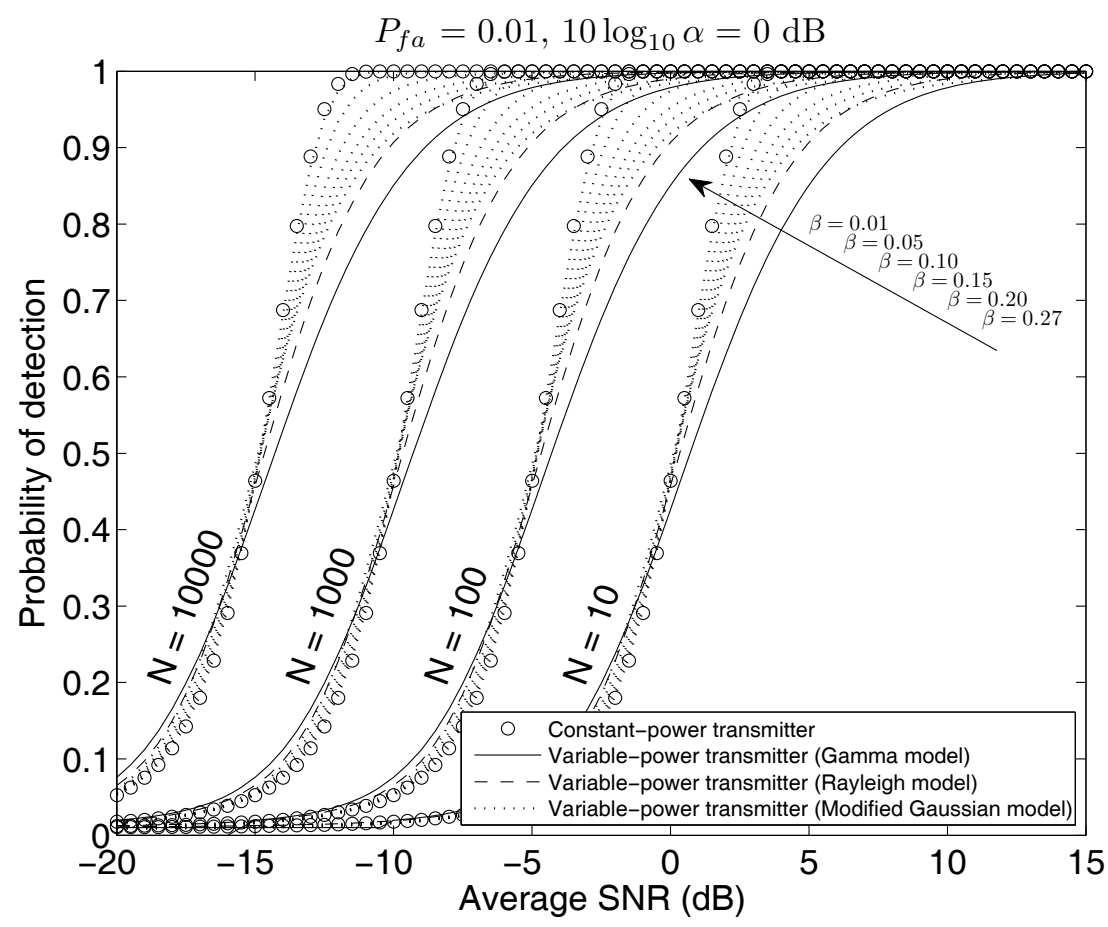

Figure 5.3: Probability of detection for constant- and variable-power transmitters when the SNR is modeled as modified Gaussian $(\beta \in[0.01,0.27])$, Rayleigh and gamma distributions.

Figure 5.3 compares Equations 4.21-4.22, 5.18 and 5.25. As it can be appreciated, the resulting probability of detection for primary signals whose received SNR can be modeled as a gamma distribution is lower than that obtained for primary signals with Rayleigh SNR distributions. This can be explained by the fact that $\sigma_{\gamma}^{2} \approx 0.27 \gamma_{0}^{2}(\beta \approx 0.27)$ for the Rayleigh case while $\sigma_{\gamma}^{2}=0.5 \gamma_{0}^{2}(\beta=0.5)$ for the gamma case. In other words, for a given average $\mathrm{SNR}$, the gamma distribution is characterized by a higher variance. This suggests that a higher signal variability can therefore be associated to a lower detection performance. In fact, the results obtained with the modified Gaussian model indicate, under constant operating conditions, that the detection performance degrades as the primary signal variability $\beta$ increases, which is in accordance with the experimental results observed in Chapter 4 . In the opposite direction, Equation 5.18 converges numerically to Equation 4.21-4.22 as the signal variability decreases. This can also be predicted analytically since:

$$
\lim _{\beta \rightarrow 0} \bar{P}_{d}^{M G}\left(\gamma_{0}\right)=\lim _{\sigma_{\gamma} \rightarrow 0} \int_{\gamma} P_{d}(\gamma) f_{\gamma}^{M G}(\gamma) d \gamma=\int_{\gamma} P_{d}(\gamma) \delta\left(\gamma-\gamma_{0}\right) d \gamma=P_{d}\left(\gamma_{0}\right)
$$

where $P_{d}(\gamma)$ is given by Equation $4.21-4.22$ and $\delta(\cdot)$ is the Dirac delta function, which can be expressed as the limit of a Gaussian PDF as its standard deviation tends to zero. For a constant-power transmitter, the received SNR is constant (neglecting the channel propagation effects), meaning that the instantaneous SNR $\gamma$ equals the average value $\gamma_{0}$ at any time, and in such a case Equations 4.21-4.22 and 5.18 coincide as indicated by Equation 
5.38. It is also worth noting that the proposed model with $\beta=0.27$ (which corresponds to a Rayleigh distribution), results in a detection probability that is quite similar to that obtained for Rayleigh SNR distributions, indicating that the proposed model is able to describe, with reasonable accuracy, the experimental probability of detection observed in practice.

\subsubsection{Impact of the sensing period}

The model of Equation 5.18 is able to describe the impact of the primary signal variability $\beta$ on the resulting detection performance of ED. However, the convergent trend observed in Figures 4.4, 4.5 and 4.6 as the sensing period $N$ increases is not described by Equation 5.18. This phenomenon can be modeled as an effective reduction of the primary signal variability $\beta$ as $N$ becomes larger, which can be expressed as $\sigma_{\gamma}^{2}=\beta \tau(N) \gamma_{0}^{2}$, where $\tau(N) \in(0,1]$ is a monotonically decreasing function of $N$.

An expression for $\tau(N)$ can be derived as follows. The ED test statistic $\mathbb{T}(\tilde{\mathbf{y}})$ as defined in Equation 4.9 represents the received primary signal energy in a sensing interval of $N$ samples, i.e. $\mathcal{E}(\tilde{\mathbf{y}})=\mathbb{T}(\tilde{\mathbf{y}})$. The received signal power can therefore be obtained as:

$$
\mathcal{P}(\tilde{\mathbf{y}})=\frac{1}{N} \mathcal{E}(\tilde{\mathbf{y}})=\frac{1}{N} \mathbb{T}(\tilde{\mathbf{y}})=\frac{1}{N} \sum_{n=1}^{N}|\tilde{y}[n]|^{2}
$$

Notice that the term $|\tilde{y}[n]|^{2}$ in Equation 5.39 is directly related to the instantaneous signal energy/power and hence the instantaneous SNR. Its variance:

$$
\operatorname{Var}\left(|\tilde{y}[n]|^{2}\right)=\operatorname{Var}\left(\left.\left[\frac{1}{N} \mathbb{T}(\tilde{\mathbf{y}})\right]\right|_{N=1}\right)
$$

is therefore associated to $\sigma_{\gamma}^{2}$, the variance of the instantaneous SNR. The reduction of the effective SNR variance as $N$ increases, $\tau(N)$, can thus be determined based on the variance of the test statistic as:

$$
\tau(N)=\frac{\operatorname{Var}\left(\left.\left[\frac{1}{N} \mathbb{T}(\tilde{\mathbf{y}})\right]\right|_{N \geq 1}\right)}{\operatorname{Var}\left(\left.\left[\frac{1}{N} \mathbb{T}(\tilde{\mathbf{y}})\right]\right|_{N=1}\right)}
$$

Both variances are related as $[211,(2.21)]$ :

$$
\begin{aligned}
\operatorname{Var}\left(\frac{1}{N} \sum_{n=1}^{N}|\tilde{y}[n]|^{2}\right) & =\frac{1}{N^{2}} \sum_{n=1}^{N} \operatorname{Var}\left(|\tilde{y}[n]|^{2}\right)+\frac{1}{N^{2}} \sum_{\substack{m=1 \\
n=1}}^{N} \sum_{\substack{n=m \\
n \neq m}}^{N} \operatorname{Cov}\left(|\tilde{y}[m]|^{2},|\tilde{y}[n]|^{2}\right) \\
& =\operatorname{Var}\left(|\tilde{y}[n]|^{2}\right)\left(\frac{1}{N}+\frac{N-1}{N} \rho\right)
\end{aligned}
$$

where it has been assumed that the samples $|\tilde{y}[n]|^{2}$ have equal variance (i.e., $\sigma_{\gamma}^{2}$ is constant over time) and $\rho$ represents the average correlation of distinct signal samples. According to Equation 5.42:

$$
\tau(N) \approx \frac{1}{N}+\frac{N-1}{N} \rho
$$




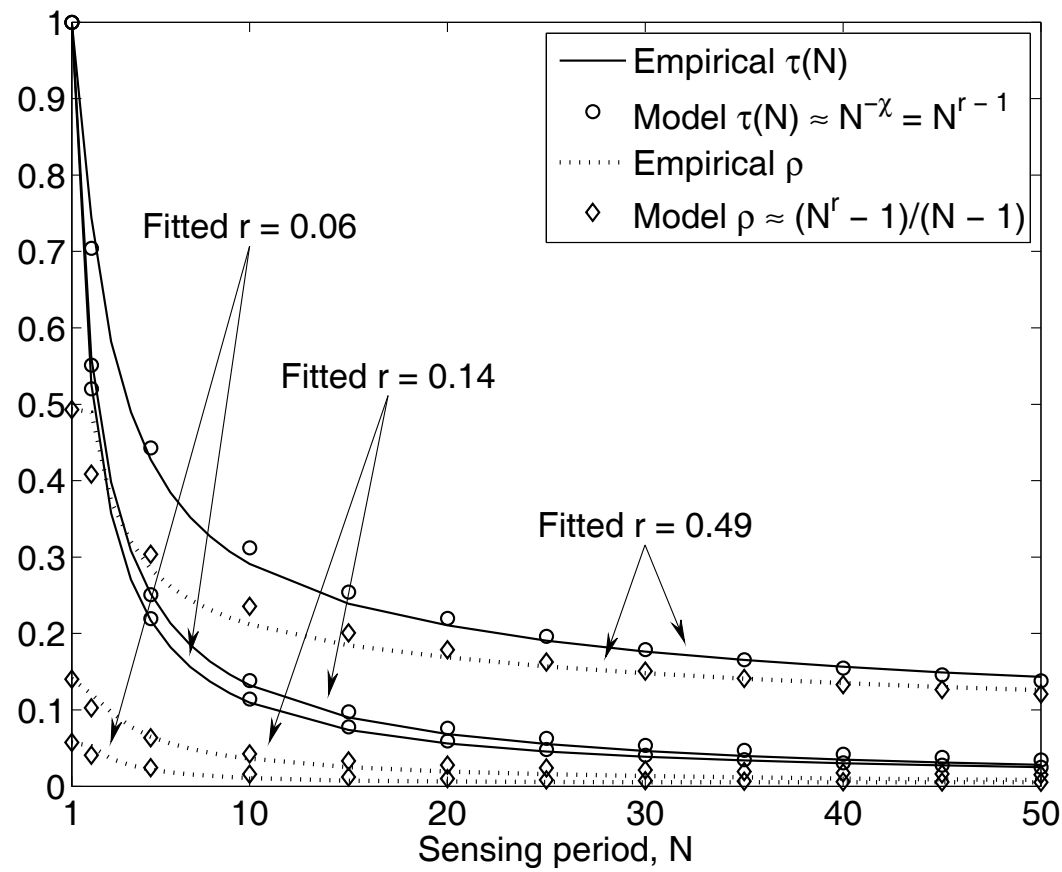

Figure 5.4: Empirical and approximated results for $\rho$ and $\tau(N)$.

In practice, however, the correlation coefficient $\rho$ is not completely independent of the sensing period $N$. Based on the empirical measurements performed in Chapter 4, and by means of curve fitting procedures, it was verified that the resulting empirical values of the average correlation coefficient can accurately be approximated as:

$$
\rho \approx \frac{N^{r}-1}{N-1}
$$

with $r$ being a fitting coefficient. Introducing this approximation into Equation 5.43 yields:

$$
\tau(N) \approx N^{-\chi}
$$

with $\chi=1-r$, which describes experimental results reasonably well (see Figure 5.4).

The parameter $\chi$ determines the rate at which the detection performance for various radio technologies converge as $N$ increases, and it will henceforth be referred to as the convergence rate. Notice that $\chi$ and $r$ are related to the correlation coefficient $\rho^{2}$, which means that the pace at which the detection performance for various radio technologies converge as $N$ increases in practice depends not only on the number of signal samples collected during the sensing period, $N$, but also on the correlation among them, which in turn depends on the sampling rate at which the samples are collected and its relation with the primary signal dynamics in the time domain. Let's denote as $T_{s}$ the sampling period, i.e. the time period

\footnotetext{
${ }^{2}$ In fact, it is interesting to note that $r=0$ for $\rho=0, r=1$ for $\rho=1$, and $r \rightarrow \rho$ as $N \rightarrow 1$.
} 
between two consecutive signal samples. It is reasonable to assume that the correlation between two consecutive signal samples decreases as $T_{s} \rightarrow \infty$. If $T_{s}$ is sufficiently large to assume that $\rho \approx 0$, then $r \approx 0$ and $\tau(N) \approx 1 / N$, which is the maximum rate at which the detection performance can converge as $N$ increases $(\chi=1)$. On the other extreme, for completely correlated samples $(\rho \approx 1)$, which corresponds to $T_{s} \rightarrow 0$, it holds that $r \approx 1$ and $\tau(N) \approx 1$, meaning that in such a case the detection performance for various primary signals would not converge $(\chi=0)$, no matter how long the sensing period is. In other words, for $\chi=0$ the convergent trend observed in Figures $4.4,4.5$ and 4.6 as the sensing period $N$ increases would not be reproduced. Instead, the distance between the performance curves for various radio technologies would remain constant, regardless of the value of $N$, as shown in Figure 5.3 (notice that this figure assumes a relation of the form $\sigma_{\gamma}^{2}=\beta \gamma_{0}^{2}$, i.e. $\tau(N)=1$ ).

In summary, the overall model for the detection performance of ED under variable primary transmission power patterns is given by Equation 5.18, where the average SNR $\gamma_{0}$ and the SNR variance $\sigma_{\gamma}^{2}$ are related as $\sigma_{\gamma}^{2}=\beta N^{-\chi} \gamma_{0}^{2}$. The signal uncertainty parameter, $\beta \geq 0$, represents the variability of the transmission power, while the convergence rate parameter, $\chi \in[0,1]$, determines the pace at which the detection performance for various primary signals converge as $N$ increases and it is related to the correlation among the signal samples.

\subsubsection{Model validation}

The overall model is validated in Figures 5.5 and 5.6, where the experimental detection performance of ED is compared with the theoretical prediction of Equation 4.21-4.22 as well as the performance predicted by the model of Equation 5.18 based on the values of $\beta$ and $\chi$ derived from empirical measurements. The results are shown for $N=100$, under the assumption of perfect calibration of the noise power in Figure 5.5 and with 1-dB noise uncertainty in Figure 5.6. As opposed to the classical theoretical result of Equation 4.21-4.22 which predicts the same detection performance for all the considered radio technologies, the proposed model is capable to capture and reproduce the impact of the variability of different transmission power patterns on the experimental performance of ED. As a result, the model of Equation 5.18 provides a more accurate estimation of the real performance of ED in practice. It is worth noting that for very short sensing periods (e.g., $N=10$ ) the model provides a slightly worse accuracy since in this case the performance curves lie in a region of SNR values (see Figures 4.4, 4.5 and 4.6) for which the assumption $\gamma \ll 1$ on which the proposed model relies is not perfectly valid. On the other hand, as the sensing period increases, the experimental performances converge on a single curve that matches with the prediction of Equation 4.21-4.22 and so does the detection performance predicted by Equation 5.18.

\subsection{Signal and noise uncertainties}

Based on the developed model, this section analyzes the impact of the unknown variability of the primary transmission power pattern (signal uncertainty) on the detection performance of ED, with and without noise uncertainty, and compares the practical consequences of both degrading effects. The design and configuration of ED is discussed as well. 

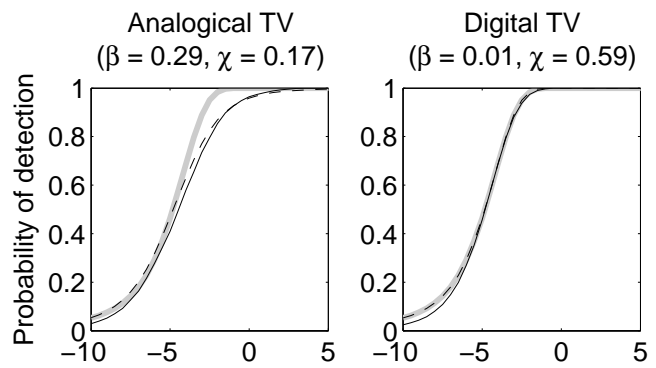

DAB-T

TETRA

E-GSM 900
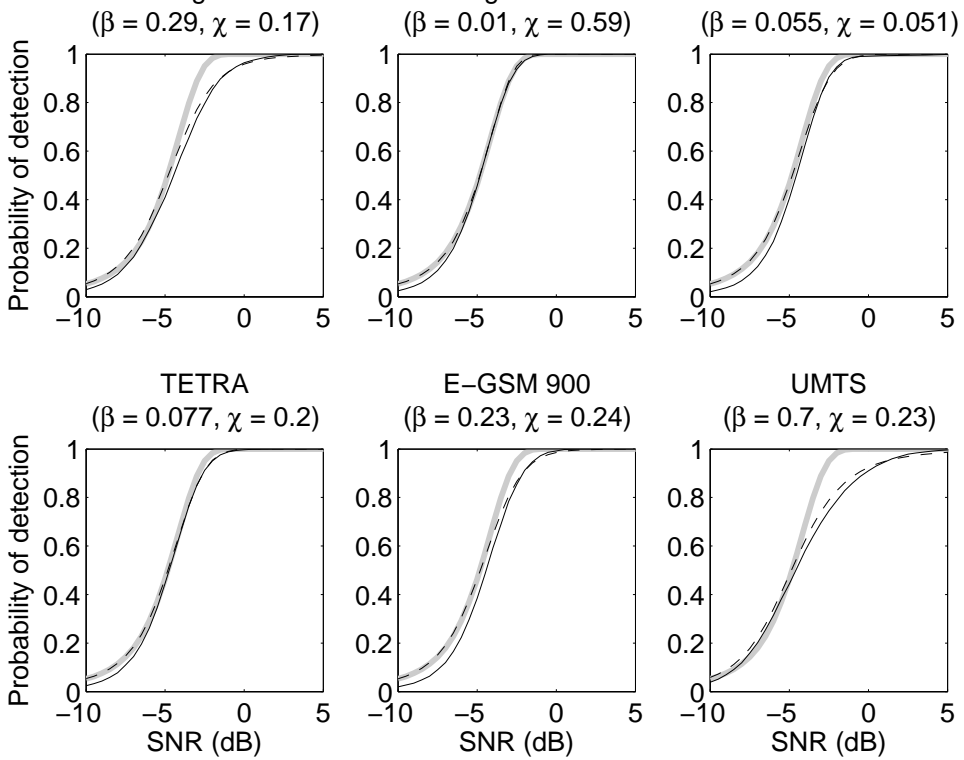

Figure 5.5: Probability of detection as a function of the SNR $\left(P_{f a}=0.01,10 \log _{10} \alpha=0 \mathrm{~dB}, N\right.$ = 100): Classical theoretical performance (light thick line), experimental performance (solid line) and proposed model (dashed line).
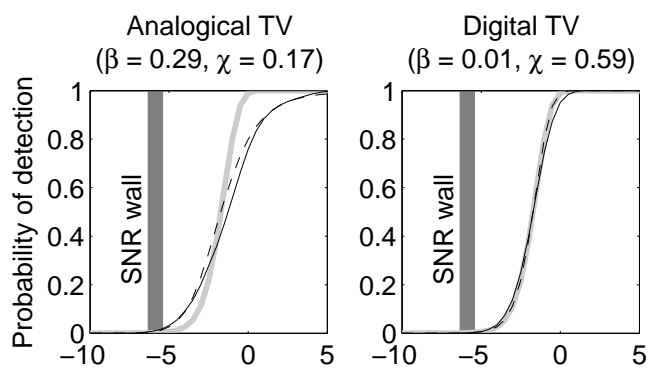

DAB-T

TETRA

E-GSM 900

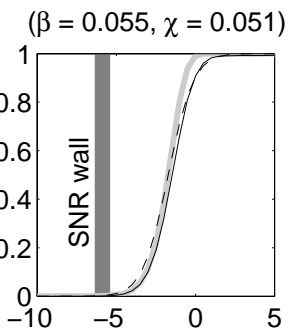

$(\beta=0.077, \chi=0.2)$

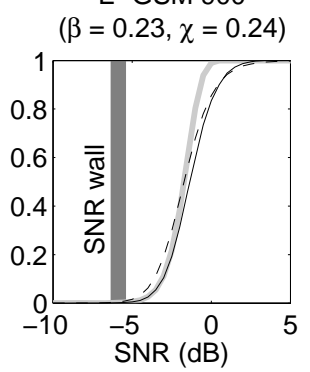

UMTS
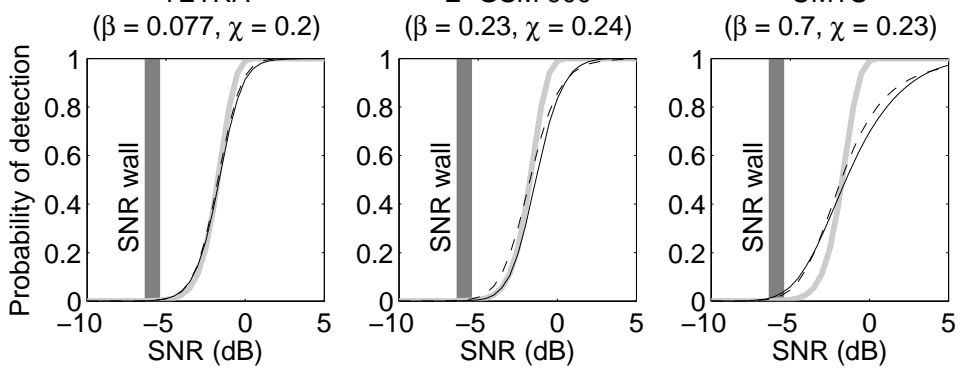

Figure 5.6: Probability of detection as a function of the SNR $\left(P_{f a}=0.01,10 \log _{10} \alpha=1 \mathrm{~dB}, N\right.$ = 100): Classical theoretical performance (light thick line), experimental performance (solid line) and proposed model (dashed line). 


\subsubsection{Performance analysis without noise uncertainty}

The maximum interference constraint for a DSA/CR network can be mapped to a minimum detection probability requirement that must be satisfied for SNR values above a predefined threshold, i.e. $\bar{P}_{d}\left(\gamma_{0}\right) \geq \bar{P}_{d}^{\min }$ for all $\gamma_{0} \geq \gamma_{0}^{\min }$. The example of this section assumes that the DSA/CR system is designed to guarantee $\bar{P}_{d}^{\min }=0.9$ at a given $\gamma_{0}^{\min }$. This requirement implies a minimum sensing period for the DSA/CR network, $N^{\min }$, which could be estimated as a function of $\gamma_{0}^{\min }$ based on the classical result of Equation 4.17-4.18 ( $\alpha=1$ in Equation 4.21-4.22, assuming a perfectly calibrated noise power), denoted as $N_{\text {classic }}^{\min }$. Based on this discourse, the equality $\bar{P}_{d}\left(\gamma_{0}\right)=\bar{P}_{d}^{\min }$ should be true for $N=N_{\text {classic }}^{\min }$. To verify this statement, and based on the model of Equation 5.18, Figure 5.7 plots the resulting $\bar{P}_{d}\left(\gamma_{0}\right)$ at the SNR values $\gamma_{0}=\gamma_{0}^{\min }$ for which $N_{\text {classic }}^{\text {min }}$ equals $10^{1}, 10^{2}, 10^{3}$ and $10^{4}$, when the target probability of detection is $\bar{P}_{d}^{\min }=0.9$ and $N=N_{\text {classic }}^{\text {min }}$. As it can be appreciated, when $N^{\text {min }}$ is computed with Equation 4.17-4.18, the equality $\bar{P}_{d}\left(\gamma_{0}\right)=\bar{P}_{d}^{\min }$ holds for constant-power transmitters only $(\beta \rightarrow 0)$. For primary signals of uncertain power variability $(\beta>0)$, the resulting $\bar{P}_{d}\left(\gamma_{0}\right)$ is lower than the target $\bar{P}_{d}^{\min }=0.9$. It is interesting to note that $\bar{P}_{d}\left(\gamma_{0}\right)$ first decreases below $\bar{P}_{d}^{\min }$ as the primary signal variability $\beta$ increases, but after reaching a minimum value it increases again. If $\beta$ were made arbitrarily large, $\bar{P}_{d}\left(\gamma_{0}\right)$ would also increase. However, it is worth noting that the maximum signal variability observed empirically in the measurements performed in Chapter 4 was $\beta=2.29$. This indicates that for practical values of $\beta$, the resulting $\bar{P}_{d}\left(\gamma_{0}\right)$ is lower than $\bar{P}_{d}^{\min }$ and the signal uncertainty phenomenon always is a degrading effect in practice, as illustrated in Figure 5.7 for various operating parameters. It is also interesting to note that the minimum value of $\bar{P}_{d}\left(\gamma_{0}\right)$ is reached at different values of $\beta$ (depending on the operating parameters), but it is always constant. In fact, it was verified that for $\bar{P}_{d}^{\min } \geq 0.9$, the worst-case performance is $\min \left\{\bar{P}_{d}\left(\gamma_{0}\right)\right\} \approx 0.8 \cdot \bar{P}_{d}^{\min }$, i.e. the worst detection probability is about $80 \%$ of the target value (this is however an optimistic estimation based on the assumption of perfectly calibrated noise power). This means that if signal uncertainty is not taken into account and the spectrum sensing function of a DSA/CR network is designed according to the classical result of Equation 4.17-4.18, then there would be a potential and unacceptable risk of harmful interference to the primary system.

\subsubsection{Performance analysis with noise uncertainty}

The results of Figure 5.7 correspond to the ideal case of no noise uncertainty, which is never true in reality. The example of this section considers that the DSA/CR terminals are assumed to operate with a small noise power calibration error $\left(10 \log _{10} \alpha=0.1 \mathrm{~dB}\right)$ and the required $N^{\min }$ is selected accordingly based on Equation 4.21-4.22. In this case, the comparison of Figures 5.7 and 5.8 indicates that the detection performance degradation due to the signal uncertainty phenomenon may become more severe in the presence of noise uncertainty. The most unfavorable case corresponds to low convergence rates, where the worst-case detection performance degrades as the sensing period increases (see Figure 5.8). This aspect should carefully be taken into account in the design of DSA/CR systems since DSA/CR devices are expected to operate in low SNR regimes, where the required sensing periods are usually high, and in the unavoidable presence of noise uncertainty. 


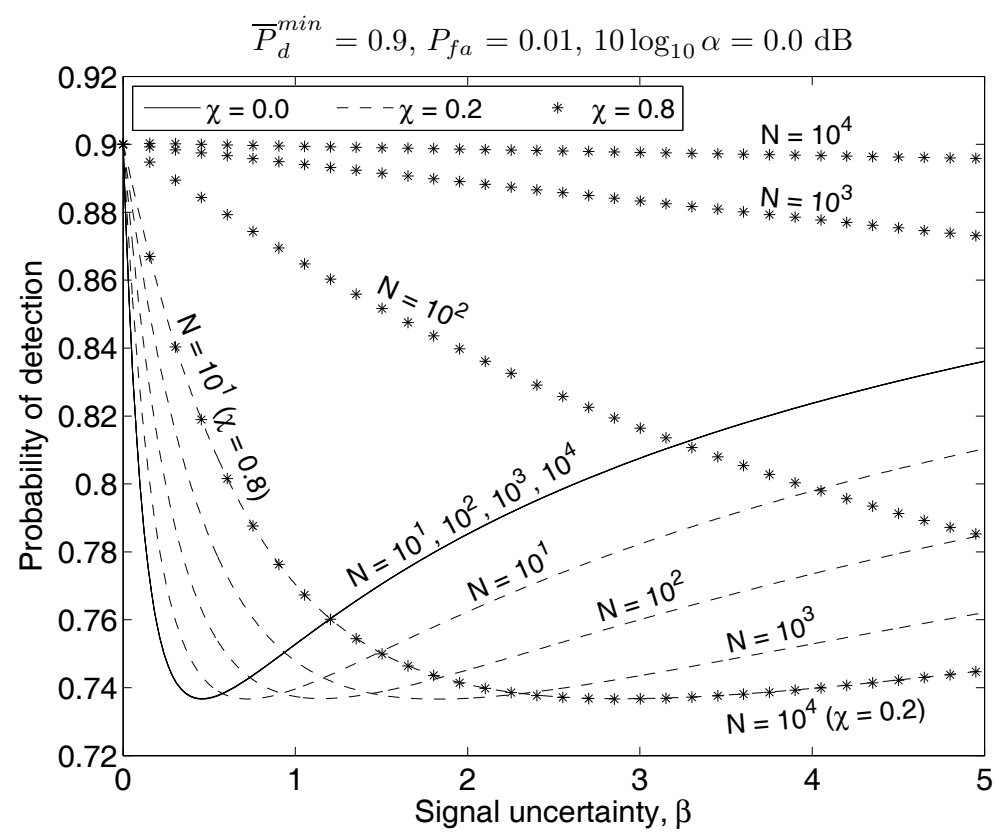

Figure 5.7: Probability of detection as a function of the signal uncertainty for various convergence rates and sensing periods $\left(\bar{P}_{d}^{\text {min }}=0.9, P_{f a}=0.01,10 \log _{10} \alpha=0.0 \mathrm{~dB}\right)$.

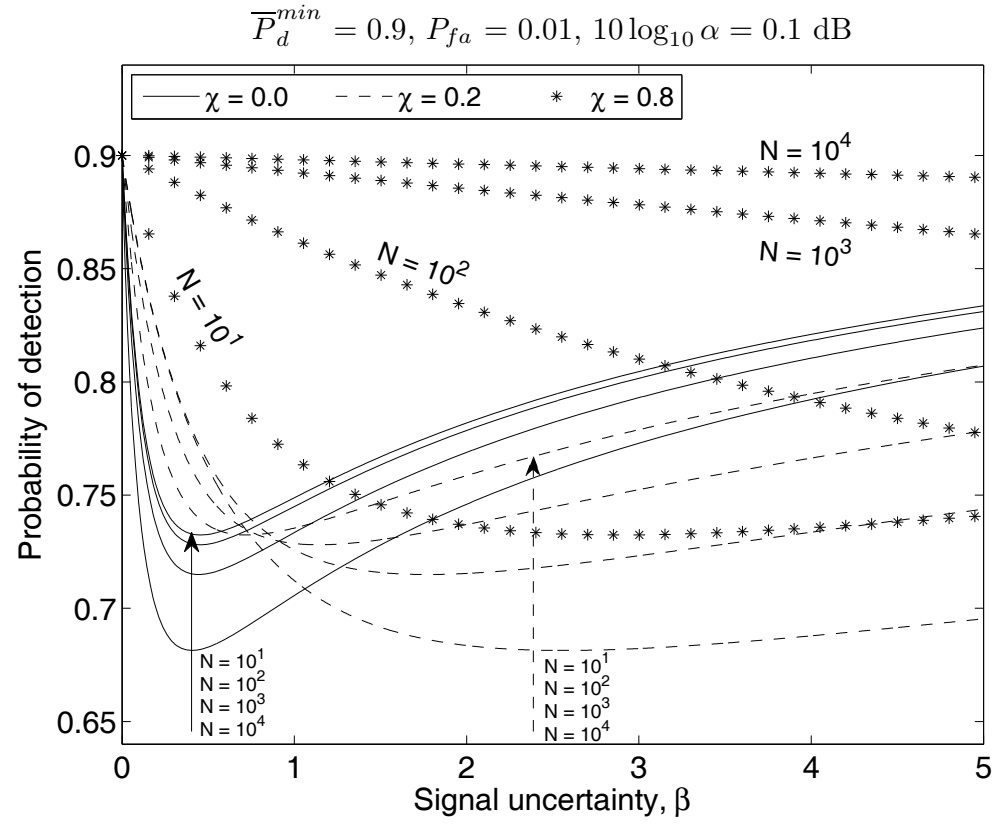

Figure 5.8: Probability of detection as a function of the signal uncertainty for various convergence rates and sensing periods $\left(\bar{P}_{d}^{\min }=0.9, P_{f a}=0.01,10 \log _{10} \alpha=0.1 \mathrm{~dB}\right)$. 
Noise uncertainty can arguably be considered as a more severe degrading phenomenon than signal uncertainty. If the noise uncertainty $\alpha$ increased arbitrarily, the SNR wall would increase unceasingly and the resulting detection probability would tend to zero. On the other hand, signal uncertainty may result in a noticeable but bounded performance degradation, which would not increase for arbitrarily large values of $\beta$ (see Figures 5.7 and 5.8). In any case, the degrading effects of the signal uncertainty phenomenon have been shown to be of notable importance and become more severe in the presence of noise uncertainty, even for very small noise power calibration errors. This highlights the importance of carefully taking into account not only the noise uncertainty phenomenon but also the primary signal uncertainty in the design and configuration of spectrum sensing in real DSA/CR systems.

\subsubsection{Design and configuration of energy detection}

To effectively achieve the desired $\bar{P}_{d}^{\text {min }}$, the required sensing period $N^{\text {min }}$ should be computed taking into account not only the presence of noise uncertainty but also the potential primary signal variability (i.e., the signal uncertainty). To this end, the model of Equation 5.18 constitutes a suitable tool and more adequate alternative than the classical theoretical result of Equations 4.17-4.18 and 4.21-4.22. Notice that Equation 5.18 and the simplified version of Equation 5.24 cannot be solved analytically for $N$ in closed form but they can be solved numerically in order to find the minimum sensing period required for a desired target performance under various operating conditions.

As an example, Figure 5.9 shows the $N^{m i n}$ required in a worst-case design (with and without noise uncertainty) as a function of the experienced $\gamma_{0}$ for a target $\bar{P}_{d}^{\min }=0.9$ and assuming a maximum expected primary signal variability of $\beta=1.0$. The prediction of the classical theoretical result of Equations 4.17-4.18 and 4.21-4.22 is also shown, indicating the SNR values $\gamma_{0}=\gamma_{0}^{\min }$ for which $N_{\text {classic }}^{\text {min }}$ equals $10^{1}, 10^{2}, 10^{3}$ and $10^{4}$. As it can be appreciated, the required $N^{\min }$ should be increased with respect to the $N_{\text {classic }}^{\text {min }}$ prediction, for which it has been shown that $\bar{P}_{d}\left(\gamma_{0}\right) \leq \bar{P}_{d}^{\text {min }}$. However, the required increment depends on the convergence rate parameter $\chi$, being greater for lower convergence rates and vice versa. This can be explained by the fact that $\bar{P}_{d}\left(\gamma_{0}\right)$ increases with $N$, but faster for higher convergence rates $(\chi=0.8,1.0)$, in which case a lower increment of $N^{\text {min }}$ may be enough to meet $\bar{P}_{d}^{\text {min }}$. Since the convergence rate $\chi$ is related to the correlation among the primary signal samples $(r$ or $\rho$ ), this means that the number of additional signal samples required for loosely correlated samples is lower than for highly correlated samples under the same operating conditions. In fact, Figure 5.7 indicates that a small number of loosely correlated samples $\left(N=10^{1}\right.$, $\chi=0.8$ ) may result in the same detection performance as a large number of highly correlated samples $\left(N=10^{4}, \chi=0.2\right)$. It is worth noting, however, that low values for the intersample correlation can be associated to large sampling periods $T_{S}$ as discussed in Section 5.2.4, meaning that the absolute sensing period in time units might require a significant increase (for both low and high correlation values) with respect to the value predicted by the classical theoretical results of Equations 4.17-4.18 and 4.21-4.22. In the worst case, Figure 5.9 indicates an increase by a factor from 2 to 4 with respect to $N_{\text {classic }}^{\min }$ under perfectly calibrated noise power and even several orders of magnitude for large sensing periods under 


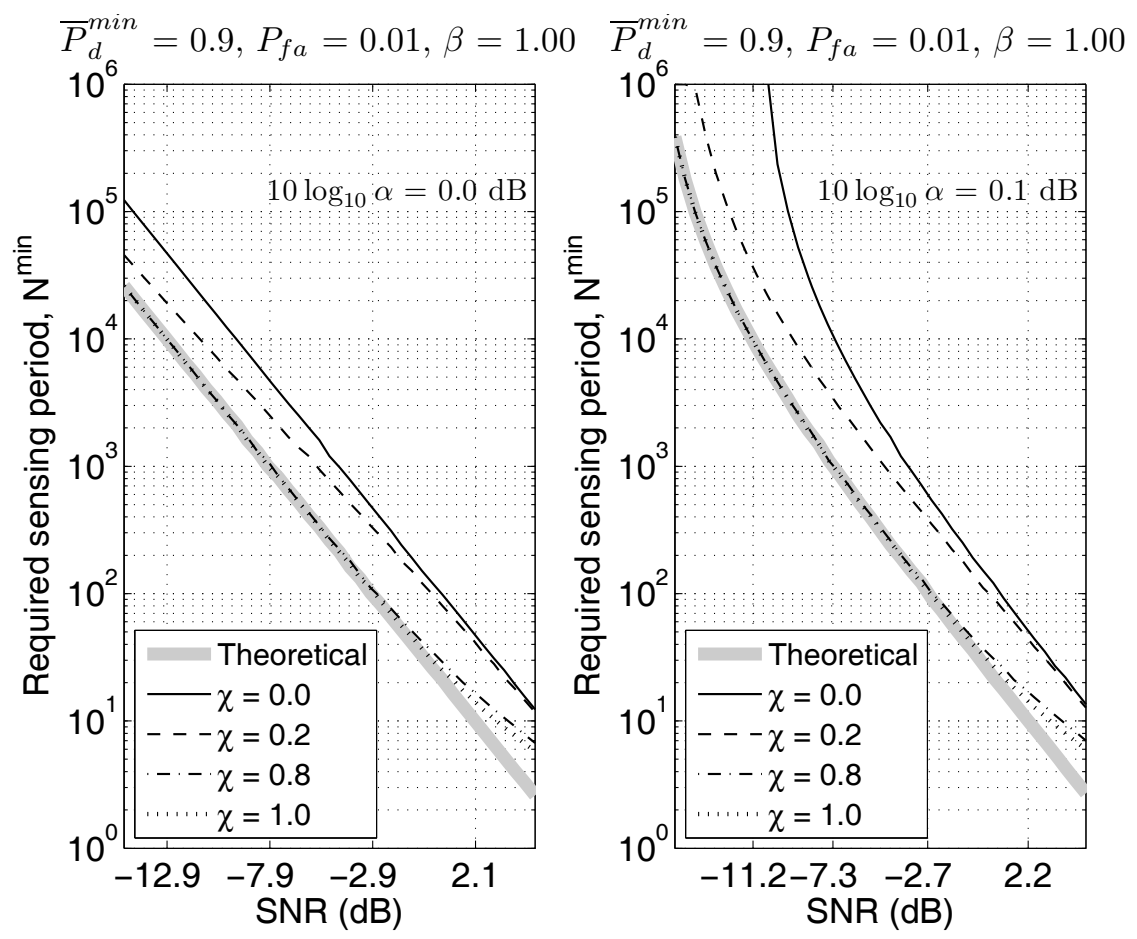

Figure 5.9: Required $N^{\text {min }}$ as a function of the experienced SNR without $\left(10 \log _{10} \alpha=0.0 \mathrm{~dB}\right)$ and with $\left(10 \log _{10} \alpha=0.1 \mathrm{~dB}\right)$ noise uncertainty $\left(\bar{P}_{d}^{\min }=0.9, P_{f a}=0.01, \beta=1.00\right)$.

noise uncertainty, where the impact of the signal uncertainty phenomenon becomes more severe. This numerical example not only highlights the importance of carefully taking into account both noise and signal uncertainties in the design of the spectrum sensing function of a DSA/CR network but also illustrates how the model proposed in this chapter can be employed to adequately configure the ED algorithm in real DSA/CR systems.

\subsection{Summary}

While the noise uncertainty phenomenon (i.e., the inability to perfectly know the system noise properties to infinite precision) has widely been studied, the impact of the signal uncertainty concept introduced in this chapter (i.e., the inability to perfectly know beforehand the primary signals that may be present in the sensed band and their properties) has not been taken into account in the analysis and performance evaluation of spectrum sensing in DSA/CR networks. In this context, this chapter has contributed a mathematical model for signal uncertainty. The proposed model is capable to capture and reproduce the impact of the variability of different transmission power patterns on the experimental performance of ED. Based on this model, the impact of signal uncertainty on the detection performance of ED has been evaluated and analyzed under the assumption of perfectly calibrated noise power and in the presence of noise uncertainty. The practical consequences of both degrad- 
ing effects (noise and signal uncertainty) have been assessed and compared. Although noise uncertainty can be considered as a more severe degrading effect, the obtained results have shown that the signal uncertainty phenomenon may also result in significant performance degradations, specially in the presence of noise uncertainty even for very small noise power calibration errors. These findings highlight the importance of carefully taking into account not only the noise uncertainty phenomenon but also the primary signal uncertainty in the design and configuration of spectrum sensing in real DSA/CR systems. 



\section{Chapter}

\section{6}

\section{IMPROVED ENERGY DETECTION}

\subsection{Introduction}

Signal detection based on ED is probably the spectrum sensing approach most widely employed in DSA/CR research. The main attractiveness of the ED method is its wide field of application since it can be employed irrespective of the signal format to be detected. Moreover, the simplicity of its operating principle results in low computational and implementation costs, specially when compared to other more sophisticated alternatives. The main drawback of the ED method, however, is its limited practical performance. Taking into account the demanding requirements imposed by the need to reliably detect the presence of primary licensed signals, ED provides a modest detection performance. Moreover, and based on the results obtained in Chapter 4, the ED performance may be particularly low for radio technologies characterized by transmission power patterns with a high level of variability, specially when employing short sensing periods. The need to guarantee a minimum detection performance implies that the sensing period cannot be shortened below a certain limit, which imposes a fundamental trade-off between detection performance and spectral agility ${ }^{1}$. A possibility to overcome these drawbacks would be to employ other more sophisticated spectrum sensing algorithms. As mentioned in Chapter 4, a wide variety of spectrum sensing methods and techniques have been proposed and proven to outperform the conventional ED scheme [151-155, 180-191]. Nevertheless, such methods have normally been devised to provide an improved detection performance for particular signal formats and structures, which restricts their field of application to a few primary radio technologies. Furthermore, the performance improvement attained by more sophisticated methods is

\footnotetext{
${ }^{1}$ The term spectral agility is employed in the context of DSA/CR to refer to the ability of a DSA/CR terminal to exploit spectrum holes across several channels by detecting their existence as soon as possible and switching seamlessly among them as fast as necessary to avoid interference. An important aspect in spectrum agility is therefore the ability to reliably detect the presence of a primary signal within the shortest possible time.
} 
normally obtained at the expense of a significant increase of the algorithm complexity and computational cost. An ideal spectrum sensing algorithm should be able to provide detection performance improvements for any primary signal and radio technology without incurring in excessive implementation and computational costs. In this context, this chapter proposes and evaluates, both analytically and experimentally, an improved spectrum sensing scheme based on the ED method. The main interest of the proposed solution relies on its capability to outperform the conventional ED scheme and cope with its degraded performance for short sensing periods while preserving a similar level of complexity and computational cost as well as the main advantages of the conventional ED method, namely its simplicity and general applicability regardless of the particular signal format or structure to be detected. The performance improvement is assessed analytically and corroborated with experimental results. The proposed algorithm will be of great utility in the processing of spectrum data in order extract the busy/idle state information for the development and validation of appropriate models.

The rest of this chapter is organized as follows. First, Section 6.2 reviews the main aspects of the Classical Energy Detection (CED) scheme and introduces the mathematical notation employed throughout this chapter. The novel ED-based spectrum sensing technique is then presented in two steps. Firstly, a Modified Energy Detection (MED) scheme motivated by the experimental results of Chapter 4 is presented in Section 6.3. Afterwards, a refined and Improved Energy Detection (IED) method is described in Section 6.4. The sample and computational complexities of the proposed IED algorithm are analyzed in Section 6.5, while a discussion on its practical use is provided in Section 6.6. Finally, Section 6.7 summarizes and concludes the chapter.

\subsection{Classical energy detection}

The CED method was presented in Chapter 4 (see Section 4.4). In order to make this chapter self-contained, this section summarizes the main aspects of the CED scheme and introduces the mathematical notation used in the remainder of this chapter. As mentioned in Section 4.4, the CED principle (see Algorithm 6.1) measures the energy received on a primary band during an observation interval of $N$ samples and declares the channel state $S_{i}$ as busy (hypothesis $\mathcal{H}_{1}$ ) if the measured energy is greater than a properly predefined threshold $\lambda$, or idle (hypothesis $\mathcal{H}_{0}$ ) otherwise. This operating principle can formally be formulated (see Equation 4.9) as:

$$
\mathbb{T}_{i}\left(\tilde{\mathbf{y}}_{i}\right)=\sum_{n=1}^{N}\left|\tilde{y}_{i}[n]\right|^{2} \stackrel{\mathcal{H}_{1}}{\underset{\mathcal{H}_{0}}{\gtrless}} \lambda
$$

where $\mathbb{T}_{i}\left(\tilde{\mathbf{y}}_{i}\right)$ is the test statistic computed in the $i$-th sensing event over the signal vector $\tilde{\mathbf{y}}_{i}=\left(\tilde{y}_{i}[1], \tilde{y}_{i}[2], \ldots, \tilde{y}_{i}[N]\right)$. Note that the sub-index $i$ has been introduced in order to make an explicit distinction between the values of the test statistic for different sensing events. The CED operating principle can alternatively be described as shown in Algorithm 6.1.

Assuming a sufficiently large sensing period $(N \gg 1)$, which is a reasonable assumption in practice as discussed in Section 4.4, the central limit theorem can be employed to 


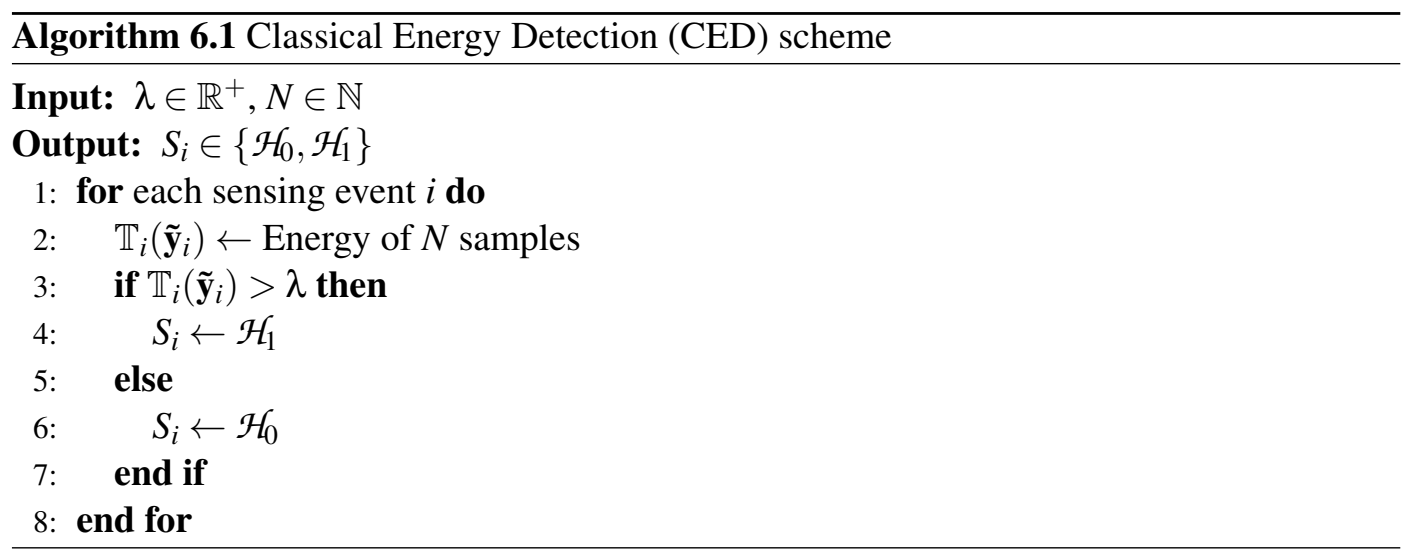

approximate the test statistic as Gaussian (see Equation 4.13):

$$
\mathbb{T}_{i}\left(\tilde{\mathbf{y}}_{i}\right) \sim \begin{cases}\mathcal{N}\left(N \sigma_{\tilde{w}}^{2}, 2 N \sigma_{\tilde{w}}^{4}\right), & \mathcal{H}_{0} \\ \mathcal{N}\left(N\left(\sigma_{\tilde{x}}^{2}+\sigma_{\tilde{w}}^{2}\right), 2 N\left(\sigma_{\tilde{x}}^{2}+\sigma_{\tilde{w}}^{2}\right)^{2}\right), & \mathcal{H}_{1}\end{cases}
$$

If only AWGN is considered, the probabilities of detection, $P_{d}^{\mathrm{CED}}$, and false alarm, $P_{f a}^{\mathrm{CED}}$, for the CED algorithm can be obtained based on $\mathbb{T}_{i}\left(\tilde{\mathbf{y}}_{i}\right)$ (see Equations 4.14 and 4.15) as:

$$
\begin{aligned}
& P_{d}^{\mathrm{CED}}=P\left\{\mathbb{T}_{i}\left(\tilde{\mathbf{y}}_{i}\right)>\lambda\right\}_{\mathcal{H}_{1}}=Q\left(\frac{\lambda-N\left(\sigma_{\tilde{x}}^{2}+\sigma_{\tilde{w}}^{2}\right)}{\sqrt{2 N\left(\sigma_{\tilde{x}}^{2}+\sigma_{\tilde{w}}^{2}\right)^{2}}}\right) \\
& P_{f a}^{\mathrm{CED}}=P\left\{\mathbb{T}_{i}\left(\tilde{\mathbf{y}}_{i}\right)>\lambda\right\}_{\mathcal{H}_{0}}=Q\left(\frac{\lambda-N \sigma_{\tilde{w}}^{2}}{\sqrt{2 N \sigma_{\tilde{w}}^{4}}}\right)
\end{aligned}
$$

where the notation $P\{A\}_{B} \triangleq P(A \mid B)$ has been introduced along with the super-index "CED" to avoid ambiguities among the algorithms treated in this chapter.

As mentioned in Section 4.4, the decision threshold $\lambda$ is normally chosen to satisfy a certain target $P_{f a}$. The decision threshold required for a specified $P_{f a \text {, target }}^{\mathrm{CED}}$ can be obtained by solving Equation 6.4 for $\lambda$ (see Equations 4.16 and 4.20):

$$
\lambda=\left(Q^{-1}\left(P_{f a, \text { target }}^{\text {CED }}\right) \sqrt{2 N}+N\right) \alpha \sigma_{\tilde{w}}^{2}
$$

where the sub-index "target" has been introduced in order to make a distinction between the target and the actually experienced $P_{f a}$ (while both agree for the CED scheme, this may not necessarily be the case in the algorithms studied in this chapter as it will be discussed in detail). Equation 6.5 assumes the noise uncertainty model $\hat{\sigma}_{\tilde{w}}^{2} \in\left[\sigma_{\tilde{w}}^{2}, \alpha \sigma_{\tilde{w}}^{2}\right]$ [207] and the worst possible case where the noise power is overestimated by a factor $\alpha>1$. 
Substituting Equation 6.5 into Equation 6.3 yields (see Equations 4.21 and 4.22):

$$
\begin{aligned}
P_{d}^{\mathrm{CED}}(\gamma) & =Q\left(\frac{\alpha Q^{-1}\left(P_{f a, \text { target }}^{\mathrm{CED}}\right) \sqrt{2 N}-N(\gamma+1-\alpha)}{\sqrt{2 N}(1+\gamma)}\right) \\
& \approx Q\left(\alpha Q^{-1}\left(P_{f a, \text { target }}^{\mathrm{CED}}\right)-\sqrt{\frac{N}{2}}(\gamma+1-\alpha)\right)
\end{aligned}
$$

which represents the detection probability of the CED algorithm as a function of the SNR. The approximation in Equation 6.7 assumes low $\operatorname{SNR}(\gamma \ll 1)$.

Without loss of generality, the results presented in this chapter assume a perfectly calibrated noise power (i.e., $\alpha=1$ ) in order to simplify the subsequent performance analysis. Therefore, the comparison of the considered ED methods will be based on numerical and experimental results obtained under the assumption of no noise uncertainty. Although this assumption is not completely valid in reality and noise uncertainty affects the performance of any spectrum sensing algorithm, the results obtained in Chapter 4 indicate that the relative performance differences of ED-based spectrum sensing among various operating conditions are preserved for various noise uncertainty levels. Figures 4.4, 4.5 and 4.6 show that when ED provides a better performance under certain operating conditions $\left(P_{f a}, N, \gamma\right)$ with no noise uncertainty $(\alpha=1)$, it also provides a better performance under the same operating conditions $\left(P_{f a}, N, \gamma\right)$ in the presence of noise uncertainty $(\alpha>1)$. Therefore, the performance comparison of the considered ED methods under no noise uncertainty will suffice to determine whether the proposed ED techniques are able to outperform the CED scheme. Nevertheless, the noise uncertainty factor $\alpha$ will be included in the analytical study in order to provide the corresponding mathematical results in their most general form.

\subsection{Modified energy detection}

\subsubsection{MED operating principle}

The experimental results obtained in Chapter 4 for the CED scheme indicated that certain inherent technology-dependent properties may result in different detection performances for various radio technologies. In particular, for signals characterized by transmission power patterns with higher levels of variability (variance) there exists a higher probability that the instantaneous signal power falls below the decision threshold, which results in a lower detection performance specially when employing short sensing periods. This observation suggests that the performance of the CED method might be improved if the misdetections caused by instantaneous signal energy (power) drops could be avoided, which motivates the development of the MED scheme (see Algorithm 6.2). Every sensing event, the MED method computes the test statistic $\mathbb{T}_{i}\left(\tilde{\mathbf{y}}_{i}\right)$ as performed by the CED method (see Equation 6.1). The main difference between the MED and the CED algorithms is that the former additionally maintains an updated list containing the test statistic values of the last $L$ sensing events ( $L$ is a configurable parameter of the MED algorithm), which is used to compute an 


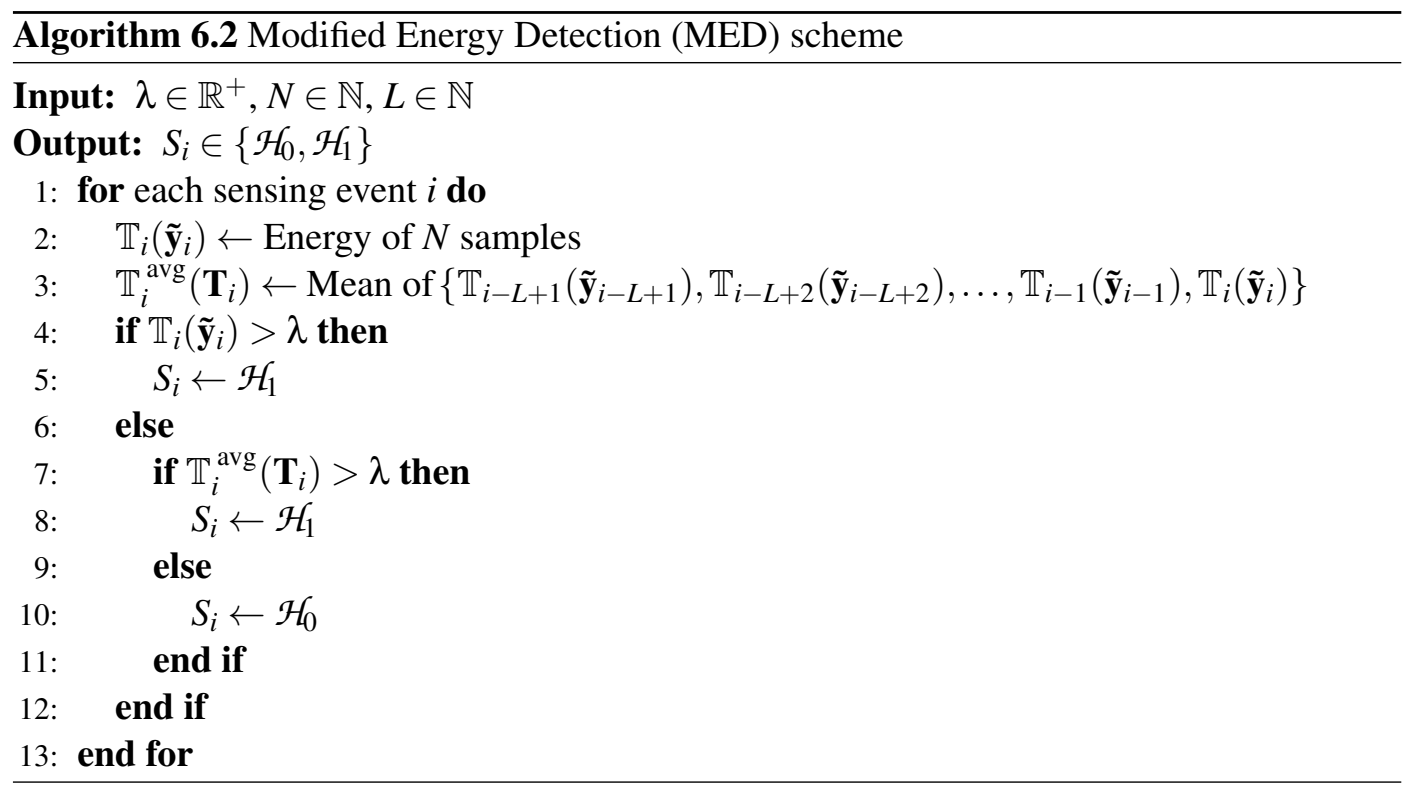

average test statistic value (line 3 in Algorithm 6.2) as follows:

$$
\mathbb{T}_{i}^{\mathrm{avg}}\left(\mathbf{T}_{i}\right)=\frac{1}{L} \sum_{l=1}^{L} \mathbb{T}_{i-L+l}\left(\tilde{\mathbf{y}}_{i-L+l}\right)
$$

where $\mathbb{T}_{i}^{\text {avg }}\left(\mathbf{T}_{i}\right)$ is the average test statistic value computed in the $i$-th sensing event based on the test statistic vector $\mathbf{T}_{i}=\left(\mathbb{T}_{i-L+1}\left(\tilde{\mathbf{y}}_{i-L+1}\right), \mathbb{T}_{i-L+2}\left(\tilde{\mathbf{y}}_{i-L+2}\right), \ldots, \mathbb{T}_{i-1}\left(\tilde{\mathbf{y}}_{i-1}\right), \mathbb{T}_{i}\left(\tilde{\mathbf{y}}_{i}\right)\right)$. In case that the test statistic $\mathbb{T}_{i}\left(\tilde{\mathbf{y}}_{i}\right)$ falls below the decision threshold $\lambda$, an additional check based on $\mathbb{T}_{i}^{\text {avg }}\left(\mathbf{T}_{i}\right)$ is then performed (line 7) before deciding the final channel state $S_{i}$. This additional check is aimed at preventing a busy channel from being declared to be idle as a result of an instantaneous signal energy (power) drop (which depends on the particular signal power variation pattern and radio propagation conditions) combined with a sufficiently short sensing period (which may be constrained by physical layer features, higher layer protocols or other circumstances). According to this additional verification, if the last sensing event reported an idle channel, $\mathbb{T}_{i}\left(\tilde{\mathbf{y}}_{i}\right)<\lambda$, but the average test statistic value (i.e., the average signal energy) of the last $L$ sensing events is greater than the decision threshold, $\mathbb{T}_{i}^{\mathrm{avg}}\left(\mathbf{T}_{i}\right)>$ $\lambda$, this means that a signal is most likely present in the sensed channel but the last sensing event resulted in $\mathbb{T}_{i}\left(\tilde{\mathbf{y}}_{i}\right)<\lambda$ due to an instantaneous energy (power) drop of the received signal combined with a sufficiently short sensing period. As a result, the channel should be declared as busy (hypothesis $\mathcal{H}_{1}$ ) in such a case (line 8). On the other hand, if the last sensing event reported an idle channel, $\mathbb{T}_{i}\left(\tilde{\mathbf{y}}_{i}\right)<\lambda$, and the average value of the test statistic based on the last $L$ sensing events also indicates an idle channel, $\mathbb{T}_{i}^{\text {avg }}\left(\mathbf{T}_{i}\right)<\lambda$, this clearly means that the channel is actually idle. In such another case hypothesis $\mathcal{H}_{0}$ can reliably be selected (line 10). With this formulation, the MED scheme aims at reducing the amount of misdetections caused by instantaneous signal energy (power) drops, which would lead to an improved detection performance. 


\subsubsection{MED theoretical performance}

The test statistic values $\mathbb{T}_{i}\left(\tilde{\mathbf{y}}_{i}\right)$ can be assumed to be normally distributed (see Equation 6.2) and mutually independent since they represent the energy (power) of the sensed signal at time instants separated by time intervals much greater than the sensing period $N$ over which the signal energy (power) is computed. Since $\mathbb{T}_{i}^{\text {avg }}\left(\mathbf{T}_{i}\right)$ is the average of independent and identically distributed (i.d.d.) Gaussian random variables, it also is normally distributed:

$$
\mathbb{T}_{i}^{\text {avg }}\left(\mathbf{T}_{i}\right) \sim \mathcal{N}\left(\mu_{\text {avg }}, \sigma_{\text {avg }}^{2}\right)
$$

where $\mu_{\text {avg }}$ and $\sigma_{\text {avg }}^{2}$ can be computed based on [211, (2.20) and (2.21)] as:

$$
\begin{aligned}
& \mu_{\mathrm{avg}}=\frac{M}{L} N\left(\sigma_{\tilde{x}}^{2}+\sigma_{\tilde{w}}^{2}\right)+\frac{L-M}{L} N \sigma_{\tilde{w}}^{2} \\
& \sigma_{\mathrm{avg}}^{2}=\frac{M}{L^{2}} 2 N\left(\sigma_{\tilde{x}}^{2}+\sigma_{\tilde{w}}^{2}\right)^{2}+\frac{L-M}{L^{2}} 2 N \sigma_{\tilde{w}}^{4}
\end{aligned}
$$

where $M \in[0, L]$ is the number of sensing events where a primary signal was actually present. Notice that the exact value of $M$ cannot be assumed to be known in practice. All that can be known is the output decisions $\mathcal{H}_{0} / \mathcal{H}_{1}$ of the spectrum sensing algorithm, which does not necessarily imply the presence/absence of a primary signal. This means that the performance of the MED algorithm cannot be predicted in practice since it depends on the particular channel occupancy pattern of the primary signal, which indeed is unknown. However, it can be lower- and upper-bounded by analyzing the extreme cases $M=0$ and $M=L$, which correspond to the case where the channel is always idle (for $M=0$ ) or always busy (for $M=L$ ) during the last $L$ sensing events.

Based on the statistics of $\mathbb{T}_{i}\left(\tilde{\mathbf{y}}_{i}\right)$ and $\mathbb{T}_{i}^{\text {avg }}\left(\mathbf{T}_{i}\right)$, the $P_{d}$ and $P_{f a}$ for the MED scheme are:

$$
\begin{aligned}
P_{d}^{\mathrm{MED}} & =P\left\{\mathbb{T}_{i}\left(\tilde{\mathbf{y}}_{i}\right)>\lambda\right\}_{\mathcal{H}_{1}}+P\left\{\mathbb{T}_{i}\left(\tilde{\mathbf{y}}_{i}\right) \leq \lambda, \mathbb{T}_{i}^{\mathrm{avg}}\left(\mathbf{T}_{i}\right)>\lambda\right\}_{\mathcal{H}_{1}} \\
& =P\left\{\mathbb{T}_{i}\left(\tilde{\mathbf{y}}_{i}\right)>\lambda\right\}_{\mathcal{H}_{1}}+P\left\{\mathbb{T}_{i}\left(\tilde{\mathbf{y}}_{i}\right) \leq \lambda\right\}_{\mathcal{H}_{1}} \cdot P\left\{\mathbb{T}_{i}^{\mathrm{avg}}\left(\mathbf{T}_{i}\right)>\lambda \mid \mathbb{T}_{i}\left(\tilde{\mathbf{y}}_{i}\right) \leq \lambda\right\}_{\mathcal{H}_{1}} \\
P_{f a}^{\mathrm{MED}} & =P\left\{\mathbb{T}_{i}\left(\tilde{\mathbf{y}}_{i}\right)>\lambda\right\}_{\mathcal{H}_{0}}+P\left\{\mathbb{T}_{i}\left(\tilde{\mathbf{y}}_{i}\right) \leq \lambda, \mathbb{T}_{i}^{\mathrm{avg}}\left(\mathbf{T}_{i}\right)>\lambda\right\}_{\mathcal{H}_{0}} \\
& =P\left\{\mathbb{T}_{i}\left(\tilde{\mathbf{y}}_{i}\right)>\lambda\right\}_{\mathcal{H}_{0}}+P\left\{\mathbb{T}_{i}\left(\tilde{\mathbf{y}}_{i}\right) \leq \lambda\right\}_{\mathcal{H}_{0}} \cdot P\left\{\mathbb{T}_{i}^{\mathrm{avg}}\left(\mathbf{T}_{i}\right)>\lambda \mid \mathbb{T}_{i}\left(\tilde{\mathbf{y}}_{i}\right) \leq \lambda\right\}_{\mathcal{H}_{0}}
\end{aligned}
$$

Notice that $\mathbb{T}_{i}^{\text {avg }}\left(\mathbf{T}_{i}\right)$ and $\mathbb{T}_{i}\left(\tilde{\mathbf{y}}_{i}\right)$ are not completely independent since the computation of the former includes the value of the latter (see Equation 6.8). However, $\mathbb{T}_{i}^{\text {avg }}\left(\mathbf{T}_{i}\right)$ needs to be computed over a representative number of test statistic values in order to provide an acceptable estimate of the average signal energy in the sensed channel. Since the average of a relatively large set of values is not significantly affected, in general, by the particular value of a single element, it is therefore reasonable to assume for $L$ sufficiently large that $\mathbb{T}_{i}^{\text {avg }}\left(\mathbf{T}_{i}\right)$ can be considered to be approximately independent of $\mathbb{T}_{i}\left(\tilde{\mathbf{y}}_{i}\right)$, regardless of the actual channel state in the $i$-th sensing event. Therefore, for $L$ sufficiently large:

$$
P\left\{\mathbb{T}_{i}^{\mathrm{avg}}\left(\mathbf{T}_{i}\right)>\lambda \mid \mathbb{T}_{i}\left(\tilde{\mathbf{y}}_{i}\right) \leq \lambda\right\}_{\mathcal{H}_{x}} \approx P\left\{\mathbb{T}_{i}^{\mathrm{avg}}\left(\mathbf{T}_{i}\right)>\lambda\right\}_{\mathcal{H}_{x}} \approx P\left(\mathbb{T}_{i}^{\mathrm{avg}}\left(\mathbf{T}_{i}\right)>\lambda\right)
$$


where $\mathcal{H}_{x}$ may be $\mathcal{H}_{0}$ or $\mathcal{H}_{1}$. Equations 6.12 and 6.13 then become:

$$
\begin{aligned}
& P_{d}^{\mathrm{MED}} \approx P_{d}^{\mathrm{CED}}+\left(1-P_{d}^{\mathrm{CED}}\right) Q\left(\frac{\lambda-\mu_{\mathrm{avg}}}{\sigma_{\mathrm{avg}}}\right) \\
& P_{f a}^{\mathrm{MED}} \approx P_{f a}^{\mathrm{CED}}+\left(1-P_{f a}^{\mathrm{CED}}\right) Q\left(\frac{\lambda-\mu_{\mathrm{avg}}}{\sigma_{\mathrm{avg}}}\right)
\end{aligned}
$$

The value of $Q\left(\left(\lambda-\mu_{\mathrm{avg}}\right) / \sigma_{\mathrm{avg}}\right)$ in Equations 6.15 and 6.16 depends on the particular channel occupancy pattern of the primary signal, which is unknown as already stated above. However, since $Q(\cdot)$ is confined within the interval $[0,1], P_{d}^{\mathrm{MED}}$ and $P_{f a}^{\mathrm{MED}}$ are bounded by:

$$
\begin{aligned}
& P_{d}^{\mathrm{CED}} \leq P_{d}^{\mathrm{MED}} \leq 1 \\
& P_{f a}^{\mathrm{CED}} \leq P_{f a}^{\mathrm{MED}} \leq 1
\end{aligned}
$$

This means that the MED algorithm is able to improve the detection performance of the CED algorithm, which was its main motivation as discussed in Section 6.3.1. However, such improvement is obtained at the expense of a false alarm probability degradation. To determine whether the MED scheme results in an overall performance improvement taking into account both $P_{d}$ and $P_{f a}$ with respect to the CED principle, Figure 6.1 depicts (based on Equations 6.3, 6.4, 6.15 and 6.16) the ROC for both algorithms when $L=3, M \in[0, L]$, $N=1000, \alpha=1$ and the SNR values for which $P_{d}^{\mathrm{CED}}=0.9(-9.15 \mathrm{~dB}), P_{d}^{\mathrm{CED}}=0.8(-10.06$ $\mathrm{dB}), P_{d}^{\mathrm{CED}}=0.7(-10.83 \mathrm{~dB})$ and $P_{d}^{\mathrm{CED}}=0.6(-11.59 \mathrm{~dB})$ when $P_{f a}^{\mathrm{CED}}=0.1^{2}$. As it can be appreciated, the MED performance depends on the activity pattern of the primary signal (implicitly represented by means of $M$ ), but it is always inferior to that of the CED method.

The results of Figure 6.1 can be explained as follows. The MED formulation is able to reduce the amount of misdetections caused by instantaneous signal energy (power) drops, which results in a $P_{d}$ improvement with respect to the CED algorithm as it has been inferred from Equation 6.15. However, the resulting $P_{f a}$ increases to a greater extent thus leading to the degraded ROC observed in Figure 6.1. The $P_{f a}$ increase can be ascribed to the additional check performed in line 7 of the MED algorithm. When the primary signal ceases after some period of activity and the channel is released, there may be some subsequent sensing events where $\mathbb{T}_{i}\left(\tilde{\mathbf{y}}_{i}\right)<\lambda$ due to the absence of the primary signal, but $\mathbb{T}_{i}^{\text {avg }}\left(\mathbf{T}_{i}\right)>\lambda$ due to the immediate past sensing events where the signal was still present. As a result, the additional check of line 7 may result in several consecutive false alarms. In the worst case, up to $L$ sensing events after the channel is released may result in false alarm decisions, depending on the particular previous occupancy history and signal energy (power). In fact, as the number of previous sensing events where the primary signal was present, $M$, increases, the resulting

\footnotetext{
${ }^{2}$ Notice that DSA/CR networks are normally constrained by a maximum interference requirement, which can be mapped to a minimum detection probability that must be satisfied for SNR values above a predefined threshold, i.e. $P_{d}(\gamma) \geq P_{d}^{\min }$ for all $\gamma \geq \gamma_{\min }$. Selecting $N=1000$ and $P_{f a}^{\mathrm{CED}}=0.1$, for a target $P_{d}^{\min }=0.9$ the corresponding SNR is $\gamma_{\min }=-9.15 \mathrm{~dB}$ (Equation 6.6 with $\alpha=1$ ). Therefore, this SNR value enables the evaluation of the potential improvements of the MED scheme with respect to the CED scheme in a realistic worst case. The SNR values for which $P_{d}^{\mathrm{CED}}=0.8,0.7$ and 0.6 enable the evaluation of the MED performance under more unfavorable operating conditions.
} 

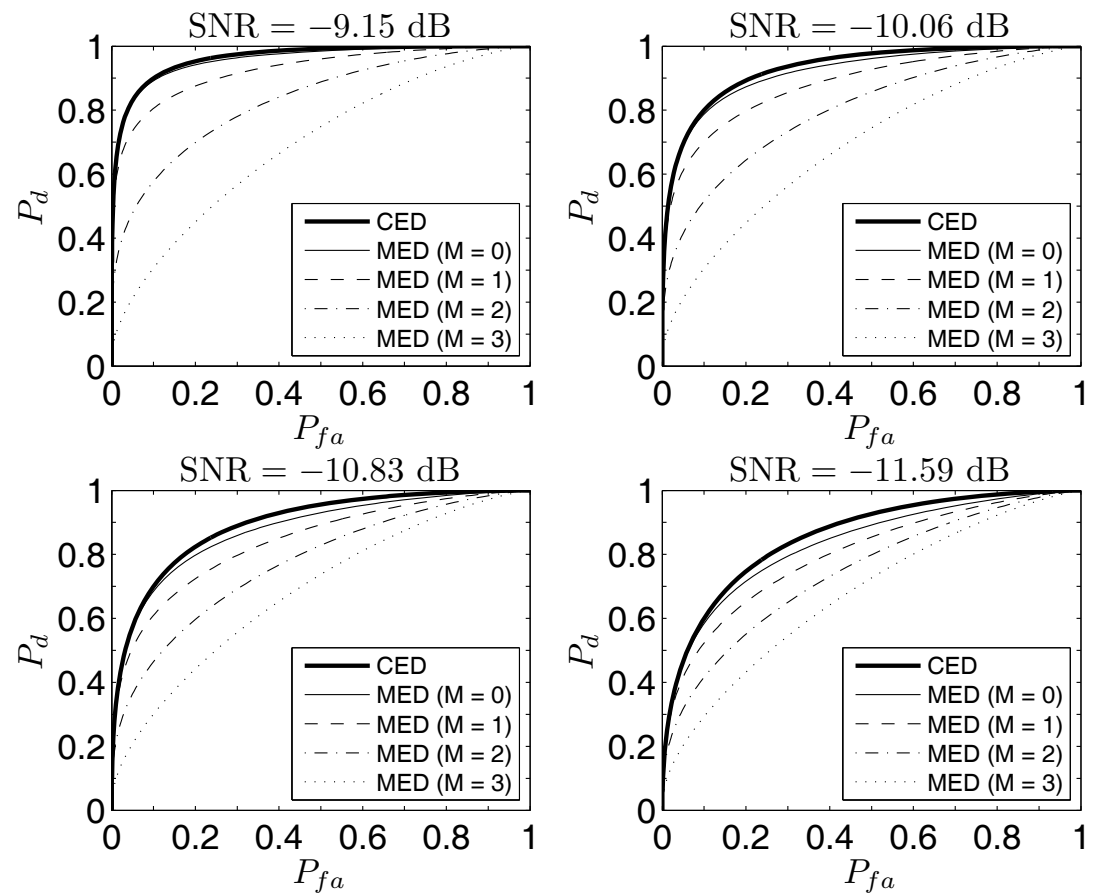

Figure 6.1: ROC curve for the CED and MED algorithms $(L=3, M \in[0, L], N=1000, \alpha=1)$.

$\mathbb{T}_{i}^{\mathrm{avg}}\left(\mathbf{T}_{i}\right)$ becomes higher and the number of false alarms after the channel is released increases, as suggested by the ROC degradation observed in Figure 6.1 as $M$ increases. On the other hand, if the channel was sparsely used by the primary signal in the previous sensing events (low $M$ ), the MED and CED behave similarly and their performances converge.

Based on the results of Figure 6.1, it can be concluded that the MED performance is upper-bounded by the CED method. However, the previous analysis motivates the development of the IED method proposed in Section 6.4 and will make its understanding simpler.

\subsection{Improved energy detection}

\subsubsection{IED operating principle}

Based on the analysis and discussion of Section 6.3.2, the IED scheme is proposed (see Algorithm 6.3) in order to reduce the false alarm rate of the MED scheme while preserving the detection performance improvement attained with respect to the CED method. The rationale of this proposal is illustrated in Figure 6.2. Two possible cases of interest are shown in sensing events number 15 (event A) and number 35 (event B). In both sensing events the MED scheme would select hypothesis $\mathcal{H}_{1}$ since $\mathbb{T}_{i}\left(\tilde{\mathbf{y}}_{i}\right)<\lambda$ and $\mathbb{T}_{i}^{\mathrm{avg}}\left(\mathbf{T}_{i}\right)>\lambda$. While this decision would result in a detection performance improvement for sensing event $\mathrm{A}$, it would lead to a more significant false alarm degradation after sensing event number 30 , where the 

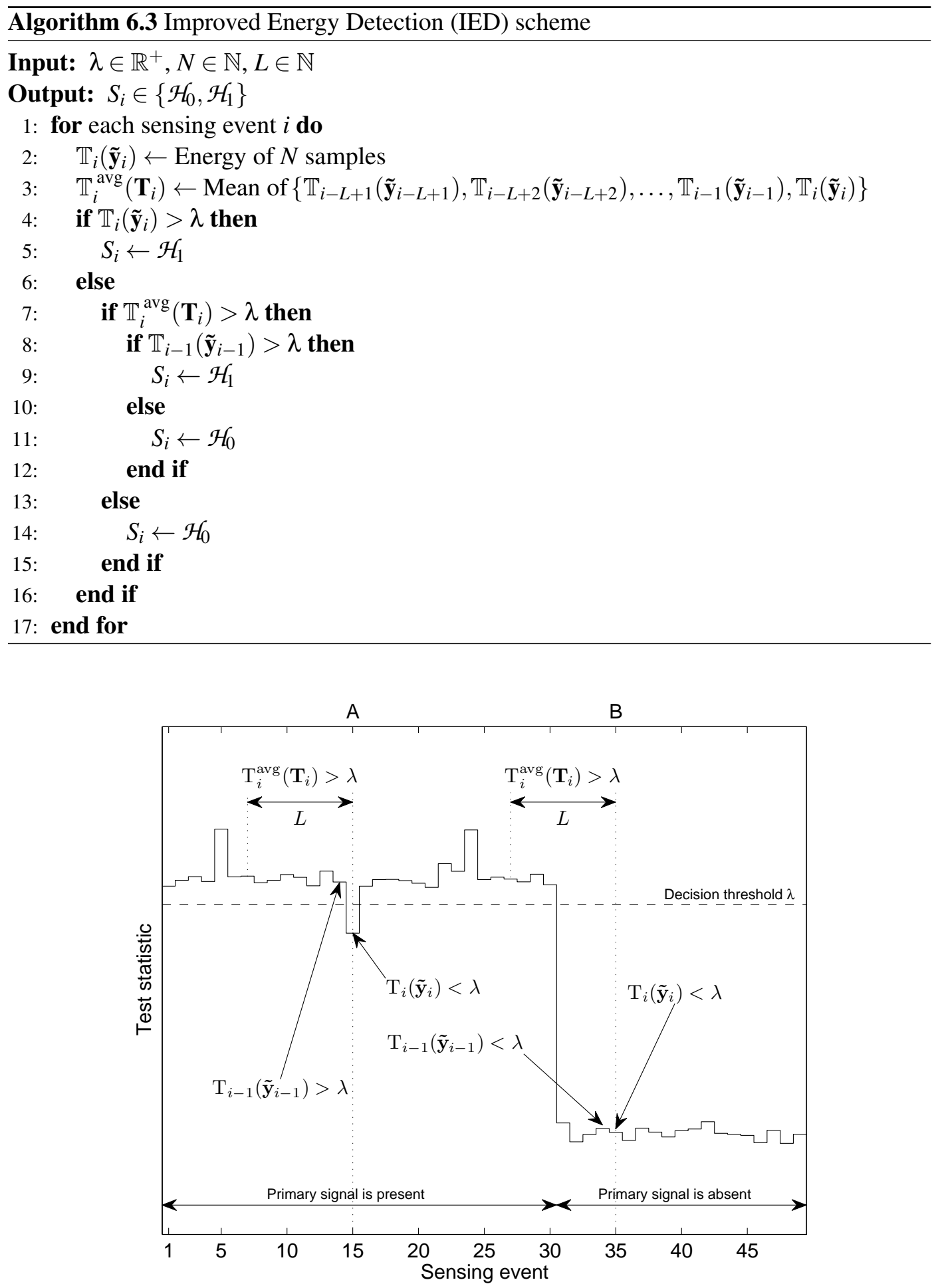

Figure 6.2: Rationale for the IED proposal. 
channel is released, up to $L$ subsequent sensing events (where event B is one of them). To avoid false alarms as those of event $\mathrm{B}$, the IED scheme performs an additional check (line 8 in Algorithm 6.3) based on the test statistic of the previous sensing event, $\mathbb{T}_{i-1}\left(\tilde{\mathbf{y}}_{i-1}\right)$. When $\mathbb{T}_{i}\left(\tilde{\mathbf{y}}_{i}\right)<\lambda$ and $\mathbb{T}_{i}^{\text {avg }}\left(\mathbf{T}_{i}\right)>\lambda$, the condition $\mathbb{T}_{i-1}\left(\tilde{\mathbf{y}}_{i-1}\right)>\lambda$ indicates that $\mathbb{T}_{i}\left(\tilde{\mathbf{y}}_{i}\right)<\lambda$ may be due to an instantaneous energy (power) drop, in which case hypothesis $\mathcal{H}_{1}$ should be selected (line 9). On the other hand, the condition $\mathbb{T}_{i-1}\left(\tilde{\mathbf{y}}_{i-1}\right)<\lambda$ suggests that $\mathbb{T}_{i}\left(\tilde{\mathbf{y}}_{i}\right)<\lambda$ may be due to the channel release, in which case hypothesis $\mathcal{H}_{0}$ should be selected (line 11). It is worth noting that the algorithm decisions could be based simply on $\mathbb{T}_{i}\left(\tilde{\mathbf{y}}_{i}\right)$ and $\mathbb{T}_{i-1}\left(\tilde{\mathbf{y}}_{i-1}\right)$. However, experimental studies demonstrated that the additional use of $\mathbb{T}_{i}^{\text {avg }}\left(\mathbf{T}_{i}\right)$ can avoid misdetections for highly variable signals where various consecutive sensing events may be affected by instantaneous energy (power) drops, in which case $\mathbb{T}_{i}\left(\tilde{\mathbf{y}}_{i}\right)<\lambda$ and $\mathbb{T}_{i-1}\left(\tilde{\mathbf{y}}_{i-1}\right)<\lambda$ even though a primary signal is actually present in the channel. Therefore, the MED and IED schemes would made the same decision in event A (hypothesis $\mathcal{H}_{1}$ ), thus improving the detection performance of the CED scheme. However, the IED scheme would select hypothesis $\mathcal{H}_{0}$ in event $\mathrm{B}$, which would also decrease the false alarm ratio of the MED method. The IED proposal is therefore expected to improve the false alarm performance of the MED algorithm while still improving the detection performance of the CED method.

\subsubsection{IED theoretical performance}

The test statistics $\mathbb{T}_{i}\left(\tilde{\mathbf{y}}_{i}\right), \mathbb{T}_{i-1}\left(\tilde{\mathbf{y}}_{i-1}\right)$ and $\mathbb{T}_{i}^{\text {avg }}\left(\mathbf{T}_{i}\right)$ can be assumed to be normally distributed as indicated by Equations 6.2 and 6.9-6.11, respectively. Based on the distributions of $\mathbb{T}_{i}\left(\tilde{\mathbf{y}}_{i}\right), \mathbb{T}_{i-1}\left(\tilde{\mathbf{y}}_{i-1}\right)$ and $\mathbb{T}_{i}^{\text {avg }}\left(\mathbf{T}_{i}\right)$, the $P_{d}$ and $P_{f a}$ for the IED algorithm are:

$$
\begin{aligned}
P_{d}^{\mathrm{IED}} & =P\left\{\mathbb{T}_{i}\left(\tilde{\mathbf{y}}_{i}\right)>\lambda\right\}_{\mathcal{H}_{1}}+P\left\{\mathbb{T}_{i}\left(\tilde{\mathbf{y}}_{i}\right) \leq \lambda, \mathbb{T}_{i}^{\mathrm{avg}}\left(\mathbf{T}_{i}\right)>\lambda, \mathbb{T}_{i-1}\left(\tilde{\mathbf{y}}_{i-1}\right)>\lambda\right\}_{\mathcal{H}_{1}} \\
& =P\left\{\mathbb{T}_{i}\left(\tilde{\mathbf{y}}_{i}\right)>\lambda\right\}_{\mathcal{H}_{1}}+P\left\{\mathbb{T}_{i}\left(\tilde{\mathbf{y}}_{i}\right) \leq \lambda\right\}_{\mathcal{H}_{1}} \cdot P\left(\mathbb{T}_{i}^{\mathrm{avg}}\left(\mathbf{T}_{i}\right)>\lambda\right) \cdot P\left\{\mathbb{T}_{i-1}\left(\tilde{\mathbf{y}}_{i-1}\right)>\lambda\right\}_{\mathcal{H}_{1}} \\
P_{f a}^{\mathrm{IED}} & =P\left\{\mathbb{T}_{i}\left(\tilde{\mathbf{y}}_{i}\right)>\lambda\right\}_{\mathcal{H}_{0}}+P\left\{\mathbb{T}_{i}\left(\tilde{\mathbf{y}}_{i}\right) \leq \lambda, \mathbb{T}_{i}^{\mathrm{avg}}\left(\mathbf{T}_{i}\right)>\lambda, \mathbb{T}_{i-1}\left(\tilde{\mathbf{y}}_{i-1}\right)>\lambda\right\}_{\mathcal{H}_{0}} \\
& =P\left\{\mathbb{T}_{i}\left(\tilde{\mathbf{y}}_{i}\right)>\lambda\right\}_{\mathcal{H}_{0}}+P\left\{\mathbb{T}_{i}\left(\tilde{\mathbf{y}}_{i}\right) \leq \lambda\right\}_{\mathcal{H}_{0}} \cdot P\left(\mathbb{T}_{i}^{\mathrm{avg}}\left(\mathbf{T}_{i}\right)>\lambda\right) \cdot P\left\{\mathbb{T}_{i-1}\left(\tilde{\mathbf{y}}_{i-1}\right)>\lambda\right\}_{\mathcal{H}_{0}}
\end{aligned}
$$

where it has been assumed, following the same argument of Section 6.3.2, that $\mathbb{T}_{i}^{\text {avg }}\left(\mathbf{T}_{i}\right)$ can be considered to be approximately independent of both $\mathbb{T}_{i}\left(\tilde{\mathbf{y}}_{i}\right)$ and $\mathbb{T}_{i-1}\left(\tilde{\mathbf{y}}_{i-1}\right)$, regardless of the actual channel state (busy or idle) in the $i$-th and $(i-1)$-th sensing events, and that the test statistics of two consecutive sensing events, $\mathbb{T}_{i}\left(\tilde{\mathbf{y}}_{i}\right)$ and $\mathbb{T}_{i-1}\left(\tilde{\mathbf{y}}_{i-1}\right)$, can also be considered to be mutually independent. Based on such assumptions, Equations 6.19 and 6.20 then become:

$$
\begin{aligned}
& P_{d}^{\mathrm{IED}} \approx P_{d}^{\mathrm{CED}}+P_{d}^{\mathrm{CED}}\left(1-P_{d}^{\mathrm{CED}}\right) Q\left(\frac{\lambda-\mu_{\mathrm{avg}}}{\sigma_{\mathrm{avg}}}\right) \\
& P_{f a}^{\mathrm{IED}} \approx P_{f a}^{\mathrm{CED}}+P_{f a}^{\mathrm{CED}}\left(1-P_{f a}^{\mathrm{CED}}\right) Q\left(\frac{\lambda-\mu_{\mathrm{avg}}}{\sigma_{\mathrm{avg}}}\right)
\end{aligned}
$$



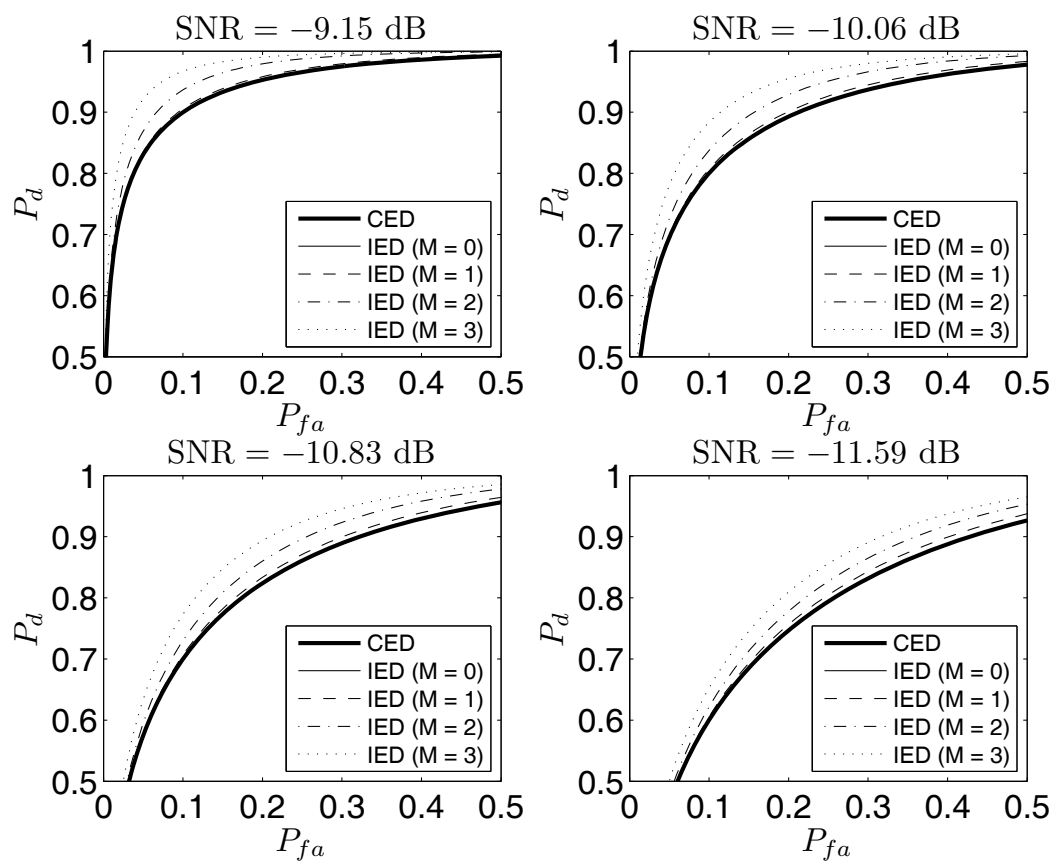

Figure 6.3: ROC curve for the CED and IED algorithms $(L=3, M \in[0, L], N=1000, \alpha=1)$.

Since $Q(\cdot) \in[0,1], P_{d}^{\mathrm{IED}}$ and $P_{f a}^{\mathrm{IED}}$ are therefore bounded by:

$$
\begin{aligned}
& P_{d}^{\mathrm{CED}} \leq P_{d}^{\mathrm{IED}} \leq 2 P_{d}^{\mathrm{CED}}-\left(P_{d}^{\mathrm{CED}}\right)^{2} \\
& P_{f a}^{\mathrm{CED}} \leq P_{f a}^{\mathrm{IED}} \leq 2 P_{f a}^{\mathrm{CED}}-\left(P_{f a}^{\mathrm{CED}}\right)^{2}
\end{aligned}
$$

meaning that the IED algorithm improves the detection performance of the CED algorithm at the expense of a false alarm probability degradation. Such degradation, however, is not as significant as in the case of the MED scheme, resulting in the improved ROC curve observed in Figure 6.3 for the IED algorithm (the figure has been obtained based on Equations 6.3, 6.4, 6.21 and 6.22, and for the same operating conditions as Figure 6.1). As it can be appreciated, the IED performance is superior to that attained by the CED method. When the channel is idle in previous sensing events $(M=0)$, the IED scheme bases its decisions mostly on the test statistic of the current sensing event, $\mathbb{T}_{i}\left(\tilde{\mathbf{y}}_{i}\right)$, since for $M=0$ it is rather unlikely that $\mathbb{T}_{i}^{\text {avg }}\left(\mathbf{T}_{i}\right)>\lambda$. In this case the IED scheme behaves similar to the CED scheme and their performances agree. However, when the channel is busy in previous sensing events $(M>0)$, the IED scheme takes profit of this additional information to avoid misdetections due to instantaneous signal energy (power) drops, as the MED scheme, but with a lower false alarm rate, which results in an overall improved performance. In fact, as $M$ increases, the IED method is able to avoid a higher amount of such misdetections. These results indicate that the IED performance is lower-bounded by the CED method: in the worst case IED performs as CED, but under favorable conditions it is able to attain significant performance improvements as highlighted by Figure 6.3. 


\subsubsection{IED threshold setting}

As mentioned in Sections 4.4 and 6.2, the ED threshold is normally chosen to satisfy a certain target $P_{f a}$, which only requires the noise power to be known. In the particular case of the CED method this is an straightforward problem that can be solved analytically based on Equation 6.4. The resulting decision threshold is given by Equation 6.5. In the case of the IED method, however, the procedure is not so straightforward because Equation 6.22 cannot be solved for $\lambda$ analytically. In principle, the decision threshold required to meet a particular target $P_{f a}$ could be computed numerically based on Equation 6.22. Nevertheless, this approach is a rather involved procedure from both computational and practical perspectives.

As an alternative, a simpler approach is proposed and analyzed. Let's assume that the decision threshold for the IED algorithm is selected according to the expression obtained for the CED scheme (see Equation 6.5):

$$
\lambda=\left(Q^{-1}\left(P_{f a, \text { target }}^{\mathrm{IED}}\right) \sqrt{2 N}+N\right) \alpha \sigma_{\tilde{w}}^{2}
$$

where $P_{f a \text {,target }}^{\mathrm{IED}}$ represents the target false alarm probability desired for the IED algorithm. Substituting Equation 6.25 into Equations 6.21 and 6.22 yields:

$$
\begin{aligned}
P_{d}^{\mathrm{IED}}(\gamma) \approx & P_{d}^{\mathrm{CED}}(\gamma)+P_{d}^{\mathrm{CED}}(\gamma)\left(1-P_{d}^{\mathrm{CED}}(\gamma)\right) \cdot \\
& \cdot Q\left(\frac{\alpha Q^{-1}\left(P_{f a, \text { target }}^{\mathrm{IED}}\right) \sqrt{2 N}+N(\alpha-1)-\frac{M}{L} N \gamma}{\sqrt{\frac{2 N}{L}\left\{1+\frac{M}{L}\left[(1+\gamma)^{2}-1\right]\right\}}}\right) \\
\approx & P_{d}^{\mathrm{CED}}(\gamma)+P_{d}^{\mathrm{CED}}(\gamma)\left(1-P_{d}^{\mathrm{CED}}(\gamma)\right) \cdot \\
& \cdot Q\left(\alpha Q^{-1}\left(P_{f a, \text { target }}^{\mathrm{IED}}\right) \sqrt{L}+\sqrt{\frac{N L}{2}}(\alpha-1)-M \sqrt{\frac{N}{2 L}} \gamma\right) \\
P_{f a}^{\mathrm{IED}}(\gamma) \approx & P_{f a, \text { target }}^{\mathrm{IED}}+P_{f a, \text { target }}^{\mathrm{IED}}\left(1-P_{f a, \text { target }}^{\mathrm{IED}}\right) \cdot \\
& \cdot Q\left(\frac{\alpha Q^{-1}\left(P_{f a, \text { target }}^{\mathrm{IED}}\right) \sqrt{2 N}+N(\alpha-1)-\frac{M}{L} N \gamma}{\sqrt{\frac{2 N}{L}\left\{1+\frac{M}{L}\left[(1+\gamma)^{2}-1\right]\right\}}}\right) \\
\approx & P_{f a, \text { target }}^{\mathrm{IED}}+P_{f a, \text { target }}^{\mathrm{IED}}\left(1-P_{f a, \text { target }}^{\mathrm{IED}}\right) \cdot \\
& \cdot Q\left(\alpha Q^{-1}\left(P_{f a, \text { target }}^{\mathrm{IED}}\right) \sqrt{L}+\sqrt{\frac{N L}{2}}(\alpha-1)-M \sqrt{\frac{N}{2 L}} \gamma\right)
\end{aligned}
$$

where $P_{d}^{\mathrm{CED}}(\gamma)$ is given by Equation 6.6, replacing $P_{f a \text {,target }}^{\mathrm{CED}}$ with $P_{f a \text {,target }}^{\mathrm{IED}}$, and Equations 6.27 and 6.29 assume low SNR regime $(\gamma \ll 1)$. It is interesting to note, as opposed to the CED scheme, that the $P_{f a}$ for the IED method depends on the SNR and therefore on the received primary signal. This dependence can be explained by the fact that each sensing decision depends on the previous sensing results, where a signal may be present even if there is no signal in the current sensing event. Equations 6.26 and 6.27 indicate that the detection probability $P_{d}^{\mathrm{IED}}(\gamma)$ would improve with respect to the CED scheme, but the resulting 
$P_{f a}^{\mathrm{IED}}(\gamma)$ would be greater than $P_{f a \text {,target }}^{\mathrm{IED}}$ according to Equations 6.28 and 6.29. In the worst possible case, which corresponds to $Q(\cdot)=1$ in Equations 6.28 and 6.29, and assuming that $P_{f a \text {,target }}^{\mathrm{IED}} \ll 1^{3}$, the resulting false alarm rate would be:

$$
\max \left\{P_{f a}^{\mathrm{IED}}(\gamma)\right\} \approx 2 P_{f a, \text { target }}^{\mathrm{IED}}-\left(P_{f a, \text { target }}^{\mathrm{IED}}\right)^{2} \approx 2 P_{f a, \text { target }}^{\mathrm{IED}}
$$

To ensure that $P_{f a}^{\mathrm{IED}}(\gamma)$ does not exceed $P_{f a \text {,target }}^{\mathrm{IED}}$ even in the worst case, the required decision threshold must then be chosen as:

$$
\lambda=\left(Q^{-1}\left(\frac{P_{f a, \text { target }}^{\mathrm{IED}}}{2}\right) \sqrt{2 N}+N\right) \alpha \sigma_{\tilde{w}}^{2}
$$

This alternative approach increases the decision threshold with respect to Equation 6.25 and reduces the experienced $P_{f a}^{\mathrm{IED}}(\gamma)$, guaranteeing that the amount of lost spectrum opportunities does not exceed the ratio $P_{f a \text {,target }}^{\mathrm{IED}}$. Due to the trade-off between $P_{f a}$ and $P_{d}$, however, this also means that the resulting detection probability would be lower than that obtained when the decision threshold is simply selected according to Equation 6.25, in which case some potential interference might be caused to the primary system. Equation 6.31 can therefore be regarded as an aggressive threshold setting approach where the maximum amount of lost spectrum opportunities is strictly constrained at the expense of some potential risk of interference to the primary network. On the other hand, Equation 6.25 can be regarded as a conservative approach because in this case a minimum detection performance is guaranteed but at the expense of sacrificing some spectrum opportunities since $P_{f a}^{\mathrm{IED}}(\gamma)>P_{f a \text {,target }}^{\mathrm{IED}}$.

The previous observations are illustrated and confirmed in Figure 6.4, where Equations 6.26 and 6.28 (IED performance) are depicted for both conservative and aggressive approaches along with Equation 6.6 (CED performance) for comparison purposes. The MED performance (Equations 6.15 and 6.16) when the decision threshold is selected according to the conservative approach is also shown in order to corroborate the discussion of Section 6.3.2. Although $P_{f a}^{\mathrm{IED}}(\gamma) \geq P_{f a \text {, target }}^{\mathrm{IED}}$ with the conservative approach, it is interesting to note that the resulting $P_{f a}$ degradation is appreciable when considering relatively large $P_{f a \text {,target }}^{\mathrm{IED}}$ values such as $P_{f a \text {,target }}^{\mathrm{IED}}=0.1$. For lower values (e.g., $P_{f a \text {, target }}^{\mathrm{IED}}=0.01$ ), the resulting $P_{f a}$ can be considered to be $P_{f a}^{\mathrm{IED}}(\gamma) \approx P_{f a \text {,target }}^{\mathrm{IED}}$ in practice. In such a case, the conservative approach is preferable over the aggressive alternative since it is able to provide the full $P_{d}$ improvements without a noticeable $P_{f a}$ degradation with respect to the CED scheme. The practical consequences of both threshold setting strategies are evaluated experimentally, based on field measurements of real signals, in Section 6.4.4.

\subsubsection{IED experimental performance}

The experimental performance of the IED scheme in terms of $P_{d}$ and $P_{f a}$ is shown in Figure 6.5. These results have been obtained based on field measurements performed with the

\footnotetext{
${ }^{3}$ For example, the IEEE 802.22 spectrum sensing requirements [200] specify that a DSA/CR terminal must be able to provide $P_{f a} \leq 0.1$. Lower values may be desirable as well in order to maximize spectrum utilization.
} 

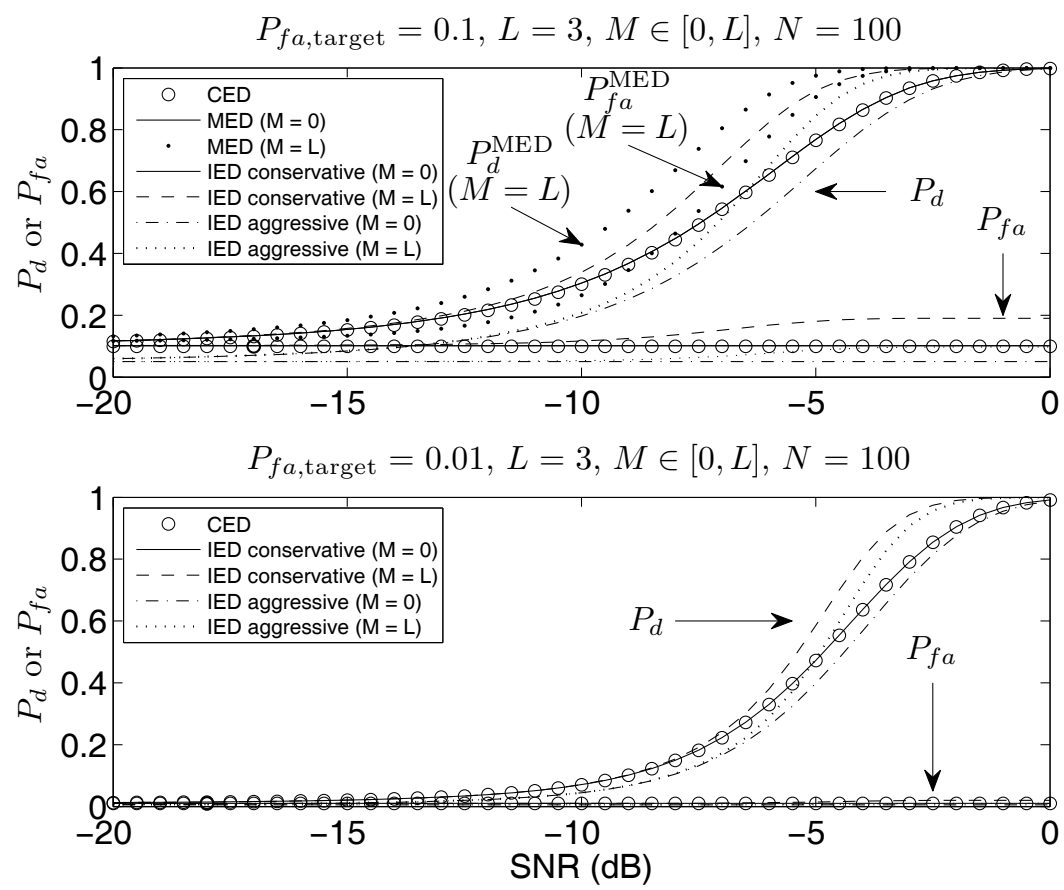

Figure 6.4: Theoretical performance for the CED, MED and IED algorithms $(\alpha=1)$.

measurement platform and evaluation methodology described in Chapter 4. The IED performance is shown as a function of the algorithm's parameter $L$. Notice that the values shown for $L=1$ correspond to the CED performance since the CED and IED schemes are practically equivalent for $L=1$. The performance has been obtained when the decision threshold is computed according to the conservative (Equation 6.25) and aggressive (Equation 6.31) approaches described in Section 6.4.3, and for various sensing periods $N$. For each sensing period, the SNR corresponding to $P_{d}^{\mathrm{CED}}=0.9$ and $P_{f a}^{\mathrm{CED}}=0.1$ is selected (according to Equation 6.6, $\mathrm{SNR}=4.29 \mathrm{~dB}$ for $N=10, \mathrm{SNR}=-3.54 \mathrm{~dB}$ for $N=100$ and $\mathrm{SNR}=-9.15$ $\mathrm{dB}$ for $N=1000$ ). The target $P_{f a}$ for the IED algorithm is $P_{f a \text {,target }}^{\mathrm{IED}}=0.1$ in all cases.

As it can be appreciated, the IED proposal clearly outperforms the conventional CED scheme ( $L=1$ in Figure 6.5) for all the considered sensing periods. As $L$ increases (i.e., the measured energy (power) of more sensing events are combined when computing $\mathbb{T}_{i}^{\mathrm{avg}}\left(\mathbf{T}_{i}\right)$ ), the true average signal energy (power) can be estimated more accurately and the actual channel state can therefore be determined more reliably, which explains the growth of $P_{d}^{\mathrm{IED}}$ as $L$ increases. However, for $L$ sufficiently large the true average signal (power) energy can be estimated with reasonable accuracy. In such a case, further increasing $L$ does not significantly contribute to the estimation accuracy. As a result, increasing $L$ beyond certain value improves $P_{d}^{\mathrm{IED}}$ marginally. Based on Figure $6.5, L=5$ can be considered as an appropriate trade-off between the obtained performance improvement and the amount of memory required to store the previous test statistic values for the sensed channels. 


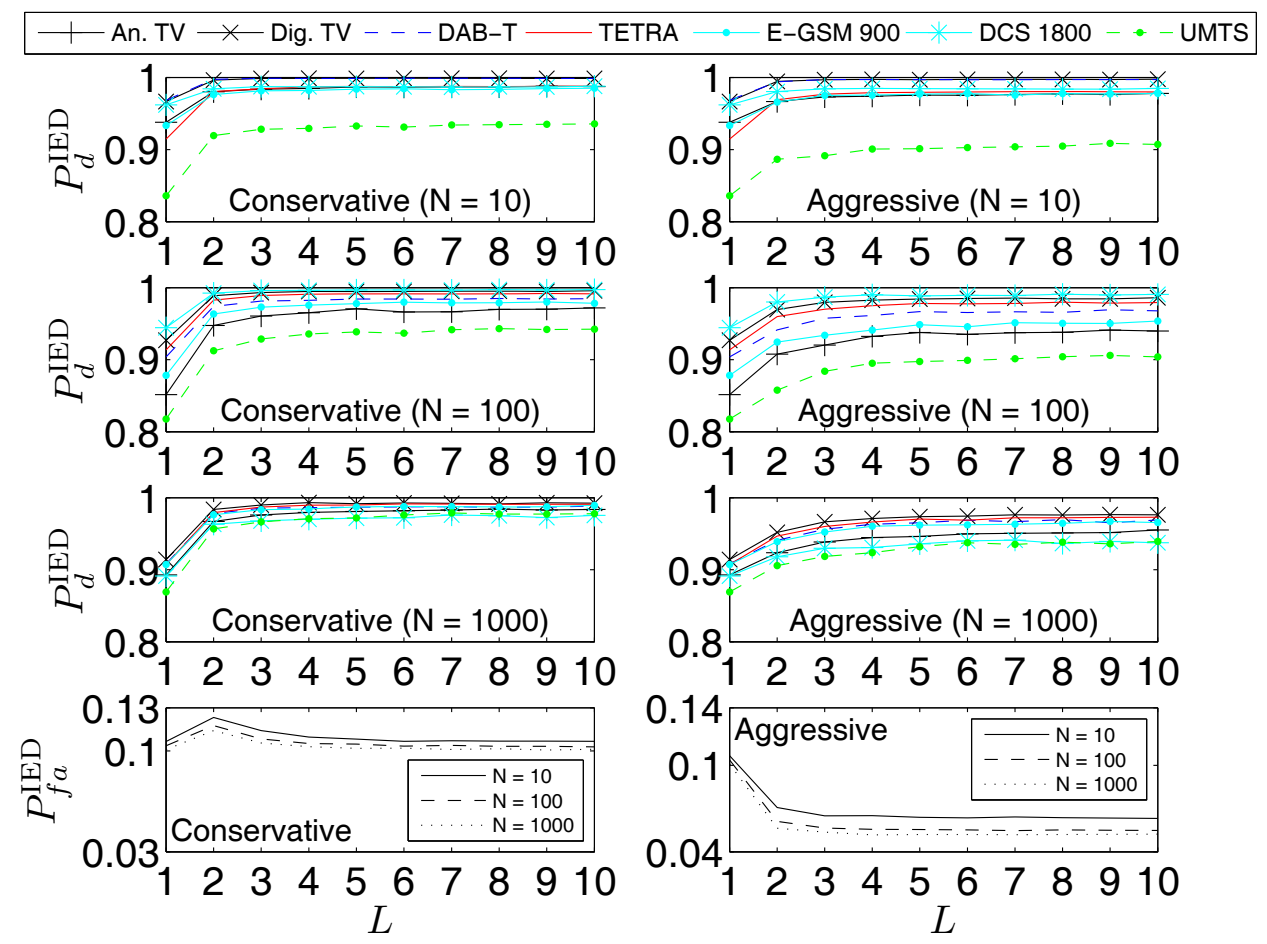

Figure 6.5: Experimental detection and false alarm performance for the IED algorithm.

It is interesting to note, as expected from the discussion in Section 6.4.3, that the detection performance for the aggressive threshold setting approach is in general lower than that of the conservative approach. This is indeed the price to be paid for the lower $P_{f a}$ attained by the aggressive approach, as shown in Figure 6.5. As it can be observed, the aggressive approach guarantees that $P_{f a \text {,target }}^{\mathrm{IED}}$ is never exceeded (which is not the case of the conservative one) at the expense of a lower detection probability and therefore an increased risk of potential interference to the primary network. Although $P_{f a}^{\mathrm{IED}} \geq P_{f a \text {, target }}^{\mathrm{IED}}$ for the conservative approach, as expected from Equation 6.28, it can be observed however that in practice the resulting $P_{f a}^{\mathrm{IED}}$ can be made to be very similar to the desired target with the conservative approach if the appropriate $L$ is selected (e.g., the value $L=5$ mentioned above constitutes a reasonable choice). This indicates that the decision threshold of the IED algorithm can be selected following the same procedure as for the CED scheme (Equation 6.25), which simplifies the design of the IED spectrum sensing algorithm.

Figure 6.6 compares the performance of the CED and IED (with $L=5$ ) algorithms in terms of the ROC. The results have been obtained by means of extensive simulations based on the same set of empirical data used to compute the results shown in Figure 6.5 and considering the same operating conditions. As it can be clearly appreciated, the IED algorithm is able to outperform the CED scheme for all the considered radio technologies and sensing periods, providing important performance gains. 

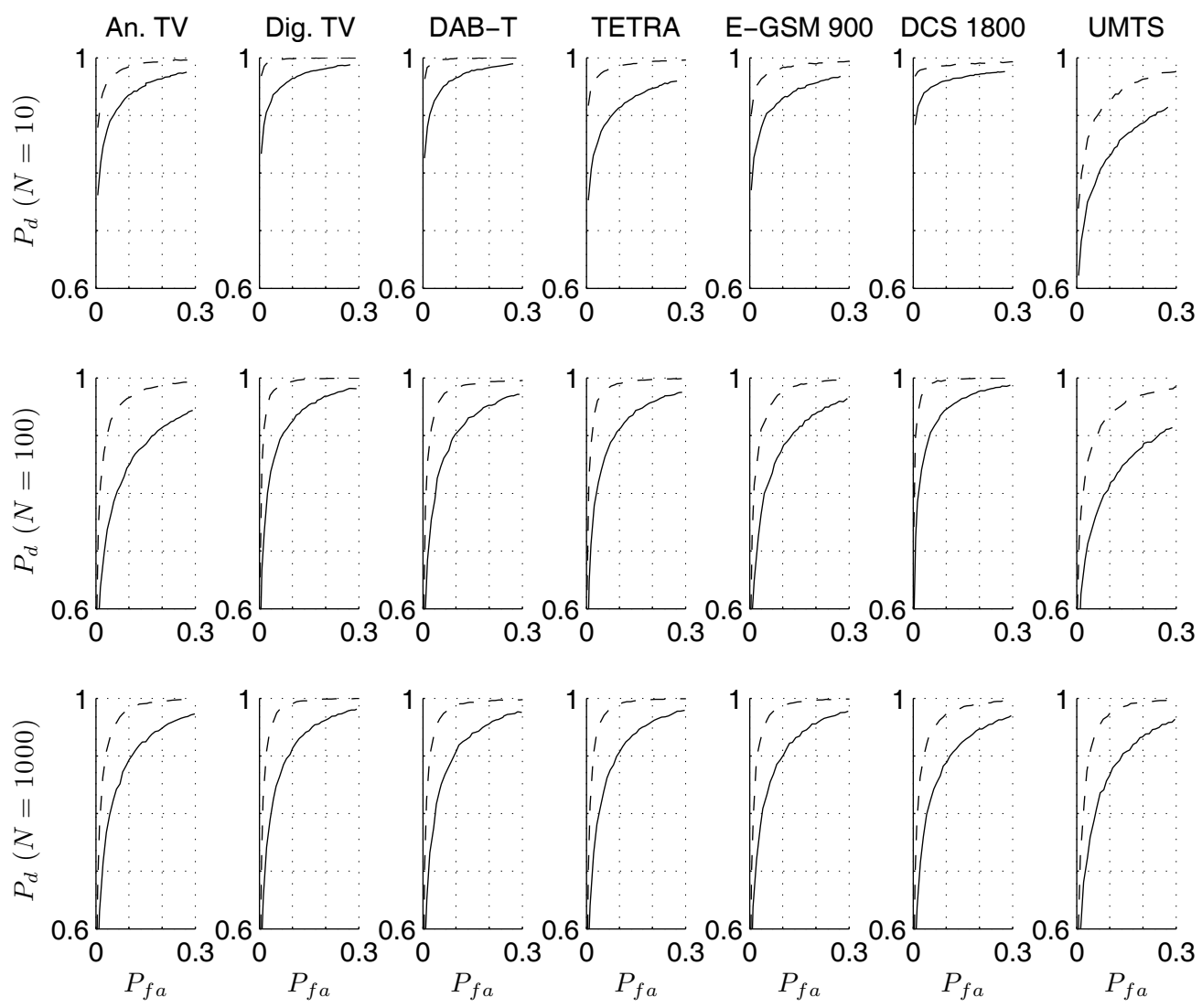

Figure 6.6: Experimental ROC for the CED (solid line) and IED (dashed line) algorithms.

Based on the results of this section, it can be concluded that the IED approach is able to achieve significant detection performance improvements with respect to the CED method. It has been shown that the observed detection enhancements are not obtained at the expense of a noticeable false alarm degradation. Moreover, the decision threshold can be selected according to the same analytical result employed for the CED method, which simplifies the design and configuration procedure for the IED scheme in a real DSA/CR system.

\subsection{Complexity analysis}

\subsubsection{Sample complexity}

The sample complexity of a spectrum sensing algorithm specifies how the sensing period $N$ required to meet a certain set of target $P_{d, \text { target }}^{\mathrm{IED}}$ and $P_{f a \text {,target }}^{\mathrm{IED}}$ scales as the experienced SNR $\gamma$ degrades. For the CED method, the sample complexity is given by (see Equation 4.19):

$$
N=2\left[\frac{Q^{-1}\left(P_{f a, \text { target }}^{\mathrm{IED}}\right)-Q^{-1}\left(P_{d, \text { target }}^{\mathrm{IED}}\right)(1+\gamma)}{\gamma}\right]^{2}
$$


Assuming low SNR regime $(\gamma \ll 1)$, the required number of samples scales as $O\left(1 / \mathrm{SNR}^{2}\right)$, which represents the sample complexity of the CED algorithm.

For the IED method, the sample complexity cannot be determined analytically by solving Equations 6.26 and 6.28 for $N$. However, Equations 6.21 and 6.22 constitute a system of two equations that can be solved numerically for $N$ and $\lambda$ under various SNR values ${ }^{4}$. The obtained $N$ versus the considered SNR is depicted in Figure 6.7, which enables the comparison of the sample complexity for the CED and IED approaches. The curves corresponding to the IED method are a shifted version of the CED curve, meaning that the IED sample complexity is also given by $O\left(1 / \mathrm{SNR}^{2}\right)$.

\subsubsection{Computational complexity}

The computational cost of the CED and IED methods can be estimated by comparing the set of operations performed by Algorithms 6.1 and 6.3. First, the computation of $\mathbb{T}_{i}\left(\tilde{\mathbf{y}}_{i}\right)$ requires $N$ products and $N-1$ sums, which is common to both algorithms. Additionally, the IED algorithm computes $\mathbb{T}_{i}^{\text {avg }}\left(\mathbf{T}_{i}\right)$, which requires $L-1$ sums and one division, performs two additional comparisons (lines 7 and 8 in Algorithm 6.3), and requires memory to store the last $L-1$ test statistic values for each channel on which the DSA/CR terminal operates. The computational cost increase of the IED method can therefore be considered to be negligible, especially when compared to other more sophisticated methods such as cyclostationary feature detectors [182, 183] or covariance-based detectors [185], which require computationally complex operations as sample autocorrelation and covariance matrices.

To compare the computational costs of the CED and IED schemes, Figure 6.7 shows the average computation times observed in the simulations performed in the context of this study, based on Matlab running in a PC with an Intel Core2 Quad processor at $2.4 \mathrm{GHz}$. As it can be appreciated, the IED computation time is approximately the same for $L=1$ and $L=100$, meaning that the computation of the average test statistic in line 3 of Algorithm 6.3 is negligible and that most of the additional time with respect to the CED scheme is due to the additional verifications performed in lines 7 and 8 of Algorithm 6.3. The time required to perform these verifications, however, becomes less significant with respect to the time required to compute $\mathbb{T}_{i}\left(\tilde{\mathbf{y}}_{i}\right)$ as the sensing period $N$ increases. As a result, the computation time of the CED and IED algorithms is approximately the same for the sensing periods normally required in practice (see Figures 4.4, 4.5 and 4.6). Despite the implemented code was not optimized and a general purpose PC was employed, these results allow to confirm that the computational cost increase of the IED algorithm is negligible in practice.

\subsection{Discussion}

The IED spectrum sensing method exploits the history of past spectrum sensing results to avoid signal misdetections caused by instantaneous energy (power) drops, which may be caused by the radio channel fading properties or the primary transmission power pattern. The use of past spectrum sensing results to infer the current channel state is sensible if the

\footnotetext{
${ }^{4}$ Notice that all the terms involved in Equations 6.21 and 6.22 implicitly depend on $N$.
} 

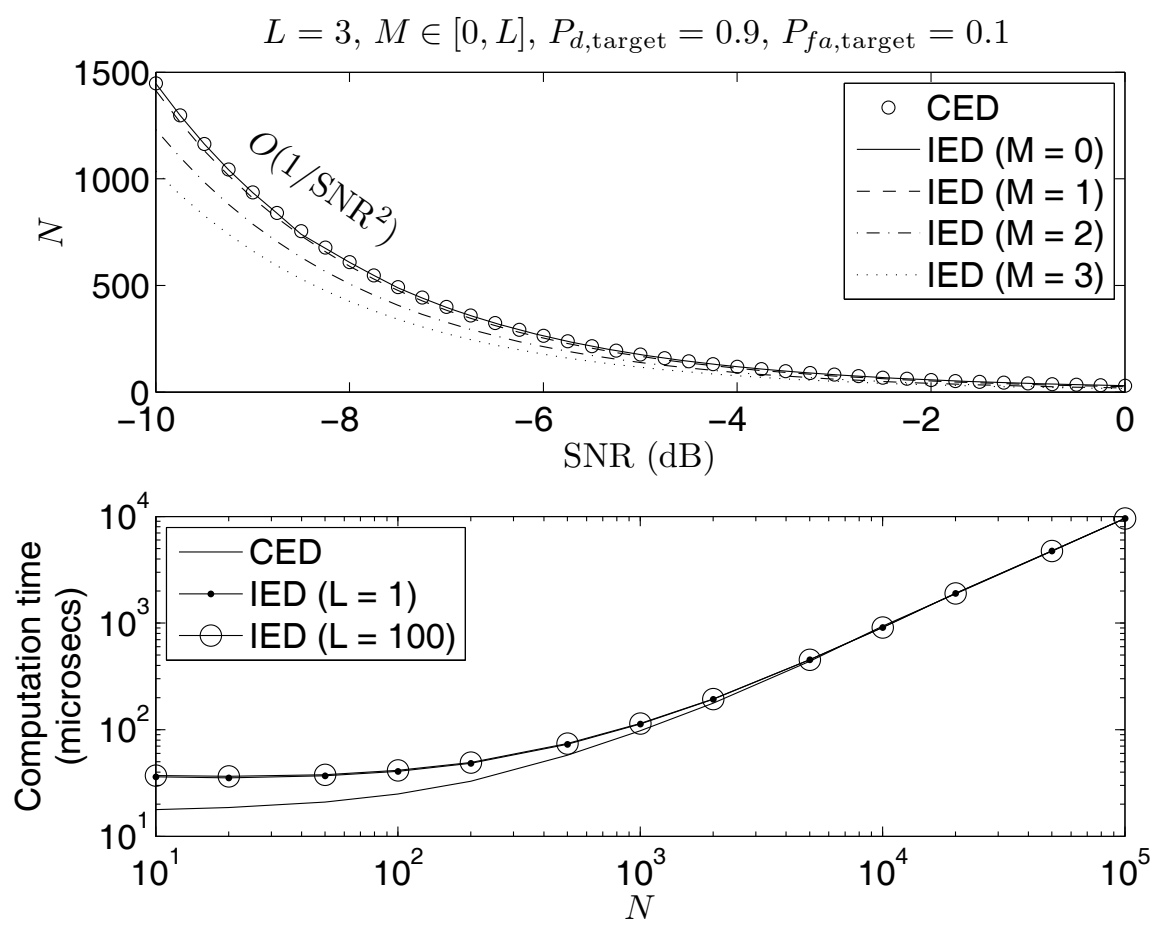

Figure 6.7: Complexities of the CED and IED algorithms (upper graph: sample complexity; lower graph: computational complexity).

sensing events are reasonably close in time. This means that the full detection performance improvement of the IED scheme would be achieved when the target channel is sensed with enough periodicity. However, if the periodicity at which the channel is sensed increases while the time period for which the channel is sensed (i.e., the sensing interval $N$ ) is kept constant, the overall time devoted to sensing increases and therefore the remaining time available for data transmission decreases, which results in a throughput degradation for the secondary system [212]. To maintain the same average throughput, the sensing interval $N$ should be decreased along with the period between two consecutive sensing events. However, if the sensing period $N$ is made sufficiently short, the test statistic values may follow the instantaneous variations of the received signal energy (power). This might result in some signal misdetections in the particular case of the CED method as discussed in Section 4.7.2, which is avoided with the IED method if the spectrum sensing operation is configured properly. Therefore, the IED improvements would be obtained with high sensing frequencies and short sensing intervals. Notice that shortening the sensing interval means that the detection probability in each isolated sensing event decreases, but if several consecutive sensing events that are sufficiently close in time are combined according to the IED principle, the same detection performance would be achieved as the obtained results indicate. In such a case, the benefit obtained would be an enhanced spectrum agility as a result of the increased sensing frequency. In other words, if the channel is sensed more frequently it is possible to provide 
shorter response times in detecting and exploiting white spaces or vacating a channel when a primary user appears ${ }^{5}$. Therefore, a proper configuration of the IED method would be able to improve the detection performance of the CED method or preserve the same performance while increasing the spectrum agility, thus enabling DSA/CR users to exploit spectrum gaps sooner and take profit of shorter spectrum opportunities. The ultimate consequence would be a notable improvement of the overall spectrum usage efficiency.

\subsection{Summary}

ED constitutes a preferred approach for spectrum sensing in DSA/CR due to its simplicity and wide field of application as well as its low computational and implementation costs. The main drawback, however, is its well known limited detection performance. Various alternative spectrum sensing methods have been shown to outperform ED, but at the expense of significantly increased complexity and limited field of applicability. In this context, this chapter has proposed an improved ED-based spectrum sensing technique that is able to outperform the conventional ED scheme while preserving a similar level of complexity and computational cost as well as its general field of application. The performance improvement has been assessed analytically and corroborated with experimental results, demonstrating appreciable performance improvements. The proposed method will enable, based on simple ED-based procedures, a more accurate estimation of the busy/idle state information from empirical spectrum data for the development and validation of spectrum usage models.

\footnotetext{
${ }^{5}$ As a numerical example, and according to Figure 6.7, the number of samples required for $\mathrm{SNR}=-10 \mathrm{~dB}$, $P_{d, \text { target }}=0.9$ and $P_{f a \text {,target }}=0.1$ would be $N^{C E D} \approx 1500$ and $N^{I E D} \approx 1000$ for the CED and IED methods, respectively. If the transmit-to-sensing time ratio is denoted as $\eta$, the time between two consecutive sensing events would be given by $N^{C E D}(1+\eta)$ and $N^{I E D}(1+\eta)$, which is 1.5 times $(33 \%)$ lower in the case of the IED method for the same $P_{d \text {,target }}, P_{f a \text {,target }}$ and $\eta$.
} 



\section{Part III}

\section{Spectrum Usage Models}

Remember that all models are wrong;

the practical question is fow wrong

do they have to be to not be useful.

George E. P. Box 



\section{Chapter}

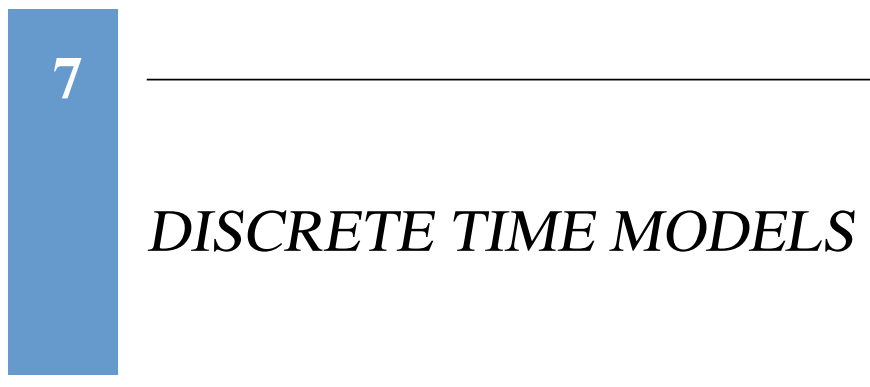

\subsection{Introduction}

The present chapter initiates a series of studies aimed at developing a holistic set of realistic models capable to accurately capture and reproduce the relevant statistical properties of spectrum usage in real radio communication systems. Relying on the findings and contributions of the studies performed in Parts I and II, the studies presented in the remainder of this dissertation develop spectrum models for the time, frequency and space dimensions.

The focus of this chapter is on the modeling of spectrum occupancy in the time domain. In particular, a discrete-time two-state Markov chain with novel deterministic and stochastic Duty Cycle (DC) models is proposed as an adequate means to accurately describe spectrum occupancy in the time domain. The validity and accuracy of the proposed modeling approach is assessed and corroborated with extensive empirical data from the multi-band spectrum measurement campaign reported in Chapter 3. The obtained results demonstrate that the proposed approach is able to accurately capture and reproduce the relevant statistical properties of spectrum usage for various radio technologies. The relevance of accurate models in DSA/CR research is illustrated with a practical case study.

The remainder of this chapter is organized as follows. First, Section 7.2 reviews the related previous work on time-dimension models of spectrum usage, identifying the existing deficiencies that motivate the investigation reported in this chapter. Section 7.3 describes the methodology employed to capture and process the empirical data used in the validation of the proposed model. Section 7.4 presents the traditional Markov chain model commonly used in the existing literature. Since such Markov model is not able to accurately capture and reproduce some relevant statistical properties of spectrum usage in the time domain, it is extended with adequate deterministic and stochastic DC models, which are presented in Sections 7.5 and 7.6, respectively. The validity and accuracy of the developed model is assessed and verified in Section 7.7. The importance of disposing of accurate models 
of spectrum usage, as the one presented in this chapter, for the design and evaluation of DSA/CR techniques and solutions is highlighted with a practical case study in Section 7.8. Finally, Section 7.9 summarizes the research presented in this chapter.

\subsection{Related work and motivation}

\subsubsection{Previous work based on continuous-time Markov chains}

From the point of view of a DSA/CR network, spectrum usage can adequately be modeled by means of a Markov chain with two states, one indicating that the channel is busy (i.e., used by a primary user and therefore not available for opportunistic access) and the other one indicating that it is idle (i.e., available for secondary use). A popular channel model in DSA/CR research is the well-known two-state Continuous-Time Markov Chain (CTMC) model, where the channel remains in one state for a random time period before switching to the other state. The state holding time or sojourn time is modeled as an exponentially distributed random variable.

The CTMC model has widely been used in the study of various DSA/CR techniques such as MAC protocols for spectrum sharing [213, 214], MAC-layer sensing schemes [215-217] and adaptive spectrum sensing solutions [218] as well as to analyze the sensing-throughput trade-off $[219,220]$ and the performance of DSA/CR sensor networks [221].

Although the CTMC model has widely been used in the literature, some works based on empirical measurements [222-226] have demonstrated that state holding times are not exponentially distributed in reality. In particular, it has been found that state holding times are more adequately described by means of generalized Pareto [222], a mixture of uniform and generalized Pareto [223, 224], hyper-Erlang [223, 224], generalized Pareto and hyperexponential [225] as well as geometric and log-normal [226] distributions. Based on these results, a more appropriate model is therefore the Continuous-Time Semi-Markov Chain (CTSMC) model, where the state holding times can follow any arbitrary distribution. As a result, some recent works have employed CTSMC models. This is the case for example of [227, 228], which have considered a CTSMC model where the busy and idle periods are modeled as exponentially and Erlang-distributed random variables, respectively.

\subsubsection{Previous work based on discrete-time Markov chains}

In the discrete-time counterpart of the CTMC, i.e. the Discrete-Time Markov Chain (DTMC) model, the time index set is discrete. According to this model, the channel remains in a certain state at each step, with the state changing randomly between steps. The behavior of the channel is described by means of a set of transition probabilities between states $^{1}$.

The DTMC model has widely been used in the DSA/CR literature as well. For instance, it has been used to analyze the performance of MAC [229] and joint MAC/sensing [230] frameworks for opportunistic spectrum access, dynamic channel selection strategies [231],

\footnotetext{
${ }^{1}$ A DTMC model could be characterized by discrete-time distributions for state holding times instead of a set of transition probabilities between states. While the former approach has received some attention [226], the latter, which has widely been employed in the literature, remains unexplored and is studied in this chapter.
} 
opportunistic scheduling policies [232], channel selection schemes [233] based on the interference temperature model [234], and to analyze the voice-service capacity of DSA/CR systems under both ideal [235] and imperfect [236] spectrum sensing conditions as well as under QoS restrictions [237].

As opposed to the continuous-time case, and to the best of the author's knowledge, the suitability of the DTMC channel model in describing the statistical properties of spectrum occupancy patterns in real systems has not been evaluated and assessed in the literature before $^{1}$. This means that an important volume of research in DSA/CR has been based on assumptions or oversimplifications that have not been validated with empirical measurement data and, more importantly, that future research based on the DTMC channel model will also suffer from the same drawback due to the lack of appropriate DTMC modeling approaches capable to capture the relevant statistical properties of spectrum occupancy in the time domain. In this context, the study reported in this chapter covers such deficiencies and fills the existing gaps by evaluating the ability of the DTMC model to reproduce the statistical properties of spectrum usage in real radio communication systems and extending the conventional DTMC model with appropriate deterministic and stochastic DC models.

\subsection{Measurement setup and methodology}

The spectrum analyzer measurement platform discussed in Chapter 2 and Appendix A was employed to perform measurements in several spectrum bands (see Table 7.1). Although this chapter does not present results for all the spectrum bands shown in Table 7.1, the proposed model was validated based on channels from all the considered radio technologies. Most of the selected bands were measured from a building rooftop (see location 1 in Figure 3.1), which represents a strategic location with direct line-of-sight to several transmitting stations located a few tens or hundreds of meters away from the antenna and without buildings blocking the radio propagation. However, the measurement equipment was placed inside the same building (see location 2 in Figure 3.1) for the DECT and ISM bands since such radio technologies are used by short-range devices more commonly deployed in indoor environments. These measurement locations were carefully selected in order to maximize the receiving SNR and hence ensure a reliable and accurate estimation of the true busy/idle states.

Based on the configuration shown in Table 3.1, each band was measured across a time span of 7 days, from Monday midnight to Sunday midnight. This measurement period enabled not only to capture a high number of signal samples (see Table 7.1), but also to appreciate any potential pattern on spectrum usage (e.g., channel use variations between weekdays and weekends as well as variations at different times along days and nights).

The captured data were used to extract the binary channel occupancy patterns from the measured channels by classifying the spectrum analyzer's power samples as either busy or idle states. Due to the heterogeneity of the measured bands and the unavailability of detailed information, an ED principle was employed to this end. As discussed in Chapter 4, ED can be applied regardless of the signal to be detected and the lack of prior information. In particular, the CED scheme described in Section 6.2 was employed to process the spectrum data on a power-sample basis (i.e., each power sample was processed individually, not in 
Table 7.1: Spectrum bands considered in this study.

\begin{tabular}{|c|c|c|c|c|}
\hline $\begin{array}{c}\text { Measured } \\
\text { band }\end{array}$ & $\begin{array}{c}\text { Frequency } \\
\text { range (MHz) }\end{array}$ & $\begin{array}{c}\text { No. of } \\
\text { channels }\end{array}$ & $\begin{array}{c}\text { No. of } \\
\text { samples }\end{array}$ & $\begin{array}{c}\text { Avg. sweep } \\
\text { time (secs) }\end{array}$ \\
\hline Amateur & $144-146$ & 79 & 234418 & 2.58 \\
\hline Paging & $157-174$ & 679 & 186628 & 3.24 \\
\hline TETRA UL & $410-420$ & 399 & 199013 & 3.04 \\
\hline TETRA DL & $420-430$ & 399 & 195956 & 3.08 \\
\hline E-GSM 900 UL & $880-915$ & 174 & 156460 & 3.86 \\
\hline E-GSM 900 DL & $925-960$ & 174 & 158147 & 3.82 \\
\hline DCS 1800 UL & $1710-1785$ & 374 & 125986 & 4.80 \\
\hline DCS 1800 DL & $1805-1880$ & 374 & 128615 & 4.70 \\
\hline DECT & $1880-1900$ & 10 & 178388 & 3.39 \\
\hline ISM & $2400-2500$ & 13 & 105940 & 5.70 \\
\hline
\end{tabular}

groups of $N$ samples as suggested by Equation 6.1). In Chapter 4 it was demonstrated that the IED scheme studied in Section 6.4 outperforms the CED scheme with a similar level of complexity. However, note that the IED algorithm makes use of past channel observations to determine the current channel state. As discussed in Section 6.6, this does not appear to be sensible when the time period between consecutive observations is in the order of several seconds as it is the case (see Table 7.1). This circumstance prevents the use of the IED scheme in this case and, as a result, the CED scheme was employed instead. The energy decision threshold was selected as the maximum noise power observed in each channel plus a 3-dB margin (i.e., a combination of the MaxNoise and $m$ - $\mathrm{dB}$ approaches described in Section 2.5). This criterion was explicitly selected in order to avoid any potential false alarms, which would have lead to wrong conclusions on the length of busy and idle periods. The detection performance loss resulting from this relatively high decision threshold was verified to be negligible since the high SNR conditions under which most of the measured signals were received guarantee that the probability of missed detections is minimized, thus resulting in a nearly ideal detection performance under such conditions. The resulting binary sequences of busy and idle states were employed to extract the lengths of busy and idle periods and validate the proposed DTMC modeling approach based on empirical data.

It is worth noting that the average sweep times shown in Table 7.1 indicate that the resulting sampling rates of swept spectrum analyzers as the one employed in this study are not comparable to that of the measurement configurations employed in other modeling studies [222-225], which may result in under-sampling of the measured signals and thus the missed detection of channel state changes between consecutive channel observations. The USRP measurement platform presented in Chapter 4 could be employed in order to increase the effective time resolution and avoid under-sampling. However, there are several reasons that motivated the use of a low time-resolution measurement platform instead. First of all, the high sampling rate of the USRP measurement platform leads to huge amounts of data that require computation times several orders of magnitude above the actual measurement pe- 
riod, which would have prevented the study of each considered band over long time periods. On the other hand, the spectrum analyzer-based platform enables the study of spectrum usage patterns over several days at affordable computational costs. As it will be shown later on, the possibility to analyze such long time periods will result in interesting findings and conclusions. Moreover, the binary occupancy pattern derived from low time-resolution measurements, although inaccurate in strict sense, is still interesting and useful due to two main reasons. The first one is that such binary pattern can be thought of as the occupancy perception of a DSA/CR user that periodically senses the channel and observes its state at discrete time instants. Therefore, spectrum analyzer measurements are useful to model spectrum occupancy from the point of view of the DSA/CR user perception. Since the overall behavior of a DSA/CR network is driven by the primary occupancy pattern as perceived by the sensing nodes, analytical studies and simulations of DSA/CR systems should rely on spectrum usage models that are able to accurately capture and reproduce the channel occupancy pattern in real channels as observed by DSA/CR terminals. On the other hand, short idle periods resulting from bursty data transmissions are difficult to exploit for secondary usage in practice $^{2}$, which from a practical point of view is equivalent to a busy channel state. Exploitable idle periods normally arise when there is no primary user making use of the channel, which leads to appreciably longer periods of inactivity that can reliably be detected with a spectrum analyzer within reasonable accuracy limits in spite of its limited time resolution. Moreover, it is worth noting that spectrum analyzers have successfully been applied in previous modeling studies [226] and have the advantage of enabling high dynamic ranges, high sensitivities and wideband measurements. The empirical data captured for various radio technologies enabled an adequate validation of the model developed in this study.

\subsection{Discrete-time Markov chain}

At a given time instant, a primary radio channel may be either busy or idle, meaning that the time occupancy pattern of a primary radio channel can adequately be modeled by means of a two-state Markov chain. The state space for a primary radio channel can be denoted as $\mathbb{S}=\left\{s_{0}, s_{1}\right\}$, where the $s_{0}$ state indicates that the channel is idle and the $s_{1}$ state indicates that the channel is busy. The channel state $S(t)$ at time $t$ can either be $S(t)=s_{0}$ or $S(t)=s_{1}$. As discussed in Section 7.2, this study focuses on the particular case of DTMCs, where the time index set is discrete, i.e. $t=t_{k}=k T_{s}$, where $k$ is a non-negative integer representing the step number and $T_{s}$ is the time period between consecutive transitions or state changes ${ }^{3}$.

The behavior of a DTMC can be described by means of a set of transition probabilities between states. If the state space $\mathbb{S}$ is finite with $n$ states, the transition probability distribution can be represented by a $n \times n$ square matrix $\mathbf{P}\left(t_{k}, t_{l}\right)=\left[p_{i j}\left(t_{k}, t_{l}\right)\right]_{n \times n}$, called the transition matrix, where the $(i, j)$-th element of $\mathbf{P}\left(t_{k}, t_{l}\right)$ is $p_{i j}\left(t_{k}, t_{l}\right)=P\left(S\left(t_{l}\right)=s_{j} \mid S\left(t_{k}\right)=s_{i}\right)$, with $t_{k}<t_{l}$. In other words, $p_{i j}\left(t_{k}, t_{l}\right)$ represents the probability that the system goes into state

\footnotetext{
${ }^{2}$ For instance, the time-slot duration is $14.167 \mathrm{~ms}$ in TETRA, $577 \mu$ s in GSM/DCS and $417 \mu$ s in DECT. The IEEE 802.11 protocol, used by wireless local area networks operating in the ISM band, defines inter-frame spaces in the order of $50 \mu$ s or less.

${ }^{3} T_{S}$ can be associated to the average sweep times shown in Table 7.1.
} 


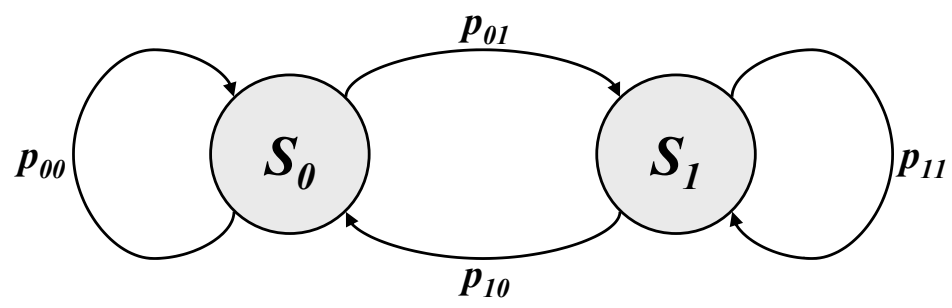

Figure 7.1: Discrete-Time Markov Chain (DTMC) model.

$s_{j}$ at $t=t_{l}$ given that it was in state $s_{i}$ at $t=t_{k}<t_{l}$. If the transition probabilities $p_{i j}\left(t_{k}, t_{l}\right)$ are independent of the time instant $t$, the Markov chain is said to be time-homogeneous or stationary. In such a case, the Markov chain is described by a single, time-independent ma$\operatorname{trix} \mathbf{P}=\left[p_{i j}\right]_{n \times n}$. Notice that $\mathbf{P}$ is a square matrix where each row consists of non-negative values summing to one, i.e. $\sum_{j=0}^{n-1} p_{i j}=1 \forall i$. In the particular case of spectrum occupancy modeling, and inasmuch as the number of channel occupancy states is $n=2$, the resulting transition matrix is given by:

$$
\mathbf{P}=\left[\begin{array}{ll}
p_{00} & p_{01} \\
p_{10} & p_{11}
\end{array}\right]
$$

The overall DTMC model describing the occupancy of a primary radio channel at discrete time instants is illustrated in Figure 7.1.

The DC of a channel, denoted as $\Psi$, is a very straightforward metric and an accurate reproduction is a minimum requirement for any time-dimension model of spectrum usage. The DC can be defined from both probabilistic and empirical perspectives. While the former results more appropriate for theoretical analyses, the latter results more convenient for validation with empirical data. From an empirical point of view, the DC can be estimated as the fraction of time that the channel is declared to be busy based on the procedure described in Section 7.3. From a probabilistic viewpoint, the DC can be defined as the probability that the channel is busy. The probabilities that the model of Figure 7.1 is in each of its states in the long term are given by [238]:

$$
\begin{aligned}
& P\left(S=s_{0}\right)=\frac{p_{10}}{p_{01}+p_{10}}=1-\Psi \\
& P\left(S=s_{1}\right)=\frac{p_{01}}{p_{01}+p_{10}}=\Psi
\end{aligned}
$$

Thus, the DTMC model can be configured to reproduce any arbitrary DC, $\Psi$, by selecting the transition probabilities as $p_{01}=p_{11}=\Psi$ and $p_{10}=p_{00}=1-\Psi$, which yields:

$$
\mathbf{P}=\left[\begin{array}{cc}
1-\Psi & \Psi \\
1-\Psi & \Psi
\end{array}\right]
$$

In order to verify the ability of the DTMC model of Equation 7.4 to reproduce the DC of real channels, the empirical data captured in the measurement campaign were used to 
estimate the transition probabilities of each channel as:

$$
\hat{p}_{i j}= \begin{cases}\frac{\eta_{i j}}{\eta_{i}}, & \eta_{i}>0 \\ 0, & \eta_{i}=0 \text { and } i=j \\ 1, & \eta_{i}=0 \text { and } i \neq j\end{cases}
$$

where $\eta_{i j}$ represents the number of transitions from state $s_{i}$ to $s_{j}$ occurring in the empirical sequences, and $\eta_{i}=\sum_{k=0}^{n-1} \eta_{i k}$ is the number of times that the channel resides in state $s_{i}$. The two last cases of Equation 7.5 are included in order to account for channels that are always busy $(\Psi=1)$ or always idle $(\Psi=0)$. The theoretical DC corresponding to the estimated probabilities $\hat{p}_{i j}$ was estimated based on Equation 7.3 and compared to the true empirical DC of the channel, appreciating a perfect agreement for channels of all the considered radio technologies. This indicates that the DTMC model of Equation 7.4 is able to accurately reproduce the mean DC of real channels ${ }^{4}$.

Nevertheless, reproducing not only the DC but also the lengths of the busy and idle periods is an important characteristic of a realistic time-dimension model of spectrum usage. While this feature is explicitly represented in the case of CTMC and CTSMC models by means of the sojourn time distributions, there is no means to account for the state holding times in the case of the DTMC model. Therefore, the DTMC model would not be expected to reproduce the statistical properties of the lengths of busy and idle periods of real channels. To verify this statement, the DTMC channel model was simulated with the transition probabilities $\hat{p}_{i j}$ estimated from empirical data for all the measured channels. During the simulation of the DTMC model, the durations of the state holding times $T_{i}$ were computed as $T_{i}=\widetilde{\eta}_{i} \cdot T_{s}$, where $\widetilde{\eta}_{i}$ represents the number of consecutive steps the channel resides in state $s_{i}$ during the simulation before switching to the other state, and $T_{s}$ is the average sweep time corresponding to the considered channel (see Table 7.1). The statistical distributions of busy and idle periods obtained by means of simulation were computed and compared to the empirical distributions of busy and idle periods observed in the measured channels. This comparison was performed for each measured channel of every radio technology. Figures 7.2-7.5 show the results obtained for some selected channels. The results are shown in terms of the Complementary Cumulative Distribution Function (CCDF) and with axes in logarithmic scale for a finer detail of appreciation. The time evolution of the DC computed over 1-hour periods is also shown. In general, the obtained results indicate, as expected, that the DTMC channel model is not able to reproduce the statistical properties of the lengths of busy and idle periods of real channels. In some cases, however, the distributions resulting from simulations show a noticeable agreement with their empirical counterparts (Figure 7.5 shows an example). After analyzing the empirical data in detail, it was observed that the convergence or divergence of empirical and simulation results can be explained in terms of the channel load variation pattern. When the channel is sparsely used (low load), the length of idle periods is significantly higher than that of busy ones. On the other hand, when the channel is subject to an intensive usage (high load), the length of busy periods increases while

\footnotetext{
${ }^{4}$ For CTMC and CTSMC models, an arbitrary DC can also be reproduced by properly selecting the parameters of the sojourn time distributions in order to provide mean values $\mathbb{E}\left\{T_{i}\right\}$ such that $\Psi=\mathbb{E}\left\{T_{1}\right\} /\left(\mathbb{E}\left\{T_{0}\right\}+\right.$ $\mathbb{E}\left\{T_{1}\right\}$ ) [238], where $\mathbb{E}\left\{T_{0}\right\}$ and $\mathbb{E}\left\{T_{1}\right\}$ are the mean sojourn times in the idle and busy states, respectively.
} 

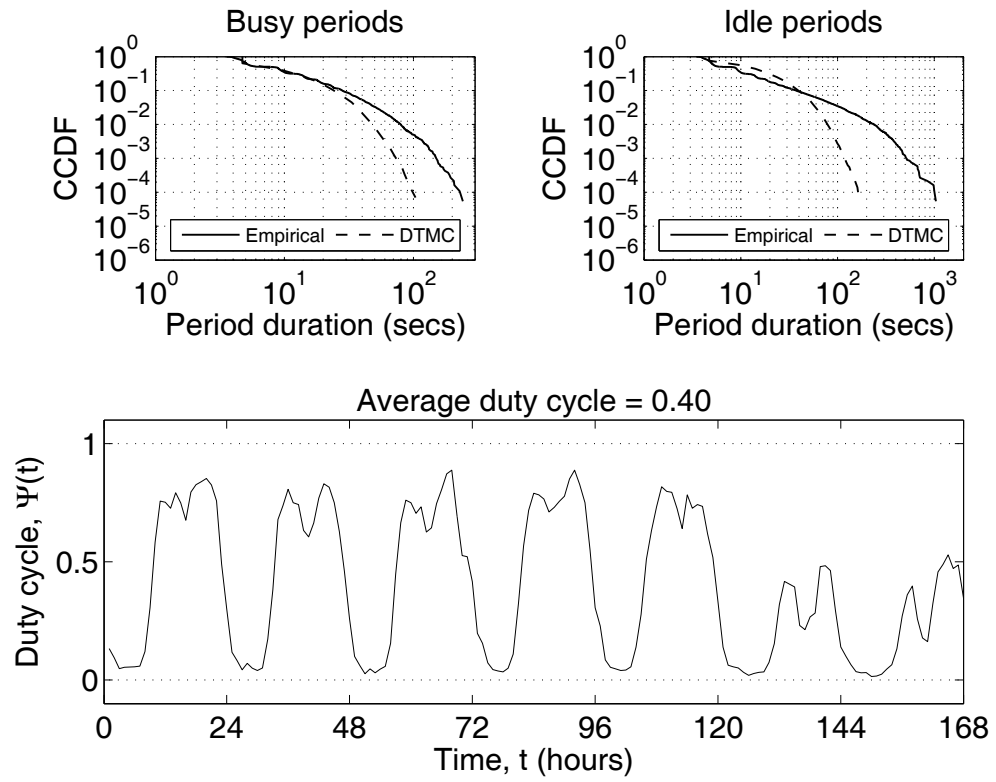

Figure 7.2: Empirical and DTMC-simulated distributions of busy and idle periods along with DC time evolution for DCS 1800 DL channel 70.
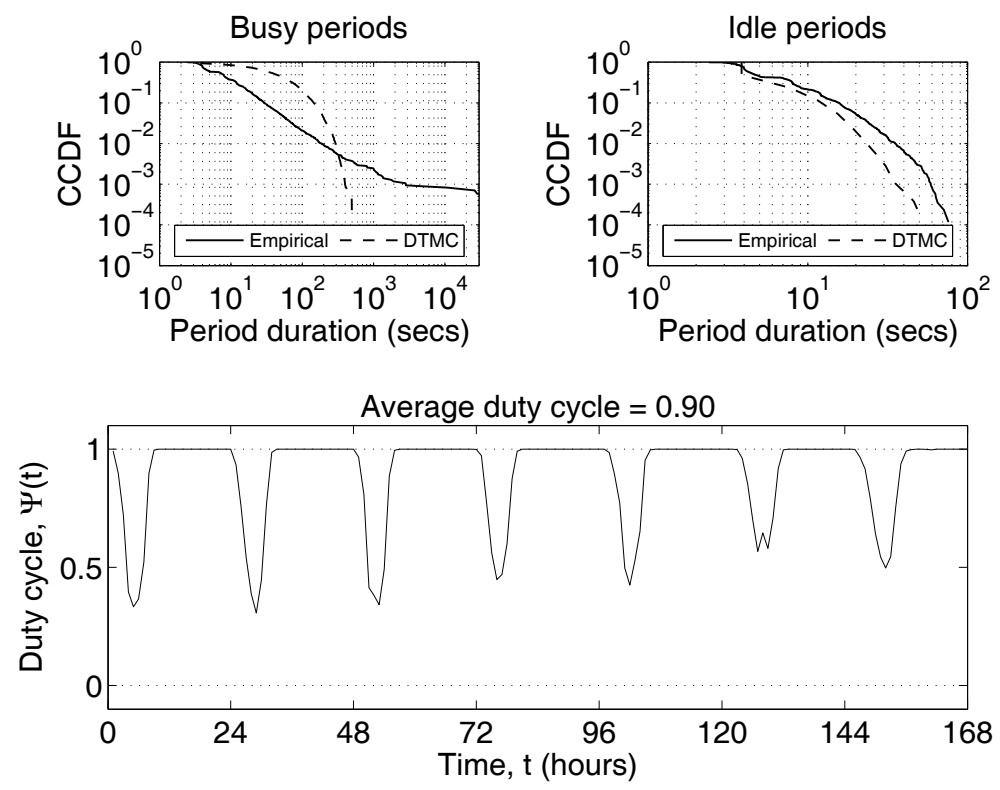

Figure 7.3: Empirical and DTMC-simulated distributions of busy and idle periods along with DC time evolution for E-GSM 900 DL channel 23. 

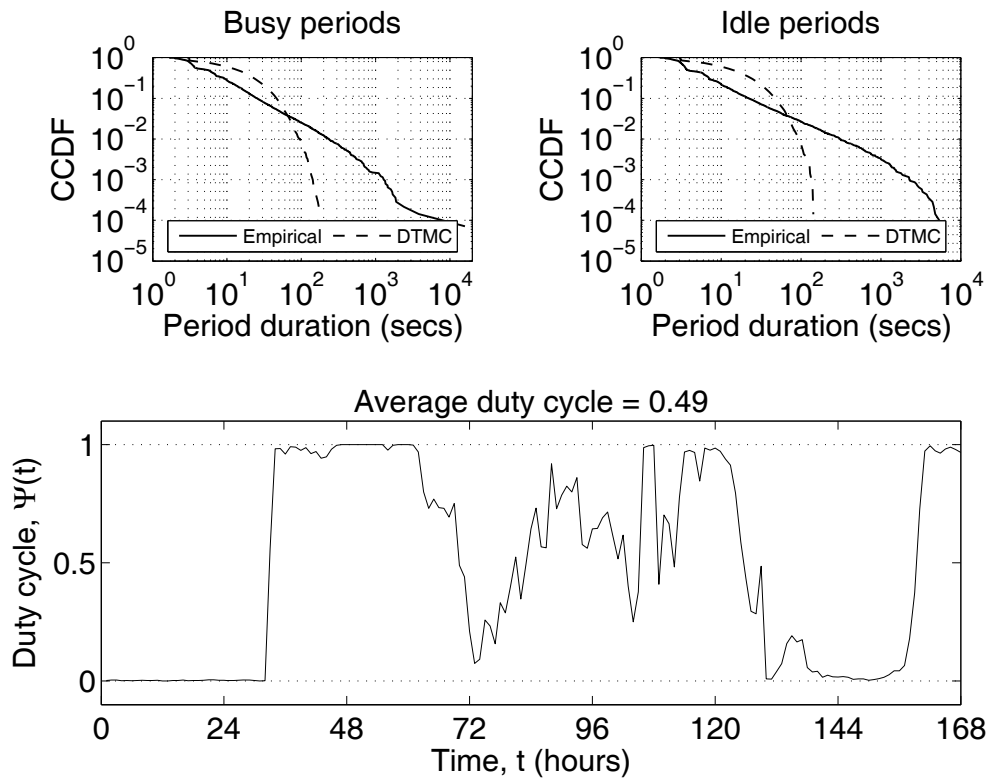

Figure 7.4: Empirical and DTMC-simulated distributions of busy and idle periods along with DC time evolution for TETRA DL channel 340.
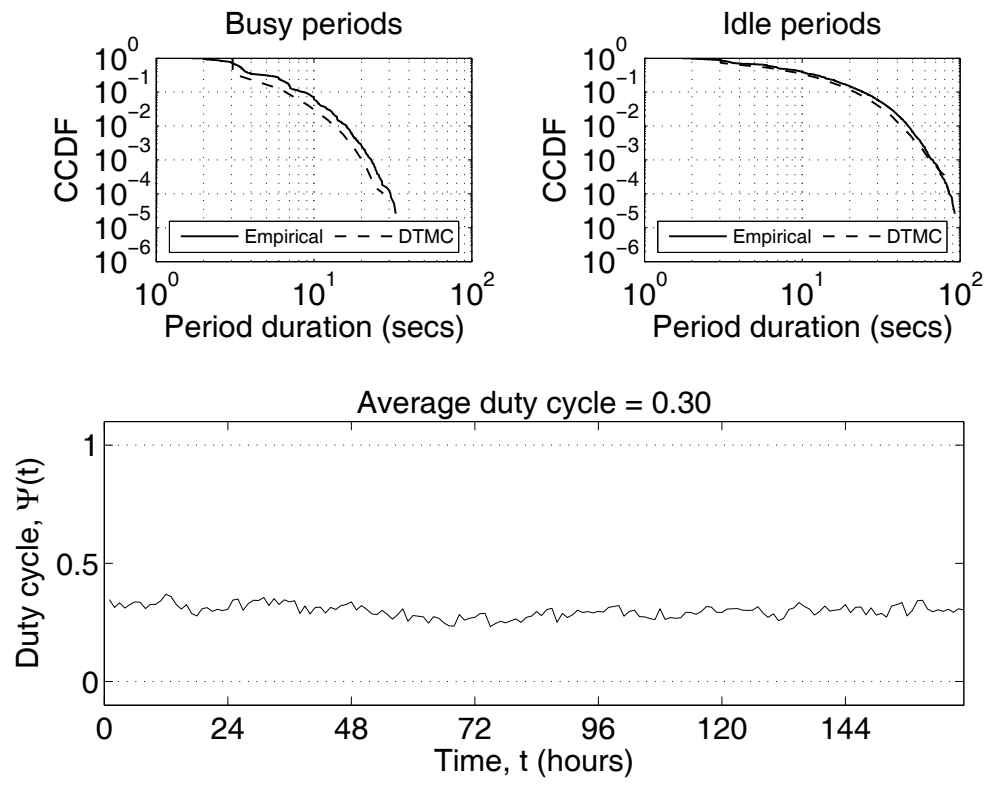

Figure 7.5: Empirical and DTMC-simulated distributions of busy and idle periods along with DC time evolution for TETRA UL channel 375. 
idle periods become notably shorter. Since the considered DTMC model is parametrized (i.e., the transition probabilities are configured) based on the long-term average load of the channel (i.e., the average DC of the whole measurement period), it is not able to capture the channel load variations and, as a result, the DTMC model cannot reproduce the resulting lengths of busy and idle periods. This can clearly be appreciated in Figures 7.2-7.4, where the channel load, characterized in terms of the DC, varies with the time and the distributions obtained by simulation diverge from the real ones. The exception corresponds to the case of channels with constant load patterns, where the average DC matches the instantaneous DC at all times, and simulation and empirical results agree as shown in Figure 7.5.

Since the probabilities of the transition matrix $\mathbf{P}$ depend on the DC, $\Psi$, and $\Psi$ changes with the time, this means that the binary occupancy pattern of real channels cannot be modeled, in general, by means of a stationary (time-homogeneous) $\mathrm{DTMC}^{5}$ as widely considered in previous DSA/CR research (see Section 7.2.2). As a result, a non-stationary (timeinhomogeneous) DTMC should be considered, with a time-dependent transition matrix:

$$
\mathbf{P}(t)=\left[\begin{array}{ll}
1-\Psi(t) & \Psi(t) \\
1-\Psi(t) & \Psi(t)
\end{array}\right]
$$

where $t=t_{k}=k T_{s}$ as previously defined.

In the stationary case of Equation 7.4, $\Psi$ represents a constant parameter. However, in the non-stationary case of Equation 7.6, $\Psi(t)$ represents a time-dependent function that needs to be characterized in order to characterize the DTMC channel model in the time domain. Appropriate and accurate DC models for $\Psi(t)$ are therefore required.

The results derived from the empirical data indicate the existence of two well-defined types of channel load variation patterns, namely patterns with an important and remarkably predominant deterministic component (e.g., Figures 7.2 and 7.3) and patterns where the carried load appears to vary following a random behavior (e.g., Figure 7.4). Based on this observation, adequate DC models of $\Psi(t)$ for both cases are developed in Sections 7.5 and 7.6 following deterministic and stochastic modeling approaches, respectively.

\subsection{Deterministic duty cycle models}

In many interesting and important cases, the load variation pattern of primary radio channels is characterized by a predominant deterministic component arising from social behavior and common habits, as it can clearly be appreciated in Figures 7.2 and 7.3. These examples correspond to cellular mobile communication systems, namely E-GSM 900 and DCS 1800. Nevertheless, it is interesting to note that similar patterns were also observed in some channels from other radio technologies such as TETRA. Moreover, deterministic patterns with different shapes were also identified in other cases. This section focuses on the analysis and modeling of the spectrum occupancy patterns commonly observed in cellular mobile communication systems, which are a clear example of predominantly deterministic behaviors.

\footnotetext{
${ }^{5}$ A stationary DTMC may be appropriate for a limited time period only if the modeled system shows approximately stationary behavior during this period. Otherwise, a non-stationary DTMC is necessary.
} 
The same modeling approach can be used and extended in order to represent other particular patterns that may be found in practice.

The load variation pattern of a cellular mobile communication system was studied in [239] by means of time series analysis and Auto-Regressive Integrated Moving Average (ARIMA) models. In this section, an alternative approach is developed based on the observation that the time evolution of $\Psi(t)$ over time periods of certain length exhibits a clear and predominant deterministic component. Moreover, the analysis of the empirical data corresponding to E-GSM 900 and DCS 1800 indicated that the variation pattern of $\Psi(t)$ is periodic with a period of one day and a slightly different shape between weekdays and weekends due to the lower traffic load normally associated with weekends. Two different shape types for $\Psi(t)$ were identified in the empirical data. The first shape type was normally observed in channels with low/medium loads (average DCs) as in the example of Figure 7.2, while the second one was more frequently observed in channels with medium/high loads as it is the case of Figure 7.3. Similar patterns were also observed in [240].

\subsubsection{Deterministic duty cycle model for low/medium loads}

The shape of $\Psi(t)$ in this case can be approximated by the summation of $M$ bell-shaped exponential terms centered at time instants $\tau_{m}$, with amplitudes $A_{m}$ and widths $\sigma_{m}$ :

$$
\Psi(t) \approx \Psi_{\min }+\sum_{m=0}^{M-1} A_{m} e^{-\left(\frac{t-\tau_{m}}{\sigma_{m}}\right)^{2}}, \quad 0 \leq t \leq T
$$

where $\Psi_{\min }=\min \{\Psi(t)\}$ and $T$ is the time interval over which $\Psi(t)$ is periodic (i.e., one day). The analysis of empirical data indicated that $\Psi(t)$ can accurately be described by means of $M=3$ terms with $\tau_{1}$ and $\tau_{2}$ corresponding to busy hours and $\tau_{0}=\tau_{2}-T$, as illustrated in Figure 7.6(a).

Based on empirical results, the approximations $A_{0}=A_{1}=A_{2}=A$ and $\sigma_{0}=\sigma_{1}=\sigma_{2}=\sigma$ are acceptable without incurring in excessive errors, which simplifies the model:

$$
\Psi(t) \approx \Psi_{\min }+A \sum_{m=0}^{M-1} e^{-\left(\frac{t-\tau_{m}}{\sigma}\right)^{2}}, \quad 0 \leq t \leq T
$$

Notice that $A$ determines the average value of $\Psi(t)$ in the time interval $[0, T]$, denoted as $\bar{\Psi}$, and it can therefore be expressed as a function of $\bar{\Psi}$ taking into account that:

$$
\bar{\Psi}=\frac{1}{T} \int_{0}^{T} \Psi(t) d t \approx \Psi_{\min }+\frac{A}{T} \sum_{m=0}^{M-1} \int_{0}^{T} e^{-\left(\frac{t-\tau_{m}}{\sigma}\right)^{2}} d t
$$

Solving Equation 7.9 for $A$ yields:

$$
A=\left(\bar{\Psi}-\Psi_{\min }\right) T\left[\sum_{m=0}^{M-1} \int_{0}^{T} e^{-\left(\frac{t-\tau_{m}}{\sigma}\right)^{2}} d t\right]^{-1}
$$




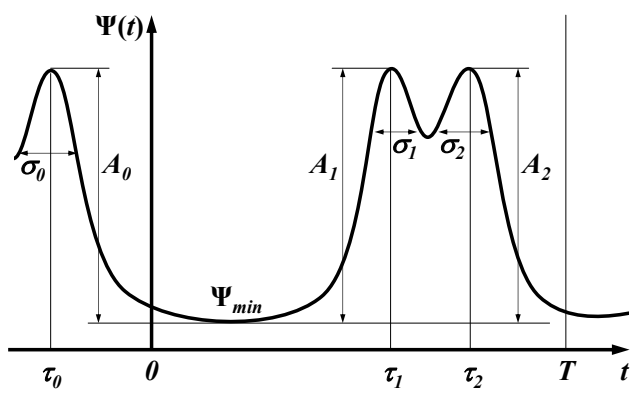

(a)

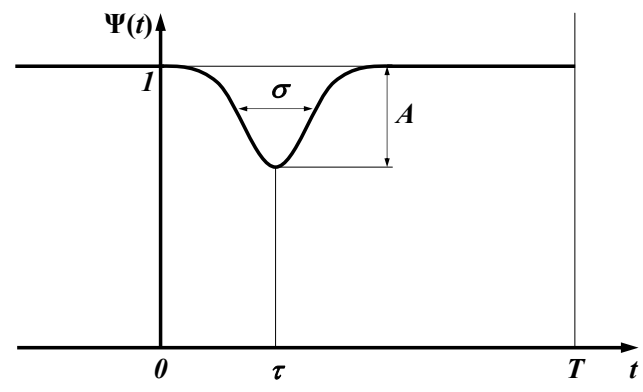

(b)

Figure 7.6: Parameters of the deterministic DC model: (a) low/medium load, (b) medium/high load.

Substituting Equation 7.10 in Equation 7.8 and solving the integral finally yields:

$$
\Psi(t) \approx \Psi_{\min }+\frac{2 T\left(\bar{\Psi}-\Psi_{\text {min }}\right)}{\sigma \sqrt{\pi}} \cdot \frac{f_{\mathrm{exp}}^{l / m}\left(t, \tau_{m}, \sigma\right)}{f_{\mathrm{erf}}^{l / m}\left(T, \tau_{m}, \sigma\right)}
$$

where $\bar{\Psi} \geq \Psi_{\min }$ and:

$$
\begin{aligned}
f_{\exp }^{l / m}\left(t, \tau_{m}, \sigma\right) & =\sum_{m=0}^{M-1} e^{-\left(\frac{t-\tau_{m}}{\sigma}\right)^{2}} \\
f_{\text {erf }}^{l / m}\left(T, \tau_{m}, \sigma\right) & =\sum_{m=0}^{M-1}\left[\operatorname{erf}\left(\frac{\tau_{m}}{\sigma}\right)+\operatorname{erf}\left(\frac{T-\tau_{m}}{\sigma}\right)\right]
\end{aligned}
$$

Equations 7.11-7.13 constitute the empirical DC model of $\Psi(t)$ for low/medium loads.

\subsubsection{Deterministic duty cycle model for medium/high loads}

The shape of $\Psi(t)$ in this case can be approximated by an expression based on a single bell-shaped exponential term centered at time instant $\tau$, with amplitude $A$ and width $\sigma$ :

$$
\Psi(t) \approx 1-A e^{-\left(\frac{t-\tau}{\sigma}\right)^{2}}, \quad 0 \leq t \leq T
$$

where $T$ is the time interval over which $\Psi(t)$ is periodic (i.e., one day). The model is illustrated in Figure 7.6(b), with $\tau$ corresponding to the time with the lowest activity levels.

As in the previous case, $A$ determines the average value of $\Psi(t)$ in the time interval $[0, T]$ and it can therefore be expressed as a function of $\bar{\Psi}$ taking into account that:

$$
\bar{\Psi}=\frac{1}{T} \int_{0}^{T} \Psi(t) d t \approx 1-\frac{A}{T} \int_{0}^{T} e^{-\left(\frac{t-\tau}{\sigma}\right)^{2}} d t
$$

Solving Equation 7.15 for $A$ yields:

$$
A=(1-\bar{\Psi}) T\left[\int_{0}^{T} e^{-\left(\frac{t-\tau}{\sigma}\right)^{2}} d t\right]^{-1}
$$




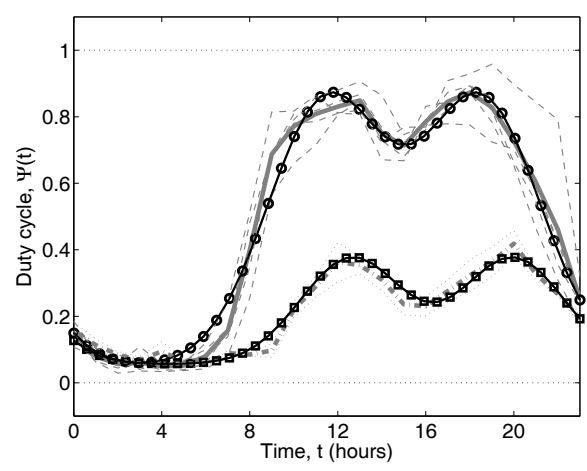

(a)

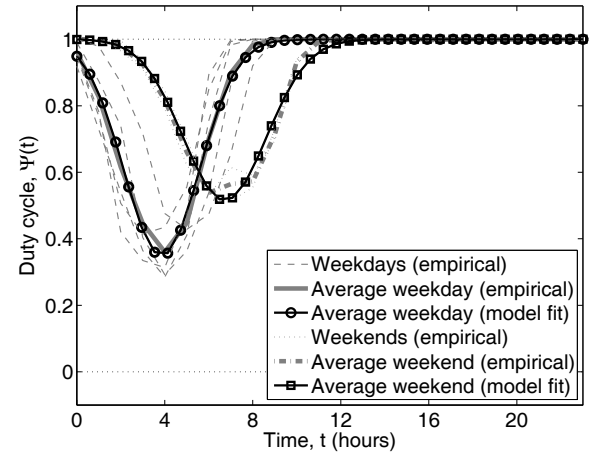

(b)

Figure 7.7: Validation of the deterministic DC model: (a) low/medium load, (b) medium/high load.

Substituting Equation 7.16 in Equation 7.14 and solving the integral finally yields:

$$
\Psi(t) \approx 1-\frac{2 T(1-\bar{\Psi})}{\sigma \sqrt{\pi}} \cdot \frac{f_{\exp }^{m / h}(t, \tau, \sigma)}{f_{\operatorname{erf}}^{m / h}(T, \tau, \sigma)}
$$

where:

$$
\begin{aligned}
f_{\exp }^{m / h}(t, \tau, \sigma) & =e^{-\left(\frac{t-\tau}{\sigma}\right)^{2}} \\
f_{\text {erf }}^{m / h}(T, \tau, \sigma) & =\operatorname{erf}\left(\frac{\tau}{\sigma}\right)+\operatorname{erf}\left(\frac{T-\tau}{\sigma}\right)
\end{aligned}
$$

Equations 7.17-7.19 constitute the empirical DC model of $\Psi(t)$ for medium/high loads.

\subsubsection{Deterministic duty cycle models validation and applicability}

The objective of this section is to evaluate the ability of the DC models of Sections 7.5.1 and 7.5.2 to describe the time evolution of $\Psi(t)$ with sufficient accuracy. To this end, the empirical values of $\Psi(t)$ were averaged among 24-hour periods of the same category (i.e., weekdays and weekends) in order to reduce the unavoidable random component of empirical data and extract the deterministic one. The mathematical expressions of Equations 7.11-7.13 and 7.17-7.19 were then fitted to the empirical data by means of curve fitting procedures. The results are shown in Figure 7.7, indicating that the proposed DC models are able to accurately reproduce the deterministic component of $\Psi(t)$ in real-world channels.

To facilitate the application of the models in analytical studies and simulations, realistic values of the models' parameters were estimated based on the empirical measurements and by means of curve fitting procedures. The fitted results are shown in Table 7.2. The values are specified in "(minimum; average; maximum)" format. To characterize the load differences between weekdays and weekends, the following parameter has been included:

$$
\kappa=\frac{\bar{\Psi}_{\text {weekends }}}{\bar{\Psi}_{\text {weekdays }}}
$$


Table 7.2: Fitted values of the deterministic DC models parameters.

\begin{tabular}{|c|c|c|c|}
\hline Load & Parameter & Weekdays & Weekends \\
\hline \multirow{4}{*}{$\begin{array}{l}\text { Low/ } \\
\text { /Med }\end{array}$} & $\Psi_{\text {min }}$ & $(0.00 ; 0.04 ; 0.31)$ & $(0.00 ; 0.05 ; 0.35)$ \\
\cline { 2 - 4 } & $\tau_{1}$ (hours) & $(10.74 ; 11.65 ; 12.28)$ & $(12.04 ; 13.03 ; 14.05)$ \\
\cline { 2 - 4 } & $\tau_{2}$ (hours) & $(17.80 ; 18.99 ; 20.09)$ & $(19.28 ; 20.42 ; 21.54)$ \\
\cline { 2 - 4 } & $\sigma$ (hours) & $(3.00 ; 3.88 ; 4.31)$ & $(2.49 ; 3.59 ; 5.83)$ \\
\cline { 2 - 4 } & $\kappa$ & \multicolumn{2}{|c|}{$(0.18 ; 0.51 ; 0.82)$} \\
\hline \multirow{3}{*}{$\begin{array}{l}\text { Med/ } \\
\text { /High }\end{array}$} & $\tau$ (hours) & $(2.94 ; 3.65 ; 4.08)$ & $(5.64 ; 6.44 ; 7.82)$ \\
\cline { 2 - 4 } & $\sigma$ (hours) & $(1.99 ; 2.81 ; 6.03)$ & $(2.29 ; 3.41 ; 8.00)$ \\
\cline { 2 - 4 } & $\kappa$ & \multicolumn{2}{|c|}{$(0.69 ; 0.97 ; 1.00)$} \\
\hline
\end{tabular}

Notice that $\bar{\Psi}$ has not been specified in Table 7.2 since this parameter is assumed to be a variable that can be configured in order to reproduce the shape of $\Psi(t)$ with any arbitrary mean $\bar{\Psi}$. Regarding this aspect, it is important to mention that, based on the captured empirical data, it was observed that the DC model for low/medium loads is valid from $\bar{\Psi}=0$ to $\bar{\Psi} \approx 0.60-0.70$. The maximum $\bar{\Psi}$ for which the model is valid depends on the particular set of selected parameters. For the average values of the fitted parameters in Table 7.2, the model is valid up to $\bar{\Psi}=0.58$ for weekdays and $\bar{\Psi}=0.55$ for weekends. On the other hand, the DC model for medium/high loads is valid from $\bar{\Psi} \approx 0.46-0.85$ to $\bar{\Psi}=1$. Again, the minimum $\bar{\Psi}$ for which the model is valid depends on the particular set of selected parameters. For the average values of Table 7.2, the model is valid down to $\bar{\Psi}=0.80$ for weekdays and $\bar{\Psi}=0.75$ for weekends. Invalid configurations can readily be identified since in these cases $\Psi(t)$ surpasses the interval $[0,1]$ within which it must mandatorily be confined.

It is worth noting that the values in Table 7.2 correspond to empirical measurements performed at a particular location and, as such, are unavoidably affected by the local habits. For example, the usual lunch time in Spain is around 2:00pm and it takes place within a lunch-break of a couple of hours. This schedule may usually be delayed about one hour on weekends. This behavior is indeed clearly appreciated in Figure 7.7(a). Habits may be different in other countries (e.g., see Figure 2 of [241] and Figure 4 of [242]), which may result in distinct shapes for $\Psi(t)$. The DC models of Equations 7.11-7.13 and 7.17-7.19 can still be valid by fitting the mathematical equations to different empirical data. For instance, an earlier lunch time would result in a lower value of $\tau_{1}$ while a shorter lunch-break (if any) would result in $\tau_{1}$ and $\tau_{2}$ being closer each other. The models however would still be valid.

Finally, the DC models of Sections 7.5.1 and 7.5.2 are envisaged to reproduce the deterministic pattern normally observed in cellular mobile communication systems such as E-GSM 900 and DCS 1800, which may also be present in other systems. Nevertheless, this does not imply that the model is always applicable to such type of systems. For instance, if the system is studied over a relatively short time period (e.g., a few hours), social behavior and external events, which may not be easily predicted, may have significant short-term impact on channel usage. This may cause the deterministic component of $\Psi(t)$ to loss importance with respect to the random one and, as a result, the occupancy of a single channel 
may experience high and unpredictable variations (e.g., see [243]). In such a case, deterministic DC models may be no longer valid and stochastic modeling approaches, as the one discussed in Section 7.6, may be a more appropriate alternative.

\subsection{Stochastic duty cycle models}

The traffic load experienced in a radio channel is normally the consequence of a significant number of random factors such as the number of incoming and outgoing users, the resource management policies employed in the system, and so forth. As a result, the channel usage level, represented by means of $\Psi(t)$, is itself a random variable (see the example of Figure 7.4). In such a case, a stochastic modeling perspective becomes a more convenient approach.

The following discussion assumes ergodicity on $\Psi(t)$, meaning that the expected values of its moments, such as its mean and variance, can be estimated as the time averages of the moments, which can be computed from a single sample (i.e, realization) of the process provided that it is sufficiently long. Notice that the sequence of $\Psi(t)$ values empirically derived from the measurements for a given channel represents a single realization of the underlying stochastic process, which is not enough to draw any conclusions on its ergodicity. Nevertheless, as it will be shown later on, the model developed under this assumption results valid and accurate in practice.

In order to determine the statistical properties of the underlying stochastic process based on the captured empirical data, $\Psi(t)$ was obtained for each channel as the time evolution of the DC computed over periods of various lengths, ranging from a few minutes up to one hour. Assuming ergodicity, the PDF of the underlying stochastic process can be estimated as the empirical PDF resulting from the empirical $\Psi(t)$ values for the considered channel. The empirical PDFs obtained with this procedure were compared to various bounded PDF models. Based on curve fitting procedures, it was found that the empirical PDFs of $\Psi(t)$ can accurately be fitted with the beta distribution [210] and the Kumaraswamy distribution [244], as it can be appreciated in the examples of Figures 7.8-7.13. The PDF for the former is given by:

$$
f_{x}^{B}(x ; \alpha, \beta)=\frac{1}{B(\alpha, \beta)} x^{\alpha-1}(1-x)^{\beta-1}, \quad x \in(0,1)
$$

where $\alpha>0$ and $\beta>0$ are shape parameters and $B(\alpha, \beta)$ is the beta function [201, 6.2.1]:

$$
B(\alpha, \beta)=\int_{0}^{1} z^{\alpha-1}(1-z)^{\beta-1} d z
$$

while the PDF for the latter is given by:

$$
f_{x}^{K}(x ; a, b)=a b x^{a-1}\left(1-x^{a}\right)^{b-1}, \quad x \in(0,1)
$$

where $a>0$ and $b>0$ are shape parameters.

The beta distribution is a well-known and widely used distribution that can be found in many popular software simulation packages, thus facilitating the implementation of the stochastic DC model in simulation tools. However, it might present some difficulties in 
analytical studies due to the complex expression of its PDF. The Kumaraswamy distribution is similar to the beta distribution, but much simpler to use in analytical studies due to the simpler closed form of its PDF [245]. Therefore, while the former may be more appropriate for simulations, the latter may be more convenient for analytical studies.

Both distributions can be configured to reproduce any arbitrary mean DC, $\bar{\Psi}$, by properly selecting the distribution's parameters. In particular, the mean value of the beta and Kumaraswamy distributions are related with their shape parameters as [210, 244]:

$$
\bar{\Psi}= \begin{cases}\frac{\alpha}{\alpha+\beta} & \text { for beta distribution } \\ b B\left(1+\frac{1}{a}, b\right) & \text { for Kumaraswamy distribution }\end{cases}
$$

with $B(\cdot, \cdot)$ given by Equation 7.22. Notice that Equation 7.24 can be satisfied for a given $\bar{\Psi}$ with different combinations of shape parameters $\alpha, \beta$ and $a, b$. The particular selection of the shape parameters determines the resulting shape of the distributions. In order to facilitate to researchers the application of the models and their configuration, an exhaustive analysis of the empirical data was performed in order to identify any potential relation between the shape parameters and the resulting channel occupancy pattern in the time domain. Based on such analysis, it was found that the PDF shapes observed in real channels can be classified into six elemental archetypes, each with a characteristic time-domain pattern. Each archetype is defined by its load level (L: low, M: medium, and H: high) as well as its load pattern (type I: very bursty, and type II: moderately bursty, but not constant). The range of shape parameters for each archetype and the corresponding time-domain pattern are (see Figures 7.8-7.13):

- Case L.I $(\alpha<1, \beta \geq 1)$ : The channel is used $(\Psi(t)>0)$ sporadically and remains unused $(\Psi(t) \approx 0)$ most of the time.

- Case L.II $(1<\alpha<\beta)$ : The channel is used $(\Psi(t)>0)$ regularly by traffic with low activity factors.

- Case M.I $(\alpha<1, \beta<1)$ : The channel is subject to an intermittent use, where high-load periods are followed by low-load periods in a similar proportion.

- Case M.II $(\alpha>1, \beta>1, \alpha \sim \beta)$ : The channel usage level oscillates weakly around the average level.

- Case H.I $(\alpha \geq 1, \beta<1)$ : The channel is used $(\Psi(t) \approx 1)$ most of the time, with some periods of lower occupancy levels $(\Psi(t)<1)$.

- Case H.II $(\alpha>\beta>1)$ : The channel is not fully used $(\Psi(t)<1)$ but subject to a constant, intesive usage.

The range of values indicated for the parameters of the beta distribution is also valid for the Kumaraswamy distribution by replacing $\alpha$ with $a$ and $\beta$ with $b$ in type-I cases. In type-II cases, the resulting Kumaraswamy distribution is more difficult to control since the same constraints on $a$ and $b$ may hold for various load levels. Based on the above archetypes and the corresponding range of shape parameters, along with Equation 7.24, the parameters of the models can be configured in order to reproduce not only arbitrary mean load levels but also various occupancy patterns observed in real channels. 

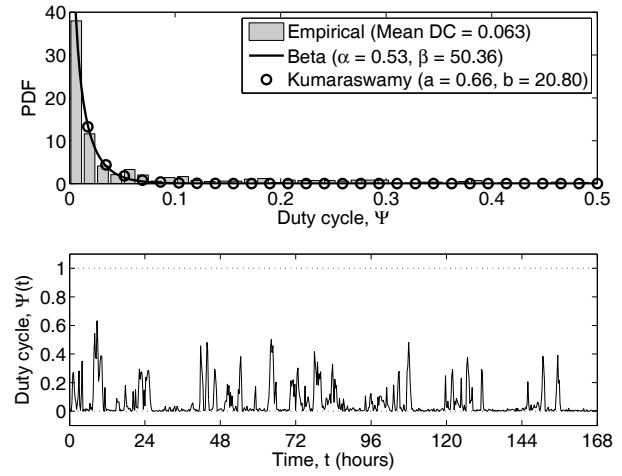

Figure 7.8: Stochastic DC models: case L.I.
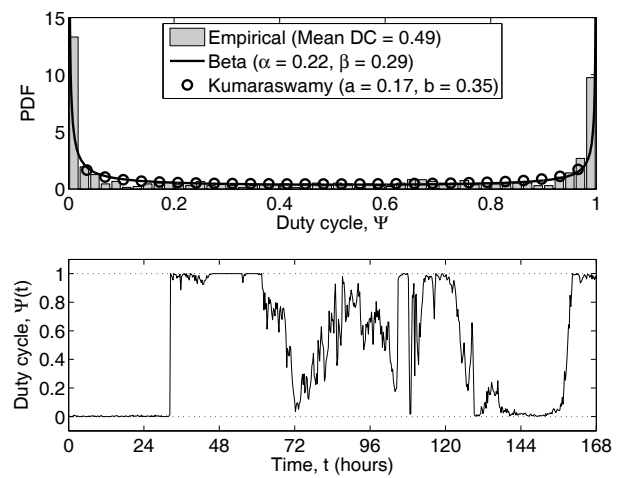

Figure 7.10: Stochastic DC models: case M.I.
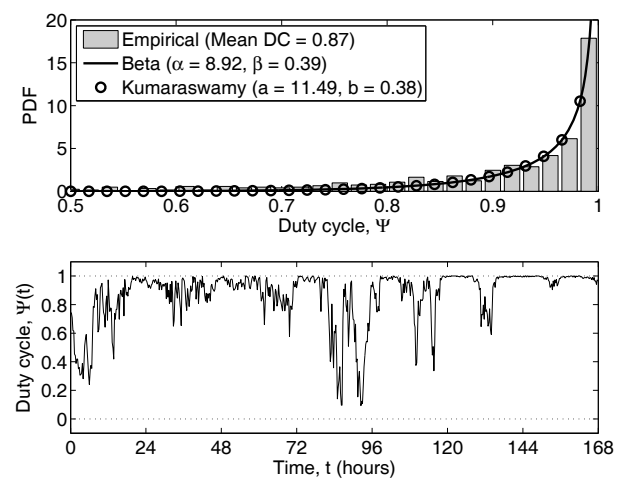

Figure 7.12: Stochastic DC models: case H.I. $\quad$ Figure 7.13: Stochastic DC models: case H.II.
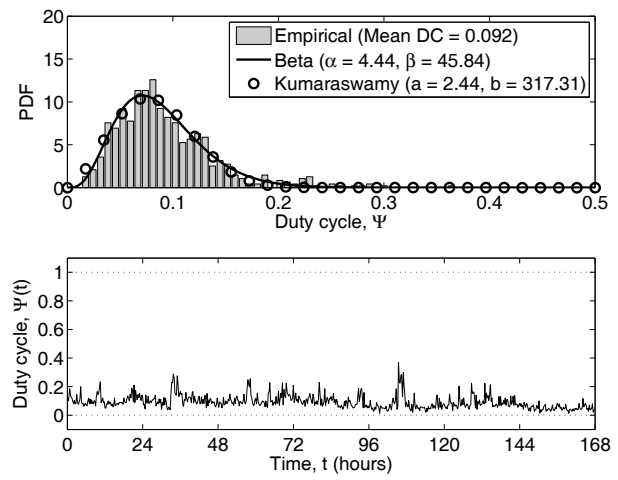

Figure 7.9: Stochastic DC models: case L.II.
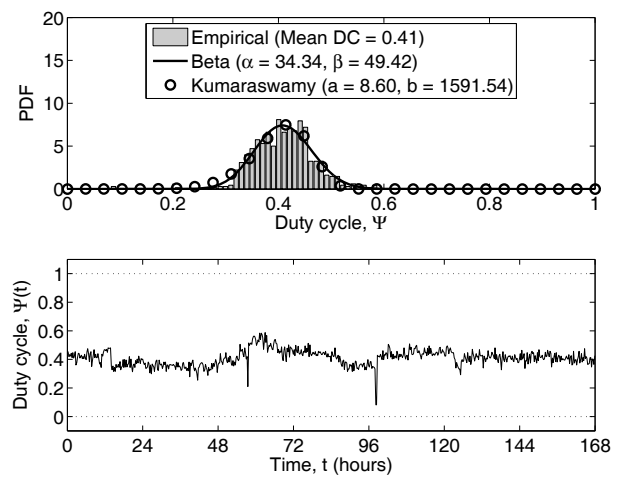

Figure 7.11: Stochastic DC models: case M.II.
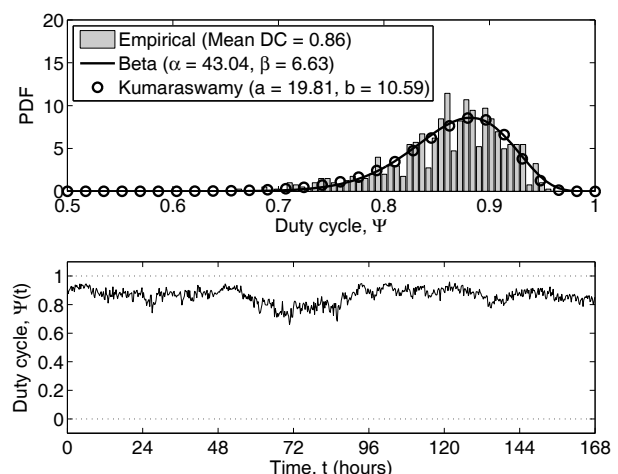


\subsection{Model validation}

The aim of this section is to assess the ability of the overall model, composed of the DTMC along with the deterministic and stochastic DC models, to reproduce with sufficient accuracy not only the mean DC but also the statistical properties of the busy and idle periods.

To this end, the DTMC model of Figure 7.1 was simulated for a sufficiently high number of iterations (transitions) and at different iterations during the simulation, the transition matrix $\mathbf{P}(t)$ (see Equation 7.6) was updated based on the DC models of Sections 7.5 and 7.6. In the deterministic case, $\Psi(t)$ is computed based on Equations 7.11 and 7.17, and taking into account the simulation time instant. In the stochastic case, $\Psi(t)$ is drawn from a beta distribution whose parameters are estimated based on the sample mean and sample variance of the empirical $\Psi(t)$. The stationary case widely considered in previous DSA/CR research (see Equation 7.4), where the DC is fixed and equal to the mean value, i.e. $\Psi(t)=\bar{\Psi} \forall t$, was also simulated. The statistical distributions obtained in both cases were compared to the real ones derived from empirical data. The obtained results are shown in Figures 7.14-7.16.

As it can be appreciated in Figures 7.14 and 7.15, the deterministic DC models are able to closely follow and reproduce the deterministic component of $\Psi(t)$ in the time domain and, as a result, the overall model is able to reproduce not only the mean DC of the channel, $\bar{\Psi}$, but also the statistical properties of busy and idle periods, which does not occur with the stationary case where the DTMC is simulated without appropriate DC models.

In the case of the stochastic DC model, the generated sequence of $\Psi(t)$ values does not follow the empirical $\Psi(t)$ values of the channel in the time domain, as it can be appreciated in Figure 7.16. However, it is important to note that the stochastic approach is not aimed at reproducing the time evolution of a particular realization of the stochastic process $\Psi(t)$, but the statistical properties thereof. The results shown in Figure 7.16 demonstrate that this modeling approach is also a valid and significantly accurate alternative to reproduce the statistical properties of busy and idle periods of real channels.

Taking into account the logarithmic axes representation of Figures 7.14-7.16, it can be appreciated that the distributions of busy and idle periods are reproduced with a significant level of precision. In order to objectively assess the accuracy, the Kolmogorov-Smirnov (KS) test [246] was performed over the obtained simulation results in order to compute the KS distances $D_{K S}\left(T_{0}\right)$ and $D_{K S}\left(T_{1}\right)$, for idle and busy periods respectively, between the empirical distribution functions and the corresponding counterparts obtained by means of simulation $^{6}$. Such study indicated for the proposed non-stationary modeling approach that $D_{K S}\left(T_{0}\right)=0.08$ and $D_{K S}\left(T_{1}\right)=0.09$ (Figure 7.14), $D_{K S}\left(T_{0}\right)=0.13$ and $D_{K S}\left(T_{1}\right)=0.06$ (Figure 7.15), while $D_{K S}\left(T_{0}\right)=0.06$ and $D_{K S}\left(T_{1}\right)=0.09$ (Figure 7.16), which highlights that the proposed modeling approach is able to achieve remarkably good accuracy levels.

Before concluding this section, it is worth noting that the practical implementation of the stochastic DC model in simulation tools may not lead to accurate results if some observations are not carefully taken into account. In particular, the DTMC has to be iterated a sufficient number of times, $N$, before updating $\mathbf{P}(t)$ according to the stochastic DC model. During

\footnotetext{
${ }^{6}$ The KS test and its associated distance metric $D_{K S} \in[0,1]$ are defined in Section 8.6. In general, the lower the $D_{K S}$, the better the fit. A value $D_{K S}=0$ would indicate an absolutely perfect fit.
} 

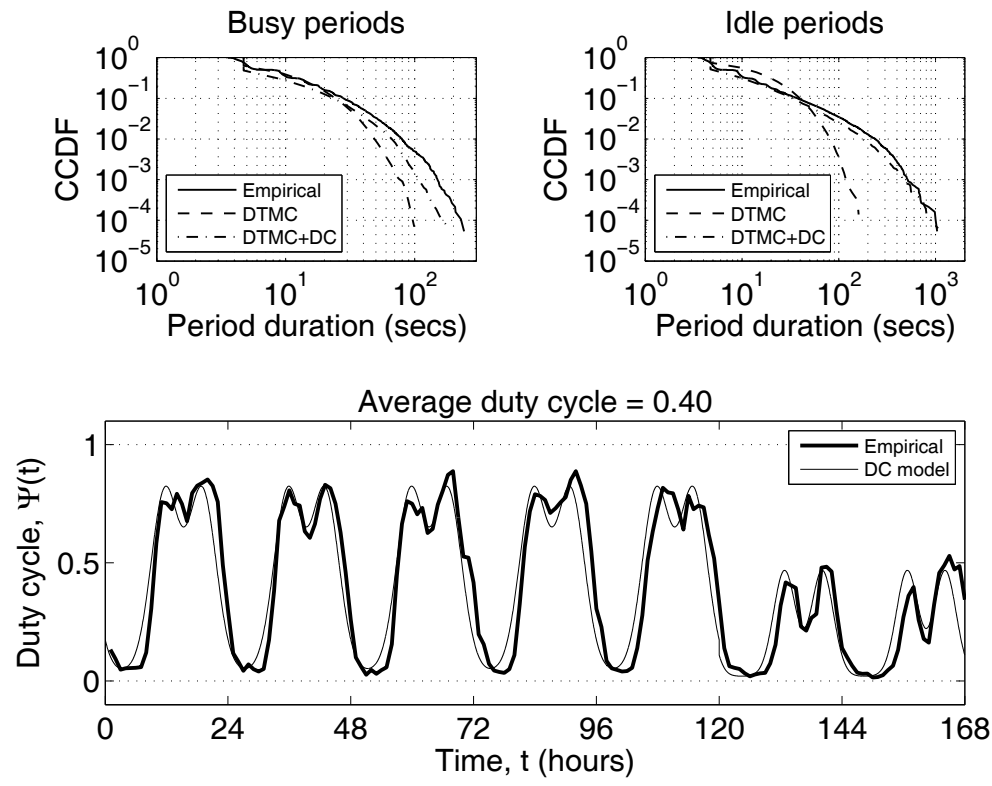

Figure 7.14: Empirical and DTMC-simulated distributions of busy and idle periods along with DC time evolution for DCS 1800 DL channel 70.
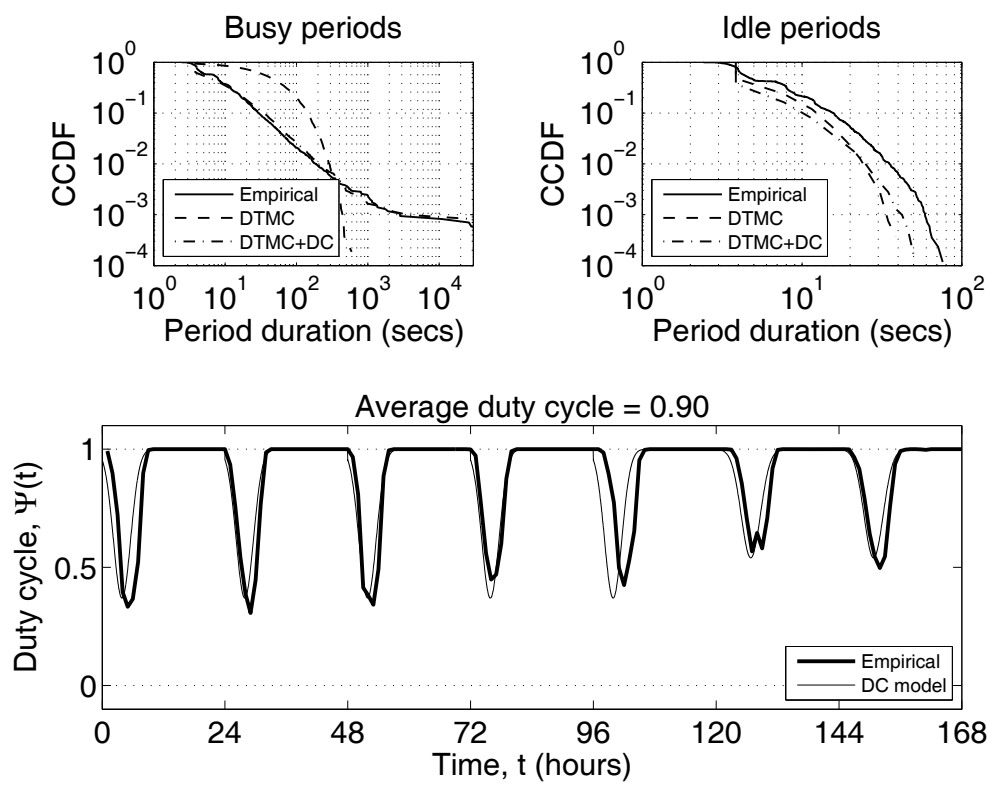

Figure 7.15: Empirical and DTMC-simulated distributions of busy and idle periods along with DC time evolution for E-GSM 900 DL channel 23. 

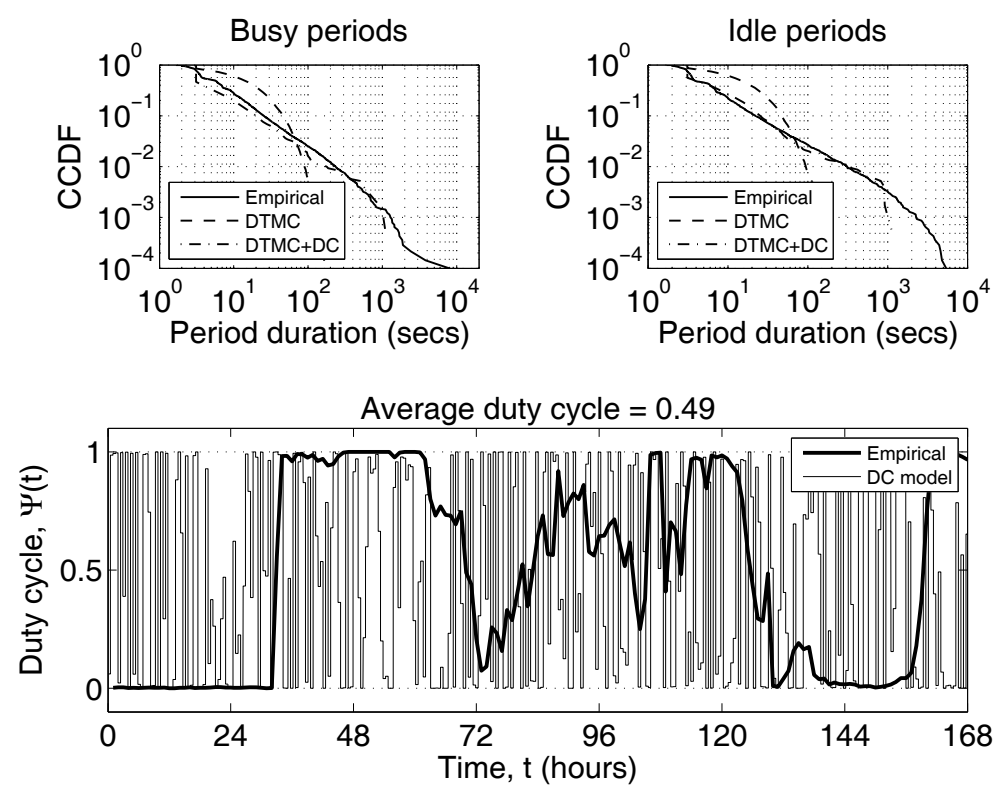

Figure 7.16: Empirical and DTMC-simulated distributions of busy and idle periods along with DC time evolution for TETRA DL channel 340.

such amount of iterations, the transition probabilities of the DTMC must remain unaltered. After such $N$ iterations, a new value of $\Psi(t)$ can be generated from a beta or Kumaraswamy distribution, and used to update the transition matrix $\mathbf{P}(t)$ for the next $N$ iterations. If the transition matrix is updated excessively fast (e.g., every iteration) the overall model may not be able to accurately reproduce the lengths of busy and idle periods.

In summary, the obtained results demonstrate that the non-stationary DTMC model along with the proposed deterministic and stochastic DC models is able to accurately reproduce not only the mean occupancy level but also the statistical properties of busy and idle periods observed in real channels.

\subsection{Case study}

The aim of this section is to demonstrate and illustrate the importance of employing realistic and accurate spectrum occupancy models in the design and evaluation of DSA/CR techniques. To this end, this section considers a simple medium access scheme where a DSA/CR terminal senses a primary radio channel periodically and accesses the channel whenever it is sensed as idle. Although the case study of this section may be considered to be trivial, it will suffice to illustrate the impact of the realism and accuracy of spectrum occupancy models on the design and performance evaluation of more sophisticated solutions such as adaptive spectrum sensing techniques, MAC protocols, MAC-layer sensing schemes, dynamic channel selection algorithms and opportunistic scheduling policies. 


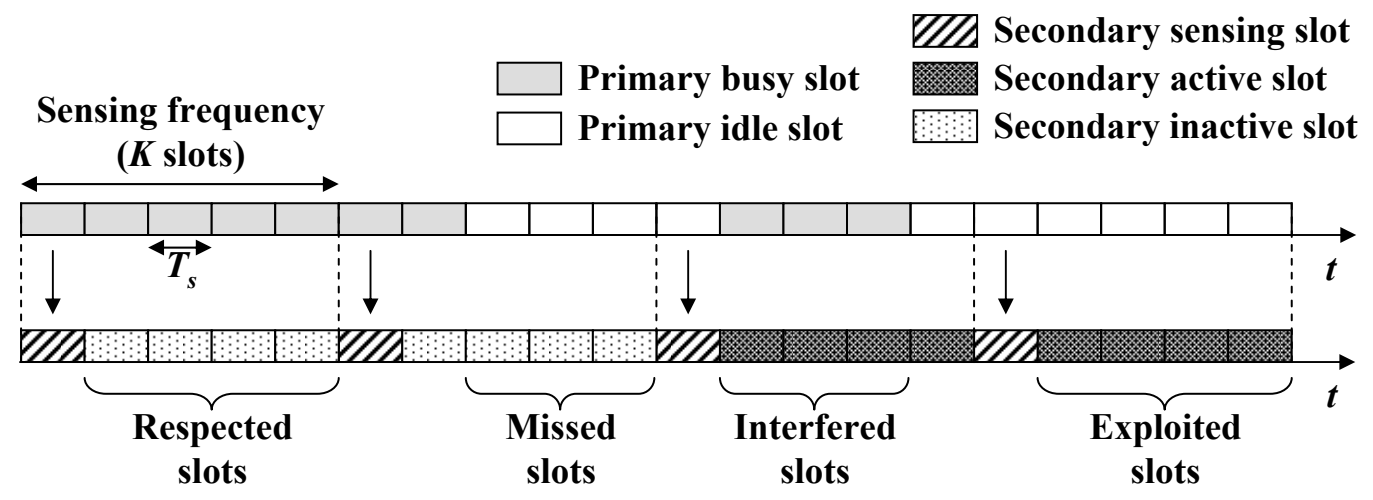

Figure 7.17: Case study description.

The considered case study assumes that the DSA/CR terminal senses and accesses the channel on a frame basis as illustrated in Figure 7.17. Each frame is composed of $K$ slots with duration $T_{s}$. The DSA/CR terminal senses the channel in the first slot of the frame and decides to transmit or not in the following $K-1$ slots based on the sensing result (perfect sensing is assumed). If the DSA/CR terminal senses the channel as busy and decides not to transmit, the following slots may be classified as respected slots if the primary user transmits in such slots, or missed slots otherwise. On the other hand, a secondary transmission may result in interfered slots if the primary user is also active, or exploited slots otherwise, as illustrated in Figure 7.17.

The objective is to evaluate the performance of the considered medium access scheme when the sequence of channel occupancy states corresponds to: a) empirical measurements of real channels, b) occupancy sequences generated with the non-stationary DTMC model along with DC models, and c) occupancy sequences generated with the stationary DTMC model alone (see Section 7.4). The comparison of the results obtained in these cases will provide a quantitative illustration of the consequences of considering (un)realistic and (in)accurate spectrum occupancy models in DSA/CR research.

Table 7.3 shows the channel access statistics obtained for the above mentioned sequences of channel occupancy states in the case of a DECT channel. As it can be appreciated, the proposed non-stationary approach (labeled as "DTMC+DC") is able to provide accurate estimations of the true channel usage statistics. The results provided by the stationary approach (labeled as "DTMC"), although less accurate, can be considered acceptable as well. This can be explained by the fact that the considered metrics represent the average number of slots for each type in the long term. As such, they depend on the average number of busy/idle slots or, in other words, the average DC of the channel. Since both DTMC models (stationary and non-stationary) are able to accurately reproduce the channel's average DC, the obtained average values for the considered metrics agree in both cases with the empirical, real ones. However, it is worth noting that in M.I-type channels (referring to the nomenclature used in Section 7.6), the stationary DTMC model was observed to fail in providing acceptable results while the proposed non-stationary DTMC model was still able to do so, as illustrated in Table 7.4. Therefore, although the stationary DTMC model is able to reproduce the true 
Table 7.3: Channel access statistics for DECT channel 9 (stochastic DC model and $K=5$ )

\begin{tabular}{|l|r|r|r|}
\hline Slot type & Empirical & DTMC+DC & DTMC \\
\hline Respected & $2.67 \%$ & $2.75 \%$ & $1.16 \%$ \\
\hline Missed & $8.34 \%$ & $8.31 \%$ & $9.95 \%$ \\
\hline Interfered & $8.25 \%$ & $8.30 \%$ & $9.73 \%$ \\
\hline Exploited & $80.74 \%$ & $80.64 \%$ & $79.16 \%$ \\
\hline
\end{tabular}

Table 7.4: Channel access statistics for TETRA DL channel 340 (stochastic DC model and $K=5$ )

\begin{tabular}{|l|r|r|r|}
\hline Slot type & Empirical & DTMC+DC & DTMC \\
\hline Respected & $41.37 \%$ & $41.15 \%$ & $23.84 \%$ \\
\hline Missed & $7.39 \%$ & $7.23 \%$ & $25.25 \%$ \\
\hline Interfered & $7.20 \%$ & $7.81 \%$ & $24.72 \%$ \\
\hline Exploited & $44.04 \%$ & $43.81 \%$ & $26.19 \%$ \\
\hline
\end{tabular}

mean DC value of the channel, this does not guarantee the reliability of average performance metrics obtained when applying such model. On the other hand, the proposed non-stationary DTMC approach provides accurate estimates of average performance metrics.

The average value of performance metrics, although useful, may not provide a full impression on the real performance of a DSA/CR technique under study. For example, let's assume that a primary user tolerates a short communication disruption provided that its duration is below a given threshold $\delta_{d}$. In such a case, the probability that the duration of interference periods exceeds $\delta_{d}$ would be a more useful performance metric than the average number of interfered slots. As shown in Figure 7.18, the distribution of interference periods resulting from the medium access technique under study is accurately reproduced by the proposed non-stationary DTMC modeling approach, which is not the case of the stationary DTMC model. As a result, the real interference to a primary user is accurately estimated with the former, while it is significantly underestimated with the latter. Concretely, Figure 7.18 indicates that the predicted interference probability estimated with the stationary DTMC model may differ up to 0.09 with respect to the real value obtained from the real channel occupancy pattern (i.e., up to $9 \%$ error), while the prediction error provided by the proposed non-stationary DTMC model is around $1 \%$ in the worst case. Therefore, even when the stationary DTMC approach is able to provide accurate estimates of average metrics, it fails in providing acceptable results for other more sophisticated performance metrics. These results demonstrate and highlight the importance of employing realistic and accurate spectrum occupancy models, as the one proposed in this chapter, for the analysis, design and performance evaluation of DSA/CR techniques. 

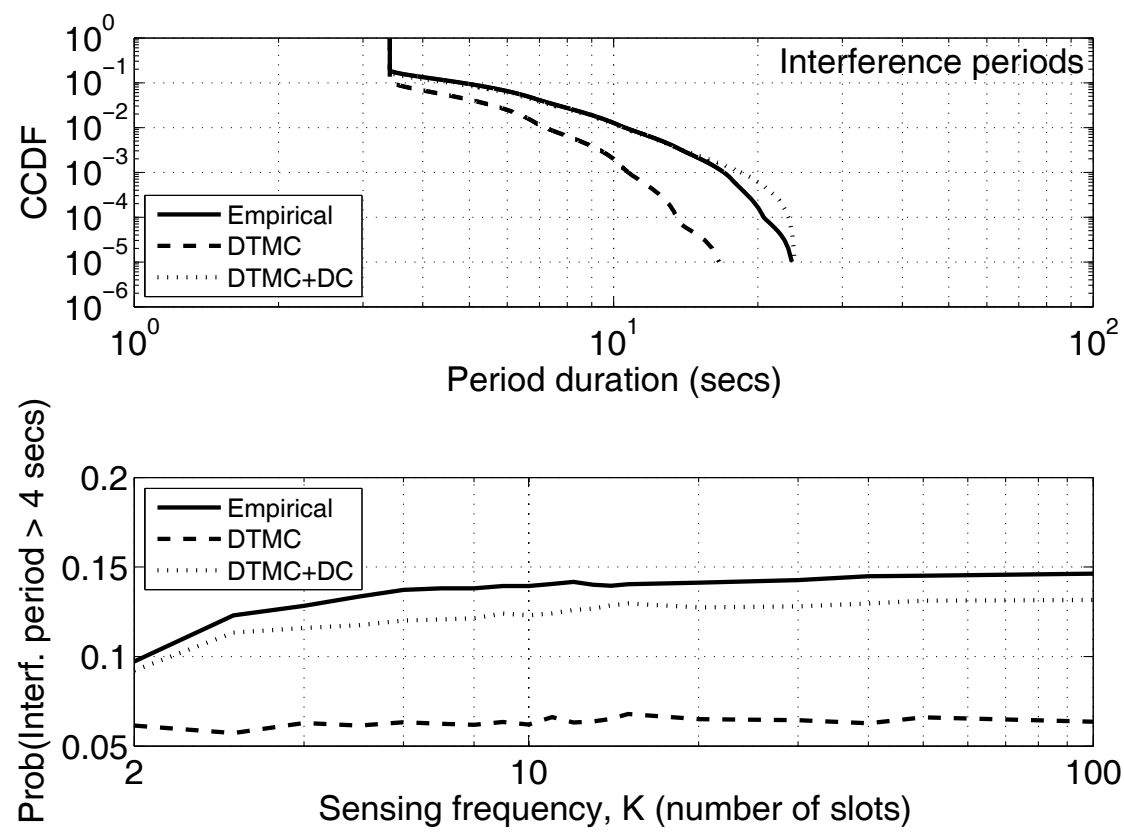

Figure 7.18: Interference results for DECT channel 9. Upper graph: distribution of interference periods $(K=5)$. Lower graph: Probability of interference as a function of the sensing frequency $\left(\delta_{d}=4\right.$ secs $)$.

\subsection{Summary}

Due to the opportunistic nature of the DSA/CR principle, the behavior and performance of a secondary network depends on the spectrum occupancy patterns of the primary system. A realistic and accurate modeling of such patterns becomes therefore essential. This chapter has demonstrated that the stationary DTMC model widely used in the DSA/CR literature in order to the describe the binary occupancy pattern of primary channels in the time domain is not able to reproduce relevant properties of spectrum usage. As a result, a non-stationary DTMC model with deterministic and stochastic DC models has been developed. The proposed approach has been validated with extensive empirical measurement results, demonstrating that it is able to accurately reproduce not only the mean occupancy level but also the statistical properties of busy and idle periods observed in real-world channels. The importance of realistically and accurately modeling spectrum usage in the design and evaluation of DSA/CR techniques has been illustrated with a practical case study. 



\section{Chapter}

8

\section{CONTINUOUS TIME MODELS}

\subsection{Introduction}

The modeling of spectrum occupancy in discrete-time by means of a Discrete-Time Markov Chain (DTMC) channel model has extensively been analyzed an discussed in Chapter 7. In contrast, this chapter focuses on modeling spectrum usage in the time domain from a continuous-time perspective. The continuous-time counterpart of the DTMC model is the well-known Continuous-Time Markov Chain (CTMC) model, where the state holding times are modeled as exponentially distributed random variables. As mentioned in Chapter 7, the CTMC model has widely been used in the literature to study various aspects of DSA/CR networks [213-221]. However, some works based on empirical measurements [222-226] have demonstrated that the lengths of busy and idle periods in real systems are not exponentially distributed, meaning that the CTMC model is unrealistic. Based on such conclusions, a more appropriate model is therefore the Continuous-Time Semi-Markov Chain (CTSMC) model, where the state holding times can follow any arbitrary distribution. Previous modeling studies have tried to find the probability distributions that can be employed for the sojourn times of the CTSMC model to accurately describe the statistical properties of spectrum usage in real systems [222-226]. Although such works are based on appropriate methodologies and procedures, they unfortunately lack of a sufficiently comprehensive treatment of the problem. The objective of the study reported in this chapter is to cover such deficiencies by performing a comprehensive, systematical and rigorous study on the probability distributions that can be employed to accurately describe the statistical properties of spectrum usage. The main drawbacks and limitations of previous studies reported in the literature are identified and the methodological approaches and procedures on which they rely are substantially extended and complemented in this study. Furthermore, this chapter also analyzes the timecorrelation properties of spectrum use in real systems and develops appropriate means to capture and reproduce such correlations, an important aspect in the field of spectrum usage modeling that has not received an adequate treatment in the existing literature. 
The remainder of this chapter is organized as follows. First, Section 8.2 reviews some previous studies on spectrum modeling and provides a detailed description of their main characteristics. Section 8.3 then summarizes the main novelties of the study presented in this chapter, highlighting the differences with respect to other similar works. The methodology employed to process the empirical data captured with the low and high time-resolution measurement platforms is described in Section 8.4. The considered probability distribution models and the goodness-of-fit metrics employed to assess their suitability in fitting the empirical data sets are presented in Sections 8.5 and 8.6, respectively. The most suitable distributions to describe the lengths of busy and idle periods in real systems are analyzed, based on low and high time-resolution measurements, in Sections 8.7 and 8.8 respectively. The study on continuous-time models is complemented in Section 8.9 with an analysis of the time-correlation properties of spectrum occupancy and the development of appropriate mathematical models as well as a simulation algorithm to reproduce such features. Based on the obtained results, Section 8.10 proposes two novel modeling approaches to simultaneously describe spectrum occupancy at both short and long time scales, including time-correlation structures. Finally, Section 8.11 summarizes and concludes the chapter.

\subsection{Previous work}

Several previous works have investigated the problem of finding the probability distributions that best fit the durations of busy and idle periods observed in various real systems and radio technologies. In [222-224], a model is proposed based on a CTSMC that statistically describes the busy and idle periods of an IEEE 802.11b Wireless Local Area Network (WLAN). The model is based on data obtained from real measurements with a vector signal analyzer in the $2.4 \mathrm{GHz}$ ISM band over an experimental controlled setup and under high SNR conditions. The experimental setup considers a traffic source of UDP packets with a constant packet length of 512 bytes and with Poisson distributed inter-departure times at different rates [222], as well as more realistic traffic sources such as FTP [223], VoIP [223] and HTTP [224] streams generated by real applications. The busy and idle duration statistics are extracted considering two different sensing strategies, namely an energy-based detection approach where the detection threshold and sensing period are determined so as to obtain the desired detection performance in terms of error probability, and a feature-based detection approach where the gathered base-band data are processed in order to detect packets more reliably and to extract the length information that is provided in the packet header. The high sampling rate provided by vector signal analyzers enable time accuracies down to the symbol level and thereby the identification of the IEEE 802.11b MAC protocol behavior in the captured traces. The sequence of states corresponding to data transmission and acknowledgment is found in [222-224] to be essentially deterministic, which results in a deterministic sojourn time in the busy state. The sojourn time in the idle state is fitted to a generalized Pareto distribution [222], a mixture of uniform distribution (associated to the effects of the contention window) and generalized Pareto distribution (associated to truly unused channels) [223, 224], and a hyper-Erlang distribution [223, 224], which represents a good trade-off between accuracy and tractability of the model. 
While [222-224] considers an interference-controlled environment with a single packet flow artificially generated, [225] analyzes the distribution of busy and idle periods in a real environment with heterogeneous wireless devices operating in the $2.4 \mathrm{GHz}$ ISM band. The study reported in [225] concludes that complex models such as the hyper-exponential distribution provide excellent fits, but simpler models such as the generalized Pareto distribution still lead to good matches with empirical data, thus providing a reasonable trade-off between complexity and accuracy.

The work reported in [226] performs a similar study over a wider set of allocated spectrum bands and based on a spectrum analyzer setup. Spectrum analyzers are characterized by significantly lower sampling rates, which may result in under-sampling of the measured signals, but enable high dynamic ranges, high sensitivity levels and wide band measurements. The work performed in [226] concludes that state holding times can appropriately be described by means of geometric distributions. For channels with low (high) loads, the duration of idle (busy) periods increases notably, leading to heavy-tailed distributions for which a log-normal model is found to provide more accurate fits.

\subsection{Novelties of this study}

Previous modeling works reported in the literature can arguably be considered to suffer from a number of drawbacks and limitations. This section identifies such deficiencies and explains how they are overcome in the study presented in this chapter.

- High time resolution measurement equipments have been employed in [222-224] (vector signal analyzer) and [225] (wireless transceiver in a laptop) in order to obtain spectrum occupancy data in the $2.4 \mathrm{GHz}$ ISM band. Although high time resolutions enable more accurate models, the studies performed in [222-225] focus on the 2.4 $\mathrm{GHz}$ ISM band exclusively. A wider set of spectrum bands have been embraced by the study performed in [226], but making use of a low time resolution measurement equipment (spectrum analyzer). The study reported in this chapter makes use of both low and high time resolution devices to measure the spectral activity in a wide range of allocated spectrum bands and radio technologies, and discusses the consequences of different levels of time resolution on the resulting models.

- As opposed to some previous works [222-224] where a single traffic flow is generated and measured, this chapter exhaustively measures the occupancy patterns of a significantly high number of channels for each analyzed spectrum band, which ensures that the resulting models are sufficiently representative of the true spectrum occupancy of channels in real wireless systems.

- Previous works have tried to fit a low number of probability distributions to empirical data, in some cases considering complex models such as phase-type distributions (e.g., hyper-Erlang or hyper-exponential) that are obtained as a linear (weighted) combination of a number of simpler distributions of the same class. Such complex models have shown to provide good accuracy levels and can be employed to provide accurate 
and realistic results in simulation tools. However, their applicability to analytical studies appears to be difficult due to their complex mathematical expressions and the high number of model parameters involved. By contrast, the study in this chapter analyzes the suitability of a wider set of simpler distributions, some of which have not been considered before, showing that adequate fits can be achieved as well.

- The parameters of various distribution models have usually been derived from the empirical data based on Maximum Likelihood Estimation (MLE) techniques [222-225]. Additionally, this chapter employs the Method Of Moments (MOM) and evaluates the resulting fits under both inference methods.

- The fits for various distribution models have solely been evaluated based on distancetype metrics (e.g., the KS distance metric). This type of metrics provide a single indication on the goodness of the fit for a certain probability model over the whole range of values of the parameter under study (i.e., the duration of busy and idle periods in this case). Although this type of metrics is also considered in the study presented in this chapter, the fit of the considered models in particular regions of the parameter under study (i.e., short and long periods) is evaluated as well.

In summary, this chapter provides a adequate treatment of the problem by performing a comprehensive, systematical and rigorous study on the probability distributions that can be employed to accurately describe the statistical properties of spectrum usage in real radio communication systems.

\subsection{Measurement setup and methodology}

Two measurement platforms, providing low and high time resolutions, are employed in this study. The first measurement setup relies on the spectrum analyzer configuration discussed in Chapter 2 and Appendix A, while the second one is based on the USRP/GNU Radio platform presented in Chapter 4. Both measurement setups were employed to record the spectral activity in the same spectrum bands studied in Chapter 7 (see Table 7.1) and considering the same measurement locations, namely a building rooftop (see location 1 in Figure 3.1) and a room inside the same building (see location 2 in Figure 3.1). The spectrum analyzer setup was employed to measure each entire band for a continuous time period of 7 days (beginning on Monday midnight and ending on Sunday midnight) with a time resolution in the order of few seconds as shown in Table 7.1. The USRP/GNU Radio platform was employed to measure selected channels within each band ${ }^{1}$ for a total time period of 20 minutes with an effective sampling rate of $10^{6}$ signal samples per second.

The spectrum data were used to extract the binary channel occupancy patterns by classifying the captured samples as either busy or idle channels. As in Chapter 7, an ED principle was employed to this end making use of the same threshold-setting strategy. Each power

\footnotetext{
${ }^{1}$ While a spectrum analyzer is capable to handle several tens to hundreds of megahertz, the bandwidth of the USRP version 1 employed in this study is $8 \mathrm{MHz}$, which allows to observe very few channels at the same time.
} 
sample provided by the spectrum analyzer was processed independently, which leads to effective time resolutions equal to the average sweep times shown in Table 7.1. The samples provided by the USRP/GNU Radio platform were processed in blocks of 128 samples (i.e., a sensing period of $N=128$ samples was applied), which results in an effective time resolution of one channel state observation every $128 \mu$ s. Notice that this time resolution enables a highly accurate estimation of the true channel activity pattern for most radio technologies ${ }^{2}$. Spectrum analyzer data were processed based on the CED scheme described in Section 6.2, while the IED scheme developed in Section 6.4 (with $L=5$ ) was used to extract binary occupancy information from the USRP/GNU Radio data. Note that the IED algorithm makes use of past channel observations to determine the current channel state. As discussed in Section 6.6, this does not appear to be sensible when the time period between consecutive observations is in the order of several seconds as it is the case of the spectrum analyzer, which prevents the use of the IED scheme in this case. On the other hand, the high time resolution of the USRP/GNU Radio platform enables the application of the IED method, which results in an improved detection performance. As illustrated in Figure 8.1, some signal missed detections may result from the selected short sensing period when the CED method is employed, which would lead to wrong conclusions on channel usage statistics (concretely, a high number of short busy/idle periods would incorrectly be extracted from a single true busy period). On the other hand, the IED algorithm is able to avoid such signal missed detections, which results in an improved detection performance and hence a more reliable and accurate estimation of the true channel occupancy periods.

Before concluding this section, it is worth discussing the impact and consequences of various time resolutions on the resulting spectrum occupancy models. On one hand, USRP measurements can be used to extract the true channel occupancy patterns with high time accuracies. On the other hand, the low effective sampling rates of spectrum analyzers result, in general, in a significant under-sampling of the measured signals, meaning that the true channel state may change between two consecutive channel observations as illustrated in Figure 8.2. The occupancy pattern observed in such a case, although inaccurate, is interesting since it can be thought of as the perception of a DSA/CR user that periodically senses the channel and observes its state at discrete time instants. Therefore, while USRP measurements are useful to accurately describe the true channel occupancy pattern at short time scales, spectrum analyzer measurements are useful to model spectrum occupancy from the point of view of the DSA/CR user perception (i.e., the spectrum occupancy pattern that would be perceived by a DSA/CR node) at longer time scales.

\subsection{Considered probability distributions}

Based on the binary occupancy patterns extracted from empirical data, the length of busy and idle periods is computed for each channel and the corresponding CDF is derived. The

\footnotetext{
${ }^{2}$ For instance, the time-slot duration is $14.167 \mathrm{~ms}$ in TETRA, $577 \mu$ s in GSM/DCS and $417 \mu$ s in DECT. Some systems may have shorter busy/idle periods, as it is the case of the MAC protocol employed by the IEEE 802.11 standard, which defines inter-frame spaces in the order of $50 \mu \mathrm{s}$ or less. The activity patterns observed under this protocol have extensively been studied in [222-225] and will not be considered in this study.
} 

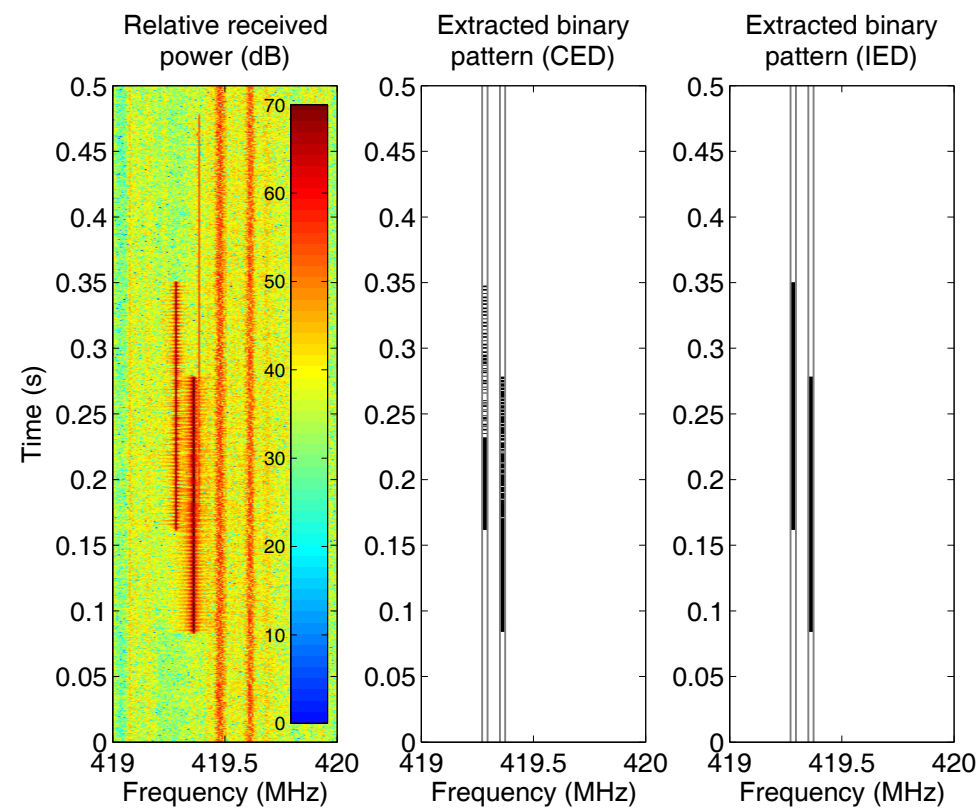

Figure 8.1: Comparison of channel occupancy patterns extracted by the CED and IED algorithms.

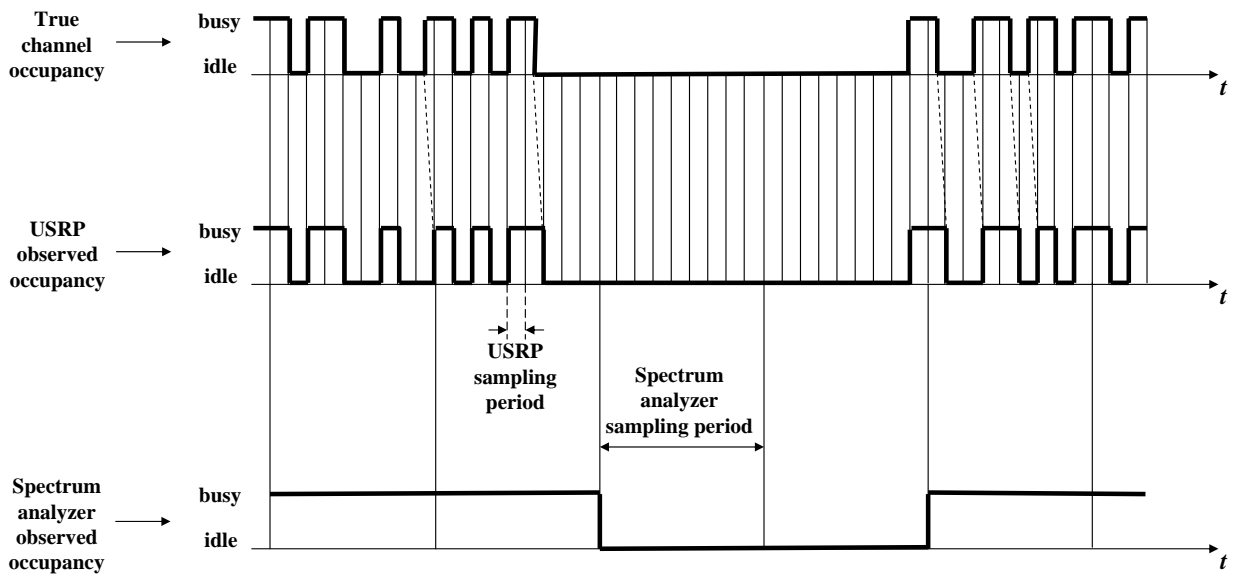

Figure 8.2: Low versus high time resolution measurements. 
empirical CDFs are then compared to a wide set of probability distribution models, which are summarized in Table 8.1. The set of analyzed CDF models includes the probability distributions analyzed in previous works plus some additional distributions that have not been considered before (generalized exponential, gamma and Weibull). Notice that some complex distributions studied in previous works (phase-type distributions such as the hyper-Erlang or hyper-exponential models) are not considered. Such distributions involve complex mathematical expressions with a high number of parameters and although they have been proven to be very accurate, their application to analytical studies seems to be difficult in practice. By contrast, the focus of this study is on simpler and more tractable models. Nevertheless, the considered CDF models are able to provide adequate fits as it will be shown later on.

The first considered model is the exponential distribution. This distribution is analyzed in order to determine whether the CTMC channel model, widely used in the literature, can be considered to be valid and realistic. It is worth noting that the study in [226] considers the geometric distribution, which represents a discrete distribution and, as such, can be employed to describe the length of busy/idle periods in terms of the number of slots. In this chapter the continuous counterpart of the geometric distribution, i.e. the exponential distribution, is considered instead. The second considered model is the generalized exponential distribution [247], a three-parameter extension to the conventional exponential distribution that has not been considered before. The interest of this distribution relies on its ability to reproduce other distributions with a single analytical expression. For example, for $\alpha=1$ it becomes the exponential distribution. Moreover, for certain ranges of the shape and scale parameters its distribution function can be very close to the corresponding distribution functions of the log-normal, gamma, and Weibull distributions. These three distributions are considered as well. The log-normal distribution has been suggested as an adequate alternative for heavytailed behaviors [226], while the suitability of the gamma and Weibull distributions has not been studied before. The Pareto and generalized Pareto distributions, considered in previous studies, are also included in the study performed in this chapter.

It is worth noting that the exponential and Pareto distributions represent particular cases of their generalized counterparts when $\alpha=1$ and $\mu=\lambda / \alpha$, respectively. In such cases, the relations $\mu_{G E}=\mu_{E}$ and $\lambda_{G E}=\lambda_{E}$ hold for the former, while $\lambda_{G P}=\lambda_{P} / \alpha_{P}$ and $\alpha_{G P}=1 / \alpha_{P}$ hold for the latter (see Table 8.1). The reason for explicitly considering the particular cases is to determine whether it is possible to fit the empirical data using simpler distributions with fewer parameters. Note that even when the particular cases are sufficient to provide an accurate fit, the numerical methods employed to estimate the parameters of the generalized distributions do not always necessarily lead to results that satisfy the relations $\alpha=1$ and $\mu=\lambda / \alpha$, hence the need to explicitly consider the particular cases in order to identify those situations where simpler distributions are enough. It is also worth noting that, in some cases, a better accuracy may be obtained with the particular cases than with the generalized distributions. This seemingly contradiction is merely an artifact of the employed numerical methods and indicates that the particular cases of the general distributions are enough to accurately fit the empirical data. This circumstance also highlights the need for explicitly considering the particular cases of the more general distributions in order to ensure obtaining the optimum fits, an aspect that seems to have been neglected in previous works. 


\begin{tabular}{|c|c|c|c|c|c|c|c|}
\hline 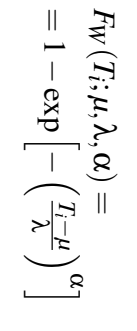 & 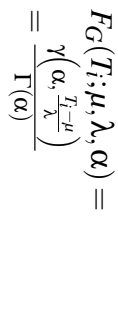 & 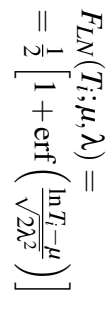 & 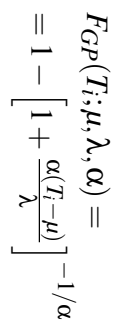 & 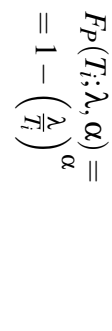 & 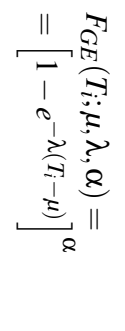 & 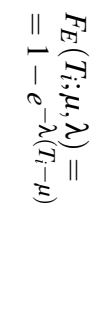 & 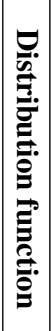 \\
\hline 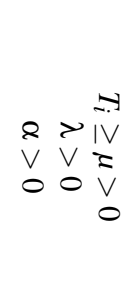 & 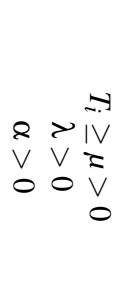 & $\begin{array}{lll}r & = \\
V & \pi & 1 V \\
0 & \emptyset & 0\end{array}$ & 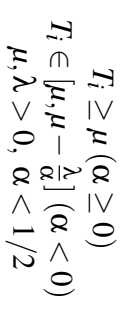 & $\begin{array}{lll}R & > & -1 \\
V & V & I V \\
N & 0 & >\end{array}$ & 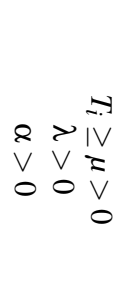 & 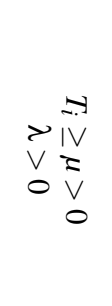 & 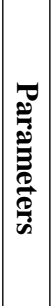 \\
\hline 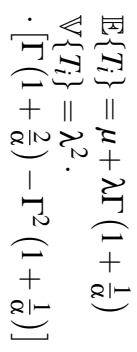 & 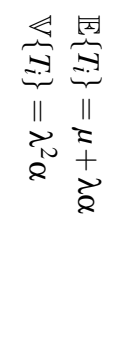 & 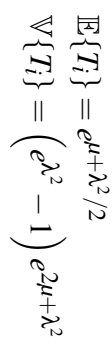 & 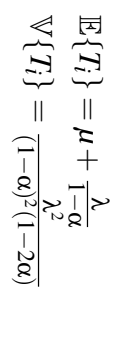 & 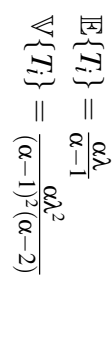 & 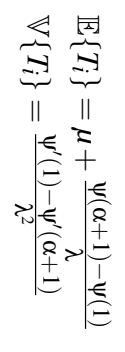 & 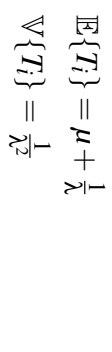 & 光 \\
\hline 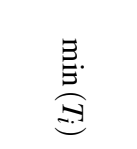 & $\stackrel{\Xi}{\mathrm{E}}$ & 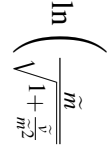 & $\stackrel{\Xi}{\Xi}$ & 1 & 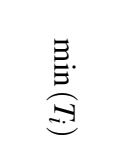 & $\stackrel{\Xi}{E}$ & $\Rightarrow$ \\
\hline 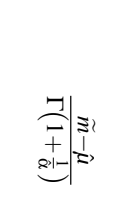 & 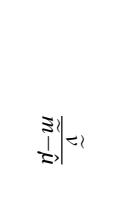 & 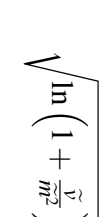 & 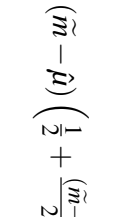 & $\stackrel{E}{E}$ & 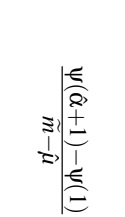 & $\begin{array}{l}5 \\
1 \\
\Rightarrow\end{array}$ & ح \\
\hline 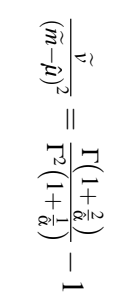 & 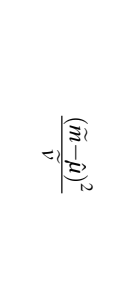 & | & 先 & 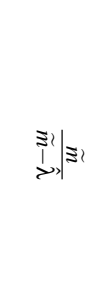 & 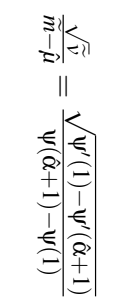 & 1 & R \\
\hline
\end{tabular}

䒕芯.

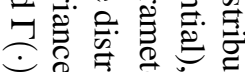

ज.

ज者

م

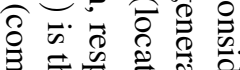

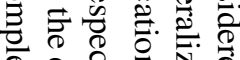

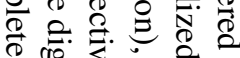

(c)

青市

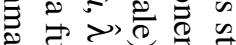

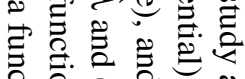

ڤ. है ह

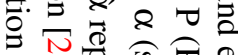

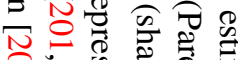

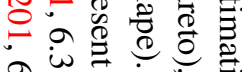
a iv

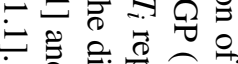

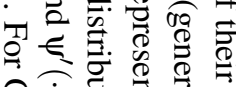

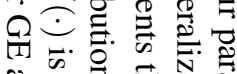
ڤँ.

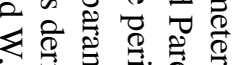
Q) 2 .

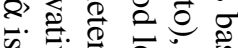
ऽ

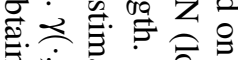

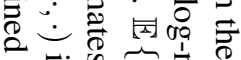

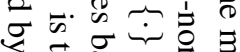

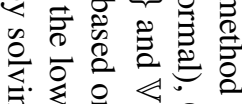

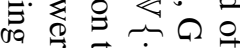

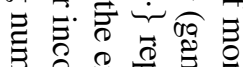
๑. के ڤ 仓 है $\overrightarrow{0} \rightleftharpoons$

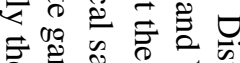

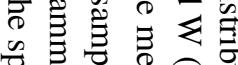
ठृ ๑.

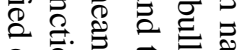

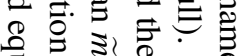
品苛

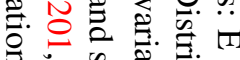
ด解苛 论戛 
As mentioned in Section 8.3, the parameters of various distribution models have usually been derived from the empirical data based on MLE techniques [222-225]. The MLE method is also employed in this chapter in order to estimate the best fitting parameters for the exponential [248-250], generalized exponential [247], Pareto [251], generalized Pareto [252-254], log-normal [248, 249, 255, 256], gamma [248, 249, 255, 256], and Weibull [248250] distributions. Additionally, MOM inference techniques are also considered, which consist in equating statistical moments with sample moments and then solving those equations for the parameters to be estimated. As shown in Table 8.1, the MOM parameter estimates are computed based on the sample mean and sample variance of the length of busy/idle periods. MLE is widely accepted as the preferred method for estimating distribution parameters since maximum likelihood estimators have higher probability of being close to the quantities to be estimated. However, as it will be shown, the MLE method not always provides the best results since the MOM approach provides better fits to empirical CDFs in some cases.

\subsection{Goodness-of-fit metrics}

In order to assess the suitability of the considered probability distributions in fitting the empirical data sets, several tests and their underlying Goodness-Of-Fit (GOF) metrics are employed. Based on the Kolmogorov-Smirnov (KS) test, the KS distance between the empirical CDF $F_{\text {emp }}\left(T_{i}\right)$ of period lengths $T_{i}\left(i \in\{0,1\}\right.$ denotes the period type, with $T_{0}$ and $T_{1}$ being idle and busy periods respectively) and the CDF model $F_{f i t}\left(T_{i}\right)$ is computed as [246]:

$$
D_{K S}=\max _{T_{i}}\left\{\left|F_{\text {emp }}\left(T_{i}\right)-F_{\text {fit }}\left(T_{i}\right)\right|\right\}
$$

A symmetric version of the Kullback-Leibler (KL) divergence [246] is also employed:

$$
\begin{aligned}
D_{K L}^{s y m} & =D_{K L}\left(f_{\text {emp }}\left(T_{i}\right) \| f_{\text {fit }}\left(T_{i}\right)\right)+D_{K L}\left(f_{\text {fit }}\left(T_{i}\right) \| f_{\text {emp }}\left(T_{i}\right)\right) \\
& =\sum_{k=1}^{K} f_{\text {emp }}\left(T_{i}^{k}\right) \ln \left(\frac{f_{\text {emp }}\left(T_{i}^{k}\right)}{f_{\text {fit }}\left(T_{i}^{k}\right)}\right)+\sum_{k=1}^{K} f_{\text {fit }}\left(T_{i}^{k}\right) \ln \left(\frac{f_{\text {fit }}\left(T_{i}^{k}\right)}{f_{\text {emp }}\left(T_{i}^{k}\right)}\right)
\end{aligned}
$$

where $f_{\text {emp }}\left(T_{i}^{k}\right)$ and $f_{f i t}\left(T_{i}^{k}\right)$ represent the empirical PDF and the evaluated PDF model, respectively, assumed to be computed for a discrete number of $K$ values of the period length $T_{i}$, $T_{i}^{k}(k=1,2, \ldots K)$ represents the $k$-th value of $T_{i}$ and $D_{K L}\left(f_{A} \| f_{B}\right)$ denotes the KL divergence between PDFs $f_{A}$ and $f_{B}$.

Finally, the Bhattacharyya distance is employed as well, which is defined as [257]:

$$
D_{B}=-\ln \left(\sum_{k=1}^{K} \sqrt{f_{\text {emp }}\left(T_{i}^{k}\right) \cdot f_{f i t}\left(T_{i}^{k}\right)}\right)
$$

As mentioned in Section 8.3, these metrics provide a single numerical value indicating the GOF of a certain probability model over the whole range of values of $T_{i}$. Additionally, the GOF of the considered models in particular regions of the CDF corresponding to low and high values of $T_{i}$ is evaluated as well. 


\subsection{Low time resolution models}

This section analyzes the suitability of the considered CDF models in describing the length of busy and idle periods based on spectrum analyzer measurements. The analysis to be performed in this section involves a high number of factors and parameters. There are seven different distributions to be fitted to the empirical length of both busy and idle periods. Moreover, the fitting needs to be performed for every channel within every measured band, by means of the MLE and MOM methods, and evaluated based on the three GOF metrics described in Section 8.6. In order to undertake the analysis, the following procedure was applied. First, the CDF models were fitted to the empirical CDFs of busy and idle periods of every individual channel within the measured bands by employing the MLE and MOM inference methods, and the resulting GOF was evaluated for each channel and inference method with the metrics described in Section 8.6. The GOF metrics obtained for each channel were then averaged over channels belonging the same spectrum band (radio technology). The average GOF results obtained for every spectrum band were observed to be in the same order of magnitude and, more importantly, the best fitting CDF model observed for individual bands agreed with the conclusions derived from the GOF metrics averaged over all bands. As an example, Table 8.2 shows the KS distance obtained for the considered distributions and some selected bands, when the idle periods are fitted based on the MLE method. As it can be appreciated, and based on the KS distance, the generalized Pareto distribution provides the best fit for idle periods for all the considered bands, which can also be concluded based on the average KS distance. Similar trends were observed for other GOF metrics and inference methods, for both busy and idle periods, thus indicating that the analysis can be performed in terms of average values since such values are sufficiently representative of the results observed for individual bands. Based on this appreciation, the GOF metrics were averaged over all bands in order to obtain the average GOF metrics shown in Table 8.3.

As it can be appreciated in Table 8.3, the three analyzed GOF metrics indicate that the MLE method results in the best fitting results for almost all the considered distributions, for both busy and idle periods. The only exception is the Weibull distribution, for which the best overall fit was observed when the distribution parameters are estimated based on the MOM. An exhaustive analysis of the GOF metrics for all the channels within every band indicated that approximately $75 \%$ of the analyzed channels were best fitted with the MLE method. In some particular cases of all the considered distributions and measured bands, the MOM approach was observed to provide a better fit. Although the MLE method does not provides the best fit always, it outperforms, in average, the MOM approach for all the considered cases excepting the Weibull distribution.

In order to compare the fitting accuracy of the considered distributions, the minimum value of the GOF metrics resulting from the MLE and MOM approaches was selected as the representative result. Notice that the best possible fit (either MLE or MOM) is the one that really indicates the ability of a distribution function to describe a set of empirical data. Thus, the comparison among the considered distributions is performed based on the minimum values obtained for each GOF metric, which are shown in bold in Table 8.3. As it can be appreciated, all the considered GOF metrics indicate that the best fit for idle periods is attained with the generalized Pareto distribution. For busy periods, the generalized Pareto 
Table 8.2: KS metric of idle periods for various distributions and bands based on the MLE method.

\begin{tabular}{|c|c|c|c|c|c|c|c|}
\hline Band/Technology & E & GE & P & GP & LN & G & W \\
\hline Amateur & 0.22 & 0.14 & 0.17 & 0.08 & 0.12 & 0.14 & 0.23 \\
\hline Paging & 0.26 & 0.17 & 0.23 & 0.13 & 0.15 & 0.16 & 0.25 \\
\hline TETRA UL & 0.25 & 0.13 & 0.17 & 0.06 & 0.10 & 0.13 & 0.17 \\
\hline TETRA DL & 0.20 & 0.13 & 0.24 & 0.13 & 0.13 & 0.13 & 0.26 \\
\hline DCS 1800 DL & 0.24 & 0.16 & 0.16 & 0.12 & 0.16 & 0.15 & 0.30 \\
\hline DECT & 0.25 & 0.15 & 0.19 & 0.07 & 0.09 & 0.14 & 0.15 \\
\hline ISM & 0.18 & 0.18 & 0.22 & 0.16 & 0.21 & 0.18 & 0.43 \\
\hline Average & 0.23 & 0.15 & 0.20 & 0.11 & 0.14 & 0.15 & 0.26 \\
\hline
\end{tabular}

Table 8.3: GOF metrics for low time resolution measurements.

\begin{tabular}{|c|c|c|c|c|c|c|c|c|c|}
\hline & & & & & & & \\
\hline & & & $\mathbf{E}$ & GE & $\mathbf{P}$ & GP & $\mathbf{L N}$ & G & $\mathbf{W}$ \\
\hline \multirow{6}{*}{ 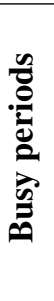 } & \multirow{2}{*}{$D_{K S}$} & MOM & 0.20 & 0.23 & 0.35 & 0.18 & 0.21 & 0.22 & 0.19 \\
\hline & & MLE & 0.20 & 0.19 & 0.23 & 0.16 & 0.20 & 0.19 & 0.43 \\
\hline & \multirow{2}{*}{$D_{K L}^{s y m}$} & MOM & 2.00 & 2.32 & 2.55 & 1.96 & 1.88 & 2.29 & 2.11 \\
\hline & & MLE & 2.00 & 1.89 & 2.22 & 1.93 & 1.94 & 1.91 & 2.63 \\
\hline & \multirow{2}{*}{$D_{B}$} & MOM & 0.25 & 0.30 & 0.32 & 0.24 & 0.32 & 0.30 & 0.27 \\
\hline & & MLE & 0.25 & 0.23 & 0.29 & 0.24 & 0.28 & 0.24 & 0.34 \\
\hline \multirow{6}{*}{$\begin{array}{l}\frac{n}{0} \\
.0 \\
\frac{0}{0} \\
\frac{0}{0} \\
\frac{0}{0}\end{array}$} & \multirow{2}{*}{$D_{K S}$} & MOM & 0.23 & 0.26 & 0.39 & 0.17 & 0.19 & 0.25 & 0.16 \\
\hline & & MLE & 0.23 & 0.15 & 0.20 & 0.11 & 0.14 & 0.15 & 0.26 \\
\hline & \multirow{2}{*}{$D_{K L}^{s y m}$} & $\mathrm{MOM}$ & 1.59 & 1.88 & 2.41 & 1.39 & 1.34 & 1.82 & 1.46 \\
\hline & & MLE & 1.59 & 1.38 & 1.64 & 1.29 & 1.32 & 1.38 & 1.70 \\
\hline & \multirow{2}{*}{$D_{B}$} & MOM & 0.19 & 0.28 & 0.31 & 0.17 & 0.23 & 0.27 & 0.19 \\
\hline & & MLE & 0.19 & 0.18 & 0.23 & 0.16 & 0.20 & 0.18 & 0.22 \\
\hline
\end{tabular}

distribution is also the most accurate model according to the KS metric, but not from the point of view of the symmetric KL divergence and the Bhattacharyya distance. However, in these two cases it is interesting to note that the generalized Pareto distribution is able to achieve a slightly worse fit than the best possible fit, thus indicating that the GOF of the generalized Pareto distribution in these cases can be considered to be acceptable as well. Therefore, and based on these observations, it can be concluded that the generalized Pareto distribution can be selected as an appropriate model for the lengths of busy and idle periods in real systems. Although other alternative models such as the generalized exponential, lognormal, gamma and Weibull distributions are able to achieve comparable GOFs in some particular cases, the generalized Pareto distribution provides, in average, the best overall fit over a wide range of spectrum bands and radio channels, for both busy and idle periods. The possibility to employ a single CDF model to characterize the lengths of busy and idle periods irrespective of the considered band and radio technology, makes of the generalized Pareto distribution an attractive alternative. 
The previous analysis has been based solely on distance metrics, which provide a single numerical value as an indication of the GOF of a certain probability model over the whole range of values of $T_{i}$. The fitting accuracy in particular regions of the CDF is analyzed in the following. Figures 8.3 and 8.4 illustrate the fitting accuracy of the considered probability models when the distribution parameters are estimated based on the MLE and MOM methods, respectively. These figures correspond to a single selected channel from a particular spectrum band. However, they are representative of the behavior observed for other channels from other bands. Each figure is composed of four graphs. Upper graphs show the statistics of busy periods, while lower graphs show the statistics of idle periods. The graphs on the left-hand side show the empirical and fitted CDFs with the abscissa axis in logarithmic magnitude, which enables a finer detail of appreciation of the fitting accuracy for low values of $T_{i}$ (i.e., short periods). The graphs on the right-hand side show the same information in terms of the CCDF with both axes in logarithmic magnitude, which allows for a finer detail of appreciation of the fitting accuracy for high values of $T_{i}$ (i.e., long periods).

It is worth noting that the results shown in Figures 8.3 and 8.4 correspond to a highly loaded channel with an average DC of 0.89 . Under high load conditions, channels remain busy most of the time and the probability of observing long busy periods increases. As a result, the distribution of busy periods shows a heavy-tailed behavior, which is not the case of idle periods as it can clearly be appreciated in Figures 8.3 and 8.4.

The first interesting observation from Figures 8.3 and 8.4 is that the exponential distribution is not able to describe with sufficient accuracy the length of busy and idle periods observed in real channels, meaning that the CTMC channel model widely employed in the literature is unrealistic ${ }^{3}$. In particular, the exponential distribution is especially unsuitable for heavy-tailed behaviors, i.e. for busy (idle) period lengths in channels with high (low) loads, where it provides one of the worst fitting accuracies. Moreover, it is important to note that the exponential distribution considered in this study (see Table 8.1) includes a location parameter $\mu$ that is not usually considered in the conventional exponential distribution widely employed in the literature. Without such location parameter (i.e., $\mu=0$ ), the resulting accuracy was observed to be appreciable worse, thus confirming that the CTMC channel model does not constitute an adequate alternative. In terms of fitting accuracy, similar comments can be made for the Pareto distribution, which suggests that simple one/two-parameter distributions do not seem to be able to describe the busy and idle periods in real channels.

For the rest of distributions, it is interesting to note, with some particular exceptions, that the best fitting accuracies in the region of short periods is normally achieved by the MLE method, while the best fit for long periods is in general attained with the MOM approach. Each inference method improves the fitting accuracy in one particular region at the expense of degrading the fit in the opposite region. From among the considered probability distributions, however, the generalized Pareto distribution is the only model that simultaneously provides a reasonable level of accuracy for both short and long periods, regardless of the inference method applied, which is not observed for any other of the considered distributions. This observation confirms the suitability of the generalized Pareto distribution in modeling

\footnotetext{
${ }^{3}$ Previous work [240] has shown that the PDF of channel vacancy durations can be fitted with an exponentiallike function of the form $f\left(T_{i}\right) \approx a+b \cdot \exp \left(-c \cdot T_{i}\right)$, with $a, b, c \in \mathbb{R}^{+}$, which is not an exponential distribution.
} 

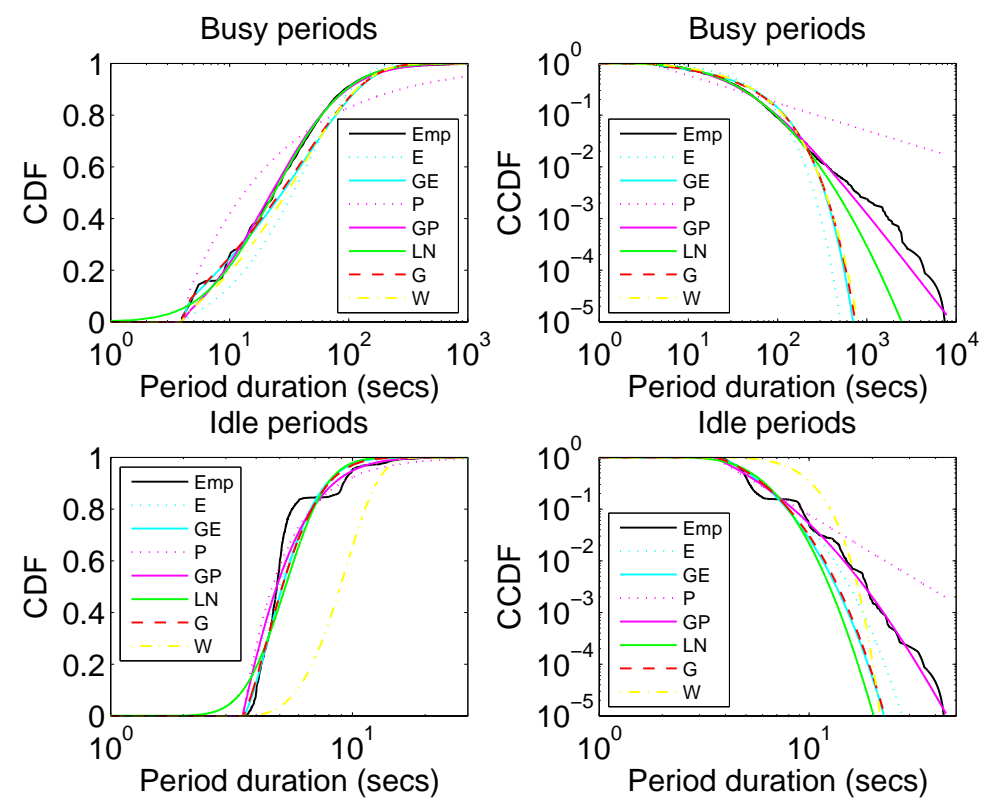

Figure 8.3: Empirical CDF and fitted distributions based on the MLE approach.
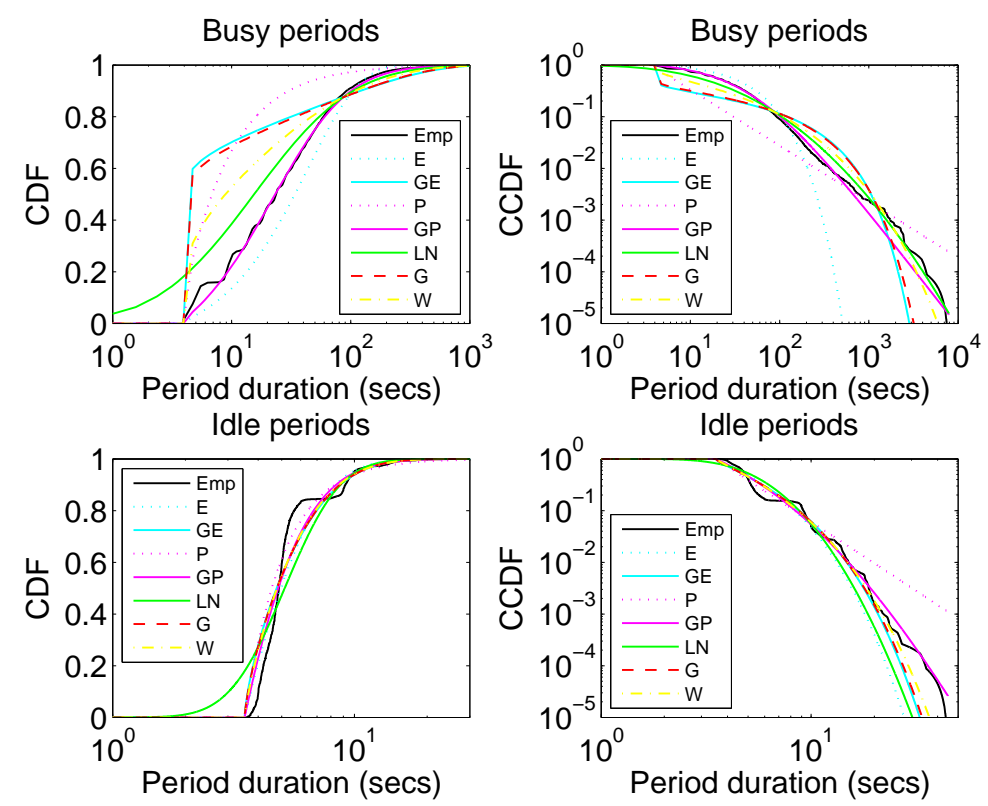

Figure 8.4: Empirical CDF and fitted distributions based on the MOM approach. 
Table 8.4: Parameters of the generalized Pareto distribution for busy and idle periods extracted from empirical measurement results by means of MLE ( $T_{i}$ in Table 8.1 expressed in seconds).

\begin{tabular}{|c|c|c|c|c|c|c|c|}
\hline \multirow{2}{*}{ Load } & \multirow{2}{*}{ Duty cycle } & \multicolumn{3}{|c|}{ Busy periods } & \multicolumn{3}{c|}{ Idle periods } \\
\cline { 3 - 8 } & & $\boldsymbol{\mu}$ (secs) & $\boldsymbol{\lambda}$ & $\boldsymbol{\alpha}$ & $\boldsymbol{\mu}$ (secs) & $\boldsymbol{\lambda}$ & $\boldsymbol{\alpha}$ \\
\hline Very low & 0.09 & 3.5150 & 1.6960 & 0.0284 & 3.6100 & 38.3633 & 0.2125 \\
\hline Low & 0.29 & 3.5150 & 2.6240 & 0.1884 & 3.5780 & 10.9356 & 0.1784 \\
\hline Medium & 0.51 & 3.5150 & 5.1483 & 0.1978 & 3.5160 & 4.6583 & 0.2156 \\
\hline High & 0.71 & 3.5470 & 10.7968 & 0.1929 & 3.5310 & 2.6272 & 0.2119 \\
\hline Very high & 0.93 & 3.5940 & 52.8611 & 0.2377 & 3.5160 & 1.6609 & 0.0068 \\
\hline
\end{tabular}

the state holding times of the CTSMC channel model. The generalized Pareto distribution provides a remarkably good fitting accuracy for both short/long busy/idle periods in channels with low/high loads over a wide range of spectrum bands and radio technologies. In order to facilitate to researchers the application of the CTSMC model, Table 8.4 provides the values of the distribution parameters extracted from field measurements for various channel loads expressed in terms of the average DC. To reproduce other arbitrary DCs, $\Psi$, the parameters of the distribution need to be chosen in such a way that the following equality holds [238]:

$$
\Psi=\frac{\mathbb{E}\left\{T_{1}\right\}}{\mathbb{E}\left\{T_{0}\right\}+\mathbb{E}\left\{T_{1}\right\}}
$$

where $\mathbb{E}\left\{T_{0}\right\}$ and $\mathbb{E}\left\{T_{1}\right\}$ represent the mean idle and busy periods, respectively, which are related with the parameters $\mu, \lambda$ and $\alpha$ as shown in Table 8.1. It is worth noting that the values shown in Table 8.4 for the location parameter $\mu$ are determined by the time resolution of the spectrum analyzer. In a DSA/CR system design, theoretical analysis or simulation tool, this parameter should be tailored to the particular scenario in accordance with the considered spectrum sensing period or the minimum period of activity/inactivity of a primary user.

\subsection{High time resolution models}

This section analyzes the suitability of the considered CDF models in describing the length of busy and idle periods based on measurements performed with the USRP/GNU Radio platform. Given the limited bandwidth capabilities of the USRP hardware, selected channels were analyzed instead of entire bands as in Section 8.7. An individual analysis for each channel was feasible in this case and, as a result, the GOF metrics were not averaged over channels belonging to the same band. Moreover, as opposed to Section 8.7, it was observed that the same distribution does not always provide an acceptable fit for all bands, thus indicating the need of an individual analysis for each considered band. Another difference with respect to the results obtained in Section 8.7 is that there is no clear predominance of an inference method over the other. The study of Section 8.7 indicated that the best fit is obtained in general when the parameters of the distributions are estimated based on the MLE method (except for the Weibull distribution) and the best accuracy for short/long periods is obtained 
with the MLE/MOM methods, respectively. Since none of these clear trends was observed in the analysis of the USRP/GNU Radio measurements, the results shown for each distribution correspond to the inference method providing the best fitting accuracy in each case. In general, the three considered GOF metrics indicated that the best fit is attained by the same inference method. In those cases where some disagreement was observed, the inference method indicated by any two of the three GOF metrics was selected (i.e., a majority vote criterion was applied). An individual analysis for each band is performed in the following.

\subsubsection{Amateur bands}

Figure 8.5 and Table 8.5 show the fitted distributions and the corresponding GOF metrics for a channel measured in the amateur band. These results are representative of other channels measured within the same band. According to Table 8.5, two of the three GOF metrics indicate that the generalized Pareto distribution can be considered as the best fit for busy periods. The KS distance metric, however, indicates that the best fit corresponds to the log-normal distribution. As appreciated in Figure 8.5, this distribution provides the best fit for long busy periods, but it cannot accurately model the minimum period duration. Therefore, it can be concluded that the generalized Pareto distribution provides a better fit over the whole range of busy period durations. For idle periods, the most precise models are the generalized Pareto and Weibull distributions, but similar accuracies are attained with the generalized exponential and gamma models, which can be considered as appropriate models as well. It is interesting to note that the exponential distribution seems to provide a reasonable fit for idle periods, which would indicate the validity of the CTMC model in this case. Nevertheless, this circumstance is due to the location parameter $\mu$ considered in this study for the exponential distribution (see Table 8.1), which is not included in the conventional exponential distribution widely employed in the literature. Without such location parameter (i.e., $\mu=0$ ), the resulting accuracy would be appreciable worse. Table 8.6 provides the values of the fitted distribution parameters.

\subsubsection{Paging bands}

Figure 8.6 and Table 8.7 show the fitted distributions and the corresponding GOF metrics for a channel measured in the paging band. These results are representative of other channels measured within the same band. The numerical results of Table 8.7 indicate that the best fit for busy periods is attained with the Weibull distribution. Nevertheless, Figure 8.6 suggests that the generalized exponential and the gamma distributions are able to provide a comparable fitting accuracy, even slightly better, over the whole range of busy period lengths. For idle periods, both Figure 8.6 and Table 8.7 agree that the best fit corresponds to the Pareto distribution. Table 8.8 provides the values of the fitted distribution parameters.

\subsubsection{Private/public access mobile radio bands}

Figure 8.7 and Table 8.9 show the fitted distributions and the corresponding GOF metrics for a channel measured in the TETRA DL band, which represents an example of a PMR/PAMR 

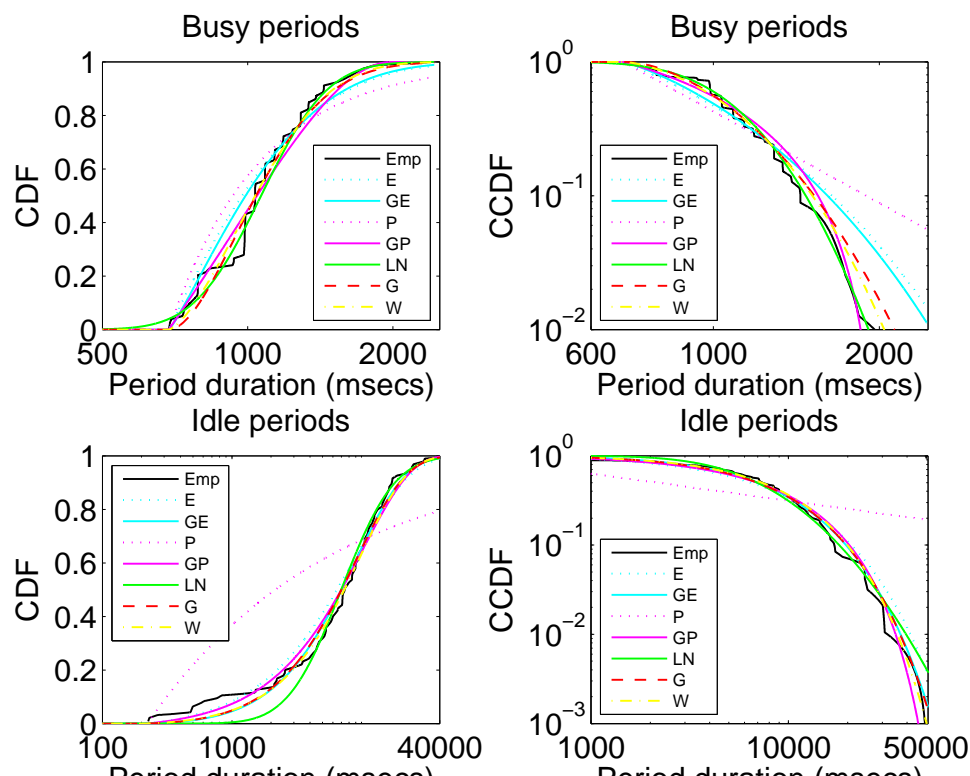

Period duration (msecs)

Period duration (msecs)

Figure 8.5: Empirical CDF and fitted distributions for amateur bands.

Table 8.5: GOF metrics for amateur bands.

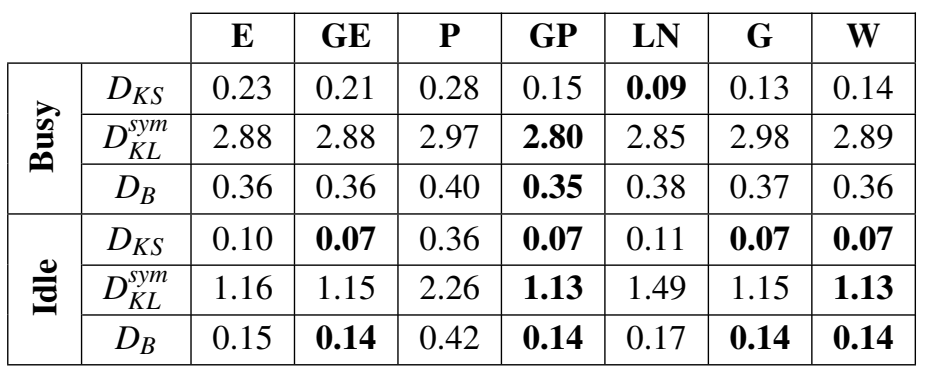

Table 8.6: Parameters of busy and idle period distributions for amateur bands ( $T_{i}$ in Table 8.1 expressed in milliseconds).

\begin{tabular}{|c|c|c|c|c|c|}
\hline \multirow{2}{*}{$\begin{array}{c}\text { Period } \\
\text { type }\end{array}$} & \multirow{2}{*}{$\begin{array}{c}\text { Fitted } \\
\text { distribution }\end{array}$} & \multicolumn{3}{|c|}{ Parameters } & \multirow{2}{*}{$\begin{array}{l}\text { Duty } \\
\text { cycle }\end{array}$} \\
\hline & & $\mu($ msecs $)$ & $\lambda$ & $\alpha$ & \\
\hline Busy & GP & $6.8659 \cdot 10^{2}$ & $6.0417 \cdot 10^{2}$ & -0.4559 & \multirow{5}{*}{0.09} \\
\hline \multirow{4}{*}{ Idle } & GP & $2.2208 \cdot 10^{2}$ & $1.0230 \cdot 10^{4}$ & -0.1395 & \\
\hline & W & $2.2208 \cdot 10^{2}$ & $9.7262 \cdot 10^{3}$ & 1.1801 & \\
\hline & GE & $2.2208 \cdot 10^{2}$ & $1.3271 \cdot 10^{-4}$ & 1.3263 & \\
\hline & G & $2.2208 \cdot 10^{2}$ & $7.0195 \cdot 10^{3}$ & 1.2790 & \\
\hline
\end{tabular}



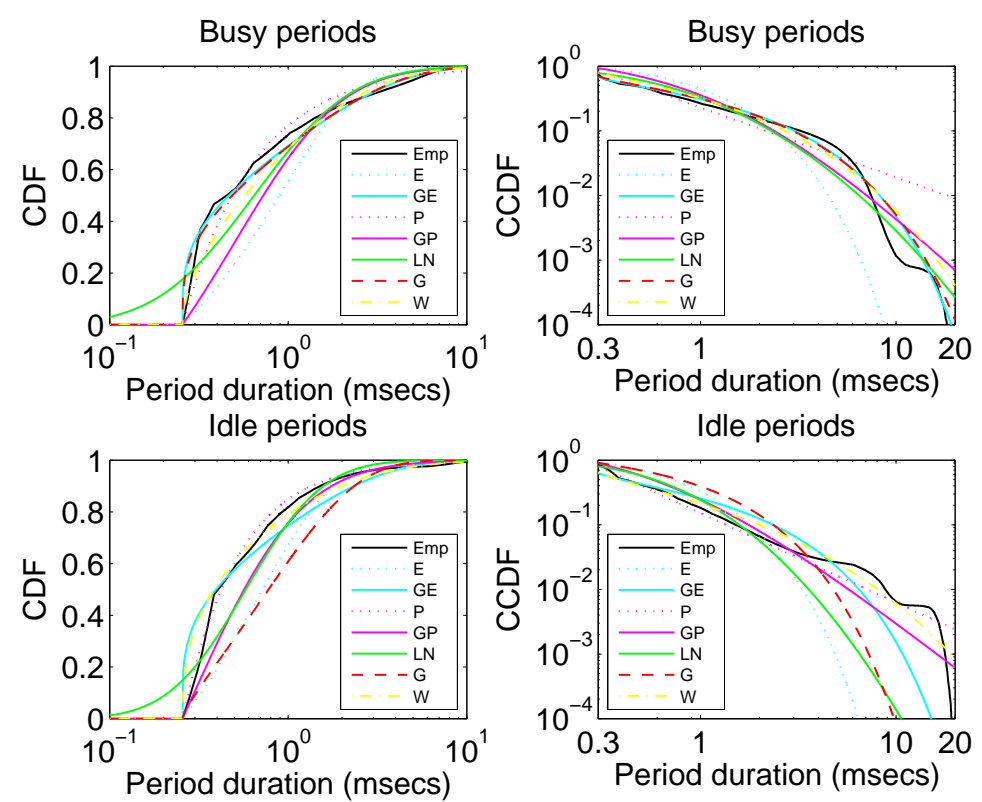

Figure 8.6: Empirical CDF and fitted distributions for paging bands.

Table 8.7: GOF metrics for paging bands.

\begin{tabular}{|c|c|c|c|c|c|c|c|c|}
\hline & & $\mathbf{E}$ & GE & $\mathbf{P}$ & GP & $\mathbf{L N}$ & $\mathbf{G}$ & $\mathbf{W}$ \\
\hline \multirow{3}{*}{$\overrightarrow{0}$} & $D_{K S}$ & 0.34 & 0.16 & 0.15 & 0.28 & 0.17 & 0.14 & 0.13 \\
\hline & $D_{K L}^{s y m}$ & 0.97 & 0.26 & 0.30 & 0.61 & 0.70 & 0.26 & 0.25 \\
\hline & $D_{B}$ & 0.13 & 0.11 & 0.05 & 0.08 & 0.19 & 0.10 & 0.05 \\
\hline \multirow{3}{*}{$\bar{\Xi}$} & $D_{K S}$ & 0.31 & 0.24 & 0.05 & 0.21 & 0.18 & 0.28 & 0.20 \\
\hline & $D_{K L}^{s y m}$ & 0.73 & 1.00 & 0.15 & 0.27 & 0.55 & 0.63 & 0.59 \\
\hline & $D_{B}$ & 0.08 & 0.12 & 0.02 & 0.03 & 0.15 & 0.08 & 0.07 \\
\hline
\end{tabular}

Table 8.8: Parameters of busy and idle period distributions for paging bands ( $T_{i}$ in Table 8.1 expressed in milliseconds).

\begin{tabular}{|c|c|c|c|c|c|}
\hline \multirow{2}{*}{$\begin{array}{c}\text { Period } \\
\text { type }\end{array}$} & $\begin{array}{c}\text { Fitted } \\
\text { distribution }\end{array}$ & \multicolumn{3}{|c|}{ Parameters } & \multirow{2}{*}{$\begin{array}{c}\text { Duty } \\
\text { cycle }\end{array}$} \\
\cline { 3 - 5 } & $\boldsymbol{\mu}$ (msecs) & $\boldsymbol{\lambda}$ & $\boldsymbol{\alpha}$ & \\
\cline { 2 - 5 } Busy & $\mathrm{W}$ & 0.2560 & 0.5922 & 0.5866 & \\
\cline { 2 - 5 } & $\mathrm{GE}$ & 0.2560 & 0.4204 & 0.2808 & \multirow{2}{*}{0.46} \\
\cline { 2 - 5 } Idle & $\mathrm{G}$ & 0.2560 & 3.0029 & 0.3058 & \\
\hline
\end{tabular}


system. These results are representative of other channels measured within the same band. The best fit for busy periods is attained with the generalized Pareto distribution. Nevertheless, the simpler Pareto distribution is able to attain a similar level of accuracy as well. For idle periods, the Weibull distribution provides the best fit to empirical data. It is worth noting that other channels within the TETRA DL band showed a slightly better fit with Weibull distributions for busy periods and (generalized) Pareto distributions for idle ones. This circumstance indicates that both busy and idle periods in the TETRA DL band can indistinctly be modeled with the Weibull and (generalized) Pareto distributions (in some cases, however, the Pareto distribution was observed not to be accurate enough and only the generalized Pareto distribution was acceptable). Table 8.10 provides the values of the fitted distribution parameters. The results in this case are shown for two different channels of the TETRA DL band with the same average DC.

\subsubsection{Cellular mobile communication bands}

Figure 8.8 and Table 8.11 show the fitted distributions and the corresponding GOF metrics for a channel measured in the E-GSM 900 DL band, which represents an example of a cellular mobile communication system. These results are representative of other channels measured within the same band and also the band allocated to the DCS 1800 system. The obtained results clearly indicate that the generalized Pareto distribution provides the best fit for busy periods over the whole range of period durations. For idle periods, the best fit is attained with the generalized exponential distribution, although the gamma distribution provides a similar accuracy. Table 8.12 provides the values of the fitted distribution parameters. The location parameter, which represents the minimum period duration, should be equal to the GSM/DCS time-slot duration (i.e., $\mu=0.577 \mathrm{~ms}$ ). The value obtained in Table 8.12, however, is affected by the measurement time resolution of $0.128 \mathrm{~ms}$ (see Section 8.4).

It is worth noting that the stair-shaped empirical CDF in Figure 8.8 is a natural consequence of the time-slotted structure employed by the multiple access mechanism of the GSM/DCS system, which defines a frame structure with 8 time slots of $577 \mu$ s each. This circumstance suggests the possibility of modeling busy/idle periods in time-slotted systems from a discrete perspective, where period lengths are described in terms of the number of time-slots. Based on this observation, and considering a time-slot duration of of $577 \mu \mathrm{s}$, the empirical CDF of Figure 8.8 was discretized and compared to several discrete-time CDF models. In particular, geometric, Poisson, binominal and negative binomial distributions (see Table 8.13) were fitted to the empirical data. Figure 8.9 and Table 8.14 show the fitted distributions and GOF metrics obtained in this case (the binomial distribution was proven to be unable to fit empirical data and it is therefore not shown). As it can be appreciated, the best fit for both busy and idle periods is attained with the negative binominal distribution, which closely resembles the empirical CDF. For busy periods, the Poisson distribution provides the same fit as the negative binominal (both distributions are indistinguishable from each other in Figure 8.9) ${ }^{4}$. For busy periods, the optimum fit with the Poisson distribution

\footnotetext{
${ }^{4}$ The negative binomial distribution converges to the Poisson distribution as its parameter $r$ tends towards infinity. Hence, they may closely resemble each other for $r$ sufficiently large.
} 

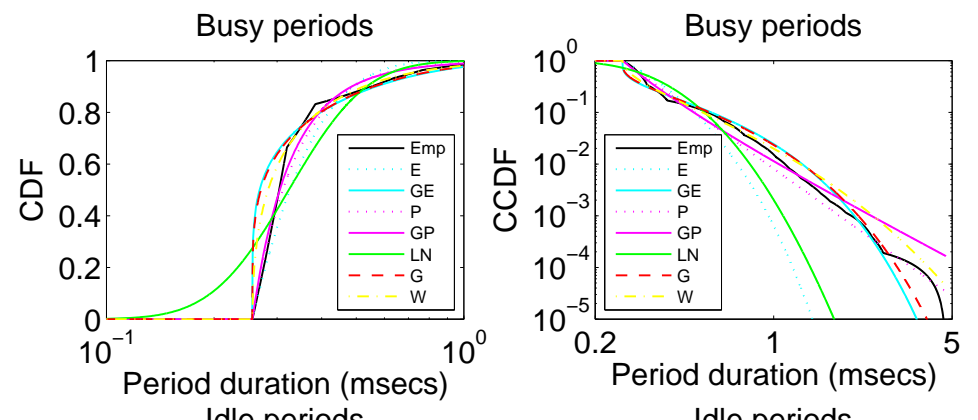

Idle periods
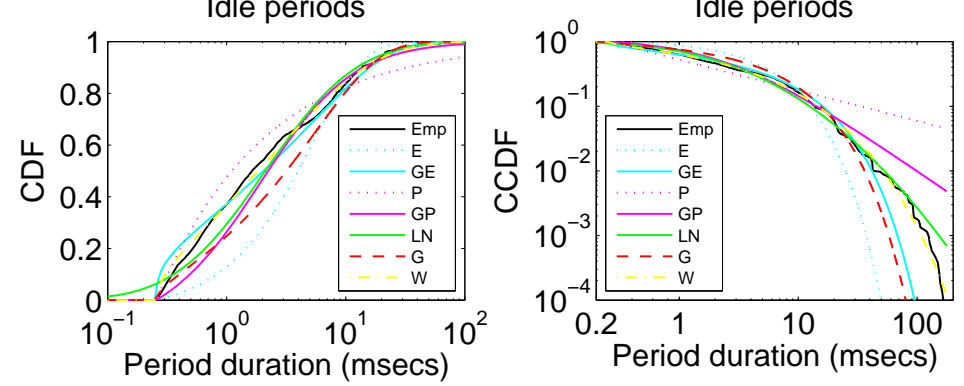

Figure 8.7: Empirical CDF and fitted distributions for PMR/PAMR bands.

Table 8.9: GOF metrics for PMR/PAMR bands.

\begin{tabular}{|c|c|c|c|c|c|c|c|c|}
\hline & & $\mathbf{E}$ & GE & $\mathbf{P}$ & GP & $\mathbf{L N}$ & G & $\mathbf{W}$ \\
\hline \multirow{3}{*}{ 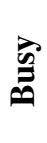 } & $D_{K S}$ & 0.20 & 0.38 & 0.12 & 0.08 & 0.27 & 0.36 & 0.24 \\
\hline & $D_{K L}^{S y m}$ & 0.45 & 0.74 & 0.24 & 0.21 & 0.81 & 0.71 & 0.51 \\
\hline & $D_{B}$ & 0.06 & 0.22 & 0.03 & 0.03 & 0.27 & 0.20 & 0.10 \\
\hline \multirow{3}{*}{ 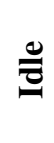 } & $D_{K S}$ & 0.27 & 0.09 & 0.11 & 0.12 & 0.08 & 0.16 & 0.04 \\
\hline & $D_{K L}^{s y m}$ & 0.79 & 0.28 & 0.35 & 0.34 & 0.35 & 0.36 & 0.23 \\
\hline & $D_{B}$ & 0.10 & 0.08 & 0.08 & 0.05 & 0.08 & 0.06 & 0.05 \\
\hline
\end{tabular}

Table 8.10: Parameters of busy and idle period distributions for PMR/PAMR bands ( $T_{i}$ in Table 8.1 expressed in milliseconds).

\begin{tabular}{|c|c|c|c|c|c|}
\hline \multirow{2}{*}{$\begin{array}{c}\text { Period } \\
\text { type }\end{array}$} & \multirow{2}{*}{$\begin{array}{c}\text { Fitted } \\
\text { distribution }\end{array}$} & \multicolumn{3}{|c|}{ Parameters } & \multirow{2}{*}{$\begin{array}{c}\text { Duty } \\
\text { cycle }\end{array}$} \\
\cline { 3 - 5 } Busy & GP & 0.2560 & 0.0621 & 0.3861 & \\
\cline { 2 - 5 } & $\mathrm{P}$ & - & 0.2560 & 3.5311 & \multirow{2}{*}{0.06} \\
\hline Idle & $\mathrm{W}$ & 0.2560 & 3.1084 & 0.5451 & \\
\hline Busy & $\mathrm{W}$ & 0.2560 & 0.4192 & 0.6656 & \multirow{2}{*}{0.06} \\
\hline Idle & $\mathrm{P}$ & - & 0.2560 & 1.0186 & \\
\hline
\end{tabular}



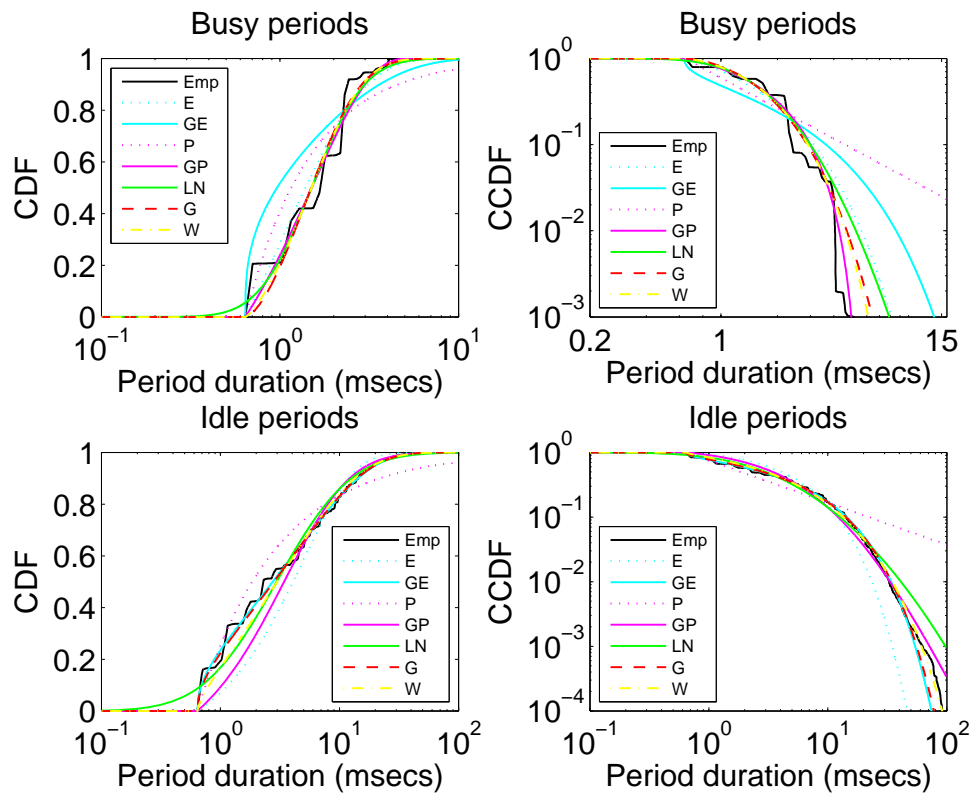

Figure 8.8: Empirical CDF and fitted distributions for cellular mobile communication bands.

Table 8.11: GOF metrics for cellular mobile communication bands.

\begin{tabular}{|c|c|c|c|c|c|c|c|c|}
\hline & & $\mathbf{E}$ & GE & $\mathbf{P}$ & GP & $\mathbf{L N}$ & $\mathbf{G}$ & $\mathbf{W}$ \\
\hline \multirow{3}{*}{$\overrightarrow{0}$} & $D_{K S}$ & 0.18 & 0.32 & 0.22 & 0.13 & 0.16 & 0.19 & 0.18 \\
\hline & $D_{K L}^{s y m}$ & 3.17 & 3.28 & 3.51 & 3.09 & 3.30 & 3.31 & 3.20 \\
\hline & $D_{B}$ & 0.38 & 0.40 & 0.44 & 0.37 & 0.41 & 0.40 & 0.39 \\
\hline \multirow{3}{*}{ 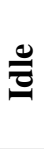 } & $D_{K S}$ & 0.23 & 0.05 & 0.13 & 0.20 & 0.13 & 0.07 & 0.12 \\
\hline & $D_{K L}^{s y m}$ & 2.70 & 2.12 & 2.32 & 2.54 & 2.42 & 2.13 & 2.25 \\
\hline & $D_{B}$ & 0.33 & 0.26 & 0.31 & 0.31 & 0.36 & 0.26 & 0.27 \\
\hline
\end{tabular}

Table 8.12: Parameters of busy and idle period distributions for cellular mobile communication bands ( $T_{i}$ in Table 8.1 expressed in milliseconds).

\begin{tabular}{|c|c|c|c|c|c|}
\hline \multirow{2}{*}{$\begin{array}{c}\text { Period } \\
\text { type }\end{array}$} & \multirow{2}{*}{$\begin{array}{c}\text { Fitted } \\
\text { distribution }\end{array}$} & \multicolumn{3}{|c|}{ Parameters } & \multirow{2}{*}{$\begin{array}{c}\text { Duty } \\
\text { cycle }\end{array}$} \\
\cline { 3 - 5 } & $\boldsymbol{\mu}$ (msecs) & $\boldsymbol{\lambda}$ & $\boldsymbol{\alpha}$ & \\
\hline \multirow{2}{*}{ Bdle } & GP & 0.5120 & 1.3692 & -0.2669 & \\
\cline { 2 - 5 } & GE & 0.5120 & 0.1141 & 0.4502 & \multirow{2}{*}{0.23} \\
\hline
\end{tabular}




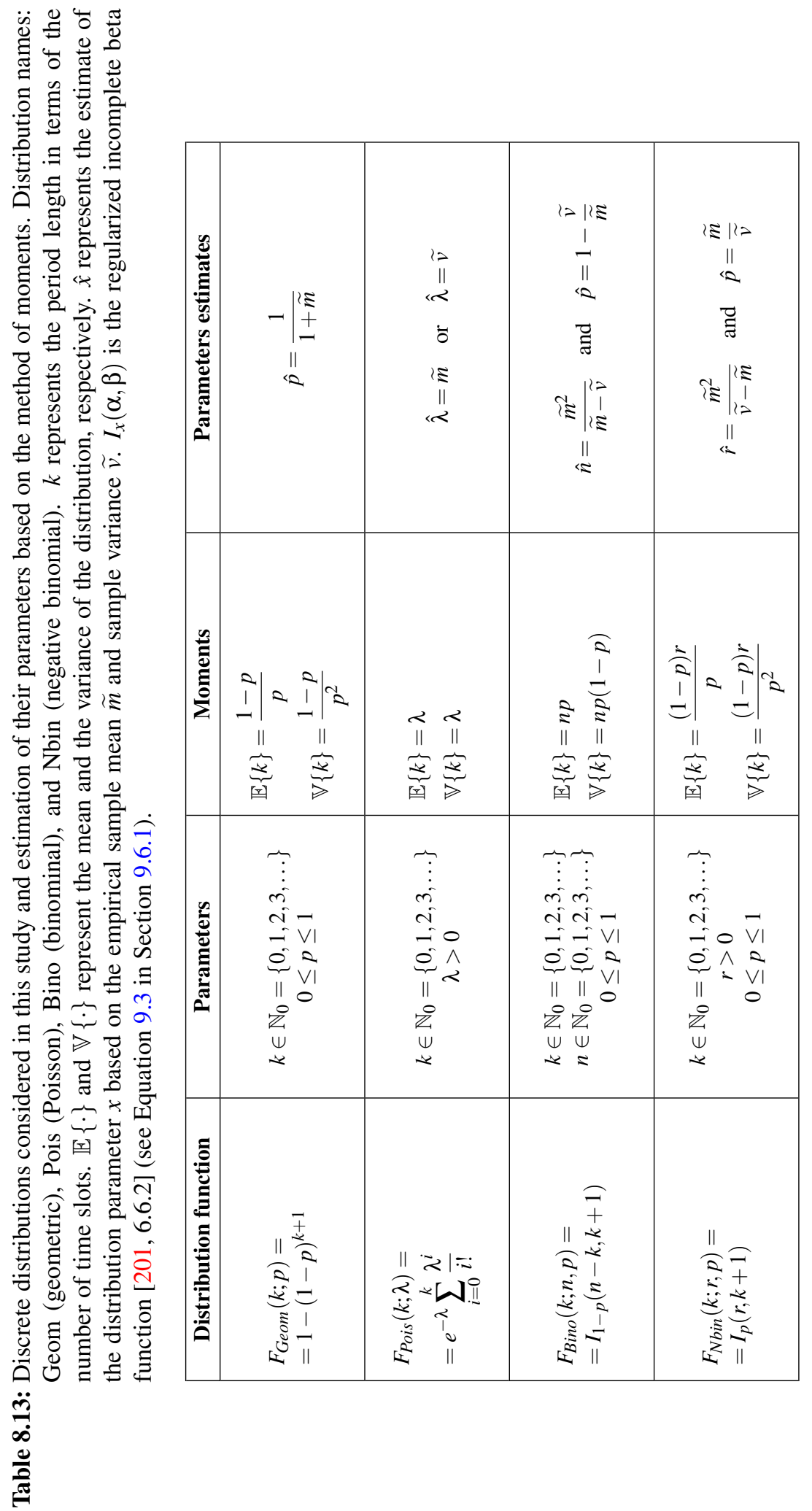




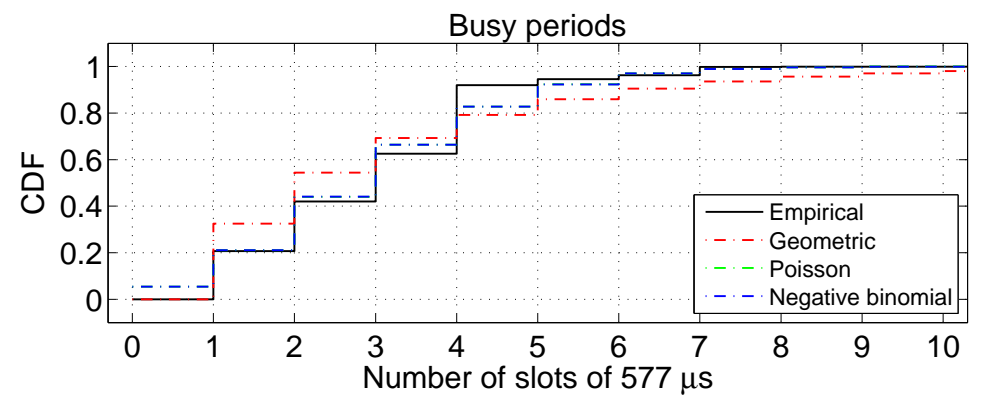

Idle periods

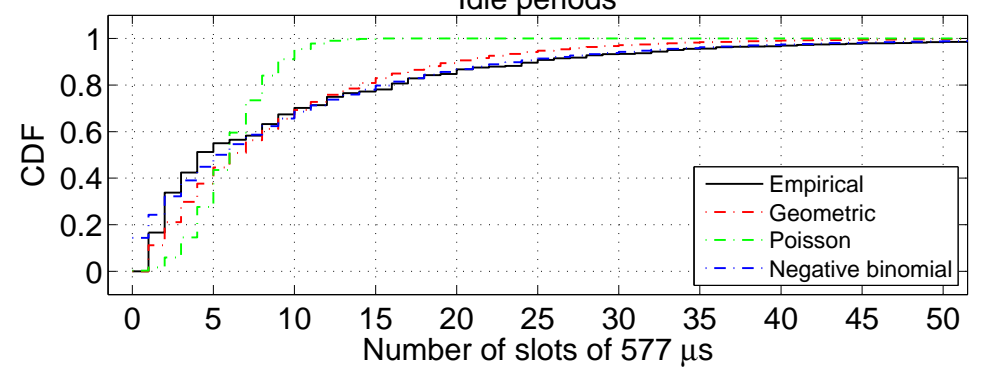

Figure 8.9: Empirical CDF and fitted distributions for cellular mobile communication bands (discrete-time models).

Table 8.14: GOF metrics for cellular mobile communication bands (discrete-time models).

\begin{tabular}{|c|c|c|c|c|}
\hline & Geometric & Poisson & Neg. binominal \\
\hline \multirow{3}{*}{ 它 } & $D_{K S}$ & 0.13 & 0.09 & 0.09 \\
\hline & $D_{K L}^{s y m}$ & 0.64 & 0.27 & 0.27 \\
\hline & $D_{B}$ & 0.09 & 0.01 & 0.01 \\
\hline \multirow{3}{*}{$\stackrel{\oplus}{\bar{\theta}}$} & $D_{K S}$ & 0.14 & 0.28 & 0.14 \\
\hline & $D_{K L}^{s y m}$ & 0.36 & 1.49 & 0.29 \\
\hline & $D_{B}$ & 0.13 & 0.32 & 0.10 \\
\hline
\end{tabular}

is obtained with parameter $\lambda=2.9239$. With the negative binomial distribution, the optimum fit is obtained with parameters $r=3.0294 \cdot 10^{3}$ and $p=0.9990$ for busy periods and parameters $r=0.7430$ and $p=0.0734$ for idle periods ${ }^{5}$.

\footnotetext{
${ }^{5}$ In its original definition, the negative binomial distribution is a discrete probability distribution of the number of successes in a sequence of Bernoulli trials before a specified (non-random) number of failures occurs. The distribution is characterized by two parameters: $r \in \mathbb{N}^{*}$ (the number of failures until the experiment is stopped) and $p \in[0,1]$ (the success probability in each experiment). The original definition can be extended to real values of the parameter $r$, which is sometimes referred to as the Pólya distribution. For the purposes of this study, the distribution is regarded as a discrete probability distribution with two parameters that are fitted to empirical data.
} 


\subsubsection{Cordless telephone bands}

The continuous-time distributions presented in Section 8.5 were fitted to the channels measured in the DECT band, which represents an example of a cordless telephone system. However, no satisfactory fits were observed for this particular system with the considered probability distributions. Since DECT employs a time-slotted frame structure ${ }^{6}$, the discrete-time distributions considered in Section 8.8.4 were then fitted to the empirical data following a similar procedure for the discretization of the continuous-time empirical CDF. As an example of the results obtained in this other case, Figure 8.10 shows the fitted distributions for a channel measured in the DECT band. These results are representative of other channels measured within the same band. As it can be appreciated, busy periods appear to be perfectly fitted with a geometric distribution. This result, however, should be interpreted carefully. The empirical CDF of Figure 8.10 indicates that the busy periods observed in the field measurements were always one time-slot long, and in this particular case the geometric distribution perfectly fits the resulting simple CDF. Nevertheless, the suitability of the geometric distribution for longer busy periods of two or more time-slots cannot be concluded from the results of Figure 8.10. Unfortunately, this statement could not be verified with empirical data since busy periods were always one time-slot long for all the measured DECT channels, which on the other hand suggests that this may be the most common case in practice. Concerning idle periods, the results of Figure 8.10 indicate that the channel inactivity periods may either be 11 or 23 time-slots long. None of the considered distributions can be fitted to the resulting empirical CDF with a reasonable level of accuracy.

Although the previous discussion indicates that a probabilistic modeling of busy and idle period lengths does not seem to be valid for DECT channels, the results of Figure 8.10 suggest an alternative, simpler modeling approach. In particular, two well-defined cases can be inferred from Figure 8.10. The first case corresponds to a base station transmitting broadcast information. A DECT base station is continuously transmitting, on at least one channel, information about the base station identity, system capabilities, status and paging information for incoming call set-up. This information is transmitted in one (busy) timeslot. If there are no active communications, the rest of downlink and uplink time-slots in the frame will be empty until the next broadcast message (time-slot). Therefore, in this case the channel occupancy pattern is composed of one busy time-slot followed by 23 idle time-slots. According to Figure 8.10, this occupancy pattern was observed for about $10 \%$ of the time in the considered channel. The second inferrable case corresponds to a single communication link between the base station and one portable device. In this case, two timeslots are used for communication, one in the downlink part of the frame and the other one in the uplink part. In this other case, the channel occupancy pattern is composed of one busy time-slot followed by 11 idle time-slots. According to Figure 8.10, this occupancy pattern was observed for about $90 \%$ of the time in the considered channel.

\footnotetext{
${ }^{6}$ The DECT radio interface [258] is based on a Multi Carrier, Time Division Multiple Access, Time Division Duplex (MC/TDMA/TDD) radio access methodology. The basic DECT frequency allocation defines 10 carrier frequencies. In the time domain, each carrier frequency is divided in 10-ms frames, which are composed of 24 time-slots of $417 \mu$ s each. The first (last) 12 time-slots of a frame are used for downlink (uplink) transmissions.
} 

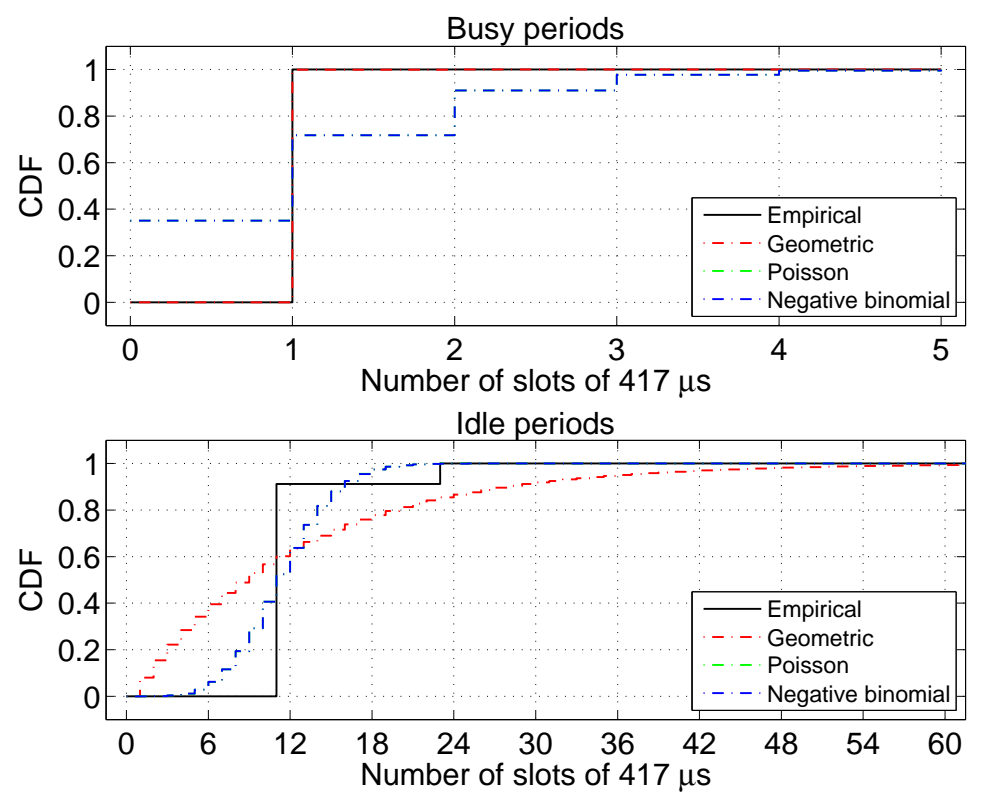

Figure 8.10: Empirical CDF and fitted distributions for cordless telephone bands (discrete-time).

Note that the two possible occupancy patterns inferred from the results of Figure 8.10 are characterized by a completely deterministic sequence of busy and idle periods, for which a probabilistic modeling may not be well suited. In the eventual case of busy periods of two or more time-slots (i.e., two or more communication links with the base station), the length of busy and idle periods would depend on the particular position of busy time-slots within the DECT frame structure. In this other case, a probabilistic modeling would be more appropriate. However, as mentioned above, busy periods of one time-slots appear to be the most common situation in real DECT systems and in such a case the deterministic modeling approach discussed before results more convenient.

Before concluding this section, it is worth noting that DECT makes use of a continuous dynamic channel selection and allocation mechanism. All DECT equipment is obliged to regularly scan its local radio environment at least once every 30 seconds. After that period of time, the system may decide to switch to a different operating carrier frequency. As a result, a particular carrier frequency may exhibit in practice long inactivity periods (while the system is operating over other carrier frequencies) followed by periods of activity (while the system is operating over that carrier frequency). During these activity periods, the carrier frequency exhibits busy/idle intervals at a much shorter time scale (i.e., at the slot level), which is due to the the two aforementioned occupancy patterns. This observation suggests that the real activity pattern of a channel may be more appropriately described by means of a two-layer approach: one modeling level would describe channel usage at a high time scale, while another modeling level would describe in detail the true occupancy pattern at a low time scale. This modeling approach will be further discussed in Section 8.10. 


\subsection{Time correlation models}

The results obtained in previous sections have indicated that the CTSMC model is able to explicitly capture and reproduce the statistical distributions of busy and idle periods as well as the mean channel occupancy level, which is also implicitly included since it depends on the mean value of the distributions (see Equation 8.5). Nevertheless, previous studies [226] have indicated that in some cases the lengths of busy and idle periods can be correlated, a feature that the CTSMC model cannot reproduce. This circumstance indicates the need of novel modeling approaches or the extension of the existing ones in order to account for more sophisticated features of spectrum usage. A modeling approach based on the aggregation and superposition of the realizations of several CTSMC processes was proposed in [259]. Although such simple model was shown to qualitatively reproduce correlations between consecutive idle periods, it suffers from some practical limitations. In particular, the resulting correlation depends on the number of aggregated processes as well as their distributions. By appropriate parameter selection various correlation values can be reproduced. However, if a particular correlation level needs to be reproduced, the number of processes to be aggregated and the required model parameters is something that cannot be determined in a straightforward manner. To this end, the use of external simulations would be required to find an adequate configuration, which complicates the application of the model in practice. Therefore, there is a clear need for simple primary user activity models featuring correlated busy/idle periods. This section explores the autocorrelation properties not only of idle periods but also of busy ones as well as the correlation properties of consecutive busy-idle periods. Based on the observed correlation properties, adequate models are developed. Furthermore, a simulation method is proposed, which is able to reproduce the observed correlation properties between consecutive busy-idle, busy-busy or idle-idle periods along with any arbitrary statistical distributions for both busy and idle periods.

\subsubsection{Correlation metrics}

The correlation properties of busy/idle periods are quantified in this section by means of the Pearson's product-moment correlation coefficient $\rho$, the Kendall's rank correlation coefficient $\tau$, and the Spearman's rank correlation coefficient $\rho_{s}$, which are defined as [260]:

$$
\begin{aligned}
\rho\left(T_{i}, T_{j}\right) & =\frac{\mathbb{C}\left\{T_{i}, T_{j}\right\}}{\sqrt{\mathbb{V}\left\{T_{i}\right\} \mathbb{V}\left\{T_{j}\right\}}}=\frac{\mathbb{E}\left\{\left(T_{i}-\mathbb{E}\left\{T_{i}\right\}\right)\left(T_{j}-\mathbb{E}\left\{T_{j}\right\}\right)\right\}}{\sqrt{\mathbb{V}\left\{T_{i}\right\} \mathbb{V}\left\{T_{j}\right\}}} \\
\tau\left(T_{i}, T_{j}\right) & =P\left(\left(T_{i}^{\prime}-T_{j}^{\prime}\right)\left(T_{i}^{\prime \prime}-T_{j}^{\prime \prime}\right)>0\right)-P\left(\left(T_{i}^{\prime}-T_{j}^{\prime}\right)\left(T_{i}^{\prime \prime}-T_{j}^{\prime \prime}\right)<0\right) \\
& =2 P\left(\left(T_{i}^{\prime}-T_{j}^{\prime}\right)\left(T_{i}^{\prime \prime}-T_{j}^{\prime \prime}\right)>0\right)-1 \\
\rho_{s}\left(T_{i}, T_{j}\right) & =\rho\left(F_{i}\left(T_{i}\right), F_{j}\left(T_{j}\right)\right)
\end{aligned}
$$

where $T_{i}$ and $T_{j}$ represent the busy/idle period lengths with CDFs $F_{i}\left(T_{i}\right)$ and $F_{j}\left(T_{j}\right),\left(T_{i}^{\prime}, T_{j}^{\prime}\right)$ and $\left(T_{i}^{\prime \prime}, T_{j}^{\prime \prime}\right)$ are two random observations of $T_{i}$ and $T_{j}$, and $\mathbb{E}\{\cdot\}, \mathbb{V}\{\cdot\}$, and $\mathbb{C}\{\cdot\}$ denote their expected value (mean), variance and covariance, respectively. If the state space of a primary radio channel is denoted as $\mathbb{S}=\left\{s_{0}, s_{1}\right\}$, with $s_{0}$ being the idle state and $s_{1}$ being the busy state, then the previous correlation coefficients represent the autocorrelation of idle 
periods when $T_{i}=T_{j}=T_{0}$, the autocorrelation of busy periods when $T_{i}=T_{j}=T_{1}$, and the correlation between consecutive busy-idle periods when $T_{i} \neq T_{j}, i, j \in\{0,1\}$.

Based on $K$ empirical samples of the period lengths $T_{i}$ and $T_{j}$, the previous correlation metrics can be estimated as:

$$
\begin{aligned}
\widehat{\rho}\left(T_{i}, T_{j}\right) & =\frac{\sum_{k=1}^{K} T_{i}^{k} T_{j}^{k}-K \widetilde{m}_{i} \widetilde{m}_{j}}{(K-1) \sqrt{\widetilde{v}_{i} \widetilde{v}_{j}}} \\
\widehat{\tau}\left(T_{i}, T_{j}\right) & =\frac{\mathcal{C}-\mathcal{D}}{\mathcal{C}+\mathcal{D}}=\frac{\mathcal{S}}{\left(\begin{array}{l}
K \\
2
\end{array}\right)}=\frac{2 \mathcal{S}}{K(K-1)} \\
\widehat{\rho}_{s}\left(T_{i}, T_{j}\right) & =\widehat{\rho}\left(F_{i}\left(T_{i}\right), F_{j}\left(T_{j}\right)\right)
\end{aligned}
$$

where $T_{i}^{k}$ or $T_{j}^{k}(k=1,2, \ldots, K), \widetilde{m}_{i}$ or $\widetilde{m}_{j}$, and $\widetilde{v}_{i}$ or $\widetilde{v}_{j}$ represent the $k$-th value, the sample mean and the sample variance of $T_{i}$ or $T_{j}$, respectively, and $S=C-\mathcal{D}$ is the difference between the number $\mathcal{C}$ of concordant pairs with $\left(T_{i}^{\prime}-T_{j}^{\prime}\right)\left(T_{i}^{\prime \prime}-T_{j}^{\prime \prime}\right)>0$ and the number $\mathcal{D}$ of discordant pairs with $\left(T_{i}^{\prime}-T_{j}^{\prime}\right)\left(T_{i}^{\prime \prime}-T_{j}^{\prime \prime}\right)<0$ observed in the sample set.

The above mentioned correlation coefficients have some similarities. All of them take values within the interval $[-1,+1]$. If both random variables $T_{i}$ and $T_{j}$ increase or decrease together, the correlation coefficients are positive. However, if one variable increases as the other decreases, then the correlation coefficients are negative. If the variables are independent, the correlation coefficients are zero (or approximately zero), but the converse is not true in general. There are, however, some important differences. First, $\rho$ is only sensitive to linear dependence relations between random variables. Thus, if the association between two random variables is purely non-linear, then $\rho=0$ even though they are not independent. On the other hand, $\tau$ and $\rho_{s}$ are sensitive and can detect some non-linear associations between variables. Moreover, $\rho$ has the unfortunate property of being sensitive (i.e., variant) under non-linear transformations of the random variables. However, $\tau$ and $\rho_{s}$ are invariant under monotone transformations. Thus, given two random variables with correlation coefficients $\rho, \tau$ and $\rho_{s}$, a transformation of the variables could (and usually does) change the value of $\rho$, but it will not affect the values of $\tau$ and $\rho_{s}$ under strictly monotone transformations.

\subsubsection{Correlation properties of spectrum usage}

\subsubsection{Correlation between busy and idle periods}

Based on the results obtained from field measurements, the correlation between busy and idle periods was evaluated for all channels within each measured band as a function of various parameters such as the channel DC as well as the mean period duration. The obtained results indicated that there is no clear relation between the empirical correlation coefficients and the considered parameters. However, consecutive busy-idle periods frequently showed non-zero correlations, meaning that they are not completely independent in reality and, as such, need to be modeled as correlated random variables.

Figure 8.11 shows the empirical PDF of the considered correlation coefficients for the channels measured within the DCS 1800 DL band with the spectrum analyzer-based platform, which constitutes a representative example of the results obtained for other spectrum 

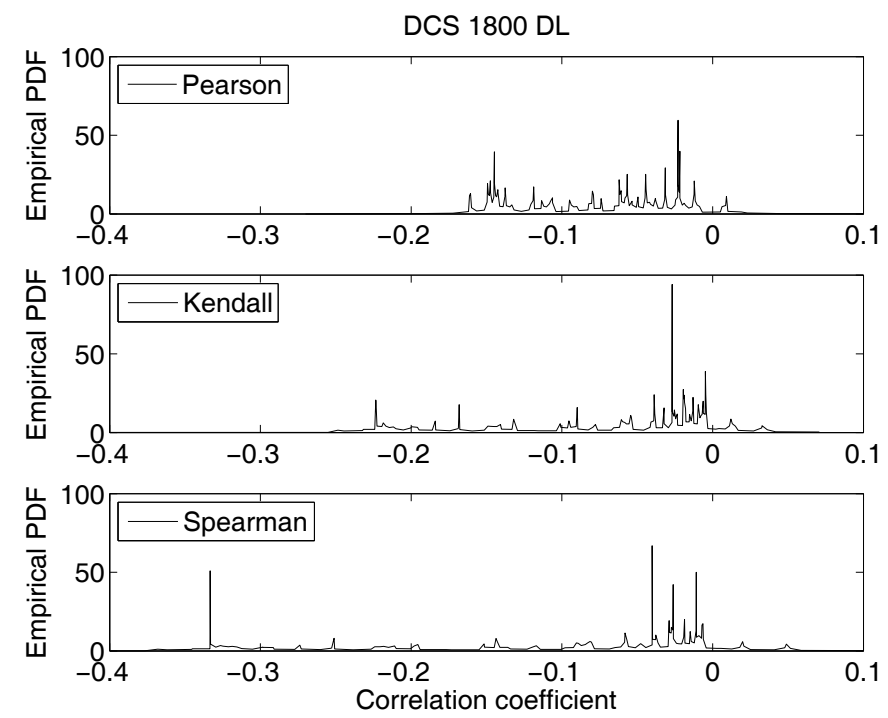

Figure 8.11: Empirical PDF of the correlation coefficients for DCS 1800 DL.

bands. As it can be appreciated, the values obtained for the three considered correlation coefficients are frequently close to zero, meaning that consecutive busy-idle periods are not tightly correlated. However, the correlation values are mostly non-zero and in some particular cases they show noticeable correlation levels. An accurate and realistic model of spectrum usage should therefore take this feature into account. In order to characterize the correlation properties of busy and idle periods, Table 8.15 shows the correlation coefficients observed for some selected spectrum bands. The results provided in Table 8.15 also correspond to field measurements performed with the spectrum analyzer-based platform. Although the number of channels measured with the USRP/GNU Radio platform did not enable the reliably computation of a similar table, it is worth noting that the correlation levels derived from the USRP measurements for individual channels were comprised within the intervals shown in Table 8.15 for each analyzed spectrum band.

It is worth noting in Figure 8.11 and Table 8.15 that empirical correlation coefficients normally take negative values, meaning that when the length of a busy period increases, the length of the next idle period tends to decrease and vice versa. This can be explained by the fact that when the channel load increases, then the fraction of time that it remains in use increases and, as a result, the duration of busy periods increases while idle periods become shorter. On the other hand, the opposite behavior is observed when the channel load decreases (i.e., the length of busy periods decreases and idle periods become longer).

\subsubsection{Autocorrelation of busy and idle periods}

The correlation between the sequence of periods of the same type (either busy or idle) of a channel and a shifted version of itself (i.e., the autocorrelation) was also evaluated based on the considered correlation metrics as a function of the distance (lag) between them. Two dif- 
Table 8.15: Busy-idle correlation coefficients for the measured bands.

\begin{tabular}{|c|c|c|}
\hline Band & Metric & (min; mean; max) \\
\hline \multirow{4}{*}{ TETRA DL } & $\rho\left(T_{0}, T_{1}\right)$ & $(-0.19 ;-0.03 ; 0.14)$ \\
\cline { 2 - 3 } & $\tau\left(T_{0}, T_{1}\right)$ & $(-0.36 ;-0.03 ; 0.13)$ \\
\cline { 2 - 3 } & $\rho_{s}\left(T_{0}, T_{1}\right)$ & $(-0.53 ;-0.04 ; 0.20)$ \\
\hline \multirow{4}{*}{ E-GSM 900 DL } & $\rho\left(T_{0}, T_{1}\right)$ & $(-0.17 ;-0.04 ; 0.01)$ \\
\cline { 2 - 3 } & $\tau\left(T_{0}, T_{1}\right)$ & $(-0.27 ;-0.16 ; 0.01)$ \\
\cline { 2 - 3 } & $\rho_{s}\left(T_{0}, T_{1}\right)$ & $(-0.39 ;-0.24 ; 0.01)$ \\
\hline \multirow{5}{*}{ DES 1800 DL } & $\rho\left(T_{0}, T_{1}\right)$ & $(-0.27 ;-0.07 ; 0.24)$ \\
\cline { 2 - 3 } & $\tau\left(T_{0}, T_{1}\right)$ & $(-0.47 ;-0.08 ; 0.07)$ \\
\cline { 2 - 3 } & $\rho_{s}\left(T_{0}, T_{1}\right)$ & $(-0.64 ;-0.12 ; 0.11)$ \\
\hline & $\rho\left(T_{0}, T_{1}\right)$ & $(-0.15 ;-0.10 ;-0.05)$ \\
\cline { 2 - 3 } & $\tau\left(T_{0}, T_{1}\right)$ & $(-0.13 ;-0.09 ;-0.04)$ \\
\cline { 2 - 3 } & $\rho_{s}\left(T_{0}, T_{1}\right)$ & $(-0.20 ;-0.14 ;-0.06)$ \\
\hline \multirow{5}{*}{ ISM } & $\rho\left(T_{0}, T_{1}\right)$ & $(-0.10 ;-0.05 ;-0.02)$ \\
\cline { 2 - 3 } & $\tau\left(T_{0}, T_{1}\right)$ & $(-0.04 ;-0.01 ; 0.02)$ \\
\cline { 2 - 3 } & $\rho_{s}\left(T_{0}, T_{1}\right)$ & $(-0.05 ;-0.02 ; 0.03)$ \\
\hline
\end{tabular}

ferent autocorrelation behaviors were empirically observed, namely one periodic and another non-periodic. While the periodic behavior was observed in the low time resolution measurements only, the non-periodic behavior was observed in the measurements performed with both the low and high time resolution measurement platforms. As an example, Figure 8.12 shows the autocorrelation function of idle periods as a function of the lag number, $m$, based on the Spearman's correlation coefficient, i.e. $\rho_{s}\left(T_{0}, T_{0} ; m\right)$. Similar trends were observed for busy periods and the rest of correlation metrics.

For channels with non-periodic autocorrelation functions (upper part of Figure 8.12), the correlation coefficient takes its maximum value $\rho_{s}^{\max }$ at $m=1$ and decreases linearly with $m$ until $m=M$, beyond which the correlation is approximately zero. This behavior can adequately be modeled as:

$$
\rho_{s}\left(T_{i}, T_{i} ; m\right)= \begin{cases}1, & m=0 \\ \rho_{s}^{\max }\left(\frac{M-m}{M-1}\right), & 1 \leq m \leq M \\ 0, & m>M\end{cases}
$$

Based on results from field measurements, it was empirically observed that $\rho_{s}^{\max } \in[0.1,0.4]$ and $M \in[2000,8000]$ in the low time resolution measurements, while the high time resolution measurements showed a higher variability with experimental values comprised within the intervals $\rho_{s}^{\max } \in[0.1,0.8]$ and $M \in[200,10000]$ approximately.

For channels with periodic autocorrelation functions (lower part of Figure 8.12) with period $M$, the correlation coefficient can be expressed as the summation of two bell-shaped 

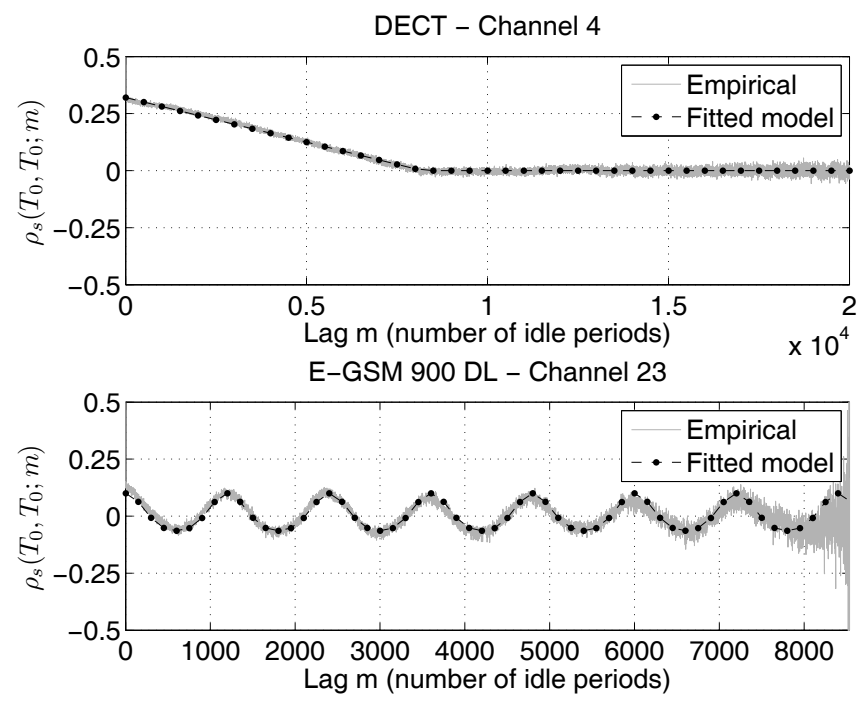

Figure 8.12: Spearman's autocorrelation functions of idle periods.

exponential terms centered at lags $m=1$ and $m=M+1$, with amplitudes $A$ and widths $\sigma$ :

$$
\rho_{s}\left(T_{i}, T_{i} ; m\right)= \begin{cases}1, & m=0 \\ \rho_{s}^{\min }+A e^{-\left(\frac{m-1}{\sigma}\right)^{2}}+A e^{-\left(\frac{m-M-1}{\sigma}\right)^{2}}, & 1 \leq m \leq M\end{cases}
$$

where $\rho_{s}^{\min }$ is the minimum correlation. This behavior was frequently observed in cellular mobile communication systems (and some TETRA channels) where the experienced loads, and thus the busy/period lengths, show a relatively similar and periodic behavior every day. The empirical results derived from the measurements performed with the spectrum analyzer-based platform indicated that $\rho_{s}^{\min } \approx-0.1$ in most cases, $A \in[0.2,0.5], M$ is very approximately equal to the average number of lags equivalent to 24 hours, and $\sigma \approx M / 4$.

As it can clearly be appreciated in Figure 8.12, the models of Equations 8.12 and 8.13 are able to accurately describe the time-domain autocorrelation properties of spectrum usage empirically observed in real radio communication systems.

\subsubsection{Random variate generation principles}

In addition to the models of Equations 8.12 and 8.13, a simulation method to reproduce the time-correlation properties of spectrum usage is also proposed, which will be described in Section 8.9.4. This section provides an overview of some fundamental results and principles from the theory of random variate generation on which such simulation method relies.

\subsubsection{The inversion method}

The inversion method [260, p. 28] can be used to generate random variates with any arbitrary distribution. This method is based on the following property. Let $F(\cdot)$ be a continuous CDF 
on $\mathbb{R}$ with inverse $\mathrm{CDF}$ given by $F^{-1}(\cdot)$. If $U$ is a uniform random variable within the interval $[0,1]$, then the $\mathrm{CDF}$ of $F^{-1}(U)$ is $F(\cdot)$. Moreover, if $X$ is a random variable with $\mathrm{CDF} F(\cdot)$, then $F(X)$ is uniformly distributed on $[0,1]$. Based on this property, any distribution $F(\cdot)$ can be generated based on random variates with uniform or any other distributions.

\subsubsection{Generation of correlated random variates}

If $Y$ and $Z$ are independent and identically distributed (iid) random variables and a new random variable $X$ is defined as:

$$
X=\rho_{0} Y+\sqrt{1-\rho_{0}^{2}} Z
$$

with $\rho_{0} \in[-1,+1]$, then $X$ and $Y$ have a Pearson's correlation coefficient $\rho(X, Y)=\rho_{0}[260$, p. 567]. This property, which can be verified from Equation 8.6, can be used to generate random variates with a specified Pearson's correlation coefficient.

It is worth mentioning that the normal distribution is one of the few distributions that is stable, meaning that a linear combination of two independent variables of such distribution also has the same distribution, up to the location and scale parameters. Therefore, if $Y$ and $Z$ are normally distributed, then $X$ is also normally distributed. Moreover, if $Y$ and $Z$ are standard (zero-mean, unit-variance) normal random variables, then $X$ is also a standard normal random variable.

A sequence $X=x_{1}, x_{2}, \ldots, x_{M}$ of $M$ standard normal random values with specified Pearson's autocorrelation function $\rho(X, X ; m)$ can be generated based on the property:

$$
\mathcal{F}\{\rho(X, X ; m)\}=|\mathcal{F}\{X\}|^{2}
$$

derived from the Wiener-Khinchin theorem, where $\mathcal{F}\{\cdot\}$ denotes the Fourier transform. Subjecting a standard Gaussian process to a linear operation (including filtering) yields another standard Gaussian process with a different autocorrelation function. Thus, an appropriate filter (derived from Equation 8.15) can be used to induce correlation on an uncorrelated Gaussian process. Concretely, if $Y=y_{1}, y_{2}, \ldots, y_{M}$ is a sequence of iid complex standard normal random values, then [261]:

$$
X=\operatorname{Re}\left\{\mathcal{F}^{-1}\{Y \odot \sqrt{|\mathcal{F}\{\rho(X, X ; m)\}|}\}\right\}
$$

is a sequence $X=x_{1}, x_{2}, \ldots, x_{M}$ of standard normal random values with Pearson's autocorrelation function $\rho(X, X ; m)$, where $\odot$ stands for Hadamard's element-wise multiplication.

\subsubsection{Relation among correlation metrics}

For normally distributed random variables $X$ and $Y$, the correlation metrics defined in Equations 8.6-8.8 are related as [262]:

$$
\rho(X, Y)=\sin \left(\frac{\pi}{2} \tau(X, Y)\right)=2 \sin \left(\frac{\pi}{6} \rho_{s}(X, Y)\right)
$$

Equation 8.17 holds if $X$ and $Y$ are normally distributed. If a monotone transformation is applied to $X$ and/or $Y, \tau(X, Y)$ and $\rho_{s}(X, Y)$ will remain unchanged but $\rho(X, Y)$ might not. 


\subsubsection{Simulation of correlation properties}

\subsubsection{Simulation method}

Based on the principles reviewed in Section 8.9.3, a simulation method is proposed in order to reproduce any arbitrary distribution of busy/idle period lengths along with the correlation properties of spectrum usage observed in Section 8.9.2 (see Algorithm 8.1). The proposed algorithm requires as input information the CDF of idle and busy periods, denoted as $F_{0}(\cdot)$ and $F_{1}(\cdot)$ respectively, the Kendall or Spearman correlation coefficient between busy/idle periods, denoted as $\tau\left(T_{0}, T_{1}\right)$ and $\rho_{s}\left(T_{0}, T_{1}\right)$ respectively, as well as the autocorrelation function (periodic or non-periodic) of idle periods in terms of the Kendall or Spearman correlation coefficients as a function of the lag number $m$, i.e. $\tau\left(T_{0}, T_{0} ; m\right)$ or $\rho_{s}\left(T_{0}, T_{0} ; m\right)$ respectively. Notice that the desired correlations to be reproduced need to be specified in terms of the Kendall or Spearman metrics since the algorithm involves some transformations of random variables that would change any specified Pearson's correlation value. The same algorithm can be used to reproduce the autocorrelation properties of busy periods, i.e. $\tau\left(T_{1}, T_{1} ; m\right)$ or $\rho_{s}\left(T_{1}, T_{1} ; m\right)$, instead of idle ones, if desired. However, idle periods represent the real spectrum opportunities for secondary users and modeling their autocorrelation properties results therefore more convenient. The proposed algorithm outputs sequences of period durations for idle periods $\left(T_{0}\right)$ and busy periods $\left(T_{1}\right)$ in blocks of $M$ values.

For periodic idle autocorrelation functions, $M$ corresponds to the function's period and determines the periodicity with which the process is repeated. For non-periodic idle autocorrelation functions, $M$ represents the lag number beyond which autocorrelation is negligible. In such a case, after generating a sequence of $M$ period lengths, a new one is generated based on different (independent) random variates.

First of all, the correlation properties specified in terms of the Kendall or Spearman metrics are converted to their Pearson counterpart based on Equation 8.17 (lines 1 and 2). Afterwards, and for every block of $M$ values, a sequence $\vartheta$ of $M$ iid complex standard normal variates is generated (line 4) and converted, based on Equation 8.16, into a sequence $\xi_{0}$ (line 5) of standard normal variates with autocorrelation function $\rho\left(T_{0}, T_{0} ; m\right)$. A sequence $\chi$ of $M$ iid standard normal variates is generated (line 6) in order to produce, based on Equation 8.14, a sequence $\xi_{1}$ (line 7) that has a correlation $\rho\left(T_{0}, T_{1}\right)$ with $\xi_{0}$. Since $\xi_{0}$ and $\xi_{1}$ are standard normal variates, the new random variables $\Phi\left(\xi_{0}\right)$ and $\Phi\left(\xi_{1}\right)$, where:

$$
\Phi(x)=\frac{1}{2}\left[1+\operatorname{erf}\left(\frac{x}{\sqrt{2}}\right)\right]
$$

is the standard normal CDF, are uniformly distributed. Thus, by the inversion principle, the transformations of lines 8 and 9 produce sequences $T_{0}$ and $T_{1}$ of $M$ period lengths with the desired CDFs. Moreover, since $\xi_{0}$ and $\xi_{1}$ are normally distributed, the desired Kendall and Spearman correlations hold between them as inferred from Equation 8.17. As a result, the monotone transformations of lines 8 and 9 preserve such correlations on $T_{0}$ and $T_{1}$. As a result, this procedure yields a sequence of idle and busy periods, $T_{0}$ and $T_{1}$ respectively, that follow the specified distributions $F_{0}(\cdot)$ and $F_{1}(\cdot)$, where idle periods are characterized by an autocorrelation function $\tau\left(T_{0}, T_{0} ; m\right)$ or $\rho_{s}\left(T_{0}, T_{0} ; m\right)$ and the correlation between busy-idle periods is given by $\tau\left(T_{0}, T_{1}\right)$ or $\rho_{s}\left(T_{0}, T_{1}\right)$. 


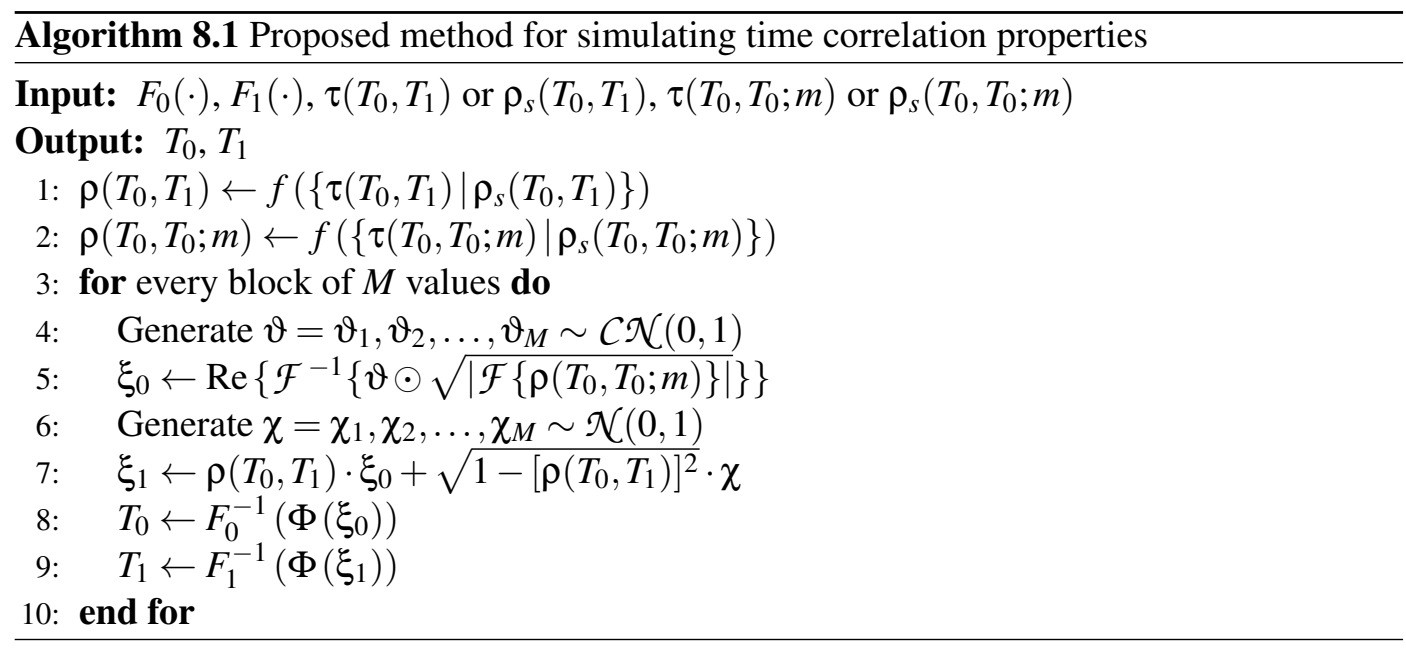

\subsubsection{Validation}

The proposed method was employed to generate sequences of idle/busy periods following generalized Pareto distributions with locations $\mu_{0}=3.5780 \mathrm{~s}$ and $\mu_{1}=3.5150 \mathrm{~s}$, scales $\lambda_{0}=$ 10.9356 and $\lambda_{1}=2.6240$ and shapes $\alpha_{0}=0.1784$ and $\alpha_{1}=0.1884$. All these values were extracted from observations of empirical measurements (see Table 8.4). The algorithm was configured in order to reproduce $\rho_{s}\left(T_{0}, T_{1}\right)=-0.3$ with both non-periodic $\left(\rho_{s}^{\max }=0.25, M=\right.$ $3000)$ and periodic $\left(\rho_{s}^{\min }=-0.1, A=0.4, M=1000, \sigma=M / 4=250\right)$ idle autocorrelation functions. Figure 8.13 shows the results obtained by averaging 10 simulations $\left(\rho_{s}\left(T_{0}, T_{1}\right) \approx\right.$ -0.29 ). As it can clearly be appreciated, the proposed method is able to accurately reproduce not only the specified statistical distributions for both busy and idle periods (shown in Figure 8.13 in terms of the $\mathrm{CCDF}$ ), but also the desired time-correlation properties.

\subsection{Proposed modeling approaches}

As mentioned in Section 8.4, the models derived from high time resolution measurements are useful to accurately describe real channel occupancy patterns at short time scales, while the models obtained from low time resolution measurements can be employed to characterize the spectrum occupancy patterns perceived by DSA/CR nodes that periodically sense the channel and observe its state at discrete time instants. In both cases, the sequence of channel states can be described by a two-state CTSMC model where the idle and busy state holding times are described by two distribution functions $F\left(T_{0}\right)$ and $F\left(T_{1}\right)$ respectively. This section explores two different extensions to this simple modeling approach to simultaneously reproduce the statistical properties of spectrum usage at long (Section 8.7) and short (Section 8.8) time scales along with the correlation properties observed in Section 8.9.

The first proposed method comprises four distribution functions, two of them are used to describe the channel usage pattern in terms of the lengths of inactivity and activity periods at long time scales, $F^{L}\left(T_{0}\right)$ and $F^{L}\left(T_{1}\right)$ respectively, while the other two describe the lengths of idle and busy periods at short time scales, $F^{S}\left(T_{0}\right)$ and $F^{S}\left(T_{1}\right)$ respectively. Ac- 

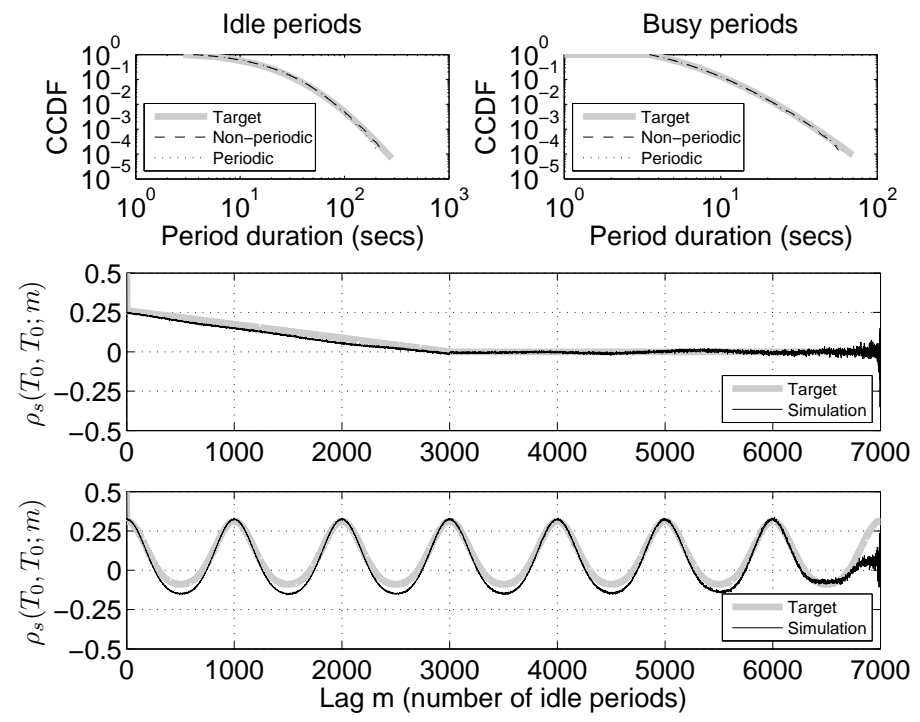

Figure 8.13: Validation of the proposed simulation method.

cording to this modeling approach, and as illustrated in Figure 8.14(a), the distribution function $F^{L}\left(T_{0}\right)$ would be used to model long inactivity periods, while the distribution function $F^{L}\left(T_{1}\right)$ would be used to characterize the length of the periods over which some primary activity exists. During such activity periods, a sequence of idle and busy periods would be present at a shorter time scale as described by the distribution functions $F^{S}\left(T_{0}\right)$ and $F^{S}\left(T_{1}\right)$. The functions $F^{L}\left(T_{0}\right)$ and $F^{L}\left(T_{1}\right)$ can be generalized Pareto distributions as derived from the low time resolution measurements in Section 8.7, while the distribution functions $F^{S}\left(T_{0}\right)$ and $F^{S}\left(T_{1}\right)$ depend on the particular radio technology under study as observed in the results obtained from high time resolution measurements in Section 8.8.

The previous modeling approach is motivated by the following observation. Low time resolution measurement devices, such as spectrum analyzers, constitute a reasonable choice to determine the length of long inactivity periods ${ }^{7}$. It is true that the real state of a channel might change between consecutive channel observations without being detected by the spectrum analyzer. However, this situation can be considered to be rather unlikely in most cases since the effective time period between observations is not excessively long and the lengths of the observed idle periods are significantly larger than those of busy periods and the effective time resolution. Therefore, it is reasonable to assume that the length of the idle periods reported by the spectrum analyzer can be considered as an acceptable estimation of the channel inactivity periods and hence $F^{L}\left(T_{0}\right)$. Spectrum analyzer measurements

\footnotetext{
${ }^{7}$ It is worth noting that the use of high time resolution devices for long measurement periods in order to extract the length of long inactivity periods would lead to huge amounts of data requiring processing times several orders of magnitude above the measurement period, even with current state-of-the-art computers. For example, the USRP/GNU Radio platform generates data files of nearly $500 \mathrm{MB}$ for a measurement period of 60 seconds. Therefore, a measurement period of 7 days as performed with the spectrum analyzer would lead to more than 10000 data files summing to more than $5 \mathrm{~TB}$ of data for every measured channel. This would require several months of computation for every single channel.
} 


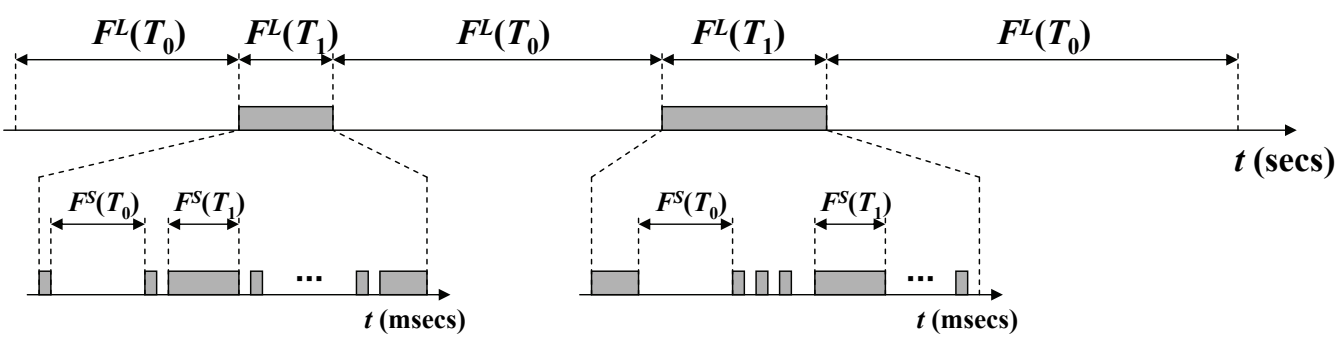

(a)

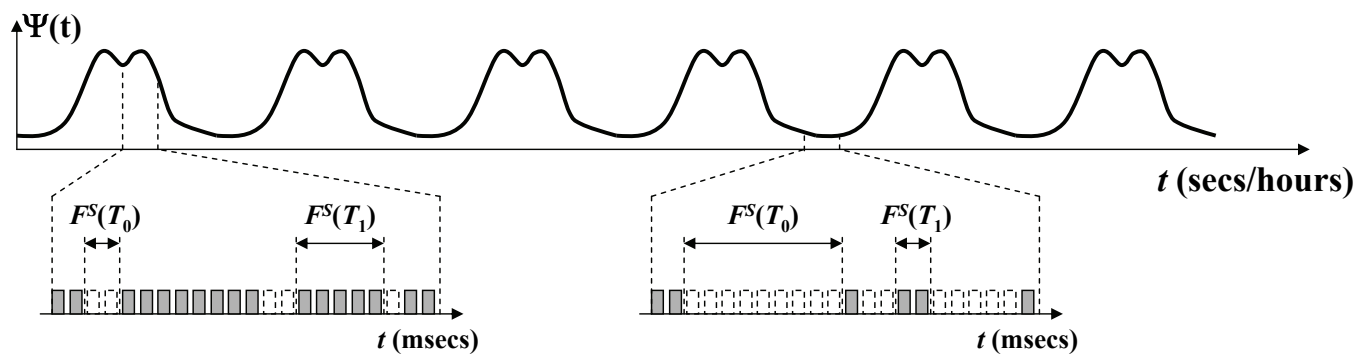

(b)

Figure 8.14: Proposed modeling approaches for spectrum occupancy patterns in the time domain: (a) general modeling approach, (b) modeling approach for cellular mobile communication systems such as GSM/DCS.

can also be employed to determine when a primary transmitter is active and therefore the length of its activity periods $F^{L}\left(T_{1}\right)$, although in this case the length of the real busy and idle periods at short time scales cannot be determined due to the limited time resolution ${ }^{8}$. High time resolution measurements can then be employed to extract the real channel occupancy pattern in terms of busy and idle periods when a primary transmitter is active, i.e. $F^{S}\left(T_{0}\right)$ and $F^{S}\left(T_{1}\right)$. Based on this discussion, the models derived from low and high time resolution measurements can be combined as mentioned above in order to jointly describe the channel occupancy patterns at different time scales.

The above modeling approach is suitable for channels that remain inactive for relatively long periods of time until a transmitter becomes active, in which case a sequence of shorter busy/idle periods follows as depicted in Figure 8.14(a). Based on the channel occupancy patterns observed for various radio technologies, it can be concluded that this modeling approach is appropriate for channels of amateur bands, paging bands, PMR/PAMR bands and

\footnotetext{
${ }^{8}$ The employed spectrum analyzer, with the selected configuration, sweeps at an approximated rate of $25 \mathrm{~ms}$ per megahertz of scanned bandwidth. This means, for instance, that a $200-\mathrm{kHz}$ GSM/DCS channel is observed for a time period of $5 \mathrm{~ms}$ (i.e., more than one GSM/DCS frame) and a 1.728-MHz DECT channel is observed for several DECT frames. If a single or a few time-slots are busy within the frame with a sufficiently high power level the spectrum analyzer will report the carrier frequency as busy. However, the exact time-slot(s) that are actually active cannot be determined.
} 
cordless telephone bands. For channels of cellular mobile communication systems such as E-GSM 900 and DCS 1800, the existence of idle periods lasting for several seconds is rather unlikely. For this particular case, a second modeling approach is proposed. This alternative considers two distribution functions to describe the length of idle and busy periods at short time scales, $F^{S}\left(T_{0}\right)$ and $F^{S}\left(T_{1}\right)$ respectively. The behavior at long time scales is included by means of a DC model that describes the channel load variation over time as illustrated in Figure 8.14(b). The DC models proposed in Section 7.5 for cellular mobile communication systems can be employed to this end. Based on this approach, the parameters of the distribution functions $F^{S}\left(T_{0}\right)$ and $F^{S}\left(T_{1}\right)$ are regularly adjusted according to Equation 8.5 so as to meet the corresponding DC, $\Psi(t)$, at any time. Based on field measurements, it can be concluded that this alternative modeling approach results more appropriate in the case of GSM/DCS systems, where at least a few slots are frequently busy with a periodicity that depends on the load supported by the channel (i.e., the higher the load, the shorter the idle periods and the longer the busy ones and vice versa).

Finally, it is worth noting that the modeling approaches depicted in Figure 8.14 are perfectly compatible with the models and the algorithm proposed in Section 8.9 to capture and reproduce the time correlation properties of spectrum usage. In particular, the modeling approaches of Figure 8.14 determine how the distribution functions $F\left(T_{0}\right)$ and $F\left(T_{1}\right)$ are combined at different time scales or adjusted along time to meet a specified DC. The distribution functions $F\left(T_{0}\right)$ and $F\left(T_{1}\right)$ obtained as a result of the application of such modeling approaches can be combined with the models developed in Section 8.9.2 in analytical studies or used as an input parameter of Algorithm 8.1 in simulation studies. In summary, the models developed in Sections 8.7, 8.8 and 8.9 can be combined in order to account for the statistical properties of spectrum usage at both short and long time scales, along with time correlation features, thus providing a holistic and realistic modeling of spectrum occupancy patterns in real radio communication systems.

\subsection{Summary}

This chapter has analyzed the spectrum occupancy patterns of various radio technologies in the time domain from a continuous-time perspective. The study reported in this chapter has overcome the deficiencies of previous works by performing a comprehensive, systematical and rigorous study on the set of probability distributions that can be employed to accurately describe the lengths of busy and idle periods in real radio communication systems. The study has been based on field measurements with various degrees of time resolution. In general, it has been corroborated that the assumption of exponentially distributed busy and idle periods is invalid, meaning that the CTMC model widely employed in the literature is unrealistic. In real systems, other distributions result more adequate. At long time scales, a single distribution function (generalized Pareto) has been proven to be capable to describe the channel usage patterns for all the considered bands. At short time scales, however, the obtained results indicate that the same distribution does not always provide the best fit, which is a attained by a particular set of distributions depending on the considered radio technology. For time-slotted systems, channel occupancy patterns can also be described from a discrete- 
time viewpoint where period lengths are expressed as an integer number of time-slots. While a probabilistic approach has been proven to be adequate for most radio technologies, it may not be appropriate in some particular cases where channel usage patterns are characterized by a strong deterministic behavior as it has been observed in this study for the DECT system. In such cases, other alternative modeling approaches taking into account technology-specific features at the physical and higher layers may result more convenient. The study has been complemented with an analysis of the time-correlation properties of spectrum occupancy and the development of appropriate mathematical models as well as a simulation algorithm to reproduce such features. Finally, a two-layer modeling approach combining the use of different models at long and short time scales has been proposed as an adequate means to describe the spectrum occupancy patterns observed in real radio communication systems. 




\section{Chapter}

9

\section{TIME-FREQUENCY MODELS}

\subsection{Introduction}

This chapter addresses the problem of jointly modeling spectrum occupancy in the time and frequency domains. Chapters 7 and 8 dealt with the question of modeling and reproducing spectrum occupancy patterns of individual channels in the time domain from discrete- and continuous-time modeling perspectives, respectively. This chapter extends the studies performed in such chapters by including the frequency dimension. The objective is to develop adequate models capable to capture and reproduce the time evolution of the occupancy patterns observed in a group of channels belonging to the same allocated spectrum band. The joint behavior of the set of channels within the same band is a statistical characteristic that should be accurately reproduced by spectrum usage models since it has a direct impact on the amount of consecutive vacant spectrum that a DSA/CR user may find as well as the time period for which spectrum holes can be exploited for opportunistic use. Realistic joint timefrequency models can also provide significant accuracy improvements in the performance evaluation of DSA/CR techniques such as, for example, adaptive and MAC-layer spectrum sensing algorithms, where DSA/CR nodes operate over a set of channels dynamically and the decisions made by every DSA/CR node may depend on the instantaneous state of the set of sensed channels. In this context, this chapter analyzes the statistical time-frequency characteristics of spectrum usage based on empirical data from extensive spectrum measurement campaigns. Based on the obtained results, adequate models are proposed. Moreover, a sophisticated procedure is developed in order to generate artificial spectrum occupancy data for simulation or other purposes. The proposed method is able to accurately capture and reproduce the statistical time-frequency characteristics of spectrum usage in real systems.

The rest of this chapter is organized as follows. First, Section 9.2 provides a brief overview of previous related works and their contributions. Afterwards, Section 9.3 describes the measurement setup and methodology considered in this study while Section 9.4 
presents the considered system model. The statistical properties of spectrum usage are then explored, first considering the time and frequency dimensions from a joint perspective in Section 9.5 and then focusing on the frequency dimension in Section 9.6. Based on the observed properties, adequate models are developed for each case. Section 9.7 discusses the applicability of the developed models and proposes a simulation method relying on such models to generate artificial spectrum data for simulation purposes or other studies. Finally, Section 9.8 summarizes and concludes the chapter.

\subsection{Previous work}

Although the work in this field is not as abundant as in the case of models for the time domain, several studies reported in the literature have addressed the problem of jointly characterizing and modeling spectrum occupancy in the time and frequency dimensions.

A joint time-frequency study is presented in [263, 264], where the continuous-time distribution of the Opportunity Time (OT) across several frequency channels is analyzed. In such study, the OT is defined as the time period elapsed between the instance when an unused channel is first available (idle) over the considered spectrum band to the instance when all channels just become unavailable for secondary usage (busy). It is found in [263, 264] that the theoretical PDF of the OT is hyper-exponentially distributed when the primary radio activities in each channel follow independent and exponentially distributed busy/idle processes. The study is extended in [265] by considering heavy-tailed distributions, more concretely Pareto and hyper-Erlang distributions for busy and idle periods, respectively. In such a case, it is found that the resulting OT PDF can be described by a gamma distribution when the primary radio activities in each channel are statistically identical and a hyper-gamma distribution in the case of statistically non-identical primary radio activities.

The study shown in [266] considers a multi-state Discrete-Time Markov Chain (DTMC) incorporating the frequency dimension. Assuming that a primary band is divided into $n$ radio channels, the DTMC model is composed of $n+1$ states, where one state is employed to represent the inactivity of the DSA/CR user due to the unavailability of transmission opportunities (the $n$ primary channels are busy), while the other $n$ states represent the opportunistic transmission of the DSA/CR user in one of the $n$ channels. Note that this DTMC model is not a primary activity model in strict sense (i.e., a channel occupancy model), but rather a secondary activity model, which is related to the former but out of the scope of this study.

Another related work is the study presented in [218], where a parametric adaptive spectrum sensing framework is proposed and evaluated based on a Continuous-Time Markov Chain (CTMC) with exponentially distributed busy periods and assuming that the mean occupancy times are uniformly distributed among the channels of the same spectrum band.

The study carried out in [226] is probably the most directly related to the objectives of this chapter. Based on field measurements, [226] analyzes the statistical properties of spectrum usage in the time and frequency domains. However, the characteristics of both domains are treated and studied in a rather isolated manner, without explicit consideration of the simultaneous time evolution of a group of channels. By contrast, this chapter performs an exhaustive analysis that takes into account this aspect. 


\subsection{Measurement setup and methodology}

The study performed in this chapter relies entirely on field measurements performed with the spectrum analyzer measurement platform discussed in Chapter 2 and Appendix A. Although the USRP/GNU Radio platform presented in Chapter 4 was initially considered as well, the spectrum analyzer-based platform was finally selected as discussed below.

On one hand, the limited bandwidth capabilities of the USRP hardware $(8 \mathrm{MHz}$ maximum) do not allow to measure all the channels within an entire spectrum band simultaneously. In principle, this limitation could be overcome to some extent by sweeping the desired band in frequency. For example, a $80-\mathrm{MHz}$ band could be scanned by dividing it into 10 chunks of $8 \mathrm{MHz}$ each and then measuring each chunk consecutively, tuning the USRP hardware to the center frequency of the new chunk every time the analysis of the previous frequency chunk is completed. On other hand, a spectrum analyzer can handle several tens and hundreds of megahertz simultaneously and can therefore measure complete bands. Nevertheless, in strict sense, the observations of different channels within the same band are not simultaneous due to the swept nature of spectrum analyzers. In spite of this, and for the bandwidths of the spectrum bands analyzed in this study, the spectrum analyzer in general performs better than sweeping the USRP in frequency as explained below.

The spectrum analyzer employed in this study provides an effective sweeping speed of about $25 \mathrm{~ms}$ per megahertz of scanned bandwidth, which is automatically adjusted based on the selected configuration parameters to the fastest speed that is able to provide reliable results. The USRP hardware, after tuning to a new center frequency, needs some transitory time before providing valid signal samples, which is caused by many delays along the digitization path (RF synthesizer settling time, FPGA processing time and USB transferring time). This means that after tuning to a new frequency it is necessary to drop the first incoming signal samples received over a specified period of time, which depends on the particular USRP daughter board. For example, for the TVRX board the minimum settling time for the RF synthesizer is around $100 \mathrm{~ms}$. Taking into account the bandwidths of the considered bands, the spectrum analyzer platform provides in general shorter effective sweeping times over frequency. Given the objectives of this study, the spectrum measurements need to provide the shortest possible difference among the time instants at which the state of the channels of a band are observed. As a result, the spectrum analyzer platform was employed to perform the measurements on which the study presented in this chapter relies.

The spectrum analyzer was employed to record the spectral activity in the same spectrum bands studied in Chapters 7 and 8 (see Table 7.1) and considering the same measurement locations, namely a building rooftop (see location 1 in Figure 3.1) and a room inside the same building (see location 2 in Figure 3.1). Based on the configuration shown in Table 3.1, each band was measured across a time span of 7 days, from Monday midnight to Sunday midnight. The captured data were used to extract the binary channel occupancy patterns from the measured channels by classifying the spectrum analyzer's power samples as either busy or idle states. To this end, the CED scheme described in Section 6.2 was employed to process the spectrum data on a power-sample basis, as in Chapters 7 and 8 . The resulting binary sequences of busy and idle states for each individual channel were compared and analyzed as detailed in the following sections. 


\subsection{System model}

The considered system model assumes that the DSA/CR network operates over a set of $C$ radio channels, denoted as $\Upsilon=\left\{v_{1}, v_{2}, \ldots, v_{c}, \ldots, v_{C}\right\}$. Let's denote as $\mathbb{S}=\left\{s_{0}, s_{1}\right\}$ the state space for a primary radio channel, where $s_{0}$ indicates that the channel is idle and $s_{1}$ that the channel is busy. The state of the $C$ channels is simultaneously observed at discrete time instants $t=t_{k}=k T_{s}$, where $k$ is a non-negative integer and $T_{s}$ is the time period elapsed between two consecutive observations. Assuming that the set of channels $\Upsilon$ is observed for a time period $K T_{s}$, each channel is characterized by a binary occupancy sequence $\left\{S_{c}\left(t_{1}\right), S_{c}\left(t_{2}\right), \ldots, S_{c}\left(t_{k}\right), \ldots, S_{c}\left(t_{K}\right)\right\}$, where $S_{c}\left(t_{k}\right)$ denotes the state of channel $v_{c}$ at time instant $t_{k}$ and it can either be $S_{c}\left(t_{k}\right)=s_{0}$ or $S_{c}\left(t_{k}\right)=s_{1}$.

\subsection{Statistical time-frequency characteristics of spectrum usage}

The aim of this section is to analyze the statistical properties of spectrum usage in the time and frequency dimensions from a joint perspective. Concretely, the first objective is to determine whether the binary time-occupancy pattern of a radio channel depends on other channels within the same band or, on the contrary, the individual channel occupancy patterns are mutually independent. In case of dependence, the second objective is to adequately model dependence relations. To this end, several metrics based on correlations and probabilistic properties are employed in order to assess the degree to which the occupancy patterns of a group of channels is mutually related.

\subsubsection{Correlation metrics}

The dependence between the occupancy patterns of any two channels, $v_{i}$ and $v_{j}$, can be evaluated by means of the correlation metrics defined in Section 8.9.1 [260], namely the Pearson's product-moment correlation coefficient $\rho\left(v_{i}, v_{j}\right)$, the Kendall's rank correlation coefficient $\tau\left(v_{i}, v_{j}\right)$ and the Spearman's rank correlation coefficient $\rho_{s}\left(v_{i}, v_{j}\right)$. If the patterns are independent, the correlation coefficients must be zero (or approximately zero), although the converse is not true in general. This means that non-zero correlations in empirical data would imply the existence of some dependence relations, which could be characterized by means of the considered correlation coefficients.

In order to apply such correlation metrics to channel occupancy sequences, the idle and busy states of every channel need to be represented by numerical values. To this end, the equivalences $s_{0} \equiv 0$ and $s_{1} \equiv 1$ are employed. Taking into account these equivalences, the following correlation matrix can be computed:

$$
\mathbf{R}=\left[r_{i j}\right]_{C \times C}=\left[\begin{array}{cccc}
r_{11} & r_{12} & \cdots & r_{1 C} \\
r_{21} & r_{22} & \cdots & r_{2 C} \\
\vdots & \vdots & \ddots & \vdots \\
r_{C 1} & r_{C 2} & \cdots & r_{C C}
\end{array}\right]
$$


where $r_{i j}(i, j=1,2, \ldots, C)$ represents the correlation between the binary occupancy sequences of channels $v_{i}$ and $v_{j}$. The elements of $\mathbf{R}$ could be computed based on the Pearson, Kendall and Spearman correlation coefficients, i.e. $r_{i j}=\rho\left(v_{i}, v_{j}\right), r_{i j}=\tau\left(v_{i}, v_{j}\right)$ and $r_{i j}=\rho_{s}\left(v_{i}, v_{j}\right)$, thus leading to correlation matrices $\mathbf{R}_{\rho}=\left[\rho\left(v_{i}, v_{j}\right)\right]_{C \times C}, \mathbf{R}_{\tau}=\left[\tau\left(v_{i}, v_{j}\right)\right]_{C \times C}$ and $\mathbf{R}_{\rho_{\mathrm{s}}}=\left[\rho_{s}\left(v_{i}, v_{j}\right)\right]_{C \times C}$, respectively. For binary sequences, however, the three correlation coefficients are equivalent and provide the same results (i.e., the computation of anyone of them is enough). Notice that $\mathbf{R}$ is a symmetric matrix since $r_{i j}=r_{j i}$ for all $i, j$. Moreover, the main diagonal of $\mathbf{R}$ is composed of ones (i.e., $r_{i j}=1$ for $i=j$ ) since the correlation of a sequence with an identical copy of itself is equal to one. The rest of elements of $\mathbf{R}$ (i.e., $r_{i j}$ for $i \neq j$ ) will enable the correlation between all possible pairs of channels to be determined.

\subsubsection{Correlation results}

Based on spectrum analyzer measurements, the matrix of Equation 9.1 was computed for various spectrum bands (see Table 7.1). Figure 9.1 shows, as an example, the results obtained for the TETRA DL band, where the elements of $\mathbf{R}$ are plotted as a function of the channel indexes $i$ and $j$. This figure is also representative of the results obtained for the rest of analyzed spectrum bands. It can be appreciated, as expected, that the matrix is symmetric and its main diagonal is composed of ones. More importantly, it can also be observed that most channel pairs show a correlation coefficient equal (or very close) to zero, except for a few particular isolated cases for which significant correlation levels are observed. The number of such cases, however, is not relevant with respect the overall number of possible channel pairs. It is also interesting to mention the existence of a region (around channel indexes 200 and 250) where the observed correlation levels, although not specially significant, are appreciably higher than in the rest of cases. The mentioned region is very close to the main diagonal, meaning that such correlation levels are observed between adjacent or nearby channels. It is also worth noting that this circumstance was not observed for other spectrum bands. Except for these particular cases, the rest of values of matrix $\mathbf{R}$ indicate that the correlation level between the occupancy patterns of any two channels within a spectrum band is in general approximately equal to zero. It is important to highlight, however, that this observation is not enough to draw any conclusions on independence since independence implies null correlation, but the opposite is not true in general. The probabilistic metrics discussed in Section 9.5.3 will determine conclusively whether the occupancy patterns for channels within a spectrum band can be considered to be mutually independent in practice.

\subsubsection{Probabilistic metrics}

The probabilistic metric defined in this section is based on the fact that two events $A$ and $B$ are independent if and only if their joint probability $P(A, B)$ equals the product of their individual probabilities $P(A)$ and $P(B)$, i.e. $P(A, B)=P(A) \cdot P(B)$. Based on this simple result from the basic theory of probability, the independence between the occupancy patterns of a pair of channels can be determined as follows. First, compute the elements of matrix $\mathbf{P}=\left[p_{i j}\right]_{C \times C}$, where $p_{i j}=P\left(S_{i}\left(t_{k}\right)=s_{1}, S_{j}\left(t_{k}\right)=s_{1}\right)$ represents the joint probability that channels $v_{i}$ and $v_{j}$ are simultaneously observed as busy at any time instant $t_{k}$. Then, compute the elements 


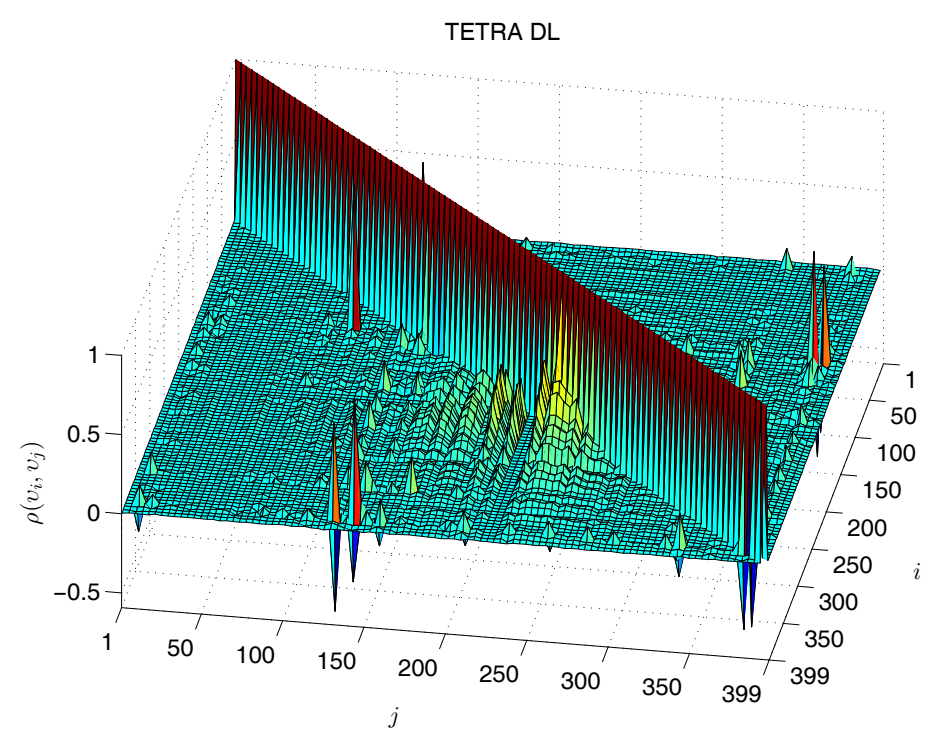

Figure 9.1: Elements of matrix R for TETRA DL based on Pearson's correlation coefficient.

of matrix $\mathbf{Q}=\left[q_{i j}\right]_{C \times C}$, where:

$$
q_{i j}= \begin{cases}\Psi_{i} \cdot \Psi_{j}, & i \neq j \\ \Psi_{i}=\Psi_{j}, & i=j\end{cases}
$$

where $\Psi_{i}$ and $\Psi_{j}$ represent the DC of channels $v_{i}$ and $v_{j}$, respectively. Finally, compute the difference between both matrices. If $\mathbf{P}-\mathbf{Q}=\mathbf{0}_{C \times C}$, where $\mathbf{0}_{C \times C}$ denotes a $C \times C$ square matrix whose elements are all zero, then the occupancy patterns of channels within the same band are mutually independent. However, if an appreciable number of elements is non-zero, then this would imply that independence is not a completely realistic assumption.

The proposed procedure is justified as follows. The elements of $\mathbf{P}$ can be expressed as $p_{i j}=P\left(S_{i}\left(t_{k}\right)=s_{1} \mid S_{j}\left(t_{k}\right)=s_{1}\right) \cdot P\left(S_{j}\left(t_{k}\right)=s_{1}\right)$. If the occupancy patterns of channels $v_{i}$ and $v_{j}$ are independent, then it must be true that $P\left(S_{i}\left(t_{k}\right)=s_{1} \mid S_{j}\left(t_{k}\right)=s_{1}\right)=P\left(S_{i}\left(t_{k}\right)=s_{1}\right)$ and in such a case $p_{i j}=P\left(S_{i}\left(t_{k}\right)=s_{1}\right) \cdot P\left(S_{j}\left(t_{k}\right)=s_{1}\right)$. Notice that the terms of the last equality represent the probability that the channels are observed as busy or in other words their DCs, i.e. $P\left(S_{i}\left(t_{k}\right)=s_{1}\right)=\Psi_{i}$ and $P\left(S_{j}\left(t_{k}\right)=s_{1}\right)=\Psi_{j}$. Therefore, if the occupancy patterns of channels $v_{i}$ and $v_{j}$ are independent, it holds that $p_{i j}=q_{i j}$ and therefore $p_{i j}-q_{i j}=0$. Notice, however, that this is only true for $i \neq j$ since the elements of the main diagonal of $\mathbf{P}$ are given by $p_{i i}=P\left(S_{i}\left(t_{k}\right)=s_{1}, S_{i}\left(t_{k}\right)=s_{1}\right)=P\left(S_{i}\left(t_{k}\right)=s_{1}\right)=\Psi_{i}$, which requires a particular definition of $q_{i j}$ in Equation 9.2 for $i=j$ in order to guarantee that the equality $\mathbf{P}-\mathbf{Q}=\mathbf{0}_{C \times C}$ holds for all elements in case of independence. 


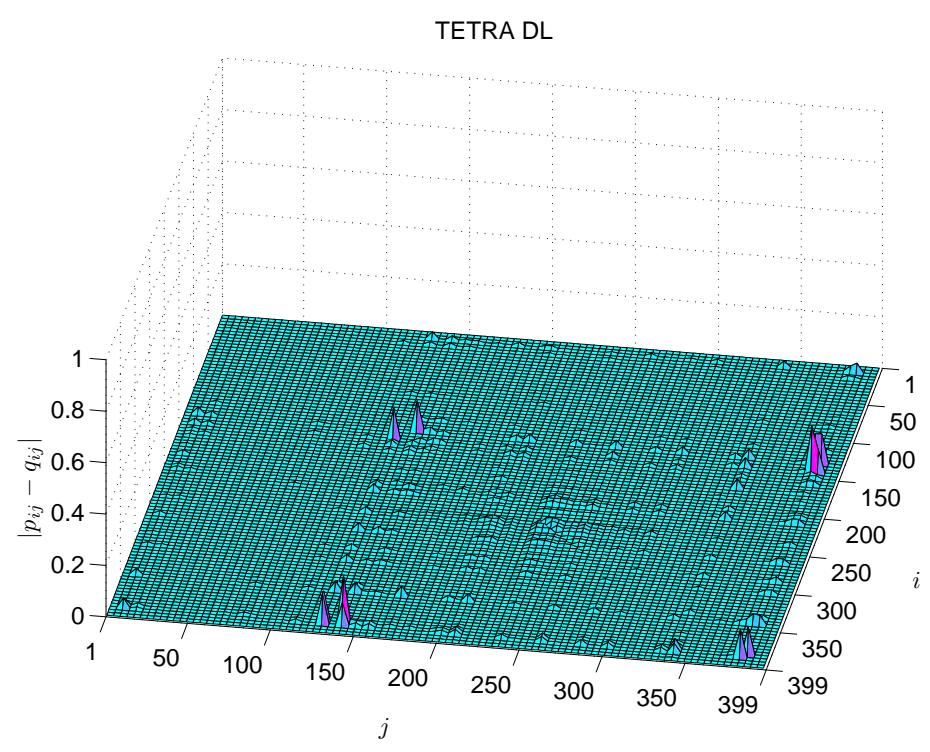

Figure 9.2: Elements of matrix abs $(\mathbf{P}-\mathbf{Q})$ for TETRA DL.

\subsubsection{Probabilistic results}

Matrices $\mathbf{P}$ and $\mathbf{Q}$ were estimated based on field measurements performed with the spectrum analyzer measurement platform. The elements of $\mathbf{P}$ were computed as the number of observations with busy states in each pair of channels divided by the total number $K$ of observations. The elements of $\mathbf{Q}$ were derived based on the empirical DCs of each individual channel, obtained as the quotient between the number of observations with busy state and the total number $K$ of observations. The difference between both matrices was computed for various spectrum bands. Figure 9.2 shows, as an example, the results obtained for the TETRA DL band, where the absolute values of the elements of the resulting difference matrix are plotted as a function of the channel indexes $i$ and $j$. This figure is also representative of the results obtained for the rest of analyzed spectrum bands. As it can be appreciated, the difference matrix is composed of zeros for all channel pairs, except for the same particular cases for which appreciable correlation levels were observed in Figure 9.1. The number of such cases, as previously mentioned, is not relevant with respect the overall number of possible channel pairs. As a result, and based on Figure 9.2, it can be concluded that the occupancy patterns for channels within a spectrum band can be considered to be mutually independent. This is a result with important implications for joint time-frequency modeling, since it implies that the instantaneous occupancy state of a channel is unrelated to the instantaneous state of the rest of channels within the considered band and, consequently, the occupancy patterns of a group of channels can be modeled independently of each other. On one hand, this enables the direct application of the time-domain models developed in Chapters 7 and 8 without any modifications or additional considerations. On the other hand, this enables the statistical properties of spectrum usage over frequency to be analyzed and studied independently of the time-dimension statistics, which is performed in Section 9.6. 


\subsection{Statistical characteristics of spectrum usage over frequency}

The results obtained in Section 9.5 indicate that the occupancy patterns of a set of channels within the same band can be modeled independently of each other. Nevertheless, there are some frequency aspects that need to be taken into account for a realistic and accurate timefrequency modeling of spectrum usage. Concretely, and after extensive analysis of empirical measurement data, two aspects deserving explicit consideration were observed. The first one is the probability distribution of DC values for channels within the same band. The second one is the DC clustering over frequency, i.e. the existence of groups of contiguous channels with similar DC values. Both aspects are discussed in detail in the following sections.

\subsubsection{Duty cycle distribution}

Given the set $\Psi=\left\{\Psi_{1}, \Psi_{2}, \ldots, \Psi_{c}, \ldots, \Psi_{C}\right\}$, where $\Psi_{c}$ is the DC of channel $v_{c}$, the probability distribution of the elements of $\boldsymbol{\Psi}$ was computed for all the analyzed bands and compared to various models. It was found that the empirical DC CDFs can accurately be fitted with the beta [210] and Kumaraswamy [244] distributions. The CDF for the former is given by:

$$
F_{B}(x ; \alpha, \beta)=I_{x}(\alpha, \beta)=\frac{B_{x}(\alpha, \beta)}{B(\alpha, \beta)}, \quad x \in(0,1)
$$

where $\alpha>0$ and $\beta>0$ are shape parameters, $I_{x}(\alpha, \beta)$ is the regularized incomplete beta function [201, 6.6.2], $B_{x}(\alpha, \beta)$ is the incomplete beta function given by [201, 6.6.1]:

$$
B_{x}(\alpha, \beta)=\int_{0}^{x} z^{\alpha-1}(1-z)^{\beta-1} d z
$$

and $B(\alpha, \beta)$ is the beta function [201, 6.2.1] given by Equation 9.4 with $x=1$ (see Equation 7.22). The CDF for the latter is given by:

$$
F_{K}(x ; a, b)=1-\left(1-x^{a}\right)^{b}, \quad x \in(0,1)
$$

where $a>0$ and $b>0$ are shape parameters.

As mentioned in Section 7.6, the beta distribution is a well-known and widely used distribution that can be found in many popular software simulation packages. However, it may present some difficulties in analytical studies due to the complex expression of its CDF. The Kumaraswamy distribution is similar to the beta distribution, but easier to use in analytical studies due to its simpler form [245]. Therefore, while the former may be more appropriate for simulations, the latter may be more convenient for analytical studies.

Figure 9.3 shows some examples of empirical DC distributions and their corresponding beta and Kumaraswamy fits. The selected bands represent examples for a wide variety of load levels, including very low (E-GSM 900 UL), low (DECT), medium (ISM) and very high (E-GSM 900 DL) average band DCs. As it can be appreciated, the beta and Kumaraswamy distributions are able to provide reasonably accurate fits in all cases. In order to facilitate the application of these models, Table 9.1 provides some reference values for the parameters of these distributions, extracted from empirical measurement data by means of MLE techniques, along with the average band DCs, computed as $\bar{\Psi}=(1 / C) \sum_{c=1}^{C} \Psi_{c}$. 


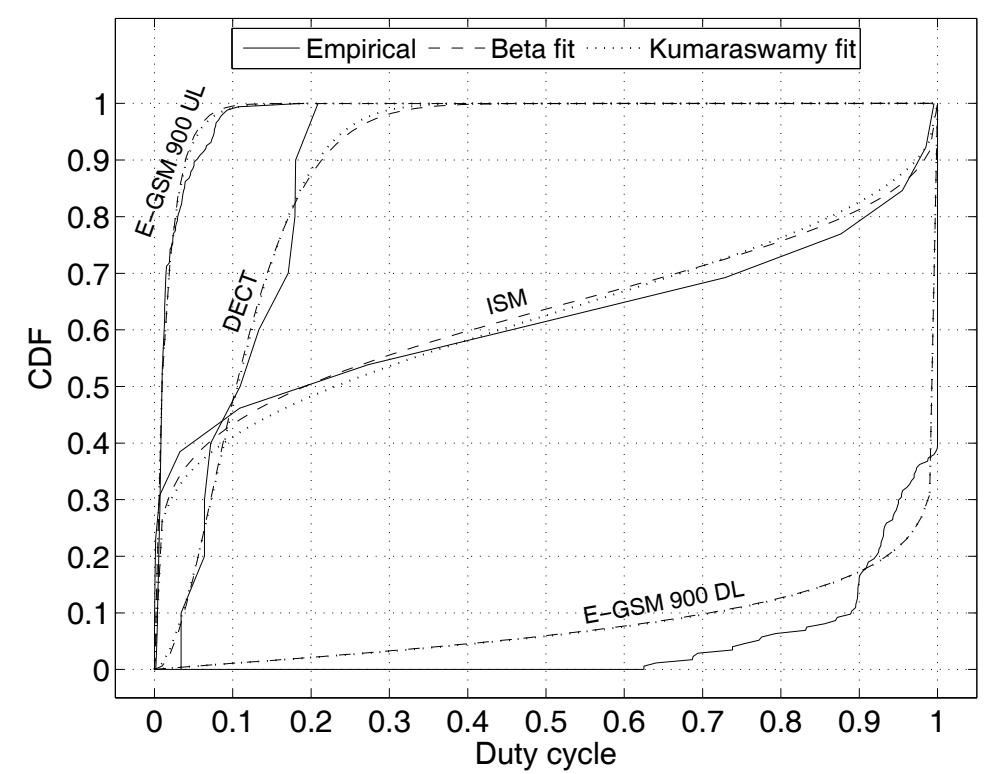

Figure 9.3: Empirical DC distributions and their corresponding beta and Kumaraswamy fits.

Table 9.1: Fitted values for the Beta and Kumaraswamy DC distributions.

\begin{tabular}{|c|c|c|c|c|c|}
\hline \multirow{2}{*}{$\begin{array}{c}\text { Measured } \\
\text { band }\end{array}$} & \multirow{2}{*}{$\begin{array}{c}\text { Average band } \\
\text { duty cycle } \bar{\Psi}\end{array}$} & \multicolumn{2}{|c|}{ Beta } & \multicolumn{2}{c|}{ Kumaraswamy } \\
\cline { 3 - 6 } & 0.17 & 0.5796 & 2.8963 & 0.6311 & 2.5599 \\
\hline Amateur & 0.28 & 1.4867 & 3.9601 & 1.3449 & 4.2382 \\
\hline Paging & 0.03 & 0.7105 & 44.0554 & 0.7849 & 26.9302 \\
\hline TETRA UL & 0.36 & 0.1840 & 0.2837 & 0.1389 & 0.4223 \\
\hline TETRA DL & 0.02 & 1.6044 & 116.6408 & 1.2690 & 208.5805 \\
\hline E-GSM 900 UL & 0.96 & 0.9119 & 0.0778 & 0.8970 & 0.0786 \\
\hline E-GSM 900 DL & 0.02 & 0.2023 & 6.0738 & 0.2545 & 2.6118 \\
\hline DCS 1800 UL & 0.44 & 0.4525 & 0.6118 & 0.4463 & 0.6846 \\
\hline DCS 1800 DL & 0.12 & 2.3217 & 17.5170 & 1.7434 & 34.2432 \\
\hline DECT & 0.42 & 0.2022 & 0.3418 & 0.1426 & 0.4155 \\
\hline ISM & & & & & \\
\hline
\end{tabular}




\subsubsection{Duty cycle clustering}

The second aspect of interest of spectrum occupancy in the frequency domain is the DC clustering over frequency, i.e. the existence of groups of contiguous channels with similar DC values. The analysis of empirical measurement data indicated that channels with similar load levels rarely occur alone, but in groups of certain size. Clearly, this aspect is a statistical property that a realistic and accurate model needs to capture and reproduce because of its direct impact on the amount of consecutive vacant spectrum that a secondary user may find.

In order to analyze and characterize the DC clustering over frequency, a set of five DC archetypes was defined, namely very low $\Psi \in[0,0.05]$, low $\Psi \in(0.05,0.40]$, medium $\Psi \in(0.40,0.60]$, high $\Psi \in(0.60,0.95]$ and very high $\Psi \in(0.95,1]$ levels. The DCs observed in the analyzed spectrum bands were computed and classified into the aforementioned archetypes. Figures 9.4 and 9.5 show some examples for the TETRA UL and TETRA DL bands, respectively. In each figure, the upper graph shows the instantaneous spectrum occupancy for each channel for a time period of 60 minutes (white/black points indicate idle/busy observations, respectively), while the lower graph shows the channel DCs and their corresponding classification into the considered archetypes (each archetype is represented by a different color). As it can clearly be appreciated, channels with similar occupancy levels appear together in blocks of certain size, i.e. the DC is clustered in the frequency domain. It is worth noting that this behavior was also observed for the rest of analyzed spectrum bands. To statistically characterize this aspect, the number of contiguous channels within each cluster (i.e., group of channels belonging to the same DC archetype) was evaluated for each measured spectrum band. The probability distribution of the resulting cluster sizes was evaluated and compared to several discrete models, from which it was observed that the empirical DC CDFs can accurately be fitted with a shifted version of the geometric distribution [210] (see Table 8.13), whose CDF is given by:

$$
F_{\text {Geom }}(k ; p)=1-(1-p)^{k}, \quad k \in \mathbb{N}^{*}=\{1,2,3, \ldots\}
$$

where $k$ represents the number of channels belonging to the same group (i.e., the cluster size) and $1 / p(0 \leq p \leq 1)$ represents its expected (mean) value, i.e. $\mathbb{E}\{k\}=1 / p$.

Figure 9.6 shows some examples of empirical distributions of the number of channels per cluster and their corresponding geometric fits. Since the number of clusters within each band is significantly low, the distribution fitting does not provide highly reliable results but clearly indicates that the geometric distribution provides reasonably accurate fits. In order to facilitate the application of this model, Table 9.2 provides some reference values for the parameter $p$ of the geometric distribution, extracted from empirical measurement data as the inverse of the average number of channels per cluster. The value of the parameter $p$ can be set following other alternative methods. For example, if a particular average number of channels per cluster, $M$, needs to be reproduced, then it can be configured as $p=1 / M$. Moreover, it was empirically observed that the relation $p \approx C \cdot 10^{-3}$, with $C$ being the number of channels, holds for many of the analyzed spectrum bands, which can also be used to select the value of the geometric distribution parameter as long as the resulting value satisfies $p \leq 1$. Another option would be to draw the value of $p$ from a uniform distribution in $[0.1,0.6]$. 

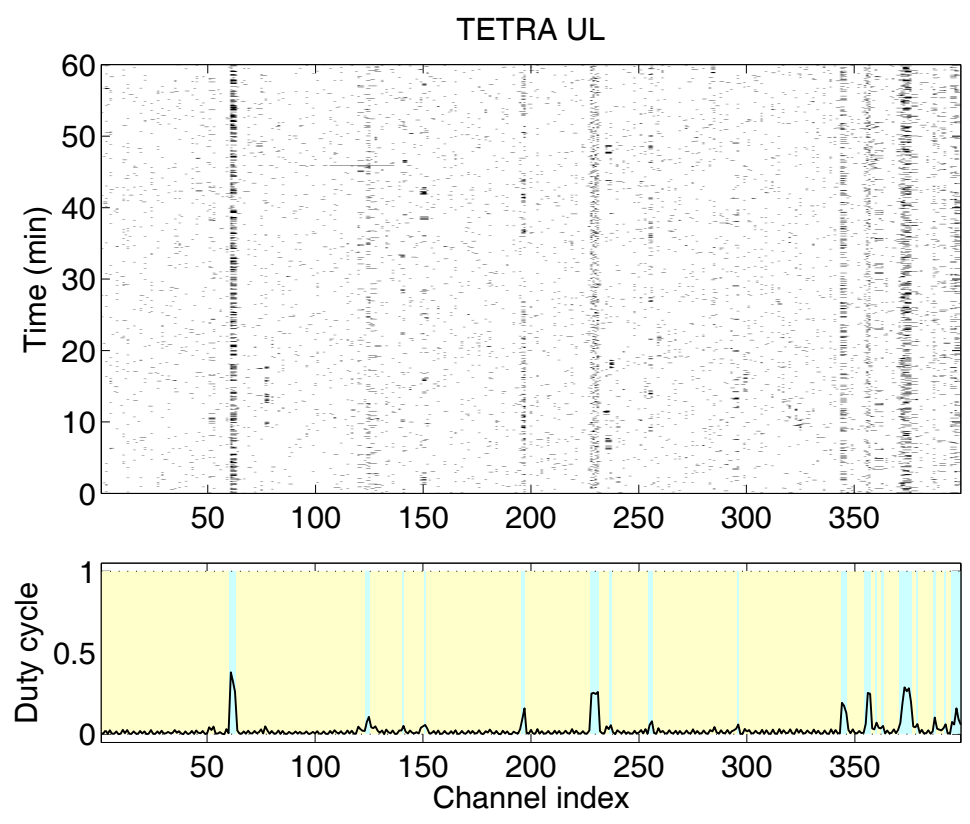

Figure 9.4: Empirical spectrum data for the TETRA UL band.
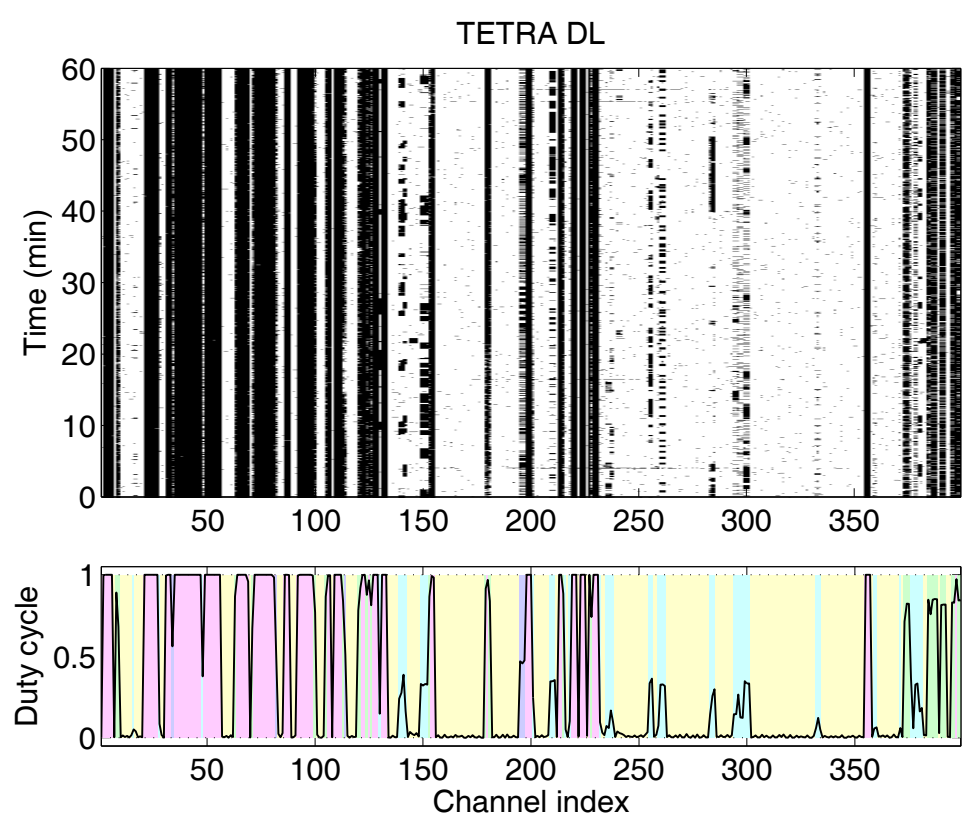

Figure 9.5: Empirical spectrum data for the TETRA DL band. 

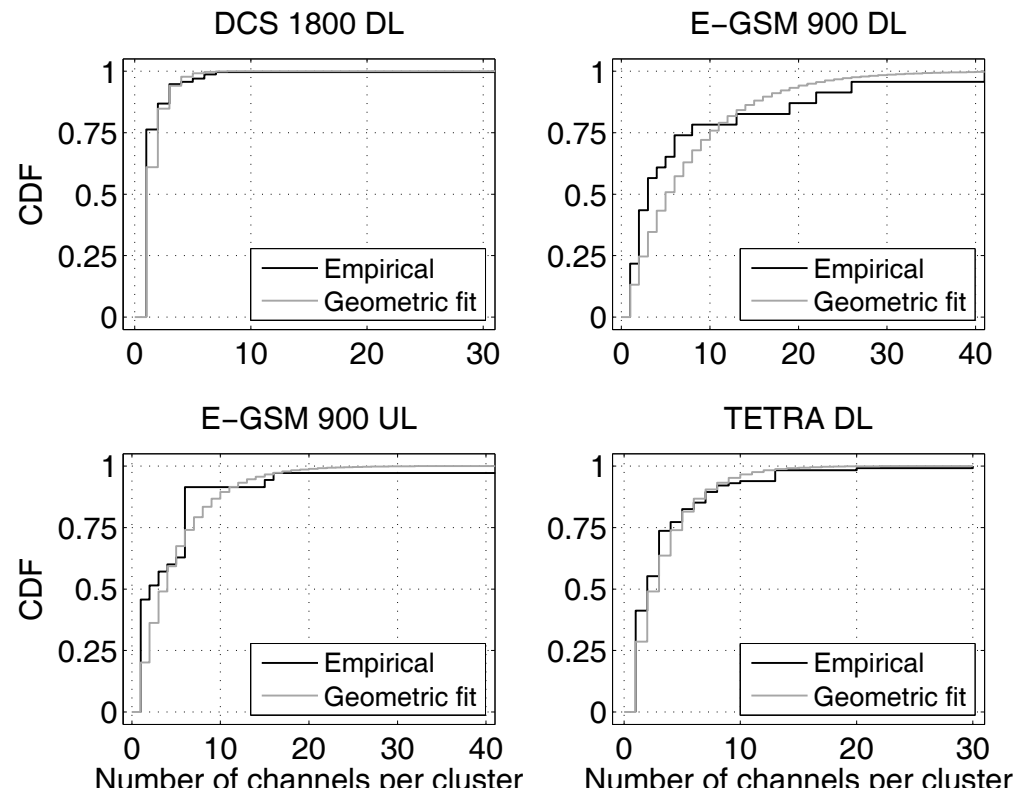

Figure 9.6: Empirical distributions of the number of channels per cluster and their corresponding geometric fits.

Table 9.2: Fitted values for the geometric distribution of number of channels per cluster.

\begin{tabular}{|c|c|}
\hline $\begin{array}{c}\text { Measured } \\
\text { band }\end{array}$ & $\begin{array}{c}\text { Parameter } \boldsymbol{p} \text { of the } \\
\text { geometric distribution }\end{array}$ \\
\hline Amateur & 0.5625 \\
\hline Paging & 0.3491 \\
\hline TETRA UL & 0.0752 \\
\hline TETRA DL & 0.2857 \\
\hline E-GSM 900 UL & 0.2011 \\
\hline E-GSM 900 DL & 0.1322 \\
\hline DCS 1800 UL & 0.3824 \\
\hline DCS 1800 DL & 0.6096 \\
\hline DECT & 0.2000 \\
\hline ISM & 0.3846 \\
\hline
\end{tabular}




\subsection{Applicability of the models and simulation method}

The study carried out in Sections 9.5 and 9.6 reveals three important conclusions on the statistical properties of spectrum usage in the time-frequency domain. First, the binary timeoccupancy patterns of the channels within a given spectrum band are mutually independent. Second, the DC of the channels within a given spectrum band follow beta or Kumaraswamy distributions. Third, the DC is clustered over frequency and the number of channels per cluster follows a geometric distribution.

These findings and the corresponding statistical models can readily be employed and applied in analytical studies involving the time-frequency dimensions of spectrum usage. However, the applicability of such models is not restricted to analytical studies. The proposed models can also be used and implemented in simulation tools for the design and dimensioning of secondary DSA/CR networks as well as the performance evaluation of DSA/CR techniques. In order to illustrate the applicability of such models in the context of simulation tools, a procedure to generate artificial spectrum data is described below. The proposed method is composed of three phases, each of which ensures that the generated data satisfy the three statistical properties mentioned above.

- Phase 1: Generation of DC values.

- Step 1.1: Specify the number $C$ of channels within the considered spectrum band.

- Step 1.2: Select a DC distribution function $F(\Psi)$ (beta or Kumaraswamy) and select appropriate values for the distribution parameters. The values provided in Table 9.1 can be used as a reference.

- Step 1.3: Based on the probability distribution resulting from Step 1.2, generate a set of $C$ independent random numbers, which will constitute the set $\widehat{\Psi}=\left\{\widehat{\Psi}_{1}, \widehat{\Psi}_{2}, \ldots, \widehat{\Psi}_{c}, \ldots, \widehat{\Psi}_{C}\right\}$ of DC values to be assigned to the $C$ channels of the considered spectrum band.

- Phase 2: Assignment of DC values to channels.

- Step 2.1: Define a set $\mathcal{A}=\left\{A_{1}, A_{2}, \ldots, A_{n}, \ldots, A_{N}\right\}$ of $N$ DC archetypes along with the corresponding set $\Lambda=\left\{\Lambda_{0}, \Lambda_{1}, \ldots, \Lambda_{n}, \ldots, \Lambda_{N}\right\}$ of $N+1 \mathrm{DC}$ thresholds, where $\Lambda_{0}=0$ and $\Lambda_{N}=1$. A DC $\widehat{\Psi}_{c}$ belongs to archetype $A_{n}$ if $\Lambda_{n-1}<\widehat{\Psi}_{c} \leq \Lambda_{n}$.

- Step 2.2: Based on the probability distribution resulting from Step 1.2, compute the elements of the set $\boldsymbol{\Pi}=\left\{\Pi_{1}, \Pi_{2}, \ldots, \Pi_{n}, \ldots, \Pi_{N}\right\}$, where $\Pi_{n}=P\left(A_{n}\right)=P\left(\Lambda_{n-1}<\widehat{\Psi}_{c} \leq \Lambda_{n}\right)=F\left(\Lambda_{n}\right)-F\left(\Lambda_{n-1}\right)$ represents the probability that a channel belongs to archetype $A_{n}$.

- Step 2.3: Classify the values of set $\widehat{\boldsymbol{\Psi}}$ into the archetypes of set $\mathcal{A}$ based on the threshold set $\Lambda$. This produces $N$ subsets $\left\{\widehat{\Psi}_{n}\right\}_{n=1, \ldots, N}$ (one per DC archetype) with $\eta_{n}=\left|\widehat{\Psi}_{n}\right|$ elements each satisfying the conditions $\bigcup_{n=1}^{N} \widehat{\boldsymbol{\Psi}}_{n}=\widehat{\boldsymbol{\Psi}}$ and $\bigcap_{n=1}^{N} \widehat{\Psi}_{n}=\emptyset$. 
- Step 2.4: Select an appropriate value for the parameter $p$ of the geometric distribution of the number of channels per cluster. The values provided in Table 9.2 as well as the alternative methods proposed in Section 9.6.2 can be used as a reference.

- Step 2.5: Set to zero the elements of $\Psi=\left\{\Psi_{1}, \Psi_{2}, \ldots, \Psi_{c}, \ldots, \Psi_{C}\right\}$, where $\Psi_{c}$ represents the DC value finally assigned to channel $v_{c}$. Set to zero the elements of the set $\boldsymbol{\alpha}=\left\{\alpha_{1}, \alpha_{2}, \ldots, \alpha_{n}, \ldots, \alpha_{N}\right\}$, where $\alpha_{n}$ represents a counter of the number of channels belonging to DC archetype $A_{n}$ with an assigned final DC value. Define the counter $\alpha_{C}=\sum_{n=1}^{N} \alpha_{n}$ for the overall number of channels with an already assigned DC value. Repeat the following points until $\alpha_{n}=\eta_{n}$ for all $n$ (i.e., $\alpha_{C}=\sum_{n=1}^{N} \eta_{n}=C$ ):

1. Decide the DC archetype $A^{\prime}=A_{n}$ for the next cluster (i.e., the next group of channels) by generating a uniformly distributed $U(0,1)$ random variate and comparing against the probabilities of set $\Pi$.

2. If this is not the first iteration of the process and the archetype $A^{\prime}$ resulting from point 1 is of the same type as the archetype $A^{\prime \prime}$ of the previously generated cluster, or if the number of channels for archetype $A^{\prime}=A_{n}$ has already been reached $\left(\alpha_{n}=\eta_{n}\right)$, go back to point 1 and recompute $A^{\prime}$ until the conditions $A^{\prime} \neq A^{\prime \prime}$ and $\alpha_{n}<\eta_{n}$ are met. The condition $A^{\prime} \neq A^{\prime \prime}$ is not necessary when there is a single DC archetype for which $\alpha_{n}<\eta_{n}$.

3. Decide the number $\chi$ of channels that belong to the new cluster of type $A^{\prime}=A_{n}$ as a random number drawn from the geometric distribution obtained in Step 2.4. If $\alpha_{n}+\chi>\eta_{n}$, then perform the correction $\chi=\eta_{n}-\alpha_{n}$ in order to meet the total number of channels per archetype.

4. Select randomly $\chi$ DC values from subset $\widehat{\mathbf{\Psi}}_{n}$ (archetype $A_{n}$ ) that have not been assigned yet in order to form the subset $\widetilde{\Psi}=\left\{\widetilde{\Psi}_{1}, \widetilde{\Psi}_{2}, \ldots, \widetilde{\Psi}_{\chi}\right\} \subseteq \widehat{\Psi}_{n}$. Append subset $\widetilde{\Psi}$ to the set of DC values already assigned, i.e. $\left\{\Psi_{\alpha_{C}+1}, \Psi_{\alpha_{C}+2}, \ldots, \Psi_{\alpha_{C}+\chi}\right\}=$ $\left\{\widetilde{\Psi}_{1}, \widetilde{\Psi}_{2}, \ldots, \widetilde{\Psi}_{\chi}\right\}=\widetilde{\Psi}$.

5. Update counters $\alpha_{n}=\alpha_{n}+\chi$ and $\alpha_{C}=\alpha_{C}+\chi$. Go to point 1 .

- Phase 3: Generation of time-domain occupancy sequences.

- Step 3.1: Select appropriate distributions $F_{0}\left(T_{0}\right)$ and $F_{1}\left(T_{1}\right)$ for the length $T_{0}$ of idle periods and the length $T_{1}$ of busy periods, respectively.

- Step 3.2: Configure the parameters of the distributions selected in Step 3.1 in such a way that the channels' average DCs meet the DC values obtained in Step 2.5, i.e. $\mathbb{E}\left\{T_{1}^{c}\right\} /\left(\mathbb{E}\left\{T_{0}^{c}\right\}+\mathbb{E}\left\{T_{1}^{c}\right\}\right)=\Psi_{c}$, where $\mathbb{E}\left\{T_{0}^{c}\right\}$ and $\mathbb{E}\left\{T_{1}^{c}\right\}$ are the mean length of idle and busy periods, respectively, for the $c$-th channel, $\mathrm{v}_{c}$. 
- Step 3.3: Generate for every channel a sequence of consecutive idle/busy periods whose lengths are drawn from the properly configured distributions $F_{0}\left(T_{0}\right)$ and $F_{1}\left(T_{1}\right)$. The sequences generated for every channel must be independent from each other. It is worth noting that the more sophisticated simulation method proposed in Section 8.9.4 can be used here in order to reproduce not only the distributions $F_{0}\left(T_{0}\right)$ and $F_{1}\left(T_{1}\right)$, but also the correlation properties of spectrum usage observed in real radio communications systems.

As it can be inferred from the proposed method, the steps conducted in the first phase guarantee that the DC values of the band follow an appropriate beta or Kumaraswamy distribution and consequently reproduce the corresponding average band DC. The second phase ensures that the DCs of contiguous channels respect the clustering properties observed in empirical measurements. Finally, the third phase provides the lengths of busy and idle periods for each channel so that not only the desired period length distributions are reproduced but also the appropriate DC distribution over frequency channels (and additionally the timecorrelation properties of spectrum usage if the method of Section 8.9.4 is employed).

In order to illustrate the proposed method, artificial spectrum data were generated for the TETRA UL and TETRA DL bands. The number of channels $C$ was set according to the spectrum regulation specifications and the parameters for the beta and geometric distributions were configured as shown in Tables 9.1 and 9.2. It is worth noting that these values correspond to the empirical measurements depicted in Figures 9.4 and 9.5. The DC archetypes considered in Section 9.6.2 were also employed in this case. Based on the conclusions reached in Section 8.7, generalized Pareto distributions were selected for both the idle and busy period lengths. For the parameters of the busy periods distribution, $F_{1}\left(T_{1}\right)$, the values shown in Table 8.4 for very high DCs were selected. For the idle periods distribution, $F_{0}\left(T_{0}\right)$, the location and shape parameters were configured as the mean of the values shown in Table 8.4, while the scale parameter was computed in order to meet the appropriate DC value as described in Step 3.2. For those channels for which this procedure resulted in negative period lengths, the scale parameter was set to 0.01 and the shape parameter was then recomputed in order to provide the required channel DC. The sequences of busy and idle periods were then generated using the simulation method described in Section 8.9.4 in order to reproduce time-correlation properties. Artificial spectrum data were generated for a time period of 60 minutes. The obtained spectrum data are shown in Figures 9.7 and 9.8 in the same format as in Figures 9.4 and 9.5. The visual inspection and comparison of these two pairs of figures suggests that the proposed method is able to reproduce the statistical properties of spectrum in the time and frequency domains. To rigorously verify this statement, the artificial spectrum data were analyzed in the same manner as the field measurement data. The analysis conducted indicated that the generated spectrum data had the same statistical properties as the empirical data in terms of the average band DC, the probability distribution for the channel DCs, the DC clustering distributions, the probability distribution for busy and idle period lengths and the time-correlation properties. This highlights the capability of the proposed models and simulation method to accurately capture and reproduce the statistical properties of spectrum usage observed for real systems in the time and frequency domains. 

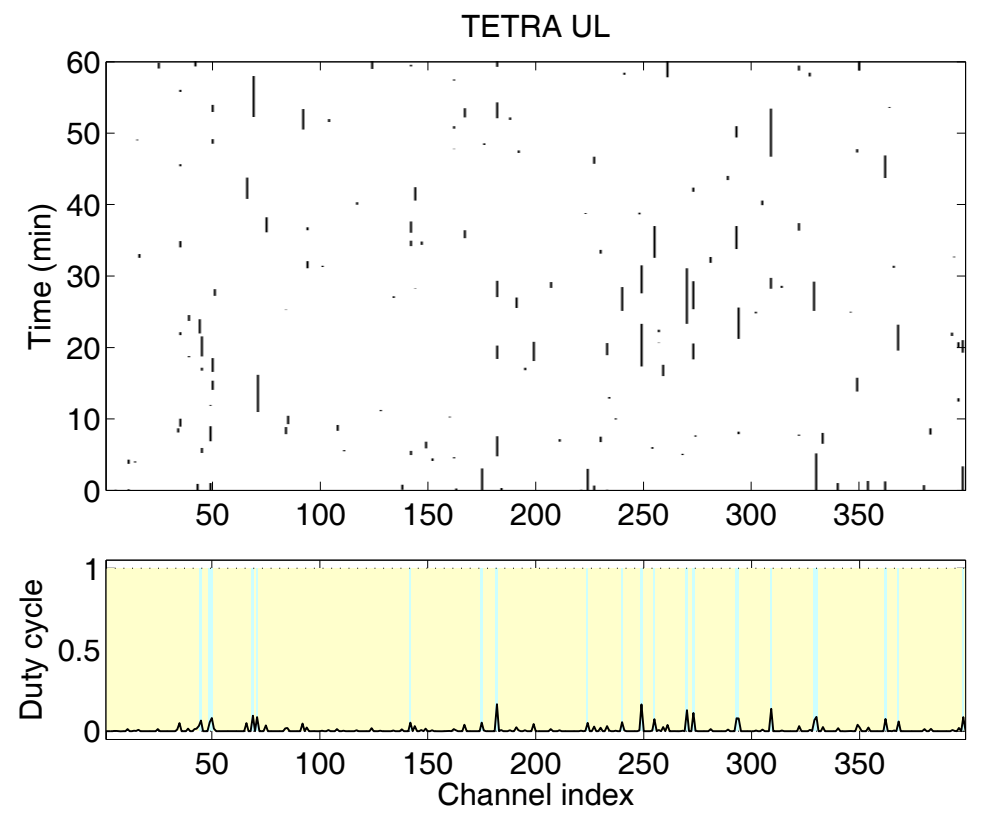

Figure 9.7: Artificially generated spectrum data for the TETRA UL band.
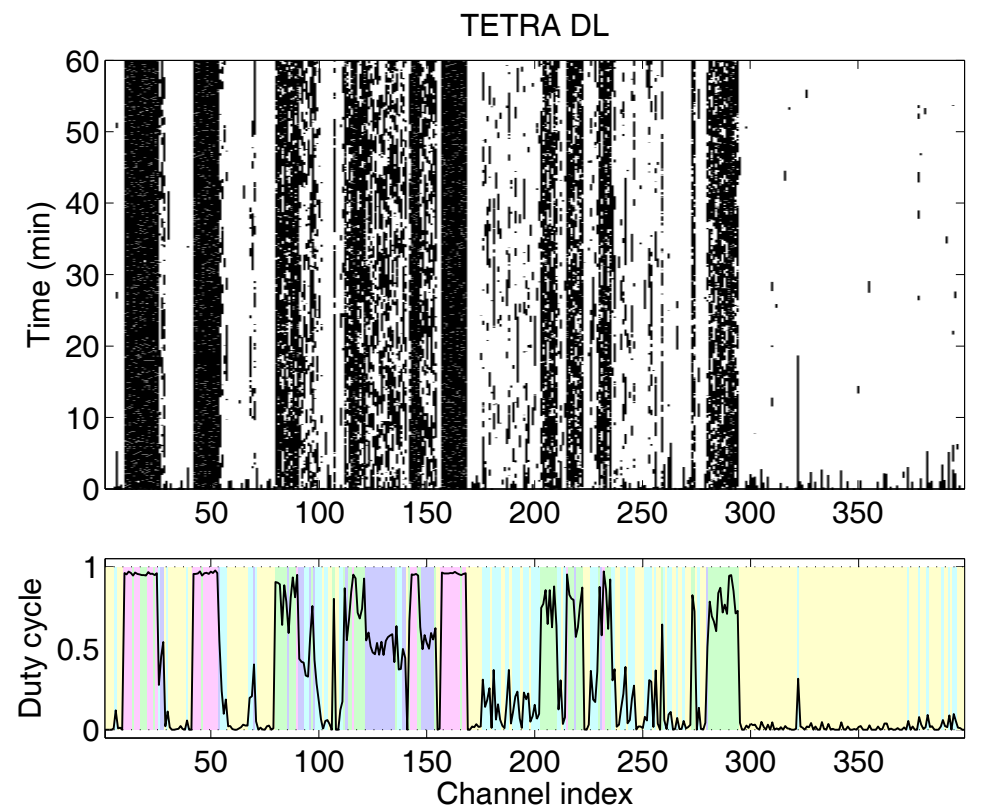

Figure 9.8: Artificially generated spectrum data for the TETRA DL band. 


\subsection{Summary}

This chapter has addressed the problem of jointly modeling spectrum occupancy in the time and frequency domains. The joint behavior of a set of channels within the same band is a feature that needs to be accurately reproduced by spectrum usage models since it has a direct impact on the amount of consecutive vacant spectrum that a DSA/CR user may find, as well as the time period for which spectrum gaps can be exploited for opportunistic use. This chapter has analyzed the statistical time-frequency characteristics of spectrum usage based on empirical data from extensive spectrum measurement campaigns. The study conducted has revealed three important aspects to be taken into account for a realistic and accurate modeling of spectrum usage. First, the binary time-occupancy patterns of the channels within a given spectrum band are mutually independent. Second, the DC of the channels within the same spectrum band follow beta or Kumaraswamy distributions. Third, the DC is clustered over frequency and the number of channels per cluster follows a geometric distribution. Based on these findings, a sophisticated procedure has been developed in order to generate artificial spectrum occupancy data for simulation and other purposes. The proposed method is able to accurately capture and reproduce the statistical time-frequency characteristics of spectrum usage observed in real radio communication systems. 



\section{Chapter}

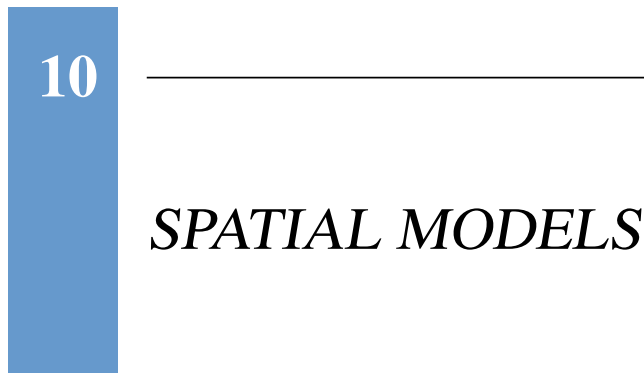

\subsection{Introduction}

The present chapter extends and complements the studies performed in Chapters 7, 8 and 9 by addressing the problem of modeling spectrum occupancy in the spatial dimension. While previous works in this area have mainly faced the problem from statistical modeling perspectives, the study reported in this chapter introduces a novel spatial model based on a different, simple, clear and intuitive theoretical approach. The proposed models are envisaged to describe the average level of occupancy (expressed in terms of the DC) that would perceived by DSA/CR users at various geographical locations based on the knowledge of some simple primary signal parameters. Moreover, an extension is proposed in order to characterize not only the average occupancy perception but also the simultaneous observations of various DSA/CR users on the spectrum occupancy pattern of the same transmitter. This extension can be useful in the study of cooperative techniques (e.g., cooperative spectrum sensing) as well as the development of innovative simulation tools. The validity and correctness of the theoretical models is evaluated and corroborated with extensive empirical measurement results. Some illustrative examples of their potential applicability are discussed as well.

The rest of this chapter is organized as follows. First, Section 10.2 reviews the related previous work on space-dimension models of spectrum usage, describing their modeling approaches and main characteristics. Section 10.3 describes the methodology employed to capture and process the empirical data used in the validation of the proposed models, which are presented in Sections 10.4 and 10.5. First, Section 10.4 presents a set of spectrum models envisaged to characterize the average spectrum occupancy perceived at different geographical locations. Section 10.5 then extends the models by including some additional considerations to characterize the concurrent observations of different DSA/CR users. The potential applicability of the developed models is illustrated in Section 10.6. Finally, Section 10.7 summarizes and concludes the investigation carried out in this chapter. 


\subsection{Previous work}

The spatial dimension of spectrum occupancy has frequently been analyzed and modeled in the existing DSA/CR literature in terms of the interference from a secondary network to a primary system as a function of relative locations, distances, transmission powers and radio propagation conditions [267]. Thus, previous works have studied for example the aggregated interference power from DSA/CR users surrounding a primary receiver [268-270], the outage probability of a primary system caused by interference from DSA/CR networks for the cases of both underlay and overlay spectrum sharing [271], the region of communication and interference for the users of a DSA/CR network that coexists with a cellular network [272], the interference to wireless microphones operating in TV bands [273] and further refinements including shadowing and fading effects [274, 275] as well as power control in the secondary network [276].

The scope of this chapter is on the busy/idle binary occupancy pattern observed by DSA/CR users as a function of their spatial location. This relevant aspect has received less attention in DSA/CR research. For example, the spatial distribution of the spectral occupancy was analyzed in [241] in the context of a cellular mobile communication system. In contrast to other similar works where a cellular network is monitored from outside the system by means of field measurements and in a limited geographical region [167, 168, 277], the study reported in [241] monitors the arrival of calls from inside the system and over hundreds of base stations simultaneously in the period of three weeks. Based on such measurements and making use of variograms, the study presented in [241] analyzes the spatial variability of the spectral occupancy for sectors of the same and different cells as well as the correlation among various cells based on the traffic supported during the minute of maximum load. Although the analyses carried out in [241] show interesting spatial properties of the spectrum utilization in cellular mobile communication systems, no model enabling the reproduction of the analyzed statistics is proposed.

The possibility to analyze and characterize the spectrum occupancy in the spatial domain by means of the PSD utilizing spatial statistics and random fields is explored in [278]. A modeling procedure based on the spatial statistics studied in [278] is proposed in [279] and complemented in [280]. The modeling procedure described in [279, 280] consists in determining the average PSD value over a set of points with a certain spatial distribution on a specified geographical area. This information can be obtained by means of field measurements as described in [279] (empirical model) or by means of simulators or radio planning software tools as detailed in [280] (deterministic model). Once the spatial values of average PSD are determined, the resulting values are employed to fit an analytic semivariogram model, which permits to reproduce the statistical properties of the average PSD values observed on a certain geographical area.

While previous works in the area of spectrum occupancy modeling in the spatial dimension have faced the problem from a statistical perspective, the study reported in this chapter introduces a novel spatial spectrum usage model based on a different, simple, clear and intuitive theoretical approach that enables modeling the occupancy level perceived at any geographical location based on the knowledge of some simple primary signal parameters. 


\subsection{Measurement setup and methodology}

The spectrum analyzer measurement platform discussed in Chapter 2 and Appendix A was employed to perform measurements in several spectrum bands (see Table 7.1) in an urban environment. Figure 3.1 shows the different geographical locations considered in this study, which embrace a wide variety of scenarios including indoor (location 2) and outdoor environments at high points (location 1), narrow streets (locations 3-7), between buildings (locations 8-10) and in open areas (locations 11-12). The considered measurement locations represent various physical scenarios of practical interest that embrace a wide range of receiving conditions and levels of radio propagation blocking, ranging from direct line of sight to severely blocked and faded signals. This variety of measurement conditions allowed to observe the same set of transmitters under different propagation conditions and with different SNR levels. Based on the configuration shown in Table 3.1, each band was measured for time periods of at least 1 hour ${ }^{1}$. The captured data were used to extract the binary channel occupancy patterns from the measured channels by classifying the spectrum analyzer's power samples as either busy or idle states. To this end, the CED scheme described in Section 6.2 was employed to process the spectrum data on a power-sample basis, as in Chapters 7,8 and 9 . The resulting binary sequences of busy and idle states for each individual channel at various locations were compared and analyzed as detailed in the following sections.

In order to validate the spectrum usage models developed for simultaneous observations of DSA/CR users in various geographical locations, some additional field measurements were performed by making use of two identical measurement suites. One measurement suite was placed in the outdoor high point (location 1 in Figure 3.1), where the receiving SNR is maximum for most of the analyzed bands, while the second measurement suite was displaced along the rest of outdoor measurement locations at the ground level (locations 3-12 in Figure 3.1). Making use of the same measurement configuration, measurements were performed concurrently for every possible pair of locations. As explained in Appendix A, the developed measurement platform is able to run synchronously with other identical units by making use of the time reference provided by an external GPS receiver. This feature can be employed when two or more measurement suites are deployed at different locations and need to be time-synchronized, which results useful to determine and compare the instantaneous activity pattern of a given transmitter as perceived at different locations. The empirical data captured with this measurement configuration were processed following the same methodological procedures as the rest of field measurements.

\subsection{Models for average spectrum occupancy perception}

The models presented in this section describe the spatial distribution of the DC. The interest of employing the DC lies in its ability to summarize the overall spectrum occupancy within

\footnotetext{
${ }^{1}$ Note that most of the measurement locations considered in this study (see Figure 3.1) are public places, which prevented unattended operation of the measurement equipment for long time periods. Since the presence of an operator was required in every measurement session, periods of 7 consecutive days as in Chapters 7,8 and 9 were infeasible and were therefore shortened to 1 hour in this case.
} 
a certain time interval and frequency range in a single numerical value. The DC has been employed in many past spectrum occupancy studies to quantify and compare the occupancy level of several spectrum bands or to compare the occupancy of the same band under different conditions or at different locations. The spatial distribution of the DC is employed in this chapter as a means to describe the spectrum occupancy that would be perceived by secondary DSA/CR terminals at different geographical locations.

It is important to make a clear distinction between the Activity Factor (AF) of a primary transmitter in a certain channel and the DC perceived by secondary DSA/CR terminals in that channel. The AF of a primary transmitter represents the fraction of time that the transmitter is active (i.e., transmitting in the channel). A DSA/CR terminal in an arbitrary location with good radio propagation conditions with respect to the primary transmitter would observe the channel as busy whenever the primary transmitter is active. However, at other locations where the propagation conditions are not so favorable, the primary signal might not be detected. In such a case, the level of spectrum activity perceived by the DSA/CR terminal in terms of the $\mathrm{DC}$ would be lower than the actual AF of the primary transmitter. While the AF is unique for a given transmitter, the DC perceived at different locations may be different. Since the propagation conditions strongly vary with the geographical location, the perceived DC will vary over space accordingly. The aim of this section is to develop a model able to describe the spatial distribution of the DC as a function of the radio propagation conditions.

\subsubsection{Received average power distribution}

The occupancy state of a channel as perceived by a DSA/CR terminal depends on the employed spectrum sensing method. The models developed in this chapter assume ED. Due to its simplicity, wide range of application and relevance, ED has been the preferred choice for DSA/CR and also constitutes the spectrum sensing method considered in this study.

According to ED, a DSA/CR terminal measures the power received in a certain frequency band over a predefined time period $2 T$, which can be expressed as:

$$
P_{R}=\frac{1}{2 T} \int_{-T}^{+T} P_{R}(t) d t
$$

where $P_{R}(t)$ is the instantaneous power received by the DSA/CR terminal (including noise) and $P_{R}$ is the average power employed as a test statistic to decide whether a primary signal is present in the sensed channel. The spectrum occupancy perception of a DSA/CR user at a particular location depends on the statistics of the received average power, $P_{R}$, which in turn depends on the radio propagation conditions resulting from the surrounding environment. The perceived spectrum occupancy can therefore be related to the received average power and studied based on the statistics of $P_{R}$, which can be inferred as discussed below.

The PDF of $P_{R}(t)$ is in general unknown since it is the result of the combined effects of the primary transmission power pattern, which in principle is unknown, and the radio propagation mechanisms. However, it is reasonable to assume that the mean value of $P_{R}(t)$, denoted as $\mu_{R, t}$, and its variance, denoted as $\sigma_{R, t}^{2}$, can be computed at any arbitrary location by employing appropriate radio propagation models. 
The instantaneous power $P_{R}(t)$ is a stochastic process ${ }^{2}$ that can be thought of as a noncountable infinity of independent and identically distributed random variables, one for each time instant $t$, with mean $\mu_{R, t}$ and variance $\sigma_{R, t}^{2}$. Since $P_{R}$ is obtained as the average of an infinite number of random variables, the central limit theorem can therefore be employed to approximate the PDF of $P_{R}$ as a normal distribution, regardless of the actual PDF of $P_{R}(t)$, with mean $\mu_{R}$ and variance $\sigma_{R}^{2}$ given by [210, pp. 523-525]:

$$
\begin{aligned}
\mu_{R} & =\mathbb{E}\left\{P_{R}\right\}=\frac{1}{2 T} \int_{-T}^{+T} \mathbb{E}\left\{P_{R}(t)\right\} d t=\mu_{R, t} \\
\sigma_{R}^{2} & =\mathbb{V}\left\{P_{R}\right\}=\frac{1}{T} \int_{0}^{2 T} C_{R}(\tau)\left(1-\frac{\tau}{2 T}\right) d \tau \\
& \approx \frac{1}{T} \int_{0}^{2 T} C_{R}(\tau) d \tau \approx \frac{1}{T} \int_{0}^{\infty} C_{R}(\tau) d \tau \\
& \approx \frac{\tau_{c}}{T} C_{R}(0)=\frac{\tau_{c}}{T} \sigma_{R, t}^{2}
\end{aligned}
$$

where $T$ has been assumed to be sufficiently large, $C_{R}(\tau)$ is the autocovariance of $P_{R}(t)$ and $\tau_{c}$ is a constant called the correlation time, which satisfies that $C_{R}(\tau) \approx 0$ for $\tau>\tau_{c}$ and is defined as the ratio [210, p. 389]:

$$
\tau_{c}=\frac{1}{C_{R}(0)} \int_{0}^{\infty} C_{R}(\tau) d \tau
$$

As it can be appreciated, the received average power distribution can be modeled as a Gaussian PDF. Its mean value equals the mean value of the instantaneous received power and its variance is directly related to the variance of the instantaneous power and depends on the sensing period and the primary signal's autocorrelation properties.

The validity of the Gaussian approximation is verified in Figure 10.1 for various radio technologies and also for the thermal noise's average power (measured by replacing the antenna with a matched load). It is worth noting that the PSD values provided by swept spectrum analyzers are obtained by sequentially tuning a narrow band filter to a set of consecutive frequency points. Every frequency point is measured for a certain time interval, thus causing some unavoidable averaging effect over the measured signal. Therefore, due to the inherent operating principle, the PSD values provided by spectrum analyzers implicitly include the averaging effect of Equation 10.1. Figure 10.1 compares the distribution of the $P_{R}$ values captured by the spectrum analyzer at some selected channels ${ }^{3}$ and the Gaussian curve corresponding to the sample mean and sample variance of the $P_{R}$ values. Although signals from different radio technologies received at different frequencies are expected to exhibit various instantaneous power patterns $P_{R}(t)$, Figure 10.1 indicates that the received average power $P_{R}$ can be modeled as a Gaussian random variable for all cases regardless of the particular instantaneous power distributions, thus verifying the formulated assumption.

\footnotetext{
${ }^{2}$ Note that even if the primary transmission power is perfectly constant, the radio propagation channel results in some random variation of the received power $P_{R}(t)$. Moreover, $P_{R}(t)$ includes the unavoidable effects of the receiver's noise, which also introduces some random component.

${ }^{3}$ Spectrum analyzers normally provide power measurements in logarithmic magnitudes (e.g., dBm). For convenience, to simplify the validation with field measurements, it is assumed hereinafter that all power values, means and variances are expressed in logarithmic magnitudes.
} 


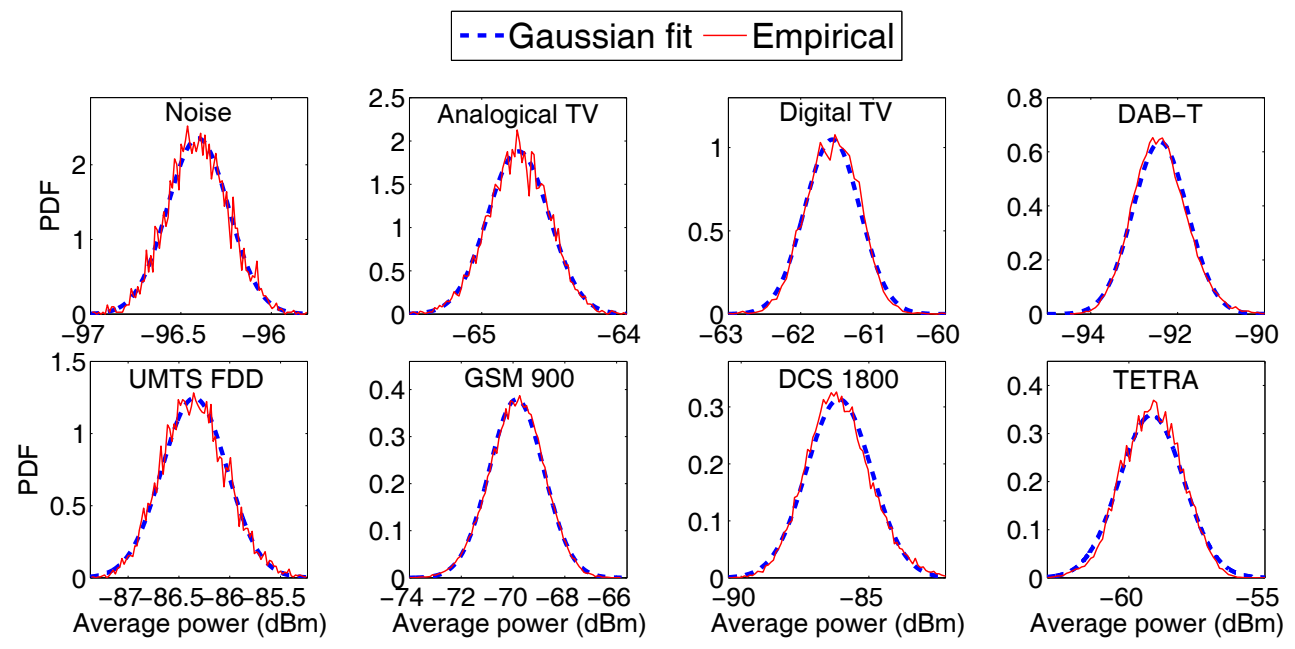

Figure 10.1: Validation of the Gaussian approximation for the PDF of the received average power. The approximation is valid for line-of-sight measurements (location 1) as well as non non-line-of-sight measurements (locations 2-12).

\subsubsection{Spatial duty cycle models}

This section presents a set of models to describe and predict the spectrum occupancy perceived at various locations in terms of the DC. The DC can be defined as the probability that the channel is observed as busy. Note that the ED method will report the channel as busy whenever the average power $P_{R}$ is above a certain decision threshold $\lambda$. Since ED is not able to distinguish between intended signals and undesired noise, the channel will be reported as busy not only if a primary signal is received above the decision threshold but also if there is no signal (or it is received below the threshold) and the noise power exceeds the threshold.

Let's denote the distribution of the noise power as $f_{N}\left(P_{N}\right) \sim \mathcal{N}\left(\mu_{N}, \sigma_{N}^{2}\right)$ and the distribution of the signal power (received in the presence of noise) as $f_{S}\left(P_{S}\right) \sim \mathcal{N}\left(\mu_{S}, \sigma_{S}^{2}\right)$. According to this formulation, $\mu_{N}$ represents the noise floor of the DSA/CR receiver and $\sigma_{N}$ denotes the standard deviation of the noise powers $P_{N}$ experienced at various sensing events (the effective noise power may be different between sensing events due to the finite averaging period $T$ or other reasons such as temperature variations). The primary power $P_{S}$ received in the presence of noise is characterized by an average value $\mu_{S}$ that depends on the transmission power and radio propagation conditions and a standard deviation $\sigma_{S}$ that is additionally affected by the noise of the DSA/CR receiver.

If the sensed channel is idle, the PDF of the observed average power, $f_{R}\left(P_{R}\right)$, will be that of the noise, $f_{N}\left(P_{N}\right)$. In such a case, the probability that the observed power is above the threshold (i.e., the perceived DC) is given by (see Figure 10.2):

$$
\Psi_{\text {idle }}=\int_{\lambda}^{\infty} f_{R}\left(P_{R}\right) d P_{R}=\int_{\lambda}^{\infty} f_{N}\left(P_{N}\right) d P_{N}=P_{f a}
$$

where it has been assumed that the decision threshold $\lambda$ is set so as to meet a specified target probability of false alarm $P_{f a}$ (see Section 4.4.3). 


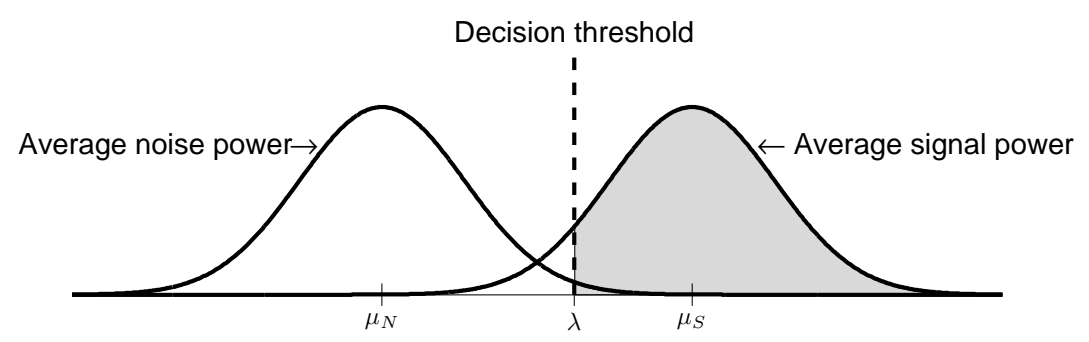

Figure 10.2: Model considered to compute the DC (shaded area).

On the other hand, if the channel is busy when it is sensed, the PDF of the observed average power, $f_{R}\left(P_{R}\right)$, will be that of the received signal, $f_{S}\left(P_{S}\right)$. Assuming an ideal situation where there is no noise, the DC perceived by the DSA/CR user would be given by:

$$
\begin{aligned}
\Psi_{\text {busy }}^{\text {ideal }} & =\int_{\lambda}^{\infty} f_{R}\left(P_{R}\right) d P_{R}=\int_{\lambda}^{\infty} f_{S}\left(P_{S}\right) d P_{S} \\
& =\frac{1}{\sqrt{2 \pi} \sigma_{S}} \int_{\lambda}^{\infty} e^{-\frac{1}{2}\left(\frac{P_{S}-\mu_{S}}{\sigma_{S}}\right)^{2}} d P_{S}=Q\left(\frac{\lambda-\mu_{S}}{\sigma_{S}}\right)
\end{aligned}
$$

where $Q(\cdot)$ represents the Gaussian $Q$-function [201, (26.2.3)]. Notice that Equation 10.6 indicates that the perceived occupancy in terms of the DC would tend to zero as the received power decreases (i.e., $\mu_{S} \rightarrow-\infty$ ). However, if the received signal power is below the receiver's noise, this situation would be equivalent to an idle channel where the receiver observes only noise. In such a case, the perceived DC should be $P_{f a}$ as indicated by Equation 10.5. A more realistic model for $f_{R}\left(P_{R}\right)$ when the channel is busy, taking into account the presence of noise, would be $f_{R}\left(P_{R}\right)=\mathcal{M}\left\{f_{N}\left(P_{N}\right), f_{S}\left(P_{S}\right)\right\}$, where $\mathcal{M}\{\cdot\}$ denotes a realization-wise maximum operator defined as follows. If $\mathcal{A}=\left\{x_{a_{1}}, x_{a_{2}}, \ldots, x_{a_{n}}, \ldots, x_{a_{N}}\right\}$ and $\mathcal{B}=\left\{x_{b_{1}}, x_{b_{2}}, \ldots, x_{b_{n}}, \ldots, x_{b_{N}}\right\}$ represent two sets of $N$ random numbers (realizations) drawn from distributions $f_{a}\left(x_{a}\right)$ and $f_{b}\left(x_{b}\right)$, respectively, then $f_{c}\left(x_{c}\right)=\mathcal{M}\left\{f_{a}\left(x_{a}\right), f_{b}\left(x_{b}\right)\right\}$ represents the distribution of the elements of the set $\mathcal{C}=\left\{x_{c_{1}}, x_{c_{2}}, \ldots, x_{c_{n}}, \ldots, x_{c_{N}}\right\}$, where $x_{c_{n}}=\max \left\{x_{a_{n}}, x_{b_{n}}\right\}$ for $n=1,2, \ldots, N$, when $N$ tends towards infinity. Notice that this operator reproduces the effect of the noise floor on the observed power (i.e., the DSA/CR user observes the received signal power when it is above the noise floor or the noise power otherwise). Therefore, this definition of $f_{R}\left(P_{R}\right)$ provides a more realistic model for the average power $P_{R}$ actually observed by the DSA/CR receiver. Based on this model, the DC perceived by the DSA/CR user when the channel is busy will then be given by:

$$
\begin{aligned}
\Psi_{\text {busy }}^{\text {real }} & =\int_{\lambda}^{\infty} f_{R}\left(P_{R}\right) d P_{R}=\int_{\lambda}^{\infty} \mathcal{M}\left\{f_{N}\left(P_{N}\right), f_{S}\left(P_{S}\right)\right\} d P_{R} \\
& =\max \left\{\int_{\lambda}^{\infty} f_{N}\left(P_{N}\right) d P_{N}, \int_{\lambda}^{\infty} f_{S}\left(P_{S}\right) d P_{S}\right\}=\max \left\{P_{f a}, Q\left(\frac{\lambda-\mu_{S}}{\sigma_{S}}\right)\right\}
\end{aligned}
$$

As it can be appreciated, this model rightly predicts that the perceived activity level is never lower than the target $P_{f a}$. The average DC perceived by the DSA/CR user will depend on the transmission power of the primary transmitter and its particular activity pattern. The next sections provide closed-form expressions for various general cases. 


\subsubsection{Constant-power continuous transmitters}

This section considers the particular case of constant-power primary transmitters with an AF of $100 \%$ (e.g., TV and DAB-T). This case provides the basis for a simple occupancy model that will be extended in the next sections for variable-power transmitters and/or discontinuous transmission patterns.

If the primary transmitter is always active, the PDF of the received average power $f_{R}\left(P_{R}\right)$ will be that of the primary signal (with noise) at the location of the DSA/CR terminal, i.e. $f_{R}\left(P_{R}\right)=\mathcal{M}\left\{f_{N}\left(P_{N}\right), f_{S}\left(P_{S}\right)\right\}$. The probability that the received average power $P_{R}$ is above the decision threshold $\lambda$ and the DSA/CR user observes the channel as busy is given by Equation 10.7. Assuming that the decision threshold is set to meet a certain $P_{f a}$ :

$$
P_{f a}=\int_{\lambda}^{\infty} f_{N}\left(P_{N}\right) d P_{N}=\frac{1}{\sqrt{2 \pi} \sigma_{N}} \int_{\lambda}^{\infty} e^{-\frac{1}{2}\left(\frac{P_{N}-\mu_{N}}{\sigma_{N}}\right)^{2}} d P_{N}=Q\left(\frac{\lambda-\mu_{N}}{\sigma_{N}}\right)
$$

Solving in Equation 10.8 for $\lambda$ yields the decision threshold:

$$
\lambda=Q^{-1}\left(P_{f a}\right) \sigma_{N}+\mu_{N}
$$

where $Q^{-1}(\cdot)$ denotes the inverse of $Q(\cdot)$. Substituting Equation 10.9 into Equation 10.7 finally yields the DC model:

$$
\Psi=\max \left\{P_{f a}, Q\left(\frac{Q^{-1}\left(P_{f a}\right) \sigma_{N}-\Gamma}{\sigma_{S}}\right)\right\}
$$

where $\Gamma=\mu_{S}-\mu_{N}$ represents the average SNR expressed in decibels, while $\sigma_{S}$ and $\sigma_{N}$ are the standard deviation of the signal and noise average powers also in decibels.

To validate Equation 10.10, Figures 10.3, 10.4, 10.5 and 10.6 depict, for selected analogical TV, digital TV, E-GSM 900 broadcast and DCS 1800 broadcast channels ${ }^{4}$, the empirical DC obtained for the 12 locations measured in Figure 3.1. Each empirical point corresponds to the result of one location in Figure 3.1. The empirical DC has been computed for the decision thresholds corresponding to $P_{f a}=1 \%$ and $P_{f a}=10 \%$, and it is shown as a function of the difference between the empirically measured average signal power $\mu_{S}$ and average noise power $\mu_{N}$ in $\mathrm{dBm}$, i.e. the SNR $\Gamma$ in $\mathrm{dB}$. The dependence of the perceived spectral activity with the geographical location is reflected in the different SNR values observed at each location. The theoretical curve of Equation 10.10 corresponding to the empirical sample means $\left(\mu_{S}, \mu_{N}\right)$ and sample standard deviations $\left(\sigma_{S}, \sigma_{N}\right)$ is also shown for comparison. As it can be appreciated, the model agrees with the empirical values in all cases. These results demonstrate that Equation 10.10 is able to accurately predict the spectral activity that would be perceived by a DSA/CR user at any position based on some basic signal parameters.

\footnotetext{
${ }^{4}$ Although GSM/DCS traffic channels cannot be considered as a case of constant-power continuous transmission, GSM/DCS broadcast channels can be classified into this category.
} 
Channel 28

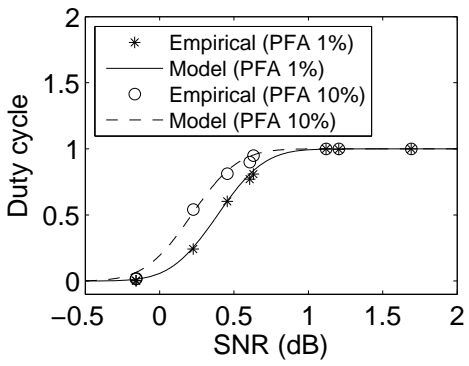

Channel 51

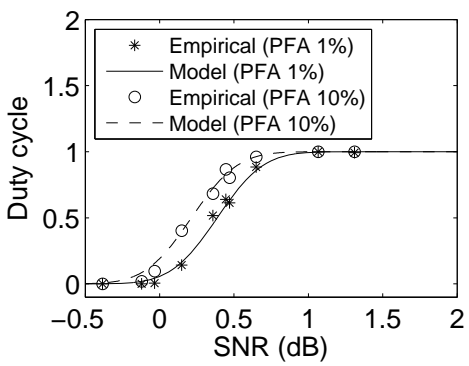

Channel 35
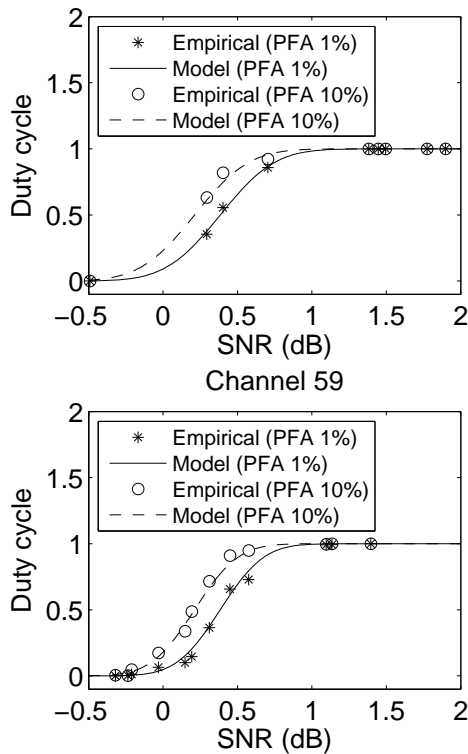

Figure 10.3: Validation of DC model for constant-power continuous transmitters (analogical TV).

Channel 22

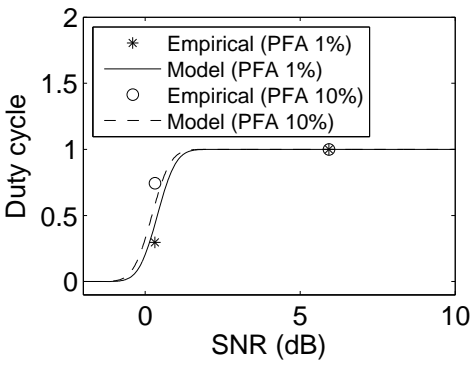

Channel 66

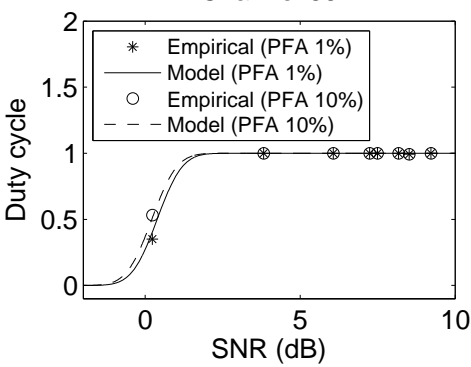

Channel 33

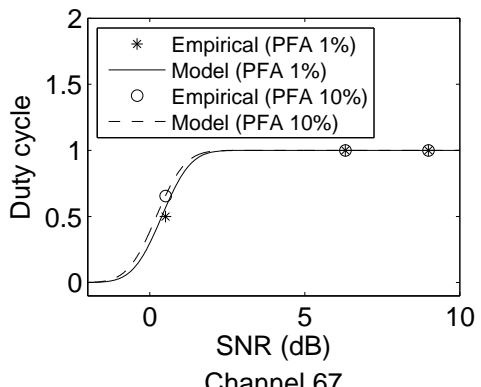

Channel 67

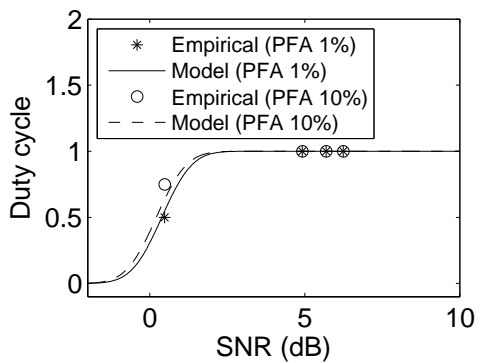

Figure 10.4: Validation of DC model for constant-power continuous transmitters (digital TV). 

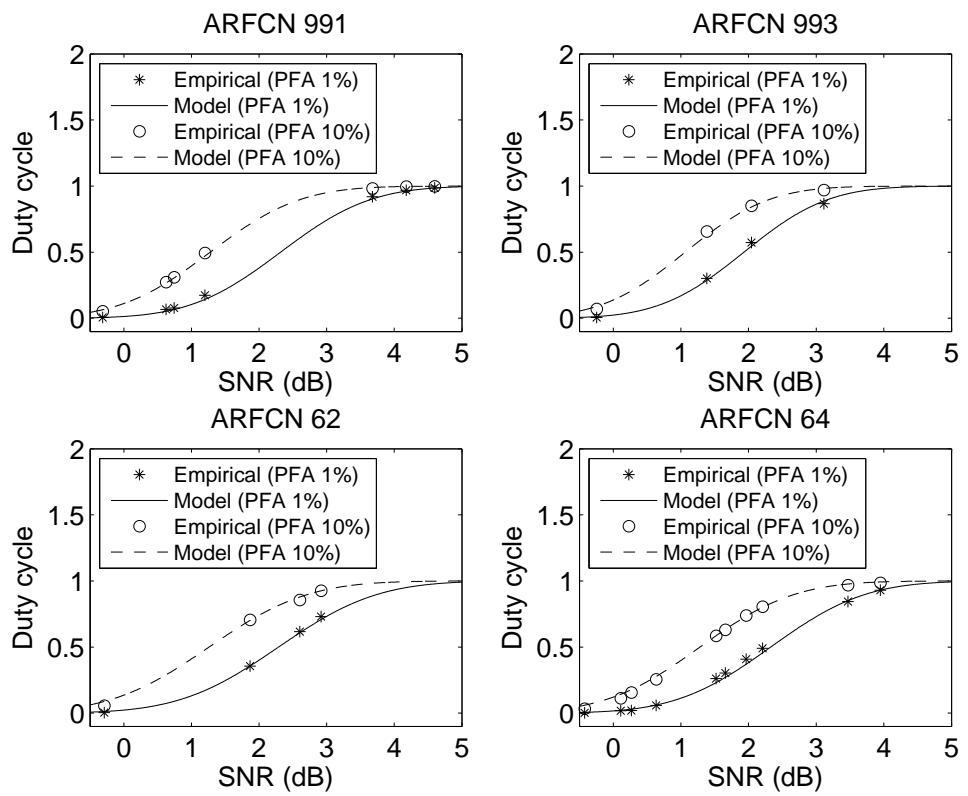

Figure 10.5: Validation of DC model for constant-power continuous transmitters (E-GSM 900 broadcast channels).
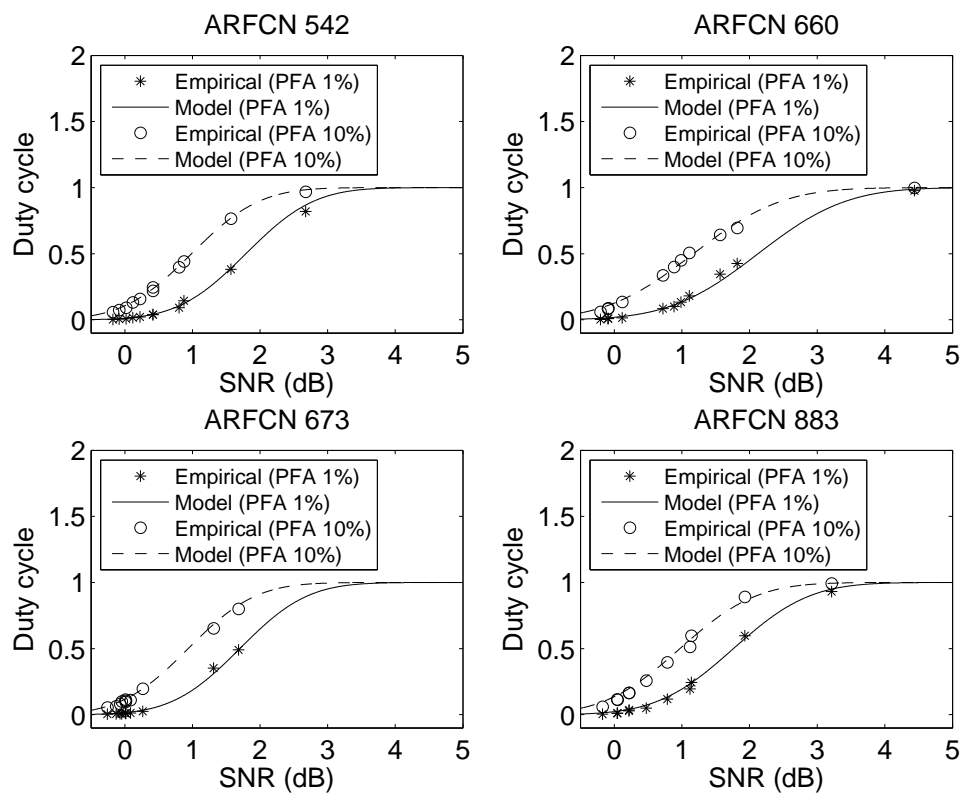

Figure 10.6: Validation of DC model for constant-power continuous transmitters (DCS 1800 broadcast channels). 


\subsubsection{Constant-power discontinuous transmitters}

This section extends the model of Equation 10.10 by including the case of constant-power but non-continuous transmitters. If the primary transmitter is characterized by a certain AF, denoted as $0<\alpha<1$, the PDF of the received average power $f_{R}\left(P_{R}\right)$ will be that of the primary signal (with noise) $\mathcal{M}\left\{f_{N}\left(P_{N}\right), f_{S}\left(P_{S}\right)\right\}$ whenever the transmitter is active (which will occur with probability $\alpha$ ) or noise $f_{N}\left(P_{N}\right)$ otherwise. Hence:

$$
f_{R}\left(P_{R}\right)=(1-\alpha) f_{N}\left(P_{N}\right)+\alpha \mathcal{M}\left\{f_{N}\left(P_{N}\right), f_{S}\left(P_{S}\right)\right\}
$$

and the resulting expression for the DC becomes:

$$
\begin{aligned}
\Psi & =\int_{\lambda}^{\infty} f_{R}\left(P_{R}\right) d P_{R} \\
& =(1-\alpha) \int_{\lambda}^{\infty} f_{N}\left(P_{N}\right) d P_{N}+\alpha \int_{\lambda}^{\infty} \mathcal{M}\left\{f_{N}\left(P_{N}\right), f_{S}\left(P_{S}\right)\right\} d P_{R} \\
& =(1-\alpha) \int_{\lambda}^{\infty} f_{N}\left(P_{N}\right) d P_{N}+\alpha \max \left\{\int_{\lambda}^{\infty} f_{N}\left(P_{N}\right) d P_{N}, \int_{\lambda}^{\infty} f_{S}\left(P_{S}\right) d P_{S}\right\} \\
& =(1-\alpha) P_{f a}+\alpha \max \left\{P_{f a}, Q\left(\frac{Q^{-1}\left(P_{f a}\right) \sigma_{N}-\Gamma}{\sigma_{S}}\right)\right\}
\end{aligned}
$$

Notice that Equation 10.10 is a particular case of Equation 10.12 with $\alpha=1$.

The validation of Equation 10.12 based on empirical measurements is not as straightforward as in the case of Section 10.4.2.1. While the values of $\mu_{N}$ and $\sigma_{N}$ (receiver's noise) can be obtained by replacing the antenna with a matched load, the values of $\mu_{S}$ and $\sigma_{S}$ cannot be obtained from the captured data sequences as in Section 10.4.2.1 because in the case of transmitters with $\alpha<1$ the captured data is composed of both signal and noise samples, which cannot be distinguished reliably. Similarly, the actual AF $\alpha$ of the transmitter is also unknown and cannot reliably be derived from the received power values without additional information. To estimate $\mu_{S}, \sigma_{S}$ and $\alpha$, an alternative procedure was followed. First, the empirical PDFs of the captured data sequences were computed and, based on curve-fitting procedures, the PDF of Equation 10.11, where $\mu_{N}$ and $\sigma_{N}$ are known, was fitted to the empirical PDFs. The set of values $\left(\mu_{S}, \sigma_{S}, \alpha\right)$ minimizing the Root Mean Square Error (RMSE) of the fits was then selected. As shown in the example of Figure 10.7, the theoretical PDF corresponding to the empirically measured $\left(\mu_{N}, \sigma_{N}\right)$ and the estimated set $\left(\mu_{S}, \sigma_{S}, \alpha\right)$ perfectly agrees with the empirical PDF, thus indicating that this procedure is able to provide accurate estimates of the true signal parameters $\left(\mu_{S}, \sigma_{S}, \alpha\right)$. Applying these values to Equation 10.12, the theoretical DC curve was obtained.

To obtain the empirical DC curve, it is important to notice that the locations considered in the measurement campaign were measured at different time instants. The actual AFs of the measured transmitters may experience some variations along time and may not be the same over different measurement sessions even for the same transmitter. When this occurs, the DC values obtained for each location/SNR are the result of different AFs and are therefore unrelated. As a result, the empirical curve obtained by plotting the empirical DC values as a function of the experienced SNR is characterized by a completely random behavior. Since 
this phenomenon was observed in the empirical data sequences, a different approach was employed to obtain the empirical DC curve. One possibility would be to deploy 12 identical measurement suites, one for each location, and perform synchronous measurements. Since this solution was infeasible, the measurements performed at location 1 (the location with the highest SNR for most of the measured bands) were selected and processed in order to emulate the corresponding signals that would be received at other locations with different SNR values. Based on the data sequence captured at location 1 with the highest SNR, different SNR values were artificially emulated by subtracting the adequate amplitude value from all the samples of the original sequence. Since signal amplitudes below the noise floor cannot be detected and are reported as noise, all the samples lying below the noise floor after subtracting the adequate amplitude value were replaced with the corresponding noise power value. However, the instantaneous noise power is not constant as discussed in Section 10.4.2. To emulate this effect, the sequence of noise power values employed in this procedure was generated as a random variable drawn from a Gaussian distribution whose mean $\mu_{N}$ and standard deviation $\sigma_{N}$ were obtained from the empirical measurements of the receiver's noise. This procedure enables to emulate the same primary signal received at various SNR levels. Since the primary signal is exactly the same for each simulated SNR value, the AF remains constant, thus overcoming the problem of field measurements performed at various time instants. The sequences obtained following this procedure were employed to compute the corresponding empirical DC value for several SNR values. This method was proven to provide realistic results. The DC curves obtained with this procedure are shown in Figure 10.8 (denoted as empiric simulated) and compared to the theoretical DC curves obtained as described above. As it can be appreciated, both curves agree perfectly, thus validating the model of Equation 10.12.

\subsubsection{Variable-power discontinuous transmitters}

This section extends the model to account for variable-power transmitters. In this case, the average transmission power is not constant but characterized by a certain PDF. To simplify the model, let's assume that the variability of the transmission power can adequately be described by a discrete set of $K$ average transmission power levels, instead of a continuous PDF. This assumption not only simplifies the analytical expressions of the model, but also enables the application of the model to the case in which a channel is time-shared by $K$ transmitters with different power levels as it may be the case of various TDMA-based systems such as GSM/DCS, TETRA, etc. The model will be developed considering both cases, i.e. a single variable-power transmitter with $K$ transmission power levels and $K$ constant-power transmitters time-sharing the channel. In both cases, the problem reduces to the possibility of observing $K$ different transmission powers in the channel.

Let's denote as $f_{S_{k}}\left(P_{S_{k}}\right)$, with mean $\mu_{S_{k}}$ and standard deviation $\sigma_{S_{k}}$, the PDF of the received average power at certain location when the $k$-th transmission power level is present in the channel $(k=1,2, \ldots, K)$. In general it can be assumed that $\mu_{S_{p}} \neq \mu_{S_{q}}$ and $\sigma_{S_{p}} \neq \sigma_{S_{q}}$ for $p \neq q$. Let's define an AF $\alpha_{k}$ for each transmission power representing the fraction of time (empirical definition) or the probability (probabilistic definition) that the $k$-th transmission power level is present in the channel. In the case of a single-transmitter with $K$ transmission 


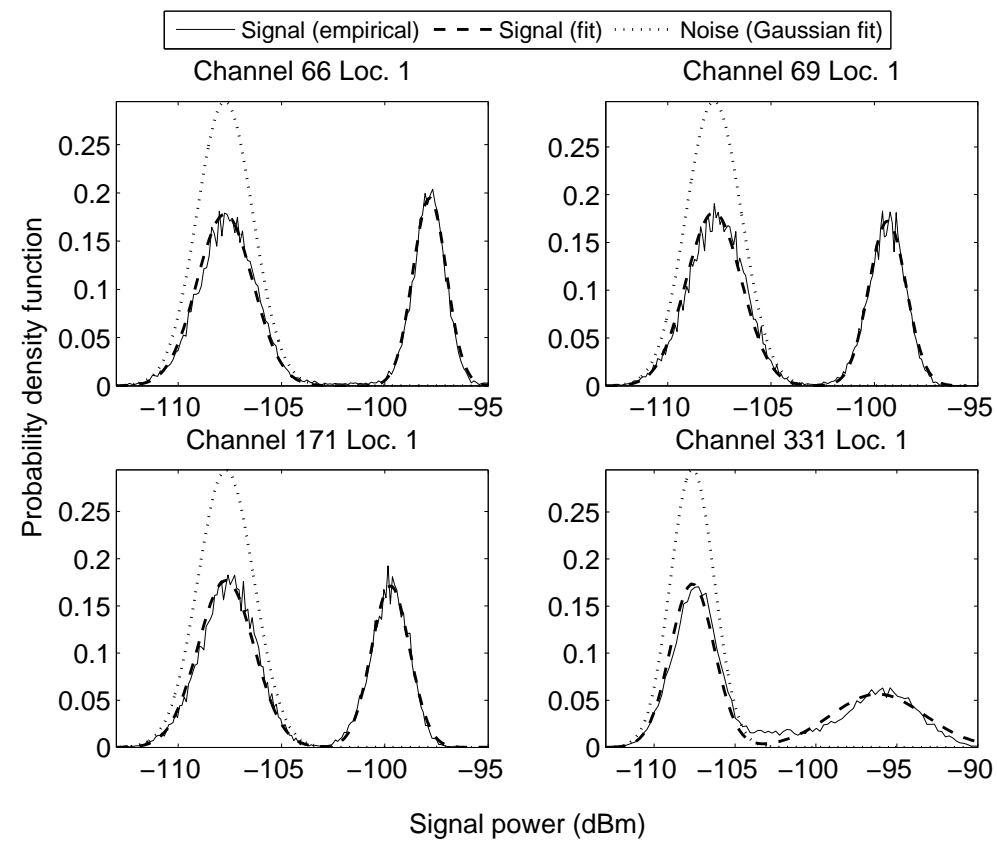

Figure 10.7: Validation of the approach employed to estimate the signal parameter's for constantpower discontinuous transmitters (TETRA channels).

Channel 66

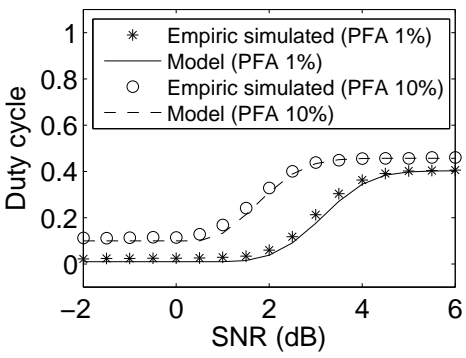

Channel 171

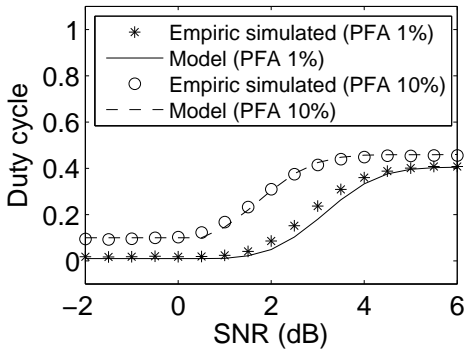

Channel 69
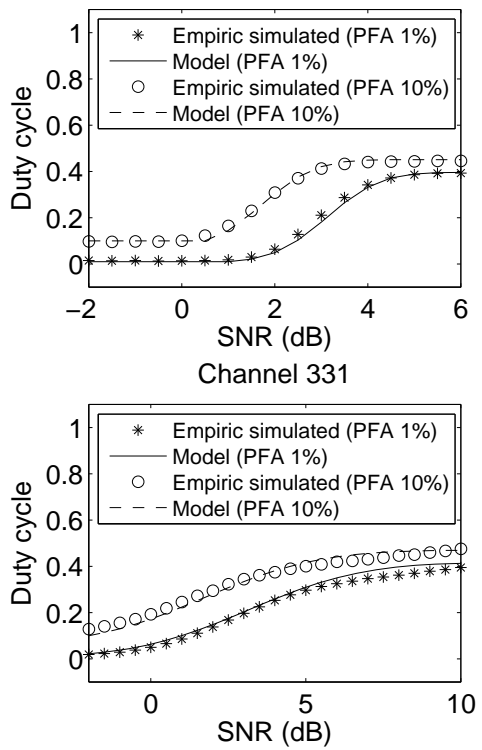

Figure 10.8: Validation of the DC model for constant-power discontinuous transmitters (TETRA channels). 
power levels, only one out of the $K$ power levels can be selected at a time. Moreover, in the case of $K$ transmitters time-sharing the channel it is reasonable to assume that there exists some MAC mechanism so that when one primary transmitter accesses the channel the rest of potential primary transmitters remain inactive. In both cases, the $K$ average power levels are mutually exclusive events. Hence:

$$
\sum_{k=1}^{K} \alpha_{k} \leq 1
$$

where the equality holds when the channel is always busy.

The left-hand side of Equation 10.13 represents the probability that any of the $K$ transmitters is active, i.e. the probability that the channel is busy, and its complementary probability $1-\sum_{k=1}^{K} \alpha_{k}$ is the probability that the channel is idle. The PDF of the received average power $f_{R}\left(P_{R}\right)$ will be that of the $k$-th primary signal (with noise) $\mathcal{M}\left\{f_{N}\left(P_{N}\right), f_{S_{k}}\left(P_{S_{k}}\right)\right\}$ whenever the $k$-th transmission power is active (which will occur with probability $\alpha_{k}$ ) or it will be noise $f_{N}\left(P_{N}\right)$ otherwise. Hence:

$$
f_{R}\left(P_{R}\right)=\left(1-\sum_{k=1}^{K} \alpha_{k}\right) f_{N}\left(P_{N}\right)+\sum_{k=1}^{K} \alpha_{k} \mathcal{M}\left\{f_{N}\left(P_{N}\right), f_{S_{k}}\left(P_{S_{k}}\right)\right\}
$$

and the resulting expression for the DC becomes:

$$
\begin{aligned}
\Psi & =\int_{\lambda}^{\infty} f_{R}\left(P_{R}\right) d P_{R} \\
& =\left(1-\sum_{k=1}^{K} \alpha_{k}\right) \int_{\lambda}^{\infty} f_{N}\left(P_{N}\right) d P_{N}+\sum_{k=1}^{K} \alpha_{k} \int_{\lambda}^{\infty} \mathcal{M}\left\{f_{N}\left(P_{N}\right), f_{S_{k}}\left(P_{S_{k}}\right)\right\} d P_{R} \\
& =\left(1-\sum_{k=1}^{K} \alpha_{k}\right) \int_{\lambda}^{\infty} f_{N}\left(P_{N}\right) d P_{N}+\sum_{k=1}^{K} \alpha_{k} \max \left\{\int_{\lambda}^{\infty} f_{N}\left(P_{N}\right) d P_{N}, \int_{\lambda}^{\infty} f_{S_{k}}\left(P_{S_{k}}\right) d P_{S_{k}}\right\} \\
& =\left(1-\sum_{k=1}^{K} \alpha_{k}\right) P_{f a}+\sum_{k=1}^{K} \alpha_{k} \max \left\{P_{f a}, Q\left(\frac{Q^{-1}\left(P_{f a}\right) \sigma_{N}-\Gamma_{k}}{\sigma_{S_{k}}}\right)\right\}
\end{aligned}
$$

where $\Gamma_{k}=\mu_{S_{k}}-\mu_{N}$ is the SNR resulting from the $k$-th average transmission power level expressed in decibels.

To validate Equation 10.15, the same approach of Section 10.4.2.2 was followed. First, the parameters required for Equation 10.15 were obtained by measuring the receiver's noise $\left(\mu_{N}, \sigma_{N}\right)$ and estimating the rest of parameters $\left(\mu_{S_{k}}, \sigma_{S_{k}}, \alpha_{k}\right)$ by fitting Equation 10.14 to the empirical PDF of the captured sequences. The number of transmitters $K$ could readily be determined by counting the number of peaks in the empirical PDF others than that of the noise. The validity of this approach is verified in Figure 10.9 (notice the presence of several transmitters in the channel, denoted as $\left.S_{1}, S_{2}, \ldots\right)$. For the part of the empirical PDF corresponding to the receiver's noise (the leftmost peak) some divergence is observed between the empirical and fitted curves. This can be explained by the presence of ambient noise. The values of $\mu_{N}$ and $\sigma_{N}$ employed in the fitting procedure were obtained by replacing the antenna 
with a matched load in order to measure the receiver's noise. In this case the ambient noise was not captured and the noise part of the fitted curve resembles that of the receiver's noise. However, when connecting the antenna, the signal captured when no primary transmission was active also included the ambient noise, thus leading to a slightly higher noise level than when measuring with the matched load. In any case, the fitting for the rest of peaks of the empirical PDF (i.e., the primary signals denoted as $S_{1}, S_{2}, \ldots$ ) is shown to be satisfactory, indicating that primary signal parameters are estimated accurately. By applying these estimated parameters to Equation 10.15, a theoretical DC curve was obtained. The empirical DC curve was obtained as in Section 10.4.2.2. Both curves are compared in Figure 10.10. Since several primary signals are present with different SNR values each, the DC is shown in this case as a function of the SNR offset, i.e. the amplitude correction factor applied to the original sequence to obtain various SNR values. As it can be appreciated, the theoretical and empirical curves agree perfectly, thus validating the model of Equation 10.15.

The validity of Equation 10.15 was corroborated by means of an additional procedure based on the field measurements performed for a time-shared TETRA channel. The empirical PDF of the power values received for such channel is shown in Figure 10.11. The figure also depicts Equation 10.14 after performing the corresponding fit to the empirical PDF curve along with the values obtained for the fitted parameters $\left(\mu_{S_{k}}, \sigma_{S_{k}}, \alpha_{k}\right)$ as well as the noise curve corresponding to the noise parameters $\left(\mu_{N}, \sigma_{N}\right)$ obtained by means of measurements of the receiver's noise. As it can be appreciated, $\alpha_{1}+\alpha_{2}=1$ meaning that the measured channel was always busy during the measurement session and therefore all the power samples captured for this channel belong to primary signals. Moreover, all the received power levels are well above the noise power, which ensures that none of the captured samples correspond to noise power samples. More interestingly, both signals are far away enough from each other to reliably classify the different received power levels as samples from each signal. For example, all power samples below $-85 \mathrm{dBm}$ can be classified as samples belonging to the first transmitter while all power samples above $-80 \mathrm{dBm}$ can be classified as samples corresponding to the second one. This observation allows to divide the original sequence of received power samples into two different sequences corresponding to each of the signals on the channel. Both sequences can then be processed separately in order to simulate any arbitrary combination of SNR values $\left(\Gamma_{1}, \Gamma_{2}\right)$ and compute the resulting DC. Following this procedure, a wide range of combinations of SNR values between -5 and $10 \mathrm{~dB}$ were simulated as already described above, and the corresponding DC was then computed. The obtained simulation results are shown in Figures 10.12(a) and 10.12(b) for target $P_{f a}$ values of $1 \%$ and $10 \%$, respectively. The DC corresponding to each simulated pair $\left(\Gamma_{1}, \Gamma_{2}\right)$ was also predicted based on Equation 10.15, making use of the fitted parameters $\left(\mu_{S_{k}}, \sigma_{S_{k}}, \alpha_{k}\right)$ shown in Figure 10.11. The differences between the DC values obtained by means of simulations and Equation 10.15 are shown in Figures 10.12(c) and 10.12(d) for the considered target $P_{f a}$ values. As it can be appreciated, the maximum absolute error for a target $P_{f a}=1 \%$ is below $1.5 \%$, while the value of the same parameter for a target $P_{f a}=$ $10 \%$ is below $8 \%$. These results not only corroborate the ability of the developed model to predict the average DC perceived by a DSA/CR user based on some basic signal parameters, but also demonstrate the accuracy attained with the proposed modeling approach. 


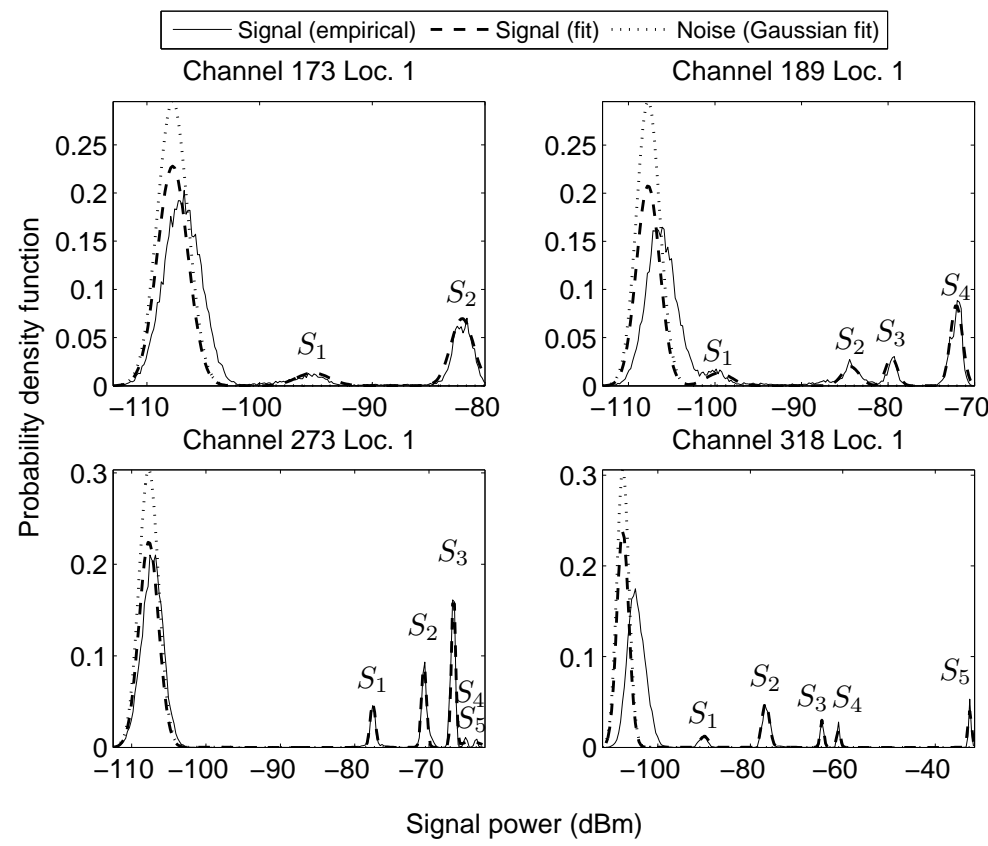

Figure 10.9: Validation of the approach employed to estimate the signal parameter's for variablepower discontinuous transmitters (TETRA channels).

Channel 173

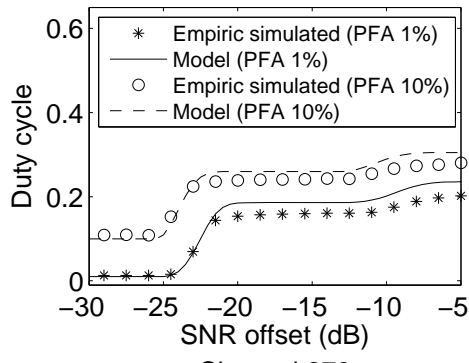

Channel 273

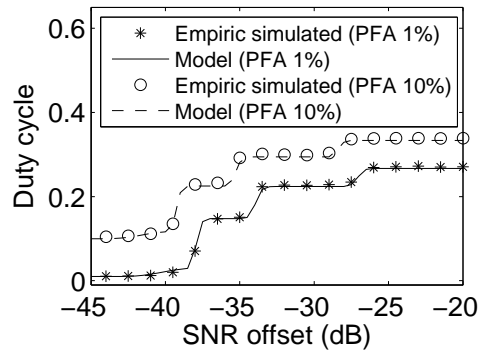

Channel 189

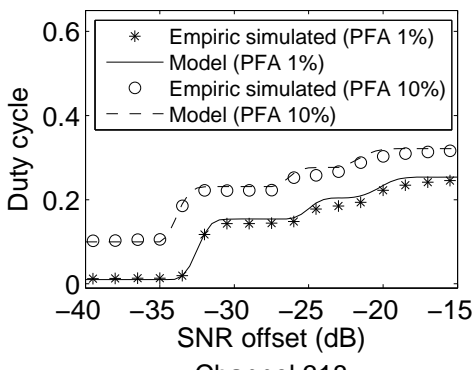

Channel 318

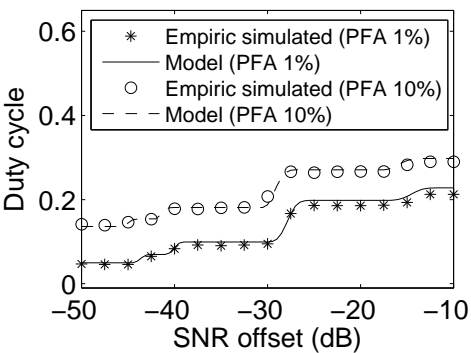

Figure 10.10: Validation of the DC model for variable-power discontinuous transmitters (TETRA channels). 


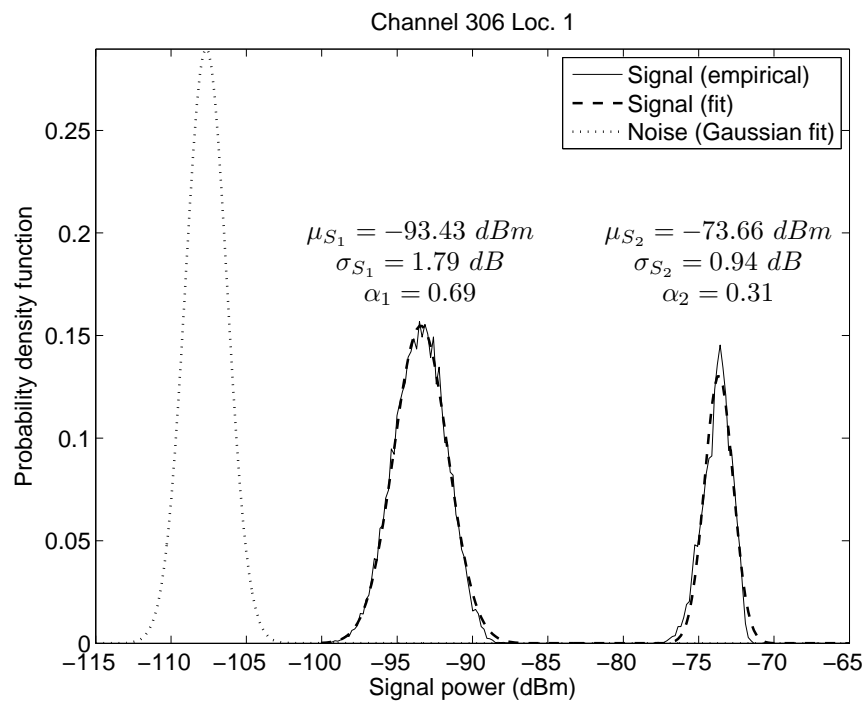

Figure 10.11: Empirical and fitted PDF of the received power for a time-shared TETRA channel.

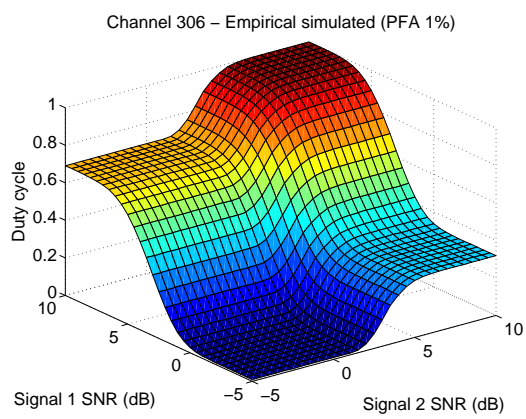

(a)

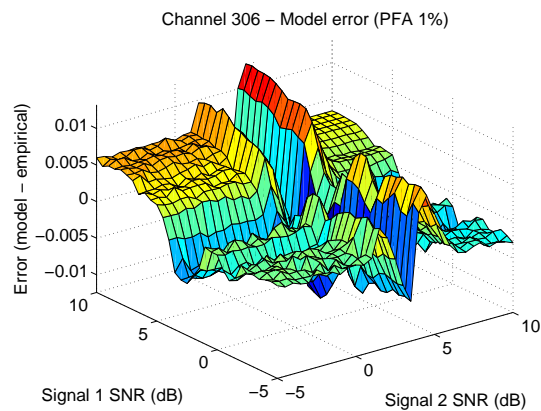

(c)

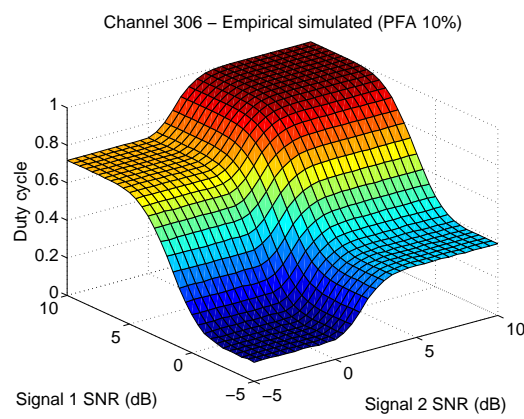

(b)

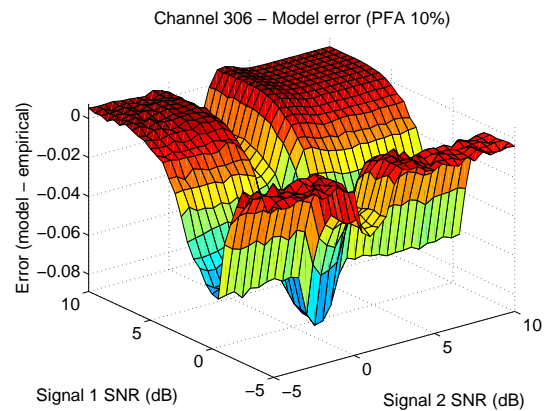

(d)

Figure 10.12: Validation of the DC model for variable-power discontinuous transmitters: (a) empirical simulated DC for $P_{f a}=1 \%$, (b) empirical simulated DC for $P_{f a}=10 \%$, (c) DC model prediction error for $P_{f a}=1 \%$, (d) DC model prediction error for $P_{f a}=10 \%$. 


\subsection{Models for concurrent snapshots observations}

The models developed in Section 10.4 are envisaged to describe the average level of occupancy (expressed in terms of the DC) that would perceived by DSA/CR users at various geographical locations based on the knowledge of some simple primary signal parameters. In some cases it can be useful to characterize not only the average level of perceived spectrum occupancy but also the simultaneous observations of several DSA/CR users at various locations. This is the case, for instance, of cooperative techniques such as cooperative spectrum sensing where the nodes of a DSA/CR network exchange sensing information (e.g., the channel state observed by each DSA/CR terminal) in order to provide, based on an appropriate processing of the gathered information, a more reliable estimation on the actual busy/idle channel state. The gain of cooperative spectrum sensing and other cooperative techniques can be characterized and analyzed in terms of simultaneous observations. For example, a group of DSA/CR nodes behind the same building would be affected by the same level of shadowing. In such a case, they would probably experience a similar average SNR and all them might not detect the presence of a primary transmission. However, other DSA/CR nodes not affected by the same building and experiencing higher SNRs might be able to detect the presence of the licensed transmission and avoid situations of harmful interference. The characterization of the simultaneous observations of various DSA/CR users as a function of their geographical locations or experienced SNRs would be of great utility in this type of studies. To this end, the model developed in Section 10.4 is extended with some additional considerations to characterize the concurrent observations of different users.

The simultaneous observations at two different locations can be characterized in terms of the joint probability that the channel is observed at both locations in certain states or the conditional probability that it is observed in a certain state in one location given that it has been observed in a specified state at the other location. This probabilistic characterization can be extended to any arbitrary number of locations by taking one location as a reference point and comparing to the rest of considered locations in pairs. The study presented in this section analyzes the joint and conditional probabilities between any two locations where one of them, the reference location, corresponds to the location where the primary signal is received at a SNR higher than that of any other location inside the geographical area under study (i.e., at the maximum experienced SNR).

The state space for a primary radio channel can be denoted as $\mathbb{S}=\left\{s_{0}, s_{1}\right\}$, where the $s_{0}$ state indicates that the channel is idle and the $s_{1}$ state indicates that the channel is busy. Let's denote as $P\left(s_{i}, s_{j}^{*}\right)$, with $i, j \in\{0,1\}$, the joint probability that the channel is simultaneously observed in state $s_{i}$ at an arbitrary location and in state $s_{j}$ at the reference location. Let's denote as $P\left(s_{i} \mid s_{j}^{*}\right)$ the conditional probability that the channel is observed in state $s_{i}$ at an arbitrary location given that it has been observed in state $s_{j}$ at the reference location. As previously mentioned, the SNR $\Gamma^{*}$ at the reference location is greater than the SNR $\Gamma$ at any other location $\left(\Gamma^{*} \geq \Gamma\right)$, which implies that the $\mathrm{DC} \Psi^{*}$ perceived at that location satisfies the condition $\Psi^{*} \geq \Psi$ for all the $\Psi$ values observed at all the other locations over the geographical area under study. The objective of this section is to derive the expressions of $P\left(s_{i}, s_{j}^{*}\right)$ and $P\left(s_{i} \mid s_{j}^{*}\right)$ for any arbitrary location as a function of the average DCs perceived at that location $(\Psi)$ and the reference location $\left(\Psi^{*}\right)$. 
The set of conditional probabilities $P\left(s_{i} \mid s_{j}^{*}\right)$ can be derived as follows. When the channel is observed as idle at the reference location, this means that the channel is actually idle or the power received at the reference location is below the decision threshold. In the latter case, the power received at any location whose receiving SNR is lower will also be below the decision threshold and the channel will also be observed as idle. However, there exists a probability $P_{f a}$ that the channel is observed as busy because of noise samples above the threshold. Thus, $P\left(s_{1} \mid s_{0}^{*}\right)=P_{f a}$ and its complementary probability is $P\left(s_{0} \mid s_{0}^{*}\right)=1-P_{f a}$. On the other hand, when the channel is observed as busy at the reference location, this means that there has been a false alarm at the reference receiver or the channel is actually busy and it has been received at the reference location with a power level above the decision threshold. In this case, the probability that the channel is observed as busy/idle at an arbitrary location depends not only on the probability of false alarm but also the experienced SNR $\Gamma$ and its relation to the reference SNR $\Gamma^{*}$. The conditional probability $P\left(s_{0} \mid s_{1}^{*}\right)$ can be derived by writing the probability $P\left(s_{0}\right)$ that the channel is observed as idle at the arbitrary location as:

$$
\begin{aligned}
P\left(s_{0}\right) & =P\left(s_{0} \mid s_{0}^{*}\right) P\left(s_{0}^{*}\right)+P\left(s_{0} \mid s_{1}^{*}\right) P\left(s_{1}^{*}\right) \\
& =\left(1-P_{f a}\right)\left(1-\Psi^{*}\right)+P\left(s_{0} \mid s_{1}^{*}\right) \Psi^{*}=1-\Psi
\end{aligned}
$$

where $P\left(s_{j}^{*}\right)$ represents the probability that the channel is observed in state $s_{j}$ at the reference location and it has been made use of the equivalence $P\left(s_{0}\right)=1-\Psi$. Solving Equation 10.16 for the desired term yields:

$$
P\left(s_{0} \mid s_{1}^{*}\right)=\frac{1-\Psi-\left(1-P_{f a}\right)\left(1-\Psi^{*}\right)}{\Psi^{*}}
$$

Following a similar procedure:

$$
\begin{aligned}
P\left(s_{1}\right) & =P\left(s_{1} \mid s_{0}^{*}\right) P\left(s_{0}^{*}\right)+P\left(s_{1} \mid s_{1}^{*}\right) P\left(s_{1}^{*}\right) \\
& =P_{f a}\left(1-\Psi^{*}\right)+P\left(s_{1} \mid s_{1}^{*}\right) \Psi^{*}=\Psi
\end{aligned}
$$

which yields:

$$
P\left(s_{1} \mid s_{1}^{*}\right)=\frac{\Psi-P_{f a}\left(1-\Psi^{*}\right)}{\Psi^{*}}
$$

The joint probabilities can readily be obtained based on their conditional counterparts as $P\left(s_{i}, s_{j}^{*}\right)=P\left(s_{i} \mid s_{j}^{*}\right) P\left(s_{j}^{*}\right)$, where $P\left(s_{0}^{*}\right)=1-\Psi^{*}$ and $P\left(s_{1}^{*}\right)=\Psi^{*}$. Table 10.1 shows the whole set of joint and conditional probabilities. These expressions combined with the analytical models developed in Section 10.4 can be employed to characterize not only the average probability that a channel is observed as busy as a function of the DSA/CR user location and some basic primary signal parameters but also the joint and conditional probability that the channel is observed in any of its states with respect to the simultaneous observation of another node at a reference location.

The validity of the expressions shown in Table 10.1 was assessed following a similar procedure as in previous sections. First, the sequence of received powers observed at location 1 (the location with the highest SNR $\Gamma^{*}$ for most of the measured bands) was selected as a reference. This sequence was then processed in order to simulate the same sequence at 
Table 10.1: Joint and conditional probabilities of simultaneous observations.

\begin{tabular}{|c|c|c|c|}
\hline$s_{\boldsymbol{i}}$ & $\boldsymbol{s}_{\boldsymbol{j}}^{*}$ & $\boldsymbol{P}\left(\boldsymbol{s}_{\boldsymbol{i}}, \boldsymbol{s}_{j}^{*}\right)$ & $\boldsymbol{P}\left(\boldsymbol{s}_{\boldsymbol{i}} \mid \boldsymbol{s}_{j}^{*}\right)$ \\
\hline$s_{0}$ & $s_{0}^{*}$ & $\left(1-P_{f a}\right)\left(1-\Psi^{*}\right)$ & $1-P_{f a}$ \\
\hline$s_{1}$ & $s_{0}^{*}$ & $P_{f a}\left(1-\Psi^{*}\right)$ & $P_{f a}$ \\
\hline$s_{0}$ & $s_{1}^{*}$ & $1-\Psi-\left(1-P_{f a}\right)\left(1-\Psi^{*}\right)$ & $\frac{1-\Psi-\left(1-P_{f a}\right)\left(1-\Psi^{*}\right)}{\Psi^{*}}$ \\
\hline$s_{1}$ & $s_{1}^{*}$ & $\Psi-P_{f a}\left(1-\Psi^{*}\right)$ & $\frac{\Psi-P_{f a}\left(1-\Psi^{*}\right)}{\Psi^{*}}$ \\
\hline
\end{tabular}

lower SNR values $\Gamma \leq \Gamma^{*}$. The reference and simulated sequences of received powers were then converted to sequences of busy/idle states by applying an ED method as mentioned in Section 10.3. Based on a direct comparison of both sequences, the joint and conditional probabilities were extracted. This procedure was repeated for a sufficiently large set of simulated SNR values $\Gamma$. The obtained results, denoted as empiric simulated, are shown in Figures 10.13 and 10.14 as a function of the SNR difference $\Gamma^{*}-\Gamma$. The theoretical curves were obtained by first estimating the required signal parameters $\left(\mu_{S_{k}}, \sigma_{S_{k}}, \alpha_{k}\right)$ by means of curve fitting procedures and the noise parameters $\left(\mu_{N}, \sigma_{N}\right)$ by means of measurements of the receiver's noise, and then employing the corresponding analytical expressions presented in Section 10.4 along with the expressions shown in Table 10.1. The obtained predictions are also shown in Figures 10.13 and 10.14 for comparison. As it can be appreciated, both curves exhibit a perfect agreement, thus indicating the validity of the proposed modeling approach.

In addition to validation procedures based on the combination of field measurements and simulation techniques, the proposed modeling approach was also validated based on measurements performed with synchronized measurement suites. As described in Section 10.3, one measurement suite was placed in the outdoor high point (location 1 in Figure 3.1) while the second measurement suite was displaced along the rest of outdoor locations at the ground level (locations 3-12 in Figure 3.1). This allowed to compare the occupancy patterns simultaneously perceived at various pairs of locations with different SNR levels. As it has already been mentioned, the main inconvenient of measuring various locations at different time instants is that the actual AF of the measured transmitter may experience some variations along time and may not be the same over different measurement sessions. However, in this case it was possible to identify a channel with a constant activity pattern (i.e., the AF inferred from the measurements was approximately constant for all measurement sessions). Based on the measurements performed over this channel it was possible to compute the curves for the joint and conditional probabilities relying solely on field measurements. The obtained empirical results are shown in Figures 10.15 and 10.16 along with the corresponding theoretical predictions. As it can be appreciated, there exists a nearly perfect agreement between the empirical and theoretical results, which confirms the validity of the proposed modeling approach and highlights its capability to provide accurate predictions of the spectrum occupancy perceived in real environments. 

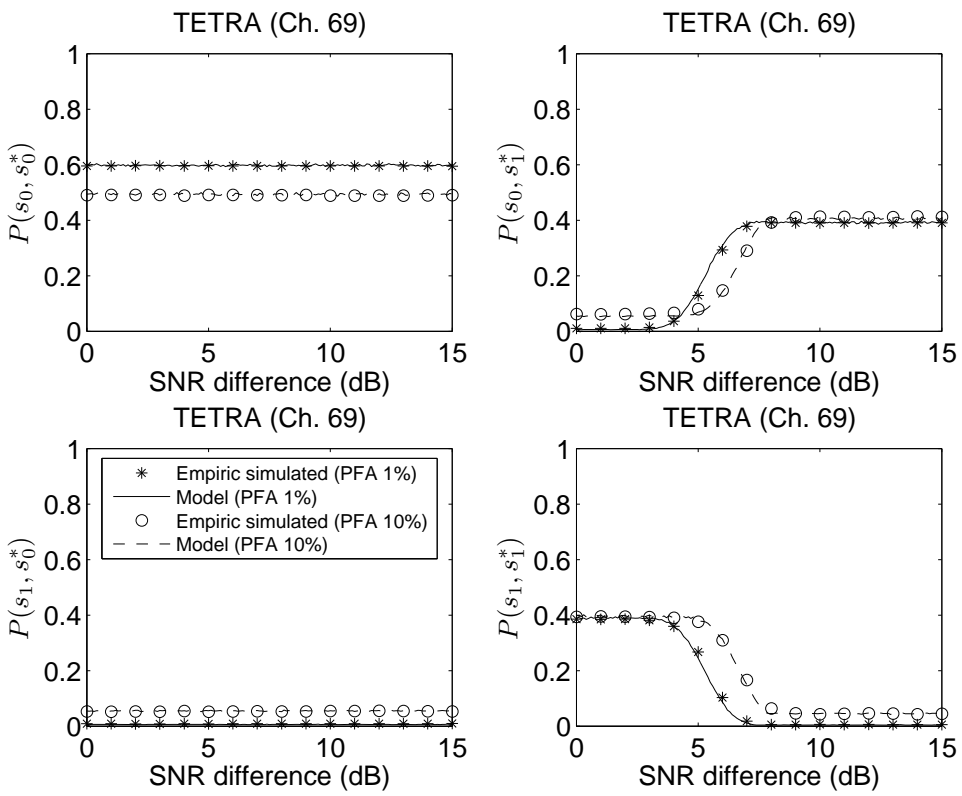

Figure 10.13: Validation of the joint probabilities.
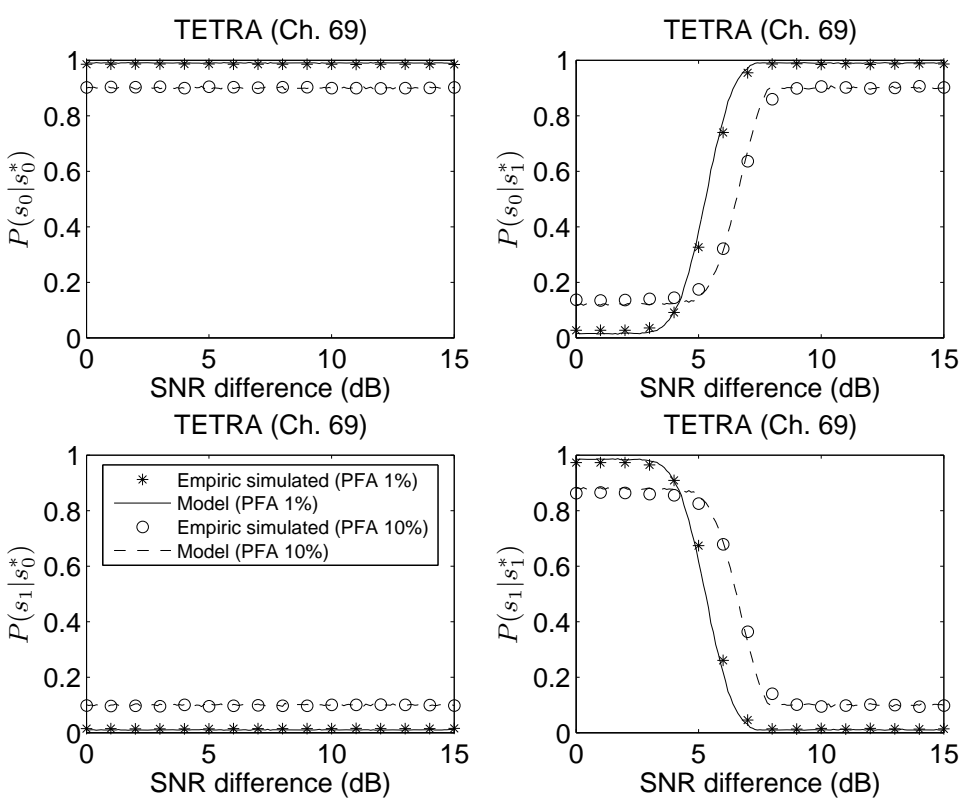

Figure 10.14: Validation of the conditional probabilities. 

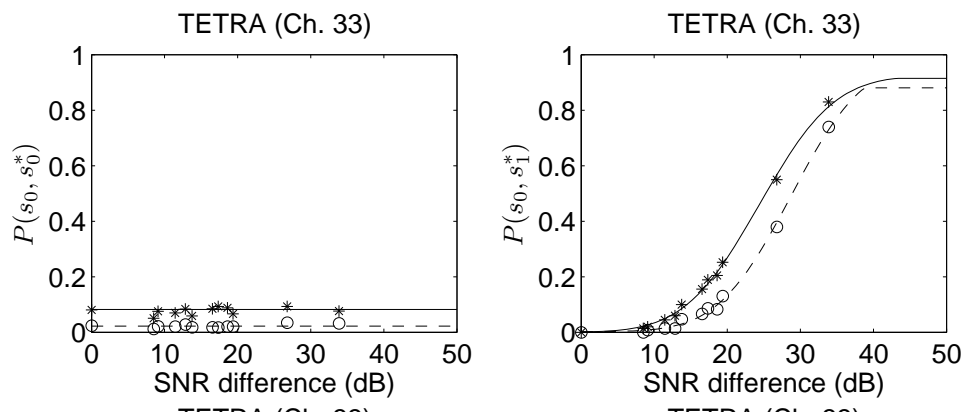

TETRA (Ch. 33)
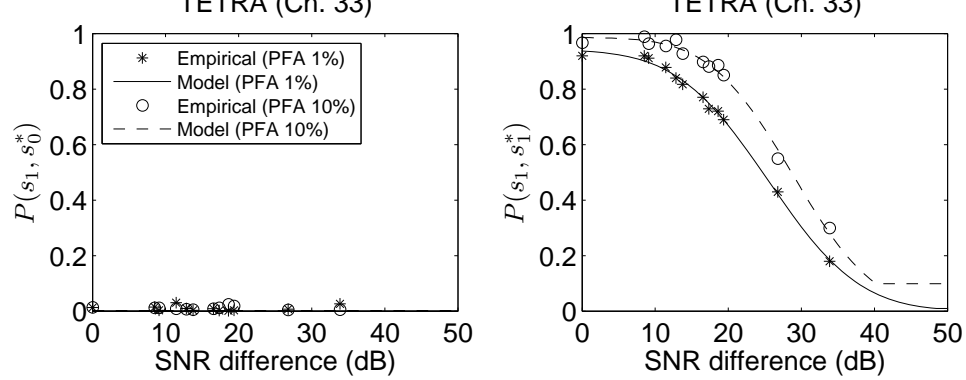

Figure 10.15: Empirical validation of the joint probabilities.

TETRA (Ch. 33)

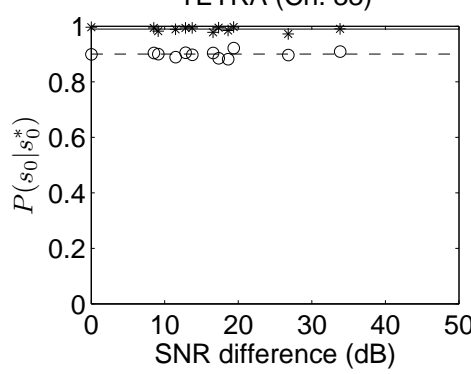

TETRA (Ch. 33)

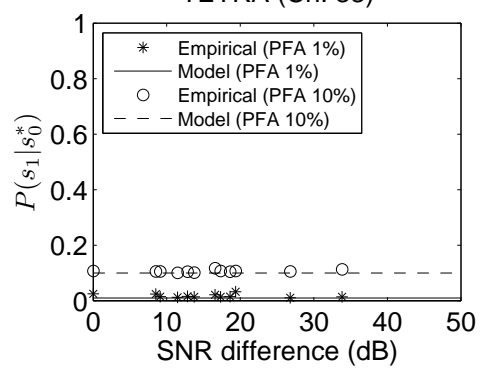

TETRA (Ch. 33)

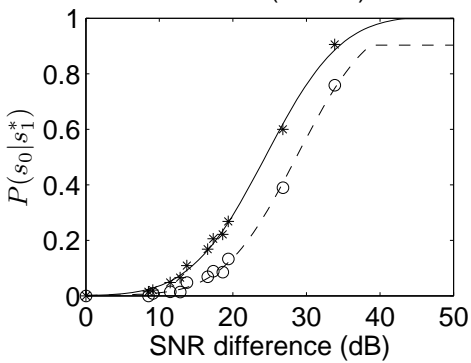

TETRA (Ch. 33)

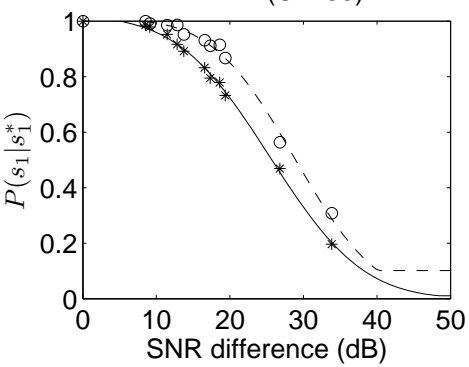

Figure 10.16: Empirical validation of the conditional probabilities. 


\subsection{Applicability of the models}

The models proposed in this chapter are theoretical in essence and provide closed-form expressions that can be employed in analytical studies. However, the applicability of the models is not restricted to analytical studies as they could find more practical applications. One possible example is the simplification of spectrum measurement campaigns in the context of DSA/CR as those detailed in Chapters 2 and 3. To obtain statistically accurate results on the occupancy level of various bands it is necessary to capture a sufficiently high number of data samples, which normally requires long measurement periods in the order of several hours, even days. If the measurements need to be repeated at different locations, the measurement campaign might require several weeks/moths. Instead of performing a high number of long measurements sessions, it would be possible to perform a single long measurement at a high-position with direct line of sight to the transmitters of interest (high SNR conditions) in order to accurately estimate the AFs $\alpha_{k}$ of the desired transmitters, and then perform some relatively short measurements at the locations of interest in order to obtain reasonable estimates of the means $\mu_{S_{k}}$ and standard deviations $\sigma_{S_{k}}$. The models proposed in this chapter could then be employed to estimate the occupancy levels that would be observed at each location. Therefore, in order to evaluate the occupancy level at $\mathcal{L}$ locations, the model could be employed to reduce the overall measurement time from $\mathcal{L}$ long measurement sessions to only one long measurement session and $\mathcal{L}$ short measurement sessions.

The proposed models can find other interesting fields of application. This section provides two detailed examples. The first example shows how the spatial models developed in this chapter can be combined with radio propagation models in order to provide a statistical characterization of the spectrum occupancy perceived at various locations within a realistic environment, while the second example illustrates how they can be implemented and employed in simulation tools.

\subsubsection{Statistical prediction of spectrum occupancy perception}

The behavior of a DSA/CR network, and consequently its impact on the primary network performance, depends on the spectrum occupancy perceived by each DSA/CR node at its local environment. The possibility of estimating the user's perceived spectrum occupancy at different realistic locations as a function of the considered scenario and the surrounding radio propagation environment can provide a valuable tool for the design, dimensioning and performance evaluation of DSA/CR networks.

The impact of considering various scenarios and propagation environments on the perceived spectrum occupancy could be estimated by means of field measurements performed at different locations. Based on experimental measurements, it is possible to infer how different operating conditions affect the perceived spectrum occupancy. One example is the study presented in Sections 3.5.3 and 3.5.4, which performed a multi-band spectrum measurement campaign and evaluated the percentage of time that various bands were observed as busy over a wide diversity of practical scenarios including indoor locations, outdoor high points and outdoor locations at the ground level (in narrow streets, between buildings and in open areas). The main advantage of field measurement-based approaches is that they im- 
plicitly account for all the potential propagation phenomena and affecting factors present in real environments. However, the user's perception as a function of the considered scenario and propagation environment can be characterized in a merely qualitative manner since the impact of particular factors or parameters (e.g., primary transmission power, operating frequency, building height, street width, etc.) cannot be isolated and quantified separately.

Another alternative approach would be to use appropriate radio propagation models in order to predict the primary signal power that would be received at various locations. Assuming that DSA/CR nodes decide on spectrum use based on ED, the power levels predicted by the propagation model would be compared to a properly set decision threshold in order to determine if the DSA/CR node would observe the sensed spectrum as busy at the considered location. Notice that this approach enables the impact of every factor or parameter considered by the propagation model to be analyzed and quantified separately. However, this method results in an oversimplified characterization of the perceived occupancy where at a given location the spectrum is always observed either as busy or idle. In practice, radio propagation phenomena such as fast (multipath) fading or slow (shadowing) fading provoke momentary signal fades. Under low SNR conditions, which is of particular interest in the study of DSA/CR, this may sometimes result in signal misdetections. Thus, the probability that the spectrum is observed as busy constitutes a more realistic parameter to characterize the spectrum occupancy perceived at various locations.

This section proposes a novel and practical approach combining radio propagation models with the developed spatial spectrum usage models in order to provide together the benefits of the aforementioned alternatives. Firstly, the proposed approach provides a probabilistic (not binary) characterization of the spectrum occupancy that would be perceived by DSA/CR nodes for different scenarios and locations. Secondly, the impact on the perceived spectrum occupancy of particular parameters of the considered scenario and radio environment can easily be isolated, quantified and analyzed. Moreover, it is possible to include the potential propagation phenomena of real environments by means of a few input parameters that can readily be obtained experimentally in practice with common general-purpose measurement devices such as off-the-shelf spectrum analyzers, thus enabling more accurate and reliable estimations. The potentials of the proposed approach are illustrated with an application to a complex and realistic scenario in a generic urban environment.

\subsubsection{Statistical prediction approach}

The proposed statistical prediction approach (illustrated in Figure 10.17) makes use of radio propagation models along with the developed DC models. In the first step, the radio propagation model is used to estimate, based on a set of input parameters $\mathbf{p}=\left(p_{1}, p_{2}, \ldots, p_{M}\right)$ such as operating frequency, distance, etc., the radio propagation loss $L$ between the primary transmitter and the DSA/CR receiver that is sensing the spectrum. Notice that the propagation model is neither specified nor constrained by the proposed method. Any model capable to estimate the radio propagation loss $L$ as a function of the scenario and environment parameters $\mathbf{p}$ can be employed. This flexibility allows the selection of the propagation model that best fits the scenario under study. The parameters $\mathbf{p}$ required to estimate $L$ are model-specific and therefore depend on the selected model. 


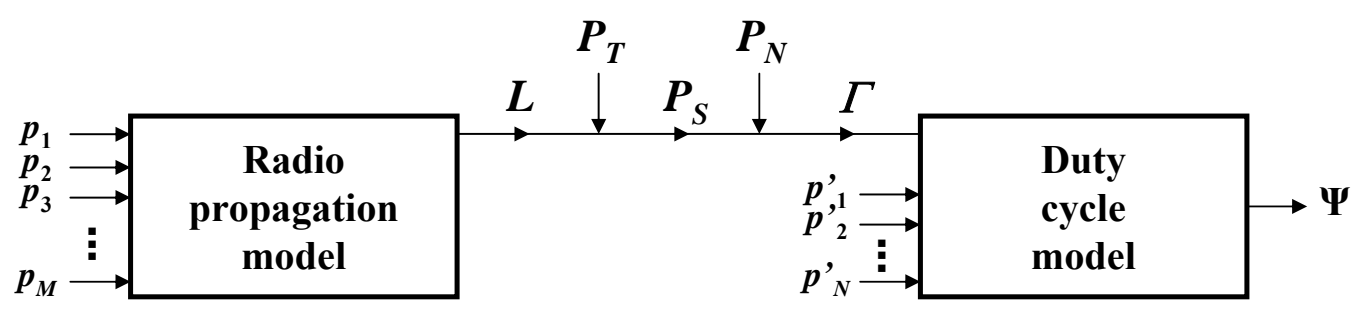

Figure 10.17: Statistical prediction approach.

Based on the primary transmission power $P_{T}$, the obtained losses $L$ are then employed to compute the primary powers $P_{S}$ that would be observed by DSA/CR nodes at various locations. The received $P_{S}$ values are translated to SNR values $\Gamma$ by making use of the noise power $P_{N}$ at the DSA/CR receiver. The resulting SNR values are then fed along with an additional set of input parameters $\mathbf{p}^{\prime}=\left(p_{1}^{\prime}, p_{2}^{\prime}, \ldots, p_{N}^{\prime}\right)$ to the DC model, which outputs an estimation $\Psi$ of the DC that would be perceived by the DSA/CR nodes at the considered geographical locations. The DC model, in its most general form, is given by Equation 10.15 and all the involved variables and parameters constitute the input vector $\mathbf{p}^{\prime}$.

The received SNR is given by $\Gamma(\mathrm{dB})=P_{S}(\mathrm{dBm})-P_{N}(\mathrm{dBm})$, where the received signal power is computed as:

$$
P_{S}(\mathrm{dBm})=P_{T}(\mathrm{dBm})-L(\mathrm{~dB})
$$

while $P_{N}$, which represents the DSA/CR terminal's noise floor created from the sum of all the receiver's noise sources (including thermal noise), can be expressed as:

$$
P_{N}(\mathrm{dBm})=-174 \mathrm{dBm} / \mathrm{Hz}+10 \log _{10} B(\mathrm{~Hz})+\mathrm{NF}(\mathrm{dB})
$$

where $-174 \mathrm{dBm} / \mathrm{Hz}$ is the thermal noise power spectral density at $290 \mathrm{~K}, B$ is the radio bandwidth of the sensed channel and NF is the DSA/CR terminal's noise figure.

The instantaneous values of signal and noise power experienced at various sensing events may suffer some fluctuations around the true mean values $P_{S}$ and $P_{N}$, respectively. In the case of the instantaneous noise power, such fluctuations are due to finite sensing times, which may not be long enough to average the instantaneous oscillations of noise. In the case of the instantaneous signal power, this may additionally be due to the fading properties of the radio channel as well as the primary transmission power pattern (i.e., the primary station may be a variable-power transmitter). To account for such fluctuations, $\sigma_{S}$ and $\sigma_{N}$ need to be considered ${ }^{5}$. Such values can be estimated or obtained experimentally in practice with common general-purpose measurement devices such as off-the-shelf spectrum analyzers. Table 10.2 shows some examples of $\sigma_{S}$ and $\sigma_{N}$ for various bands, which have been derived from the field measurements. Notice that the received signal is always affected by the receiver's noise and, as a result, $\sigma_{S}>\sigma_{N}$. The spectrum analyzer employed in this study is characterized by an approximated average sweep speed of $25 \mathrm{~ms} / \mathrm{MHz}$, which results in the sensing times shown in Table 10.2. As it can be appreciated, there is a clear and direct relation between

\footnotetext{
${ }^{5}$ Notice that $\sigma_{S}$ and $\sigma_{N}$ are some of the input parameters of vector $\mathbf{p}^{\prime}=\left(p_{1}^{\prime}, p_{2}^{\prime}, \ldots, p_{N}^{\prime}\right)$ in Figure 10.17.
} 
Table 10.2: Experimental values of $\sigma_{S}$ and $\sigma_{N}$.

\begin{tabular}{|c|c|c|c|c|}
\hline Band & $\boldsymbol{B}(\mathbf{H z})$ & Sensing time $(\mathbf{m s})$ & $\boldsymbol{\sigma}_{\boldsymbol{S}}(\mathbf{d B})$ & $\boldsymbol{\sigma}_{\boldsymbol{N}}(\mathbf{d B})$ \\
\hline $\mathrm{TV}$ & $8 \cdot 10^{6}$ & 200 & 0.5252 & 0.1679 \\
\hline $\mathrm{UMTS}$ & $5 \cdot 10^{6}$ & 125 & 0.4138 & 0.2093 \\
\hline $\mathrm{DAB}-\mathrm{T}$ & $1.7 \cdot 10^{6}$ & 42.5 & 0.8298 & 0.3640 \\
\hline $\mathrm{GSM} / \mathrm{DCS}$ & $200 \cdot 10^{3}$ & 5 & 1.6421 & 0.8921 \\
\hline TETRA & $25 \cdot 10^{3}$ & 0.625 & 2.0469 & 1.3624 \\
\hline
\end{tabular}

the effective sensing time per channel and the resulting $\sigma_{N}$. The trend is not so well-defined for $\sigma_{S}$ because in this case the obtained values also depend on the channel fading properties at various frequencies as well as the particular power pattern of each radio technology.

\subsubsection{Considered scenario and propagation models}

The considered scenario consists on a generic urban environment where buildings of height $h_{r}(\mathrm{~m})$ are deployed following a uniform layout with inter-building separation $b(\mathrm{~m})$ and street width $w(\mathrm{~m})$ as shown in Figure 10.18. The area under study comprises a grid of $5 \times 5$ buildings. A TV transmitter with height $h_{b}(\mathrm{~m})$ and operating frequency $f=800 \mathrm{MHz}$ is located $d(\mathrm{~km})$ apart (taking as a reference the geometrical center of the area under study). Within this scenario, DSA/CR nodes with antenna height $h_{m}=2 \mathrm{~m}$ may be located at the center of building rooftops (height is $h_{r}+h_{m}$ ), at the ground level between buildings (height is $h_{m}$ ) or inside buildings at any floor (height is $n \cdot h+h_{m}$, with $n$ being the floor number and $h=3$ $\mathrm{m} /$ floor). The considered locations represent various cases of practical interest and embrace a wide range of receiving conditions, ranging from direct line-of-sight at rooftops to severely blocked and faded signals at the ground level and inside buildings.

DSA/CR nodes intend to opportunistically access the spectrum band used by the primary transmitter and to this end they sense the spectrum. The aim is to determine the spectrum occupancy that would be perceived by the DSA/CR nodes within this scenario based on the approach presented in Section 10.6.1.1.

The propagation losses $L$ between the TV station and the different scenario's locations are estimated as follows. For DSA/CR nodes in building rooftops, the Okumura-Hata model [281, 282] is employed. This model is intended for receivers at the ground level or low heights. However, since it does not take into account the receiver's environment, it has been employed to estimate the received power at building rooftops. In order to reduce the estimation error, the Hata's correction factor for open areas [282, (19-20)] has been employed. For DSA/CR nodes at the ground level, the received power is computed based on the COST231 Walfisch-Ikegami model [283], which accounts for the receiver's surrounding environment to provide more accurate estimations. Both models were envisaged for cellular mobile communication systems but are valid for the considered operating frequency. In both cases, the expressions corresponding to large metropolitan areas are considered. For indoor DSA/CR nodes, losses are estimated based on the building penetration loss model for non-line-of- 


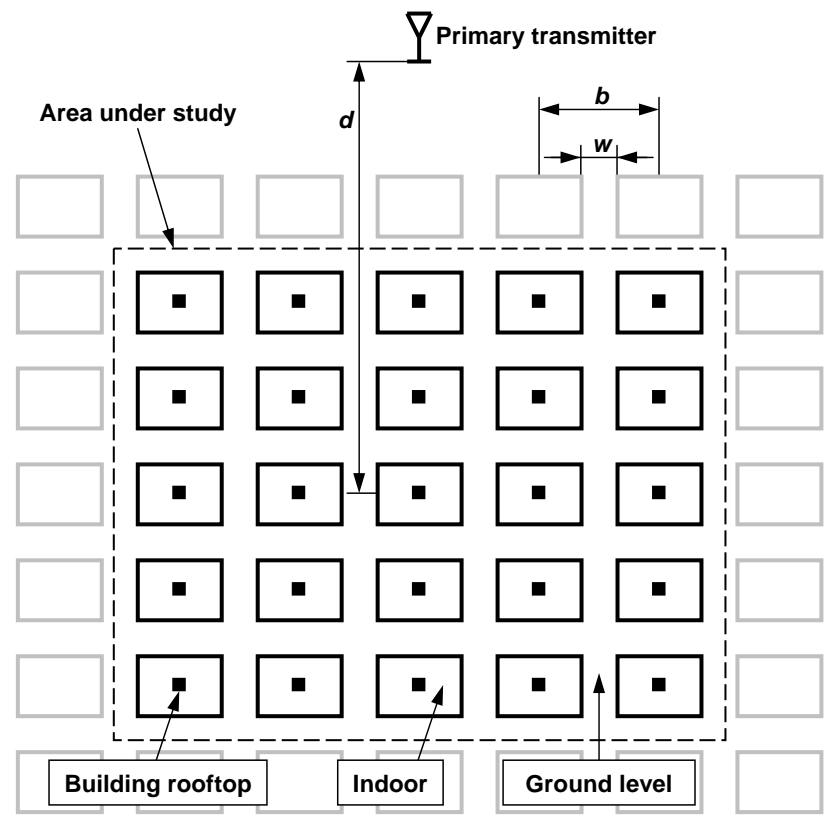

Figure 10.18: Considered scenario.

sight conditions described in [283]:

$$
L(\mathrm{~dB})=L_{\text {outside }}(\mathrm{dB})+W_{e}+W_{g e}+\beta \cdot l-h \cdot G_{h}
$$

where $L_{\text {outside }}$ is the outside loss from the TV transmitter to the building's external wall at $h_{m}=2 \mathrm{~m}$ height above the ground (based on the COST231 Walfisch-Ikegami model), $W_{e}=7$ $\mathrm{dB}$ is the loss in the externally illuminated wall at perpendicular penetration of $\phi=90^{\circ}$, $W_{g e}=4 \mathrm{~dB}$ is the additional loss in the external wall when $\phi=0^{\circ}, \beta=0.6 \mathrm{~dB} / \mathrm{m}$ is the loss per distance between adjacent walls, $l(\mathrm{~m})$ is the perpendicular distance from the externally illuminated wall, $h=3 \mathrm{~m} /$ floor and $G_{h}=1.6 \mathrm{~dB} / \mathrm{m}$ is the height gain.

\subsubsection{Numerical results}

This section analyzes how the proposed approach characterizes and describes the spectrum occupancy perceived by the nodes of a DSA/CR network. Moreover, the users' perceived spectrum occupancy at different realistic locations as a function of the considered scenario and the surrounding radio propagation environment is also investigated.

Figure 10.19 shows the primary signal power $P_{S}(\mathrm{dBm})$ received at various locations within the area under study, which has been computed based on the radio propagation models mentioned in Section 10.6.1.2. As it can be appreciated, the highest power level is observed at rooftops. It is interesting to note that the signal strength at that locations is slightly above the sensitivity of conventional TV receivers $(\approx-85 \mathrm{dBm})$, meaning that the area under study (with the selected configuration parameters) can be considered to correspond to the border of the coverage area intended for the primary transmitter. As a result, primary 
users may be present within the area under study. The results of Figure 10.19 also indicate that the primary signal power received at the ground level and inside buildings suffers important attenuations with respect to rooftops, in the order of 10 and $20 \mathrm{~dB}$ respectively. This suggests that DSA/CR nodes within the same geographical area may experience quite dissimilar perceptions depending on their particular locations and propagation conditions.

To estimate the spectrum occupancy perceived within the considered scenario, and as a first approach, the power levels $P_{S}(\mathrm{dBm})$ of Figure 10.19 are compared to a decision threshold in order to determine at which locations a DSA/CR node would decide that the spectrum is being used by the primary network. The decision threshold is set according to Equation 10.9 in order to provide a probability of false alarm $P_{f a}=1 \%$, which yields $\lambda \approx-96 \mathrm{dBm}$ for the considered parameters' values. The results are shown in Figure 10.20, where the white and gray colors indicate that the primary signal is declared to be present or absent, respectively, at the corresponding location. At rooftops, where the highest power levels are observed, the primary signal is always detected according to this prediction approach. On the other hand, the signal appears to always be undetected in all indoor locations, where the lowest power levels are observed (excepting some buildings where the signal can be detected in indoor locations near to the externally illuminated wall). At the ground level, the considered prediction approach indicates that the primary signal may be detected depending on the DSA/CR node's location. These results indicate that the considered simple approach is able to predict that, in the border of the primary coverage area (i.e., under low SNR conditions), there may be situations where primary users are present but secondary DSA/CR nodes may misdetect their transmissions, which would lead to potential situations of harmful interference. It is important to note, however, that the resulting characterization of the perceived spectrum occupancy is not only excessively simple but also unrealistic. In fact, Figure 10.20 shows, at the ground level, that this prediction approach indicates the existence of a hard limit such that the primary signal is always detected at locations slightly above but never detected at locations slightly below.

In order to provide a more sophisticated and realistic characterization of the perceived spectrum occupancy, the statistical prediction approach of Section 10.6.1.1 is proposed. As shown in Figure 10.17, the $P_{S}(\mathrm{dBm})$ values of Figure 10.19 are further processed in order to compute the probability that the sensed spectrum is observed as busy at various locations, based on the DC model of Equation 10.15. This approach has been applied to the considered scenario with the same configuration parameters of Figure 10.20, resulting in the spectrum occupancy characterization shown in Figure 10.21. The same results are shown in Figure 10.22 in a three-dimensional view for ease of appreciation. Comparing Figure 10.20 with Figures 10.21 and 10.22 it can be observed that the two considered prediction approaches agree for locations at building rooftops and indoor environments, which can be considered as extreme cases in the sense that the highest and lowest primary signal powers are observed, respectively, at such locations. Thus, at rooftops (highest primary power), the simple approach predicts that the sensed spectrum is always observed as busy while the proposed approach indicates that this occurs with probability equal to one. At indoor locations (lowest primary power), the simple approach indicates that spectrum is always observed as idle, which is also corroborated by the proposed approach with a probability of observing the spectrum as 


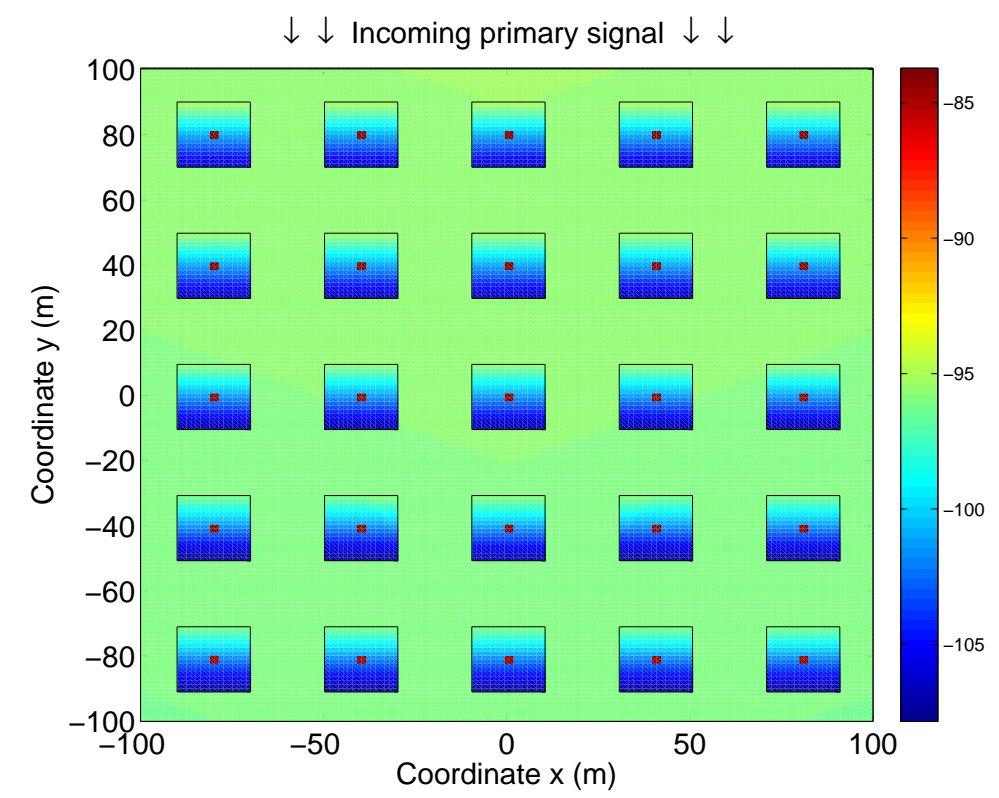

Figure 10.19: Primary signal power $P_{S}(\mathrm{dBm})$ received at various locations in the area under study $\left(P_{T}=60 \mathrm{dBm}, \sigma_{S}=0.5252 \mathrm{~dB}, \sigma_{N}=0.1679 \mathrm{~dB}, d=4.8 \mathrm{~km}, b=40 \mathrm{~m}, w=20 \mathrm{~m}, h_{b}\right.$ $\left.=50 \mathrm{~m}, h_{r}=40 \mathrm{~m}, h_{m}=2 \mathrm{~m}, n=3\right)$.

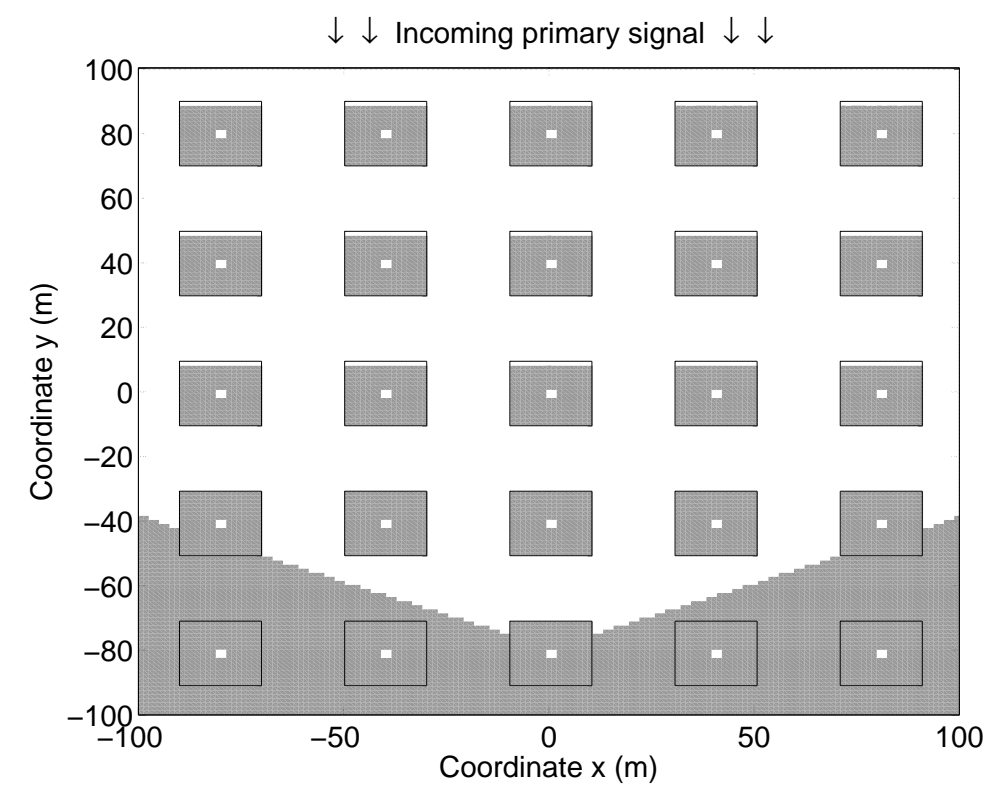

Figure 10.20: Binary spectrum occupancy pattern perceived at various locations in the area under study $\left(P_{T}=60 \mathrm{dBm}, \sigma_{S}=0.5252 \mathrm{~dB}, \sigma_{N}=0.1679 \mathrm{~dB}, P_{f a}=0.01, \mathrm{NF}=8.6 \mathrm{~dB}, d=\right.$ $\left.4.8 \mathrm{~km}, b=40 \mathrm{~m}, w=20 \mathrm{~m}, h_{b}=50 \mathrm{~m}, h_{r}=40 \mathrm{~m}, h_{m}=2 \mathrm{~m}, n=3\right)$. 
$\downarrow \downarrow$ Incoming primary signal $\downarrow \downarrow$

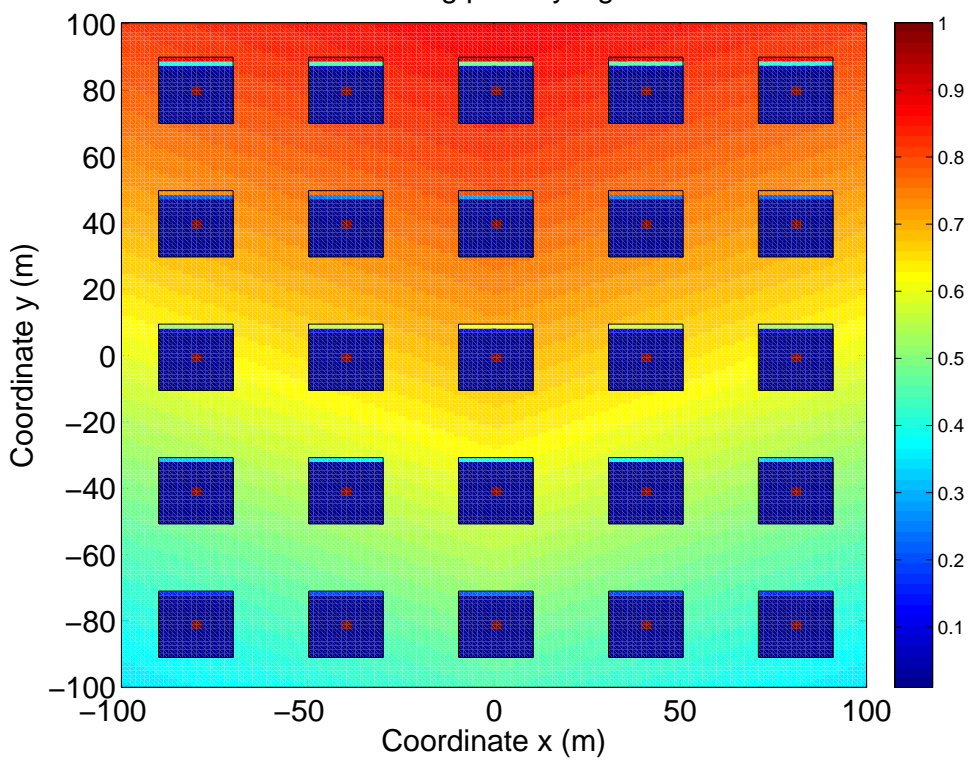

Figure 10.21: Probabilistic spectrum occupancy pattern perceived at various locations in the area under study $\left(P_{T}=60 \mathrm{dBm}, \sigma_{S}=0.5252 \mathrm{~dB}, \sigma_{N}=0.1679 \mathrm{~dB}, P_{f a}=0.01, \mathrm{NF}=8.6\right.$ $\left.\mathrm{dB}, d=4.8 \mathrm{~km}, b=40 \mathrm{~m}, w=20 \mathrm{~m}, h_{b}=50 \mathrm{~m}, h_{r}=40 \mathrm{~m}, h_{m}=2 \mathrm{~m}, n=3\right)$.

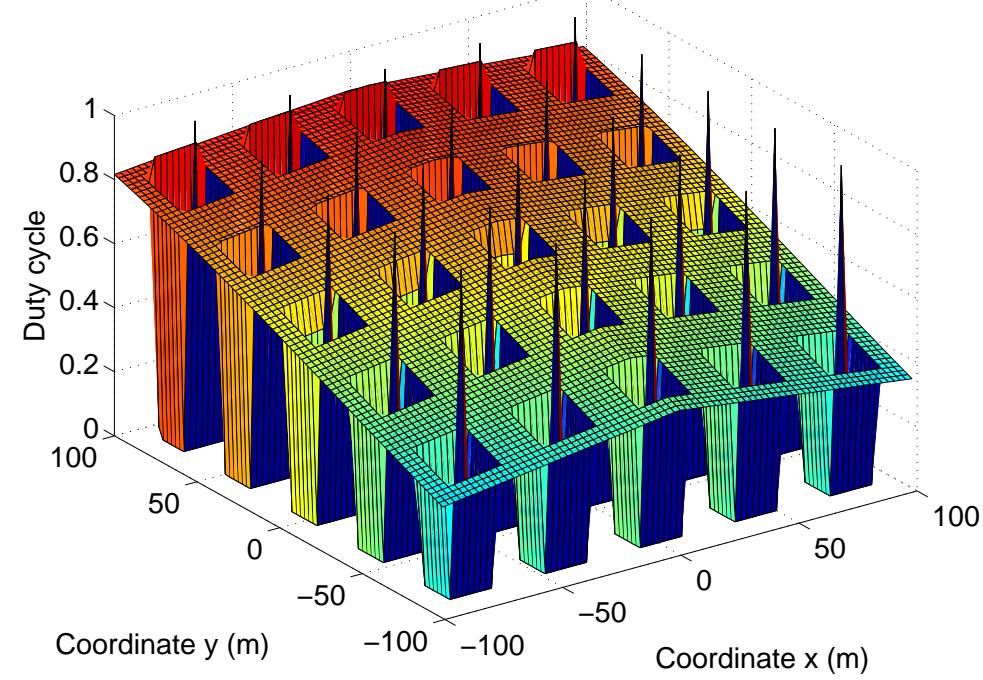

Figure 10.22: Probabilistic spectrum occupancy pattern perceived at various locations in the area under study $\left(P_{T}=60 \mathrm{dBm}, \sigma_{S}=0.5252 \mathrm{~dB}, \sigma_{N}=0.1679 \mathrm{~dB}, P_{f a}=0.01, \mathrm{NF}=8.6\right.$ $\left.\mathrm{dB}, d=4.8 \mathrm{~km}, b=40 \mathrm{~m}, w=20 \mathrm{~m}, h_{b}=50 \mathrm{~m}, h_{r}=40 \mathrm{~m}, h_{m}=2 \mathrm{~m}, n=3\right)$. 
busy approximately equal to zero. The differences between both approaches are observed in the spectrum occupancy perception predicted at the ground level. While the simple approach provides a simple binary characterization with a hard borderline, the proposed approach provides a more sophisticated characterization by means of the probability that the spectrum is observed as busy, which increases progressively as the considered location approaches the primary transmitter (without observing any abrupt transitions). It is also interesting to note that, in some locations where the simple approach predicts the channel as always idle (busy), the proposed approach indicates that the probability of observing the channel as busy is low (high) but not equal to zero (one). This example shows how the proposed statistical prediction approach is able to provide a sophisticated and realistic characterization of the perceived spectrum occupancy as a function of the considered propagation scenario.

The proposed approach can also be used to study the impact of certain scenario and propagation parameters on the user's perception. Figures 10.23, 10.24 and 10.25 show some examples. Figure 10.23 evaluates the impact of the transmitter and receiver antenna heights when the DSA/CR node is at the ground level. As expected, the detection performance improves as antenna heights increase, which results from the reduction of the radio propagation blocking caused by buildings. However, there are other interesting observations that can be inferred from the prediction provided by the proposed approach, and that would not have been possible with the simple approach considered as a reference. For example, Figure 10.23 indicates that the primary signal would not be detected for $h_{b}<42 \mathrm{~m}$, but it would always be detected for $h_{b}>46 \mathrm{~m}$, regardless of $h_{m}$. To guarantee that the primary signal is detected at the ground level with a probability of 0.9 , the primary antenna height should be around $10 \mathrm{~m}$ above the rooftop level (i.e., $h_{b} \approx h_{r}+10 \mathrm{~m}$ ) for the selected parameters. For fixed $h_{b}$, the detection performance can be improved by increasing $h_{m}$. In this sense, it is interesting to note that Figure 10.23 indicates that the DSA/CR node's antenna height, $h_{m}$, should be increased about $1 \mathrm{~m}$ for Medium-Small Cities (MSC) with respect to Large Cities (LC) in order to obtain the same detection performance. This can be explained by the fact that the COST231 Walfisch-Ikegami model employed to obtain Figure 10.23 includes an additional attenuation factor for the higher amount of vegetation usually present in MSCs.

Figure 10.24 shows the impact of building height and street width on the perceived spectrum occupancy for DSA/CR nodes at the ground level. Again, the detection performance improves as the radio propagation blocking becomes less significant, which in this case occurs for smaller buildings and wider streets. For example, for a fixed building height (e.g., $h_{r}$ $=40 \mathrm{~m})$, the primary signal might be detected with probability close to one in wide streets $(\mathrm{w}$ $=25 \mathrm{~m}$ ) but it might be completely undetected in narrower streets $(w=15 \mathrm{~m})$. In general, the perceived spectral activity is higher in open areas than in narrow streets between buildings. It is worth noting that the trend shown in Figure 10.24 was also observed qualitatively in the experimental study presented in Section 3.5.4.

Finally, Figure 10.25 explores the spectrum occupancy perceived in indoor environments as a function of the distance from the externally illuminated wall $l$ and floor number $n$. Compared to the user's perception at the ground level, the received primary signal strength in the indoor environment decreases faster with the distance. In fact, the signal would be detected close to the wall, but would be missed a few meters apart. The exact point where 


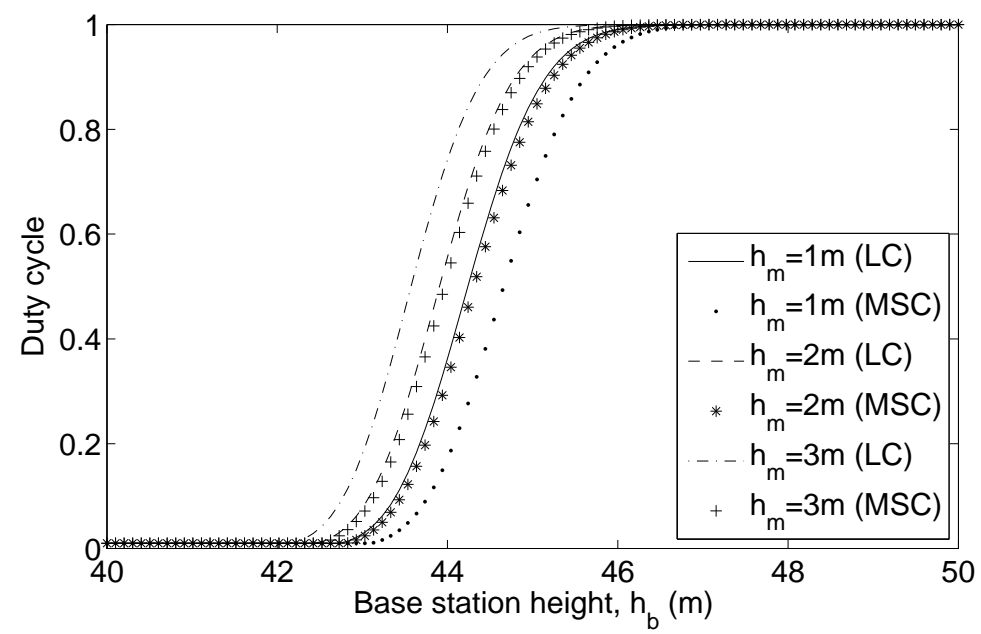

Figure 10.23: Perceived spectrum occupancy at the ground level $\left(P_{T}=60 \mathrm{dBm}, \sigma_{S}=0.5252 \mathrm{~dB}, \sigma_{N}\right.$ $\left.=0.1679 \mathrm{~dB}, P_{f a}=0.01, \mathrm{NF}=8.6 \mathrm{~dB}, d=5 \mathrm{~km}, b=40 \mathrm{~m}, w=20 \mathrm{~m}, h_{r}=35 \mathrm{~m}\right)$.

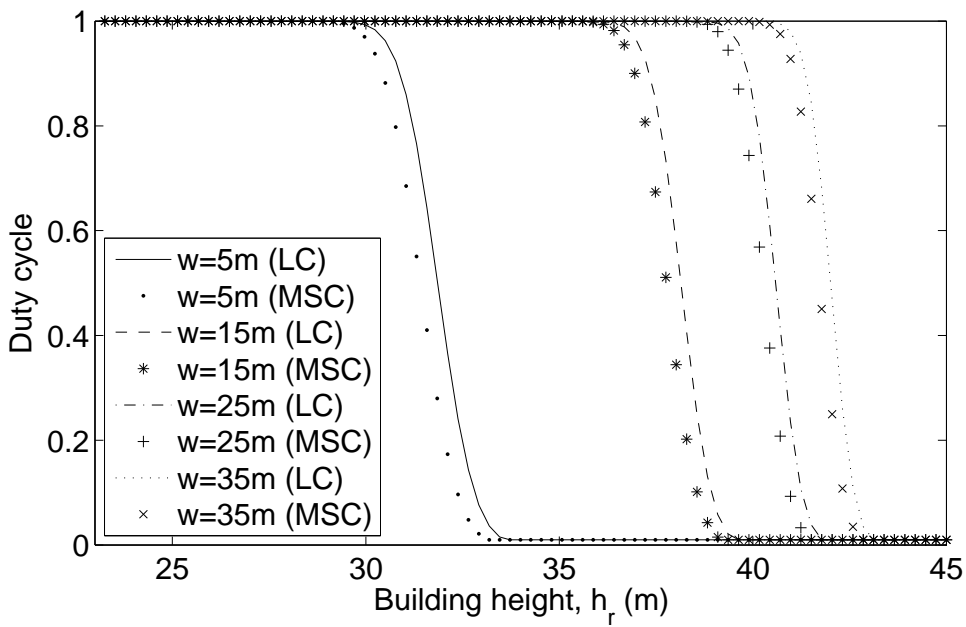

Figure 10.24: Perceived spectrum occupancy at the ground level $\left(P_{T}=60 \mathrm{dBm}, \sigma_{S}=0.5252 \mathrm{~dB}, \sigma_{N}\right.$ $\left.=0.1679 \mathrm{~dB}, P_{f a}=0.01, \mathrm{NF}=8.6 \mathrm{~dB}, d=5 \mathrm{~km}, b=40 \mathrm{~m}, h_{b}=50 \mathrm{~m}, h_{m}=2 \mathrm{~m}\right)$. 


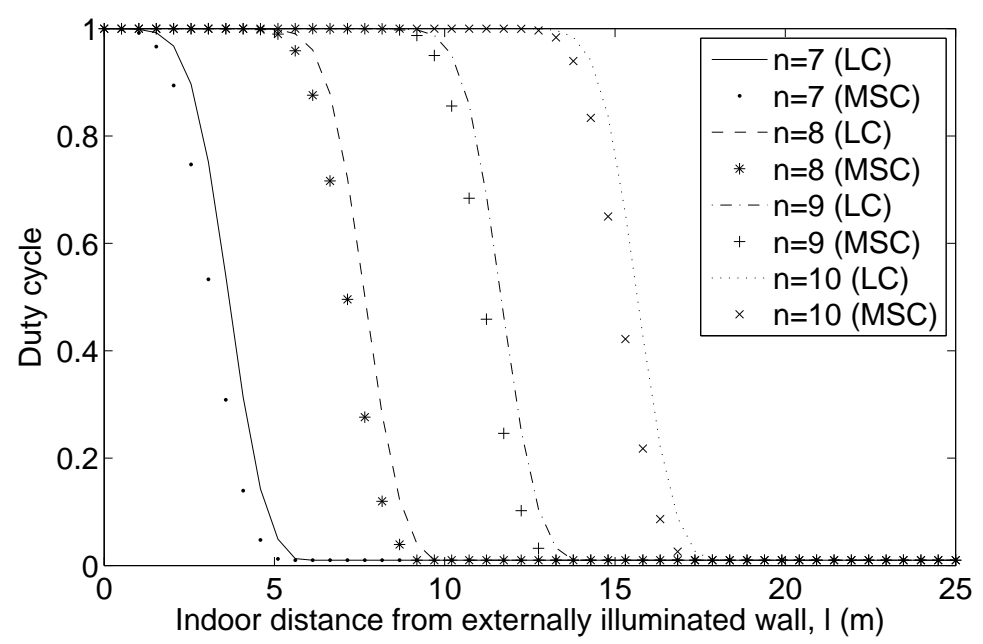

Figure 10.25: Perceived spectrum occupancy at indoor locations $\left(P_{T}=60 \mathrm{dBm}, \sigma_{S}=0.5252 \mathrm{~dB}, \sigma_{N}\right.$ $=0.1679 \mathrm{~dB}, P_{f a}=0.01, \mathrm{NF}=8.6 \mathrm{~dB}, d=5 \mathrm{~km}, b=40 \mathrm{~m}, w=20 \mathrm{~m}, h_{b}=50 \mathrm{~m}, h_{r}=$ $\left.40 \mathrm{~m}, h_{m}=2 \mathrm{~m}\right)$.

the signal begins to be undetected depends on the considered floor. As it can be observed, the perceived spectral activity is more significant in higher floors, which again can be related to a lower radio propagation blocking.

These few examples illustrate how the proposed approach can be employed not only to provide a statistical prediction of the spectral activity perceived by DSA/CR nodes but also to quantify and analyze the impact of certain particular scenario and propagation parameters on the user's perception, which constitutes an important aspect in the design and dimensioning of DSA/CR systems in real deployments.

\subsubsection{Snapshot-based simulation of spatial spectrum usage}

Another illustrative example of the applicability of the models developed in this chapter is the simplification of simulation platforms. Let's assume two system level simulators integrated into a single simulation platform and running together. One of them emulates a whole primary network in order to know what primary channels are actually busy at any time instant during the simulation. This information is used by the second simulator reproducing a secondary DSA/CR network to decide if the DSA/CR terminals at different locations observe the primary channels as busy or idle and therefore if they transmit or remain inactive. The models developed in this chapter can be used to replace the primary network simulator and simplify the overall simulation platform. Based on the locations of the primary transmitters, the knowledge of some basic signal parameters and the use of radio propagation models, it is possible to determine the average DC that would be perceived at each location inside the simulation scenario as illustrated in Figures 10.21 and 10.22. This value can be thought of as the probability that the primary channel is observed as busy at each location. Based on the perceived DC, the local decisions of DSA/CR terminals can be obtained for 
example by comparing the DC value $\Psi$ computed at their locations with a random value $X_{0}$ drawn from a uniform distribution $U(0,1)$. If $X_{0} \leq \Psi$, then the DSA/CR terminal would observe the channel as busy. Otherwise, the channel would be observed as idle. Replacing the primary network simulator with this simulation model would result in a more efficient simulation platform and therefore in significantly reduced simulation times.

Although the aforementioned simulation approach would provide the right average level of perceived spectrum occupancy in terms of the DC in the long-term, the simultaneous observations of various DSA/CR users at particular time instants would be independent of each other. The described simulation approach can be refined by additionally taking the expressions shown in Table 10.1 into account. The refined approach consists in identifying the location within the simulated geographical region where the received SNR is maximum. Selecting such location as a reference, the instantaneous channel state observed in the rest of the simulated locations is determined as follows. When the channel is observed as idle at the reference location, the rest of locations may observe the channel as busy with probability $P\left(s_{1} \mid s_{0}^{*}\right)=P_{f a}$ and idle with probability $P\left(s_{0} \mid s_{0}^{*}\right)=1-P_{f a}$. On the other hand, when the channel is perceived as busy at the reference location, the rest of locations may observe the channel as busy with probability $P\left(s_{1} \mid s_{1}^{*}\right)=\left[\Psi-P_{f a}\left(1-\Psi^{*}\right)\right] / \Psi^{*}$ and idle with probability $P\left(s_{0} \mid s_{1}^{*}\right)=\left[1-\Psi-\left(1-P_{f a}\right)\left(1-\Psi^{*}\right)\right] / \Psi^{*}$. These probabilities can be used to generate a sequence of random simulation snapshots that can adequately characterize the simultaneous observations at various locations and are able to reproduce, in the long-term, the average DC corresponding to each location within the simulation scenario.

An open question in this simulation approach is how to decide the observed channel state at the reference location. One possibility is to make use of random values $X_{0}$ drawn from a uniform distribution $U(0,1)$. If $X_{0} \leq \Psi^{*}$, then the channel is observed as busy at the reference location. Otherwise, it is observed as idle. Based on the channel state observed at the reference location and following a similar procedure the observations in the rest of locations can be generated based on the corresponding conditional probabilities $P\left(s_{i} \mid s_{j}^{*}\right)$. Another possibility is to make use of a time-dimension model to generate the sequence of channel states observed at the reference location and then decide the observations in the rest of geographical locations. This alternative as well as other integrated approaches will be further discussed in Chapter 11.

As an illustrative example, Figures 10.26 and 10.27 show some random simulation snapshots obtained by means of the aforementioned simulation method. When the channel is idle at the reference location (Figure 10.26), it is also observed as idle in most locations excepting some particular cases where it is observed as busy as a result of some false alarms. On the other hand, when the channel is perceived as busy at the reference location (Figure 10.27), the rest of locations may observe the channel as busy or idle depending on the particular user location and the corresponding conditional probabilities. In general, in areas close to the primary transmitter the probability to observe the channel as busy is higher and as a result there is a higher number of locations where it is detected in such state. This example illustrates how the proposed models can be combined and employed in the implementation and development of simulation tools for DSA/CR networks. 


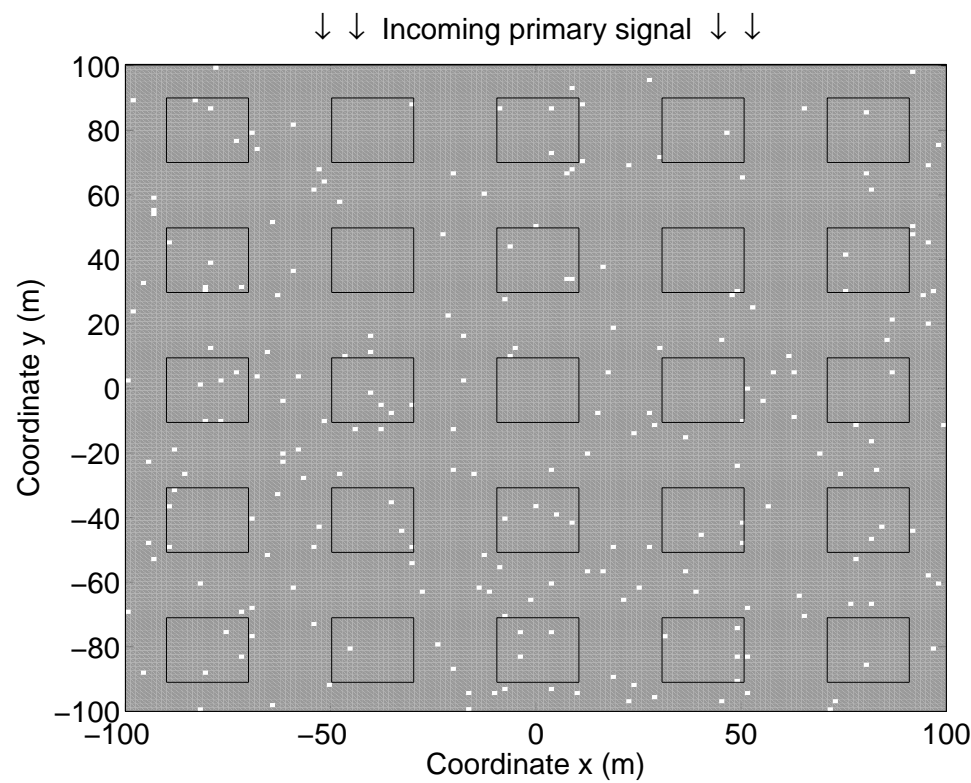

Figure 10.26: Random simulation snapshot when the channel is observed as idle at the reference location $\left(P_{T}=60 \mathrm{dBm}, \sigma_{S}=0.5252 \mathrm{~dB}, \sigma_{N}=0.1679 \mathrm{~dB}, P_{f a}=0.01, \mathrm{NF}=8.6 \mathrm{~dB}, d\right.$ $\left.=4.8 \mathrm{~km}, b=40 \mathrm{~m}, w=20 \mathrm{~m}, h_{b}=50 \mathrm{~m}, h_{r}=40 \mathrm{~m}, h_{m}=2 \mathrm{~m}, n=3\right)$.

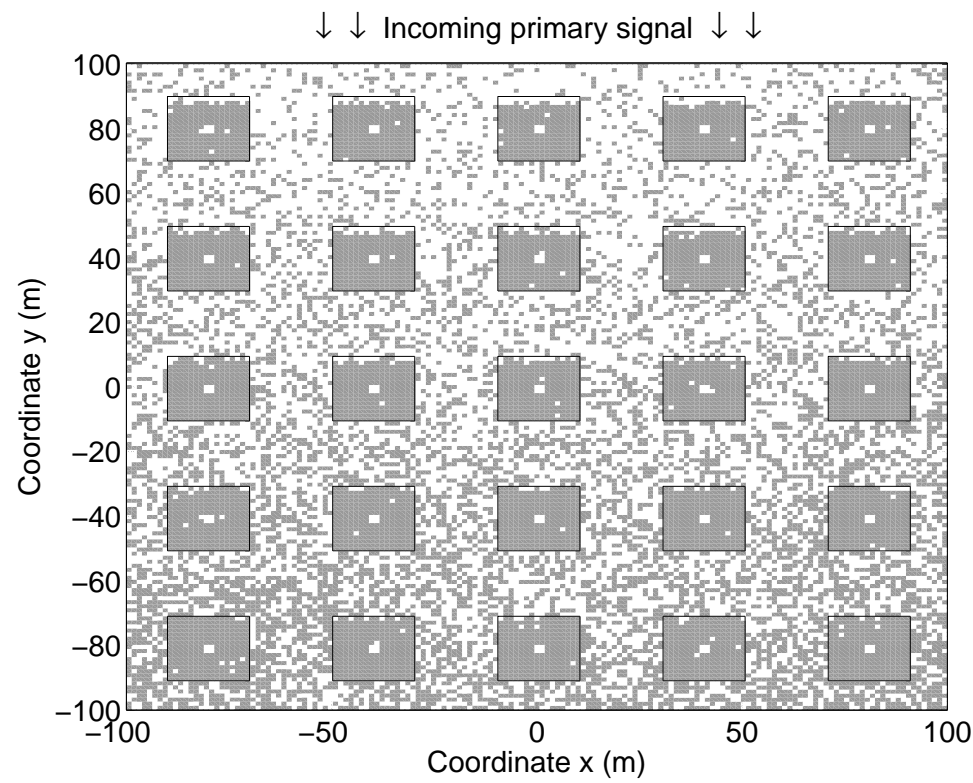

Figure 10.27: Random simulation snapshot when the channel is observed as busy at the reference location $\left(P_{T}=60 \mathrm{dBm}, \sigma_{S}=0.5252 \mathrm{~dB}, \sigma_{N}=0.1679 \mathrm{~dB}, P_{f a}=0.01, \mathrm{NF}=8.6 \mathrm{~dB}, d\right.$ $\left.=4.8 \mathrm{~km}, b=40 \mathrm{~m}, w=20 \mathrm{~m}, h_{b}=50 \mathrm{~m}, h_{r}=40 \mathrm{~m}, h_{m}=2 \mathrm{~m}, n=3\right)$. 


\subsection{Summary}

This chapter has addressed the problem of modeling spectrum usage in the spatial domain by introducing a novel set of models that describe the average spectrum occupancy level (expressed in terms of the DC) that would be perceived by DSA/CR users at any geographical location based on the knowledge of some simple primary signal parameters. An extension has also been proposed in order to characterize not only the average occupancy perception but also the simultaneous observations of various DSA/CR users on the spectrum occupancy pattern of the same transmitter. The validity and correctness of the proposed modeling approaches have been evaluated and corroborated with extensive empirical measurement results. Some illustrative examples of their potential applicability have been presented as well. 




\section{Chapter}

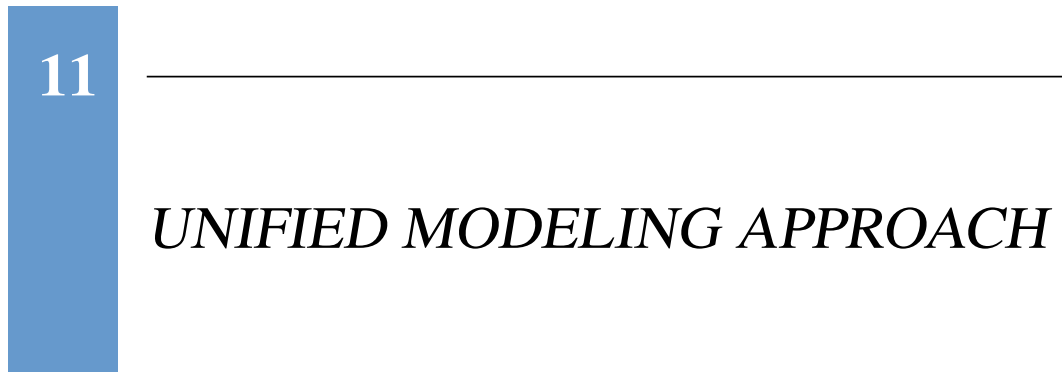

\subsection{Introduction}

Chapters 7, 8, 9 and 10 have dealt with the development of realistic and accurate models for the time (from both discrete- and continuous-time perspectives), frequency and space dimensions of spectrum usage, respectively. The validity and correctness of the proposed modeling approaches have been evaluated with extensive empirical measurements and the obtained results have demonstrated that the developed models are able to precisely reproduce relevant statistical properties of spectrum usage in real radio communication systems. However, each dimension of spectrum usage has been analyzed separately in its corresponding chapter. The purpose of this short chapter is to discuss how the models that have been proposed and developed separately can been combined and integrated into a unified modeling approach where the time, frequency and space dimensions of spectrum usage can simultaneously be taken into account and accurately reproduced. This chapter analyzes the integration of the proposed models in the context of two important fields of application, namely the realization of analytical studies, which is discussed in Section 11.2, and the development of simulation tools, which is treated in Section 11.3.

\subsection{Integration of the proposed models in analytical studies}

The models developed in this dissertation are characterized by closed-form expressions describing relevant aspects and properties of spectrum usage, which can be employed in analytical studies of DSA/CR systems. Chapter 7 has presented deterministic and stochastic DC models to be combined with DTMCs. While the deterministic models are characterized by their particular mathematical expressions, the considered stochastic models (i.e., beta and Kumaraswamy distributions) are characterized by the corresponding PDFs and CDFs, 
which can be expressed in closed-form. Similarly, in Chapter 8 it has been analyzed the suitability of various probability distributions to describe the statistical properties of busy and idle periods in real systems. The mathematical expressions for the associated PDFs and CDFs can be employed in analytical studies as well. The time-correlation properties of spectrum usage have also been analyzed in Chapter 8 and adequate mathematical expressions for the observed correlation patterns have been proposed. In Chapter 9 it has been highlighted the existence of two important aspects to be accounted for in the frequency dimension of spectrum usage, namely the DC distribution over frequency, which can be characterized by means of beta and Kumaraswamy distributions, and the DC clustering over frequency, which can be described by means of a geometric distribution. The mathematical expressions for the associated PDFs and CDFs can be employed in analytical studies related to the frequency domain of spectrum usage. Finally, the study reported in Chapter 10 has contributed a set of mathematical expressions to characterize and predict not only the average level of spectrum occupancy (expressed in terms of the DC) but also the simultaneous observations that would be perceived by DSA/CR users at various geographical locations.

The mathematical expressions developed or presented throughout this dissertation can be employed to study and analyze the behavior and performance of a DSA/CR technique in the time, frequency and space dimensions. An analytical study taking together into account aspects of various dimensions should rely on an adequate use and combination of the mathematical expressions associated to each dimension of spectrum usage (i.e., time, frequency and space). However, the concrete way in which such expressions should be combined and employed in an analytical study is a problem-specific aspect that depends on the particular scenario under consideration. It is worth noting that the availability of models capable to describe separately the relevant statistical properties of spectrum usage in its various dimensions is something that can facilitate and simplify their combination and joint use. If the relevant statistical properties of spectrum usage and their corresponding mathematical models are taken into account and adequately combined in the context of an analytical study, the obtained results and conclusions will be more realistic and reliable.

\subsection{Integration of the proposed models in simulation tools}

Another important field of application of spectrum usage models is the development of simulation tools for the performance evaluation of DSA/CR networks and their associated techniques. Some simulation methods have already been proposed throughout this dissertation in order to illustrate the implementation of the proposed models in simulation tools. This section provides a more detailed description of how the developed models can be combined and used together in order to generate artificial spectrum data capable to reproduce the statistical properties of spectrum usage in the time, frequency and space dimensions. It is worth noting that the procedure described in this section should not be interpreted as a dogmatic method but rather as an illustrative example. The spectrum usage models developed in this dissertation could be combined and used together following other approaches. Moreover, some aspects of the simulation methodology proposed in this section are based on arbitrary decisions and might need some modifications in order to meet particular simulation needs. 


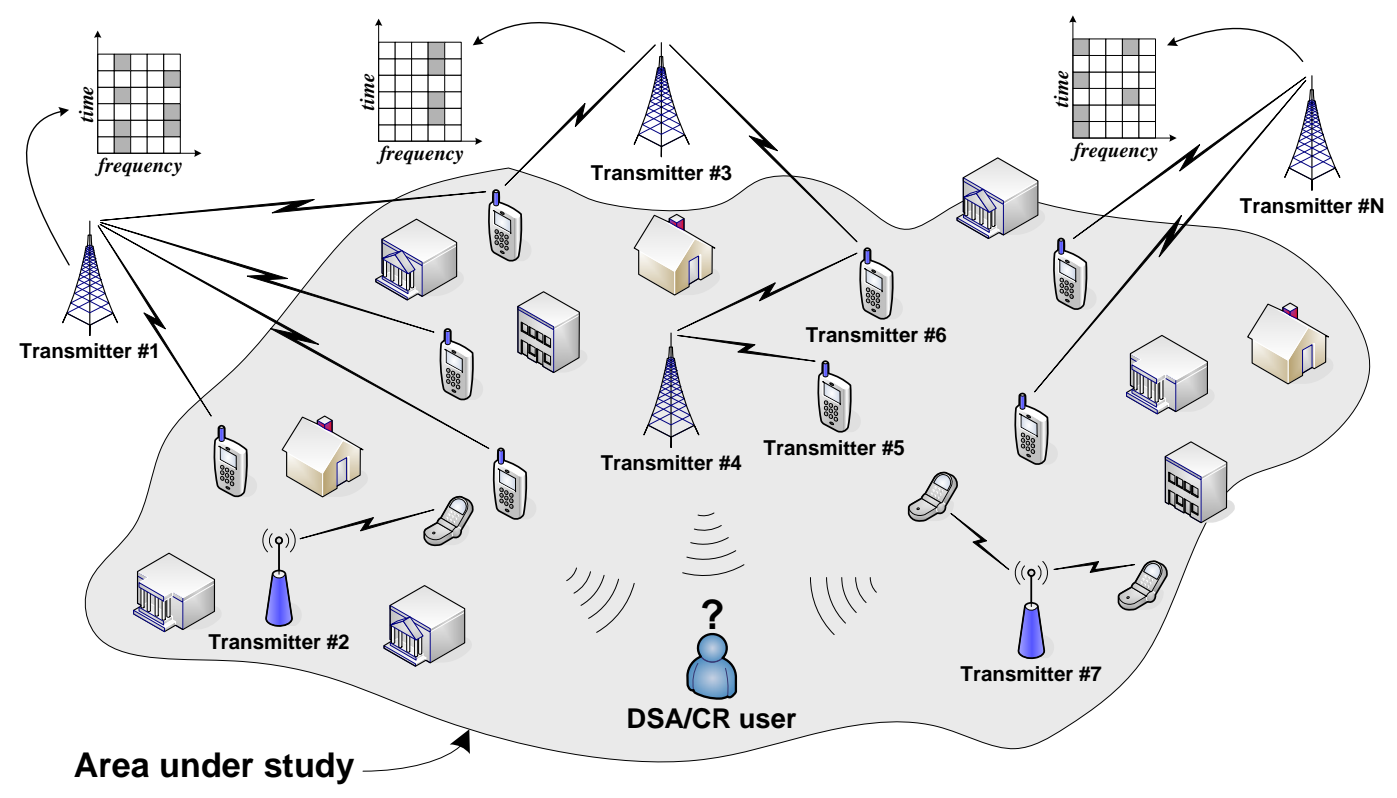

Figure 11.1: Generic simulation scenario.

The main objective of this section is to highlight the possibility to combine the spectrum usage models that have been developed independently into a unified simulation procedure that takes into account all the aspects analyzed in separate studies.

A generic simulation scenario is shown in Figure 11.1. The simulation scenario considers a specified geographical region where secondary DSA/CR users are assumed to be present. There exists a set of $N$ primary transmitters whose intended coverage areas overlap, totally or partially, with the considered geographical region. The activity of these primary transmitters needs therefore to be taken into account. Each primary transmitter is characterized by a certain location within the simulation scenario and a particular time-frequency transmission pattern as illustrated in Figure 11.1. Primary transmitters may be located inside or outside the geographical area under study and may be fixed or mobile. A mobile transmitter would imply that its location needs be recomputed periodically during the simulation according to a certain mobility model (see e.g. [284, 285]). The time-frequency pattern of each primary transmitter is defined by the set of radio channels over which the transmitter operates (note that a single primary transmitter may operate over several radio channels), the binary busy/idle occupancy sequence of each radio channel as well as the employed transmission powers. Notice that the transmission power may not be unique, for example, in the case of a time-slotted downlink channel where various slots are allocated to various receivers at different locations requiring different transmission powers. The objective is to determine the time-frequency pattern that would be perceived by a DSA/CR user over the whole spectrum band, at any arbitrary location within the simulated scenario, based on the time-frequency patterns of all primary transmitters. 
The simulation method proposed in order to generate artificial spectrum data based on the developed models is illustrated in Figure 11.2. The first step is to generate a timefrequency map of spectrum usage based on the simulation method described in Section 9.7. Such method is divided in three phases. The first two phases ensure that the generated spectrum data reproduce two relevant properties of spectrum usage in the frequency domain, namely the DC distribution (first phase of the algorithm) and the DC clustering (second phase of the algorithm). The third phase deals with the generation of individual busy/idle occupancy sequences for each of the radio channels within the considered spectrum band according to the average DC assigned to each channel as a result of the two previous phases. In this third phase, the simulation method described in Section 8.9.4 can be employed in order to reproduce not only certain specified statistical distributions for the lengths of busy and idle periods but also the desired time-correlation properties. The two-level modeling approaches proposed in Section 8.10 can also be employed in conjunction with the simulation method of Section 8.9.4 in order to generate time-occupancy sequences with specific characteristics in the short- and long-terms, including the desired statistical distributions and time-correlation properties for busy and idle periods (see Section 8.10 for details). The final result of the aforementioned simulation methods is a single time-frequency map consisting of a time-domain binary occupancy sequence for each of the radio channels within the considered primary spectrum band. As an illustrative example, Figure 11.3 shows a time-frequency map of spectrum usage generated for the TETRA DL band along with the corresponding DC distribution over frequency. Figure 11.3 has been generated based on the simulation method of Section 9.7 (including the algorithm presented in Section 8.9.4) and making use of the same configuration parameters employed to generate Figure 9.8.

The occupancy sequence observed in each radio channel of the obtained time-frequency map is the result of the activity pattern of at least one primary transmitter. The next step is to decide the primary transmitter(s) associated to each radio channel along with the corresponding location(s) and transmission power(s). These parameters can be selected so as to reproduce specific network deployments or in order to meet particular configurations or simulation needs. Another option is to select them randomly ${ }^{1}$. In the illustrative example of this section it is assumed that all radio channels belong to a single primary transmitter. Therefore, the location and transmission power associated to each channel is the same for all of them. The consequence of this simplistic assumption is that a change in the considered DSA/CR user location will result in the same SNR increase/reduction for all radio channels. In a more realistic configuration where different radio channels belong to various primary transmitters at different locations, a displacement of the DSA/CR user would result in approaching or moving away from various transmitters and hence different SNR increases/reductions for each radio channel. Although more realistic configurations are possible, this simple approach will suffice to illustrate the proposed modeling approach.

\footnotetext{
${ }^{1}$ It is interesting to mention that some works have recently proposed the use of image processing techniques to estimate the location of a primary transmitter based on spatially distributed power measurements [286, 287]. The application of such techniques to the field measurements performed in the context of this dissertation might be considered in order to statistically characterize the spatial distribution of primary transmitters in real systems. This information would be useful in simulation tools in order to randomly select the location of the primary transmitters. Although this is an interesting aspect, it is out of the scope and main objectives of this dissertation.
} 


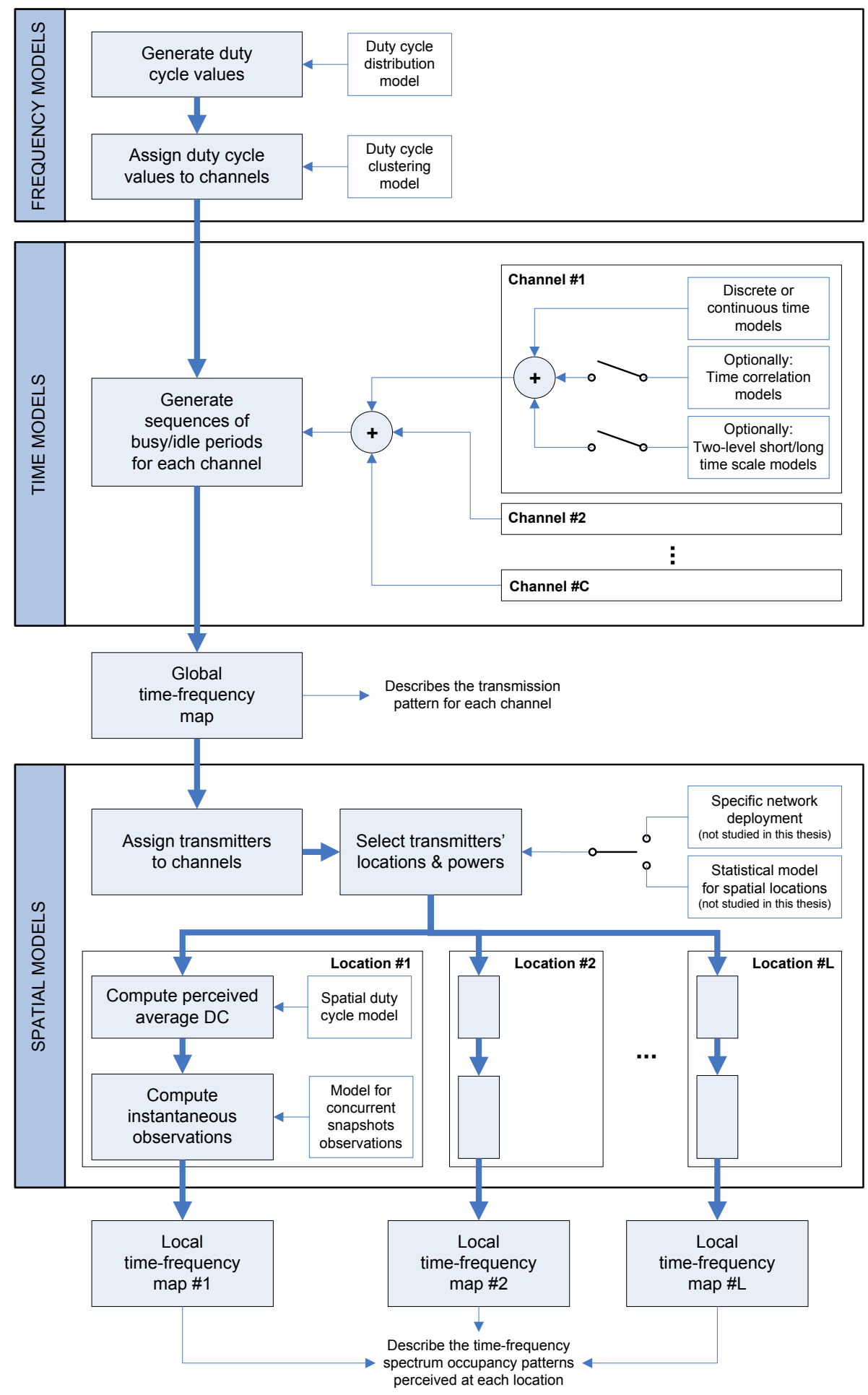

Figure 11.2: Unified simulation approach. 
It is worth noting that the generated time-frequency map can be thought of as the superposition of the spectrum occupancy patterns of all the primary transmitters, where the individual occupancy sequence at each radio channel is indeed the transmission sequence of one primary transmitter.

After generating the time-frequency map and selecting the primary transmitters, locations and transmission powers, the next step is to determine how the time-frequency map (i.e., the set of primary transmissions) is perceived by DSA/CR users at arbitrary locations within the area under study. This can be accomplished by means of the spatial simulation method described in Section 10.6.2, where the perceptions at arbitrary locations are determined based on the observations at one reference location where the receiving SNR is maximum. If the locations of the primary transmitters can be assumed to be known, then the simulation method is greatly simplified since (for each radio channel) the reference location is indeed the location of the primary transmitter (i.e., where the SNR is maximum) and the AF and reference DC values are identical (i.e., $\alpha=\Psi^{*}$ ) and equal to the average DC observed in the time-frequency map. The only unknown parameter is the average DC perceived at each location of interest, which can be computed based on the locations and transmission powers of the primary transmitters by making use of the expressions presented in Section 10.4.2. The time-frequency map perceived at every location can then be determined based on the method of Section 10.6.2 as follows: whenever the time-frequency map indicates a busy state, the channel may be observed as busy at an arbitrary location with probability $P\left(s_{1} \mid s_{1}^{*}\right)$ and whenever the map indicates an idle state, the channel may be observed as busy with probability $P\left(s_{1} \mid s_{0}^{*}\right)$. Following this procedure, the time-frequency map can be extrapolated to any arbitrary location within the area of study based on the corresponding conditional probabilities $P\left(s_{i} \mid s_{j}^{*}\right)$, which are provided in Table 10.1.

As an example, Figures 11.4, 11.5 and 11.6 show the time-frequency map of Figure 11.3 as perceived at arbitrary locations where the receiving SNR is $10 \mathrm{~dB}, 3 \mathrm{~dB}$ and $0 \mathrm{~dB}$, respectively. These results have been obtained by making use of the values shown in Table 10.2 for TETRA and assuming $P_{f a}=10 \%$ (the target $P_{f a}$ has intentionally be set to this high value to clearly show its impact). As it can be appreciated in Figure 11.4, under high SNR conditions the channels are observed as busy whenever they are actually busy. However, there is an appreciable number of points indicating that, in some cases, the channel is detected as busy when it is actually idle. These points correspond to false alarms where the noise power of the receiver surpasses the decision threshold. In fact, while the DC shown in Figure 11.3 takes values within the interval $[0,1]$, in Figure 11.4 it is above $10 \%$ (i.e., the $P_{f a}$ ). For locations where the experienced SNR is low, the primary signal may be received below the decision threshold, in which case it is misdetected. This is clearly shown in Figure 11.5 where the perceived DC is notably lower than in Figure 11.4. Finally, at locations with very low SNRs as in the example of Figure 11.6 the primary signals can hardly be detected and only false alarms are observed (i.e., $\Psi \approx P_{f a}$ ). It is worth noting in the examples of Figures 11.4, 11.5 and 11.6 that all channels experience a similar reduction of the DC as the SNR decreases because all them have been assumed to belong to the same primary transmitter. As mentioned above, in a more realistic scenario the DC may increase for some channels and decrease for some others at the same time as the DSA/CR user moves along the area under study. 

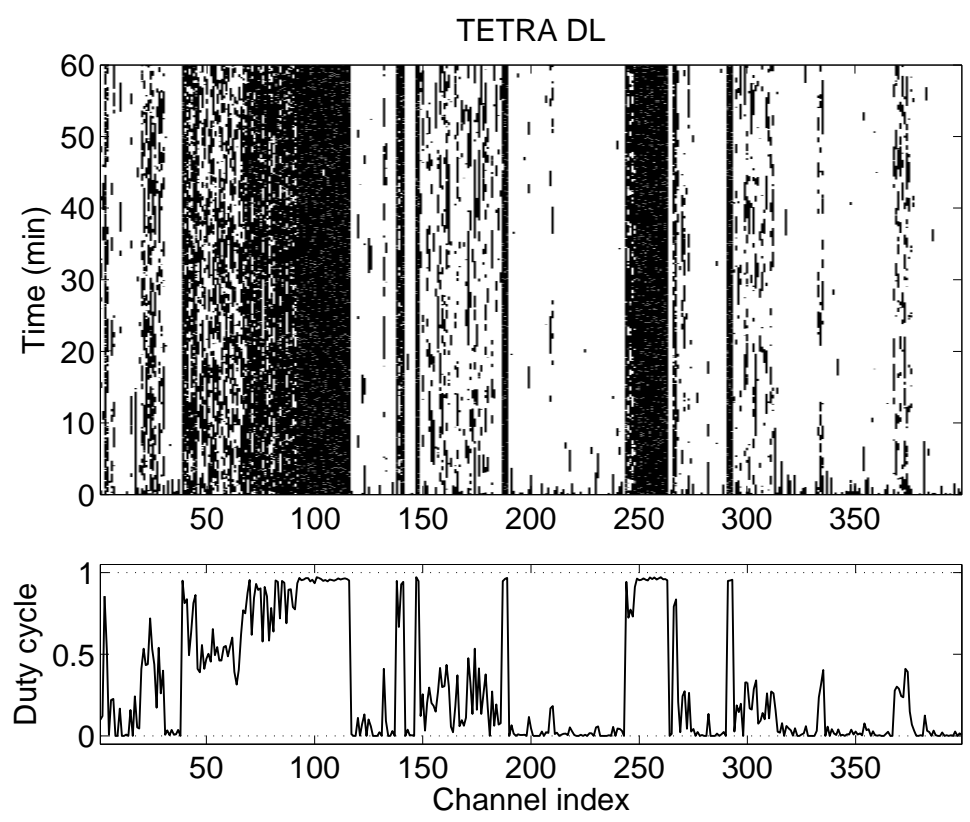

Figure 11.3: Time-frequency map of spectrum occupancy.
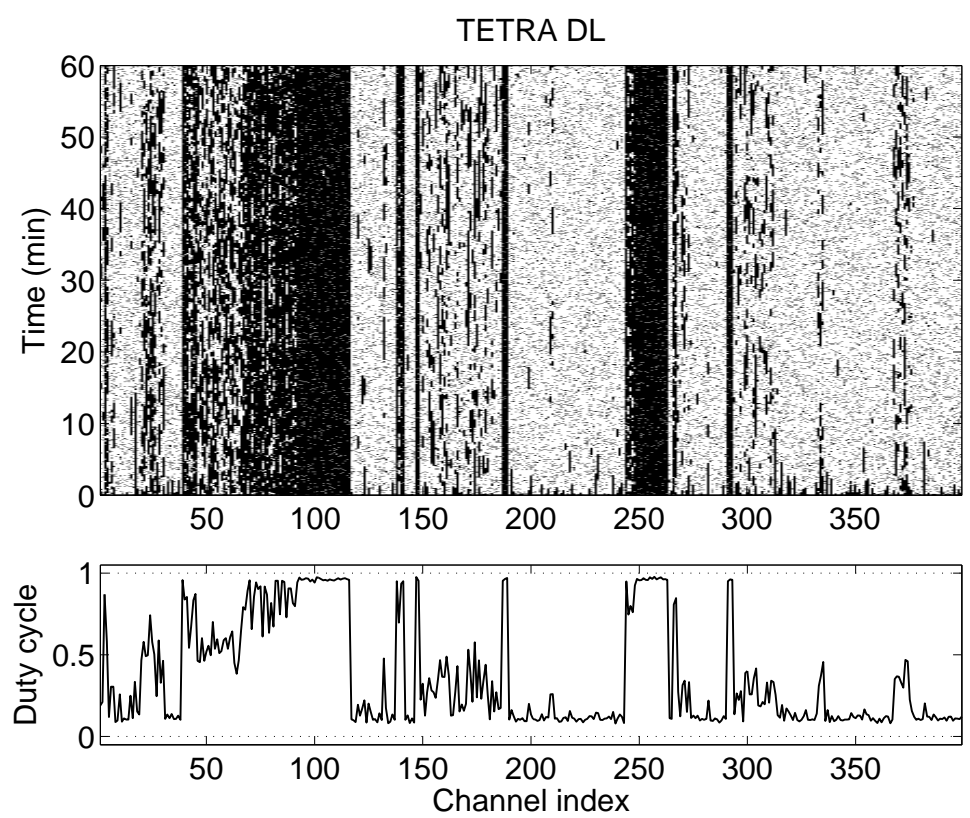

Figure 11.4: Time-frequency map of spectrum occupancy as perceived at 10-dB SNR. 
TETRA DL
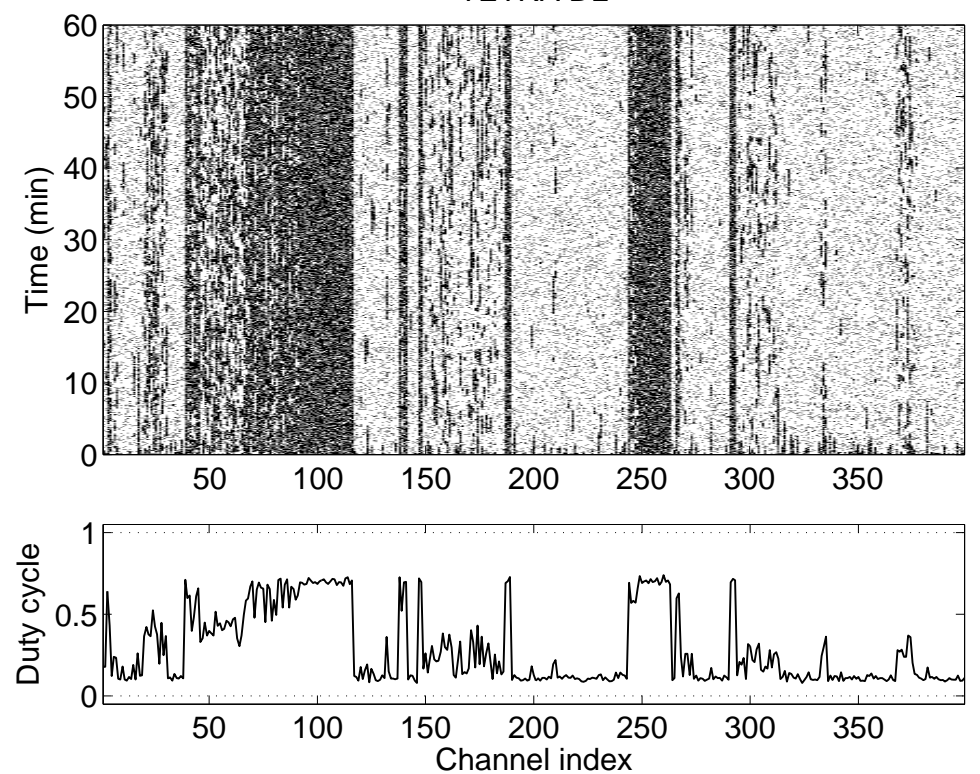

Figure 11.5: Time-frequency map of spectrum occupancy as perceived at 3-dB SNR.

TETRA DL
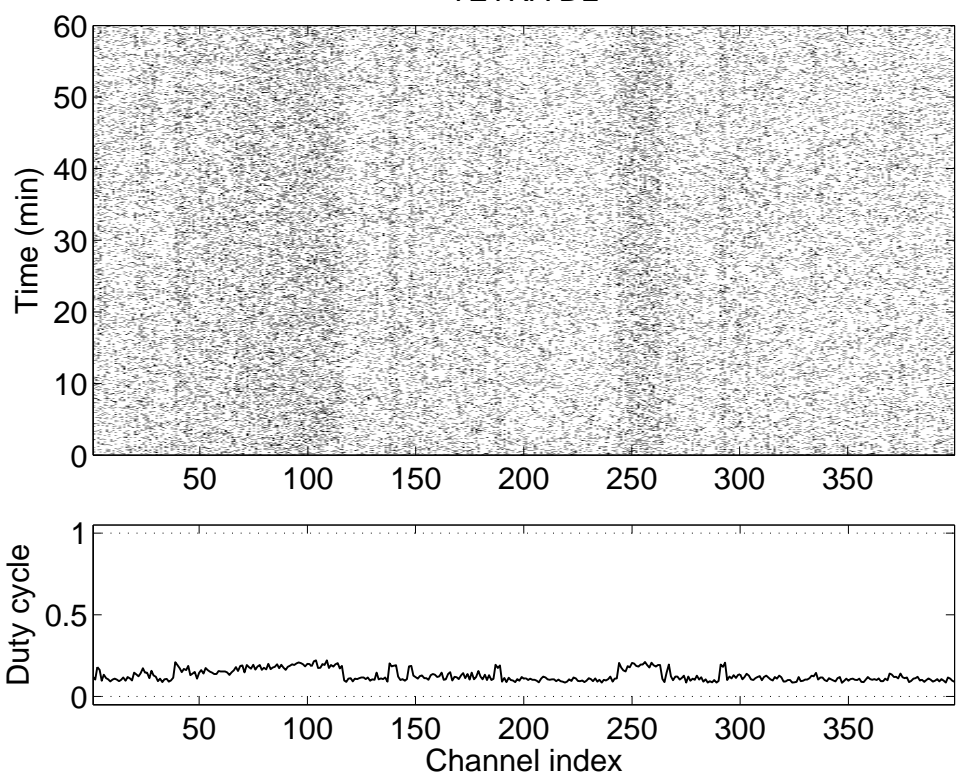

Figure 11.6: Time-frequency map of spectrum occupancy as perceived at 0-dB SNR. 
Regarding the implementation of the unified modeling approach in simulation tools, it is worth noting that time-frequency maps can be precomputed off-line for a set of predefined locations within the simulated scenario (for example according to a regular grid) and loaded into the simulator during its initialization. During the execution of the simulator, the location of each DSA/CR user can be approximated to the nearest location for which a time-frequency map has been precomputed and, based on the associated map, the spectrum occupancy that would be perceived by each DSA/CR user can be determined. Another option is to implement the whole map generation method in the simulation tool and compute on-demand time-frequency maps during the execution as required. While the former approach may result in more efficient simulations and hence shorter execution times, the latter may provide more accurate results since the exact location of the DSA/CR user is employed instead of the closest point of a grid. However, both approaches would be valid in order to include in the simulations the statistical properties of spectrum usage observed for real radio communication systems in the time, frequency and space domains.

\subsection{Summary}

The models contributed by this dissertation have been developed by analyzing the statistical properties of each spectrum usage dimension (i.e., time, frequency and space) independently and in an isolated manner. Nevertheless, the proposed models can been combined and integrated into a unified modeling approach where the time, frequency and space dimensions of spectrum usage can simultaneously be taken into account and accurately reproduced. This chapter has discussed the integration of the proposed models in the context of two important fields of application, namely the realization of analytical studies and the development of simulation tools. Some illustrative examples have been provided as well. In summary, and in spite of the independent modeling studies carried out throughout this dissertation, the proposed models can be gathered into a unified modeling approach in order to provide a complete and holistic characterization of spectrum usage in real systems for the analysis, design and simulation of DSA/CR networks. 



\section{Part IV}

\section{Conclusions and Future Work}

There is a way to do it better - find it.

Thomas A. Edison

All of old. $\mathcal{N}$ otting else ever. Ever tried. Ever failed.

No matter. Try again. Fail again. Fail better.

Samuel Beckett 



\section{Chapter}

\section{2}

\section{CONCLUSIONS AND FUTURE WORK}

The DSA/CR concept has received increasing attention in the last years for its promising potential to conciliate the existing conflicts between the ever-increasing spectrum demand growth and the currently inefficient spectrum utilization. DSA/CR aims at improving spectrum use efficiency by allowing unlicensed (secondary) users to access in an opportunistic and non-interfering manner some licensed bands temporarily unoccupied by the licensed (primary) users. This conceptually simple but innovative and challenging spectrum access paradigm is expected to enable a more efficient use and exploitation of the spectrum bands with commercially attractive radio propagation characteristics, thus providing the means for the commercial rollout of new emerging services, radio access technologies and operators.

Due to the opportunistic operating principle of the DSA/CR concept, the behavior and performance of a secondary network depends on the spectrum occupancy patterns of the primary system. A realistic and accurate modeling of such patterns becomes therefore essential and extremely useful in the domain of DSA/CR research. The potential applicability of spectrum usage models ranges from analytical studies to the design and dimensioning of secondary networks as well as the development of innovative simulation tools and more efficient DSA/CR techniques. However, the utility of such models depends on their realism and accuracy. Unfortunately, the models for spectrum usage commonly used to date in DSA/CR research are limited in scope and based on oversimplifications or assumptions that have not been validated with empirical measurement data. In this context, this dissertation has addressed the problem of modeling spectrum usage in the context of DSA/CR networks. A comprehensive and holistic set of realistic models has been contributed. The proposed models are capable to accurately capture and reproduce the relevant statistical properties of spectrum usage observed in real radio communication systems in the time, frequency and space dimensions. This chapter summarizes the main conclusions derived from the investigation carried out in this dissertation and discusses possible directions for future work. 


\subsection{Conclusions}

The first part of this dissertation has addressed the development of a unified methodological framework for spectrum measurements in the context of DSA/CR. Although several spectrum measurement campaigns have been performed in the context of DSA/CR, there is a lack of common and appropriate evaluation methodology, which would be desirable not only to prevent inaccurate results but also to enable the direct comparison of results from different sources. In this sense, this dissertation has presented a comprehensive and in-depth discussion of several important methodological aspects that need to be carefully taken into account when evaluating spectrum occupancy. A quantitative evaluation of the impact of different individual factors on the obtained results along with various useful guidelines have been provided as well. Relying on the findings of such study, this dissertation has presented the results of a broadband spectrum measurement campaign conducted in the frequency range 75-7075 MHz over a wide variety of scenarios in the metropolitan area of Barcelona, Spain. To the best of the author's knowledge, this is the first study of these characteristics performed under the scope of the Spanish spectrum regulation and one of the earliest studies in Europe. The obtained results have indicated that the actual utilization of spectrum is not uniform: some spectrum bands are subject to intensive usage while some others show moderate utilization levels, are sparsely used and, in some cases, are not used at all. However, the overall level of utilization has been verified to be significantly low. Most of allocated spectrum offers interesting deployment possibilities for DSA/CR systems, even those bands with the highest observed activity levels.

The second part of this dissertation has dealt with the study of various specific aspects related to the processing of the measurements in order to extract the spectrum occupancy patterns, which is largely similar to the problem of spectrum sensing in DSA/CR systems. The performance of ED, the most widely employed spectrum sensing technique in DSA/CR, has been assessed experimentally. Despite the simplicity and generality of its operating principle, the obtained results have indicated that certain inherent technology-dependent properties may result in different detection performances for various radio technologies. The detection performance differences are more noticeable for short sensing periods, where it has been observed that transmission power patterns with higher (lower) levels of variability result in a lower (higher) detection performance. As the sensing interval increases, the ED performance becomes more similar for different radio technologies and thus more independent of the signal to be detected. The outcome of this study has highlighted two important practical aspects. Firstly, the classical theoretical results are not able to predict the dependence of the ED performance on the variability of the primary transmission power pattern, which limits their applicability in the design of real DSA/CR systems. This drawback has been overcome by contributing a more accurate theoretical-empirical model for the performance of ED, which has introduced the concept of signal uncertainty in spectrum sensing. Secondly, the dependence of the ED performance on the variability of the sensed signal may result in a specially degraded detection performance for highly variable signals. This another inconvenient has been solved by developing an improved ED scheme capable to cope with the degraded performance of the classical ED scheme for short sensing periods while providing a similar level of complexity, computational cost and field of application. 
The findings of the aforementioned studies have been applied in the third part of this dissertation to the development of innovative spectrum usage models. The developed models have been proven to accurately capture and reproduce relevant statistical properties of spectrum usage in the time, frequency and space domains.

First, this dissertation has addressed the problem of modeling spectrum usage in the time domain from a discrete-time perspective. The stationary DTMC model widely used in the DSA/CR literature in order to the describe the binary occupancy pattern of primary channels in the time domain has been proven to be unable to reproduce important characteristics of spectrum usage. As a result, a non-stationary DTMC model with deterministic and stochastic DC models has been developed. The proposed modeling approach has been validated with extensive empirical measurement results, demonstrating that it is able to accurately reproduce not only the mean occupancy level but also the statistical properties of busy and idle periods observed in real-world channels.

The continuous-time case has then been treated by performing a comprehensive, systematical and rigorous study on the set of probability distributions that can be employed to accurately describe the lengths of busy and idle periods in real radio communication systems. The study has been based on field measurements with various degrees of time resolution. In general, it has been corroborated that the assumption of exponentially distributed busy and idle periods is invalid, meaning that the CTMC model widely employed in the literature is unrealistic. In real systems, other distributions result more adequate. At long time scales, a single distribution function (generalized Pareto) has been proven to be capable to describe the channel usage patterns for all the considered bands. At short time scales, however, the obtained results indicate that the same distribution does not always provide the best fit, which is a attained by a particular set of distributions depending on the considered radio technology. For time-slotted systems, channel occupancy patterns can also be described from a discretetime viewpoint where period lengths are expressed as an integer number of time-slots. The study has been complemented with an analysis of the time-correlation properties of spectrum occupancy and the development of appropriate mathematical models as well as a simulation algorithm to reproduce such features. A two-layer modeling approach combining the use of different models at long and short time scales has been proposed as an adequate means to describe the spectrum occupancy patterns observed in real radio communication systems.

The joint analysis of the statistical time-frequency properties of spectrum usage has revealed three important aspects to be taken into account for a realistic and accurate modeling of spectrum usage. First, the binary time-occupancy patterns of the channels within a given spectrum band are mutually independent. Second, the DC of the channels within the same spectrum band follow beta or Kumaraswamy distributions. Third, the DC is clustered over frequency and the number of channels per cluster follows a geometric distribution. Based on these findings, a sophisticated procedure has been developed in order to generate artificial spectrum occupancy data for simulation and other purposes.

The study performed for the spatial domain of spectrum usage has contributed a set of novel models that describe the average spectrum occupancy level (expressed in terms of the DC) that would be perceived by DSA/CR users at any geographical location based on the knowledge of some simple primary signal parameters. An extension has also been 
proposed in order to characterize not only the average occupancy perception but also the simultaneous observations of various DSA/CR users on the spectrum occupancy pattern of the same transmitter. The applicability of the developed models has been illustrated by proposing a novel approach for the statistical prediction of spectrum occupancy perception and developing a method for snapshot-based simulations.

Finally, the integration of the proposed models has been discussed in the context of two important fields of application, namely the realization of analytical studies and the development of innovative simulation tools. Although each dimension of spectrum usage (i.e., time, frequency and space) has been analyzed and modeled independently, the proposed models can been combined and integrated into a unified modeling approach where the time, frequency and space dimensions can simultaneously be taken into account and accurately reproduced, thus providing a complete and holistic characterization of spectrum usage in real systems for the analysis, design and simulation of DSA/CR networks.

\subsection{Future work}

The research conducted in the context of this dissertation opens new horizons for future works. The discussion below suggests some possible directions to extend the studies performed in each of the three parts of this dissertation.

One important aspect in the practical development of the DSA/CR technology, in general, and realistic spectrum usage models, in particular, is the need for a clear understanding of the dynamic use of spectrum in real radio communication systems. In this context, the measurement of real network activities constitutes a valuable tool. Spectrum utilization has already been evaluated and analyzed in various measurement campaigns all over the world. However, there are some aspects not covered in this work that still remain unexplored. One of them is the perceived spectrum occupancy as a function of the time resolution of the measurements. High time resolution measurements allow to extract the true occupancy pattern of a channel with high time accuracies. However, the amount of spectrum data generated under high sampling rates may require processing times several orders of magnitude above the actual measurement period. On the other hand, low effective sampling rates enable the possibility to perform long measurements (in order to appreciate potential daily or weekly patterns) and process the captured data within reasonable computation times. However, this may result in a significant under-sampling of the measured signals, meaning that the true channel state may change between two consecutive channel observations. This dissertation has discussed a novel two-level modeling approach envisaged to simultaneously describe the statistical properties and occupancy patterns of spectrum usage at both short and long time scales. This modeling approach, however, has been developed based spectrum measurements performed independently with low and high time resolutions. It would be extremely useful to have spectral occupancy measurements performed over long time periods (e.g., several days, weeks or even months) with high levels of temporal resolution. On one hand, this would enable to appreciate potential long-term or seasonal trends on spectrum usage that otherwise would not be noticed in short measurement sessions. On the other hand, the high time resolution would enable an accurate estimation of the true channel occupancy 
patterns at short time scales and their study and analysis as a function of the long-term or seasonal characteristics. In summary, this would enable the development of spectrum occupancy models able to simultaneously describe the properties of spectrum usage at short and long time scales. The main drawback, however, is that the amount of data generated after performing high time resolution measurements over several days, weeks or months would incur in extremely high computational costs. This study is proposed as a future work.

Another important aspect in the development of spectrum usage models based on field measurements is the need to process the captured spectrum data in order to extract the channel occupancy patterns. This problem is related to the problem of spectrum sensing in DSA/CR networks, where an abundant amount of literature exists. A significant number of spectrum sensing techniques for DSA/CR has been proposed and new methods and variations thereof still continue to appear in the literature with relative frequency. Most of the existing proposals have been aimed at improving the detection performance of the conventional ED scheme. However, this has frequently been accomplished by exploiting particular features of specific radio technologies and making use of significantly complex methods and signal processing techniques, which have normally lead to a limited field of applicability of the proposed solutions and a significantly increased computational cost. By contrast, this dissertation has proposed an improved ED scheme that outperforms the conventional scheme while preserving a similar level of complexity, computational cost and field of application. This spectrum sensing method should serve as a motivation for the development of new spectrum sensing techniques in the future following similar approaches, where the objective should be to improve the detection performance but bearing in mind the need to preserve a wide field of application (in order to detect the presence of any primary signal irrespective of its radio technology and signaling format) as well as a reasonable computational cost (in order to reduce the complexity and manufacturing cost of DSA/CR terminals and thus enable a large scale deployment of the DSA/CR technology).

The main approach employed in this dissertation when developing spectrum occupancy models has been to design generic models that can be employed to describe the spectrum occupancy pattern of any radio technology by simply configuring the models' parameters according to the radio technology under study. However, some systems may exhibit particular properties for which generic modeling approach might not be the optimum solution. In such cases, other alternative modeling alternatives taking into account detailed technologyspecific features at the physical and higher layers might result more convenient. The development of this type of models, however, is out of the scope of this dissertation and is therefore proposed as a future work.

Finally, the utility of the models developed in this dissertation has extensively been illustrated with discussions on their applicability to analytical studies as well as a wide range of numerical examples and detailed algorithms for their implementation in simulation tools. However, it is worth noting that the field of application of the proposed models is not confined within these examples. On the contrary, the models presented in this dissertation open new research possibilities in the context of the DSA/CR technology. Realistic spectrum usage models can be exploited in the development of new DSA/CR techniques envisaged to predict the spectrum occupancy pattern of a primary system, or some statistical properties 
thereof, in the time, frequency and space dimensions. The availability of some predictions on the behavior of the primary system could be exploited by the secondary network in order to anticipate to the potential release of spectral resources or the appearance of primary users within certain time intervals, spectrum bands or geographical regions. Spectrum usage models can indeed constitute a key element in the development of the recently emerging concept of Radio Environment Map (REM). REM is envisaged as a broad integrated database that characterizes the environment of a DSA/CR terminal. REM consists of multi-domain environmental information such as geographical features, available services, spectral regulations or locations of relevant entities and their properties (e.g., primary transmitters along with their estimated locations, radio technologies, transmission powers, radio propagation conditions and spectrum occupancy patterns). REM information may be updated periodically with observations from DSA/CR nodes and disseminated through the DSA/CR network. Knowledge of the radio environment can be exploited to significantly enhance the associated decision-making and radio resource management processes (e.g., selection of transmission power and modulation, guiding of the sensing process or configuration of the MAC-layer parameters, to name a few examples). In the context of REMs, spectrum usage models can be employed to concisely characterize and represent the statistical properties of the spectrum in the radio environment surrounding a DSA/CR network. The REM concept can enormously benefit from the availability of realistic and accurate spectrum usage models, as those developed in this dissertation, which can be used not only to predict possible future trends on the utilization of spectrum but also to support the associated decision-making and radio resource management processes of the DSA/CR network, which would ultimately result not only in an enhanced performance but also in a more efficient use of the spectrum. 




\section{Part V}

\section{Appendices}

First define, then refine.

Bill Guild 



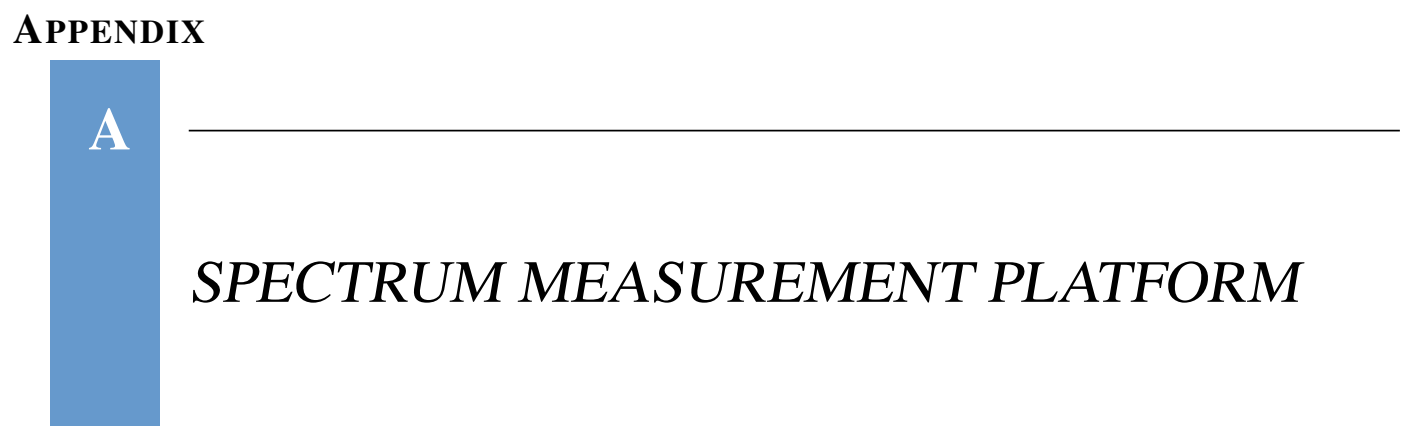

\section{A.1 Introduction}

The utilization of appropriate measurement equipment for the evaluation of spectrum occupancy is essential to ensure accurate and reliable results. This appendix presents a sophisticated radio spectrum measurement platform that has explicitly been designed for spectrum surveys and studies in the context of DSA/CR. The developed platform constitutes a flexible measurement tool that combines a powerful RF measurement system with intelligent computer control and data processing. An exhaustive and in-depth description is provided, with a special emphasis on implementation details and lessons learned during the development.

The description of this appendix corresponds to the measurement platform considered in Part I. The measurement platform considered in Part II is presented in Chapter 4.

\section{A.2 Measurement platform overview}

The presented platform is based on a spectrum analyzer setup, where different external devices have been added in order to improve the detection capabilities of the system and hence obtain more reliable and accurate results. A detailed scheme is shown in Figure A.1. The design is composed of two broadband discone-type antennas covering the frequency range from 75 to $7075 \mathrm{MHz}$, a switch to select the desired antenna, several filters to remove undesired signals, a low-noise pre-amplifier to enhance the overall sensitivity and thus the ability to detect weak signals, a high-performance spectrum analyzer to record the spectral activity, and a laptop (not shown in Figure A.1) running a tailor-made software that controls the measurement process. All the components integrating the platform can be divided into four modules (see Figure A.2), namely the antenna subsystem, the RF subsystem, the capturing subsystem, and the control subsystem, which are detailed in the following sections. 


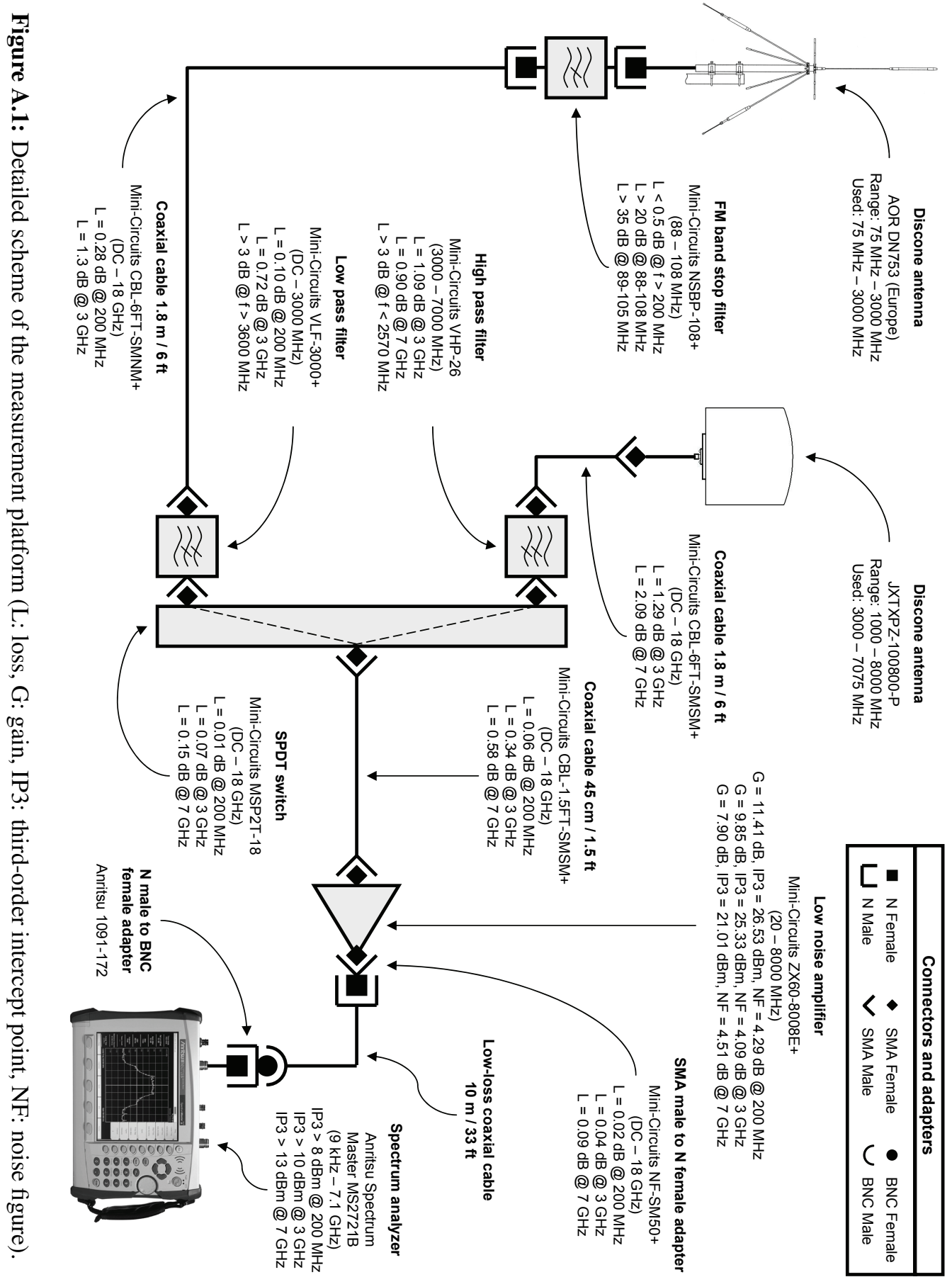



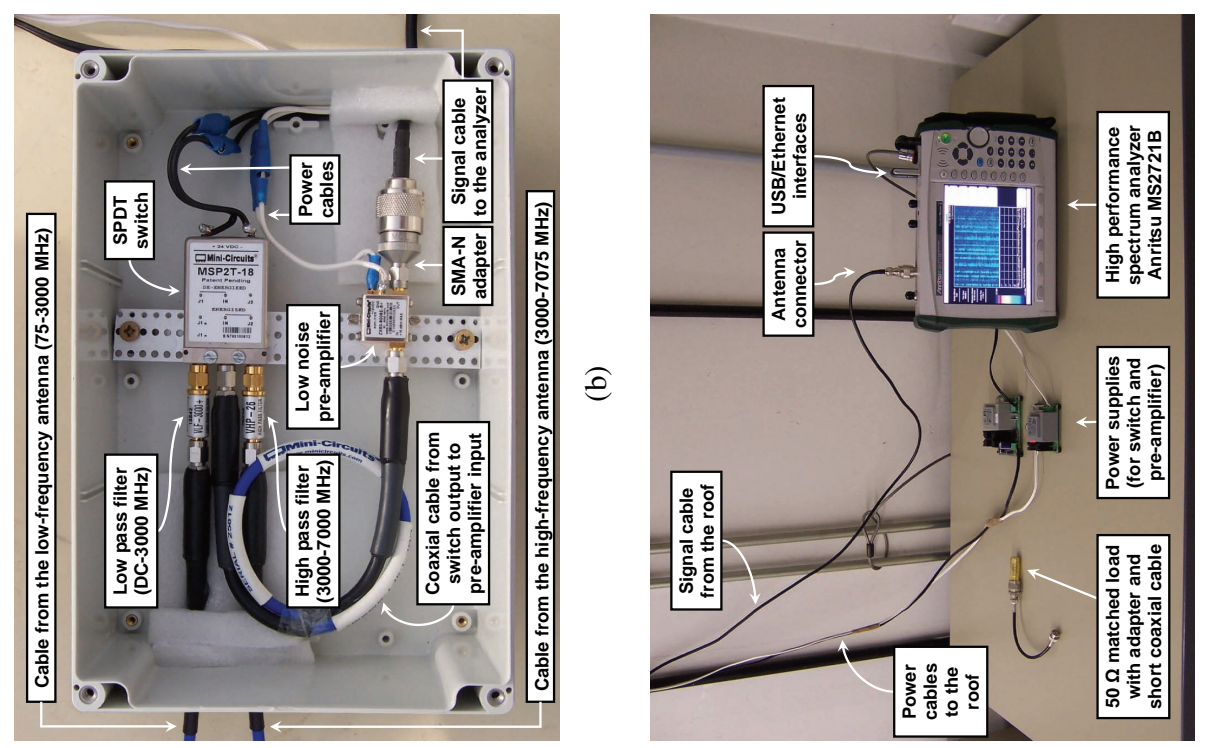

产

$\frac{1}{2}$
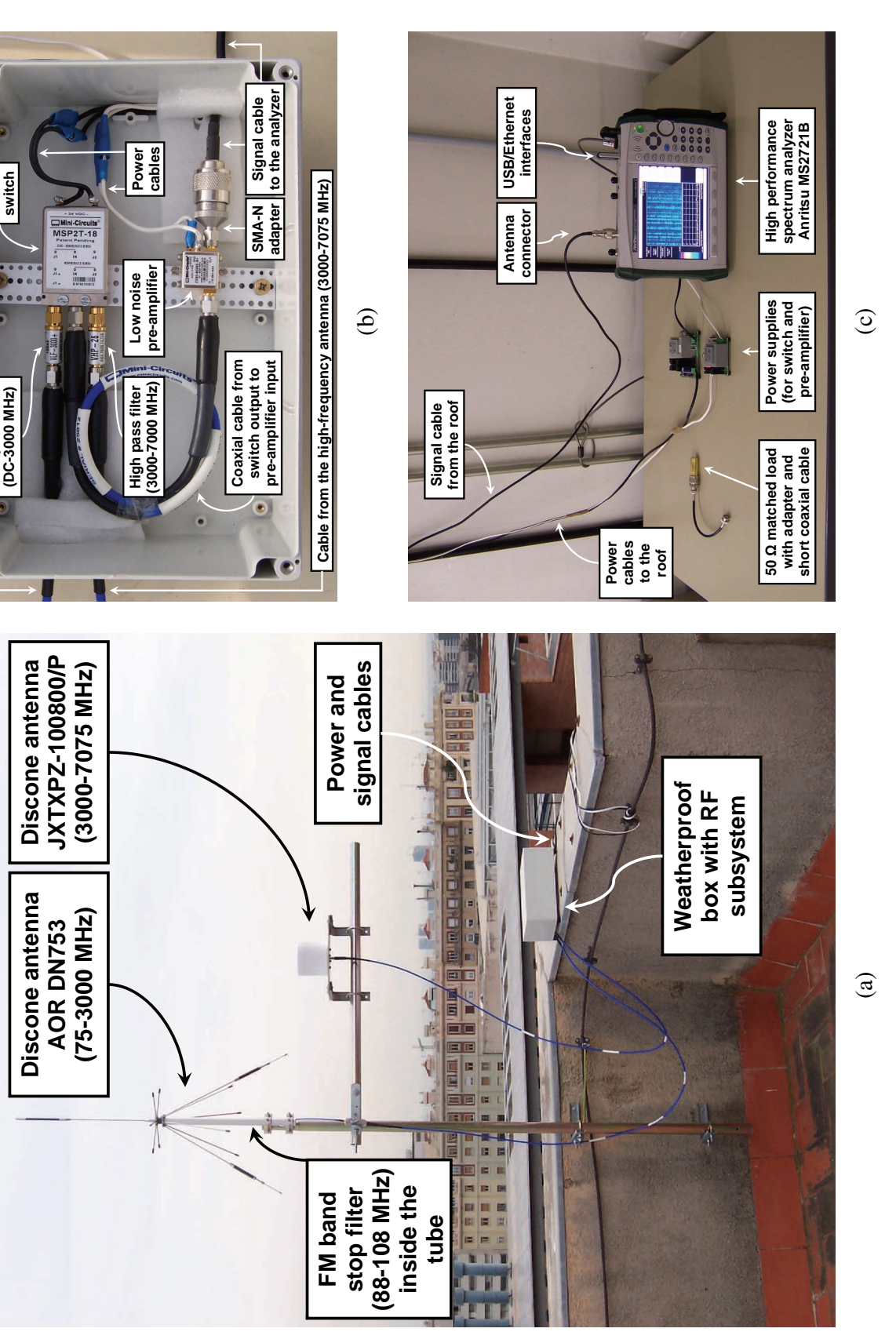


\section{A.3 Antenna subsystem}

When covering small frequency ranges or specific licensed bands a single antenna may suffice. However, in broadband spectrum measurements from a few megahertz up to several gigahertz, two or more broadband antennas are required in order to cover the whole frequency range. The antenna subsystem, shown in Figure A.2(a), is composed of two broadband discone-type antennas covering the frequency range from 75 to $7075 \mathrm{MHz}$. The first antenna (AOR DN753) is used between 75 and $3000 \mathrm{MHz}$, while the second antenna (A-INFO JXTXPZ-100800/P) covers the frequency range 1-8 GHz but is employed between 3000 and $7075 \mathrm{MHz}$. Discone antennas are broadband antennas with vertical polarization and omni-directional receiving pattern in the horizontal plane. Even though some transmitters may be horizontally polarized, they usually are high-power stations (e.g., TV stations) that can be detected even with vertically polarized antennas. The exceptionally wide band coverage (allowing a reduced number of antennas in broadband studies) and the omni-directional feature (allowing the detection of licensed signals coming for any directions) make discone antennas an attractive option in radio scanning and monitoring applications.

\section{A.4 Radio frequency subsystem}

The RF subsystem is in charge of performing antenna selection, filtering and amplification. The RF module is shown in Figure A.2(b).

The desired antenna is selected by means of a Single Pole Double Throw (SPDT) switch. An electromechanical switch (MiniCircuits MSP2T-18) has been selected because of its high isolation (90-100 dB) and low insertion loss (0.1-0.2 dB). When compared to other switch types, electromechanical switches in general provide slower switching times and shorter lifetimes. Nevertheless, this choice results appropriate since antenna switching is always performed off-line by turning the switch on/off between measurement sessions.

To remove undesired signals, three filters are employed. A band stop filter (MiniCircuits NSBP-108+) blocks signals in the frequency range of Frequency Modulation (FM) broadcast stations (87.5-108 MHz). Such stations usually are high power transmitters that may induce overload in the receiver, thus degrading the reception performance with an increased noise floor (which prevents the receiver from detecting the presence of weak signals) or with the appearance of spurious signals (which may be misinterpreted as true signals). Since the FM band is of presumably low interest for opportunistic use due to its usually high transmission powers and occupancy rates, a FM band stop filter is employed in order to remove FM signals and avoid overload problems, improving the detection of weak signals at other frequencies. Low pass (MiniCircuits VLF-3000+) and high pass (MiniCircuits VHP-26) filters have been used to remove out-of-band signals and reduce potential intermodulation products.

To compensate for device and cable losses and increase the system sensitivity, a lownoise pre-amplifier is employed. It is important to note that higher amplification gains result in better sensitivities at the expense of reduced dynamic ranges. Since very different signal levels may be present in broadband spectrum surveys, the existing trade-off between sensitivity and dynamic range must therefore be taken into account. The selected mid-gain 
amplifier (MiniCircuits ZX60-8008E+) provides significant sensitivity improvements while guaranteeing a SFDR [148] of $75 \mathrm{~dB}$, which was observed to be enough in practical measurement conditions. Although the employed spectrum analyzer includes a high-gain builtin amplifier, the use of an additional external pre-amplifier closer to the antenna subsystem results in an improved overall noise figure (8-9 dB lower than in the case where only the internal amplifier is employed). For measurements below $3 \mathrm{GHz}$, where some overloading signals may be present, only the external amplifier is used. For measurements above $3 \mathrm{GHz}$, where the received powers are lower due to the attenuation of higher frequencies, both the external and the spectrum analyzer's internal amplifier are employed.

\section{A.5 Capturing subsystem}

A high performance handheld spectrum analyzer (Anritsu Spectrum Master MS2721B) is employed to provide power spectrum measurements and record the spectral activity over the complete frequency range (see Figure A.2(c)). This spectrum analyzer provides a measurement range from $9 \mathrm{kHz}$ to $7.1 \mathrm{GHz}$, low noise levels and a built-in pre-amplifier (which facilitates the detection of weak signals), fast sweep speeds automatically adjusted, and various communication interfaces enabling the connection of external USB storage devices as well as controlling instruments. Moreover the handheld, battery-operated design simplifies the displacement of the equipment to different measurement locations.

In spectrum analyzers, a tunable receiver tunes continuously across the selected frequency span, beginning at the lowest frequency of the span and increasing in frequency until the highest frequency of the span is reached. Due to the swept operating principle of spectrum analyzers, the time interval between two consecutive samples of a given frequency channel may be notably high, in the order of several seconds depending on the width of the selected frequency span and the bandwidth of the selected intermediate frequency filter (referred to as resolution bandwidth). This means that the effective sampling rate of individual channels cannot be compared to that attained with other capturing devices such as vector signal analyzers or digital sampling cards. Nevertheless, spectrum analyzers have the advantage of providing high sensitivity levels (ability to detect the presence of weak licensed signals), high dynamic ranges (ability to simultaneously detect the presence of signals with very dissimilar power levels) and wide band measurements (ability to observe the occupancy state of all the radio channels within an entire band), which are fundamental requirements in order to obtain a meaningful and comprehensive picture of spectrum usage.

\section{A.6 Control subsystem}

The control subsystem, shown in Figure A.3, is in charge of supervising the measurement process, retrieving the measurement data from the spectrum analyzer and saving the results in an appropriate format for off-line data post-processing.

The control subsystem is mainly composed of a laptop, which is connected to the spectrum analyzer via an Ethernet interface. The laptop runs a tailor-made script under Matlab's 


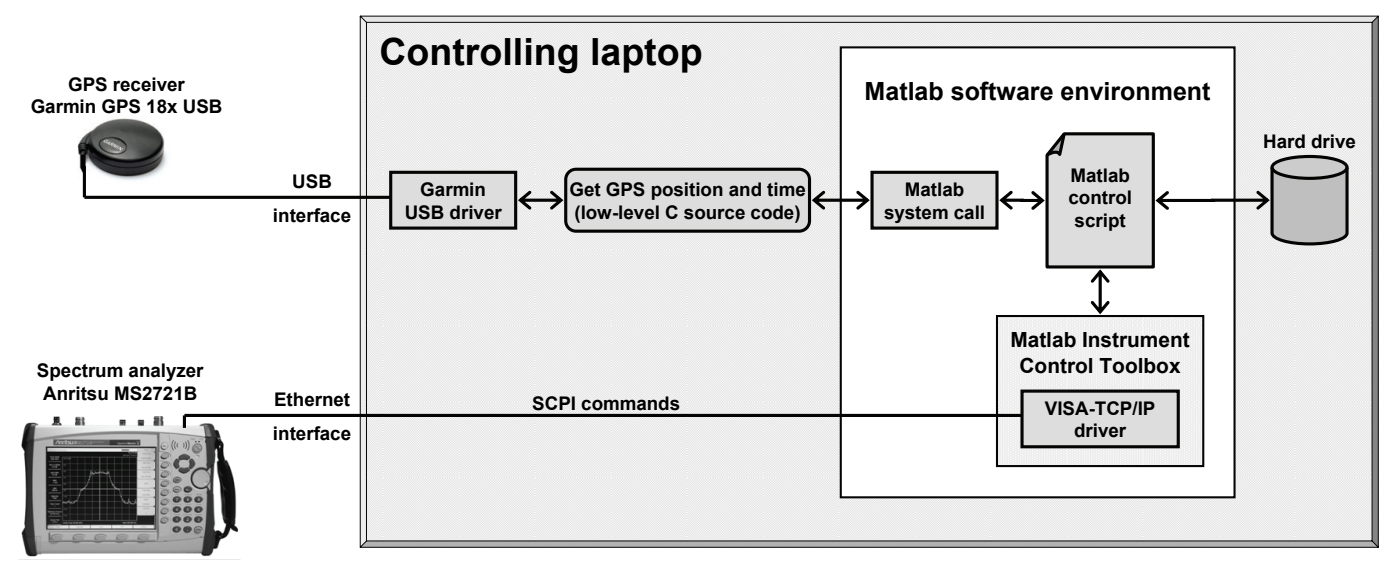

Figure A.3: Control subsystem.

software environment, which controls the measurement process. The control script communicates with the spectrum analyzer by means of the Matlab's Instrument Control Toolbox and making use of commands in SCPI (Standard Commands for Programmable Instruments) format with the VISA (Virtual Instrument Standard Architecture)-TCP/IP interface.

The script receives the following data as input parameters from the user:

- ip_address: The IP address configured in the spectrum analyzer.

- $f \_s t a r t:$ The lowest frequency in MHz of the band/span to be measured.

- $f \_s t o p:$ The highest frequency in $\mathrm{MHz}$ of the band/span to be measured.

- $t$ _start: The time instant to begin measurements, specified in year-month-day-hourminute-second (YYYY/MM/DD/HH/MM/SS) format.

- $t \_s t o p:$ The time instant to end measurements, specified in year-month-day-hourminute-second (YYYY/MM/DD/HH/MM/SS) format.

- file_name: The root/base name for the generated data files.

- nof_traces_per_file: Number of traces/sweeps saved in each generated file. To avoid excessively large (computationally intractable) files when the measurement period is long (e.g., hours or days), the data are split into several files.

Based on the received input information, the measurement process is controlled as follows (see Algorithm A.1). First of all, the script tries to establish communication with the spectrum analyzer at the specified IP address (line 1) by sending the appropriate commands [288]. If the connection establishment is successful, the set of configuration parameters (including the specified frequency band to be measured and some others) are then sent to the spectrum analyzer (line 2). After initializing the counters and variables employed in the 
measurement process (lines 3-7), the script then waits until the time for beginning the measurement session is reached (lines 8-10). When the start time is reached, the measurement process begins and it is performed repeatedly until the specified stop time is reached (lines 11-30). Every cycle consists in the realization of one sweep and its storage. The current time at the beginning of the cycle is stored and used as a time stamp for the current sweep (line 12). A new sweep is then immediately commanded to the spectrum analyzer (line 13). The script then waits for the sweep to be complete by continuously monitoring the corresponding status bits of the spectrum analyzer (lines 14-16). When the sweep is complete, the measured data are retrieved (line 17). After removing headers, the measured power values are extracted from the data provided by the spectrum analyzer in comma-separated ASCII format (line 18). The measured power values (line 19) along with the corresponding time stamp (line 20) are concatenated to the appropriate matrices. Since one sweep is completed at this time, the trace counter is increased (line 21) and compared to the number of traces to be saved in each generated file (line 22). In case that a new file needs to be created, the file counter is updated (line 23) and the matrices containing the measured power values (line 24) and their corresponding time stamps (line 25) are then saved, after which the counter and the matrices are reset (lines 26-28). When one cycle (lines 11-30) is finished, another one is started immediately, and the process is repeated cyclically until the stop time is reached. The set of remaining traces/sweeps at this time, lower than nof_traces_per_file, is saved into new files (lines 32-34) along with the frequency vector containing the exact values for the frequency points that have been measured within the band of interest (line 36). The communication with the spectrum analyzer is finally closed and terminated (line 37).

The time reference employed to determine the beginning and the ending of the measurement session, as well as the time stamps, is obtained from the laptop's internal clock. Alternatively, the time reference can be obtained from an external GPS receiver. This option is useful when two or more measurement suites are deployed at different locations and need to be synchronized among them. In this case, the control script is slightly different (see Algorithm A.2). First, an additional input parameter $T_{\text {_sweep }}$ indicating the time period between two consecutive sweep cycles needs to be specified by the user. A new variable (line 8) is used to store the start time for the next sweep cycle and control the exact time instant at which a new sweep cycle is initiated. Before a new sweep cycle is started, the control script remains idle waiting for the next_sweep_time instant (lines 10-12). When the next_sweep_time instant is reached, a new sweep is then initiated and the time instant for the next sweep is updated (line 14) according to the specified $T_{\text {_sweep. The specified }}$ time period must be long enough to allow for a complete cycle (lines 9-32) to be performed and enables various measurement suites to be synchronized on a sweep basis. This type of measurements is interesting, for example, in spatial spectrum occupancy studies where the objective is to determine how several nodes of a DSA/CR network at different locations would perceive the spectral activity of the same primary transmitter, thus identifying potential dependence and/or correlation patterns among the observations. Additionally, the external GPS receiver can also be used to easily determine the location where a measurement is being performed and study such patterns as a function of the distance between the DSA/CR nodes. This operation mode may find other interesting applications as well. 


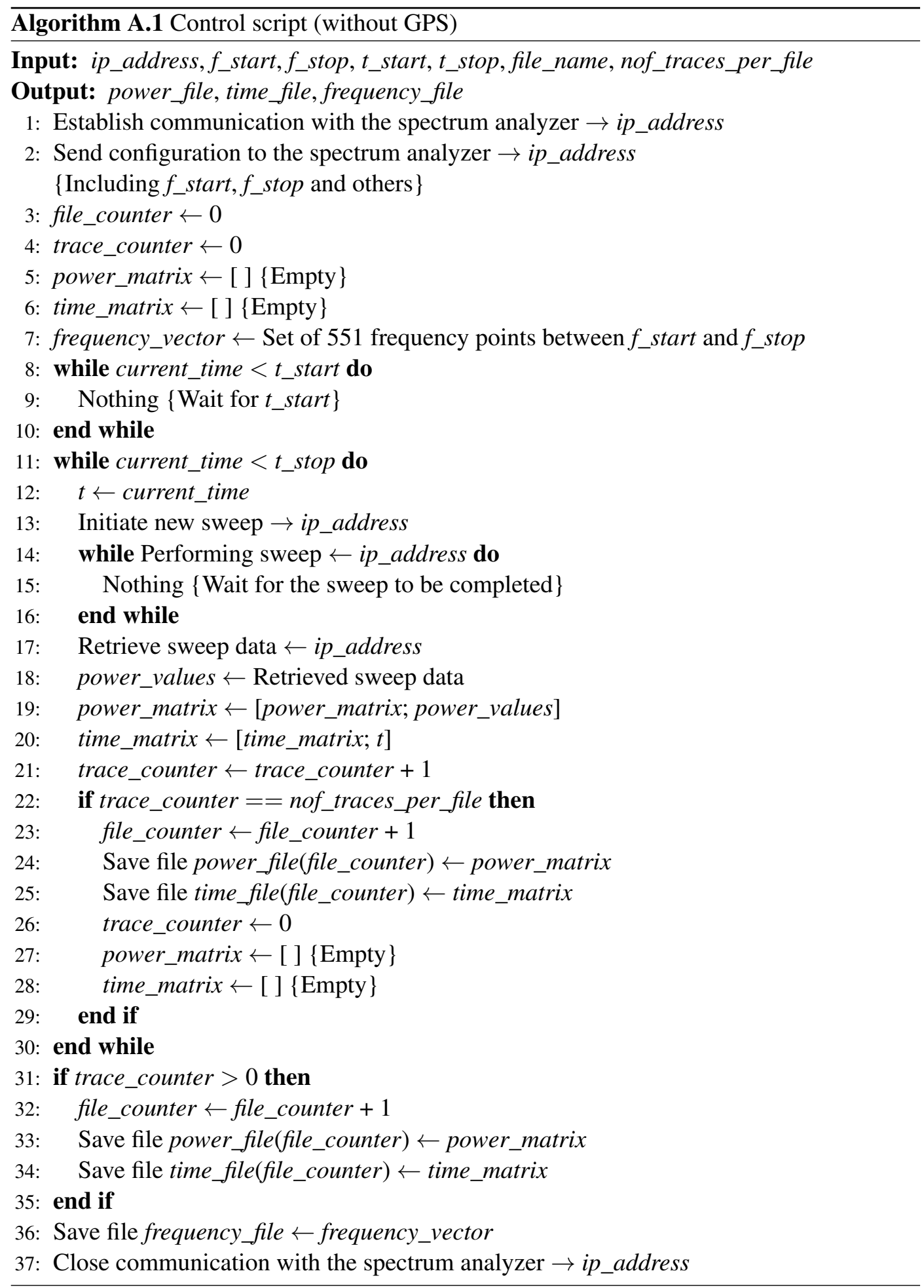




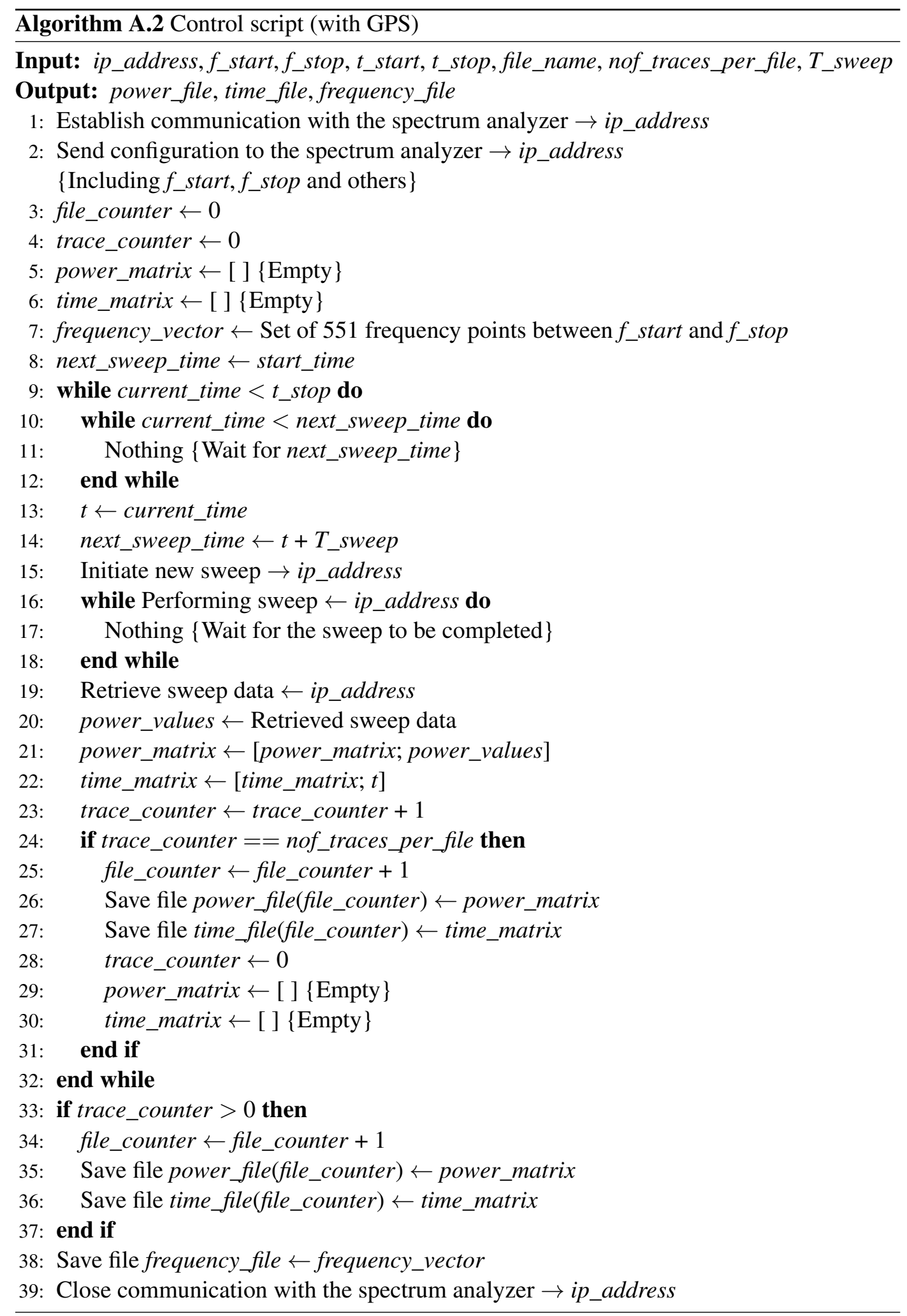


The device employed in the presented implementation (Garmin GPS 18x USB) is a small and highly accurate GPS receiver with high sensitivity levels $(-184 \mathrm{dBW})$ and a maximum acquisition time of around 45 seconds. As shown in Figure A.3, the GPS receiver requires a specific USB driver to be installed in the controlling laptop. This driver is used by a set of three executable files, written in C source code, containing a low-level implementation of the proprietary Garmin USB communication protocol [289]. Each file executes a different set of commands, depending on its finality. The first file checks the connectivity with the GPS receiver to verify that it is properly connected and working. The other two files retrieve the current GPS location and current GPS time, respectively, from the GPS receiver. These files are executed from the operating system's command line and invoked from Matlab by means of a system call with the system function. Each executable file returns the result of the corresponding operation (i.e., GPS receiver status, GPS location or GPS time) as a character string with a predefined format, which is processed by the control script in order to extract the desired information. While the files providing the GPS receiver status and location are invoked once at the beginning of each measurement session, the GPS time is requested repeatedly in order to obtain the desired time reference.

As shown in Algorithm A.1, the control script generates, for each measurement session, one file storing the frequency points measured within the band of interest (frequency_file) and a set of files containing the measured power values (power_file) and the corresponding time stamps (time_file). The data formats for each file and the corresponding relations are illustrated in Figure A.4. The frequency file contains a $1 \times 551$ row vector including the frequency points, in $\mathrm{MHz}$, that have been measured by the spectrum analyzer. This vector length corresponds to the number of points per sweep provided by the selected spectrum analyzer. Each generated power_file contains a nof_traces_per_file $\times 551$ matrix whose values correspond to the power level recorded at each one of the 551 measured frequency points for a total amount of nof_traces_per_file sweeps. The default unit for expressing the recorded power levels is $\mathrm{dBm}$ but it can be modified to represent both power and voltage amplitude values in various orders of magnitude (e.g., $\mathrm{mW}$ and $\mathrm{W}$ ) and either in linear or logarithmic magnitude (e.g., $\mathrm{mW}$ and $\mathrm{dBm}$ ). For each generated power_file there exists a corresponding time_file containing a nof_traces_per_file $\times 6$ matrix, where the $n$-th row contains the time stamp (in YYYY-MM-DD-HH-MM-SS format) for the sweep reported in the $n$-th row of the associated power_file.

Notice that the selected data formats provide some interesting advantages for data storage and data post-processing. On one hand, the frequency_file and the time_file can be employed to search for the empirical data corresponding to particular time periods and frequency ranges. Since these matrices are significantly small, the files where they are stored can be loaded and processed very fast. This enables particular sets of empirical data of interest to be rapidly found within the set of matrices contained in the power_file files, which are of significantly higher sizes. Once the subset of empirical data of interest is identified by exhaustive searching within the corresponding set of frequency_file and time_file files, only the power_file file(s) containing the desired data need to be loaded and processed. Moreover, the size of each power_file depends on the value of the parameter nof_traces_per_file provided as an input to the control script, which can be flexibly configured depending on the 


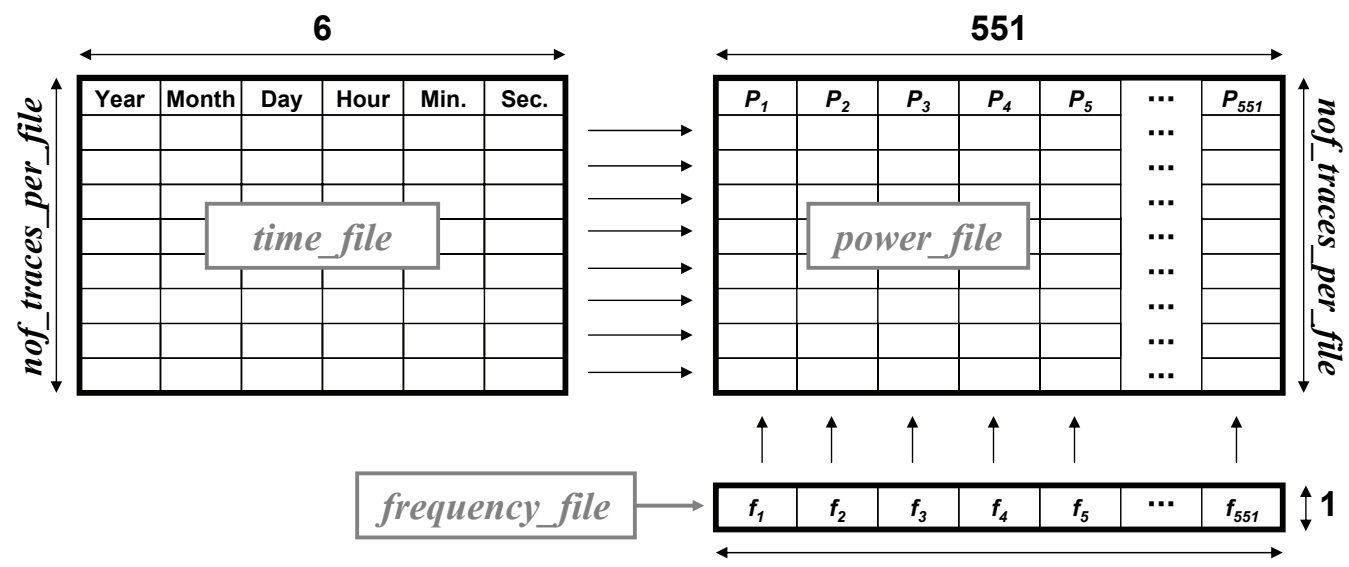

551

Figure A.4: Data formats.

available computational capabilities. For powerful computers able to simultaneously handle very high data volumes, this parameter can be set to higher values, thus reducing the amount of required data files and storage space. In conclusion, the selected data formats enable large volumes of empirical data not only to be stored and structured systematically, but also to be accessed and processed in an easy, fast and efficient manner.

\section{A.7 Summary}

This appendix has presented a sophisticated radio spectrum measurement platform specifically envisaged and designed for spectrum occupancy surveys in the context of the DSA/CR technology. The presented tool is a very flexible system combining a powerful radio measurement design with intelligent computer control and data processing. The developed platform can be employed to carry out extensive spectrum measurement campaigns, with significant sensitivity levels, and providing a set of structured empirical data that can be analyzed and processed in an easy, fast and efficient manner. An exhaustive and in-depth description of the developed measurement platform has been provided, with a special emphasis on implementation details that may result helpful to other researchers and engineers in the development of similar radio spectrum measurement tools. 



\section{APPENDIX}

B

\section{DUTY CYCLE COMPUTATION}

This appendix provides a detailed description of the procedure employed to compute the Duty Cycle (DC) based on field measurements. The DC is used throughout this dissertation as an evaluation metric to quantify to degree to which a channel or spectrum band is used.

The DC is computed based on a finite set of discrete measurements collected along a range of frequencies $F_{\text {span }}=f_{\text {stop }}-f_{\text {start }}$ (frequency span) and over a period of time $T_{\text {span }}=$ $t_{\text {stop }}-t_{\text {start }}$ (time span).

The measured discrete time instants $t_{i}\left(t_{\text {start }} \leq t_{i}<t_{\text {stop }}\right)$ are given by:

$$
t_{i}=t_{\text {start }}+(i-1) \cdot T_{r}, \quad i=1,2, \ldots, N_{t}
$$

where $T_{r}$ represents the time resolution and is given by the spectrum analyzer's sweep time, which in turn depends on the selected configuration parameters. For a given time resolution $T_{r}$, the number of traces $N_{t}$ collected within a time span $T_{\text {span }}$ is given by $N_{t}=T_{\text {span }} / T_{r}$.

The measured discrete frequency points $f_{j}\left(f_{\text {start }} \leq f_{j}<f_{\text {stop }}\right)$ are given by:

$$
f_{j}=f_{\text {start }}+(j-1) \cdot F_{r}, \quad j=1,2, \ldots, N_{f}
$$

where the frequency resolution $F_{r}=F_{s p a n} / N_{f}$ is given by the frequency bin, determined by the selected frequency span $F_{\text {span }}$ and the number of points $N_{f}$ measured by the spectrum analyzer (as mentioned in Appendix A, $N_{f}=551$ for the employed spectrum analyzer).

The set of PSD samples collected by a spectrum analyzer over a time span $T_{\text {span }}$ and along a frequency span $F_{\text {span }}$ can be represented by a $N_{t} \times N_{f}$ matrix $\mathbf{M}$ as:

$$
\mathbf{M}=\left[M\left(t_{i}, f_{j}\right)\right]_{N_{t} \times N_{f}}
$$

where each element $M\left(t_{i}, f_{j}\right)$ represents the PSD sample captured at time instant $t_{i}(i=$ $\left.1,2, \ldots, N_{t}\right)$ and frequency point $f_{j}\left(j=1,2, \ldots, N_{f}\right)$. 
To compute the DC, the presence or absence of a signal needs to be determined for each PSD sample $M\left(t_{i}, f_{j}\right)$. In other words, for each captured PSD sample it is necessary to determine whether the sample corresponds to a licensed signal sample or a noise sample. Several signal detection principles have been proposed in the literature to perform such task. However, as discussed in Chapter 2, when only power measurements of the spectrum utilization are available, the ED method is the only possibility left. ED compares the received signal energy in a certain frequency range to a predefined threshold. If the signal energy lies above the threshold, a licensed signal is declared to be present. Otherwise, the measured frequency range is supposed to be idle. Following this principle, a binary spectral occupancy matrix

$$
\boldsymbol{\Omega}=\left[\Omega\left(t_{i}, f_{j}\right)\right]_{N_{t} \times N_{f}}
$$

is defined, where each element $\Omega\left(t_{i}, f_{j}\right) \in\{0,1\}$ is computed as:

$$
\Omega\left(t_{i}, f_{j}\right)=\xi\left(M\left(t_{i}, f_{j}\right), \lambda\left(f_{j}\right)\right)
$$

with $\lambda\left(f_{j}\right)$ being an energy decision threshold for frequency point $f_{j}$ and $\xi(x, y)$ a function defined as:

$$
\xi(x, y)= \begin{cases}0, & x<y \\ 1, & x \geq y\end{cases}
$$

Each element $\Omega\left(t_{i}, f_{j}\right)$ of $\boldsymbol{\Omega}$ represents the presence $\Omega\left(t_{i}, f_{j}\right)=1$ or absence $\Omega\left(t_{i}, f_{j}\right)=0$ of a licensed signal at time instant $t_{i}$ and frequency point $f_{j}$, according to the ED principle based on an energy decision threshold $\lambda\left(f_{j}\right)$. Unless otherwise stated, the decision threshold is set according to the PFA $1 \%$ criterion (see Chapter 2).

For each measured frequency point $f_{j}$, the $\mathrm{DC} \Psi_{f_{j}}$ is computed as the proportion of PSD samples, out of all the PSD samples recorded at that frequency, that lie above the decision threshold $\lambda\left(f_{j}\right)$ and thus are considered as samples of busy channels:

$$
\Psi_{f_{j}}=\frac{1}{N_{t}} \sum_{i=1}^{N_{t}} \Omega\left(t_{i}, f_{j}\right)
$$

For a frequency point $f_{j}$, this metric represents the fraction of time that the frequency is considered to be busy. For a certain frequency span (i.e., range of frequencies $j=1,2, \ldots, N_{f}$ ), the average DC $\Psi$ of the band is computed by averaging the $\mathrm{DC} \Psi_{f_{j}}$ of all the $N_{f}$ frequency points measured within the band:

$$
\Psi=\frac{1}{N_{f}} \sum_{j=1}^{N_{f}} \Psi_{f_{j}}=\frac{1}{N_{t}} \frac{1}{N_{f}} \sum_{i=1}^{N_{t}} \sum_{j=1}^{N_{f}} \Omega\left(t_{i}, f_{j}\right)
$$

This metric represents the average degree of spectrum utilization within certain time $\left(T_{\text {span }}\right)$ and frequency $\left(F_{\text {span }}\right)$ spans. The DC, $\Psi \in[0,1]$, is sometimes specified as a percentage. 




\section{APPENDIX}

C

\section{APPROXIMATION TO THE GAUSSIAN Q-FUNCTION}

\section{C.1 Introduction}

The Gaussian $Q$-function $Q(x)$ [201, (26.2.3)], the directly related error function $\operatorname{erf}(x)$ [201, (7.1.1)], and its complementary error function $\operatorname{erfc}(x)[201,(7.1 .2)]$ are of paramount importance in many practical problems found in electrical engineering and other related fields, where unknown factors under study are frequently modeled as Gaussian random variables in order to make them mathematically tractable (the most clear example is the thermal noise present in any communication system). Unfortunately, no exact and simple closed-form expressions, appropriate for mathematical manipulations, are known for these functions. In many cases it is useful (and enough) to have closed-form approximations in order to facilitate analytical manipulations. As a result, and besides efficient numerical methods and infinite series proposed for the calculation of the $Q$-function [201, 290-294], several empirical approximations have been presented in the literature [295-300] providing different trade-offs between estimation accuracy and analytical tractability. Some of them offer simple mathematical expressions that can easily be employed in analytical studies, at the cost of a limited accuracy [296]. On the other hand, some others are able to provide accurate estimates of the true $Q$-function's values [295]. These approximations have been developed bearing in mind applications that require high estimation accuracies (e.g., derivation of the error probability for digital modulation schemes in fading channels, which may be in the order of $10^{-6}$ to $10^{-9}$ ). Unfortunately, the associated mathematical expressions are too complex to be easily employed in many other analytical studies, even if they do not require such a high accuracy. In this appendix, a simple, yet accurate mathematical approximation to the Gaussian $Q$-function is developed. Its simplicity enables its application over a wide range of analytical studies at reasonable accuracy levels. As an illustrative example, the proposed approximation is employed to obtain a new and simple closed-form expression for the probability of detection of an energy detector under Rayleigh fading channels. 


\section{C.2 Previous work}

Previous approximations for the Gaussian $Q$-function are reviewed in this section. In 1979, Börjesson et al. proposed the following tight approximation [295, (13)]:

$$
Q(x) \approx \frac{1}{\sqrt{2 \pi}} \cdot \frac{1}{(1-a) x+a \sqrt{x^{2}+b}} \cdot e^{-x^{2} / 2}, \quad x \geq 0
$$

where $a=0.339$ and $b=5.510$ are computed to minimize the maximum absolute relative error. A simpler, but less accurate approximation, was proposed by Chiani et al. [296, (14)]:

$$
\operatorname{erfc}(x) \approx \frac{1}{6} e^{-x^{2}}+\frac{1}{2} e^{-4 x^{2} / 3}, \quad x \geq 0
$$

Prony (sum-of-exponentials) approximations were also proposed by Loskot et al. [297, $(13 \mathrm{c}-\mathrm{d})]$ :

$$
\begin{aligned}
& Q(x) \approx 0.208 e^{-0.971 x^{2}}+0.147 e^{-0.525 x^{2}}, \quad x \geq 0 \\
& Q(x) \approx 0.168 e^{-0.876 x^{2}}+0.144 e^{-0.525 x^{2}}+0.002 e^{-0.603 x^{2}}, \quad x \geq 0
\end{aligned}
$$

A novel approximation aimed at increasing the tightness in the region of small function's arguments was contributed by Karagiannidis et al. [298, (6)]:

$$
\operatorname{erfc}(x) \approx \frac{1-e^{-A x}}{B} \cdot \frac{e^{-x^{2}}}{\sqrt{\pi} x}, \quad x \geq 0
$$

where $A$ and $B$ are computed so as to minimize the integral of the absolute error (for $x \in$ $[0,20], A=1.98$ and $B=1.135$ ). A modified version of Equation C.5 was proposed by Isukapalli et al. with the aim of providing an easily integrable expression for any $m$ of a Nakagami- $m$ fading distribution while preserving the tightness of the approximation [299, (3)]:

$$
Q(x) \approx e^{-x^{2} / 2} \sum_{n=1}^{n_{a}} \frac{(-1)^{n+1}(A)^{n}}{B \sqrt{\pi}(\sqrt{2})^{n+1} n !} x^{n-1}, \quad x \geq 0
$$

where $n_{a}$ is the number of terms considered after truncating the infinite series.

A polynomial approximation, based on the observation that a Gaussian random variable can be represented by a sum of $n$ uniform random variables, was proposed by Chen et al. $[300,(4)]$ for analytical derivations of error rates in log-normal channels:

$$
\begin{gathered}
Q(x) \approx 1-\sum_{m=0}^{n} \sum_{p=0}^{n} \frac{(-1)^{m+p}\left(\begin{array}{l}
n \\
p
\end{array}\right)}{m !(n-m) !}\left(\frac{n}{12}\right)^{\frac{p}{2}}\left(\frac{n}{2}-m\right)^{n-p} x^{p} \\
\cdot U\left(x-\sqrt{\frac{12}{n}}\left(\frac{n}{2}-m\right)\right), \quad|x|<\sqrt{3 n}
\end{gathered}
$$

where $U(\cdot)$ represents the unit step function [201, (29.1.3)]. 


\section{C.3 Proposed approximation}

The existing approximations are well suited for their particular applications. However, they are too complex to be easily employed over a wide variety of analytical studies. To cope with this drawback, a mathematical model based on a second-order exponential function is here proposed:

$$
Q(x) \approx e^{a x^{2}+b x+c}, \quad x \geq 0
$$

where $a, b, c \in \mathbb{R}$ are fitting parameters. The main attractiveness of Equation C.8 is its analytical simplicity. Notice, for instance, that the extension to any power of $Q(x)$ is trivial. However, the interest of this approximation lies not only on its mathematical simplicity but also, as it will be shown, in its ability to capture the behavior of the $Q$-function with significant accuracy levels.

The proposed model is characterized by only three fitting parameters, the optimum value of which can be determined according to different criteria. Two different fitting criteria are considered in this appendix to this end. The first one computes the optimum values of fitting parameters $\left(a^{*}, b^{*}, c^{*}\right)$ so as to minimize the Sum of Square Errors (SSE):

$$
\left(a^{*}, b^{*}, c^{*}\right)=\arg \min _{(a, b, c)}\left\{\sum_{n=1}^{N}\left[Q\left(x_{n}\right)-e^{a x_{n}^{2}+b x_{n}+c}\right]^{2}\right\}
$$

where $N$ is the number of numerical values $x_{n}$ employed for the argument $x$ in the fitting process. This approach, which minimizes the overall absolute error over the range of considered arguments, will henceforth be referred to as the min-SSE criterion.

The second fitting criterion consists in minimizing the Maximum Absolute Relative Error (MARE). The optimum values of fitting parameters $\left(a^{*}, b^{*}, c^{*}\right)$ are thus computed as:

$$
\left(a^{*}, b^{*}, c^{*}\right)=\arg \min _{(a, b, c)}\left\{\max _{n}\left\{\left|\frac{Q\left(x_{n}\right)-e^{a x_{n}^{2}+b x_{n}+c}}{Q\left(x_{n}\right)}\right|\right\}\right\}
$$

This approach, which minimizes the MARE observed for all $n$, will henceforth be referred to as the min-MARE criterion.

\section{C.4 Accuracy analysis and comparison}

The optimum values of fitting parameters $\left(a^{*}, b^{*}, c^{*}\right)$ are shown in Table C.1. The fitting process has been performed over different argument ranges, denoted as $\hat{x}$. These argument ranges represent the interval for which the obtained fit is optimum according to the corresponding criterion. The Goodness-Of-Fit (GOF) is evaluated in Figure C.1 in terms of the absolute relative error as a function of the argument $x$. As it can be appreciated, the GOF for the min-MARE criterion is clearly dependent on the argument range $\hat{x}$ for which the fit is optimized, while in the case of the min-SSE criterion it is significantly independent, excepting the case $\hat{x} \in[0,2]$ where a slightly different result is observed. The accuracy attained with min-SSE is noticeably good for small arguments $(x \leq 1.5)$, but degrades as the argument 
Table C.1: Optimum values of fitting parameters $\left(a^{*}, b^{*}, c^{*}\right)$ for different fitting criteria and optimized argument ranges $\hat{x}$.

\begin{tabular}{|c|c|c|c|c|c|c|c|}
\cline { 2 - 8 } \multicolumn{1}{c|}{} & $\hat{x} \in[0,2]$ & $\hat{x} \in[0,4]$ & $\hat{x} \in[0,6]$ & $\hat{x} \in[0,8]$ & $\hat{x} \in[0,10]$ & $\hat{x} \in[0,20]$ \\
\hline \multirow{4}{*}{$\min -S S E$} & $a^{*}$ & -0.3807 & -0.3847 & -0.3846 & -0.3846 & -0.3845 & -0.3842 \\
\cline { 2 - 8 } & $b^{*}$ & -0.7674 & -0.7632 & -0.7633 & -0.7634 & -0.7635 & -0.7640 \\
\cline { 2 - 8 } & $c^{*}$ & -0.6960 & -0.6966 & -0.6966 & -0.6966 & -0.6966 & -0.6964 \\
\hline \multirow{4}{*}{$\min -$ MARE } & $a^{*}$ & -0.3976 & -0.4369 & -0.4577 & -0.4698 & -0.4774 & -0.4920 \\
\cline { 2 - 8 } & $b^{*}$ & -0.7418 & -0.6511 & -0.5695 & -0.5026 & -0.4484 & -0.2887 \\
\cline { 2 - 8 } & $c^{*}$ & -0.7019 & -0.7358 & -0.7864 & -0.8444 & -0.9049 & -1.1893 \\
\hline
\end{tabular}

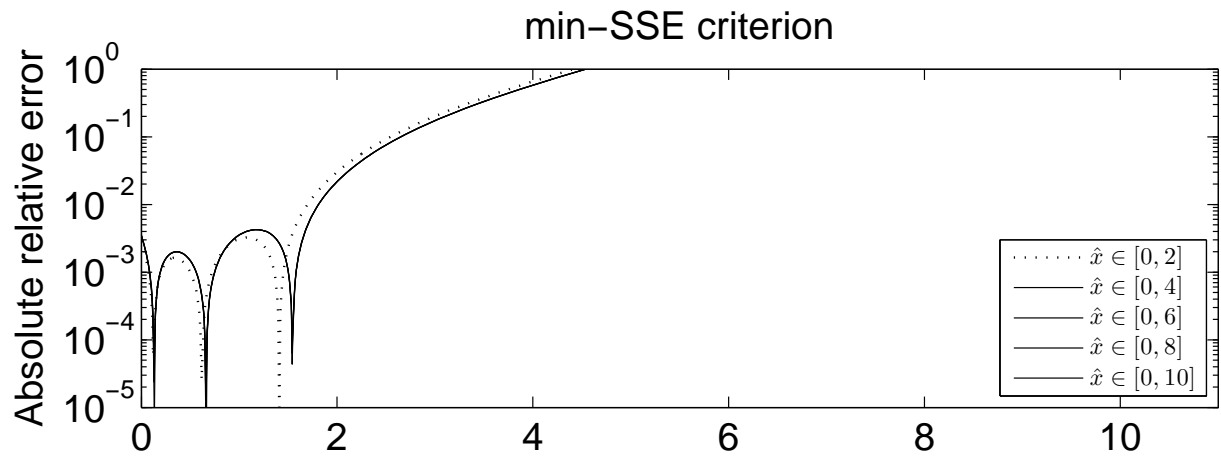

min-MARE criterion

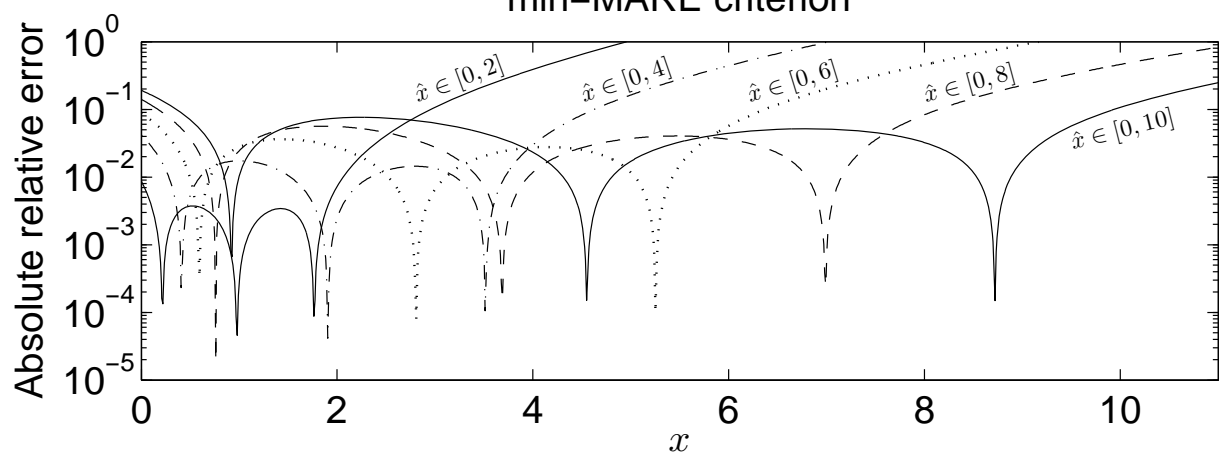

Figure C.1: Absolute relative error of the proposed exponential approximations for various optimized argument ranges $\hat{x}$. 
values increase. On the other hand, the min-MARE criterion in general provides coarser, yet reasonably accurate estimates over a wider range of arguments. It is interesting to note, in the latter case, how the accuracy degrades for argument values above the optimized interval $\hat{x}$. Moreover, as the length of the optimized interval $\hat{x}$ decreases, the overall relative error within the interval $\hat{x}$ improves.

The proposed approximation is compared with other existing solutions in Figures C.2, C. 3 and C. 4 within an optimized interval $\hat{x}=[0,6]$. The impact of larger optimized intervals is discussed where appropriate. Among the previously proposed approximations, the Börjesson's approximation can arguably be considered as the most accurate one for almost all arguments. Other proposed approximations, in general, are not able to provide its level of accuracy. Nevertheless, it is interesting to note, for small arguments $(x \leq 1.5)$, that the min-SSE criterion provides similar accuracy levels and within a limited region $(x \leq 0.8)$ even outperforms the Börjesson's approximation. From the comparison of Equations C.1 and C.8 it is clear that the proposed exponential approximation provides a much simpler and analytically tractable expression at a reasonable accuracy.

The Chen's polynomial approximation was proposed for analytical derivations in lognormal channels, for which exponential and rational approximations are not well suited. Besides this particular scenario, the simpler proposed approximation appears to be applicable over a wider range of analytical problems. In terms of accuracy, Figure C.2 shows that the proposed approximation with the min-SSE criterion is comparable $(x \gtrsim 1.75)$ or even better $(x \lesssim 1.75)$. The accuracy of the Chen's approximation can be improved by increasing $n$ in Equation C.7. However, an important increase of $n$ does not seem to provide a significant accuracy improvement. Therefore, the proposed approximation is able to provide a comparable (even better) accuracy level with a much simpler mathematical expression (see Equations C.7 and C.8).

When compared to the Karagiannidis' approximation, the obtained accuracy depends on the considered configuration and argument range. The min-SSE criterion provides better accuracy for low arguments $(x \leq 1.88)$ while the min-MARE criterion provides better accuracy for large arguments $(x \geq 2.14$ for $\hat{x} \in[0,6], x \geq 3.21$ for $\hat{x} \in[0,10], x \geq 6.33$ for $\hat{x} \in[0,20])$. Between both argument ranges, there exists a limited region where the Karagiannidis' approximation provides a slightly better accuracy, which is comparable to that of the proposed exponential approximation. Depending on the particular argument range involved in the problem under study, the proposed exponential approximation can be configured to provide a comparable level of accuracy with a simpler and more tractable analytical expression (see Equations C.5 and C.8).

The Isukapalli's approximation constitutes a simplification of the Karagiannidis' approximation, by means of a truncated infinite series, with the explicit purpose of analytical tractability. Hence, it is expected that the former cannot outperform the latter in terms of accuracy, which is corroborated in Figure C.3. In fact, it can be verified, as expected, that the accuracy of Equation C.6 tends asymptotically to that of Equation C.5 as $n_{a} \rightarrow \infty$. As a result, accuracy improvements observed for the proposed approximation with respect to the Karagiannidis' approximation are also guaranteed with respect to the Isukapalli's one. In terms of analytical complexity, the proposed approximation provides a mathematical expres- 


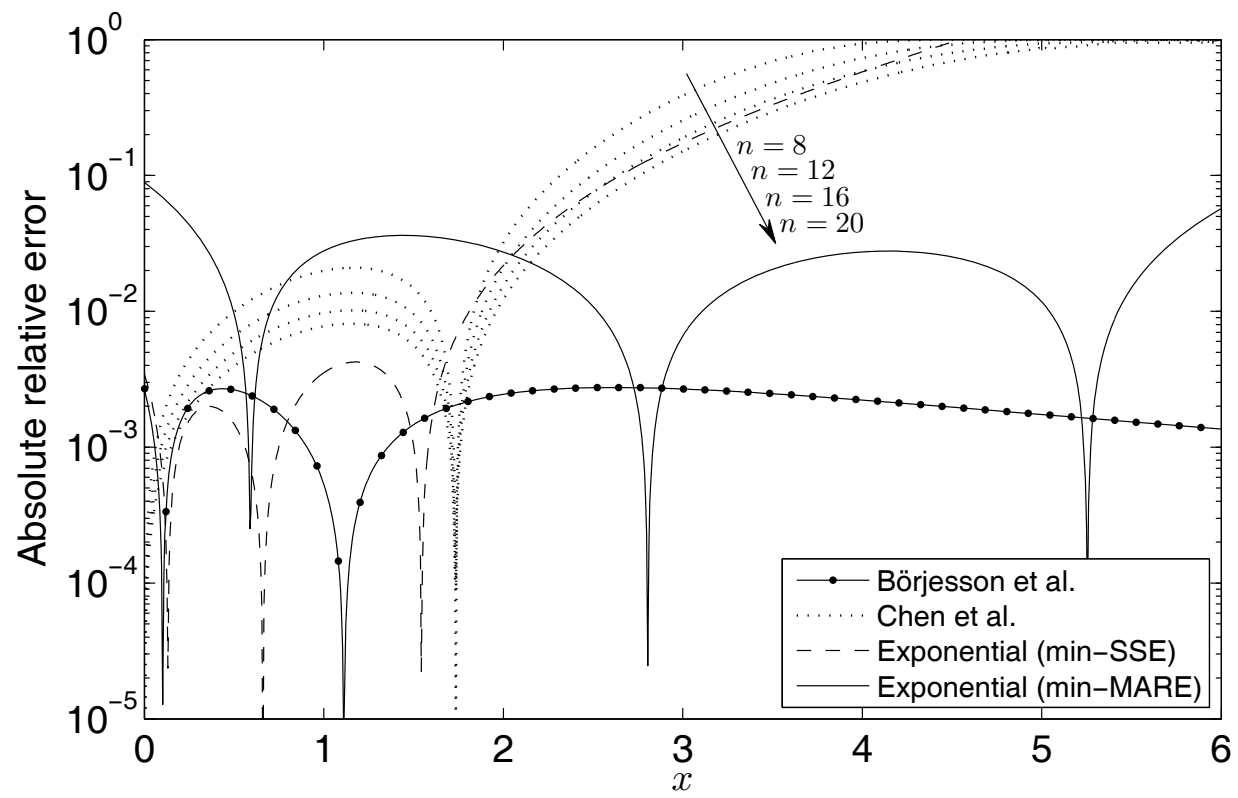

Figure C.2: Absolute relative error of the proposed exponential, Börjesson et al., and Chen et al. approximations $(\hat{x} \in[0,6])$.

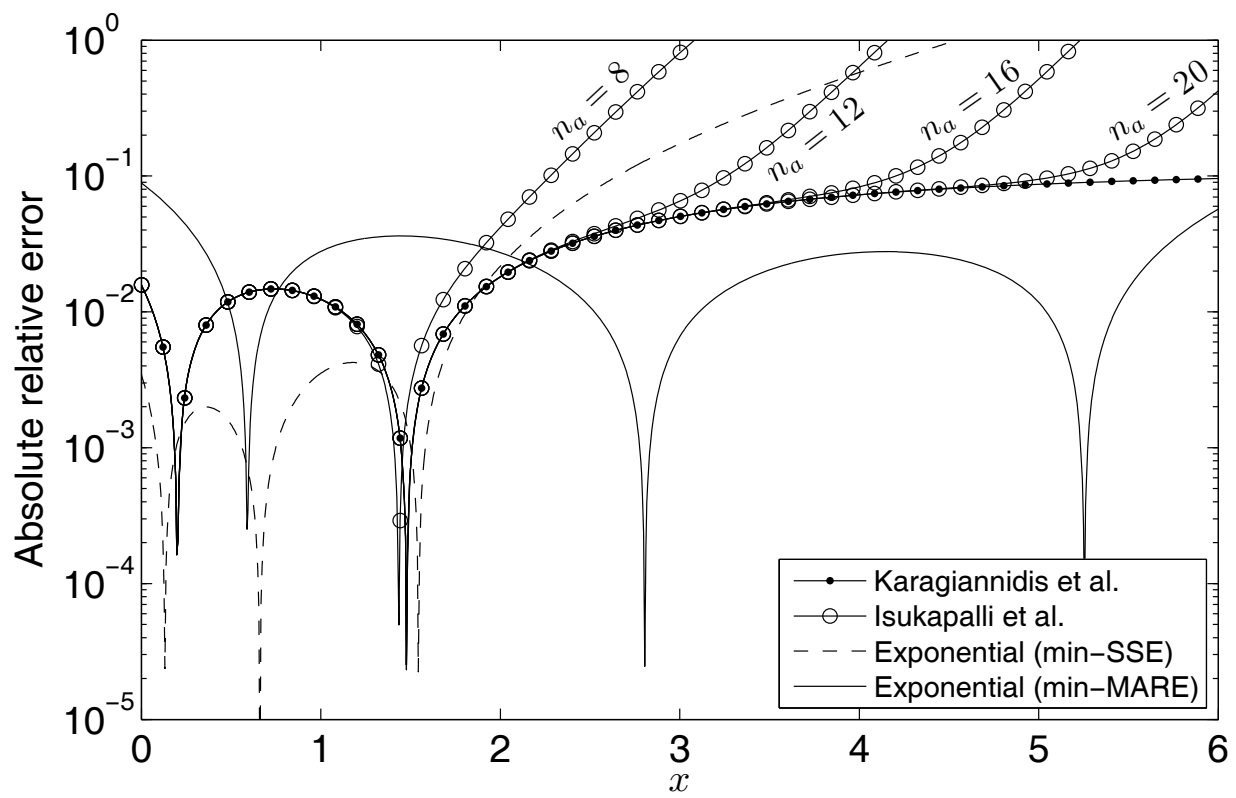

Figure C.3: Absolute relative error of the proposed exponential, Karagiannidis et al., and Isukapalli et al. approximations $(\hat{x} \in[0,6])$. 


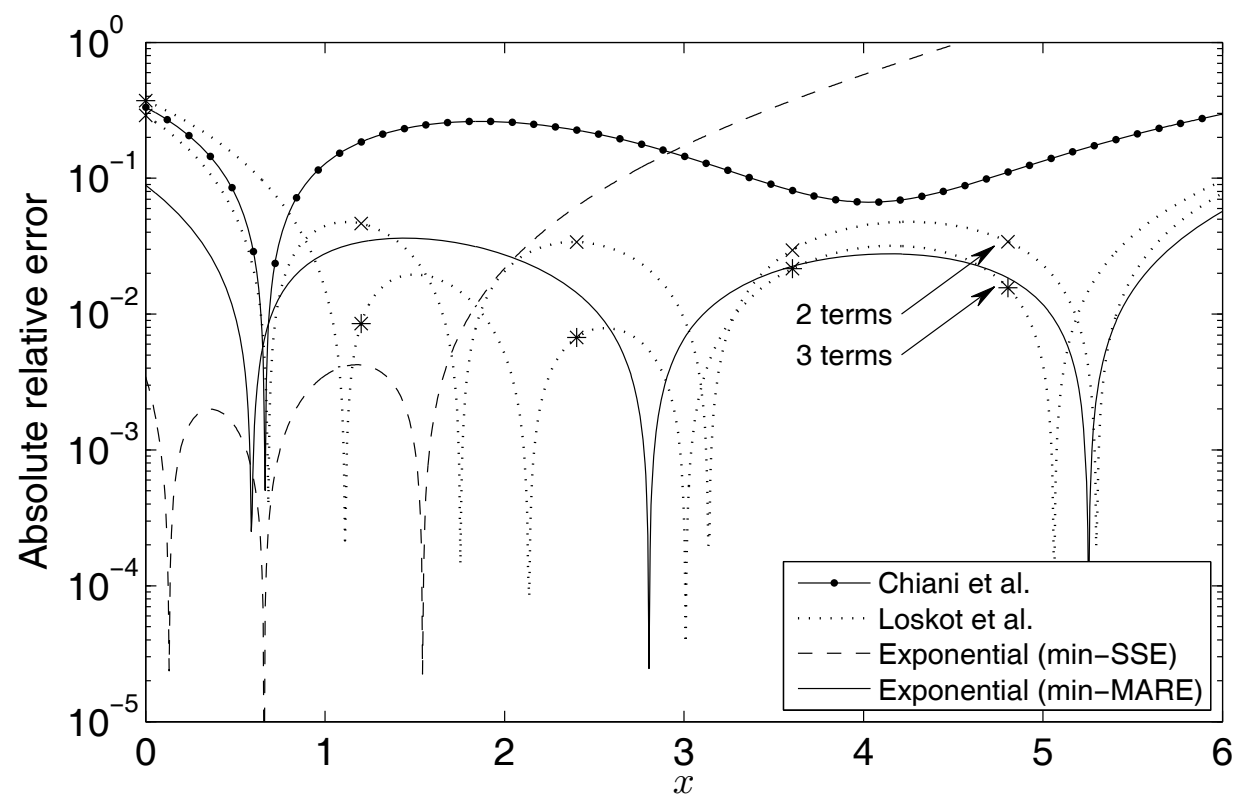

Figure C.4: Absolute relative error of the proposed exponential, Chiani et al., and Loskot et al. approximations $(\hat{x} \in[0,6])$.

sion simpler than the Isukapalli's approximation, thus resulting in a more adequate balance between accuracy and analytical tractability.

Finally, the main attractiveness of the Chiani's approximation is its analytical simplicity, which comes at the cost of limited accuracy (see Figure C.4). The obtained results indicate that the proposed exponential approximation with the min-MARE criterion provides a mathematical expression with a similar complexity, in some cases even slightly simpler (only one single exponential term), that clearly outperforms the Chiani's approximation for almost all arguments. The Loskot's approximation with two exponential terms outperforms the Chiani's approximation with the same analytical complexity. However, the proposed approximation still provides a more adequate balance between accuracy and analytical tractability since it attains a comparable level of accuracy with a single exponential term. The accuracy of the Loskot's approximation can be enhanced with a third term (see Equation C.4). However, the improvement obtained in this case comes at the expense of a slightly increased complexity.

Based on the previous analysis, it can be concluded that the proposed exponential approximation for the Gaussian $Q$-function constitutes an adequate balance between accuracy and analytical tractability. On one hand, such approximation provides a simpler analytical expression than other proposals, thus enabling its use over a wider range of analytical studies. On the other hand, the resulting accuracy is similar or even better than that attained by other existing proposals based on more complex mathematical expressions. 


\section{C.5 Applicability example}

Previous approximations to the Gaussian $Q$-function have mainly been developed to evaluate the bit, symbol or block error probabilities in many communication theory problems. The approximation proposed in this appendix could be employed to solve many of such problems as well. However, in order to illustrate its versatility and applicability, this section considers a different problem related to the field of DSA in CR networks. Concretely, this section employs the approximation proposed in this appendix to easily compute the ED's average probability of detection under Rayleigh fading environments. This problem was studied in [197], resulting in mathematical expressions of notable complexity. This section obtains a much simpler alternative closed-form expression and illustrates the potential applicability and usefulness of the proposed approximation with a practical case study.

The probability of detection of ED can be approximated (see Chapter 4) as:

$$
P_{d}(\gamma)=Q\left(\frac{Q^{-1}\left(P_{f a}\right) \sqrt{2 N}-N \gamma}{\sqrt{2 N}(1+\gamma)}\right)=Q(\zeta(\gamma))
$$

where $N$ is the number of signal samples collected during the sensing interval, $P_{f a}$ is the target probability of false alarm, $\gamma$ is the SNR and, assuming the common case of low SNR regime in DSA/CR $(\gamma \ll 1), \zeta(\gamma)$ can be approximated as:

$$
\zeta(\gamma)=\frac{Q^{-1}\left(P_{f a}\right) \sqrt{2 N}-N \gamma}{\sqrt{2 N}(1+\gamma)} \approx Q^{-1}\left(P_{f a}\right)-\sqrt{\frac{N}{2}} \gamma
$$

Equation C.11 gives the probability of detection conditioned on the instantaneous value of $\gamma$. Under varying SNR, a more useful performance parameter is the average probability of detection $\bar{P}_{d}$ experienced for an average SNR $\gamma_{0}$, which may be derived by averaging Equation C.11 over the SNR statistics:

$$
\bar{P}_{d}\left(\gamma_{0}\right)=\mathbb{E}\left[P_{d}(\gamma)\right]=\int_{\gamma} P_{d}(\gamma) f_{\gamma}(\gamma) d \gamma=\int_{\gamma} Q(\zeta(\gamma)) f_{\gamma}(\gamma) d \gamma
$$

where $f_{\gamma}(\gamma)$ is the PDF of the received SNR. If the signal amplitude follows a Rayleigh distribution, the SNR then follows an exponential PDF given by:

$$
f_{\gamma}(\gamma)=\frac{1}{\gamma_{0}} \exp \left(-\frac{\gamma}{\gamma_{0}}\right), \quad \gamma \geq 0
$$

Equation C.13 can be simplified and solved by applying the approximation of Equation C.8. Notice that $\zeta(\gamma)$, the argument of $Q(x)$, may take both positive and negative values even though $\gamma \geq 0$ (Equation C.12). Since the approximation in Equation C.8 is valid for positive 
arguments, the property $Q(x)=1-Q(-x)$ must be used for negative values of $\zeta(\gamma)$. Thus:

$$
\begin{aligned}
\bar{P}_{d}\left(\gamma_{0}\right)= & \int_{\zeta(\gamma)<0}[1-Q(-\zeta(\gamma))] f_{\gamma}(\gamma) d \gamma+\int_{\zeta(\gamma) \geq 0} Q(\zeta(\gamma)) f_{\gamma}(\gamma) d \gamma \\
\approx & \int_{\sqrt{\frac{2}{N}} Q^{-1}\left(P_{f a}\right)}^{\infty} \frac{1}{\gamma_{0}} \exp \left(-\frac{\gamma}{\gamma_{0}}\right) d \gamma \\
& -\int_{\sqrt{\frac{2}{N}} Q^{-1}\left(P_{f a}\right)}^{\infty} \frac{1}{\gamma_{0}} \exp \left(-\frac{\gamma}{\gamma_{0}}\right) \exp \left(a[\zeta(\gamma)]^{2}-b \zeta(\gamma)+c\right) d \gamma \\
& +\int_{0}^{\sqrt{\frac{2}{N}} Q^{-1}\left(P_{f a}\right)} \frac{1}{\gamma_{0}} \exp \left(-\frac{\gamma}{\gamma_{0}}\right) \exp \left(a[\zeta(\gamma)]^{2}+b \zeta(\gamma)+c\right) d \gamma \\
\approx & \int_{\sqrt{\frac{2}{N}} Q^{-1}\left(P_{f a}\right)}^{\infty} \frac{1}{\gamma_{0}} \exp \left(-\frac{\gamma}{\gamma_{0}}\right) d \gamma \\
& -\int_{\sqrt{\frac{2}{N}} Q^{-1}\left(P_{f a}\right)}^{\infty} \frac{1}{\gamma_{0}} \exp \left(-\Omega \gamma^{2}-\Xi \gamma-\Theta\right) d \gamma \\
& +\int_{0}^{\sqrt{\frac{2}{N}} Q^{-1}\left(P_{f a}\right)} \frac{1}{\gamma_{0}} \exp \left(-\Omega \gamma^{2}-\Psi \gamma-\Phi\right) d \gamma
\end{aligned}
$$

where

$$
\begin{aligned}
& \Omega=-a N / 2 \\
& \Xi=a Q^{-1}\left(P_{f a}\right) \sqrt{2 N}-b \sqrt{N / 2}+1 / \gamma_{0} \\
& \Psi=a Q^{-1}\left(P_{f a}\right) \sqrt{2 N}+b \sqrt{N / 2}+1 / \gamma_{0} \\
& \Theta=-a\left[Q^{-1}\left(P_{f a}\right)\right]^{2}+b Q^{-1}\left(P_{f a}\right)-c \\
& \Phi=-a\left[Q^{-1}\left(P_{f a}\right)\right]^{2}-b Q^{-1}\left(P_{f a}\right)-c
\end{aligned}
$$

Taking into account the approximation in Equation C.12, Equation C.15 is composed of three definite exponential integrals that can readily be solved in terms of exponential, error and complementary error functions:

$$
\begin{aligned}
\bar{P}_{d}\left(\gamma_{0}\right) \approx & \exp \left(-\frac{1}{\gamma_{0}} \sqrt{\frac{2}{N}} Q^{-1}\left(P_{f a}\right)\right) \\
& -\frac{1}{2 \gamma_{0}} \sqrt{\frac{\pi}{\Omega}} \exp \left(\frac{\Xi^{2}}{4 \Omega}-\Theta\right) \operatorname{erfc}\left(\sqrt{\frac{2 \Omega}{N}} Q^{-1}\left(P_{f a}\right)+\frac{\Xi}{2 \sqrt{\Omega}}\right) \\
& +\frac{1}{2 \gamma_{0}} \sqrt{\frac{\pi}{\Omega}} \exp \left(\frac{\Psi^{2}}{4 \Omega}-\Phi\right)\left[\operatorname{erf}\left(\sqrt{\frac{2 \Omega}{N}} Q^{-1}\left(P_{f a}\right)+\frac{\Psi}{2 \sqrt{\Omega}}\right)-\operatorname{erf}\left(\frac{\Psi}{2 \sqrt{\Omega}}\right)\right]
\end{aligned}
$$

Although the analytical result of Equation C.21 is slightly complex for mathematical manipulations, it is important to note that solving Equation C.13 with some of the approximations presented in Section C. 2 would have lead to much more complex solving procedures 
Table C.2: Comparison of the exact and approximated probability of detection of an energy detector under Rayleigh fading $\left(P_{f a}=0.01\right.$ and $\left.N=1000\right)$.

\begin{tabular}{|c|c|c|c|c|c|c|c|c|}
\hline & \multicolumn{7}{|c|}{ SNR (dB) } \\
\hline & & -20 & -15 & -10 & -5 & 0 & 5 & 10 \\
\hline \multirow{12}{*}{ 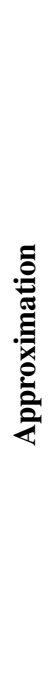 } & Exact & 0.0205 & 0.0929 & 0.3886 & 0.7263 & 0.9019 & 0.9677 & 0.9896 \\
\hline & $\min -\mathrm{SSE}$ & 0.0210 & 0.0931 & 0.3887 & 0.7263 & 0.9019 & 0.9677 & 0.9896 \\
\hline & min-MARE & 0.0219 & 0.0935 & 0.3884 & 0.7263 & 0.9019 & 0.9677 & 0.9896 \\
\hline & Börjesson & 0.0206 & 0.0929 & 0.3886 & 0.7263 & 0.9019 & 0.9677 & 0.9896 \\
\hline & Chiani & 0.0255 & 0.0998 & 0.3916 & 0.7269 & 0.9020 & 0.9677 & 0.9896 \\
\hline & Loskot (2 terms) & 0.0203 & 0.0927 & 0.3883 & 0.7263 & 0.9019 & 0.9677 & 0.9896 \\
\hline & Loskot (3 terms) & 0.0206 & 0.0906 & 0.3865 & 0.7259 & 0.9019 & 0.9677 & 0.9896 \\
\hline & Karagiannidis & 0.0202 & 0.0930 & 0.3888 & 0.7264 & 0.9019 & 0.9677 & 0.9896 \\
\hline & Isukapalli $\left(n_{a}=8\right)$ & 0.0196 & 0.0926 & 0.3888 & 0.7265 & 0.9020 & 0.9677 & 0.9896 \\
\hline & Isukapalli $\left(n_{a}=20\right)$ & 0.0202 & 0.0930 & 0.3888 & 0.7264 & 0.9019 & 0.9677 & 0.9896 \\
\hline & Chen $(n=8)$ & 0.0198 & 0.0931 & 0.3890 & 0.7264 & 0.9019 & 0.9677 & 0.9896 \\
\hline & Chen $(n=20)$ & 0.0203 & 0.0930 & 0.3788 & 0.7264 & 0.9019 & 0.9677 & 0.9896 \\
\hline
\end{tabular}

and resulting mathematical expressions. This indicates the ability of the approximation proposed in Equation C.8 to simplify the analytical resolution of some problems for which the approximations of Section C.2 were not envisaged. It is worth noting, moreover, that the two last terms of Equation C.21 lead to similar numerical values, specially for high values of SNR, so that they approximately cancel out each other. As a result, the simplification given in Equation C.22, which is valid over a wide range of SNR values (and tighter for high $N$ and low $P_{f a}$ values), can be employed in analytical studies:

$$
\bar{P}_{d}\left(\gamma_{0}\right) \approx \exp \left(-\frac{1}{\gamma_{0}} \sqrt{\frac{2}{N}} Q^{-1}\left(P_{f a}\right)\right)
$$

To comparatively assess the accuracy of the proposed approximation in evaluating the performance of ED under Rayleigh fading, Equation C.13 was solved numerically employing the exact values of the Gaussian $Q$-function as well as the approximated values provided by Equations C.1-C.8. The results are shown in Table C.2. As it can be appreciated, all the analyzed approximations provide exact values for high SNR values but diverge as the SNR value decreases. This can be explained by the fact that $\zeta(\gamma)$, the argument of the $Q$-function in Equation C.13, increases as the SNR decreases and some of the considered approximations provide higher estimation errors for larger arguments (see Figures C.2, C.3 and C.4). Therefore, in the case study of this section, the accuracy of the analyzed approximations is better appreciated at low SNR values. In such region, it can be observed that the Börjesson and Chiani approximations result, respectively, in the most and least accurate estimates, which is in accordance with the relative errors observed in Figures C.2 and C.4. Regarding 
the proposed approximation, the best estimate is provided in this case by the min-SSE criterion. Although other approximations are able to provide more accurate results, the accuracy of the proposed approximation can arguably be considered as sufficient for most practical problems. Moreover, it is worth emphasizing that the analytical evaluation of Equation C.13 with other approximations would have resulted in much more complex solving procedures, which highlights the ability of the proposed approximation to simplify analytical studies at reasonable accuracy levels.

\section{C.6 Summary}

This appendix has presented a novel exponential approximation for the Gaussian $Q$-function providing an adequate balance between accuracy and analytical tractability. The approximation provides a simple mathematical expression that enables its use over a wide range of analytical studies. The resulting accuracy is similar or even better than that attained by other existing proposals based on more complex mathematical expressions. As an example of its versatility, the proposed approximation has been used to derive a new closed-form approximation to the probability of detection of an energy detector under Rayleigh fading. 



\section{REFERENCES}

[1] J. C. Maxwell, "A dynamical theory of the electromagnetic field," Philosophical Transactions of the Royal Society of London, vol. 155, pp. 459-512, 1865.

[2] H. R. Hertz, Electric waves: being researches on the propagation of electric action with finite velocity through space. Cornell University Library, 1893, (translated by D. E. Jones).

[3] R. W. Lucky, "The precious radio spectrum," IEEE Spectrum, vol. 38, no. 9, p. 90, Sep. 2001.

[4] Federal Communications Commission (FCC), Spectrum Policy Task Force, "Report of the spectrum efficiency working group," Nov. 2002.

[5] Federal Communications Commission (FCC), "ET Docket no. 03-322: Notice of proposed rule making and order," Dec. 2003.

[6] J. A. Hoffmeyer, "Regulatory and standardization aspects of DSA technologies global requirements and perspective," in Proceedings of the First IEEE International Symposium on New Frontiers in Dynamic Spectrum Access Networks (DySPAN 2005), Nov. 2005, pp. 700-705.

[7] R. Ercole, "Innovation, spectrum regulation, and DySPAN technologies access to markets," in Proceedings of the First IEEE International Symposium on New Frontiers in Dynamic Spectrum Access Networks (DySPAN 2005), Nov. 2005, pp. 494-511.

[8] C. Ting, S. S. Wildman, and J. M. Bauer, "Government policy and the comparative merits of alternative governance regimes for wireless services," in Proceedings of the First IEEE International Symposium on New Frontiers in Dynamic Spectrum Access Networks (DySPAN 2005), Nov. 2005, pp. 401-419. 
[9] N. Jesuale, "Overview of state and local government interests in spectrum policy issues," in Proceedings of the First IEEE International Symposium on New Frontiers in Dynamic Spectrum Access Networks (DySPAN 2005), Nov. 2005, pp. 476-485.

[10] A. Gad and F. Digham, "Impact of DSA on current regulatory regimes," in Proceedings of the Third IEEE International Symposium on New Frontiers in Dynamic Spectrum Access Networks (DySPAN 2008), Oct. 2008, pp. 1-6.

[11] M. J. Marcus, "Cognitive radio under conservative regulatory environments: lessons learned and near term options," in Proceedings of the Fourth IEEE International Symposium on New Frontiers in Dynamic Spectrum Access Networks (DySPAN 2010), Apr. 2010, pp. 1-5.

[12] H. R. Karimi, M. Fenton, G. Lapierre, and E. Fournier, "European harmonized technical conditions and band plans for broadband wireless access in the $790-862 \mathrm{MHz}$ digital dividend spectrum," in Proceedings of the Fourth IEEE International Symposium on New Frontiers in Dynamic Spectrum Access Networks (DySPAN 2010), Apr. 2010, pp. 1-9.

[13] M. Cooper, "The economics of collaborative production in the spectrum commons," in Proceedings of the First IEEE International Symposium on New Frontiers in Dynamic Spectrum Access Networks (DySPAN 2005), Nov. 2005, pp. 379-400.

[14] D. Grandblaise, C. Kloeck, K. Moessner, V. Rodriguez, E. Mohyeldin, M. K. Pereirasamy, J. Luo, and I. Martoyo, "Techno-economic of collaborative based secondary spectrum usage - E2R research project outcomes overview," in Proceedings of the First IEEE International Symposium on New Frontiers in Dynamic Spectrum Access Networks (DySPAN 2005), Nov. 2005, pp. 318-327.

[15] J. M. Marcus, "Real time spectrum markets and interruptible spectrum: new concepts of spectrum use enabled by cognitive radio," in Proceedings of the First IEEE International Symposium on New Frontiers in Dynamic Spectrum Access Networks (DySPAN 2005), Nov. 2005, pp. 512-517.

[16] C. Bazelon, "Licensed or unlicensed: the economic considerations in incremental spectrum allocations," in Proceedings of the Third IEEE International Symposium on New Frontiers in Dynamic Spectrum Access Networks (DySPAN 2008), Oct. 2008, pp. $1-8$.

[17] M. Nekovee, "Cognitive radio access to TV white spaces: spectrum opportunities, commercial applications and remaining technology challenges," in Proceedings of the Fourth IEEE International Symposium on New Frontiers in Dynamic Spectrum Access Networks (DySPAN 2010), Apr. 2010, pp. 1-10.

[18] C. Jackson, "Dynamic sharing of radio spectrum: a brief history," in Proceedings of the First IEEE International Symposium on New Frontiers in Dynamic Spectrum Access Networks (DySPAN 2005), Nov. 2005, pp. 445-466. 
[19] Q. Zhao and A. Swami, "A survey of dynamic spectrum access: signal processing and networking perspectives," in Proceedings of the IEEE International Conference on Acoustics, Speech and Signal Processing (ICASSP 2007), vol. 4, Apr. 2007, pp. IV/1349-/IV-1352.

[20] Q. Zhao and B. M. Sadler, "A survey of dynamic spectrum access," IEEE Signal Processing Magazine, vol. 24, no. 3, pp. 79-89, May 2007.

[21] M. M. Buddhikot, "Understanding dynamic spectrum access: taxonomy, models and challenges," in Proceedings of the 2nd IEEE International Symposium on New Frontiers in Dynamic Spectrum Access Networks (DySPAN 2007), Apr. 2007, pp. 649-663.

[22] J. M. Peha, "Sharing spectrum through spectrum policy reform and cognitive radio," Proceedings of the IEEE, vol. 97, no. 4, pp. 708-719, Apr. 2009.

[23] D. N. Hatfield and P. J. Weiser, "Property rights in spectrum: taking the next step," in Proceedings of the First IEEE International Symposium on New Frontiers in Dynamic Spectrum Access Networks (DySPAN 2005), Nov. 2005, pp. 43-55.

[24] L. Xu, R. Tönjes, T. Paila, W. Hansmann, M. Frank, and M. Albrecht, "DRiVE-ing to the internet: dynamic radio for IP services in vehicular environments," in Proceedings of the 25th Annual IEEE Conference on Local Computer Networks (LCN 2000), Nov. 2000, pp. 281-289.

[25] P. Leaves, K. Moessner, R. Tafazolli, D. Grandblaise, D. Bourse, R. Tönjes, and M. Breveglieri, "Dynamic spectrum allocation in composite reconfigurable wireless networks," IEEE Communications Magazine, vol. 42, no. 5, pp. 72-81, May 2004.

[26] R. Keller, T. Lohmar, R. Tonjes, and J. Thielecke, "Convergence of cellular and broadcast networks from a multi-radio perspective," IEEE Personal Communications Magazine, vol. 8, no. 2, pp. 51-56, Apr. 2001.

[27] P. Demestichas, G. Vivier, K. El-Khazen, and M. Theologou, "Evolution in wireless systems management concepts: from composite radio environments to reconfigurability," IEEE Communications Magazine, vol. 42, no. 5, pp. 90-98, May 2004.

[28] B. Aazhang, J. Lilleberg, and G. Middleton, "Spectrum sharing in a cellular system," in Proceedings of the IEEE Eighth International Symposium on Spread Spectrum Techniques and Applications (ISSSTA 2004), Aug. 2004, pp. 355-359.

[29] C. A. Nissen and G. M. Butler, "A technology enabled framework for dynamic allocation of the radio frequency spectrum," The MITRE Corporation, Bedford (USA), Tech. Rep., Nov. 2004.

[30] V. Rodriguez, K. Moessner, and R. Tafazolli, "Market-driven dynamic spectrum allocation: optimal end-user pricing and admission control for CDMA," in Proceedings of the 14th IST Mobile \& Wireless Communications Summit, Jun. 2005, p. 5. 
[31] Y. Xing, R. Chandramouli, S. Mangold, and S. S. N, "Dynamic spectrum access in open spectrum wireless networks," IEEE Journal on Selected Areas in Communications, vol. 24, no. 3, pp. 626-637, Mar. 2006.

[32] L. Kovacs and A. Vidacs, "Interference-tolerant spatio-temporal dynamic spectrum allocation," in Proceedings of the 2nd IEEE International Symposium on New Frontiers in Dynamic Spectrum Access Networks (DySPAN 2007), Apr. 2007, pp. 403-411.

[33] W. Lehr and J. Crowcroft, "Managing shared access to a spectrum commons," in Proceedings of the First IEEE International Symposium on New Frontiers in Dynamic Spectrum Access Networks (DySPAN 2005), Nov. 2005, pp. 420-444.

[34] G. Hardin, "The tragedy of the commons," Science, vol. 162, no. 3859, pp. 12431248, Dec. 1968.

[35] _ "Extensions of "the tragedy of the commons"," Science, vol. 280, no. 5364, pp. 682-683, May 1998.

[36] C. Raman, R. D. Yates, and N. B. Mandayam, "Scheduling variable rate links via a spectrum server," in Proceedings of the First IEEE International Symposium on New Frontiers in Dynamic Spectrum Access Networks (DySPAN 2005), Nov. 2005, pp. $110-118$.

[37] O. Ileri, D. Samardzija, and N. B. Mandayam, "Demand responsive pricing and competitive spectrum allocation via a spectrum server," in Proceedings of the First IEEE International Symposium on New Frontiers in Dynamic Spectrum Access Networks (DySPAN 2005), Nov. 2005, pp. 194-202.

[38] V. Brik, E. Rozner, S. Banerjee, and P. Bahl, "DSAP: a protocol for coordinated spectrum access," in Proceedings of the First IEEE International Symposium on New Frontiers in Dynamic Spectrum Access Networks (DySPAN 2005), Nov. 2005, pp. 611-614.

[39] R. Etkin, A. Parekh, and D. Tse, "Spectrum sharing for unlicensed bands," IEEE Journal on Selected Areas in Communications, vol. 25, no. 3, pp. 517-528, Apr. 2007.

[40] J. Huang, R. A. Berry, and M. L. Honig, "Spectrum sharing with distributed interference compensation," in Proceedings of the First IEEE International Symposium on New Frontiers in Dynamic Spectrum Access Networks (DySPAN 2005), Nov. 2005, pp. 88-93.

[41] N. Clemens and C. Rose, "Intelligent power allocation strategies in an unlicensed spectrum," in Proceedings of the First IEEE International Symposium on New Frontiers in Dynamic Spectrum Access Networks (DySPAN 2005), Nov. 2005, pp. 37-42. 
[42] C. Santivanez, R. Ramanathan, C. Partridge, R. Krishnan, M. Condell, and S. Polit, "Opportunistic spectrum access: challenges, architecture, protocols," in Proceedings of the 2nd Annual International Workshop on Wireless Internet (WICON 2006), Aug. 2006, p. 13.

[43] S. Ball, A. Ferguson, and T. W. Rondeau, "Consumer applications of cognitive radio defined networks," in Proceedings of the First IEEE International Symposium on New Frontiers in Dynamic Spectrum Access Networks (DySPAN 2005), Nov. 2005, pp. $518-525$.

[44] J. A. Stine, "Spectrum management: the killer application of ad hoc and mesh networking," in Proceedings of the First IEEE International Symposium on New Frontiers in Dynamic Spectrum Access Networks (DySPAN 2005), Nov. 2005, pp. 184193.

[45] P. Pawelczak, R. V. Prasad, L. Xia, and I. G. M. M. Niemegeers, "Cognitive radio emergency networks - requirements and design," in Proceedings of the First IEEE International Symposium on New Frontiers in Dynamic Spectrum Access Networks (DySPAN 2005), Nov. 2005, pp. 601-606.

[46] A. Gorcin and H. Arslan, "Public safety and emergency case communications: opportunities from the aspect of cognitive radio," in Proceedings of the Third IEEE International Symposium on New Frontiers in Dynamic Spectrum Access Networks (DySPAN 2008), Oct. 2008, pp. 1-10.

[47] J. Mitola, "Software radios - survey, critical evaluation and future directions," in Proceedings of the National Telesystems Conference (NTC 1992), May 1992, pp. 13/15$13 / 23$.

[48] J. Mitola and G. Q. Maguire, "Cognitive radio: making software radios more personal," IEEE Personal Communications Magazine, vol. 6, no. 4, pp. 13-18, Aug. 1999.

[49] J. Mitola, Software Radio Architecture. Wiley-Interscience, Nov. 2000.

[50] —_, Cognitive Radio Architecture. Wiley-Interscience, Oct. 2006.

[51] S. Haykin, "Cognitive radio: brain-empowered wireless communications," IEEE Journal on Selected Areas in Communications, vol. 23, no. 2, pp. 201-220, Feb. 2005.

[52] F. K. Jondral, "Software-defined radio - basics and evolution to cognitive radio," EURASIP Journal on Wireless Communications and Networking, vol. 2005, no. 3, p. 9, 2005.

[53] K. G. Shin, H. Kim, A. W. Min, and A. Kumar, "Cognitive radios for dynamic spectrum access: from concept to reality," IEEE Wireless Communications, vol. 17, no. 6, pp. 64-74, Dec. 2010. 
[54] I. F. Akyildiz, W.-Y. Lee, M. C. Vuran, and S. Mohanty, "NeXt generation/dynamic spectrum access/cognitive radio wireless networks: a survey," Computer Networks, vol. 50, no. 13, pp. 2127-2159, Sep. 2006.

[55] — - "A survey on spectrum management in cognitive radio networks," IEEE Communications Magazine, vol. 46, no. 4, pp. 40-48, Apr. 2008.

[56] M. J. Marcus, "CR: cooperative radio or confrontational radio," in Proceedings of the 2nd IEEE International Symposium on New Frontiers in Dynamic Spectrum Access Networks (DySPAN 2007), Apr. 2007, pp. 208-211.

[57] O. Sallent, J. Pérez-Romero, R. Agustí, and P. Cordier, "Cognitive pilot channel enabling spectrum awareness," in Proceedings of the IEEE International Conference on Communications Workshops (ICC Workshops 2009), Jun. 2009, pp. 1-6.

[58] J. Pérez-Romero, O. Sallent, R. Agustí, and L. Giupponi, "A novel on-demand cognitive pilot channel enabling dynamic spectrum allocation," in Proceedings of the 2nd IEEE International Symposium on New Frontiers in Dynamic Spectrum Access Networks (DySPAN 2007), Apr. 2007, pp. 46-53.

[59] M. Filo, A. Hossain, A. R. Biswas, and R. Piesiewicz, "Cognitive pilot channel: enabler for radio systems coexistence," in Proceedings of the Second International Workshop on Cognitive Radio and Advanced Spectrum Management (CogART 2009), Jun. 2009, pp. 17-23.

[60] S. M. Mishra, A. Sahai, and R. W. Brodersen, "Cooperative sensing among cognitive radios," in Proceedings of the IEEE International Conference on Communications (ICC 2006), vol. 4, Jun. 2006, pp. 1658-1663.

[61] A. Ghasemi and E. S. Sousa, "Collaborative spectrum sensing for opportunistic access in fading environments," in Proceedings of the First IEEE International Symposium on New Frontiers in Dynamic Spectrum Access Networks (DySPAN 2005), Nov. 2005, pp. 131-136.

[62] G. Ganesan and L. Ye, "Cooperative spectrum sensing in cognitive radio, Part I: two user networks," IEEE Transactions on Wireless Communications, vol. 6, no. 6, pp. 2204-2213, Jun. 2007.

[63] — - "Cooperative spectrum sensing in cognitive radio, Part II: multiuser networks," IEEE Transactions on Wireless Communications, vol. 6, no. 6, pp. 2214-2222, Jun. 2007.

[64] C. Cormio and K. R. Chowdhury, "A survey on MAC protocols for cognitive radio networks," Ad Hoc Networks, vol. 7, no. 7, pp. 1315-1329, Sep. 2009.

[65] Federal Communications Commission (FCC), "ET Docket no. 03-287: Notice of proposed rule making and order," Nov. 2003. 
[66] T. A. Weiss and F. K. Jondral, "Spectrum pooling: an innovative strategy for the enhancement of spectrum efficiency," IEEE Communications Magazine, vol. 42, no. 3, pp. S8-S14, Mar. 2004.

[67] F. Capar, I. Martoyo, T. Weiss, and F. K. Jondral, "Comparison of bandwidth utilization for controlled and uncontrolled channel assignment in a spectrum pooling system," in Proceedings of the IEEE 55th Vehicular Technology Conference (VTC 2002 Spring), vol. 3, May 2002, pp. 1069-1073.

[68] T. A. Weiss, J. Hillenbrand, A. Krohn, and F. K. Jondral, "Efficient signaling of spectral resources in spectrum pooling systems," in Proceedings of the 10th Symposium on Communications and Vehicular Technology (SCVT 2003), Nov. 2003, pp. 1-6.

[69] T. Weiss, M. Spiering, and F. K. Jondral, "Quality of service in spectrum pooling systems," in Proceedings of the 15th IEEE International Symposium on Personal, Indoor and Mobile Radio Communications (PIMRC 2004), vol. 1, Sep. 2004, pp. 345-349.

[70] T. Weiss, J. Hillenbrand, A. Krohn, and F. K. Jondral, "Mutual interference in OFDMbased spectrum pooling systems," in Proceedings of the IEEE 59th Vehicular Technology Conference (VTC 2004 Spring), vol. 4, May 2004, pp. 1873-1877.

[71] I. Budiarjo, H. Nikookar, and L. P. Ligthart, "Combined spectrum pooling and adaptive bit loading for cognitive radio OFDM based system," in Proceedings of the 13th Symposium on Communications and Vehicular Technology (SCVT 2006), Nov. 2006, pp. 73-76.

[72] A. V. Adamis and P. Constantinou, "Performance study of CSMA/CA over spectrum pooling environment for cognitive radios," in Proceedings of the Third IEEE International Conference on Wireless and Mobile Computing, Networking and Communications (WiMOB 2007), Oct. 2007, pp. 31-31.

[73] R. W. Brodersen, A. Wolisz, D. Cabric, S. M. Mishra, and D. Willkomm, "Corvus: a cognitive radio approach for usage of virtual unlicensed spectrum," White paper, Jul. 2004.

[74] D. Cabric, S. M. Mishra, D. Willkomm, R. Brodersen, and A. Wolisz, "A cognitive radio approach for usage of virtual unlicensed spectrum," in Proceedings of the 14th IST Mobile Wireless Communications Summit, Jun. 2005, p. 4.

[75] D. Willkomm, J. Gross, and A. Wolisz, "Reliable link maintenance in cognitive radio systems," in Proceedings of the First IEEE International Symposium on New Frontiers in Dynamic Spectrum Access Networks (DySPAN 2005), Nov. 2005, pp. 371-378.

[76] M. M. Buddhikot, P. Kolodzy, S. M. adn K. Ryan, and J. Evans, "DIMSUMnet: new directions in wireless networking using coordinated dynamic spectrum," in Proceedings of the Sixth IEEE International Symposium on a World of Wireless Mobile and Multimedia Networks (WoWMoM 2005), Jun. 2005, pp. 78-85. 
[77] Y. Zeng, Y.-C. Liang, Z. Lei, S. W. Oh, F. Chin, and S. Sun, "Worldwide regulatory and standardization activities on cognitive radio," in Proceedings of the Fourth IEEE International Symposium on New Frontiers in Dynamic Spectrum Access Networks (DySPAN 2010), Apr. 2010, pp. 1-9.

[78] R. V. Prasad, P. Pawelczak, J. A. Hoffmeyer, and H. S. Berger, "Cognitive functionality in next generation wireless networks: standardization efforts," IEEE Communications Magazine, vol. 46, no. 4, pp. 72-78, Apr. 2008.

[79] M. Sherman, A. N. Mody, R. Martinez, C. Rodriguez, and R. Reddy, "IEEE standards supporting cognitive radio and networks, dynamic spectrum access, and coexistence," IEEE Communications Magazine, vol. 46, no. 7, pp. 72-79, Jul. 2008.

[80] C. Cordeiro, K. Challapali, D. Birru, and N. S. Shankar, "IEEE 802.22: the first worldwide wireless standard based on cognitive radios," in Proceedings of the First IEEE International Symposium on New Frontiers in Dynamic Spectrum Access Networks (DySPAN 2005), Nov. 2005, pp. 328-337.

[81] — - "IEEE 802.22: an introduction to the first wireless standard based on cognitive radios," Journal of Communications, vol. 1, no. 1, pp. 38-47, Apr. 2006.

[82] C. Stevenson, G. Chouinard, Z. Lei, W. Hu, S. Shellhammer, and W. Caldwell, "IEEE 802.22: the first cognitive radio wireless regional area network standard," IEEE Communications Magazine, vol. 47, no. 1, pp. 130-138, Jan. 2009.

[83] C. Cordeiro, K. Challapali, and M. Ghosh, "Cognitive PHY and MAC layers for dynamic spectrum access and sharing of TV bands," in Proceedings of the First International Workshop on Technology and Policy for Accessing Spectrum (TAPAS 2006), Aug. 2006, p. 3.

[84] K. Challapali, C. Cordeiro, and D. Birru, "Evolution of spectrum-agile cognitive radios: first wireless internet standard and beyond," in Proceedings of the 2nd Annual International Workshop on Wireless Internet (WICON 2006), Aug. 2006, p. 27.

[85] B. Scott and M. Calabrese, "Measuring the TV white space available for unlicensed wireless broadband," Free Press and New America Foundation, Tech. Rep., Nov. 2005.

[86] M. Mishra and A. Sahai, "How much white space is there?" Electrical Engineering and Computer Sciences, University of California at Berkeley, Berkeley, CA, USA, Tech. Rep. UCB/EECS-2009-3, Jan. 2009.

[87] M. Nekovee, "Quantifying the availability of TV white spaces for cognitive radio operation in the UK," in Proceedings of the IEEE International Conference on Communications Workshops (ICC Workshops 2009), Jun. 2009, pp. 1-5. 
[88] S. Buljore, V. Merat, H. Harada, S. Filin, P. Houze, K. Tsagkaris, V. Ivanov, K. Nolte, T. Farnham, and O. Holland, "IEEE P1900.4 system overview on architecture and enablers for optimised radio and spectrum resource usage," in Proceedings of the Third IEEE International Symposium on New Frontiers in Dynamic Spectrum Access Networks (DySPAN 2008), Oct. 2008, pp. 1-8.

[89] A. Stirling, "White spaces - the new Wi-Fi?" International Journal of Digital Television, vol. 1, no. 1, pp. 69-83, 2010.

[90] S. Deb, V. Srinivasan, and R. Maheshwari, "Dynamic spectrum access in DTV whitespaces: design rules, architecture and algorithms," in Proceedings of the 15th Annual International Conference on Mobile Computing and Networking (MobiCom 2009), Sep. 2009, pp. 1-16.

[91] P. Piggin and K. L. Stanwood, "Standardizing WiMAX solutions for coexistence in the $3.65 \mathrm{GHz}$ band," in Proceedings of the Third IEEE International Symposium on New Frontiers in Dynamic Spectrum Access Networks (DySPAN 2008), Oct. 2008, pp. 1-7.

[92] ITU, "Software defined radio in IMT-2000, the future development of IMT-2000 and systems beyond IMT-2000," International Telecommunication Union, Tech. Rep. ITU-R M.2063, 2005.

[93] _ _ "Software-defined radio in the land mobile service," International Telecommunication Union, Tech. Rep. ITU-R M.2064, 2005.

[94] ETSI, "Reconfigurable Radio Systems (RRS); Summary of feasibility studies and potential standardization topics," European Telecommunications Standards Institute, Tech. Rep. ETSI TR 102838 v1.1.1, Oct. 2009.

[95] J. Wang, M. S. Song, S. Santhiveeran, K. Lim, G. Ko, K. Kim, S. H. Hwang, M. Ghosh, V. Gaddam, and K. Challapali, "First cognitive radio networking standard for personal/portable devices in TV white spaces," in Proceedings of the Fourth IEEE International Symposium on New Frontiers in Dynamic Spectrum Access Networks (DySPAN 2010), Apr. 2010, pp. 1-12.

[96] ECMA, "MAC and PHY for operation in TV white space," European association for standardizing information and communication systems, Tech. Rep. ECMA-392, Dec. 2009.

[97] S. Probasco, G. Bajko, and B. Rosen, "Protocol to access white space database: problem statement and requirements," Feb. 2011, available at the following URL: http://tools.ietf.org/html/draft-patil-paws-problem-stmt-01.

[98] T. Derryberry and B. Patil, "Protocol to access white space database: overview and use case scenarios," Mar. 2011, available at the following URL: http://tools.ietf.org/ html/draft-probasco-paws-overview-usecases-00. 
[99] S. M. Mishra, D. Cabric, C. Chang, D. Willkomm, B. van Schewick, A. Wolisz, and R. W. Brodersen, "A real time cognitive radio testbed for physical and link layer experiments," in Proceedings of the First IEEE International Symposium on New Frontiers in Dynamic Spectrum Access Networks (DySPAN 2005), Nov. 2005, pp. $562-567$.

[100] K. E. Nolan, P. D. Sutton, and L. E. Doyle, "Dynamic spectrum access and coexistence experiences involving two independently developed cognitive radio testbeds," in Proceedings of the 2nd IEEE International Symposium on New Frontiers in Dynamic Spectrum Access Networks (DySPAN 2007), Apr. 2007, pp. 270-275.

[101] E. Pappas, S. Boettcher, C. Mascaro, and S. Thai, "Spectrum simulation testbed for dynamic spectrum access," in Proceedings of the Third IEEE International Symposium on New Frontiers in Dynamic Spectrum Access Networks (DySPAN 2008), Oct. 2008, pp. 1-5.

[102] L. E. Doyle, P. D. Sutton, K. E. Nolan, J. Lotze, B. Ozgul, T. W. Rondeau, S. A. Fahmy, H. Lahlou, and L. A. DaSilva, "Experiences from the Iris testbed in dynamic spectrum access and cognitive radio experimentation," in Proceedings of the Fourth IEEE International Symposium on New Frontiers in Dynamic Spectrum Access Networks (DySPAN 2010), Apr. 2010, pp. 1-8.

[103] S. Seidel and R. Breinig, "Autonomous dynamic spectrum access system behavior and performance," in Proceedings of the First IEEE International Symposium on New Frontiers in Dynamic Spectrum Access Networks (DySPAN 2005), Nov. 2005, pp. $180-183$.

[104] H. Harada, "Software defined radio prototype toward cognitive radio communication systems," in Proceedings of the First IEEE International Symposium on New Frontiers in Dynamic Spectrum Access Networks (DySPAN 2005), Nov. 2005, pp. 539-547.

[105] Y. Yuan, P. Bahl, R. Chandra, P. A. Chou, J. I. Ferrell, T. Moscibroda, S. Narlanka, and Y. Wu, "KNOWS: cognitive radio networks over white spaces," in Proceedings of the 2nd IEEE International Symposium on New Frontiers in Dynamic Spectrum Access Networks (DySPAN 2007), Apr. 2007, pp. 416-427.

[106] M. McHenry, K. Steadman, A. E. Leu, and E. Melick, "XG DSA radio system," in Proceedings of the Third IEEE International Symposium on New Frontiers in Dynamic Spectrum Access Networks (DySPAN 2008), Oct. 2008, pp. 1-11.

[107] R. Ahuja, R. Corke, and A. Bok, "Cognitive radio system using IEEE 802.11a over UHF TVWS," in Proceedings of the Third IEEE International Symposium on New Frontiers in Dynamic Spectrum Access Networks (DySPAN 2008), Oct. 2008, pp. 19.

[108] P. Amini, E. Azarnasab, S. Akoum, and B. Farhang-Boroujeny, "An experimental cognitive radio for first responders," in Proceedings of the Third IEEE International 
Symposium on New Frontiers in Dynamic Spectrum Access Networks (DySPAN 2008), Oct. 2008, pp. 1-6.

[109] H. Harada, "A feasibility study on software defined cognitive radio equipment," in Proceedings of the Third IEEE International Symposium on New Frontiers in Dynamic Spectrum Access Networks (DySPAN 2008), Oct. 2008, pp. 1-12.

[110] R. J. DeGroot, D. P. Gurney, K. Hutchinson, M. L. Johnson, S. Kuffner, A. Schooler, S. D. Silk, and E. Visotsky, "A cognitive-enabled experimental system," in Proceedings of the First IEEE International Symposium on New Frontiers in Dynamic Spectrum Access Networks (DySPAN 2005), Nov. 2005, pp. 556-561.

[111] F. W. Seelig, "A description of the august 2006 XG demonstrations at Fort A.P. Hill," in Proceedings of the 2nd IEEE International Symposium on New Frontiers in Dynamic Spectrum Access Networks (DySPAN 2007), Apr. 2007, pp. 1-12.

[112] M. McHenry, E. Livsics, T. Nguyen, and N. Majumdar, "XG dynamic spectrum access field test results," IEEE Communications Magazine, vol. 45, no. 6, pp. 51-57, Jun. 2007.

[113] A. D. Spaulding and G. H. Hagn, "On the definition and estimation of spectrum occupancy," IEEE Transactions on Electromagnetic Compatibility, vol. EMC-19, no. 3, pp. 269-280, Aug. 1977.

[114] P. J. Laycock, M. Morrell, G. F. Gott, and A. R. Ray, "A model for HF spectral occupancy," in Proceedings of the Fourth International Conference on HF Radio Systems and Techniques, 1988, pp. 165-171.

[115] G. F. Gott, C. R. Poole, P. J. Laycock, S. K. Chan, and A. R. Ray, "Spectral occupancy - measurement system and mathematical models," in Proceedings of the Fifth International Conference on HF Radio Systems and Techniques, 1991, pp. 332-336.

[116] A. J. Gibson and L. Arnett, "Measurements and statistical modelling of spectrum occupancy," in Proceedings of the Sixth International Conference on HF Radio Systems and Techniques, 1994, pp. 150-154.

[117] — , "Statistical modelling of spectrum occupancy," Electronics Letters, vol. 29, no. 25, pp. 2175-2176, Dec. 1993.

[118] C. A. Pantjiaros, P. J. Laycock, G. F. Gott, and S. K. Chan, "Development of the Laycock-Gott occupancy model," IEE Proceedings-Communications, vol. 144, no. 1, pp. 33-39, Feb. 1997.

[119] D. J. Percival, M. Kraetzl, and M. S. Britton, "A model for HF spectral occupancy in Central Australia," in Proceedings of the IEEE Military Communications Conference (MILCOM 1997), 1997, pp. 346-350. 
[120] —-, "A Markov model for HF spectral occupancy in Central Australia," in Proceedings of the Seventh International Conference on HF Radio Systems and Techniques, 1997, pp. 14-18.

[121] L. V. Economou, H. Haralambous, C. A. Pantjairos, P. R. Green, G. F. Gott, P. J. Laycock, M. Broms, and S. Boberg, "Models of HF spectral occupancy over a sunspot cycle," IEE Proceedings-Communications, vol. 152, no. 6, pp. 980-988, Dec. 2005.

[122] R. J. Matheson, "A radio spectrum measurement system for frequency management data," IEEE Transactions on Electromagnetic Compatibility, vol. EMC-19, no. 3, pp. 225-230, Aug. 1977.

[123] F. H. Sanders and V. S. Lawrence, "Broadband spectrum survey at Denver, Colorado," National Telecommunications and Information Administration (NTIA), Tech. Rep. 95-321, Sep. 1995.

[124] F. H. Sanders, B. J. Ramsey, and V. S. Lawrence, "Broadband spectrum survey at San Diego, California," National Telecommunications and Information Administration (NTIA), Tech. Rep. 97-334, Dec. 1996.

[125] — - "Broadband spectrum survey at Los Angeles, California," National Telecommunications and Information Administration (NTIA), Tech. Rep. 97-336, May 1997.

[126] F. H. Sanders, "Broadband spectrum surveys in Denver, CO, San Diego, CA, and Los Angeles, CA: methodology, analysis, and comparative results," in Proceedings of IEEE International Symposium on Electromagnetic Compatibility (EMC 1998), vol. 2, Aug. 1998, pp. 988-993.

[127] M. A. McHenry and K. Steadman, "Spectrum occupancy measurements, location 1 of 6: Riverbend Park, Great Falls, Virginia, April 7, 2004," Shared Spectrum Company, Tech. Rep., Aug. 2005, available at: http://www.sharedspectrum.com.

[128] —-, "Spectrum occupancy measurements, location 2 of 6: Tyson's Square Center, Vienna, Virginia, April 9, 2004," Shared Spectrum Company, Tech. Rep., Aug. 2005, available at: http://www.sharedspectrum.com.

[129] M. A. McHenry and S. Chunduri, "Spectrum occupancy measurements, location 3 of 6: National Science Foundation building roof, April 16, 2004, revision 2," Shared Spectrum Company, Tech. Rep., Aug. 2005, available at: http://www.sharedspectrum. com.

[130] M. A. McHenry, D. McCloskey, and G. Lane-Roberts, "Spectrum occupancy measurements, location 4 of 6: Republican National Convention, New York City, New York, August 30, 2004 - September 3, 2004, revision 2," Shared Spectrum Company, Tech. Rep., Aug. 2005, available at: http://www.sharedspectrum.com. 
[131] M. A. McHenry and K. Steadman, "Spectrum occupancy measurements, location 5 of 6: National Radio Astronomy Observatory (NRAO), Green Bank, West Virginia, October 10 - 11, 2004, revision 3," Shared Spectrum Company, Tech. Rep., Aug. 2005, available at: http://www.sharedspectrum.com.

[132] M. A. McHenry, D. McCloskey, and J. Bates, "Spectrum occupancy measurements, location 6 of 6: Shared Spectrum building roof, Vienna, Virginia, December 15-16, 2004," Shared Spectrum Company, Tech. Rep., Aug. 2005, available at: http://www. sharedspectrum.com.

[133] M. A. McHenry, P. A. Tenhula, D. McCloskey, D. A. Roberson, and C. S. Hood, "Chicago spectrum occupancy measurements \& analysis and a long-term studies proposal," in Proceedings of the First International Workshop on Technology and Policy for Accessing Spectrum (TAPAS 2006), Aug. 2006, pp. 1-12.

[134] M. A. McHenry and D. McCloskey, "Spectrum occupancy measurements: Chicago, Illinois, November 16-18, 2005," Shared Spectrum Company, Tech. Rep., Dec. 2005, available at: http://www.sharedspectrum.com.

[135] T. Erpek, K. Steadman, and D. Jones, "Spectrum occupancy measurements: Dublin, Ireland, April 16-18, 2007," Shared Spectrum Company, Tech. Rep., Nov. 2007, available at: http://www.sharedspectrum.com.

[136] T. Erpek, M. Lofquist, and K. Patton, "Spectrum occupancy measurements: Loring Commerce Centre, Limestone, Maine, September 18-20, 2007," Shared Spectrum Company, Tech. Rep., Nov. 2007, available at: http://www.sharedspectrum.com.

[137] A. Petrin and P. G. Steffes, "Analysis and comparison of spectrum measurements performed in urban and rural areas to determine the total amount of spectrum usage," in Proceedings of the International Symposium on Advanced Radio Technologies (ISART 2005), Mar. 2005, pp. 9-12.

[138] A. Petrin, "Maximizing the utility of radio spectrum: broadband spectrum measurements and occupancy model for use by cognitive radio," Ph.D. dissertation, School of Electrical and Computer Engineering, Georgia Institute of Technology, Aug. 2005.

[139] M. Wellens, J. Wu, and P. Mähönen, "Evaluation of spectrum occupancy in indoor and outdoor scenario in the context of cognitive radio," in Proceedings of the Second International Conference on Cognitive Radio Oriented Wireless Networks and Communications (CrowCom 2007), Aug. 2007, pp. 1-8.

[140] R. I. C. Chiang, G. B. Rowe, and K. W. Sowerby, "A quantitative analysis of spectral occupancy measurements for cognitive radio," in Proceedings of the IEEE 65th Vehicular Technology Conference (VTC 2007 Spring), Apr. 2007, pp. 3016-3020. 
[141] M. H. Islam, C. L. Koh, S. W. Oh, X. Qing, Y. Y. Lai, C. Wang, Y.-C. Liang, B. E. Toh, F. Chin, G. L. Tan, and W. Toh, "Spectrum survey in Singapore: occupancy measurements and analyses," in Proceedings of the 3rd International Conference on Cognitive Radio Oriented Wireless Networks and Communications (CrownCom 2008), May 2008, pp. 1-7.

[142] P. G. Steffes and A. J. Petrin, "Study of spectrum usage and potential interference to passive remote sensing activities in the $4.5 \mathrm{~cm}$ and $21 \mathrm{~cm}$ bands," in Proceedings of the IEEE International Geoscience and Remote Sensing Symposium (IGARSS 2004), vol. 3, Sep. 2004, pp. 1679-1682.

[143] J. Do, D. M. Akos, and P. K. Enge, "L and S bands spectrum survey in the San Francisco bay area," in Proceedings of the Position Location and Navigation Symposium (PLANS 2004), Apr. 2004, pp. 566-572.

[144] M. Biggs, A. Henley, and T. Clarkson, "Occupancy analysis of the $2.4 \mathrm{GHz}$ ISM band," IEE Proceedings on Communications, vol. 151, no. 5, pp. 481-488, Oct. 2004.

[145] S. W. Ellingson, "Spectral occupancy at VHF: implications for frequency-agile cognitive radios," in Proceedings of the IEEE 62nd Vehicular Technology Conference (VTC 2005 Fall), vol. 2, Sep. 2005, pp. 1379-1382.

[146] S. D. Jones, E. Jung, X. Liu, N. Merheb, and I.-J. Wang, "Characterization of spectrum activities in the U.S. public safety band for opportunistic spectrum access," in Proceedings of the 2nd IEEE International Symposium on New Frontiers in Dynamic Spectrum Access Networks (DySPAN 2007), Apr. 2007, pp. 137-146.

[147] R. J. Matheson, "Strategies for spectrum usage measurements," in Proceedings of the IEEE International Symposium on Electromagnetic Compatibility (EMC 1988), Aug. 1988, pp. 235-241.

[148] W. F. Egan, Practical RF system design. Wiley-IEEE Press, 2003.

[149] Agilent, Application note 150: spectrum analysis basics, available at: http://www. agilent.com.

[150] R. B. Bacchus, A. J. Fertner, C. S. Hood, and D. A. Roberson, "Long-term, wideband spectral monitoring in support of dynamic spectrum access networks at the IIT spectrum observatory," in Proceedings of the 3rd IEEE Symposium on New Frontiers in Dynamic Spectrum Access Networks (DySPAN 2008), Oct. 2008, pp. 1-10.

[151] J. Ma, G. Y. Li, and B. H. Juang, "Signal processing in cognitive radio," Proceedings of the IEEE, vol. 97, no. 5, pp. 805-823, May 2009.

[152] Y. Zeng, Y.-C. Liang, A. T. Hoang, and R. Zhang, "A review on spectrum sensing for cognitive radio: challenges and solutions," EURASIP Journal on Wireless Communications and Networking, vol. 2010, pp. 1-15, 2010. 
[153] T. Yücek and H. Arslan, "A survey of spectrum sensing algorithms for cognitive radio applications," IEEE Communications Surveys and Tutorials, vol. 11, no. 1, pp. 116130, First Quarter 2009.

[154] D. D. Ariananda, M. K. Lakshmanan, and H. Nikookar, "A survey on spectrum sensing techniques for cognitive radio," in Proceedings of the Second International Workshop on Cognitive Radio and Advanced Spectrum Management (CogART 2009), May 2009, pp. 74-79.

[155] D. Noguet et al., "Sensing techniques for cognitive radio - state of the art and trends," Oct. 2009, IEEE SCC 41 P1900.6 White paper, available at http://grouper.ieee.org/ groups/scc41/6/documents/white_papers/P1900.6_WhitePaper_Sensing_final.pdf.

[156] H. Urkowitz, "Energy detection of unknown deterministic signals," Proceedings of the IEEE, vol. 55, no. 4, pp. 523-531, Apr. 1967.

[157] M. A. McHenry, K. Steadman, and M. Lofquist, "Determination of detection thresholds to allow safe operation of television band "white space" devices," in Proceedings of the Third IEEE International Symposium on New Frontiers in Dynamic Spectrum Access Networks (DySPAN 2008), Oct. 2008, pp. 1-12.

[158] Radiocommunications Bureau, Handbook on spectrum monitoring, International Telecommunication Union (ITU), 2002.

[159] N. Otsu, "A threshold selection method from gray-level histograms," IEEE Transactions on Systems, Man, and Cybernetics, vol. 9, no. 1, pp. 62-66, Jan. 1979.

[160] D. Datla, A. M. Wyglinski, and G. J. Minden, "A spectrum surveying framework for dynamic spectrum access networks," IEEE Transactions on Vehicular Technology, vol. 58, no. 8, pp. 4158-4168, Oct. 2009.

[161] K. A. Qaraqe, H. Celebi, A. Gorcin, A. El-Saigh, H. Arslan, and M.-S. Alouini, "Empirical results for wideband multidimensional spectrum usage," in Proceedings of the IEEE 20th International Symposium on Personal, Indoor and Mobile Radio Communications (PIMRC 2009), Sep. 2009, pp. 1262-1266.

[162] A. Martian, I. Marcu, and I. Marghescu, "Spectrum occupancy in an urban environment: a cognitive radio approach," in Proceedings of the Sixth Advanced International Conference on Telecommunications (AICT 2010), May 2010, pp. 25-29.

[163] R. Schiphorst and C. H. Slump, "Evaluation of spectrum occupancy in Amsterdam using mobile monitoring vehicles," in Proceedings of the IEEE 71st Vehicular Technology Conference (VTC 2010 Spring), May 2010, pp. 1-5.

[164] V. Valenta, R. Maršálek, G. Baudoin, M. Villegas, M. Suarez, and F. Robert, "Survey on spectrum utilization in Europe: measurements, analyses and observations," in Proceedings of the Fifth International Conference on Cognitive Radio Oriented Wireless Networks \& Communications (CROWNCOM 2010), Jun. 2010, pp. 1-5. 
[165] S. Pagadarai and A. M. Wyglinski, "A quantitative assessment of wireless spectrum measurements for dynamic spectrum access," in Proceedings of the 4th International Conference on Cognitive Radio Oriented Wireless Networks and Communications (CrownCom 2009), Jun. 2009, pp. 1-5.

[166] Spanish Ministry of Industry, Tourism and Commerce, State Agency for Telecommunications and Information Society (Secretaría de Estado de Telecomunicaciones y para la Sociedad de la Información), "Table of National Frequency Allocations (Cuadro Nacional de Atribución de Frecuencias)," Nov. 2007.

[167] O. Holland, P. Cordier, M. Muck, L. Mazet, C. Klöck, and T. Renk, "Spectrum power measurements in $2 \mathrm{G}$ and $3 \mathrm{G}$ cellular phone bands during the 2006 Football World Cup in Germany," in Proceedings of the 2nd IEEE International Symposium on New Frontiers in Dynamic Spectrum Access Networks (DySPAN 2007), Apr. 2007, pp. $575-578$.

[168] T. Renk, C. Kloeck, F. K. Jondral, P. Cordier, O. Holland, and F. Negredo, "Spectrum measurements supporting reconfiguration in heterogeneous networks," in Proceedings of the 16th IST Mobile and Wireless Communications Summit (IST Mobile Summit 2007), Jul. 2007, pp. 1-5.

[169] R. de Francisco and A. Pandharipande, "Spectrum occupancy in the 2.36-2.4 GHz band: measurements and analysis," in Proceedings of the 16th European Wireless Conference (EW 2010), Jun. 2010, pp. 231-237.

[170] M. Matinmikko, M. Mustonen, M. Höyhtyä, T. Rauma, H. Sarvanko, and A. Mämmelä, "Distributed and directional spectrum occupancy measurements in the $2.4 \mathrm{GHz}$ ISM band," in Proceedings of the 7th International Symposium on Wireless Communication Systems (ISWCS 2010), Sep. 2010, pp. 976-980.

[171] J. Palicot, C. Moy, and R. Hachemani, "Multilayer sensors for the sensorial radio bubble," Physical Communication, Special Issue on Cognitive Radio Networks: algorithms and System Design, vol. 2, no. 1-2, pp. 151-165, Mar. 2009.

[172] E. N. Skomal, Man-made radio noise. Van Nostrand Reinhold, 1978.

[173] R. J. Matheson, "Measurements of electromagnetic noise radiated from automotive ignition systems," National Telecommunications and Information Administration (NTIA), Tech. Rep. 80-54, Nov. 1980.

[174] A. D. Spaulding and R. T. Disney, "Man-made radio noise, part 1: estimates for business, residential, and rural areas," Office of Telecommunications (OT), Tech. Rep. 74-38, Jun. 1974.

[175] R. J. Achatz and R. A. Dalke, "Man-made noise power measurements at VHF and UHF frequencies," National Telecommunications and Information Administration (NTIA), Tech. Rep. 02-390, Dec. 2001. 
[176] R. Dalke, R. Achatz, Y. Lo, P. Papazian, and G. Hufford, "Measurement and analysis of man-made noise in VHF and UHF bands," in Proceedings of Wireless Communications Conference, Aug. 1997, pp. 229-233.

[177] P. Constantinou, D. Apostolakis, and M. Katsikis, "Man made noise measurements," in Proceedings of the 41st IEEE Vehicular Technology Conference (VTC 1991), May 1991, pp. 475-476.

[178] K. N. Steadman, A. D. Rose, and T. T. N. Nguyen, "Dynamic spectrum sharing detectors," in Proceedings of the 2nd IEEE International Symposium on New Frontiers in Dynamic Spectrum Access Networks (DySPAN 2007), Apr. 2007, pp. 276-282.

[179] A. Ghasemi and E. S. Sousa, "Spectrum sensing in cognitive radio networks: requirements, challenges and design trade-offs," IEEE Communications Magazine, vol. 46, no. 4, pp. 32-39, Apr. 2008.

[180] R. Price and N. Abramson, "Detection theory," IEEE Transactions on Information Theory, vol. 7, no. 3, pp. 135-139, Jul. 1961.

[181] J. G. Proakis, Digital communications, 5th ed. McGraw-Hill, 2008.

[182] W. A. Gardner, "Signal interception: a unifying theoretical framework for feature detection," IEEE Transactions on Communications, vol. 36, no. 8, pp. 897-906, Aug. 1988.

[183] W. A. Gardner and C. M. Spooner, "Signal interception: performance advantages of cyclic-feature detectors," IEEE Transactions on Communications, vol. 40, no. 1, pp. 149-159, Jan. 1992.

[184] M. Naraghi-Pour and T. Ikuma, "Autocorrelation-based spectrum sensing for cognitive radios," IEEE Transactions on Vehicular Technology, vol. 59, no. 2, pp. 718-733, Feb. 2010.

[185] Y. Zeng and Y.-C. Liang, "Spectrum-sensing algorithms for cognitive radio based on statistical covariances," IEEE Transactions on Vehicular Technology, vol. 58, no. 4, pp. 1804-1815, May 2009.

[186] — "Maximum-minimum eigenvalue detection for cognitive radio," in Proceedings of the IEEE 18th International Symposium on Personal, Indoor and Mobile Radio Communications (PIMRC 2007), Sep. 2007, pp. 1-5.

[187] A. Kortun, T. Ratnarajah, M. Sellathurai, and C. Zhong, "On the performance of eigenvalue-based spectrum sensing for cognitive radio," in Proceedings of the Fourth IEEE International Symposium on New Frontiers in Dynamic Spectrum Access Networks (DySPAN 2010), Apr. 2010, pp. 1-6.

[188] A. Kortun, T. Ratnarajah, M. Sellathurai, C. Zhong, and C. B. Papadias, "On the performance of eigenvalue-based cooperative spectrum sensing for cognitive radio," 
IEEE Journal of Selected Topics in Signal Processing, vol. 5, no. 1, pp. 49-55, Feb. 2011.

[189] C. Liu, Y. Zeng, and S. Attallah, "Max-to-mean ratio detection for cognitive radio," in Proceedings of the IEEE Vehicular Technology Conference (VTC 2008 Spring), May 2008, pp. 1959-1963.

[190] D. J. Thomson, "Spectrum estimation and harmonic analysis," Proceedings of the IEEE, vol. 70, no. 9, pp. 1055-1096, Sep. 1982.

[191] B. Farhang-Boroujeny, "Filter bank spectrum sensing for cognitive radios," IEEE Transactions on Signal Processing, vol. 56, no. 5, pp. 1801-1811, May 2008.

[192] D. Cabric, A. Tkachenko, and R. W. Brodersen, "Experimental study of spectrum sensing based on energy detection and network cooperation," in Proceedings of the First International Workshop on Technology and Policy for Accessing Spectrum (TAPAS 2006), Aug. 2006, pp. 1-8.

[193] S. W. Oh, A. A. S. Naveen, Y. Zeng, V. P. Kumar, T. P. C. Le, K. J. M. Kua, and W. Zhang, "White-space sensing device for detecting vacant channels in TV bands," in Proceedings of the 3rd International Conference on Cognitive Radio Oriented Wireless Networks and Communications (CrownCom 2008), May 2008, pp. 1-6.

[194] V. Considine, "Digital complex sampling," Electronics Letters, vol. 19, no. 16, pp. 608-609, Aug. 1983.

[195] T. E. Thiel and G. J. Saulnier, "Simplified complex digital sampling demodulator," Electronics Letters, vol. 26, no. 7, pp. 419-421, Mar. 1990.

[196] E. Hermanowicz, "Practical digital complex sampling scheme," Electronics Letters, vol. 27, no. 5, pp. 460-462, Feb. 1991.

[197] F. F. Digham, M.-S. Alouini, and M. K. Simon, "On the energy detection of unknown signals over fading channels," IEEE Transactions on Communications, vol. 55, no. 1, pp. 21-24, Jan. 2007.

[198] I. S. Gradshteyn and I. M. Ryzhik, Table of integrals, series, and products, 6th ed. Academic, 2000.

[199] A. H. Nuttall, "Some integrals involving the $Q_{M}$-function," IEEE Transactions on Information Theory, vol. 21, no. 1, pp. 95-96, Jan. 1975.

[200] S. Shellhammer and G. Chouinard, "Spectrum sensing requirements summary," Jul. 2006, IEEE 802.22-06/0089r5.

[201] M. Abramowitz and I. A. Stegun, Handbook of mathematical functions with formulas, graphs, and mathematical tables, 10th ed. New York: Dover, 1972. 
[202] J. J. Lehtomaki, M. Juntti, H. Saarnisaari, and S. Koivu, "Threshold setting strategies for a quantized total power radiometer," IEEE Signal Processing Letters, vol. 12, no. 11, pp. 796-799, Nov. 2005.

[203] S. M. Kay, Fundamentals of statistical signal processing: detection theory. Prentice Hall, 1998, vol. 2.

[204] H. V. Poor, An introduction to signal detection and estimation, 2nd ed. Springer, 1998, vol. 2.

[205] R. Tandra and A. Sahai, "SNR walls for signal detection," IEEE Journal of Selected Topics in Signal Processing, vol. 2, no. 1, pp. 4-17, Feb. 2008.

[206] — "SNR walls for feature detectors," in Proceedings of the 2nd IEEE International Symposium on New Frontiers in Dynamic Spectrum Access Networks (DySPAN 2007), Apr. 2007, pp. 559-570.

[207] A. Sahai, N. Hoven, and R. Tandra, "Some fundamental limits on cognitive radio," in Proceedings of the Forty-second Allerton Conference on Communications, Control, and Computing (Allerton Conference 2004), Sep. 2004, pp. 1-10.

[208] D. Cabric, A. Tkachenko, and R. W. Brodersen, "Spectrum sensing measurements of pilot, energy, and collaborative detection," in Proceedings of the IEEE Military Communications Conference (MILCOM 2006), Oct. 2006, pp. 1-7.

[209] ETSI EN 300 392-2, v2.5.2, “Terrestrial Trunked Radio; Voice plus Data; Part 2: Air Interface," Nov. 2005.

[210] A. Papoulis and S. U. Pillai, Probability, random variables, and stochastic processes, 4th ed. Boston: McGraw-Hill, 2002.

[211] D. Blumenfeld, Operations research calculations handbook. CRC Press, 2001.

[212] Y.-C. Liang, Y. Zeng, E. C. Y. Peh, and A. T. Hoang, "Sensing-throughput tradeoff for cognitive radio networks," IEEE Transactions on Wireless Communications, vol. 7, no. 4, pp. 1326-1337, Apr. 2008.

[213] H. Nan, T.-I. Hyon, and S.-J. Yoo, "Distributed coordinated spectrum sharing MAC protocol for cognitive radio," in Proceedings of the 2nd IEEE International Symposium on New Frontiers in Dynamic Spectrum Access Networks (DySPAN 2007), Apr. 2007, pp. 240-249.

[214] S. Huang, X. Liu, and Z. Ding, "On optimal sensing and transmission strategies for dynamic spectrum access," in Proceedings of the 3rd IEEE International Symposium on New Frontiers in Dynamic Spectrum Access Networks (DySPAN 2008), Oct. 2008, pp. $1-5$. 
[215] L. Yang, L. Cao, and H. Zheng, "Proactive channel access in dynamic spectrum networks," in Proceedings of the 2nd International Conference on Cognitive Radio Oriented Wireless Networks and Communications (CrownCom 2007), Aug. 2007, pp. 487-491.

[216] M. Hamid, A. Mohammed, and Z. Yang, "On spectrum sharing and dynamic spectrum allocation: MAC layer spectrum sensing in cognitive radio networks," in Proceedings of the 2nd International Conference on Communications and Mobile Computing (CMC 2010), Apr. 2010, pp. 183-187.

[217] C. Guo, T. Peng, Y. Qi, and W. Wang, "Adaptive channel searching scheme for cooperative spectrum sensing in cognitive radio networks," in Proceedings of the IEEE Wireless Communications and Networking Conference (WCNC 2009), Apr. 2009, pp. $1-6$.

[218] D. Datla, R. Rajbanshi, A. M. Wyglinski, and G. J. Minden, "Parametric adaptive spectrum sensing framework for dynamic spectrum access networks," in Proceedings of the 2nd IEEE International Symposium on New Frontiers in Dynamic Spectrum Access Networks (DySPAN 2007), Apr. 2007, pp. 482-485.

[219] Y. Pei, A. T. Hoang, and Y.-C. Liang, "Sensing-throughput tradeoff in cognitive radio networks: How frequently should spectrum sensing be carried out?" in Proceedings of the IEEE 18th International Symposium on Personal, Indoor and Mobile Radio Communications (PIMRC 2007), Sep. 2007, pp. 1-5.

[220] Y. Xu, J. Wang, and Q. Wu, "Interference-throughput tradeoff in dynamic spectrum access: Analysis based on discrete-time queuing subjected to bursty preemption," in Proceedings of the 4th International Conference on Cognitive Radio Oriented Wireless Networks and Communications (CrownCom 2009), Jun. 2009, pp. 1-6.

[221] Z. Liang and D. Zhao, "Quality of service performance of a cognitive radio sensor network," in Proceedings of the IEEE International Conference on Communications (ICC 2010), May 2010, pp. 1-5.

[222] S. Geirhofer, L. Tong, and B. M. Sadler, "A measurement-based model for dynamic spectrum access in WLAN channels," in Proceedings of the IEEE Military Communications Conference (MILCOM 2006), Oct. 2006, pp. 1-7.

[223] — - "Dynamic spectrum access in WLAN channels: Empirical model and its stochastic analysis," in Proceedings of the First International Workshop on Technology and Policy for Accessing Spectrum (TAPAS 2006), Aug. 2006, pp. 1-10.

[224] _ _ "Dynamic spectrum access in the time domain: Modeling and exploiting white space," IEEE Communications Magazine, vol. 45, no. 5, pp. 66-72, May 2007.

[225] L. Stabellini, "Quantifying and modeling spectrum opportunities in a real wireless environment," in Proceedings of the IEEE Wireless Communications and Networking Conference (WCNC 2010), Apr. 2010, pp. 1-6. 
[226] M. Wellens, J. Riihijärvi, and P. Mähönen, "Empirical time and frequency domain models of spectrum use," Physical Communication, vol. 2, no. 1-2, pp. 10-32, Mar. 2009.

[227] H. Kim and K. G. Shin, "Efficient discovery of spectrum opportunities with MAClayer sensing in cognitive radio networks," IEEE Transactions on Mobile Computing, vol. 7, no. 5, pp. 533-545, May 2008.

[228] P. K. Tang and Y. H. Chew, "Modeling periodic sensing errors for opportunistic spectrum access," in Proceedings of the IEEE 72nd Vehicular Technology Conference (VTC 2010 Fall), Sep. 2010, pp. 1-5.

[229] A. Motamedi and A. Bahai, "MAC protocol design for spectrum-agile wireless networks: Stochastic control approach," in Proceedings of the 2nd IEEE International Symposium on New Frontiers in Dynamic Spectrum Access Networks (DySPAN 2007), Apr. 2007, pp. 448-451.

[230] Q. Zhao, L. Tong, A. Swami, and Y. Chen, "Decentralized cognitive MAC for opportunistic spectrum access in ad hoc networks: A POMDP framework," IEEE Journal on Selected Areas in Communications, vol. 25, no. 3, pp. 589-600, Apr. 2007.

[231] P. N. Anggraeni, N. H. Mahmood, J. Berthod, N. Chaussonniere, L. My, and H. Yomo, "Dynamic channel selection for cognitive radios with heterogenous primary bands," Wireless Personal Communications, vol. 45, no. 3, pp. 369-384, May 2008.

[232] R. Urgaonkar and M. J. Neely, "Opportunistic scheduling with reliability guarantees in cognitive radio networks," IEEE Transactions on Mobile Computing, vol. 8, no. 6, pp. 766-777, Jun. 2009.

[233] M. Sharma, A. Sahoo, and K. D. Nayak, "Channel selection under interference temperature model in multi-hop cognitive mesh networks," in Proceedings of the 2nd IEEE International Symposium on New Frontiers in Dynamic Spectrum Access Networks (DySPAN 2007), Apr. 2007, pp. 133-136.

[234] P. J. Kolodzy, "Interference temperature: a metric for dynamic spectrum utilization," International Journal of Network Management, vol. 16, no. 2, pp. 103-113, Mar. 2006.

[235] H. Lee and D.-H. Cho, "VoIP capacity analysis in cognitive radio system," IEEE Communications Letters, vol. 13, no. 6, pp. 393-395, Jun. 2009.

[236] — "Capacity improvement and analysis of VoIP service in a cognitive radio system," IEEE Transactions on Vehicular Technology, vol. 59, no. 4, pp. 1646-1651, May 2010.

[237] P. Wang, D. Niyato, and H. Jiang, "Voice-service capacity analysis for cognitive radio networks," IEEE Transactions on Vehicular Technology, vol. 59, no. 4, pp. 17791790, May 2010. 
[238] O. C. Ibe, Markov processes for stochastic modeling. Academic Press, 2009.

[239] Z. Wang and S. Salous, "Spectrum occupancy statistics and time series models for cognitive radio," Journal of Signal Processing Systems, vol. 62, no. 2, pp. 145-155, Feb. 2011.

[240] D. Chen, S. Yin, Q. Zhang, M. Liu, and S. Li, "Mining spectrum usage data: a largescale spectrum measurement study," in Proceedings of the 15th ACM Annual International Conference on Mobile Computing and Networking (MobiCom 2009), Sep. 2009, pp. 13-24.

[241] D. Willkomm, S. Machiraju, J. Bolot, and A. Wolisz, "Primary users in cellular networks: A large-scale measurement study," in Proceedings of the 3rd IEEE International Symposium on New Frontiers in Dynamic Spectrum Access Networks (DySPAN 2008), Oct. 2008, pp. 1-11.

[242] L. M. Correia, D. Zeller, O. Blume, D. Ferling, Y. Jading, I. Gódor, G. Auer, and L. van der Perre, "Challenges and enabling technologies for energy aware mobile radio networks," IEEE Communications Magazine, vol. 48, no. 11, pp. 66-72, Nov. 2010.

[243] V. Blaschke, H. Jaekel, T. Renk, C. Kloeck, and F. K. Jondral, "Occupation measurements supporting dynamic spectrum allocation for cognitive radio design," in Proceedings of the 2nd International Conference on Cognitive Radio Oriented Wireless Networks and Communications (CrownCom 2007), Aug. 2007, pp. 50-57.

[244] P. Kumaraswamy, "A generalized probability density function for double-bounded random processes," Journal of Hydrology, vol. 46, no. 1-2, pp. 79-88, Mar. 1980.

[245] M. C. Jones, "Kumaraswamy's distribution: A beta-type distribution with some tractability advantages," Statistical Methodology, vol. 6, no. 1, pp. 70-81, Jan. 2009.

[246] W. H. Press, S. A. Teukolsky, W. T. Vetterling, and B. P. Flannery, Numerical recipes: The art of scientific computing, 3rd ed. Cambridge University Press, 2007.

[247] R. D. Gupta and D. Kundu, "Generalized exponential distributions," Australian and New Zealand Journal of Statistics, vol. 41, no. 2, pp. 173-188, Jun. 1999.

[248] J. F. Lawless, Statistical models and methods for lifetime data. Wiley, 1982.

[249] W. Q. Meeker and L. A. Escobar, Statistical methods for reliability data. Wiley, 1988.

[250] M. J. Crowder, A. C. Kimber, R. L. Smith, and T. J. Sweeting, Statistical analysis of reliability data. Chapman and Hall, 1991.

[251] D. C. Montgomery and G. C. Runger, Applied statistics and probability for engineers, 3rd ed. John Wiley \& Sons, 2003. 
[252] T. Öztekin, "Comparison of parameter estimation methods for the three-parameter generalized Pareto distribution," Turkish Journal of Agriculture and Forestry, vol. 29, no. 6, pp. 419-428, Dec. 2005.

[253] P. Embrechts, C. Klüppelberg, and T. Mikosch, Modelling extremal events for insurance and finance. Springer, 1997.

[254] S. Kotz and S. Nadarajah, Extreme value distributions: Theory and applications. World Scientific Publishing Company, 2001.

[255] N. G. Cadigan and R. A. Myers, "A comparison of gamma and lognormal maximum likelihood estimators in a sequential population analysis," Canadian Journal of Fisheries and Aquatic Sciences, vol. 58, no. 3, pp. 560-567, Mar. 2001.

[256] M. Evans, N. Hastings, and B. Peacock, Statistical distributions, 2nd ed. Wiley, 1993.

[257] A. Bhattacharyya, "On a measure of divergence between two statistical populations defined by their probability distributions," Bulletin of the Calcutta Mathematical Society, vol. 35, pp. 99-109, 1943.

[258] ETSI ETS 300 175-2, "Radio Equipment and Systems (RES); Digital European Cordless Telecommunications (DECT); Common interface; Part 2: Physical layer," Oct. 1992.

[259] M. Wellens, J. Riihijärvi, and P. Mähönen, "Modelling primary system activity in dynamic spectrum access networks by aggregated ON/OFF-processes," in Proc. Fourth IEEE Workshop on Networking Technologies for Software Defined Radio Networks (SDR 2009), Jun. 2009, pp. 1-6.

[260] L. Devroye, Non-uniform random variate generation. Springer-Verlag, 1986.

[261] G. N. Tavares and A. Petrolino, "On the generation of correlated Gaussian random variates by inverse DFT," IEEE Transactions on Communications, vol. 59, no. 1, pp. 45-51, Jan. 2011.

[262] H. Hotelling and M. R. Pabst, "Rank correlation and tests of significance involving no assumption of normality," Annals of Mathematical Statistics, vol. 7, no. 1, pp. 29-43, Mar. 1936.

[263] P. K. Tang, Y. H. Chew, and L. C. Ong, "On the time distribution of white space access opportunities," in Proceedings of the IEEE Wireless Communications and Networking Conference (WCNC 2008), Apr. 2008, pp. 729-734.

[264] _ - "On the distribution of opportunity time for the secondary usage of spectrum," IEEE Transactions on Vehicular Technology, vol. 58, no. 3, pp. 1517-1527, Mar. 2009. 
[265] P. K. Tang and Y. H. Chew, "Study of non-Markovian distributed primary radio activities on the opportunity time for secondary usage of spectrum," in Proceedings of the IEEE Military Communications Conference (MILCOM 2009), Oct. 2009, pp. 1-7.

[266] A. Pereira, J. Bastos, P. Marques, and A. Gameiro, "Analysis of communication opportunities in UMTS cellular networks," in Proceedings of the 2nd IEEE International Symposium on New Frontiers in Dynamic Spectrum Access Networks (DySPAN 2007), Apr. 2007, pp. 99-102.

[267] X. Hong, C.-X. Wang, H.-H. Chen, and Y. Zhang, "Secondary spectrum access networks: Recent developments on the spatial models," IEEE Vehicular Technology Magazine, vol. 4, no. 2, pp. 36-43, Jun. 2009.

[268] N. Hoven and A. Sahai, "Power scaling for cognitive radio," in Proceedings of the International Conference on Wireless Networks, Communications and Mobile Computing (WNCMC), Jun. 2005, pp. 250-255.

[269] M. Timmers, S. Pollin, A. Dejonghe, A. Bahai, L. van der Perre, and F. Catthoor, "Accumulative interference modeling for cognitive radios with distributed channel access," in Proceedings of the 3rd International Conference on Cognitive Radio Oriented Wireless Networks and Communications (CrownCom 2008), May 2008, pp. $1-7$.

[270] —- "Accumulative interference modeling for distributed cognitive radio networks," Journal of Communications, vol. 4, no. 3, pp. 175-185, Apr. 2009.

[271] R. Menon, R. M. Buehrer, and J. Reed, "Outage probability based comparison of underlay and overlay spectrum sharing techniques," in Proceedings of the First IEEE International Symposium on New Frontiers in Dynamic Spectrum Access Networks (DySPAN 2005), Nov. 2005, p. $101 U ̋ 109$.

[272] T. Kamakaris, D. Kivanc-Tureli, and U. Tureli, "Interference model for cognitive coexistence in cellular systems," in Proceedings of the IEEE Global Telecommunications Conference (GLOBECOM 2007), Nov. 2007, pp. 4175-4179.

[273] R. S. Dhillon and T. X. Brown, "Models for analyzing cognitive radio interference to wireless microphones in TV bands," in Proceedings of the 3rd IEEE Symposium on New Frontiers in Dynamic Spectrum Access Networks (DySPAN 2008), Oct. 2008, pp. $1-10$.

[274] X. Hong, C.-X. Wang, and J. S. Thompson, "Interference modeling of cognitive radio networks," in Proceedings of the IEEE Vehicular Technology Conference (VTC 2008 Spring), May 2008, p. 1851 Ü1855.

[275] R. Menon, R. Buehrer, and J. Reed, "On the impact of dynamic spectrum sharing techniques on legacy radio systems," IEEE Transactions on Wireless Communications, vol. 7, no. 11, pp. 4198-4207, Nov. 2008. 
[276] Z. Chen, C.-X. Wang, X. Hong, J. Thompson, S. Vorobyov, and X. Ge, "Interference modeling for cognitive radio networks with power or contention control," in Proceedings of the IEEE Wireless Communications and Networking Conference (WCNC 2010), Apr. 2010, pp. 1-6.

[277] T. Kamakaris, M. M. Buddhikot, and R. Iyer, "A case for coordinated dynamic spectrum access in cellular networks," in Proceedings of the First IEEE International Symposium on New Frontiers in Dynamic Spectrum Access Networks (DySPAN 2005), Nov. 2005, pp. 289-298.

[278] J. Riihijärvi, P. Mähönen, M. Wellens, and M. Gordziel, "Characterization and modelling of spectrum for dynamic spectrum access with spatial statistics and random fields," in Proceedings of 1st International Workshop on Cognitive Radios and Networks (CRNETS 2008), Sep. 2008, pp. 1-6.

[279] M. Wellens, J. Riihijärvi, M. Gordziel, and P. Mähönen, "Spatial statistics of spectrum usage: From measurements to spectrum models," in Proceedings of the IEEE International Conference on Communications (ICC 2009), Jun. 2009, pp. 1-6.

[280] M. Wellens, J. Riihijärvi, and P. Mähönen, "Spatial statistics and models of spectrum use," Computer Communications, vol. 32, no. 18, pp. 1998-2011, Dec. 2009.

[281] Y. Okumura, E. Ohmori, T. Kawano, and K. Fukuda, "Field strength and its variability in VHF and UHF land-mobile radio service," Review of the Electrical Communications Laboratory, vol. 16, no. 9-10, pp. 825-873, Sep. 1968.

[282] M. Hata, "Empirical formula for propagation loss in land mobile radio services," IEEE Transactions on Vehicular Technology, vol. VT-29, no. 3, pp. 317-325, Aug. 1980.

[283] D. J. Cichon and T. Kürner, "Propagation prediction models (Digital mobile radio towards future generation systems, chapter 4)," COST231, Tech. Rep., Nov. 1998.

[284] J. Pons-Puig, "Application of link adaptation to evolved TDMA mobile communications systems," Ph.D. dissertation, University of Strathclyde, 1999.

[285] M. López-Benítez, M. C. Lucas-Estañ, and J. Gozálvez, "A dynamic radio simulation platform for the study of radio resource management techniques in heterogeneous wireless systems," in Proceedings of the 9-th ACM/IEEE International Symposium on Modeling, Analysis and Simulation of Wireless and Mobile Systems (MSWiM 2006), Oct. 2006, pp. 143-171.

[286] J. Pérez-Romero, O. Sallent, and R. Agustí, "On the applicability of image processing techniques in the radio environment characterisation," in Proceedings of the IEEE 69th Vehicular Technology Conference (VTC 2009 Spring), Apr. 2009, pp. 1-5. 
[287] L. Bolea, J. Pérez-Romero, R. Agustí, and O. Sallent, "Primary transmitter discovery based on image processing in cognitive radio," in Proceedings of the 15th Eunice International Workshop (EUNICE 2009), Lecture Notes in Computer Science, vol. 5733, Sep. 2009, pp. 178-187.

[288] Programming manual for MS272XB series spectrum analyzers, Anritsu, Jan. 2007.

[289] Garmin Device Interface Specification, Garmin, May 2006.

[290] S. A. Dyer and J. S. Dyer, "Approximations to error functions," IEEE Instrumentation \& Measurement Magazine, vol. 10, no. 6, pp. 45-48, Dec. 2007.

[291] W. J. Cody, "Rational Chebyshev approximations for the error function," Mathematics of Computation, vol. 23, no. 107, pp. 631-637, Jul. 1969.

[292] N. C. Beaulieu, "A simple series for personal computer computation of the error function $Q(\cdot)$, , IEEE Transactions on Communications, vol. 37, no. 9, pp. 989-991, Sep. 1989.

[293] C. Tellambura and A. Annamalai, "Efficient computation of erfc $(x)$ for large arguments," IEEE Transactions on Communications, vol. 48, no. 4, pp. 529-532, Apr. 2000.

[294] G. T. F. de Abreu, "Jensen-Cotes upper and lower bounds on the Gaussian $Q$-function and related functions," IEEE Transactions on Communications, vol. 57, no. 11, pp. 3328-3338, Nov. 2009.

[295] P. O. Börjesson and C.-E. W. Sundberg, "Simple approximations of the error function $Q(x)$ for communications applications," IEEE Transactions on Communications, vol. COM-27, no. 3, pp. 639-643, Mar. 1979.

[296] M. Chiani, D. Dardari, and M. K. Simon, "New exponential bounds and approximations for the computation of error probability in fading channels," IEEE Transactions on Wireless Communications, vol. 2, no. 4, pp. 840-845, Jul. 2003.

[297] P. Loskot and N. C. Beaulieu, "Prony and polynomial approximations for evaluation of the average probability of error over slow-fading channels," IEEE Transactions on Vehicular Technology, vol. 58, no. 3, pp. 1269-1280, Mar. 2009.

[298] G. K. Karagiannidis and A. S. Lioumpas, "An improved approximation for the Gaussian $Q$-function," IEEE Communications Letters, vol. 11, no. 8, pp. 644-646, Aug. 2007.

[299] Y. Isukapalli and B. D. Rao, "An analytically tractable approximation for the Gaussian $Q$-function," IEEE Communications Letters, vol. 12, no. 9, pp. 669-671, Sep. 2008.

[300] Y. Chen and N. C. Beaulieu, "A simple polynomial approximation to the Gaussian $Q$-function and its application," IEEE Communications Letters, vol. 13, no. 2, pp. 124-126, Feb. 2009. 




\section{SHORT BIOGRAPHICAL SKETCH}

Miguel López-Benítez received a B.Sc. degree (2003) and a M.Sc. degree (2006) in Telecommunications Engineering, both with First-Class Honors, from Universidad Miguel Hernández (UMH), Elche, Spain. He was the recipient of the 2003 and 2006 University Education National Awards, a distinction from the Spanish Ministry of Education and Science to the best national academic records, as well as some other distinctions from the Spanish professional association of telecommunications engineers. His M.Sc. thesis was awarded a national research prize from the France Telecom foundation (today Orange foundation) in the context of the 5th Archimedes University Competition, the main young scientist contest in Spain organized by the Spanish Ministry of Education and Science (more than 100 works were presented). From February 2003 to July 2006 he collaborated in several Spanish national and regional research projects in what today is the Ubiquitous Wireless Communications Research (Uwicore) laboratory at UMH. In September 2006 he joined the Mobile Communication Research Group (GRCM) at the Department of Signal Theory and Communications (TSC) of the Universitat Politècnica de Catalunya (UPC), Barcelona, Spain, where he has pursued a Ph.D. degree in Telecommunications Engineering and collaborated as a teaching assistant with the support of a FPU grant from the Spanish Ministry of Education and Science. He has been actively involved in European-funded projects AROMA, NEWCOM++ and FARAMIR along with Spanish projects COGNOS and ARCO. His research interests include the field of mobile radio communication systems, with a special emphasis on radio resource management, heterogeneous wireless systems, quality of service provisioning, spectrum modeling and opportunistic/dynamic spectrum access in cognitive radio networks. For more details, please visit http://www. lopezbenitez.es. 